\title{
Österreichischer Forschungs- und Technologiebericht
}

2013

Lagebericht gem. § 8 (1) FOG über die aus Bundesmitteln geförderte Forschung, Technologie und Innovation in Österreich 
Der vorliegende Bericht ist im Auftrag der Bundesministerien für Wissenschaft und Forschung (BMWF), Verkehr, Innovation und Technologie (BMVIT) und Wirtschaft, Familie und Jugend (BMWFJ) entstanden. Die Erstellung des Berichts erfolgte durch JOANNEUM RESEARCH (JR), dem Austrian Institute of Technology (AIT), dem Zentrum für Europäische Wirtschaftsforschung (ZEW) sowie unter Beteiligung der Statistik Austria.

AutorInnenteam: Andreas Schibany (Koordination, JR), Martin Berger (JR), Bernhard Dachs (AIT), Brigitte Ecker (JR), Jürgen Egeln (ZEW), Helmut Gassler (JR), Sandra Gottschalk (ZEW), Karl-Heinz Leitner (AIT), Bettina Müller (ZEW), Christian Rammer (ZEW), Christian Reiner (JR), Gerhard Streicher (JR), Georg Zahradnik (AIT)

Impressum

Medieninhaber (Verleger):

Bundesministerium für Wissenschaft und Forschung gemeinsam mit Bundesministerium für Verkehr, Innovation und Technologie sowie Bundesministerium für Wirtschaft, Familie und Jugend

1010 Wien

Alle Rechte vorbehalten

Auszugsweiser Nachdruck nur mit Quellenangabe gestattet

Gestaltung und Produktion:

Peter Sachartschenko \& Mag. Susanne Spreitzer OEG, Wien

Umschlagfotos: @ DrHitch - Fotolia.com

Druck:

Gugler GmbH, 3390 Melk/Donau

Wien, 2013 


\section{Vorwort}

Der Österreichische Forschungs- und Technologiebericht 2013 widmet sich als Lagebericht gemäß $\S 8$ (1) FOG den aktuellen nationalen und internationalen forschungs- und technologiepolitischen Herausforderungen durch Analysen aktueller Entwicklungen und Trends und die Aufbereitung umfangreichen Datenmaterials zu Forschung und Entwicklung sowie zu speziellen Schwerpunktthemen.

Wie die Statistik Austria in ihrer jüngst publizierten Globalschätzung prognostizierte, werden die gesamten Ausgaben für Forschung und Entwicklung in Österreich 2013 um 2,9 \% steigen und einen Rekordwert von voraussichtlich $€ 8,96$ Mrd. erreichen. Die Forschungsquote als Prozentanteil am Bruttoinlandsprodukt, das zuletzt wieder merklich angestiegen ist, beträgt voraussichtlich 2,81 \%. Damit liegt Österreich im internationalen Vergleich deutlich über dem EUDurchschnitt von 2,03\% und weist innerhalb der EU-27 die fünfthöchste Forschungsquote auf.

Diese erfreuliche Entwicklung spiegelt das verstärkte Engagement des Bundes der vergangenen Jahre wider: Der Bund hat seine Forschungsinvestitionen während der Wirtschaftskrise antizyklisch erhöht und 2012 seine F\&E-Investitionen gegenüber 2011 um 14,2 \% angehoben.

Genau so erfreulich ist es, dass 2013 die Investitionen in Forschung und Entwicklung von Seiten der Unternehmen wieder leicht steigen und mit einem prognostizierten dreiprozentigen Wachstum rd. $€ 3,93$ Mrd. betragen werden. Rechnet man die Auslandsfinanzierung von rd. $€ 1,3$ Mrd., die zum überwiegenden Teil von ausländischen Unternehmen an österreichische Tochterfirmen fließt, den Unternehmen hinzu, wird hier ein Anteil von rd. $59 \%$ an der gesamten F\&E-Finanzierung erreicht. Die F\&E-Ausga- ben des Bundes werden um 2,8\% auf einen neuen Höchstwert von $€ 3,09$ Mrd. steigen. Der gesamte öffentliche Sektor wird rd. $41 \%$ der F\&EAusgaben finanzieren.

Der diesjährige Forschungs- und Technologiebericht fasst zusammen, welche Schritte unter anderem im letzten Jahr gesetzt wurden, um das in der Strategie für Forschung, Technologie und Innovation (FTI-Strategie) der Bundesregierung festgelegte Ziel, bis $2020 \mathrm{zu}$ einem der innovativsten Länder der EU zu zählen, zu erreichen. Dazu gehören die im vorliegenden Bericht behandelten Themen der Schaffung neuer wichtiger Governance- und Finanzierungsinstrumente für die österreichischen Hochschulen durch den Hochschulplan, der Implementierung einer kapazitätsorientierten, auf Studierende bezogenen Universitätsfinanzierung, der Abstimmung bei der Profil- und Schwerpunktsetzung im Bereich F\&E, der Erstellung eines Forschungsinfrastrukturplans sowie der Restrukturierungsprozesse der Österreichischen Akademie der Wissenschaften. Ein weiteres Thema, das national und international immer bedeutender wird, ist das Publizieren wissenschaftlicher Ergebnisse im Internet (Open access). Es wird im Bericht umfassend präsentiert, die Vor- und Nachteile werden analysiert sowie unterschiedliche Strategien im Umgang mit Open Access vorgestellt.

Ein weiteres wichtiges Schwerpunktthema im aktuellen Bericht ist die Rolle der Industrie im Innovationssystem. Die weltweite Wirtschaftsund Finanzkrise hat $z u$ einer Neubeurteilung wirtschaftspolitischer Optionen und des Strukturwandels geführt, und der Beitrag der Industrie für Innovationen, Exporte und Beschäftigung steht erneut im Zentrum wirtschaftspolitischer 
Diskussionen. Zahlreiche Länder, aber auch die Europäische Kommission verfolgen eine Strategie der Reindustrialisierung. Österreich gehört im internationalen Vergleich zur Gruppe jener Länder mit den höchsten Industrieanteilen gemessen an der gesamten Wertschöpfung. Österreich ist bei den von der Europäischen Union definierten Schlüsseltechnologien gut positioniert. Dies gilt insbesondere auch für das rasch wachsende Segment der Umweltindustrie.

Insgesamt zeigt das Innovationsverhalten der österreichischen Unternehmen, auch der Kleinund Mittelbetriebe, ein erfreuliches Bild. Österreich verfügt über ein horizontal ausgerichtetes Fördersystem, das bewirkt, dass die Unterschiede zwischen KMU und Großunternehmen im Innovationsverhalten geringer sind als in den meisten anderen hoch entwickelten europäischen Industrieländern. Der Anteil innovierender Unterneh-

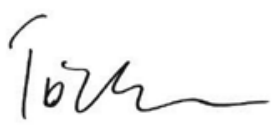

o. Univ.-Prof. Dr. Karlheinz Töchterle Bundesminister für Wissenschaft und Forschung men liegt in Österreich deutlich über dem Schnitt der EU-27, wobei die Innovatorenquote in allen Branchen hoch ist.

Durch den diesjährigen Forschungs- und Technologiebericht wird deutlich, dass wir konsequent an der Umsetzung der FTI-Strategie arbeiten. Bis 2020 bedarf es aber noch weiterer außerordentlicher Anstrengungen und gezielter Investitionen, um Österreich an die Forschungsspitze der EU zu führen. Evaluierungen, Jahresberichte und statistische Erhebungen helfen uns bei der Standortbestimmung in diesem aufstrebenden Prozess. Sie runden gemeinsam mit Berichten der einschlägigen Förderagenturen FWF, FFG, AWS, der CDG sowie von PROVISO zu EU-Förderungen das Bild über die aktuellen Entwicklungen von Forschung, Technologie und Innovation in Österreich $2013 \mathrm{ab}$.

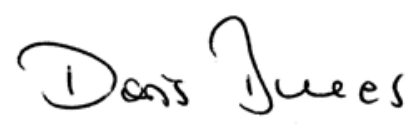

Doris Bures

Bundesministerin für

Verkehr, Innovation und Technologie 


\section{Inhalt}

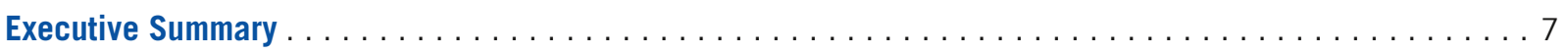

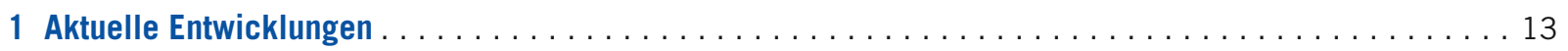

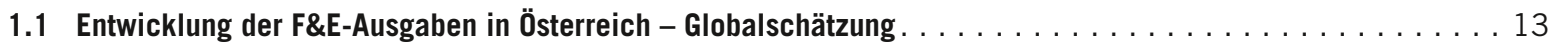

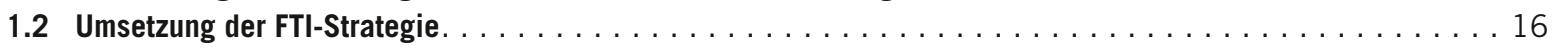

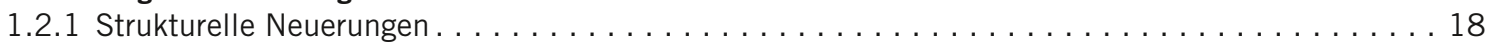

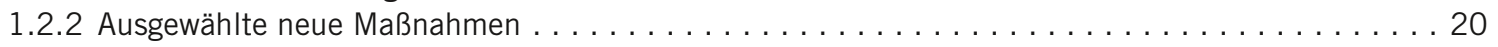

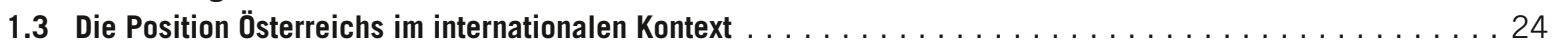

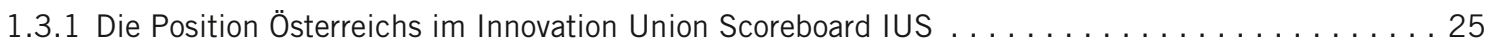

1.3.2 „Innovationsindikator" der Deutschen Telekom Stiftung und des

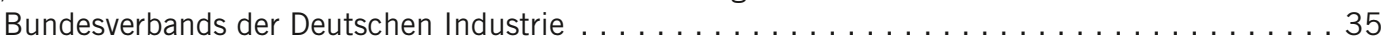

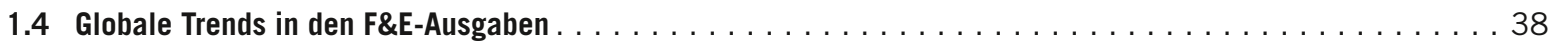

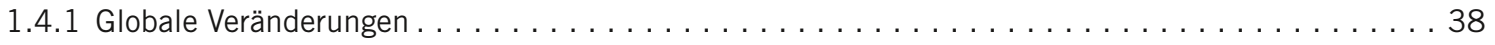

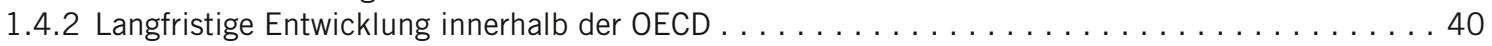

1.4.3 Verschiebungen der Ausgaben für F\&E innerhalb der Europäischen Union . . . . . . . . . . . 41

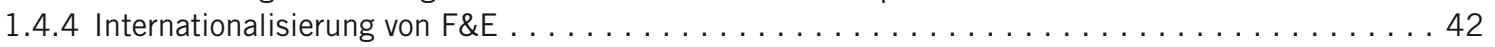

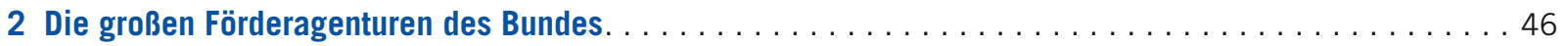

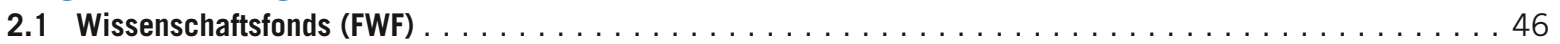

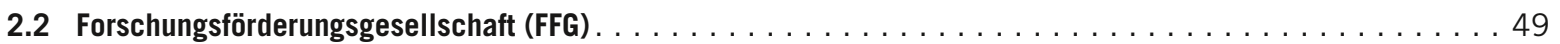

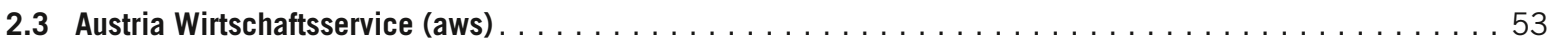

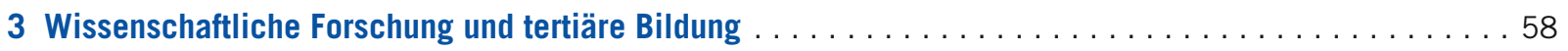

3.1 Hochschulplan und Hochschulkonferenz als neue Governance-Instrumente

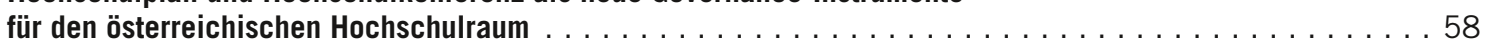

3.2 Die Finanzierung der Universitäten in den Jahren 2013 bis $2015 \ldots \ldots \ldots \ldots \ldots \ldots \ldots \ldots \ldots \ldots$

3.2.1 Abschluss der Leistungsvereinbarungen 2013 bis $2015 \ldots \ldots \ldots \ldots \ldots \ldots \ldots \ldots \ldots \ldots \ldots$

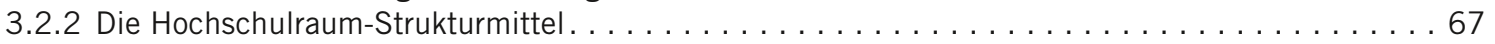

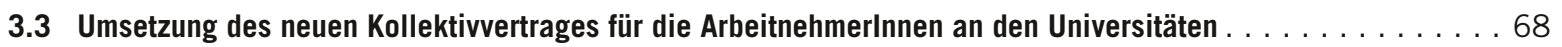

3.4 Externe Qualitätssicherung und Agentur für Qualitätssicherung und Akkreditierung Austria $\ldots \ldots \ldots \ldots \ldots 2$

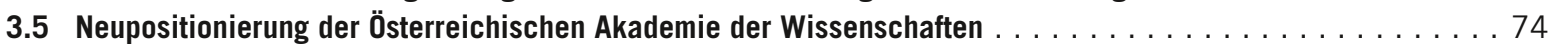

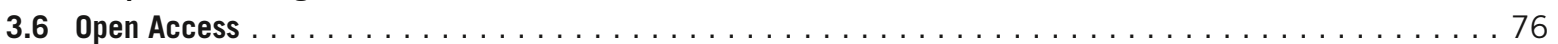

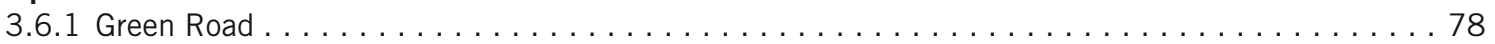

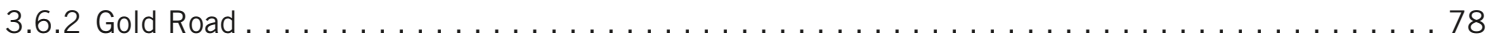

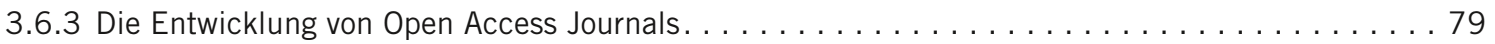

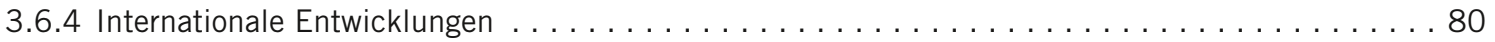

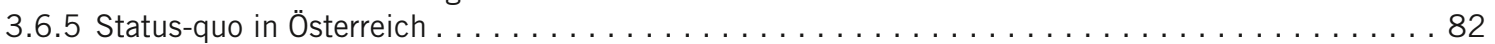

3.6.6 Maßnahmen der österreichischen Universitäten. . . . . . . . . . . . . . . . . . . . . 84

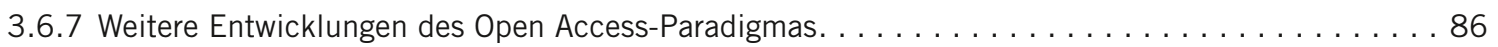

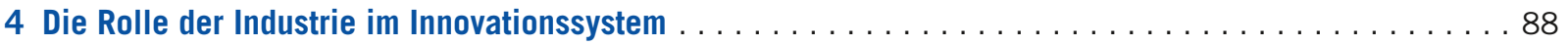

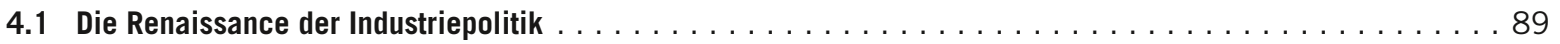

4.2 Globale Verschiebungen von industriellen Produktionskapazitäten $\ldots \ldots \ldots \ldots \ldots \ldots \ldots \ldots \ldots \ldots 1$

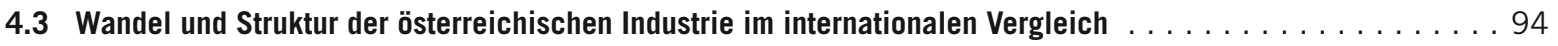

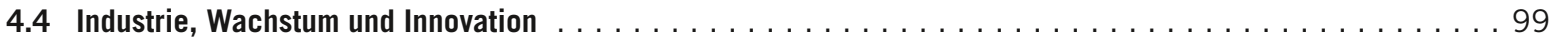

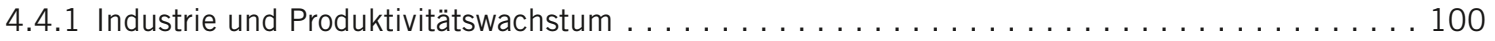

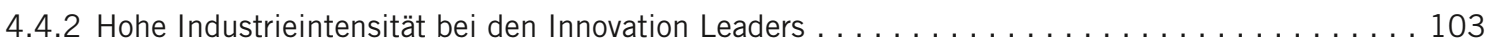

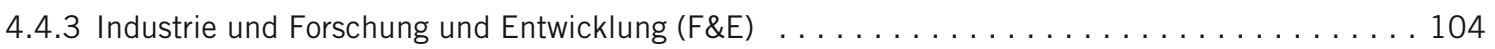

4.4.4 Innovationen und Innovationskooperationen im Industrie- und Dienstleistungssektor . . . . . 106 


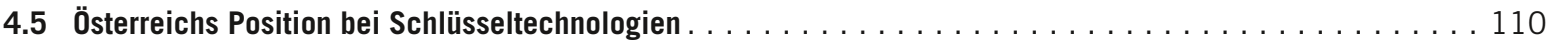

4.5.1 Bedeutung von Schlüsseltechnologien für industrielle Innovationen . . . . . . . . . . . 111

4.5.2 Position Österreichs bei Patentanmeldungen im Bereich von Schlüsseltechnologien . . . . . . . . 112

4.5.3 Position Österreichs im Außenhandel mit schlüsseltechnologiebasierten Produkten . . . . . . . 121

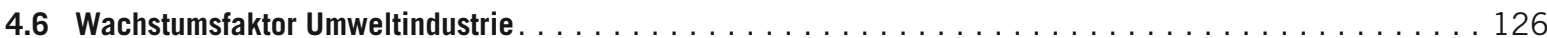

4.6.1 Marktentwicklung und Marktpotenzial für Umweltschutzgüter. . . . . . . . . . . . . . . . . 126

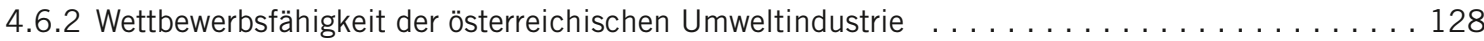

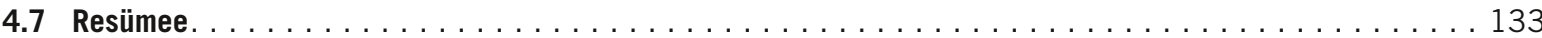

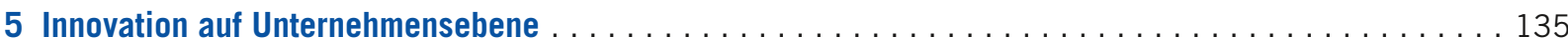

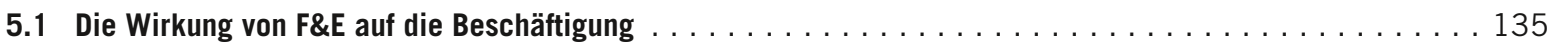

5.1.1 F\&E-Intensität und Beschäftigungswachstum vor und nach der Krise . . . . . . . . . . . . 136

5.1.2 F\&E-Intensität und Beschäftigungswachstum nach Unternehmensgröße . . . . . . . . . . . 137

5.1.3 F\&E-Intensität und Beschäftigungswachstum nach Unternehmensalter . . . . . . . . . . . . . . 139

5.1 .4 F\&E-Intensität und Exportquote nach Unternehmensgröße . . . . . . . . . . . . . . . . . . . 140

5.2 Innovationsaktivitäten im Unternehmenssektor und die Rolle von KMU . . . . . . . . . . . . . . . . 141

5.2.1 Die Innovationsperformance im europäischen Vergleich . . . . . . . . . . . . . . . . . . . . . 141

5.2 .2 Branchenspezifische Ergebnisse für Österreich . . . . . . . . . . . . . . . . . . . . . . . . 147

5.2 .3 Innovationsstrategien der kleinen und mittleren Unternehmen . . . . . . . . . . . . . . . . . . . 149

5.2 .4 Besonderheiten des Innovationsverhaltens von KMU in Österreich. . . . . . . . . . . . . . . . 155

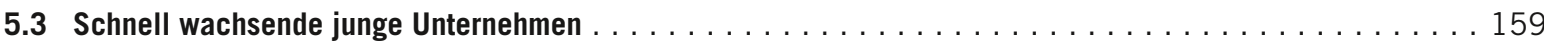

5.3.1 Beschäftigungsentwicklung junger Unternehmen in Österreich . . . . . . . . . . . . . . 161

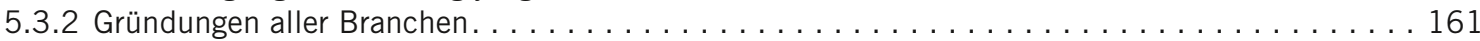

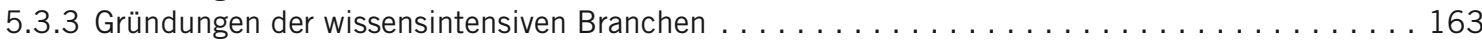

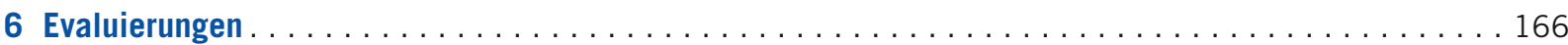

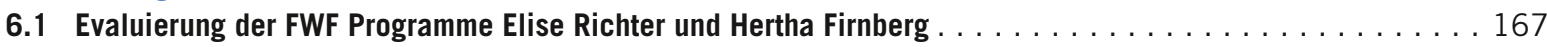

6.2 Zwischenevaluierung der Dienstleistungsinitiative $\ldots \ldots \ldots \ldots \ldots \ldots$

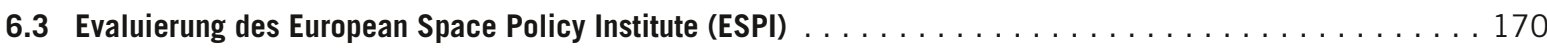

6.4 Evaluierung der Strategieprogramme IV2S und IV2Splus $\ldots \ldots \ldots \ldots \ldots \ldots \ldots$

6.5 Interimsevaluation des österreichischen Sicherheitsforschungsprogramms KIRAS $\ldots \ldots \ldots \ldots$

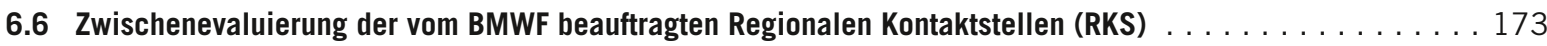

7 Literatur . . . . . . . . . . . . . . . . . . . . . . . . . . . . . . . 176

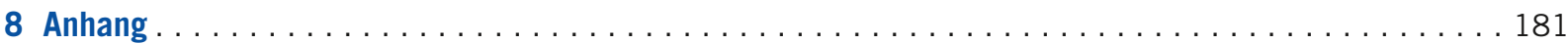

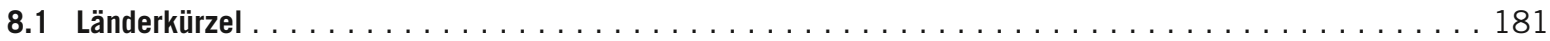

8.2 Forschungsschwerpunkte an Österreichs Universitäten in der Periode der

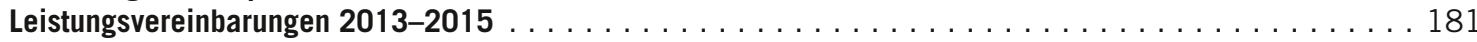

8.3 Liste der Einzelindikatoren des „Innovationsindikators“ der Deutschen Telekom Stiftung und des BDI . . . . 183

8.4 Klassifikation von potenziellen Umweltschutzgütern $\ldots \ldots \ldots \ldots \ldots$

8.5 Abgrenzung von Branchen nach Technologieintensität gemäß OECD-Klassifikation auf Ebene der

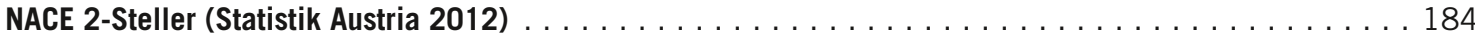

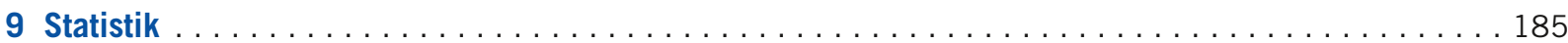

Tabellenübersicht $\ldots \ldots \ldots \ldots \ldots \ldots \ldots \ldots \ldots \ldots$ 


\section{Executive Summary}

Der Forschungs- und Technologiebericht 2013 ist ein Lagebericht über die aus Bundesmitteln geförderte Forschung, Technologie und Innovation in Österreich. Auf Basis aktueller Daten und Befunde werden relevante Entwicklungstrends und ausgewählte Themen des österreichischen Innovationssystems beschrieben und vor einem internationalen Kontext reflektiert. Der vorliegende Bericht ist im Auftrag der Bundesministerien für Wissenschaft und Forschung (BMWF), Verkehr, Innovation und Technologie (BMVIT) und Wirtschaft, Familie und Jugend (BMWFJ) erarbeitet worden. Sämtliche Beiträge wurden in interministeriellen Arbeitsgruppen, in denen die genannten Ressorts eingebunden waren, diskutiert und akkordiert.

\section{Globalschätzung der F\&E-Ausgaben für 2013}

Gemäß der aktuellen Globalschätzung der Statistik Austria vom April 2013 werden die gesamten Ausgaben für Forschung und Entwicklung in Österreich im Jahr 2013 voraussichtlich 8,96 Mrd. $€$ betragen. Gegenüber dem Jahr 2012 bedeutet dies eine Zunahme von nominell ca. 255 Mio. $€$ bzw. 2,9 \%. Durch das für $2013 \mathrm{zu}$ erwartende nominelle Wachstum des BIP von $3 \%$ auf 319,15 Mrd. $€^{1}$ entspricht dies einer Forschungsquote (Bruttoinlandsausgaben für Forschung und Entwicklung im Verhältnis zum Bruttoinlandsprodukt) von $\mathbf{2 , 8 1} \%$. Für 2012 wurde die Forschungsquote auf ebenfalls 2,81\% (von 2,80 \% in der Globalschätzung 2012) revidiert, 2011 betrug sie aufgrund des stärkeren Anstiegs des Bruttoinlandsprodukts 2,72\%. Seit dem Beginn der Finanz- und Wirtschaftskrise konnten die F\&E-Ausgaben gesteigert und die Forschungsquote auf konstanter Höhe gehalten werden.

Mit einem Plus von $3 \%$ verzeichnet im Jahr 2013 der Finanzierungssektor Unternehmen die höchste Wachstumsrate. Dieser Sektor finanziert ein Volumen von 3,93 Mrd. $€$ und trägt damit knapp $44 \%$ zur Finanzierung der österreichischen Forschung und Entwicklung bei. Somit weist der Unternehmenssektor den höchsten Finanzierungsanteil auf. Zwar sind die Wachstumsraten der Forschungsfinanzierung des Unternehmenssektors geringer als in den Jahren vor der Finanz- und Wirtschaftskrise, die Stagnation in den unmittelbaren Krisenjahren 2009 und 2010 ist jedoch überwunden und die Forschungsfinanzierung durch den Unternehmenssektor befindet sich wieder auf einem Wachstumspfad, der jenem des (nominellen) BIP-Wachstums entspricht.

Der Bund finanziert mit 3,09 Mrd. $€$ im Jahr 2013 ca. 34 \% der gesamten Ausgaben für Forschung und Entwicklung in Österreich. In absoluten Zahlen bedeutet dies gegenüber 2012 einen Anstieg von etwa 83 Mio. $€$ bzw. 2,8 \%. Dieser Anstieg ist vor dem Hintergrund zu sehen, dass der Bund (nicht zuletzt durch die Erhöhung der Forschungsprämie von $8 \%$ auf $10 \%$ ) im Jahr 2012 eine Zunahme seiner F\&E-Finanzierung von $14,2 \%$ bzw. plus 374 Mio. $€$ in absoluten Zahlen verzeichnete. Insgesamt hat der öffentliche Sektor (wobei der Bund hier den überwältigenden Anteil trägt) seine F\&E-Finanzierung seit der Krise stark ausgeweitet. Die F\&E-Finan-

1 Siehe WIFO-Konjunkturprognose März 2013. 
zierung des öffentlichen Sektors wird im Jahr 2013 nominell um ca. 36 \% höher sein als noch im Rezessionsjahr 2009.

Der Finanzierungssektor Ausland (hauptsächlich ausländische Unternehmen, die F\&E ihrer österreichischen Tochterunternehmen mitfinanzieren sowie zu einem kleineren Teil auch Rückflüsse aus den EU-Forschungsrahmenprogrammen) trägt mit 1,36 Mrd. $€$ ca. $15 \%$ zur Finanzierung der österreichischen Forschung und Entwicklung bei.

Insgesamt übertrifft Österreich im Jahr 2011 (dem letzten Jahr, für das internationale Vergleichszahlen verfügbar sind) deutlich den EUDurchschnitt von 2,03 \%, wenn auch die Forschungsquoten von Finnland, Schweden und Dänemark über $3 \%$ liegen. Nach Deutschland, das mit 2,84 \% knapp vor Österreich liegt, weist Österreich somit die fünfthöchste Quote innerhalb der EU-27 auf.

\section{Die Umsetzung der FTI-Strategie der Österreichischen Bundesregierung}

Die im März 2011 verabschiedete FTI-Strategie der Bundesregierung verfolgt einen breiten, systemischen Ansatz zur Unterstützung und Strukturierung des Innovationsystems. Zeitgemäße FTI-Politik ist nicht ausschließlich auf Wissenschafts- und Technologieförderung konzentriert, sondern erfolgt in Abstimmung mit anderen Politikfeldern wie Bildung, Wettbewerbspolitik und korrespondierenden Regelungsmaterien wie etwa dem Steuer- und Finanzierungssystem und berücksichtigt wechselseitig Wirkungszusammenhänge.

Daher wurde zur Konkretisierung und Koordination der Umsetzung der Strategie auf hoher Verwaltungsebene unter dem Vorsitz des Bundeskanzleramtes, gemeinsam mit den relevanten Bundesministerien, die Task Force FTI eingerichtet. Ein kontinuierlicher Austausch dieses Gremiums erfolgt umfassend auch unter Einbeziehung des Rates für Forschung und Technologieentwicklung. Darüber hinaus wurden unter dem Dach der FTI-Strategie alle FTI-relevanten
Programme und Initiativen zusammengeführt und neun Arbeitsgruppen in spezifischen wichtigen Bereichen eingerichtet.

\section{Österreichs Position im Innovation Union Scoreboard}

Der Innovation Union Scoreboard (IUS) stellt eine jährliche Bewertung der Innovationsperformance der EU-Staaten dar. Österreich nimmt im aktuellen Innovation Union Scoreboard den 9. Rang ein und bleibt damit fest in der ersten Hälfte der Gruppe der „Innovation Followers“ verankert (gemeinsam mit den Niederlanden, Luxemburg, Belgien, Großbritannien und Irland auf den Plätzen 5 bis 10). Diese Gruppenzugehörigkeit ist seit einigen Jahren recht stabil, Verschiebungen der Positionen innerhalb dieser Teilgruppe, wie sie im Jahresvergleich immer wieder vorkommen, sollten nicht allzu hoch bewertet werden. Die absoluten Unterschiede bezüglich des Gesamtindikators sind innerhalb dieser Gruppe nämlich sehr gering. Die Einzelindikatoren bestätigen das von früheren Ausgaben des IUS bzw. des EIS (European Innovation Scoreboard als Vorläufer des IUS) bekannte Stärken/Schwächen-Muster Österreichs: Stärken sind bei den wissenschaftlichen Publikationen, den F\&E-Ausgaben der Unternehmen sowie den Indikatoren zum geistigen Eigentum festzustellen.

Gewisse Schwächen zeigen sich weiterhin in der tertiären Ausbildung, in der Risikokapitalausstattung, bei den Lizenz- und Patenteinnahmen sowie den wissensintensiven Dienstleistungsexporten. Bei den aus dem Community Innovation Survey (CIS) abgeleiteten Indikatoren (von den sechs aus dem CIS entnommenen Indikatoren betreffen vier das Innovationsverhalten von KMU) weist Österreich im Zeitablauf merkliche Schwankungen auf; diese sind aber nicht zuletzt durch geänderte Rahmenbedingungen bei Design und Durchführung dieser Befragung zurückzuführen. Anzumerken ist, dass der IUS auf strukturelle Aspekte des Innovationssystems abzielt. Dementsprechend weisen viele der Indikatoren eine langfristige Perspektive auf. 
Unmittelbare Reaktionen auf veränderte Politikbedingungen, in Form kurzfristiger substanzieller Verbesserungen im IUS, sind daher nur bedingt $\mathrm{zu}$ erwarten.

\section{Globalisierung und Internationalisierung von F\&E}

Die weltweiten F\&E-Ausgaben sind in den Jahren 2002 bis 2009 um mehr als die Hälfte gestiegen, 2009 betrugen die globalen F\&E-Ausgaben mehr als 1,2 Billionen US \$ (zu Kaufkraftparitäten) gegenüber knapp 800 Mrd. US \$ im Jahr 2002. Besonders die schnell wachsenden asiatischen Volkswirtschaften, insbesondere China, konnten in den letzten Jahren deutlich ihre Anteile an den globalen F\&E-Ausgaben erhöhen, während die entsprechenden Anteile der USA, Japans und der EU-27 zurückgingen. Vor diesem Hintergrund ist es bemerkenswert, dass es Österreich als einem der wenigen EU-Länder gelang, seinen Anteil an den weltweiten F\&E-Ausgaben stabil zu halten. Dank des starken Wachstums seiner F\&E-Ausgaben erhöhte sich der Anteil Österreichs an der gesamten F\&E der EU-27 sowie auch der OECD-Staaten deutlich, und zwar auf $3 \%$ gemessen an den EU-27 und 0,75\% gemessen an den OECD-Staaten.

Der Anstieg der weltweiten F\&E-Ausgaben war begleitet von einer Internationalisierung der F\&E-Aktivitäten, getrieben vor allem durch die großen multinationalen Unternehmen, die heute vielfach ein weltumspannendes Netz von Forschungsstandorten betreiben. Die Bedeutung dieser ausländischen F\&E-Investitionen ist vor allem in kleineren und mittleren EU-Staaten sehr hoch. Im Fall von Österreich tragen Unternehmen, die im ausländischen Besitz sind, bereits rund ein Drittel zu den gesamten F\&E-Ausgaben bei und sind somit ein maßgeblicher Treiber des starken Anstiegs der F\&E-Ausgaben im Zeitraum von 2002 bis 2009. ${ }^{\text {la }}$ Einen besonderen Stellenwert nimmt dabei das Nachbarland Deutschland ein. Nach den USA ist Österreich das zweitwichtigste Zielland für grenzüber- schreitende F\&E-Ausgaben deutscher Unternehmen. Der Anteil deutscher Unternehmen an allen ausländischen F\&E-Investitionen in Österreich beträgt mehr als $50 \%$.

\section{Neue Governance-Instrumente für den österreichischen Hochschulraum}

Die im Jahr 2011 eingeleitete Entwicklung des Hochschulplans ist ein wichtiges wissenschaftsund bildungspolitisches Vorhaben mit dem Ziel, durch abgestimmte Kooperation, Profilbildung und der Bündelung von Ressourcen die höchste Qualität von Lehre und Forschung sicherzustellen. Die zunehmende Ausdifferenzierung der Akteure, die Internationalisierung der Forschung, veränderte Finanzierungssysteme, Initiativen und Politiken auf europäischer Ebene und ambitionierte forschungspolitische Ziele erfordern, dass sich die Hochschulpartner und die Politik abstimmen und durch die Bündelung von Ressourcen geschärft und weiterentwickelt werden. Der Hochschulplan versteht sich als ein rollierendes weiterzuentwickelndes Planungsinstrument unter Einbeziehung wichtiger Akteure im Hochschulbereich. Die Koordination im Bereich der Profilbildung, Lehre und Forschung ist dabei eine zentrale Herausforderung. Sowohl im Bereich der Schwerpunktbildung in der Forschung, als auch bei der Planung des Fächerangebots, ist hier eine Abstimmung und ein Abgleich zwischen den Universitäten und insbesondere zwischen den Standorten notwendig. Ein prioritäres Ziel der nationalen Hochschulpolitik ist auch die Einführung der Studienplatzfinanzierung, deren Umsetzung bis zum Vollausbau in mehreren Phasen bis zum Jahr 2021 geplant ist.

Um die Realisierung der im Hochschulplan formulierten Ziele zu unterstützen und die koordinierenden Maßnahmen umzusetzen, wurde im Frühjahr 2012 die Hochschulkonferenz konstituiert. Es handelt sich dabei um ein koordinierendes beratendes Gremium, das Stellung-

1a Dieser Wert ist nicht zu verwechseln mit der Finanzierung der F\&E durch den sogenannten Auslandssektor, bei der es sich um tatsächliche Mittelzuflüsse aus dem Ausland nach Österreich handelt. 
nahmen zu wichtigen wissenschaftspolitischen Themen erarbeitet, Problembereiche priorisiert und Empfehlungen und Lösungsvorschläge als Input für den Hochschulplan einbringt.

\section{Die Finanzierung der Universitäten}

Die Finanzierung der Universitäten für die Jahre 2013 bis 2015 ist durch zwei wesentliche Merkmale geprägt: Zum einen gehen die Universitäten eine neue dreijährige Leistungsvereinbarungsperiode ein und zum anderen wird das formelgebundene Budget durch das neue Instrument der Hochschulraum-Strukturmittel abgelöst.

Die Leistungsvereinbarungen sind das zentrale Instrument für die Finanzierung und Steuerung der österreichischen öffentlichen Universitäten, mit deren Abschluss Globalbudgets über eine Periode von drei Jahren allokiert werden. Seitens des Bundes stellen der Hochschulplan, aber auch die FTI-Strategie den strategischen Rahmen zur Steuerung der Universitäten dar. Die wichtigsten Ziele im Bereich Forschung, auf welche die Universitäten mitunter auch im Rahmen ihrer Leistungsvereinbarung Bezug nehmen, sind dabei: die Fortführung der Schwerpunkt- und Profilbildung, die Intensivierung der Kooperationen, der Ausbau der Internationalisierung, der strategische Ausbau der Forschungsinfrastruktur, sowie die weitere (quantitative und qualitative) Entwicklung der Drittmitteleinwerbung. Von Seiten der Universitäten werden die in den Leistungsvereinbarungen angestrebten Ziele und Maßnahmen durch die Erstellung von Entwicklungsplänen begleitet.

Im Rahmen einer Novelle des UG wurde im Sommer 2012 das Instrument der Hochschulraum-Strukturmittel eingeführt. Damit wurde die leistungsorientierte Finanzierung in Form des Formelbudgets durch einen neuen Allokationsmechanismus abgelöst und der zur Finanzierung der Universitäten zur Verfügung stehende Gesamtbetrag um die Hochschulraum-Strukturmittel ergänzt. Ziel der neuen Regelung ist es, die bisherige komplexe indikatorenbezogene Finanzierung über das formelgebundene Budget an
Hand von wenigen Indikatoren nachvollziehbar zu berechnen. Der den Universitäten zur Verfügung stehende Gesamtbetrag setzt sich daher für die LV-Periode 2013 bis 2015 aus einem Teilbetrag für die Grundbudgets und einem Teilbetrag für die Hochschulraum-Strukturmittel zusammen. Neben dem Grundbudget, das weiterhin auf Basis von Leistungsvereinbarungen definiert wird, werden für die LV-Periode 2013 und 2015 Hochschulraum-Strukturmittel in der Höhe von 450 Mio. $€$ verteilt.

\section{Neupositionierung der Österreichischen Akademie der Wissenschaften}

Die Österreichische Akademie der Wissenschaften hat eine lange Tradition im österreichischen Wissenschaftssystem. Im Jahr 1847 gegründet, besteht sie heute aus einer Gelehrtengesellschaft, einer Forschungsträgereinrichtung sowie einer Nachwuchsförder- und Serviceeinrichtung. Seit 2000 ist insbesondere die Forschungsträgerorganisation beachtlich gewachsen. Darunter sind zahlreiche wissenschaftlich höchst erfolgreiche Forschungsinstitute, welche in Gebieten wie der Molekularbiologie, Biomedizin, Physik, angewandten Mathematik, Weltraumforschung, Materialwissenschaften sowie den Geistes-, Sozial- und Kulturwissenschaften tätig sind. Dieser enorme Ausbau des wissenschaftlich sehr erfolgreichen ÖAW-Forschungsträgers machte es auch notwendig, die Managementstrukturen der ÖAW zu modernisieren und anzupassen. Im Jahr 2011 traten daher eine neue Satzung und Geschäftsordnung in Kraft, welche die Implementierung moderner und transparenter Strukturen auf der Managementebene nach sich zogen. Auch wurde als wesentlicher Schritt erstmals in der Geschichte der ÖAW eine Leistungsvereinbarung geltend für die Periode 2012 bis 2014 abgeschlossen.

\section{Renaissance der Industriepolitik}

Die weltweite Wirtschafts- und Finanzkrise hat $z u$ einer Neubeurteilung wirtschaftspolitischer 
Optionen und des Strukturwandels geführt. Im Zentrum dieser global beobachtbaren Re-Orientierung steht insbesondere der industrielle Sektor. Ein großer Industriesektor galt lange Zeit als Zeichen eines verzögerten Strukturwandels. Dieses Urteil hat sich mittlerweile geradezu in sein Gegenteil verkehrt. Neben einer Renaissance der Industriepolitik ist die Industrie durch einen parallel ablaufenden technologischen $\mathrm{Pa}$ radigmenwandel gekennzeichnet, der auch als "Dritte industrielle Revolution" bezeichnet wird. Diese ist vor allem durch eine Konvergenz verschiedener Technologien wie etwa Materialtechnologien, Internet, 3D-Printing oder Technologien im Zusammenhang mit erneuerbaren Energien gekennzeichnet. Die Rolle der Industrie und deren Beitrag für Innovation, Exporte und Beschäftigung stehen heute erneut wieder im Zentrum wirtschaftspolitischer Debatten. So verfolgen zahlreiche Länder, aber auch die Europäische Kommission selbst, aktuell eine Strategie der Reindustrialisierung.

\section{Die Rolle der Industrie im österreichischen Innovationssystem}

Österreich gehört im internationalen Vergleich zur Gruppe der Länder mit den höchsten Industrieanteilen gemessen an der gesamten Wertschöpfung. Dennoch hat der Industrieanteil im langfristigen Vergleich abgenommen, wenngleich seit etwa 15 Jahren, ähnlich wie in Deutschland oder der Schweiz, eine Stabilisierung des Industrieanteils an der Wertschöpfung stattgefunden hat. Die Beschäftigung in der Industrie hingegen fällt sowohl nach der absoluten Zahl als auch nach dem Anteil an der Gesamtbeschäftigung. In Bezug auf den technologischen Wandel innerhalb der Industrie ergibt sich eine starke Zunahme von Industrien im mittleren Technologiesegment bei vergleichsweise geringem Anteil des Hochtechnologiesegments. Allerdings stellt sich die mitteltechnologische Industrie in Österreich als relativ F\&E-intensiv dar. Generell erlebte die österreichische Industrie seit 1990 einen äußerst erfolgreichen Pro- zess der Internationalisierung, der Ausdruck ihrer hohen Wettbewerbsfähigkeit ist und der ein weiteres Abschmelzen des industriellen Kerns verhindert hat.

Die Industrie trägt in deutlich überproportionalem Ausmaß zum technologischen Wandel einer Volkswirtschaft bei. Der Großteil der F\&Eund Innovationsaktivitäten Österreichs wird von Industrieunternehmen - freilich oftmals in enger Kooperation mit Dienstleistungsunternehmen - durchgeführt. Diese überdurchschnittliche Innovationsperformance setzt sich in dem Maße fort, als die Industrie ein Motor des Produktivitätswachstums Österreichs ist. Die Rolle der Industrie für F\&E sowie Innovation wird auch dadurch deutlich, dass die Innovation Leaders über durchwegs höhere Industrieanteile als die Innovation Followers, Moderate Innovators und Modest Innovators verfügen.

\section{Österreichs Position bei Schlüsseltechnologien}

Österreich ist sowohl hinsichtlich seiner F\&Eals auch seiner Produktionskapazitäten gut bei den Schlüsseltechnologien, wie sie jüngst von Seiten der Europäischen Union definiert wurden, positioniert. In den vergangenen zehn Jahren haben die österreichischen Industrieunternehmen ihre Patentaktivitäten im Bereich industrieller Schlüsseltechnologien deutlich ausgeweitet. Dadurch hat Österreich an Gewicht innerhalb der weltweiten Produktion neuen technologischen Wissens in diesen besonders wichtigen Technologiefeldern gewonnen. Damit ging auch eine kräftige Ausweitung der Exporte von schlüsseltechnologiebasierten Produkten Österreichs einher.

Ebenso erfreulich ist die gute Performance der österreichischen Industrie in einem besonders rasch wachsenden Segment, nämlich der Umweltindustrie. Eine empirische Analyse der Internationalisierung der österreichischen Umweltindustrie zeigt eine positive Dynamik und eine über die Zeit zunehmende Wettbewerbsfähigkeit. Hohe Wachstumserwartungen für Umweltschutzgüter auf den Weltmärkten eröffnen hier 
Optionen für eine Expansion moderner und ökologisch nachhaltiger industrieller Aktivitäten.

\section{Österreichs Industrie als Erfolgsgeschichte}

Die österreichische Industrie ist insgesamt eine Erfolgsgeschichte. Ihr Erfolg beruht wesentlich auf motivierten, leistungsbereiten und kompetenten UnternehmerInnen sowie qualifizierten Fachkräften. Hinzu kommen Rahmenbedingungen, die insbesondere durch die Sozialpartnerschaft und die europäische Integration vorteilhaft gestaltet sind. Die Ostöffnung und der europäische Binnenmarkt haben durch den steigenden Wettbewerbsdruck zu einer Dynamisierung der heimischen Industrieunternehmen beigetragen. In Zukunft wird eine weitere Verbesserung des Humankapitals von entscheidender Bedeutung sein. Schulen und Lehrlingsausbildung, aber auch die Ausbildung von NaturwissenschaftlerInnen und IngenieurInnen an Universitäten und Fachhochschulen ist besondere Aufmerksamkeit zu schenken. Die Industriepolitik, die in Österreich im Wesentlichen und richtigerweise Innovationspolitik ist, kann Impulse und Anreize für eine weitere Verbesserung der internationalen Wettbewerbsfähigkeit setzen. Dafür sind die notwendigen Instrumente und Institutionen vorhanden. Es gilt also den erfolgreichen Weg der letzten Jahre weiter zu gehen und flexibel und koordiniert auf neue Herausforderungen für die österreichische Industrie zu reagieren.

\section{Innovation als treibende Kraft für einen dauerhaften Unternehmenserfolg}

Die kontinuierliche Umsetzung von Innovationen in marktfähige Produkte und Dienstleistungen ist die treibende Kraft für einen dauerhaften Unternehmenserfolg und somit letztlich für wirtschaftliches Wachstum und Beschäftigung.
Empirische Analysen der Europäischen Innovationserhebung (CIS) zeigen, dass Österreich im europäischen Vergleich eine gute (bis sehr gute) Position einnimmt, insbesondere auch in Hinblick auf die Performance des österreichischen KMU-Sektors. Der Anteil innovierender Unternehmen liegt in Österreich deutlich über dem Schnitt der EU-27, wobei die Innovatorenquote in allen Branchen hoch ist.

Gleichzeitig sind die Innovationsausgaben durch ein hohes Gewicht von F\&E-Ausgaben gekennzeichnet. Auch eine Analyse des Zusammenhangs von F\&E-Intensität und Beschäftigungsentwicklung deutet auf die große Bedeutung von $\mathrm{F} \& \mathrm{E}$ hin. F\&E-intensive Unternehmen weisen ein deutlich höheres Beschäftigungswachstum auf. Österreich ist somit durch ein modernes Innovationssystem geprägt, dessen Unternehmen ständig neues Wissen durch eigene F\&E-Aktivitäten generieren und am Markt in Form von neuen Produkten bzw. Dienstleistungen umsetzen und platzieren. Dabei sind Österreichs Unternehmen gut mit ihren Zulieferern und KundInnen, aber auch mit den Hochschulen in Innovationsnetzwerken verbunden. Die österreichische Wirtschaftspolitik hat bereits seit langem den hohen Stellenwert von unternehmerischen Innovationen erkannt und fördert das unternehmerische Innovationsverhalten mit vielfältigen Instrumenten. Österreich hat damit ein Fördersystem mit einer großen Reichweite, d.h. Innovationen werden horizontal adressiert. Der Anteil der Unternehmen, die innovationsspezifische Förderungen erhalten, zählt zu den höchsten innerhalb der EU. Diese Breitenwirkung der Förderung trägt wesentlich dazu bei, dass die Unterschiede zwischen KMU und Großunternehmen im Innovationsverhalten in Österreich geringer sind als in den meisten anderen hoch entwickelten europäischen Industrieländern. 


\section{Aktuelle Entwicklungen}

\subsection{Entwicklung der F\&E-Ausgaben in Österreich - Globalschätzung}

Gemäß der aktuellen Globalschätzung der Statistik Austria vom April 2013 werden die gesamten Ausgaben für Forschung und Entwicklung in Österreich im Jahr 2013 voraussichtlich 8,96 Mrd. $€$ betragen. Gegenüber dem Jahr 2012 bedeutet dies eine Zunahme von ca. 255 Mio. $€$ bzw. 2,9 \%. Durch das für $2013 \mathrm{zu}$ erwartende nominelle Wachstum des BIP von $3 \%$ auf 319,15 Mrd. $€^{2}$ entspricht dies einer Forschungsquote (Bruttoinlandsausgaben für Forschung und Entwicklung im Verhältnis zum Bruttoinlandsprodukt) von $2,81 \%$. Für 2012 wird die Forschungsquote nunmehr auf ebenfalls 2,81 \% (von 2,80\% in der Globalschätzung 2012) revidiert, 2011 betrug sie aufgrund des stärkeren Anstiegs des Bruttoinlandsprodukts 2,72 \%. Die Entwicklung der Forschungsquote sowie der absoluten Beiträge der einzelnen Finanzierungssektoren ist in Abb. 1 dargestellt. Seit dem Ausbruch der Finanz- und

Abb. 1: Ausgaben für Forschung und Entwicklung in Österreich nach Finanzierungssektoren

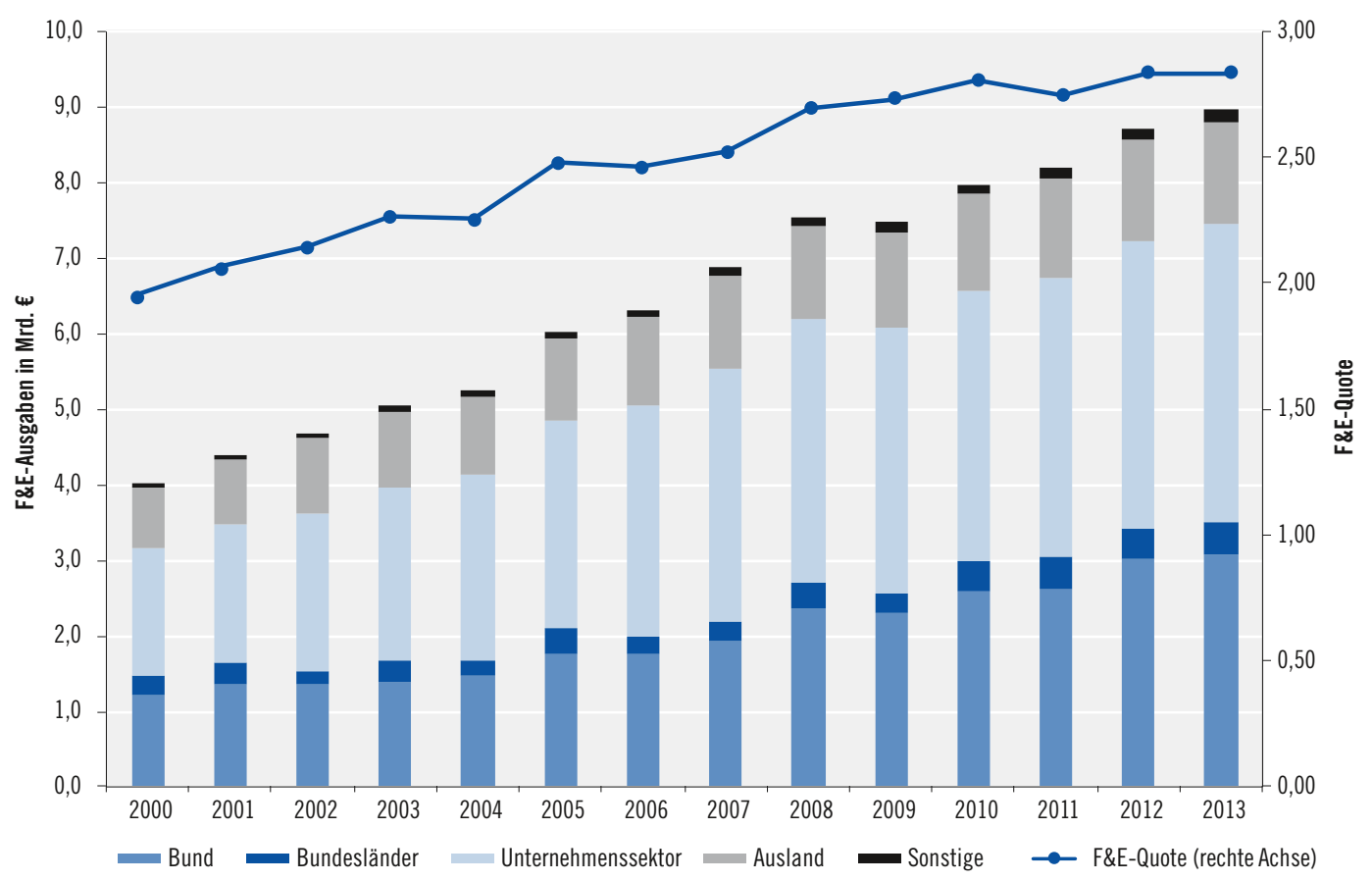

Quelle: Statistik Austria, Globalschätzung vom Stand 11. April 2013, nominelle Werte.

2 WIFO-Konjunkturprognose März 2013. 
Wirtschaftskrise konnten die F\&E-Ausgaben gesteigert und die Forschungsquote auf konstanter Höhe gehalten werden. Mit der prognostizierten Forschungsquote von 2,81 \% liegt Österreich deutlich über dem Durchschnitt der EU-27, der 2011 bei 2,03 \% lag und befindet sich damit an fünfter Stelle hinter Finnland, Schweden, Dänemark und Deutschland.

Betrachtet man die Gesamtausgaben für Forschung und Entwicklung in Österreich differenziert nach den verschiedenen Finanzierungssektoren ergibt sich folgendes Bild (vgl. Abb. 2 und Abb. 3):

- Der Bund finanziert mit 3,09 Mrd. € im Jahr 2013 voraussichtlich 34,4 \% der gesamten Ausgaben für Forschung und Entwicklung in Österreich. In absoluten Zahlen bedeutet das gegenüber 2012 einen Anstieg von ca. 83 Mio. $€$ bzw. 2,8 \%. Dieser Anstieg ist vor dem Hintergrund zu sehen, dass der Bund (nicht zuletzt durch die Erhöhung der Forschungsprämie von $8 \%$ auf $10 \%$ ) im Jahr 2012 eine Steigerung seiner F\&E-Finanzierung von 14,2 \% (bzw. plus 374 Mio. $€$ in absoluten Zahlen) verzeichnete. Insgesamt hat der öffentliche Sektor (wobei der Bund hier den überwältigenden Anteil trägt) seine F\&E-Finanzierung seit der Krise (und auch als wirtschaftspolitische Antwort darauf) enorm ausgeweitet. Die F\&E-Finanzierung des öffentlichen Sektors wird im Jahr 2013 nominell um ca. 36 \% höher sein als noch im Rezessionsjahr 2009 (siehe Abb. 2).

- Der Unternehmenssektor finanziert im Jahr 2013 voraussichtlich 3,39 Mrd. € an Forschung und Entwicklung und weist mit 43,9\% den höchsten Anteil aller Finanzierungssektoren auf. Gegenüber 2012 ist die Finanzierung um ca. 115 Mio. $€$ gestiegen, was einem prozentuellen Wachstum von $3 \%$ entspricht, also entsprechend dem geschätzten nominellen BIPWachstum. Bemerkenswert ist, dass - nachdem das nominelle Wachstum in den Jahren 2009 und 2010 als unmittelbare Folge der Krise nur jeweils unter 1,5\% blieb - die Zunahme der F\&E-Finanzierung von Seiten des Unternehmenssektors mittlerweile seit 2011 wieder auf einen Wachstumspfad von $3 \%$ und mehr zum Liegen kommt (allerdings kommen diese Wachstumsraten nicht an das Niveau der Vorkrisenzeit heran).

- Der Finanzierungssektor Ausland (dabei handelt es sich überwiegend um mit österreichischen Unternehmen verbundene ausländische Unternehmen sowie zu einem kleineren Teil auch um Rückflüsse aus den EU-Forschungsrahmenprogrammen) trägt mit 1,36 Mrd. $€$ 15,2 \% zum Gesamtvolumen der österreichischen Forschung und Entwicklung bei. In absoluten Zahlen beträgt das Wachstum 36 Mio. $€$ bzw. plus 2,7 \% relativ gesehen.

- Die Bundesländer finanzieren voraussichtlich 0,43 Mrd. $€$ und weisen damit einen Finanzierungsanteil von $4,8 \%$ auf. Ihr Wachstum gegenüber 2012 beträgt 15 Mio. $€$ bzw. 3,6 \%.

- Der Sektor Sonstige /sonstige öffentliche Finanzierung z.B. durch Gemeinden oder Sozialversicherungsträger, privater gemeinnütziger Sektor) trägt mit $0,15 \mathrm{Mrd}$. $€$ etwa $1,7 \%$ zum Gesamtvolumen der österreichischen Forschungsfinanzierung bei. Sein Wachstum wird für 2013 auf 5,5 Mio. $€$ bzw. 3,7 \% geschätzt.

Betrachtet man die Finanzierungsstruktur der F\&E-Ausgaben in Österreich, so zeigt sich, dass der mit der Krise einsetzende Trend des relativen Rückgangs des Finanzierungsanteils des Unternehmenssektors mittlerweile gestoppt werden konnte (vgl. Abb. 3). Hatte der Unternehmenssektor vor der Krise im Jahr 2007 noch einen Anteil von $48,7 \%$, so ging mit Einsetzen der Krise dieser Anteil sukzessive auf 43,8 \% zurück. Da für das Jahr 2013 in der Globalschätzung der Statistik Austria für den Unternehmenssektor ein stärkeres Wachstum der F\&E-Finanzierung prognostiziert wird, steigt nun dieser Anteil wieder geringfügig auf 43,9 \%. Rechnet man das Ausland mit hinzu (dessen Finanzierung ja zum überwiegenden Anteil von ausländischen Unternehmen stammt, aus großteils internationalen Konzernen, die Forschungs- und Entwicklungsvorhaben ihrer österreichischen Tochterunternehmen mitfinanzieren), ergibt sich ein Anteil 
Abb. 2: Entwicklung der F\&E in Österreich nach Finanzierungssektoren (Index, 2007=100)

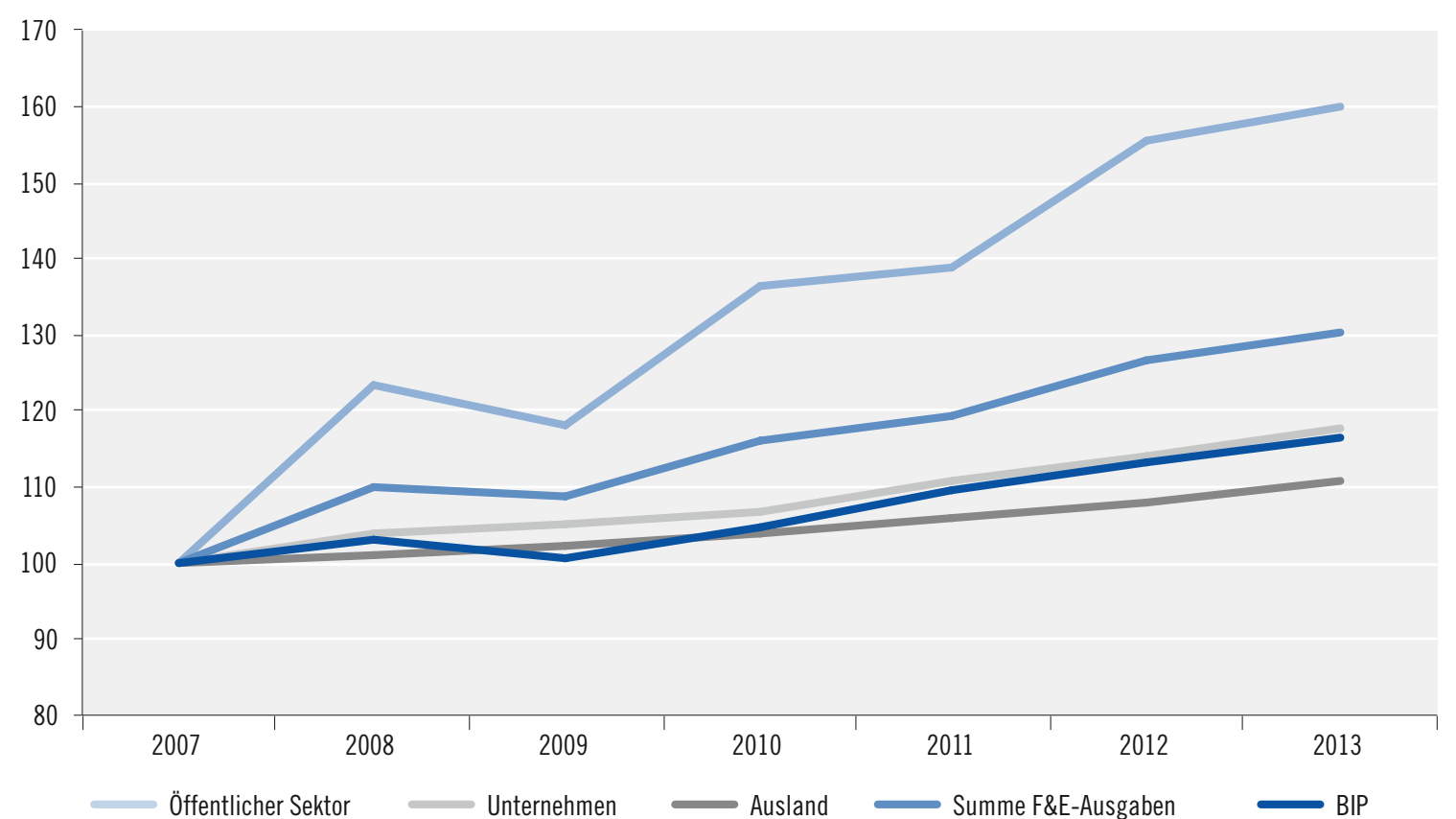

Anm.: Der Finanzierungssektor „Sonstige“ (der u.a. die Gemeinden oder die Sozialversicherungsträger sowie den privaten gemeinnützigen Sektor umfasst) wurde hier zum „Öffentlichen Sektor" gezählt.

Quelle: Statistik Austria, Globalschätzung vom Stand 11. April 2013.

Abb. 3: Finanzierungsanteile für F\&E in Österreich nach Finanzierungssektoren (in Prozent)

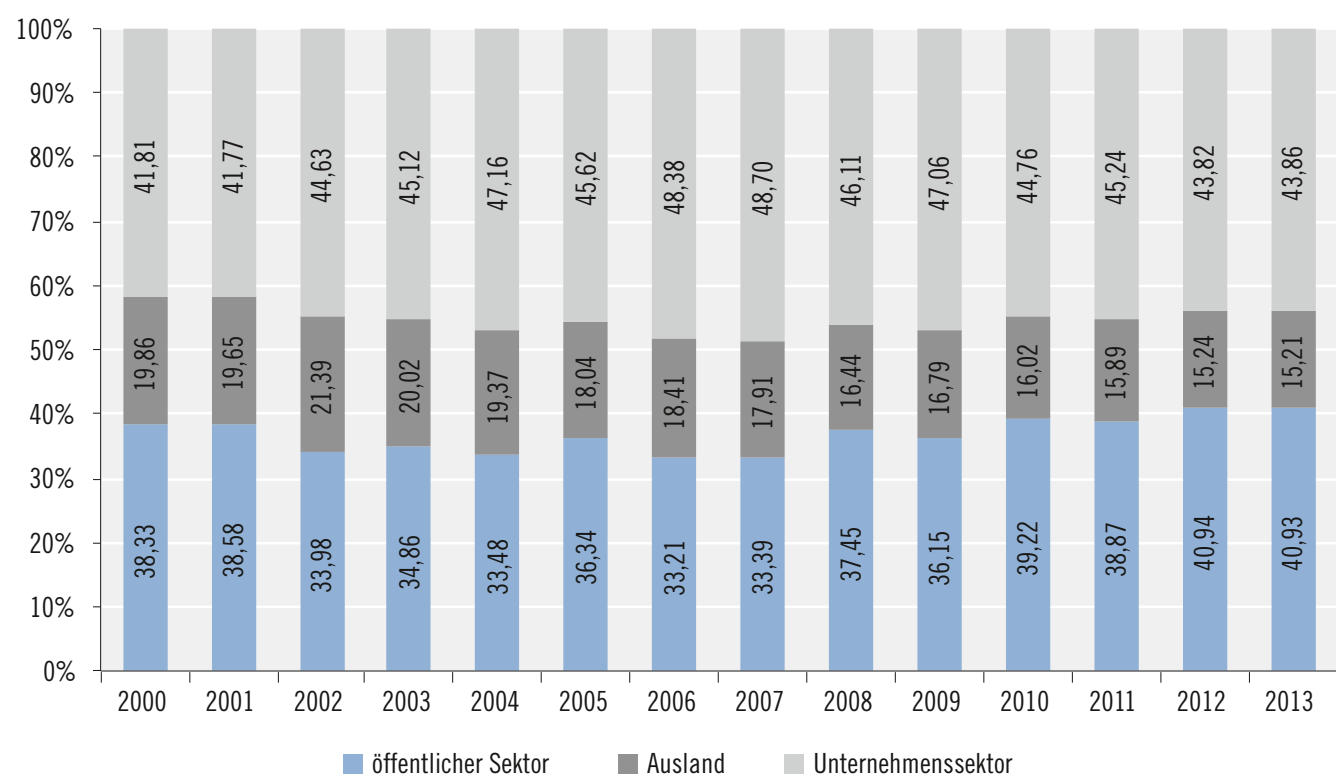

Quelle: Statistik Austria, Globalschätzung vom Stand 11. April 2013. 
des privaten Sektors von annähernd $60 \%$. Somit ist die Finanzierungsstruktur der Forschung und Entwicklung in Österreich derzeit nicht allzu weit vom generellen Ziel der Forschungs- und Technologiepolitik der Europäischen Union als auch des Ziels der FTI-Strategie der österreichischen Bundesregierung, nämlich einer ungefähren Verteilung der Finanzierungsanteile auf zwei Drittel privat und ein Drittel öffentlich, entfernt.

\section{$1.2 \quad$ Umsetzung der FTI-Strategie}

Die im März 2011 verabschiedete FTI-Strategie der Bundesregierung verfolgt - wie bereits im vergangenen Forschungs- und Technologiebericht ausführlich dargelegt - einen breiten, systemischen Ansatz zur Unterstützung und Strukturierung des Innovationsystems. Zeitgemäße FTI-Politik ist nicht ausschließlich auf Wissenschafts- und Technologieförderung konzentriert, sondern erfolgt in Abstimmung mit anderen Politikfeldern wie Bildung, Wettbewerb und korrespondierenden Regelungsmaterien wie etwa dem Steuer- und Finanzierungssystem, und berücksichtigt wechselseitige Wirkungszusammenhänge.

Daher wurde zur Konkretisierung und Koordination der Umsetzung der Strategie auf hoher Verwaltungsebene unter dem Vorsitz des Bundeskanzleramtes, gemeinsam mit den relevanten Bundesministerien, die Task Force FTI eingerichtet. Ein kontinuierlicher Austausch dieses Gremiums erfolgt umfassend auch unter Einbeziehung des Rates für Forschung und Technologieentwicklung.

Die FTI-Strategie ist grundsätzlich bis zum Jahr 2020 ausgerichtet, was bedeutet, dass die Gesamtentwicklung und die Wirkung der einzelnen Maßnahmen vielfach erst längerfristig eingeschätzt werden kann.

Welche Implementierungsschritte wurden nun im vergangenen Jahr konkret gesetzt?

Insgesamt wurde die gut etablierte Zusammenarbeit der FTI-relevanten Ressorts stärker strukturiert, intensiviert und weiter ausgebaut und ist $\mathrm{zu}$ einem wesentlichen Instrument ge- worden, um der steigenden Komplexität von Forschung, Technologie und Innovation sinnvoll zu begegnen.

Konkret bedeutete dies zunächst das Zusammenführen aller FTI-relevanten Programme und Initiativen unter dem Dach der FTI-Strategie. Nach Diskussion sämtlicher Ziele und Maßnahmen der FTI-Strategie aus strategischer Perspektive wurden neun Arbeitsgruppen (AG) in spezifischen wichtigen Bereichen eingerichtet:

- Die AG 1 „Humanpotenziale“ beschäftigt sich mit der Bedeutung von Bildung als Fundament für ein leistungsfähiges Innovationssystem. Ein erster zentraler Schwerpunkt liegt in der Kooperation aller Ressorts im Rahmen von Maßnahmen und Projekten, die MINT-Disziplinen (Mathematik, Informatik, Naturwissenschaften, Technik) betreffen. Dabei geht es vor allem um die Begeisterung für, die Heranführung an und die Lehr- und Lernmethoden für die MINT-Disziplinen. Das gilt natürlich für alle Stufen der Bildungslaufbahn, hat engste Wechselwirkungen $\mathrm{zu}$ den damit assoziierten Berufsbildern und damit zu deren Attraktivität für Männer und insbesondere Frauen. Damit wird ein für den Innovationsstandort Österreich langfristiger wichtiger Kulturwandel in den Bereichen Ausbildung, Bildung, Forschung und Arbeitswelt eingeleitet. Ziel der AG sind dabei vor allem die Kohärenz und Nachhaltigkeit dieser strukturverändernden Maßnahmen.

- AG 2 und 3 beschäftigen sich mit den in der FTI-Strategie festgelegten ressortübergreifenden Schwerpunktthemen „Klimawandel/ knappe Ressourcen" (AG 2) und „Lebensqualität und demographischer Wandel" (AG 3): In diesen AGs wurden zunächst alle FTI-Initiativen $\mathrm{zu}$ diesen jeweiligen Themenbereichen zusammengeführt, mit dem Ziel, ein klares Gesamtbild der bereits existierenden Programme zu etablieren und Abstimmungs- und Kooperationspotenziale auszuloten. In beiden Arbeitsgruppen steht auch im Vordergrund, das Themenmanagement der Ressorts dort, wo es Sinn macht, zu verschränken und ver- 
stärkt Synergien im Fördersystem zu nutzen. Als ein zentrales Thema im Bereich der AG 3 „Lebensqualität und demographischer Wandel" hat sich "aktive Mobilität" herausgegliedert.

- AG 4 beschäftigt sich im Wesentlichen mit Großforschungsinfrastrukturen (über 100.000,- €). Moderne, hochtechnologische Forschungsinfrastrukturen, bzw. der Zugang zu diesen, sind eine essentielle Grundlage für exzellente Forschung und konkurrenzfähige Technologieentwicklung in Österreich. Diese bedürfen jedoch kostenintensiver Investitionen mit längeren Investitions- und Nutzungszeiträumen. Ziel der AG ist es daher, ein effizientes, ressortübergreifendes Monitoring zur Implementierung der in der FTI-Strategie diesbezüglich genannten Maßnahmen sicherzustellen. Die AG beschäftigt sich mit der Vernetzung von Infrastrukturen und dem Ausbau der Kooperationen im Bereich Infrastrukturnutzung zwischen den forschenden Einrichtungen sowie mit Finanzierungsmodellen und rechtlichen Rahmenbedingungen für die Nutzung von Forschungsinfrastrukturen. Die AG nimmt dafür ein Mapping bestehender oder kurzfristig umzusetzender Maßnahmen vor und wird einen Vorschlag im Sinne einer nationalen Roadmap für Forschungsinfrastrukturen vorlegen.

- AG 5 „Wissenstransfer und Gründungen“ wurde vor dem Hintergrund etabliert, dass die Gründungsdynamik innovativer neuer Unternehmen in Österreich unterdurchschnittlich ist, diese für den Strukturwandel in Richtung wertschöpfungsintensiverer und FTI-intensiverer Produkte jedoch sehr wichtig wäre. Aufgabe von AG 5 ist es in diesem Zusammenhang, die Fördermaßnahmen verschiedener Ressorts besser aufeinander abzustimmen, Lücken zu schließen und Synergieeffekte zu lukrieren.

- AG 6 „Unternehmensforschung“ dient im Wesentlichen der weiteren Optimierung der Zusammenarbeit zwischen Wissenschaft und Wirtschaft. Im Konkreten bedeutet dies: Die
AG widmet sich der Weiterentwicklung des kooperativen Sektors in Zusammenhang mit der Entwicklung der Universitäten und der Ausarbeitung von Vorschlägen, wie die steuerliche Forschungsförderung effizienter und effektiver werden kann.

- Die AG 7a „Internationalisierung und FTI-Außenpolitik“ und AG 7b "Aktionsplan Österreich und der Europäische Wissensraum 2020" arbeiten an Strategien zur Positionierung Österreichs im europäischen Forschungsraum bzw. an einer globalen österreichischen FTIAußenpolitik und -präsenz. Die Arbeitsgruppen werden von renommierten außeruniversitären Forschungsinstituten durch Analysen unterstützt. Auch sind die zentralen österreichischen FTI-Stakeholder aktiv in den Prozess eingebunden. Beide Arbeitsgruppen werden im Juli 2013 Endberichte mit Empfehlungen an die Task Force FTI vorlegen.

- AG 8 „Internationale Rankings“ dient der Rezeption diverser nationaler und internationaler FTI-Rankings und Indikatoren. Ziel ist hier vor allem, ein gemeinsames Verständnis über Potentiale und Mängel dieser Instrumente zu entwickeln. Im vergangenen Jahr hat sich diese Gruppe auch intensiv mit dem Indikatorenset auseinandergesetzt, das im ersten Monitoringbericht des Rates für Forschung und Technologieentwicklung enthalten war und nun noch weiter spezifiziert wurde.

Mitglieder aller AGs sind jedenfalls die betroffenen Bundesministerien, in vielen AGs sind aber auch Stakeholder und Forschungsinstitute eingebunden. Wichtig bei der Etablierung der AGs war außerdem, dass keine bestehenden Abstimmungsprozesse dupliziert werden. Alle neun Arbeitsgruppen stehen in enger Verbindung mit der Task Force FTI bzw. sind Teil der Task Force FTI. Im Juni 2012 fand zu diesem Zweck ein gemeinsamer Workshop der Vorsitzenden der AGs mit den Mitgliedern der Task Force statt, bei dem die ersten Zwischenergebnisse und die weiteren Pläne intensiv diskutiert und abgestimmt wurden. 
Die Task Force diskutiert laufend auch jene Maßnahmen und Ziele der FTI-Strategie, die thematisch nicht in den Arbeitsgruppen enthalten sind, und wägt ab, in welchen Bereichen bereits Maßnahmen gesetzt wurden und inwieweit kurz-, mittel- oder längerfristig neue Initiativen $\mathrm{zu}$ überlegen sind. Insbesondere jene Projekte, die mehrere Ressorts berühren, sind regelmäßig Gegenstand gemeinsamer Abstimmung, dazu zählten im vergangenen Jahr beispielsweise das Projekt der "Langen Nacht der Forschung", das OST (Office of Science and Technology) China oder verschiedene Forschungsförderprogramme.

Darüber hinaus behandelt die Task Force regelmäßig die Empfehlungen des Rates für Forschung und Technologieentwicklung. Dabei werden die Empfehlungen zunächst auf ExpertInnenebene unter allen Ressorts erörtert, abschließend nimmt die Task Force FTI schriftlich dazu Stellung und übermittelt diese an den Rat.

Die aktuellen Innovation Leader aus Skandinavien zeigen anschaulich, dass kleine, offene und innovative Volkswirtschaften nicht nur im globalen Wettbewerb bestehen, sondern auch ihren gesellschaftlichen Wohlstand weiter ausbauen können. Mit der Umsetzung der FTI-Strategie ist Österreich weiterhin auf diesem Weg.

Im Folgenden wird ein Überblick über die Umsetzung FTI-relevanter Maßnahmen und Initiativen der Ressorts im vergangenen Jahr gegeben. Dem Überblick über strukturelle Maßnahmen folgt eine Zusammenschau über die wichtigsten Projekte und neuen Programme.

\subsubsection{Strukturelle Neuerungen}

\section{Steuerliche Forschungsförderung}

Mit dem Budgetbegleitgesetz 2011 wurden die Forschungsfreibeträge abgeschafft und die Forschungsprämie von $8 \%$ auf $10 \%$ angehoben. Im Zuge des 1. Stabilitätsgesetzes 2012, das seit 1 . April 2012 in Kraft ist, wurde im Rahmen der steuerlichen Forschungsförderung ein neues Verfahren eingeführt, wodurch eine strengere Kontrolle der Anspruchsvoraussetzungen gewährleis- tet werden soll: Bei der Prüfung der Anträge auf Forschungsprämie wird nunmehr die Forschungsförderungsgesellschaft (FFG) eingebunden. Die FFG soll die Förderwürdigkeit der Forschungsprojekte und die Qualität der eigenbetrieblichen Forschung beurteilen. Die bescheidmäßige Forschungsbestätigung als Anspruchsvoraussetzung für die Forschungsprämie wird weiters durch die Möglichkeit einer bescheidmäßigen Feststellung über die Höhe der Bemessungsgrundlage für die Forschungsprämie ergänzt: Die neuen Regelungen betreffend Beantragung, Bestätigung und Gewährung der Forschungsprämie gelten ab 01.01.2013 für die Wirtschaftsjahre ab 2012.

\section{Universitätsfinanzierung NEU}

Mit der UG-Novelle 2013 zur schrittweisen Implementierung der kapazitätsorientierten, studierendenbezogenen Universitätsfinanzierung (,Studienplatzfinanzierung“) wird die Universitätsfinanzierung in Österreich auf eine neue Basis gestellt. Ziel ist eine transparentere sowie stärker als bisher auf Leistung, Qualität und Kapazität hin ausgerichtete Gestaltung der Finanzierung der Universitäten. Gleichzeitig soll in einer Testphase die Betreuungssituation in fünf stark nachgefragten Studienfeldern verbessert werden - einerseits durch zusätzliche Personalressourcen, andererseits durch die Festlegung von Kapazitäten für StudienanfängerInnen und die Möglichkeit von Zugangsregelungen, wenn diese überschritten werden. Wesentliche Prämisse dabei ist, die Zahl der Studierenden österreichweit insgesamt nicht zu verringern.

Insgesamt wird die Studienplatzfinanzierung über mehrere Leistungsvereinbarungsperioden umgesetzt. Die am 27.02.2013 im Nationalrat beschlossene Gesetzesnovelle schafft dafür die Grundlagen. Der Vollausbau ist ab der Leistungsvereinbarungsperiode 2019 bis 2021 geplant. Für die fünf besonders stark nachgefragten Studienfelder - Wirtschaft, Architektur, Informatik, Biologie und Pharmazie - wurde ein „Qualitätspaket" geschnürt, das die Betreuungssituation verbessern soll: einerseits durch zusätzliche Perso- 
nalressourcen (insgesamt wurden ca. 36 Mio. $€$ im Kontext der Leistungsvereinbarungen für zusätzliche ProfessorInnen bzw. Personaläquivalente in den Jahren 2013 bis 2015 zur Verfügung gestellt) und andererseits durch die Festlegung von Studienanfängerplätzen, bei deren Überschreitung die Universitäten Zugangsregelungen einführen können, welche seitens der Universitäten weitgehend autonom gestaltet werden können. Langfristiges Ziel ist es, eine ausreichende Zahl an Studienplätzen unter im internationalen Vergleich adäquaten Studienbedingungen zur Verfügung zu stellen. Damit soll ein effizienterer Lehrbetrieb und Studierenden ein Studienabschluss in angemessener Zeit ermöglicht werden. Die Anzahl von Plätzen für StudienanfängerInnen in den genannten Fächern wird gesetzlich festgelegt. Der Zielwert liegt bei österreichweit 20.220 Studienanfängerplätzen - das entspricht den Anfängerzahlen 2011/12. Wie diese auf die einzelnen Universitäten aufgeteilt werden, wird jeweils im Rahmen der Leistungsvereinbarungen geregelt.

\section{Hochschulraum-Strukturmittel}

Im Rahmen einer Novelle des UG wurde im Sommer 2012 das Instrument der Hochschulraum-Strukturmittel eingeführt. Damit wurde die leistungsorientierte Finanzierung in Form des Formelbudgets durch einen neuen Allokationsmechanismus abgelöst und der zur Finanzierung der Universitäten zur Verfügung stehende Gesamtbetrag um die Hochschulraum-Strukturmittel ergänzt. Ziel der neuen Regelung ist es, die bisherige komplexe indikatorenbezogene Finanzierung über das formelgebundene Budget an Hand von wenigen Indikatoren nachvollziehbar zu berechnen. Der den Universitäten zur Verfügung stehende Gesamtbetrag setzt sich für die LV-Periode 2013 bis 2015 somit aus einem Teilbetrag für die Grundbudgets und einem Teilbetrag für die Hochschulraum-Strukturmittel zusammen. Eine ausführliche Darstellung dieses neuen Governance-Instruments findet sich in Kapitel 3.2.2.
Strukturelle Reform der Österreichischen

Akademie der Wissenschaften (ÖAW)

Rund ein Jahr nach Unterzeichnung der Leistungsvereinbarung mit dem Bundesministerium für Wissenschaft und Forschung ist im Dezember 2012 durch den Abschluss der Übertragung von 13 ÖAW-Einrichtungen und ÖAW-Forschungsgruppen an neun österreichische Universitäten ein wichtiges Ziel im Restrukturierungsprozess der ÖAW, nämlich die Fokussierung des Forschungsträgers, erreicht worden. Damit wird die Profilbildung an Österreichs Hochschul- und Forschungseinrichtungen gefördert und bestehende Stärken an Universitäten können weiter ausgebaut werden. Die ÖAW wiederum konzentriert sich dadurch verstärkt auf ihre in der Leistungsvereinbarung verankerten Kerngebiete. Statt 63 Forschungseinheiten Anfang des Jahres 2012 sind ein Jahr später 28 Institute unter dem Dach der ÖAW versammelt.

Seit Mitte 2012 wird eine strukturelle Reform der Akademie diskutiert; Ziel des Reformprozesses ist die Entflechtung von Gelehrtengesellschaft und Forschungsträger unter dem gemeinsamen ÖAW-Dach. Der diesbezügliche Grundsatzbeschluss wurde im Oktober 2012 von der Gesamtsitzung der Akademie gefasst. Die Gelehrtengesellschaft soll in Zukunft ihr Engagement in der Gesellschafts- und Politikberatung verstärken. Zur strukturellen Reform der ÖAW siehe die weiteren Ausführungen, insbesondere zu den Performance-Indikatoren, in Kapitel 3.5.

\section{Innovationsfördernde öffentliche Beschaffung (IÖB)}

Im September 2012 hat der Österreichische Ministerrat das Leitkonzept für eine innovationsfördernde öffentliche Beschaffung (IÖB) verabschiedet, das von BMWFJ und BMVIT im Zuge eines Stakeholderprozesses ausgearbeitet wurde. Als konkrete Maßnahme, um in Zukunft das öffentliche Beschaffungswesen innovationsfördernder zu gestalten, wurden in dem IÖB-Leitkonzept u.a. Folgendes vereinbart: Das Bundes- 
vergabegesetz soll um „Innovation“ als sekundäres Beschaffungskriterium erweitert werden; es soll eine zentrale Servicestelle für IÖB eingerichtet werden; es sollen Themenworkshops mit Anbietern und Beschaffern innovativer Produkte und Dienstleistungen etabliert und regelmäßig Informations- und Koordinationsveranstaltungen durchgeführt werden. Die 2011 gestartete Pilotausschreibung Verkehrsinfrastrukturforschung befindet sich in der 2. Phase, der Prototypenentwicklung. Die erstmalige Anwendung dieses Instruments der vorkommerziellen Beschaffung brachte wichtige Erkenntnisse für die Akteure (BMVIT, ÖBB, ASFINAG und FFG), welche die Ausschreibung finanzierten bzw. durchführten. Forschungsergebnisse werden erst für 2014 erwartet. Zum Thema Kälte/Wärme in historischen Gebäuden wird ein Pilotvorhaben der Burghauptmannschaft Österreich in Kooperation mit der BBG gestartet.

\subsubsection{Ausgewählte neue Maßnahmen}

\section{MINDT-Initiative}

In dem seit etwa zehn Jahren in mehreren Phasen laufenden Projekt „Innovationen Machen Schule Top" (IMST) wurden wesentliche Bausteine für einen verbesserten Unterricht in den Fächern Mathematik, Informatik, Naturwissenschaften, Deutsch und Technik (MINDT) entwickelt. Ab 2013 sorgt das BMUKK über eine neue MINDTInitiative für eine Verbreitung der Ergebnisse an weiteren österreichischen Schulen und auch bei jenen Lehrkräften, die bisher noch nicht mit IMST in Kontakt gekommen sind. Dabei werden in Kooperationen zwischen Pädagogischen Hochschulen und Universitäten 80 bis 100 Schulprojekte durchgeführt und wissenschaftlich begleitet sowie die bisher bereits agierenden 17 regionalen Fachdidaktikzentren an Pädagogischen Hochschulen und Universitäten quantitativ und qualitativ weiter ausgebaut. Auch die in allen neun Bundesländern etablierten regionalen Netzwerke (wie auch einige Bezirksnetzwerke und ein Gender-Netzwerk) werden weiter unter- stützt, da sie ganz wesentlich zu einer regionalen Weiterentwicklung des MINDT-Unterrichts und einer Vernetzung der Akteure in der Region beitragen.

\section{Verstärkte Förderung von DoktorandInnen und Nachwuchswissenschaftlerlnnen}

Die Doktoratskollegs (DKs) des FWF sind ein bewährtes Instrument zur Schaffung qualitätsgesicherter, international sichtbarer Ausbildungszentren für den hoch qualifizierten wissenschaftlichen Nachwuchs an Österreichs Forschungsstätten mit Promotionsrecht. Im Gegensatz zur klassischen Doktoratsausbildung werden DissertantInnen im Rahmen eines DKs von einem ganzen Team betreut. Dieses setzt sich aus fünf bis 20 Faculty-Mitgliedern zusammen, die auf Basis eines klar definierten, idealerweise disziplinenübergreifenden Forschungsschwerpunkts im intensiven Austausch zusammenarbeiten. Die maximale Laufzeit eines DK beträgt drei Förderperioden zu je vier Jahren. Seit dem Jahr 2004 hat der FWF 38 DKs mit knapp 108 Mio. $€$ finanziert; derzeit werden rund 380 DissertantInnen in den strukturierten Doktorandenprogrammen ausgebildet. In Zukunft werden dem FWF - mittels der neuen Initiative „DK Profil“ - zusätzlich 18 Mio. $€$ für DKs zur Verfügung gestellt, die von bis zu 100 neue Stellen für DoktorandInnen schaffen werden.

Das von der Nationalstiftung für Forschung, Technologie und Entwicklung mit 8 Mio. $€$ geförderte ÖAW-Impulsprogramm „New Frontiers Groups" wurde im Jahr 2012 erstmals ausgeschrieben. Ziel ist es, besonders begabten jungen WissenschaftlerInnen den Aufbau und die Leitung einer Forschungsgruppe unter dem Dach der ÖAW bei freier Wahl der Thematik und bei Verwendung der zuerkannten Mittel nach eigenem Ermessen zu ermöglichen. Mit dem auf Cutting Edge Forschung ausgerichteten Programm sollen neue, zukunftsträchtige und gesellschaftlich relevante Themen aufgegriffen werden, die zur stetigen Erneuerung des Forschungsportfolios der ÖAW beitragen. Die Lauf- 
zeit einer New Frontiers Group beträgt dabei maximal fünf Jahre mit einer Förderhöhe von höchstens 4 Mio. $€$.

\section{Patentierungs- und Verwertungsstrukturen an Universitäten}

In den letzten Jahren ist es erfolgreich gelungen, gut funktionierende Verwertungsstrukturen an den Universitäten einzurichten und diese auch in den Leistungsvereinbarungen mit den Universitäten zu verankern. Ein wichtiger Schwerpunkt der Leistungsvereinbarungen 2013-2015 ist daher die professionelle Weiterentwicklung der universitären Schutzrechts- und Verwertungsstrategien und damit der gezielte Ausbau des universitären Wissens- und Technologietransfers.

Damit soll ein vergleichbares, angemessenes und nachgewiesenes Professionalisierungsniveau im Wissenstransfer der öffentlichen Forschungseinrichtungen sichergestellt werden. Die Etablierung klarer Regelungen im Umgang mit geistigem Eigentum (insbesondere auch bei Gründungen) ist ein wichtiger Bestandteil der Schutzrechts- und Verwertungsstrategien. Die Universitäten leisten dadurch mitunter einen erheblichen Beitrag zur Umsetzung der EU-Empfehlungen (IP-Recommendation). Das bedeutet: Soweit keine berechtigten universitären Geheimhaltungsinteressen entgegenstehen, sollen die Strategien sowohl universitätsintern als auch -extern veröffentlicht werden, um dadurch eine möglichst offene Kommunikation insbesondere mit den Partnern aus der Wirtschaft sicherzustellen.

\section{Neue Josef Ressel-Zentren}

Josef Ressel-Zentren sollen forschungserprobte Fachhochschulen über stabile und langfristige F\&E-Kooperationen als regionale F\&E-Partner für die Wirtschaft stärken. Der Kompetenzaufbau in der angewandten Forschung soll gleichzeitig auch die Qualität der FH-Ausbildung verbessern (insbesondere hinsichtlich Nachwuchsförderung und Bereitstellung attraktiver Forschungsarbeitsplätze für die Region). Nach einer positiven Evaluierung wurden alle drei laufenden Josef Ressel-Zentren der Pilotaktion auf die Gesamtlaufzeit von fünf Jahren verlängert. Mit Jahresbeginn 2012 startete auch die Neuauflage des Josef Ressel-Zentren-Programms.

Die neuen Josef Ressel-Zentren werden von der Christian Doppler Forschungsgesellschaft im Rahmen eines eigenständigen Programms betreut. Ausrichtung und Abwicklung orientieren sich in wesentlichen Punkten am bewährten CDG-Modell. Wie bei den CD-Labors werden nach einem kompetitiven Auswahlverfahren zeitlich befristete Zentren an Fachhochschulen gefördert, die intensiv in Forschungsfragen mit Unternehmen kooperieren und konkrete Lösungen für technische Probleme in der Wirtschaft erarbeiten. Die Laufzeit ist mit fünf Jahren etwas kürzer als bei den CD-Labors und der Fokus liegt auf der angewandten Forschung mit hohem Niveau. Im Vergleich zu den CD-Labors ist das Fördervolumen pro Zentrum kleiner dimensioniert. Des Weiteren sind keine in-kind Leistungen von Unternehmenspartnern vorgesehen, sodass eine Beteiligung grundsätzlich nur in Form von Geldleistungen möglich ist.

\section{Produktion der Zukunft}

Um den gegenwärtigen Herausforderungen (Globalisierung, demografische Entwicklungen und alternde Gesellschaft und Arbeitsmarkt, zunehmende Ressourcenverknappung, notwendige Energieeffizienz und höchste Umweltstandards) angemessen begegnen zu können, hat das BMVIT mit ExpertInnen aus Forschung und Industrie eine Strategie für eine Rahmeninitiative „Produktion der Zukunft" erarbeitet. Ziel ist es, in Österreich Themen und Fragestellungen der Produktionsforschung - mehr als bisher - gezielt aufzugreifen und strategisch an der Schnittstelle zwischen Wirtschaft und Wissenschaft zu fördern. Im Jahr 2011 konnte eine erste, sehr erfolgreiche Ausschreibung gestartet werden. Es wurden Projekte mit insgesamt 50 Mio $€$ gefördert. Die Nachfrage der Unternehmen war sehr hoch, das Förderungsvolumen konnte 2012 weiter auf 95 
Mio $€$ gesteigert werden. Dies zeigt das große Interesse der Industrie an Förderungen für innovative Produkte, Prozesse, Technologien und Geschäftsmodelle zur Verbesserung der Wettbewerbsfähigkeit.

\section{Jungunternehmerinitiative}

Vor dem Hintergrund der aktuellen Konjunkturlage sind GründerInnen oftmals mit Finanzierungsbarrieren für neue Geschäftsideen konfrontiert. Ebenfalls stark betroffen von der Wirtschafts- und Finanzkrise ist der heimische Risikokapitalmarkt, welcher in Österreich traditionell schlecht entwickelt ist. Vor diesem Hintergrund wurde mit der neuen Initiative ein Gesamtpaket im Umfang von 110 Mio. $€$ beschlossen, welches ab Anfang 2013 in Kraft trat und für UnternehmensgründerInnen in der besonders schwierigen Anfangsphase im Schnitt ein doppelt so hohes jährliches Unterstützungsvolumen wie bisher ermöglicht. Zwei ergänzende Fonds unterstützen dieses Ziel:

Gründerfonds: Der mit insgesamt 65 Mio. $€$ ausgestattete Gründerfonds richtet sich an innovative Unternehmen maximal fünf Jahre nach deren Gründung und ist branchenoffen. Die Beteiligungshöhe liegt zwischen 100.000 bis maximal einer Mio. $€$. Die Dauer der Beteiligung von maximal zehn Jahren ermöglicht Investitions- und Planungssicherheit für das Unternehmen; gleichzeitig ist es das Ziel, möglichst früh das Interesse von privaten Investoren zu wecken.

Business Angel Fund: Ein mit insgesamt 45 Mio. $€$ ausgestatteter Business Angel Fund erhöht die Risikokapitalversorgung, indem die Investitionskraft von Business Angels verdoppelt wird. Für jeden Euro, den Privatinvestoren in junge Unternehmen investieren, wird ein weiterer $€$ der öffentlichen Hand investiert. Durch die Einbindung der Europäischen Investitionsbank sowie der privaten Business Angels kann eine Hebel- wirkung für den Einsatz der öffentlichen Mittel erzielt werden. Das bedeutet, in Summe stammen 15 Mio. $€$ aus öffentlichen Mitteln in Österreich, 7,5 Mio. $€$ vom Europäischen Investitionsfonds und 22,5 Mio. $€$ von Investments durch Business Angels. Der Fond richtet sich ebenfalls an innovative Unternehmen maximal fünf Jahre nach deren Gründung, wobei die Investitionshöhe zwischen 150.000 und $300.000 €$ pro Unternehmen liegt. Es wird eine Beteiligungsdauer zwischen drei bis fünf Jahre erwartet, mit insgesamt 30 bis 50 Beteiligungen pro Jahr bzw. rund 300 Beteiligungen bis 2020. Bei einem Verkauf fließen die öffentlichen Anteile in den Fund zurück und ermöglichen damit weitere Investments.

\section{Markt.Start}

Die öffentliche Finanzierung von F\&E-Projekten beschränkt sich in der Regel auf die technischen Entwicklungen und endet mit einem funktionellen Prototyp. Der Weg zum Markt, der mit geringem technischen, aber mit hohem wirtschaftlichen Risiko verbunden ist, wird nicht gefördert. Hier setzt die Förderungsmaßnahme Markt.Start $\mathrm{an}^{3}$ und unterstützt nach erfolgreichem technischem Abschluss eines durch die FFG unterstützten F\&E-Projektes der experimentellen Entwicklung den Eintritt in den Markt. Markt.Start fokussiert sich auf die Unterstützung von jungen, technologieorientierten Start Up-Unternehmen, die als Kleinunternehmen (KU) eingestuft werden. Gefördert werden die Markteinführung und Umsetzung innovativer Produkt-, Verfahrens- und Dienstleistungsentwicklungen im Rahmen einer Unternehmensförderung, welche ausschließlich aus Darlehen von bis zu einer Mio. $€$ besteht.

\section{Frontrunner - Initiative}

Die Frontrunner - Initiative im Umfang von 20 Mio. $€$ wird von der FFG gemeinsam mit der aws abgewickelt und richtet sich an Unternehmen

3 www.ffg.at/marktstart 
mit Sitz in Österreich, die eine technologische Spitzenposition innehaben oder gerade auf dem Sprung dorthin sind und ihre Position durch eine offensive Frontrunner-Strategie stärken möchten. Sogenannte Frontrunner bewegen sich in einem kompetitiven Marktumfeld und müssen daher laufend innovative Produkte oder Prozesse entwickeln bzw. auch neue Märkte ansprechen. Die dadurch notwendige offensive Unternehmens- bzw. Geschäftsfeldstrategie (Frontrunner - Strategie) basiert dabei oft auf bahnbrechenden und riskanten F\&E Projekten, deren spezifische Risiken durch das spezielle Förderangebot in Form einer Zuschussförderung abgefedert werden sollen.

\section{Kreativwirtschaftsscheck}

Die Kreativwirtschaft ist ein immer wichtiger werdender Treiber für Innovationen in anderen wirtschaftlichen Bereichen. Jedoch besteht empirische Evidenz, dass diese Dienstleistungen von KMU, besonders von Kleinst- und Kleinunternehmen, noch zu wenig genützt werden. Aus diesem Grund wurde mit Februar 2013 das Programm „Kreativwirtschaftsscheck" gestartet. Ziel ist es, die Nachfrageseite in der Kreativwirtschaft zu unterstützen und künftig kleineren und mittleren Unternehmen einen finanziellen Anreiz zu bieten, mit Kreativwirtschaftsunternehmen zu kooperieren. Es soll die Innovationstätigkeit von KMU gesteigert, die Inanspruchnahme von Kreativleistungen durch KMU erhöht und damit die Kreativwirtschaft selbst gestärkt werden. Gefördert werden die Kosten der kreativwirtschaftlichen Leistungen zur Ideengenerierung, Konzeption, Entwicklung, Anwendung, Umsetzung und/oder Marktüberleitung im Rahmen einer konkreten unternehmerischen Innovationsaktivität eines KMU. Die Förderung beträgt maximal $5.000 €$ (Gesamtkosten sind bis zu dieser Höhe zu 100 \% förderbar). Aufgrund des großen Erfolges wurde das ursprüngliche Budget von 1,5 Mio. $€$ verdoppelt, wodurch für 2013 3 Mio. $€$ zur Verfügung stehen. Da das Kontingent bereits überzeichnet ist, sind derzeit keine weiteren Einreichungen möglich. Das Programm soll aber - bei positiver Evaluierung - ab 2014 fortgesetzt werden.

\section{Energieforschungsinitiative (EFI)}

Die mit 12 Mio. $€$ dotierte Energieforschungsinitiative $^{4}$ ist eine neue Initiative zur Förderung von innovativen Energieforschungsprojekten und wird im Rahmen von laufenden Förderinstrumenten der FFG und der aws implementiert. Die neue Energieforschungsinitiative soll österreichische Unternehmen dazu motivieren, neue Wege in der Energieforschung einzuschlagen und Forschungsergebnisse und damit verbundenen technologischen Fortschritt im Rahmen der „Europäischen Energy Roadmap 2050" zu ihrem Wettbewerbsvorteil zu machen.

Die Forschungsinitiative unterstützt die Nutzung erneuerbarer Elektrizität und trägt zu einer Steigerung des Anteils erneuerbarer Energien gemessen am österreichischen Gesamtverbrauch bei. Die Initiative fördert nicht zuletzt die Entwicklung neuer Verfahren zur CO2-freien Erzeugung von Wasserstoff und den industriellen Einsatz von $\mathrm{CO}_{2}$ als Rohstoff für die Herstellung von wirtschaftlich vermarktbaren Produkten bzw. für den Einsatz in industriellen Prozessen. Hauptziel dieser Initiative ist die Realisierung von Prototyp- bis Demoanlagen für eine mögliche großtechnische industrielle Verwertung.

\section{Climate Change Centre Austria (CCCA)}

Das im Sommer 2011 eingerichtete $\mathrm{CCCA}^{5}$ ist Anlaufstelle für Forschung, Politik, Medien und Öffentlichkeit für alle Fragen der Klimaforschung in Österreich und fördert somit einen nachhalti-

4 www.ffg.at/efi/initiative

5 www.ccca.boku.ac.at 
gen Klimadialog und fungiert als koordinierende Einrichtung zur Förderung der Klimaforschung in Österreich. Dabei verfolgt das CCCA die folgenden Ziele: die Stärkung der Klimaforschungslandschaft in Österreich, die Förderung des wissenschaftlichen Nachwuchses und die Unterstützung des Wissenstransfers sowie die Beratung von Politik und Gesellschaft und adressiert damit eine der "Grand Challenges" unserer Gesellschaft. Neben der CCCA Geschäftsstelle wurde das CCCA Servicezentrum mit dem Ziel eingerichtet, Climate Services - also Informationen und Daten zum Klimawandel, seiner Ursache und Folgen - für Forschung und Gesellschaft aufzubereiten und zur Verfügung zu stellen.

\section{Vorbereitungen für HORIZON 2020}

In den letzten Monaten wurde intensiv über das künftige EU-Rahmenprogramm HORIZON 2020 verhandelt. HORIZON 2020 wird die Förderung der gesamten Innovationskette von der Grundlagenforschung bis zur Markteinführung neuer Produkte und Dienstleistungen ermöglichen. Darüber hinaus wird die Erforschung gesellschaftlicher Herausforderungen („Grand Challenges") ein Schwerpunkt des nächsten Förderprogramms sein.

Österreich hat auf Grundlage seiner im österreichischen Ministerrat vom 14. Februar 2012 beschlossenen Verhandlungsposition aktiv an den europäischen Verhandlungen teilgenommen und unter anderem die folgenden Änderungen erwirkt:

- Österreich hat die Herausforderung „Gesundheit, demographischer Wandel und Wohlergehen" zwar in den Grundzügen begrüßt, hat aber festgehalten, dass diese Programmschiene (mit damals 16 Einzelmaßnahmen) nicht kohärent gestaltet war. Angesichts dessen hat Österreich Vorschläge für eine kohärentere Gestaltung vorgelegt - mit Erfolg, wurde doch mit der nunmehrigen Struktur entlang von sieben Einzelmaßnahmen eine deutliche Verbesserung erzielt.
- Im Zuge der Verhandlungen über den Verordnungstext hat Österreich eine Teilung der sechsten Herausforderung der dritten Säule befürwortet - mit dem positiven Ergebnis, dass es nun jeweils eine Herausforderung für die Geistes-, Sozial- und Kulturwissenschaften sowie für die Sicherheitsforschung gibt.

- Österreich hat sich mit anderen Mitgliedsstaaten gemeinsam dafür stark gemacht, dass KMU in Zukunft mehr Fördermittel erhalten - mit Erfolg, werden doch 20 \% der Fördermittel aus den Säulen „Industrial Leadership" und "Societal Challenges" speziell für KMU zur Verfügung gestellt werden.

Der Verhandlungsprozess wird im Herbst 2013 planmäßig abgeschlossen sein, wobei in den kommenden Monaten die Einigung zwischen Rat und Europäischem Parlament angestrebt wird. Wichtig ist in diesem Zusammenhang vor allem die Verteilung der Budgetmittel auf die verschiedenen Bereiche von HORIZON 2020. In Österreich wird währenddessen mit der FFG über eine Beauftragung über die Beratung und Betreuung der österreichischen FTI-Akteure in HORIZON 2020 verhandelt.

\subsection{Die Position Österreichs im internationalen Kontext}

Die Innovationsleistung eines Landes wird von einer Vielzahl von Faktoren bestimmt. Neben der Innovationsfähigkeit der Wirtschaft spielen u.a. Bildung und Wissenschaft, gesellschaftliche Einstellungen, die Rahmenbedingungen auf den Faktor- und Gütermärkten sowie die staatlichen Maßnahmen zur Förderung von Forschung und Innovation eine wesentliche Rolle. Um angesichts dieser Multidimensionalität einen zusammenfassenden Befund zur Position eines Landes im internationalen Innovationswettbewerb anzubieten, wurden in den vergangenen Jahren verschiedene Indikatorensysteme entwickelt. ${ }^{6}$ Ihnen gemeinsam ist, dass sie unterschiedliche Einzelindikatoren zur Innovationsleistung $\mathrm{zu}$ einem Gesamtindikator bündeln. Damit erlauben sie 
nicht nur Rückschlüsse auf die Position eines Landes im Innovationswettbewerb, sondern spiegeln auch aktuelle Trends wider und geben Hinweise auf mögliche Ansatzpunkte für innovationspolitische Maßnahmen. Im Folgenden wird die Position Österreichs im Innovation Union Scoreboard (IUS) 2013 der EU-Kommission analysiert. Der IUS ist von besonderem Interesse, da sich die österreichische Bundesregierung zum Ziel gesetzt hat, dass Österreich mittelfristig in diesem Ranking zur Gruppe der "Innovation Leader" aufschließt. Ergänzend dazu wird ein weiteres Ranking betrachtet, der „Innovationsindikator" der Deutschen Telekom Stiftung und des Bundesverbands der Deutschen Industrie. Dieses Ranking setzt im Vergleich zum IUS etwas andere Prioritäten und ermöglicht damit Rückschlüsse auf die Bedeutung methodischer Unterschiede für die Positionierung von Ländern in internationalen Innovationsvergleichen.

\subsubsection{Die Position Österreichs im Innovation Union Scoreboard (IUS)}

Der IUS stellt eine Weiterentwicklung des Europäischen Innovationsanzeigers (European Innovation Scoreboard - EIS) dar und wurde erstmals 2010 dem europäischen Innovationsvergleich zugrunde gelegt. Der IUS wird auf der Basis der Kommunikation der Europäischen Kommission zur „Europe 2020 Flagship Initiative Innovation Union" eingesetzt, um die Innovationsentwicklung innerhalb der EU-27 sowie der EU gegenüber anderen Volkswirtschaften (v.a. USA und Japan) einschätzen und vergleichen zu können. ${ }^{7}$

Der IUS stellt eine (quantifizierbare) Performancedarstellung auf Basis bestimmter Indikatoren dar, die im Laufe der Jahre mit dem Ziel wei- terentwickelt wurden, eine realistische und vergleichbare Einschätzung der Innovationsentwicklung zu erhalten. ${ }^{8}$ Verbesserungen in der Datenbasis sowie die konstante Weiterentwicklung der Analysemethoden (und nicht zuletzt die zunehmende Länge der Betrachtungsperiode) ließen die Vergleichbarkeit zwischen den Ländern und damit die Aussagekraft des IUS/EIS mit der Zeit steigen.

Trotz dieser Verbesserungen müssen jedoch auch die Grenzen von indikatorenbasierten Vergleichen von Innovationssystemen berücksichtigt werden, zumal die im IUS verwendeten Einzelindikatoren $z u$ einem Summary Innovation Index (SII) zusammengefasst werden, woraus sich die Notwendigkeit einer höchst vorsichtigen Interpretation dieser Zahl ergibt - nicht alle Determinanten und Einflussgrößen lassen sich mittels quantifizierbarer Indikatoren erfassen. Aber diese Grenzen berücksichtigend, hat sich der IUS als geeignetes Instrument erwiesen, um Entwicklungen nachzuzeichnen und eine Basis für Vergleichsmöglichkeiten in den Bereichen F\&E und Innovation zu erzielen. ${ }^{9}$

Der europäische Scoreboard (EIS und IUS) erfuhr im Laufe der Zeit Veränderungen und Verbesserungen; so verkürzte sich die Liste an verwendeten Indikatoren beispielsweise auf 25. Diese decken die relevanten Bereiche von Forschung und Innovation ab. ${ }^{10}$ Dabei wird zwischen drei Typen von Indikatoren (Enablers, Firm activities und Outputs) sowie acht Dimensionen unterschieden. Eine Beschreibung der Indikatoren sowie der eingesetzten Methode findet sich in Hollanders und Tarantola (2011).

Tab. 1 gibt einen Überblick über die dem IUS 2013 zugrundeliegenden Indikatoren sowie deren Quellen.

6 Hierzu zählen u.a. das Innovation Union Scoreboard der EU-Kommission, der Global Competitiveness Report des World Economic Forums, der World Competitiveness Report des IMD World Competitiveness Centre, der Global Innovation Index von INSEAD sowie verschiedene Einzelstudien, wie z.B. Institut der deutschen Wirtschaft Köln (2012), Economist Intelligence Unit (2009), Boston Consulting Group (2009) und Information Technology and Innovation Foundation (2009).

7 Europäische Kommission (2010a).

8 Eine ausführliche Diskussion des EIS findet sich im Forschungs- und Technologiebericht 2008 (S. 17ff).

9 Für eine ausführliche Diskussion dieser Aspekte siehe Schibany und Streicher (2008).

10 Für Details siehe die Dokumentation auf http://www.proinno-europe.eu/metrics 


\section{Aktuelle Entwicklungen}

Tab. 1: Die Indikatoren des IUS 2013

\begin{tabular}{|c|c|c|}
\hline Indicator & Data Source & $\begin{array}{c}\text { Years } \\
\text { covered }\end{array}$ \\
\hline \multicolumn{3}{|l|}{ ENABLERS } \\
\hline \multicolumn{3}{|l|}{ Human resources } \\
\hline 1.1.1 New doctorate graduates (ISCED 6) per 1000 population aged 25-34 & Eurostat & $2006-2010$ \\
\hline 1.1.2 Percentage population aged $30-34$ having completed tertiary education & Eurostat & $2007-2011$ \\
\hline 1.1.3 Percentage youth aged 20-24 having attained at least upper secondary level education & Eurostat & $2007-2011$ \\
\hline \multicolumn{3}{|l|}{ Open, excellent and attractive research systems } \\
\hline 1.2.1 International scientific co-publications per million population & Science-Metrix (Scopus) & $2007-2011$ \\
\hline 1.2.2 Scientific publications among the top $10 \%$ most cited publications worldwide as \% of total scientific publications of the country & Science-Metrix (Scopus) & $2004-2008$ \\
\hline 1.2.3 Non-EU doctorate students as a \% of all doctorate students & Eurostat & $2006-2010$ \\
\hline \multicolumn{3}{|l|}{ Finance and support } \\
\hline 1.3.1 R\&D expenditure in the public sector as \% of GDP & Eurostat & $2007-2011$ \\
\hline 1.3.2 Venture capital investment as \% of GDP & Eurostat & $2007-2011$ \\
\hline \multicolumn{3}{|l|}{ FIRM ACTIVITIES } \\
\hline \multicolumn{3}{|l|}{ Firm investments } \\
\hline 2.1.1 R\&D expenditure in the business sector as \% of GDP & Eurostat & $2007-2011$ \\
\hline 2.1.2 Non-R\&D innovation expenditures as \% of turnover & Eurostat (CIS) & $2006,2008,2010$ \\
\hline \multicolumn{3}{|l|}{ Linkages \& entrepreneurship } \\
\hline 2.2.1 SMEs innovating in-house as \% of SMEs & Eurostat (CIS) & $2006,2008,2010$ \\
\hline 2.2.2 Innovative SMEs collaborating with others as \% of SMEs & Eurostat (CIS) & $2006,2008,2010$ \\
\hline 2.2.3 Public-private co-publications per million population & CWTS (Thomson Reuters) & 2007, 2011 \\
\hline \multicolumn{3}{|l|}{ Intellectual assets } \\
\hline 2.3.1 PCT patents applications per billion GDP (in PPSE) & Eurostat & 2005,2009 \\
\hline 2.3.2 PCT patent applications in societal challenges per billion GDP (in PPSE) (environment-related technologies; health) & OECD / Eurostat & 2005,2009 \\
\hline 2.3.3 Community trademarks per billion GDP (in PPS€) & OHIM2 / Eurostat & 2007, 2011 \\
\hline 2.3.4 Community designs per billion GDP (in PPSE) & OHIM / Eurostat & 2007, 2011 \\
\hline \multicolumn{3}{|l|}{ OUTPUTS } \\
\hline \multicolumn{3}{|l|}{ Innovators } \\
\hline 3.1.1 SMEs introducing product or process innovations as \% of SMEs & Eurostat (CIS) & $2006,2008,2010$ \\
\hline 3.1.2 SMEs introducing marketing or organisational innovations as \% of SMEs & Eurostat (CIS) & $2006,2008,2010$ \\
\hline 3.1.3 High-growth innovative firms & N/A & N/A \\
\hline \multicolumn{3}{|l|}{ Economic effects } \\
\hline 3.2.1 Employment in knowledge-intensive activities (manufacturing and services) as \% of total employment & Eurostat & 2007, 2011 \\
\hline 3.2.2 Contribution of medium and high-tech product exports to the trade balance & UN & 2007,2011 \\
\hline 3.2.3 Knowledge-intensive services exports as \% total service exports & UN / Eurostat & 2006,2010 \\
\hline 3.2.4 Sales of new to market and new to firm innovations as \% of turnover & Eurostat (CIS) & $2006,2008,2010$ \\
\hline 3.2.5 License and patent revenues from abroad as \% of GDP & Eurostat & 2007,2011 \\
\hline
\end{tabular}

Quelle: InnoMetrics. 
Die "Innovationskraft" jedes Landes wird auf der Basis der zugrundeliegenden 25 Indikatoren zu einem composite indicator (Summary Innovation Index - SII) zusammengefasst. Das Ranking der 27 Mitgliedsstaaten wird sodann auf Grundlage dieser SII-Werte bestimmt.

Die grundlegende Reihung der EU-Mitgliedsländer im IUS/EIS ist seit der Einführung dieses Benchmarks im Wesentlichen gleichgeblieben: Die Gruppe der „Innovation Leaders“ umfasst vier Länder: Schweden, Deutschland, Dänemark und Finnland. In der Gruppe der „Innovation Followers" finden sich mit Niederlande, Luxemburg, Belgien, Großbritannien, Österreich, Ir- land, Frankreich, Slowenien, Zypern und Estland zehn Länder, die noch über (bzw. knapp unter) dem Durchschnitt der 27 EU-Mitgliedsstaaten liegen.

Die Gruppe der "Moderate Innovators" umfasst die Länder Italien, Spanien, Portugal, Tschechische Republik, Griechenland, Slowakische Republik, Ungarn, Malta und Litauen (Positionen 15-23); die Gruppe der "Modest Innovators" umfasst schließlich Polen, Lettland, Rumänien und Bulgarien.

Diese Gruppen sind im Zeitablauf sehr stabil; Änderungen in der relativen Positionierung erfolgen ganz überwiegend innerhalb dieser Grup-

\section{Abb. 4: Ländervergleich auf Basis von IUS 2013 und IUS 2011 11}

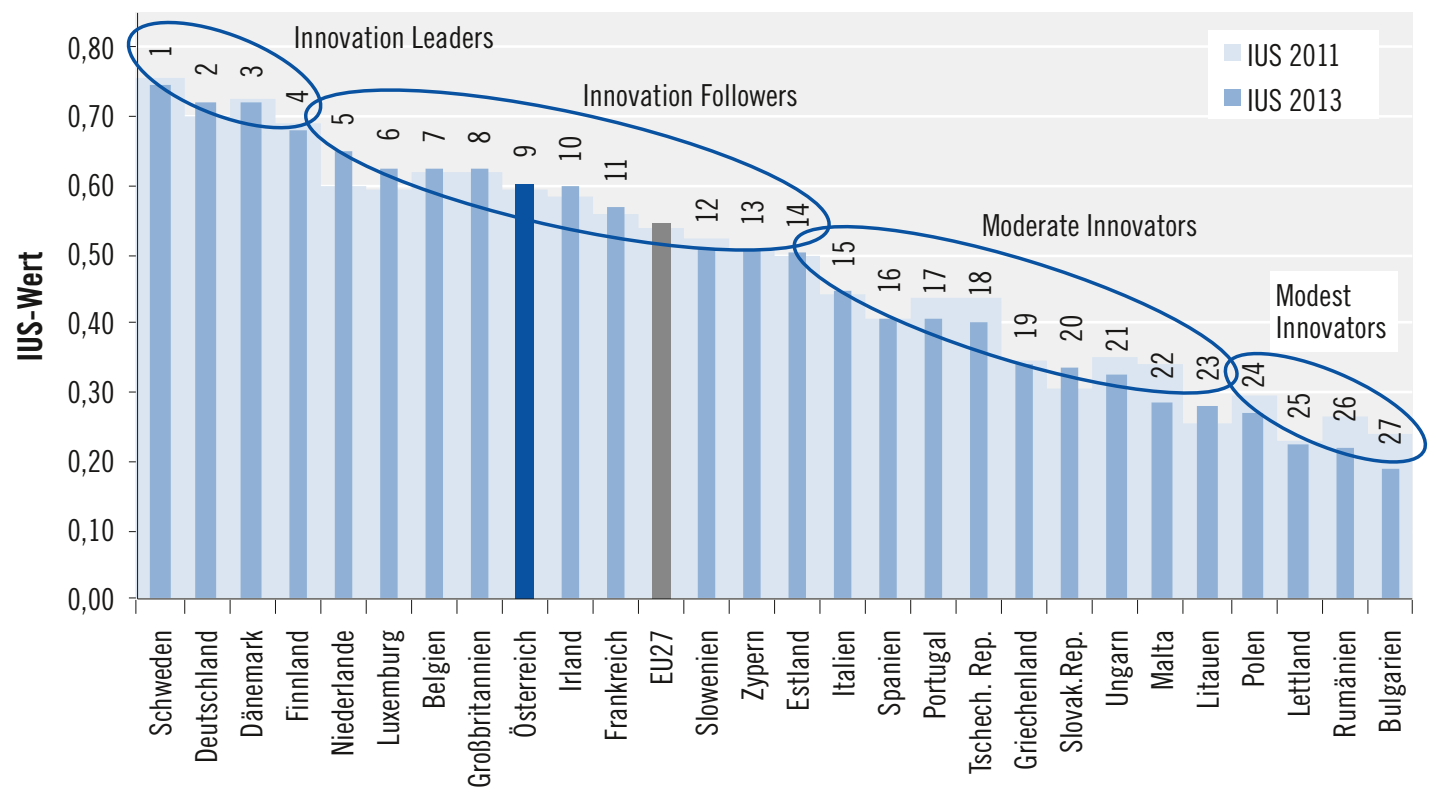

Quelle: InnoMetrics. Darstellung JOANNEUM RESEARCH.

11 Vorbemerkung zur Nomenklatur: Der neue IUS wird als „IUS 2013“ bezeichnet, bezieht sich also auf das Publikationsjahr t. Dies ist eine Neuerung: Bisher wurde der IUS (früher EIS) auf das Publikations-Vorjahr t-1 bezogen - der letzte IUS ist also nicht der IUS 2012, sondern der IUS 2011. Dies gibt natürlich Anlass zu Verwirrung, die nicht geringer wird, wenn man bedenkt, dass sich weder die neue noch die alte Nomenklatur auf das letzte Datenjahr beziehen, das für die Berechnung des Scoreboard verwendet wurde - diese war in der alten Nomenklatur das Jahr t-1 (der IUS 2011 hatte also als letztes Datenjahr 2010); im neuen IUS ist es entsprechend t-2 (der IUS 2013 bezieht sich also auf 2011 als aktuellstes Datenjahr). Um hier eine einheitliche Sicht zu gewährleisten, beziehen sich im Folgenden die Jahresangaben (etwa bei der Darstellung von zeitlichen Verläufen) auf das Publikationsjahr von IUS/EIS. 
Abb. 5: Publizierte Rangordnung der 27 EU-Staaten im Zeitablauf (Publikationsjahre 2008-2013)

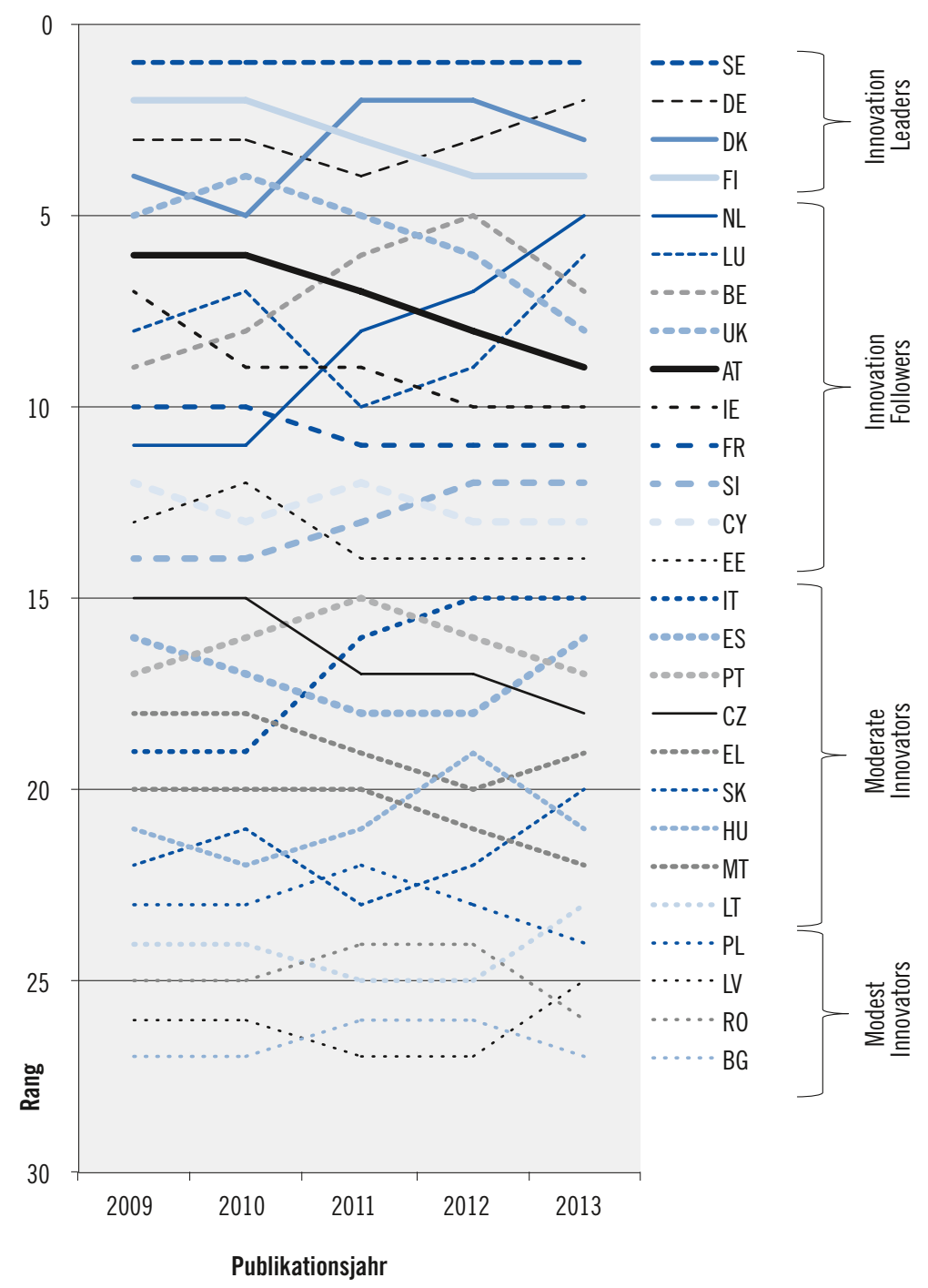

Quelle: InnoMetrics. Darstellung JOANNEUM RESEARCH.

pen. Für Österreichs Position in den jeweiligen Publikationsjahren bedeutet dies Folgendes:

Österreich belegt aktuell den 9. Platz und hat sich damit nominell im Laufe der letzten Jahre kontinuierlich vom 6. auf den 9. Platz verschlechtert. Gegenüber dem Vorjahr konnte Österreich zwar seinen SII-Wert etwas steigern, wurde aber trotzdem von Luxemburg (das nach dem 9. nun aktuell den 6 . Rang belegt) eingeholt. Wie bereits in den vergangenen Technologieberichten dargelegt, ist dies allerdings mit Vorsicht zu interpretieren, nicht zuletzt, da die offiziellen Reihungen in den jeweiligen Publikationsjahren auf der in dem jeweiligen Jahr verfügbaren Datenbasis beruhen. Wenn ein Indikatorwert für ein Land noch nicht verfügbar ist, wird er durch den letzten verfügbaren Wert substituiert. Dies bedeutet, dass sich die Indikatoren nicht für alle Länder auf das- 
selbe Datenjahr beziehen (müssen) - wenn zu einem späteren Zeitpunkt der Indikator dann doch verfügbar wird, kann es passieren, dass sich die relativen Positionen der Länder verändern. Des Weiteren ist zu bedenken, dass durch das Ranking eine kontinuierliche Variable /der Summary Innovation Index) in eine diskrete Variable (die Position eines Landes innerhalb der EU-27) übersetzt wird; das Ausmaß der SII-Differenz geht dabei verloren.

Die aktuellen Positionen und - noch mehr leichte Veränderungen im Länder-Ranking sind daher immer mit größter Vorsicht (und sehr unaufgeregt) zu interpretieren. Relevanter als die exakte Position ist die Ländergruppe, in der sich Österreich befindet: Wie aus Abb. 4 ersichtlich, liegt Österreich in der Gruppe der „Innovation Followers", und zwar in der ersten Hälfte dieser Gruppe; an dieser Gruppenzugehörigkeit hat sich in den letzten Jahren nichts geändert. Diese Gruppe ist durch sehr ähnliche Zahlenwerte des Summary Innovation Index gekennzeichnet; bereits leichte Änderungen in diesem führen daher zu merklichen Änderungen in der Positionierung eines Landes (und genau das passiert, wenn aufgrund mangelnder Datenverfügbarkeit in einem Publikationsjahr alte Indikatorwerte fortgeschrieben werden). Die Gruppe der Länder, welche die Plätze 5-10 belegen, unterscheidet sich in ihren SII-Werten um nur 0,05; Rang 4 (Finnland) weist hingegen einen um fast 0,04 höheren Wert als Rang 5 (Niederlande) auf (die SII-Differenz zwischen Irland auf Platz 10 und Frankreich auf Platz 11 beträgt 0,03$)$. Die Länder auf den Plätzen 5-10 können (und sollten) daher als eine recht homogene Gruppe betrachtet werden, in der sich bereits geringfügige Datenveränderungen in merklichen Rangverschiebungen auswirken können (und das auch tun).

Ein Blick auf die Einzelindikatoren (in der folgenden Abbildung sind die österreichischen Werte zusammen mit den Minima bzw. Maxima der
EU-27 dargestellt, jeweils bezogen auf den Durchschnitt der verfügbaren EU-27-Länder) zeigt ein verglichen mit den Ergebnissen der letzten Jahre praktisch unverändertes Bild: Bei sieben Einzelindikatoren liegt Österreich mehr als $10 \%$ unter dem EU-27-Schnitt (bei weiteren 5 liegt Österreich in einer +/- $10 \%$-Bandbreite um den Durchschnitt herum); bei 12 Indikatoren weist Österreich deutlich überdurchschnittliche Werte auf. Die Stärken und Schwächen sind durchaus wohlbekannt: Bei den Tertiärabschlüssen in der Gruppe der 30 bis 34-Jährigen liegt Österreich doch deutlich $(-30 \%)$ unter dem EU-Schnitt (überdurchschnittlich hingegen die Position Österreichs bei den Doktoratsabschlüssen und beim Bevölkerungsanteil mit zumindest Sekundarstufe II-Abschluss).

Die Qualität der wissenschaftlichen Publikationen ist überdurchschnittlich: Im Fall der „meistzititierten Publikationen" befindet sich Österreich zwar fast genau im EU-Schnitt, die internationalen Co-Publikationen betragen aber fast das Vierfache des EU-27-Schnitts. Bei den DoktoratsstudentInnen aus Nicht-EU-Ländern liegt Österreich hingegen mehr als $50 \%$ unter dem Durchschnitt (Dies verbirgt allerdings einen hohen Anteil von „normalen" StudentInnen aus dem EUAusland, speziell aus Deutschland). Die Werte der einzelnen Länder in diesem Indikator sind allerdings sehr ungleich verteilt und werden von einigen wenigen Ländern deutlich dominiert - was dadurch verdeutlicht wird, dass Österreich, trotz des deutlich unterdurchschnittlichen Zahlenwerts, bei diesem Indikator an 10. Stelle liegt.

In der Gruppe der 9 unternehmensbezogenen Indikatoren liegt Österreich nur bei einem einzigen Wert unter dem Durchschnitt (hier allerdings deutlich), nämlich bei den Ausgaben für nichtF\&E-bezogene Innovationen.

Schwächere Positionen zeigen sich hingegen bei den Exporten in wissensintensiven Dienstleistungen $^{12}$ sowie den Umsätzen mit innovativen

12 Die oft konstatierte Schwäche Österreichs bei den High-Tech-Sachgüterexporten findet sich im IUS nicht, da hier neben den HighTech auch die Medium-Tech-Exporte betrachtet werden, also auch Maschinen-, Anlagen- und Fahrzeugbau inkludiert sind - jene Bereiche, in denen Österreich starke Strukturen aufweist. 
Abb. 6: Detaillierte Ergebnisse des IUS 2013: Österreich versus Minimum/Maximum der EU-27

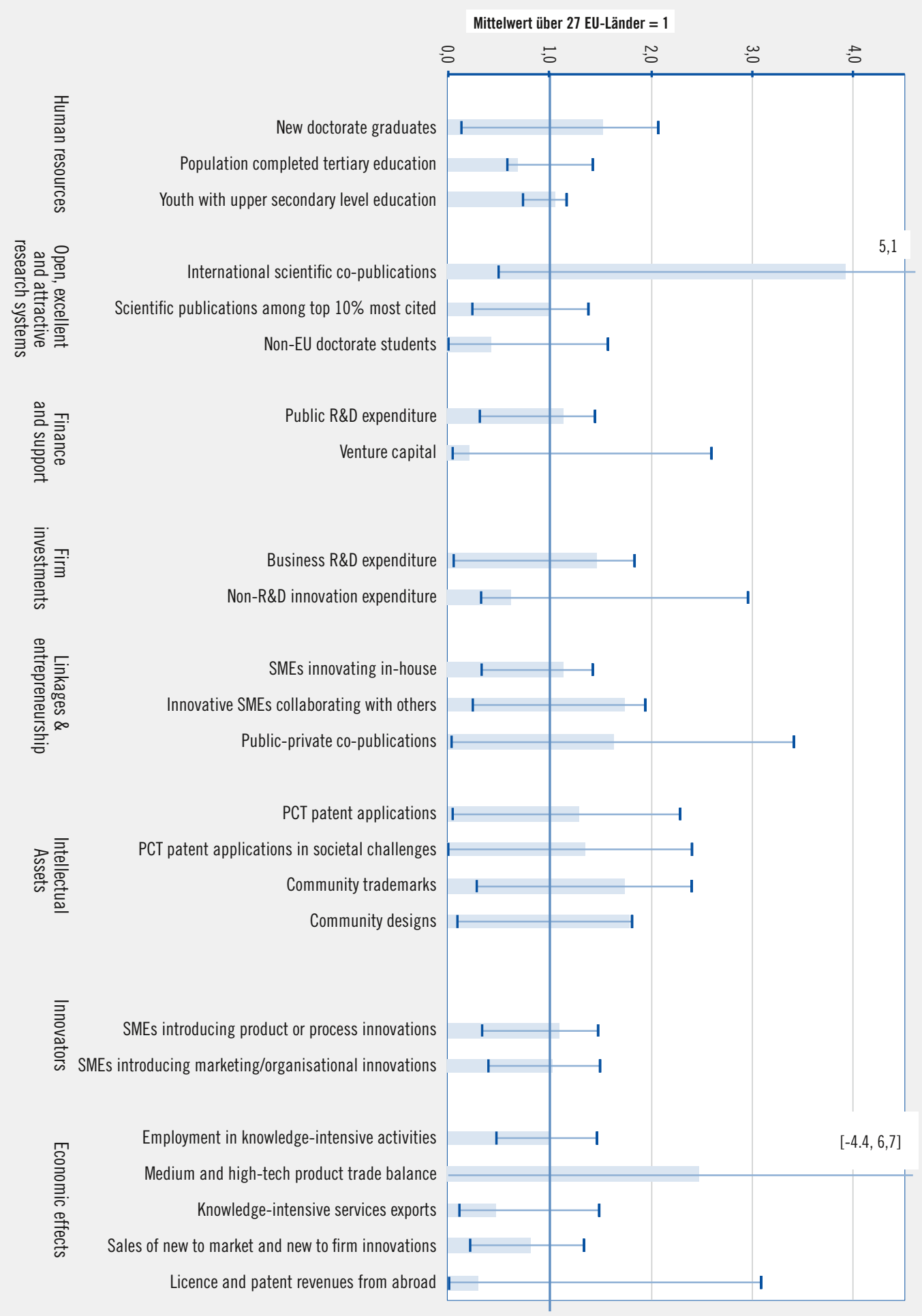

Quelle: InnoMetrics. Darstellung JOANNEUM RESEARCH. 
Produkten und den Lizenzeinnahmen aus dem Ausland: Dies bildet einen gewissen Widerspruch zu der guten Positionierung in Patenten, Trademarks und KMU-Innovatoren.

\section{Die Einzelindikatoren im Zeitablauf}

Im Folgenden wird die zeitliche Entwicklung der Einzelindikatoren von Österreich mit jener der Innovation Leaders und Innovation Followers (jeweils ungewichtete Mittelwerte) verglichen. Der Vergleich wird auf Basis der originalen Indikatorwerte durchgeführt (in manchen Jahren fehlende Werte wurden dabei durch den letzten verfügbaren Wert approximiert) und für die gesamte in der Datenbasis des IUS 2013 verfügbare Zeitperiode 2001-2011 dargestellt.

Bei den meisten Indikatoren zeigen Innovation Leaders bzw. Followers und Österreich ähnliche Tendenzen - dies bedeutet, dass die weiter oben beschriebenen relativen Stärken-Schwächen-Strukturen Österreichs in den betrachteten fünf bis zehn Jahren recht stabil waren. Eine gewisse Ausnahme bildet die Gruppe jener Indikatoren, die dem Community Innovation Survey (CIS) entnommen sind (dabei handelt es sich um die Indikatoren 2.1.2, 2.2.1, 2.2.2, 3.1.1, 3.1.2 und 3.2.4 - jene Indikatoren, die „Innovation" im Titel führen). Diese weisen für Österreich doch merkliche Sprünge auf. Der Grund dafür liegt in der Erhebungsmethodik: Aufgrund geänderter Rahmenbedingungen sind CIS 2006 und CIS 2008 in Österreich nur sehr bedingt vergleichbar. Dazu schreibt die Statistik Austria in Innovation 2006-2008 - Ergebnisse der Sechsten Europäischen Innovationserhebung (CIS 2008):
„Aus verschiedenen Gründen (zum Teil stark veränderter Fragenprogramme, einer veränderten Stichprobenmethodik und einer verbesserten Durchführung der Non-Response-Analyse [...], einer neuen Wirtschaftsklassifikation und nicht zuletzt einer starken Ausweitung des Innovationsbegriffs) sind die Vergleichsmöglichkeiten über die Jahre aber stark eingeschränkt. Für die Vergleichbarkeit zwischen den vorliegenden Ergebnissen und jenen des CIS 2006 gelten insbesondere die letzten beiden aufgezählten Gründe. "13

Bei den Indikatoren 1.3.1 und 2.1.1 (F\&E-Ausgaben der öffentlichen Hand bzw. des Unternehmenssektors) weist Österreich sich ziemlich genau in der Mitte zwischen Innovation Leaders und Followers auf (und weist zudem eine sehr ähnliche Dynamik auf). Im Vergleich sehr gering ist allerdings die österreichische Venture Capital-Ausstattung (1.3.2): Mit nur 0,02 \% des BIP liegt es bei den aktuellsten Zahlen um einen Faktor 6 unter den Vergleichsdurchschnitten (zeigt dafür aber einen weniger markanten Rückgang in den letzten Jahren).

Besondere Betrachtung erfordert Indikator 1.1.2, der Anteil von tertiär Ausgebildeten in der Gruppe der 30 bis 34-Jährigen. Österreich weist mit $24 \%$ einen deutlich geringeren Anteil auf als der Durchschnitt von sowohl Innovation Leaders als auch Innovation Followers (mit jeweils über $40 \%$ ); der EU-Schnitt liegt bei $35 \%$. Der Grund liegt nicht zuletzt in Zuordnungsproblemen zur ISCED 1997, wobei hier die Klassifikation der BHS-AbsolventInnen Probleme bereitet. ${ }^{14}$ Würden diese klassifikatorischen Unklarheiten zugunsten einer Zuordnung $\mathrm{zu}$ den tertiären $\mathrm{Ab}$ schlüssen gelöst, wäre der tertiäre Anteil in Ös-

\footnotetext{
13 Statistik Austria (2010), S. 15.

14 Im Hinblick auf die Vergleichbarkeit problematisch [ist] allerdings die BHS-Matura, die nach Besuch der fünfjährigen Hauptform einer HTL, HAK usw. als Abschluss der Ebene ISCED 4a klassifiziert ist, während die Sonderformen der BHS (Kolleg, Aufbaulehrgang, BHS für Berufstätige) unter die Kategorie ISCED 5b fallen und dem Tertiärbereich zugerechnet werden. Das passiert aus der Logik heraus, dass Bildungsgänge - die sequentiell aufeinander folgen und hierarchisch abgestuft sind - für die ISCED die Einheiten der Klassifikation darstellen.
} 
terreich bei $36,8 \%{ }^{15}$ - er wäre damit zwar über dem Schnitt der EU-27, aber doch noch merklich unter dem Niveau der Innovation Leaders und Followers ${ }^{16}$. Und auch die zeitliche Entwicklung zeigt in Österreich eine recht moderate Dynamik: Zwischen 2001 und 2011 stieg der Anteil in Österreich von 21 auf $24 \%$, beim Schnitt der Innovation Followers hingegen von 29 auf $42 \%$ (bei den Innovation Leaders von 33 auf $41 \%$ ). Sehr hoch ist in Österreich hingegen der Anteil der 20 bis 24-Jährigen mit zumindest gehobener Sekundarstufenausbildung (1.1.3); neben den Skandinaviern sind es übrigens die "neuen" Mitglieder aus Osteuropa, die hier die höchsten Werte aufweisen.

Interessant - und durchaus erfreulich - ist das Abschneiden Österreichs in den Patentindikatoren: Bei den beiden Indikatoren zu den $\mathrm{PCT}^{17} \mathrm{~Pa}$ tentapplikationen (2.3.1 und 2.3.2) liegt Österreich über den Innovation Followers (wenn auch deutlich hinter den Innovation Leaders); bei den Indikatoren zu Community Designs und Community Trademarks liegt Österreich sogar über dem Durchschnittsniveau der Innovation Leaders. Markant unterdurchschnittlich ist jedoch das österreichische Lizenz- und Patenteinkommen aus dem Ausland (3.2.5): Der österreichische Wert beträgt nicht einmal ein Viertel der Innovation Leaders und Followers (und auch nur ein Drittel des Gesamt-EU-Schnitts).

Merklich sind die Unterschiede bei den beiden Indikatoren „Beitrag der Medium- und HighTech-Exporte zur Handelsbilanz" (3.2.2) und "Anteil der wissensintensiven Dienstleistungsexpor- te" (3.2.3): Kann Österreich im ersten Fall sehr gut mithalten (und sogar höhere Beiträge aufweisen als die Durchschnitte von Innovation Leaders und Followers), liegt im zweiten Fall der österreichische Wert um mehr als $50 \%$ unter dem jeweiligen Durchschnitt der Vergleichsländer. Hier ist allerdings zu beachten, dass Dienstleistungen, die von ausländischen Touristen in Österreich nachgefragt werden, als Dienstleistungsexporte gezählt werden, und der Tourismussektor in Österreich eine überdurchschnittliche Rolle in der Gesamtwirtschaft einnimmt.

\section{Zusammenfassung}

Österreich nimmt im aktuellen Innovation Union Scoreboard (IUS 2013) den 9. Rang ein (nach Platz 8 im letztjährigen IUS $2011^{18}$ und Platz 7 im IUS 2010) und bleibt damit fest in der (ersten Hälfte der) Gruppe der "Innovation Followers" verankert. Diese Gruppenzugehörigkeit ist seit einigen Jahren recht stabil, Verschiebungen der Positionen innerhalb dieser (Teil)Gruppe, wie sie im Jahresvergleich immer wieder vorkommen, sollten nicht allzu hoch bewertet werden (das gilt allerdings nicht nur für „Verschlechterungen", sondern sollte auch bei allfälligen Verbesserungen im Ranking bedacht werden). Österreich ist in guter Position innerhalb der Innovation Followers /gemeinsam mit den Niederlanden, Luxemburg, Belgien, Großbritannien und Irland auf den Plätzen 5 bis 10), die als Gruppe allerdings auch deutlich hinter den Innovation Leaders (Schweden, Deutschland, Dänemark und Finnland) zurückliegen.

15 Siehe dazu die Pressemitteilung 10.485-061/13 der Statistik Austria vom 19.03.2013: „Trotz Anstiegs der Tertiärquote liegt Österreich auch weiterhin deutlich unter dem EU-Schnitt. Bei internationalen Vergleichen zählen neben Hochschul-, Akademie- und Kollegabschlüssen auch Meister- und Werkmeisterprüfungen zu den Tertiärabschlüssen. Im Sinne dieser Klassifikation konnten im Jahr 2010 19,3\% der österreichischen Bevölkerung im Alter von 25 bis 64 Jahren einen Tertiärabschluss vorweisen. Im Schnitt jener 21 EUStaaten, die auch gleichzeitig OECD-Mitglied sind, verfügten allerdings 27,6 \% dieser Altersgruppe über einen Tertiärabschluss. Da die Tertiärquote in vielen Ländern rascher ansteigt als in Österreich, vergrößerte sich der Abstand zum EU-21-Durchschnitt in den letzten Jahren kontinuierlich. Betrachtet man allerdings die aus Sicht des Europa 2020-Ziels relevante Gruppe der 30 bis 34-Jährigen, so liegt die Tertiärquote unter Einbeziehung äquivalenter Bildungsabschlüsse mit 36,8 \% knapp über dem EU-Durchschnitt und in Reichweite des Zielwerts von $40 \%$."

16 Auf die Positionierung Österreichs im IUS 2013 hat dieses Problem nur geringe Auswirkungen: Zwar würde sich bei Unterstellung einer Tertiärquote von 36,9 \% der SII-Wert Österreichs an jenen von Großbritannien (Platz 8) deutlich annähern, für einen Positionswechsel würde es jedoch nicht reichen.

17 Patent Cooperation Treaty (PCT) ist ein Zusammenschluß von 16 nationalen/regionalen Patentämtern.

18 Zur Erklärung, warum dem IUS 2013 der IUS 2011 vorausgeht, sei auf die in der Fußnote 11 erwähnte Änderung in der Nomenklatur verwiesen. 
Abb. 7: Zeitliche Entwicklung der Einzelindikatoren, Teil 1: Österreich versus Innovation Leaders bzw. Innovation Followers

1.1.1 New doctorate graduates (ISCED 6) per 1000 population aged $25-34$

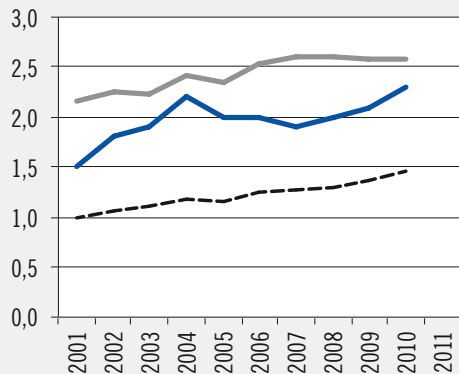

1.2.1 International scientific co-publications per million population

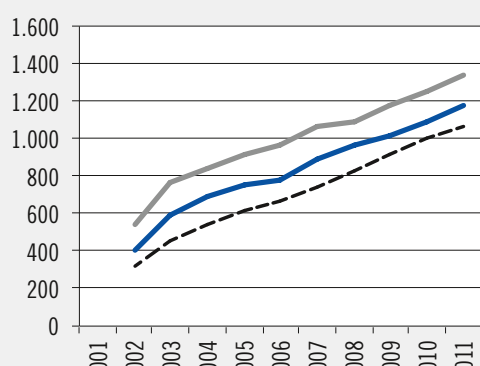

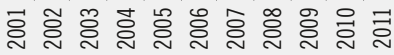

1.3.1 Public R\&D expenditures as \% of GDP

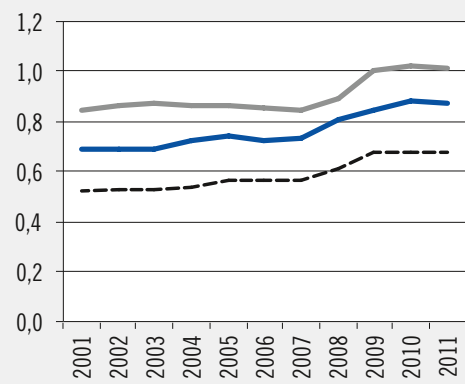

2.1.2 Non-R\&D innovation expenditures as $\%$ of turnove

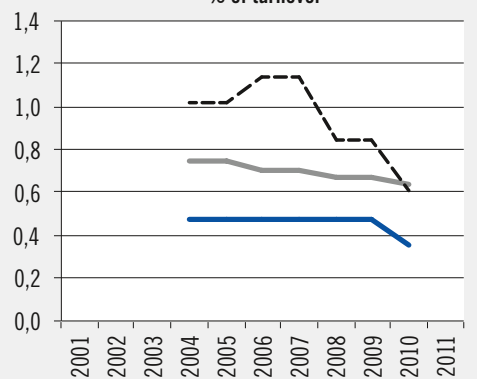

1.1.2 Percentage population aged 30-34 having completed tertiary education

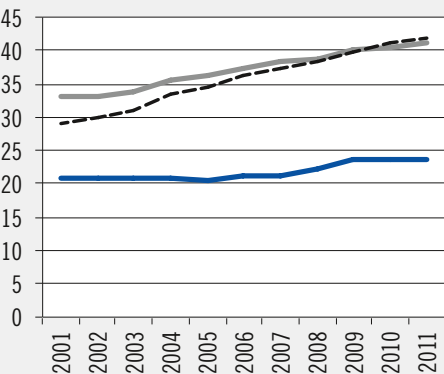

1.2.2 Scientific publications among the top $10 \%$ most cited publications worldwide as $\%$ of total scientific publications of the country

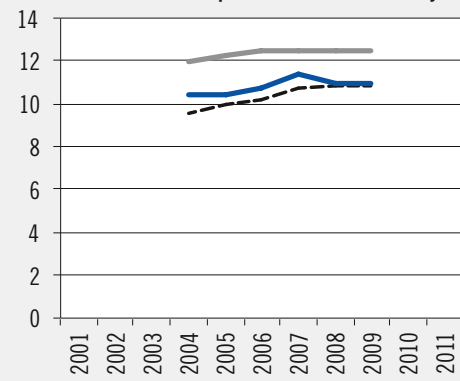

1.3.2 Venture capital (early stage, expansion and replacement) as \% of GDP

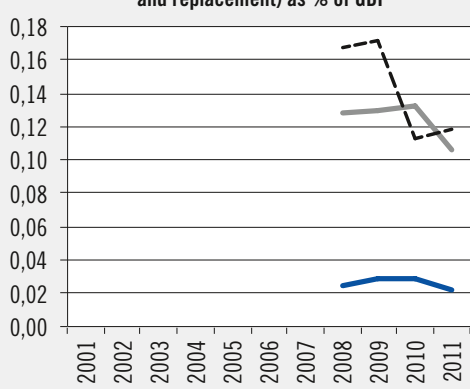

2.2.1 SMEs innovating in-house as \% of SMEs

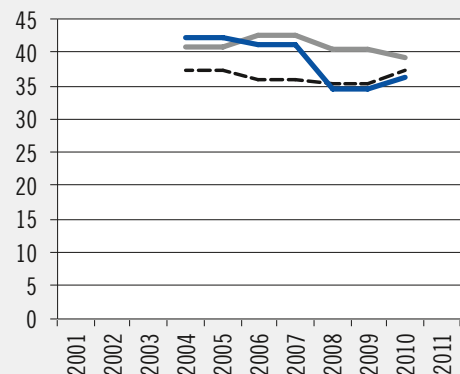

1.1.3 Percentage youth aged 20-24 having attained at least upper secondary level education

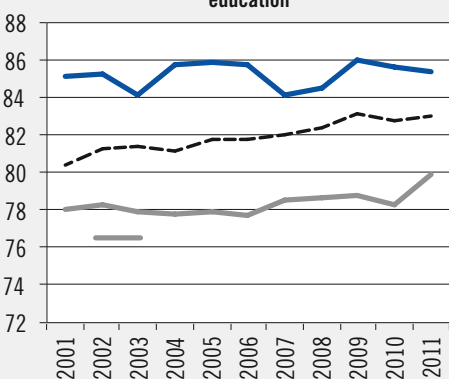

1.2.3 Non-EU doctorate students 3 as a $\%$ of all doctorate students

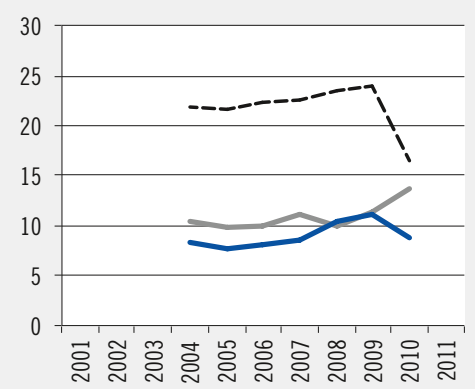

2.1.1 Business R\&D expenditures as \% of GDP

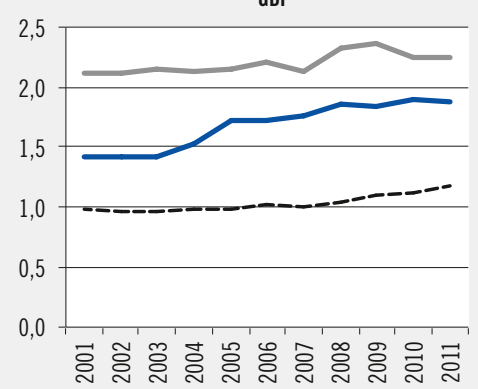

2.2.2 Innovative SMEs collaborating with others as \% of SMEs

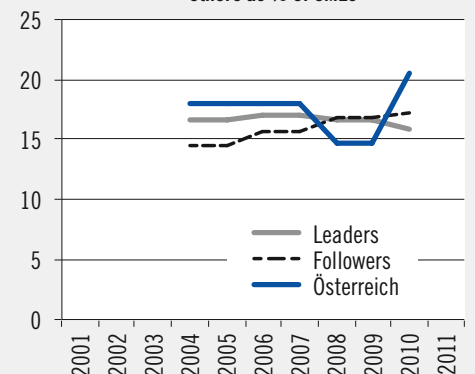

Quelle: InnoMetrics. Darstellung JOANNEUM RESEARCH. 
Abb. 8: Zeitliche Entwicklung der Einzelindikatoren, Teil 2: Österreich versus Innovation Leaders bzw. Innovation Followers

2.2.3 Public-private co-publications per million population

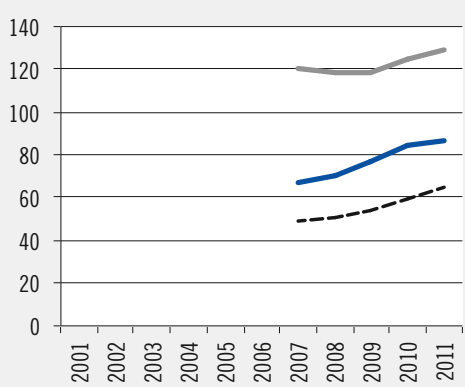

2.3.3 Community trademarks per billion GDP (in PPS €)

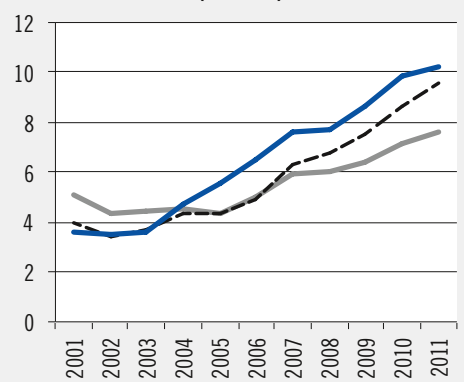

3.1.2 SMEs introducing marketing or organisational innovations as \% of SMEs

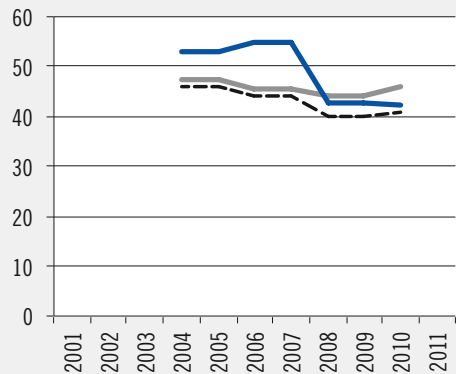

3.2.4 Sales of new to market and new to firm innovations as \% of turnover

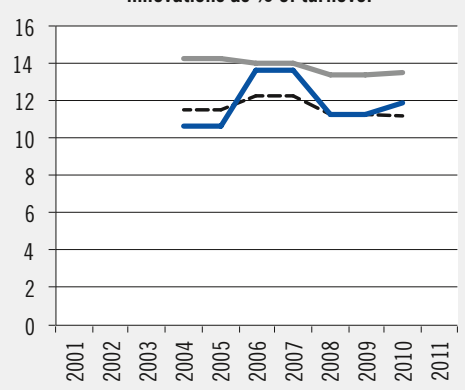

2.3.1 PCT patents applications per billion GDP (in PPS €)

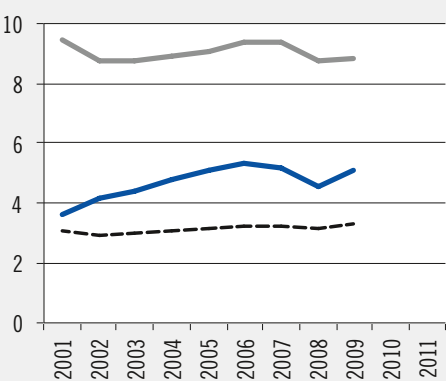

2.3.4 Community designs per billion GDP (in PPS €)

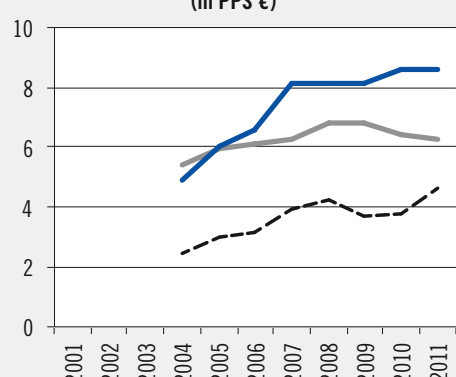

3.2.2 Contribution of medium and high-tech product exports to trade balance

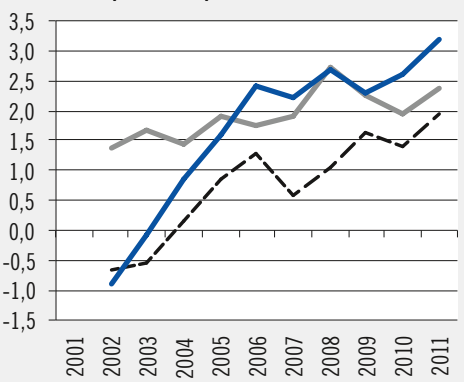

3.2.5 License and patent revenues from abroad as \% of GDP

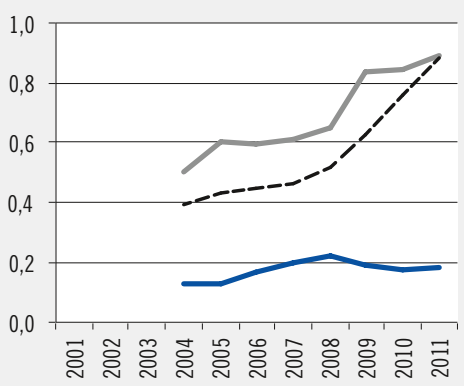

2.3.2 PCT patent applications in societal challenges per billion GDP (in PPS €) (climate change mitigation; health)

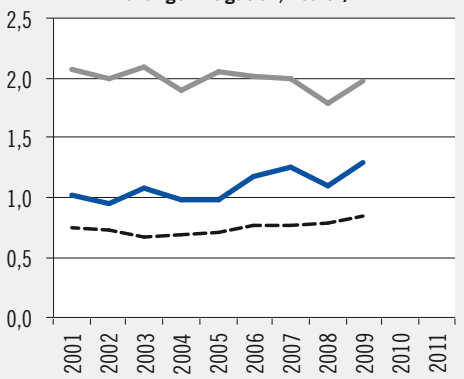

3.1.1 SMEs introducing product or process innovations as $\%$ of SMEs

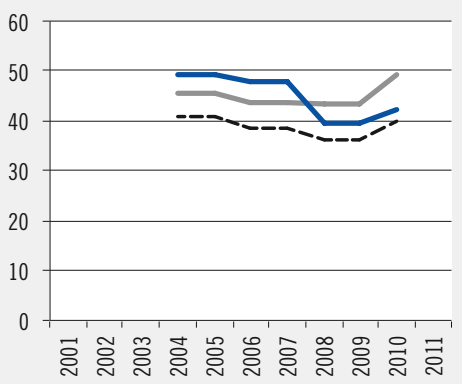

3.2.3 Knowledge-intensive services exports as $\%$ total service exports

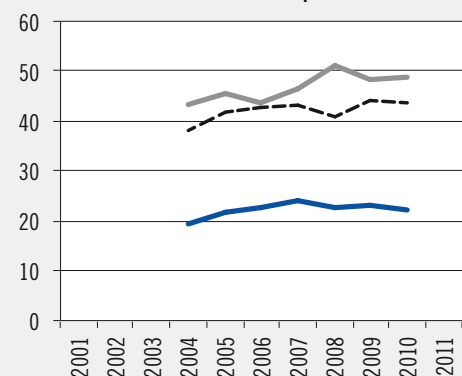

- Leaders

Österreich 
Die Einzelindikatoren bestätigen das von früheren Analysen von IUS/EIS bekannte Stärken/ Schwächen-Muster: Gewisse Schwächen zeigen sich weiterhin in der tertiären Ausbildung, der Risikokapitalausstattung, den Lizenz- und Patenteinnahmen und wissensintensiven Dienstleistungsexporten. Stärken sind bei den Sekundärabschlüssen, den wissenschaftlichen Publikationen, den F\&E-Ausgaben der Unternehmen, den Medium- und High-Tech-Sachgüterexporten sowie beim geistigen Eigentum festzustellen. Bei den aus dem Community Innovation Survey (CIS) abgeleiteten Indikatoren (von den sechs aus dem CIS entnommenen Indikatoren betreffen vier das Innovationsverhalten von KMU) weist Österreich im Zeitablauf merkliche Schwankungen auf; diese sind aber auf geänderte Rahmenbedingungen bei Design und Durchführung dieser Befragung zurückzuführen. Bei vielen der $25 \mathrm{im}$ IUS versammelten Indikatoren zeigen Innovation Leaders bzw. Followers und Österreich ähnliche Tendenzen - dies bedeutet, dass die Stärken/ Schwächen-Strukturen Österreichs in den letzten 5-10 Jahren relativ stabil waren.

Abschließend sei erwähnt, dass von seiner Idee und Durchführung her der IUS auf strukturelle Aspekte abzielt; dementsprechend weisen viele der Indikatoren eine langfristige Perspektive auf. Unmittelbare Reaktionen auf veränderte Politikbedingungen, in Form kurzfristiger substanzieller Verbesserungen im IUS, sind daher nur bedingt $\mathrm{zu}$ erwarten; der IUS (wie auch ähnliche andere Benchmark-Studien) soll hingegen strukturelle Schwächen und Stärken aufzeigen, um daraus langfristige Perspektiven ableiten zu können.

\subsection{2 "Innovationsindikator" der Deutschen Telekom Stiftung und des Bundesverbands der Deutschen Industrie}

Der "Innovationsindikator" der Deutschen Telekom Stiftung und des Bundesverbands der Deut- schen Industrie (BDI) wurde erstmals 2005 vorgelegt und im Jahr 2011 grundlegend überarbeitet. Er vergleicht die Innovationsleistung von 28 Industrie- und Schwellenländern anhand von 38 Einzelindikatoren, die fünf Feldern (Wirtschaft, Wissenschaft, Bildung, Staat und Gesellschaft) zugeordnet sind. Er beruht auf einer ähnlichen methodischen Basis wie des IUS. Im Gegensatz zum IUS spielen allerdings „weiche“ Faktoren wie Einschätzungen von ExpertInnen, gesellschaftliche Einstellungen zu Innovation sowie die Vernetzung von Innovationsakteuren eine größere Rolle, während die Innovationsleistung von KMU nicht gesondert betrachtet wird und Indikatoren aus den Community Innovation Surveys außen vor bleiben. Außerdem wird die Position eines Landes nicht im Vergleich zu allen anderen Ländern bestimmt, sondern gegenüber einer Gruppe von besonders leistungsstarken bzw. größeren Ländern (USA, Japan, Deutschland, Frankreich, Großbritannien, Italien und der Schweiz), d.h. es wird ein anspruchsvollerer Maßstab angelegt.

Österreich nimmt im aktuellen „Innovationsindikator 2012" den 11. Platz ein (siehe Abb. 9). Im Vergleich zum Vorjahresranking verlor Österreich drei Plätze, da einige im Jahr 2011 noch knapp hinter Österreich gelegene Länder (Belgien, USA und Dänemark) ihre Indikatorwerte erhöhen konnten, während Österreichs Wert konstant blieb. Im längerfristigen Vergleich zählt Österreich allerdings zu den Ländern, die ihre Position im „Innovationsindikator" deutlich verbessern konnten. Der Gesamtindikatorwert Österreichs stieg zwischen 2000 und 2011 von 44 auf 53 Punkte. ${ }^{19}$ In Europa ist dies hinter den Niederlanden, Norwegen und Irland die größte Verbesserung. Damit konnte Österreich seinen Abstand zu mehreren Ländern, die Anfang der 2000er Jahre noch zu den Innovationsführern zählten, wie Schweden, die USA und Finnland, deutlich verringern. Gleichwohl liegt Österreich

19 Die Indikatorwerte werden ähnlich wie im IUS über eine Normierung der Einzelindikatoren an den höchsten und niedrigsten Werten der führenden Innovationsnationen und eine Gleichgewichtung berechnet. Der Wertebereich der Indikatoren liegt zwischen 0 und 100. Eine Erhöhung bedeutet somit, dass sich ein Land gegenüber den führenden Innovationsnationen verbessert hat. 
Abb. 9 Länderranking im „Innovationsindikator“ 2000 und 2011

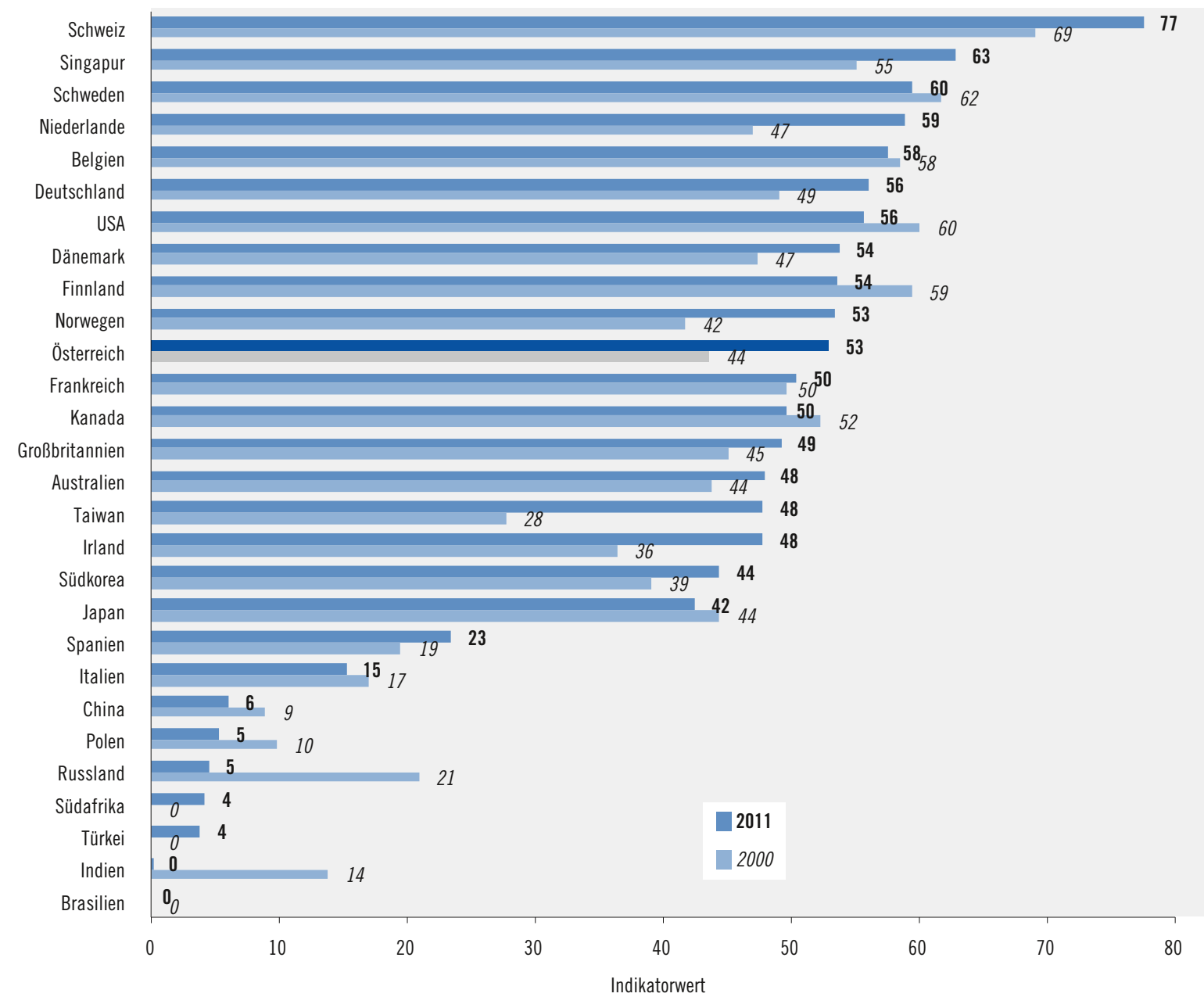

Anm.: Alle Indikatorwerte auf Basis der 2011 vorgenommenen methodischen Revision.

Quelle: Deutsche Telekom Stiftung/BDI: Innovationsindikator 2012. Berechnungen des Fraunhofer-ISI und ZEW

auch beim „Innovationsindikator" am Ende einer Gruppe von „Innovation Followers". Der Abstand zu den Ländern mit den höchsten Indikatorwerten - 2012 waren dies die Schweiz und Singapur - ist weiterhin beträchtlich und hat sich in den vergangenen zehn Jahren nicht merklich verringert.

Österreichs Stärken im „Innovationsindikator" liegen in der Wissenschaft (2012: Rang 10) und im Bereich der gesellschaftlichen Einstellungen zu Innovation (2012: Rang 7), wobei der letztgenannte Subindikator nur ein geringes Gewicht für die Performance insgesamt hat. In der Wissenschaft konnte Österreich seinen Indikatorwert seit Mitte der 1990er Jahre deutlich und beinahe kontinuierlich erhöhen. Hinter dieser Entwicklung steht u.a. die Ausweitung der Patentaktivitäten der Universitäten und staatlichen Forschungseinrichtungen, die Zunahme des Anteils ausländischer Studierender, die Erhöhung der Anzahl Promovierter in den Natur- und Ingenieurwissenschaften sowie die verstärkte Zitierung von Publikationen österreichischer WissenschaftlerInnen. 
Ebenfalls verbessert hat sich Österreich beim Subindikator "Wirtschaft", wenngleich hier in den jüngsten Jahren kaum noch eine Erhöhung des Indikatorwerts beobachtet werden konnte. Neben der Steigerung der F\&E-Ausgaben der Wirtschaft, hohen Produktivitätszuwächsen sowie einer Verbesserung der Handelsbilanz bei Hochtechnologiegütern zählte lange Zeit auch die steuerliche F\&E-Förderung zu den Pluspunkten der positiven Entwicklung Österreichs. Bei der steuerlichen F\&E-Förderung ist Österreich allerdings jüngst insofern zurückgefallen, als einige andere Länder aus der Referenzgruppe - namentlich Frankreich und Japan - ihr Fördersystem deutlich generöser ausgestaltet haben. Dadurch ist auch der Indikatorwert im Bereich Staat nach 2007 deutlich zurückgegangen, da die steuerliche F\&E-Förderung ein wesentliches Element der Innovationspolitik ist. Der Rückgang des Indikator- werts bedeutet allerdings nicht, dass sich die staatliche Unterstützung für F\&E in Österreich verschlechtert hätte, sondern dass andere Länder an dieser Stelle an Attraktivität gewonnen haben.

Ein weiterer Grund für die Abnahme des Indikatorwerts im Subsystem Staat sind relative Positionsverschlechterungen im staatlichen Bildungsbereich. Im Vergleich zu anderen Ländern waren die Hochschulausgaben je Studierenden weniger dynamisch, außerdem haben sich die Experteneinschätzungen zur Qualität von staatlichen Bildungs- und Forschungseinrichtungen verschlechtert. Generell ist der Subindikator Bildung eher eine Schwachstelle der österreichischen Performance im Innovationsindikator. In den vergangenen 15 Jahren konnte hier keine nachhaltige Verbesserung der Indikatorwerte erreicht werden.

Abb. 10: Indikatorwerte Österreichs im „Innovationsindikator“ 1990-2011 nach Subindikatoren

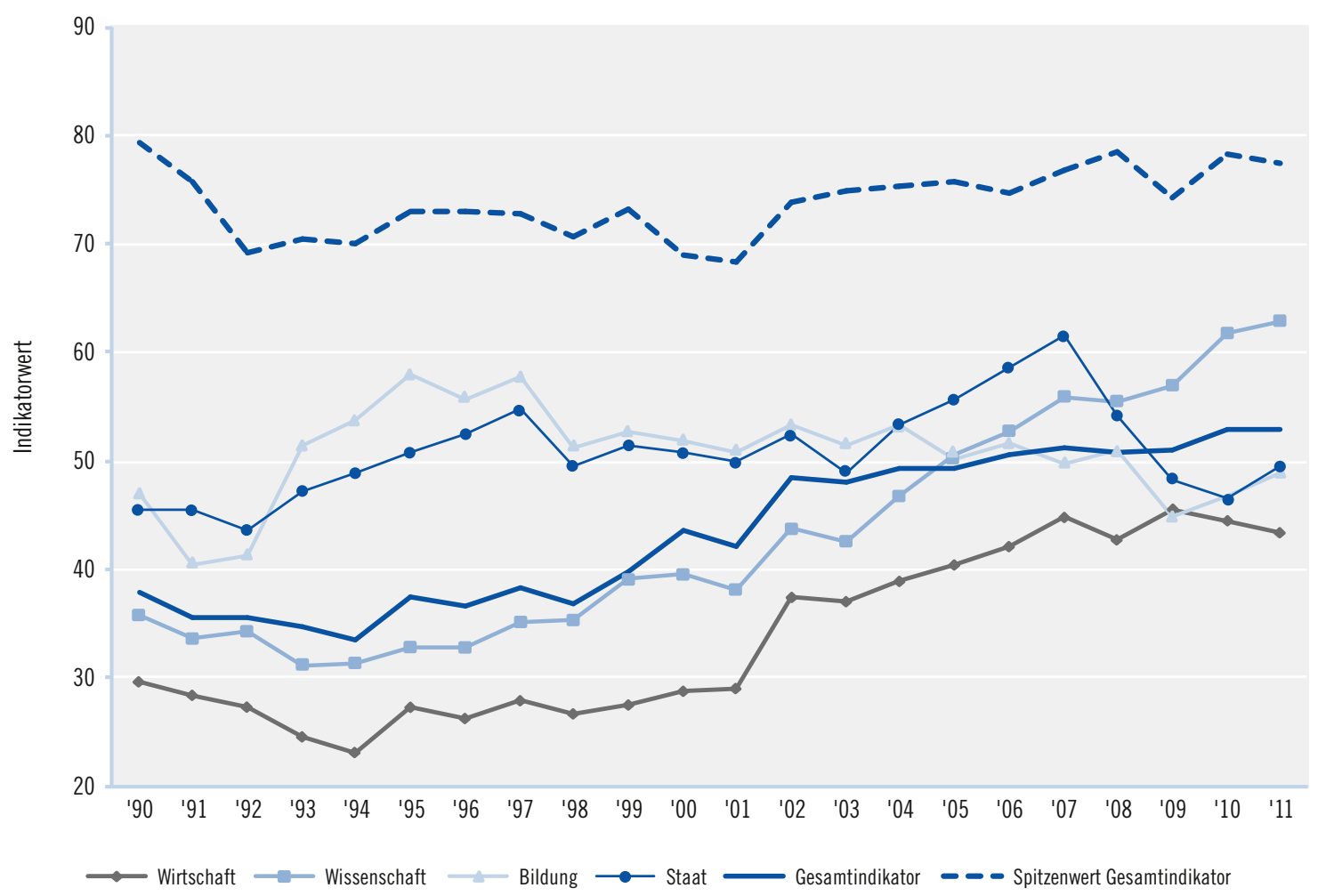

Anm.: Alle Indikatorwerte auf Basis der 2011 vorgenommenen methodischen Revision.

Quelle: Deutsche Telekom Stiftung/BDI: Innovationsindikator 2012. Berechnungen des Fraunhofer-ISI und ZEW. 
Der "Innovationsindikator" unterscheidet zwischen Input- und Outputindikatoren ${ }^{20}$. Inputindikatoren repräsentieren Investitionen in das Innovationssystem, während Outputindikatoren das Ergebnis von Forschungsund Innovationsbemühungen widerspiegeln. Österreich zeichnet sich durch ein besonders günstiges Verhältnis zwischen Outputs und Inputs aus, das als eine hohe Produktivität des Innovationssystems interpretiert werden kann. Diese "Systemproduktivität" konnte von sehr niedrigen Werten Anfang der 1990er Jahre deutlich gesteigert werden, wobei sich die Outputindikatoren stärker verbessert haben als die Inputindikatoren. So konnte sich Österreich vom 15. Rang im Jahr 1995 auf den 7. Rang im Jahr 2011 nach vorne arbeiten.

\subsection{Globale Trends in den F\&E-Ausgaben}

Die weltweiten Ausgaben für F\&E sind in den vergangenen zehn Jahren trotz der schweren Wirtschafts- und Finanzkrise um mehr als die Hälfte gestiegen. Gleichzeitig hat sich die Verteilung der weltweiten F\&E-Ausgaben deutlich zugunsten schnell wachsender Länder - insbesondere im Raum Asien - verschoben. Der folgende Abschnitt zeigt diese Veränderungen auf und geht der veränderten Rolle Österreichs und der Europäischen Union (EU-27) in diesem Prozess nach.

Um die globale Dimension und einen möglichst großen Zeitraum abzudecken, kommen dabei unterschiedliche Datenquellen (UNESCO, OECD, Eurostat und Daten nationaler statistischer Ämter) zur Anwendung. Zumindest bei den Daten der EU-27 und OECD-Mitgliedsstaaten ist eine internationale Vergleichbarkeit durch die Anwendung des Frascati-Handbuchs ${ }^{21}$ in der Datenerhebung gewährleistet. Dieses definiert Forschung und experimentelle Entwicklung (F\&E) als: „(...) schöpferische Tätigkeit, welche auf systematische Weise unter Verwendung wissenschaftlicher Methoden mit dem Ziel durchgeführt wird, den Stand des Wissens zu vermehren sowie neue Anwendungen dieses Wissens zu erarbeiten". Betrachtet werden die gesamten F\&EAusgaben (Gross Expenditure on R\&D - GERD) in allen Durchführungssektoren (Unternehmenssektor, Hochschulsektor, Sektor Staat und Privater Gemeinnütziger Sektor). Nicht-OECD-Mitglieder folgen nur zum Teil den einheitlichen Definitionen, Standards und Methoden des Frascati Manuals. Ein Vergleich von OECD- und NichtOECD-Ländern ist daher mit einer gewissen Vorsicht zu interpretieren. Des Weiteren ist die Datenverfügbarkeit für OECD bzw. EU-Länder deutlich besser als für Nicht-OECD-Mitglieder und über längere Zeiträume möglich.

\subsubsection{Globale Veränderungen}

Im folgenden Abschnitt wird zunächst die weltweite Verteilung der F\&E-Ausgaben (und deren Veränderung) auf die großen Weltregionen zwischen 2002 und 2009 betrachtet. In den sieben Jahren von 2002 bis $2009^{22}$ sind laut Daten der UNESCO die weltweiten F\&E-Ausgaben von 788 Mrd. PPP US \$ ${ }^{23}$ auf 1.277 Mrd. PPP US \$ um rund $62 \%$ gestiegen.

Betrachtet man zunächst die Daten für 2009 (siehe Abb. 11), das aktuellste Jahr mit vollständigen weltweiten Daten, zeigt sich annähernd eine Drittelung der weltweiten F\&E-Ausgaben zwischen Nordamerika $(32,7 \%)$, Europa (inkl. europäischer Staaten, die keine EU-Mitglieder sind) $(28,5 \%)$ und Asien (33,1 \%). Südamerika, Afrika und Ozeanien spielen mit einem gemeinsamen Anteil von 5,7 \% eine vergleichsweise kleine Rolle. Seit 2002 zeigt sich für sämtliche Weltregionen ein deutliches absolutes Wachstum. Der Umfang dieses Wachstums war aber äußerst unterschied-

20 Die Liste der Einzelindikatoren findet sich im Anhang I.

21 OECD (2002), S. 30

22 Nur für die Jahre 2002, 2007 und 2009 liegen vollständige Daten zu den weltweiten F\&E-Ausgaben nach Ländern und Regionen vor.

23 PPP US \$: Purchasing power parity US \$ (Kaufkraftparitäten US \$) zu laufenden Preisen. 
Abb. 11: F\&E-Ausgaben (GERD), 2002, 2007 und 2009

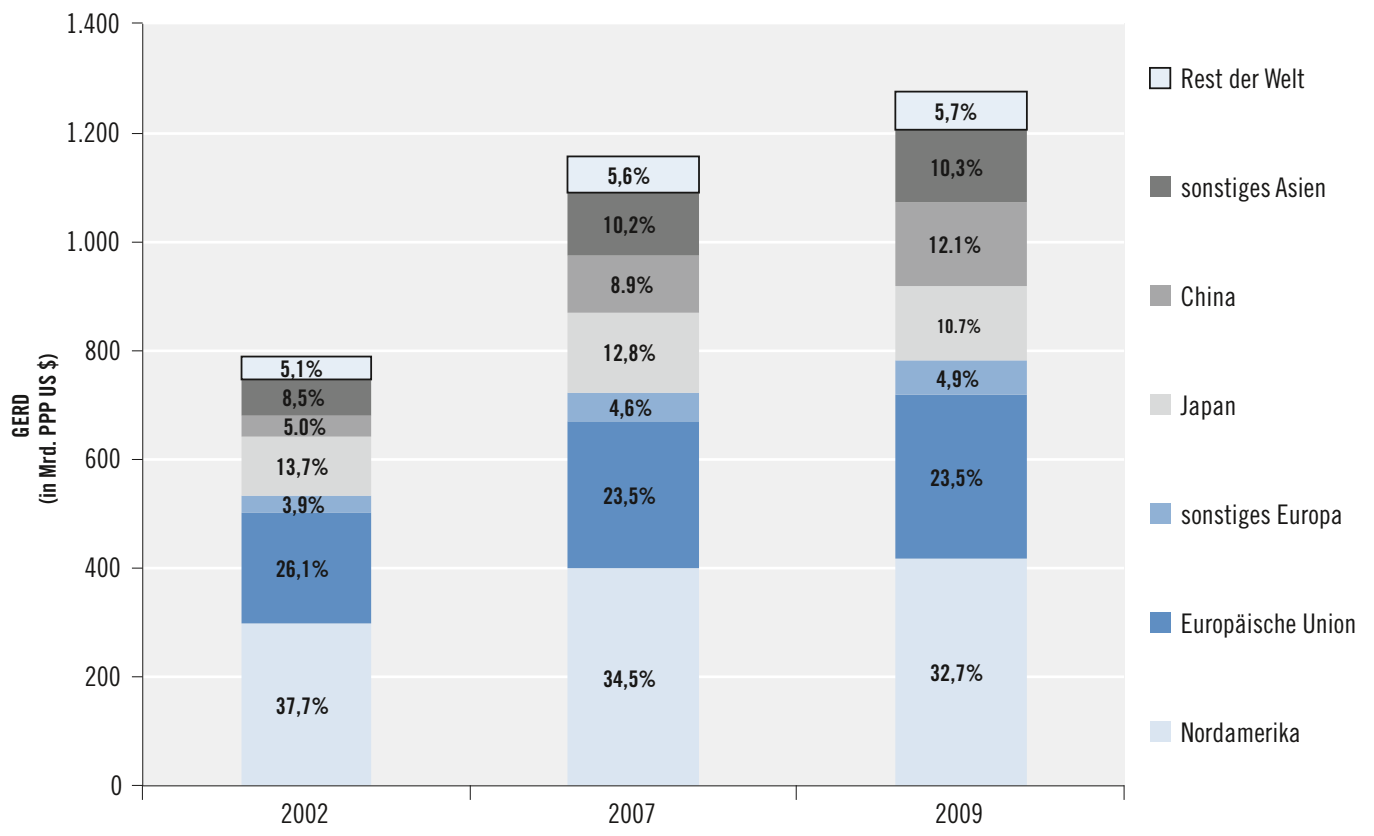

Anm.: sonstiges Asien: Hong Kong ,Indonesien, Malaysia, Philippinen, Südkorea und Singapur.

Quelle: UNESCO. Berechnungen AIT.

lich und führte zu einer Verschiebung der Anteile der betrachteten Länder und Regionen an den weltweiten F\&E-Ausgaben, in erster Linie weg von Nordamerika und Europa hin in Richtung Asien.

Mit einer annähernden Vervierfachung der F\&E-Ausgaben weist China dabei von den größeren Volkswirtschaften das mit Abstand höchste relative Wachstum auf; auch andere asiatische Wachstumsmärkte wie Indien oder Korea weisen ein überdurchschnittliches Wachstum der F\&EAusgaben auf. Gleichzeitig ist mit Japan ein asiatisches Land auch das mit dem geringsten relativen Anstieg (+27\%) im Beobachtungszeitraum. Neben dem Bedeutungsgewinn von Asien kam es somit auch zu einer deutlichen Verschiebung innerhalb Asiens. Der relative Anstieg der F\&EAusgaben in der EU-27 lag hingegen mit $+46 \%$ zwar etwas unter dem weltweiten Wert, jedoch nicht nur deutlich über Japan, sondern auch über dem Vergleichswert für Nordamerika (40 \%).

Das hohe relative Wachstum Chinas ist zum
Teil auch bedingt durch das vergleichsweise geringe Ausgangsniveau. Betrachtet man die absoluten Zuwächse, so sind diese in Nordamerika mit 120 Mrd. PPP US \$ am höchsten, gefolgt von China mit 115 Mrd. PPP US \$ und der Europäischen Union mit 94 Mrd. PPP US \$. Mit insgesamt 127 Mrd. PPP US \$ Zuwachs liegt Europa (inkl. europäischer Staaten, die keine EUMitglieder sind) absolut sogar knapp über den entsprechenden Werten für Nordamerika und China.

Als Folge des hohen Wachstums der chinesischen F\&E-Ausgaben hat sich der Anteil Chinas an den weltweiten F\&E-Ausgaben von $5 \%$ im Jahr 2002 auf 12 \% im Jahr 2009 erhöht (Abb. 11). Dieser Bedeutungsgewinn Chinas und anderer asiatischer Volkswirtschaften (exkl. Japan) führte zunächst von 2002 bis 2007 zu einem Rückgang des Anteils Nordamerikas (3\%-Punkte), der EU-27 (3 \%-Punkte) und Japans (1\%-Punkte) an den weltweiten F\&E-Ausgaben. In den beiden folgenden Jahren bis 2009 war der 
Anteil der EU-27 stabil, während Nordamerika einen weiteren $2 \%$-Punkteanteil und Japan ebenfalls einen $2 \%$-Punkteanteil an den weltweiten F\&E-Ausgaben einbüßte. Trotz des bemerkenswerten Anstiegs der Bedeutung Chinas lagen im Jahr 2009 die F\&E-Ausgaben gemessen in PPP US \$ in der EU-27 immer noch etwa auf dem doppelten Niveau von China.

\subsubsection{Langfristige Entwicklung innerhalb der OECD}

Unterscheidet man bei der Betrachtung der weltweiten F\&E-Ausgaben zwischen der OECD und Nicht-OECD-Mitgliedsländern, so verfügen die OECD-Staaten im Jahr 2009 über einen Anteil von $75 \%$ an den weltweiten F\&E-Ausgaben. Bedingt durch die steigende Bedeutung Chinas und anderer stark wachsenden Wirtschaften außerhalb der OECD entspricht dies einem deutlichen Rückgang gegenüber 2002, als die OECD-Länder in Summe noch über einen Anteil von 85 \% verfügten. Auf Grund der Datenverfügbarkeit und besseren Vergleichbarkeit der Erhebungsmethodik ist eine Analyse der Entwicklung der F\&EAusgaben vor dem Jahr 2002 nur für die OECDMitgliedsstaaten möglich (Abb. 12).

Über den gesamten 30-jährigen Beobachtungszeitraum bleibt der Anteil der EU-27 an den F\&EAusgaben in der OECD äußerst stabil und liegt stets zwischen 30 und $35 \%$. Während in den

Abb. 12: Anteile an den gesamten F\&E-Ausgaben der OECD (in PPP US \$, 1981 bis 2010)

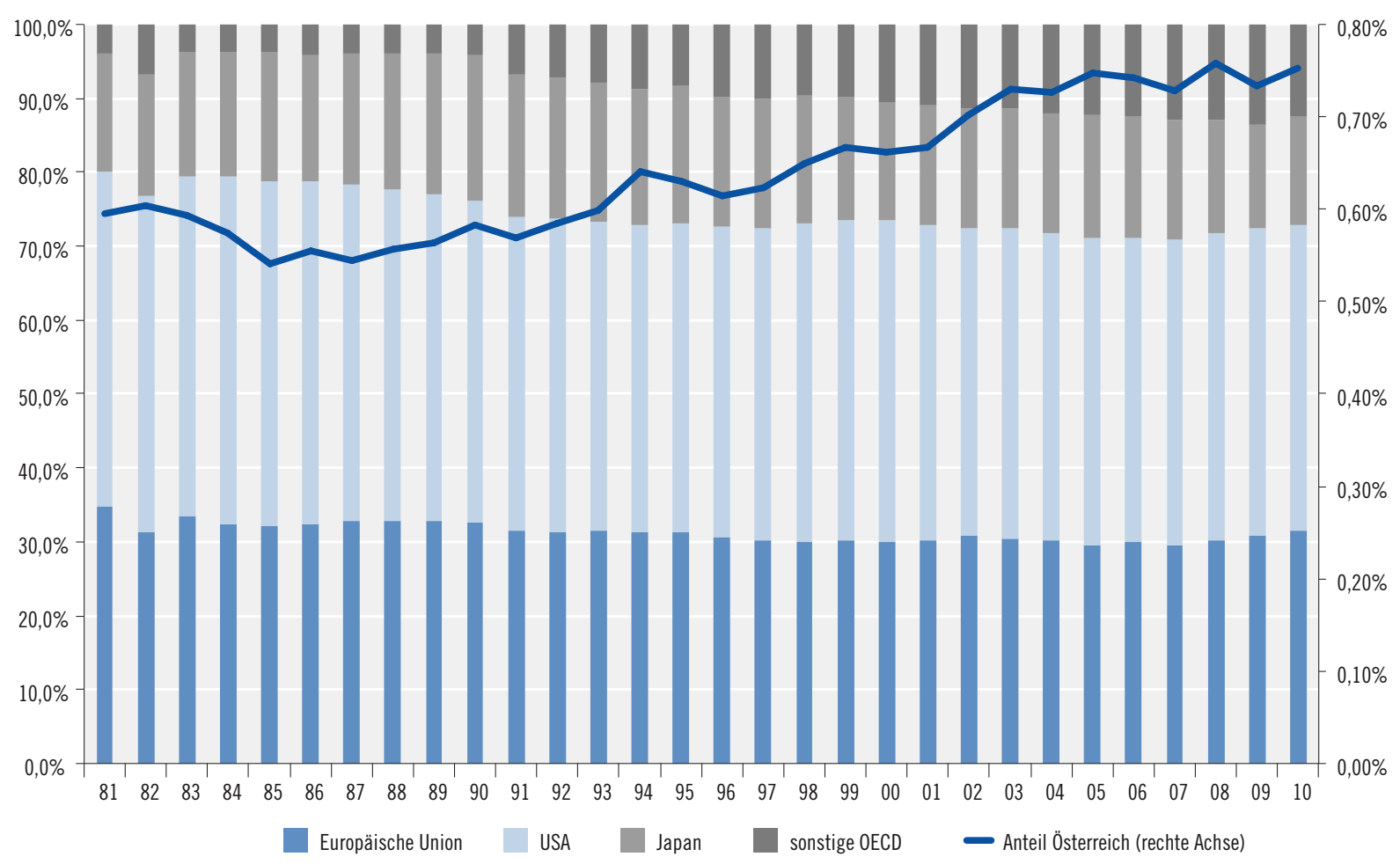

Quelle: OECD. Berechnungen AIT. 
1990ern eine leicht absteigende Tendenz zu erkennen ist, steigt der Anteil ab dem Jahr 2000 wieder leicht an und liegt im letzten Beobachtungsjahr 2010 mit 31,5 \% fast exakt auf demselben Niveau wie im Jahr 1982 mit 31,4 \%.

Mit einem Anteil von 41,5 \% im Jahr 2010 sind die USA das Land mit dem mit Abstand größten Anteil an den gesamten F\&E-Ausgaben im OECD-Raum und an den gesamten weltweiten F\&E-Ausgaben. Dieser Anteil lag in den 1980er Jahren mit rund 45 \% noch etwas höher und ist dann wie in der EU in den 1990ern leicht zurückgegangen. Im Gegensatz dazu schaffte es die USA jedoch nur kurzfristig - um das Jahr 2000 - ihren Anteil wieder zu erhöhen und liegt zuletzt wieder etwa beim selben Anteil wie in den frühen 1990ern. Über die gesamten 30 Jahre hinweg bewegte sich somit auch der Anteil der USA innerhalb eines relativ engen Bereichs zwischen $41 \%$ und $46 \%$ der F\&E-Ausgaben innerhalb der OECD.

Etwas größere Veränderungen in der Bedeutung sind für Japan zu konstatieren. Zunächst stieg der Anteil Japans an den gesamten F\&EAusgaben in der OECD von 1981 bis 1990 kontinuierlich von $16,0 \%$ auf $19,7 \%$. Danach folgte eine Phase des ebenso kontinuierlichen Rückgangs an diesem Anteil auf zuletzt nur mehr $14,5 \%$.

Deutlich zugenommen hat in den vergangen 30 Jahren die Bedeutung der sonstigen OECDLänder. Diese Gruppe umfasst einerseits mit Kanada und Australien große traditionelle Industrieländer, beinhaltet aber auch Schwellenländer wie Korea oder Chile. Zum Teil sind diese Länder auch erst im Beobachtungszeitraum der OECD beigetreten und wurden erst ab diesem Beitritt in den Daten berücksichtigt. Es ist daher von einer leichten Überschätzung des Wachstums der sonstigen OECD-Staaten auszugehen.

Vor dem Hintergrund des leicht sinkenden Anteils der EU-27 an den F\&E-Ausgaben der OECD-Staaten ist der gleichzeitige Anstieg des Anteils Österreichs bemerkenswert. Während in den 1980ern nur rund 0,6 \% der F\&E-Ausgaben in der OECD auf Österreich entfielen, stieg dieser Anteil seit den 1990er-Jahren kontinuierlich auf zuletzt rund $0,75 \%$. In absoluten Zahlen sind die österreichischen F\&E- Ausgaben von gut fünf Mrd. PPP US \$ auf fast acht Mrd. PPP US \$ gewachsen. Damit wuchsen die F\&E-Ausgaben in Österreich deutlich über dem OECD- oder EUSchnitt und konnten somit mit den hohen globalen Wachstumsraten Schritt halten.

\subsubsection{Verschiebungen der Ausgaben für F\&E innerhalb der Europäischen Union}

In den 15 Jahren von 1995 bis 2010 sind die gesamten F\&E-Ausgaben der EU-27 von $138 \mathrm{Mrd}$. PPP US \$ auf 305 Mrd. PPP US \$ auf mehr als das Doppelte gestiegen. Während dabei alle Mitgliedsstaaten ihre absoluten F\&E-Ausgaben steigerten, kam es auch innerhalb der EU-27 zu starken Verschiebungen der Anteile der Länder an den gesamten F\&E-Ausgaben. Tab. 2 stellt daher diese Anteile der EU-Staaten dar. Zusätzlich wird die Veränderung in \%-Punkten von 1995 bis 2010 sowie die Positionierung der Länder im Innovation Union Scoreboard ${ }^{24}$ (IUS) gezeigt.

Der Anteil Österreichs an den gemeinsamen F\&E-Ausgaben der EU-27 hat sich dabei in den letzten Jahren deutlich von 2,1 \% im Jahr 1995 auf $3 \%$ im Jahr 2010 erhöht. Dabei handelt es sich um den zweitgrößten Anstieg gemessen in \%-Punkten in der EU-27, nur Spanien konnte deutlich stärker anteilsmäßig gewinnen. Aus der Gruppe der Innovation Leader (Finnland, Dänemark, Deutschland und Schweden) konnten Dänemark und Finnland den Anteil in ähnlichem Ausmaß steigern. Im Gegensatz dazu gingen die Anteile Deutschlands und Schwedens - allerdings von einem hohen Niveau - leicht zurück. Innerhalb der Gruppe der Innovation Follower (IUS Rang 5 bis 14), zu der auch Österreich gehört, ist Österreich das einzige Land mit einem deutlich steigenden Anteil an den F\&E-Ausgaben der EU-27. Die beiden größten Volkswirtschaften

24 Siehe Europäische Kommission (2011a). 
Tab. 2: Länderanteile an den gesamten EU-27 F\&E-Ausgaben (in PPP US \$), 1995, 2000, 2005 und 2010

\begin{tabular}{|c|c|c|c|c|c|c|}
\hline & uc Danr & 1005 & בnחמ & 2005 & 2010 & Veränderung \\
\hline & IUS Kallig & 1990 & 2000 & 2000 & 2010 & 2010 zu 1995 \\
\hline Spanien & 17 & $3,6 \%$ & $4,2 \%$ & $5,8 \%$ & $6,7 \%$ & $3,1 \%$ \\
\hline Österreich & 8 & $2,1 \%$ & $2,4 \%$ & $2,9 \%$ & $3,0 \%$ & $0,9 \%$ \\
\hline Finnland & 4 & $1,6 \%$ & $2,4 \%$ & $2,4 \%$ & $2,5 \%$ & $0,9 \%$ \\
\hline Portugal & 15 & $0,5 \%$ & $0,7 \%$ & $0,8 \%$ & $1,4 \%$ & $0,9 \%$ \\
\hline Dänemark & 2 & $1,6 \%$ & $1,9 \%$ & $1,9 \%$ & $2,2 \%$ & $0,7 \%$ \\
\hline Polen & 23 & $1,3 \%$ & $1,4 \%$ & $1,3 \%$ & $1,8 \%$ & $0,5 \%$ \\
\hline Irland & 9 & $0,6 \%$ & $0,7 \%$ & $0,9 \%$ & $1,0 \%$ & $0,5 \%$ \\
\hline Tschechische Republik & 16 & $0,9 \%$ & $1,0 \%$ & $1,3 \%$ & $1,4 \%$ & $0,4 \%$ \\
\hline Ungarn & 18 & $0,5 \%$ & $0,5 \%$ & $0,7 \%$ & $0,8 \%$ & $0,3 \%$ \\
\hline Baltische Staaten & 13,25 und 27 & n.V. & $0,2 \%$ & $0,3 \%$ & $0,4 \%$ & $0,2 \%$ \\
\hline Belgien und Luxemburg* & 5 und 9 & $2,7 \%$ & $3,2 \%$ & $2,9 \%$ & $2,9 \%$ & $0,2 \%$ \\
\hline Griechenland & 19 & $0,5 \%$ & $0,6 \%$ & $0,7 \%$ & $0,6 \%$ & $0,1 \%$ \\
\hline Slowenien & 11 & $0,3 \%$ & $0,3 \%$ & $0,3 \%$ & $0,4 \%$ & $0,1 \%$ \\
\hline Malta und Zypern & 20 und 12 & n.V. & n.V. & $0,1 \%$ & $0,1 \%$ & $0,0 \%$ \\
\hline Bulgarien & 26 & $0,2 \%$ & $0,1 \%$ & $0,2 \%$ & $0,2 \%$ & $0,0 \%$ \\
\hline Slowakei & 22 & $0,3 \%$ & $0,2 \%$ & $0,2 \%$ & $0,3 \%$ & $0,0 \%$ \\
\hline Rumänien & 24 & $0,7 \%$ & $0,3 \%$ & $0,4 \%$ & $0,5 \%$ & $-0,2 \%$ \\
\hline Schweden & 1 & $4,5 \%$ & $5,1 \%$ & $4,6 \%$ & $4,1 \%$ & $-0,4 \%$ \\
\hline Niederlande & 7 & $4,7 \%$ & $4,7 \%$ & $4,7 \%$ & $4,3 \%$ & $-0,5 \%$ \\
\hline Italien & 14 & $8,4 \%$ & $8,3 \%$ & $7,8 \%$ & $8,0 \%$ & $-0,5 \%$ \\
\hline Deutschland & 3 & $29,0 \%$ & $28,5 \%$ & $28,0 \%$ & $28,3 \%$ & $-0,8 \%$ \\
\hline Vereinigtes Königreich & 6 & $15,8 \%$ & $15,2 \%$ & $14,8 \%$ & $12,8 \%$ & $-3,0 \%$ \\
\hline Frankreich & 10 & $19,8 \%$ & $17,9 \%$ & $17,1 \%$ & $16,4 \%$ & $-3,5 \%$ \\
\hline
\end{tabular}

in dieser Gruppe, Frankreich und das Vereinigte Königreich, sind hingegen die beiden Länder mit dem stärksten Rückgang.

Während große Volkswirtschaften wie Deutschland, Frankreich und das Vereinigte Königreich innerhalb der EU an Bedeutung verlieren, steigt tendenziell der Anteil von kleineren und mittleren Staaten. Neben Spanien, Österreich, Dänemark und Finnland gewannen in erster Linie die zwölf neuen EU-Mitgliedsstaaten an Bedeutung. Trotz dieses Bedeutungsgewinns ist deren Anteil mit in Summe 5,7 \% im Jahr 2010 weiterhin vergleichsweise gering.

Eine direkte Folge der Zuwächse kleiner Länder ist die Abnahme der Konzentration der F\&EAusgaben in der EU-27. Der Anteil der vier größ- ten Mitgliedsstaaten - Deutschland, Frankreich, Italien und das Vereinigte Königreich - an den gesamten F\&E-Ausgaben sank von $73 \%$ im Jahr 1995 auf 65,5 \% im Jahr 2010. Die Entwicklung der F\&E-Quote auf EU-Ebene wird daher im zunehmenden Maß auch von den Entwicklungen in kleinen und mittleren Mitgliedsstaaten bestimmt.

\subsubsection{Internationalisierung von F\&E}

Treibende Kraft bei der Steigerung der F\&E-Ausgaben weltweit waren die Ausgaben des Unternehmenssektors für F\&E. Dabei wuchsen die F\&E-Ausgaben von Unternehmen außerhalb des jeweiligen Heimatlandes besonders stark, ein 
Phänomen, das auch als Internationalisierung bzw. Globalisierung von F\&E bezeichnet wird. Es stellt sich somit die Frage, inwieweit die F\&EAusgaben ausländischer Firmen $z u$ den massiven Anstiegen der gesamten F\&E-Ausgaben und zu den beobachteten Verschiebungen beigetragen haben.

Abb. 13 stellt dafür den Anteil der F\&E-Ausgaben ausländischer Unternehmen an der gesamten F\&E-Quote dar. Es zeigt sich, dass die auslandsfinanzierte Forschung in einigen kleineren und mittleren Ländern für einen wesentlichen Teil der F\&E-Quote verantwortlich ist. Gleichzeitig spielt diese Forschung durch ausländische Unternehmen in den großen Ländern wie den USA, Japan oder Deutschland, die für große Teile der gesamten F\&E-Ausgaben verantwortlich sind, eine eher geringe Rolle. Vollständige Daten für F\&E-Ausgaben ausländischer Unternehmen für China liegen nicht vor, aber basierend auf Daten US-amerikanischer Unternehmen, die in China F\&E betreiben, wurden die F\&E-Ausgaben ausländischer Unternehmen in China auf zwischen 2,3 und 6,1 Mrd. $€$ geschätzt ${ }^{25}$. Dies entspricht einem Anteil von unter $10 \%$ an den gesamten F\&E-Ausgaben in China. Trotz der zunehmenden Bedeutung Chinas als F\&E-Standort für ausländische Unternehmen kann somit der starke Anstieg der F\&E in China nicht nur durch die F\&E-Aktivitäten ausländischer Firmen in China erklärt werden. Neben dem direkten Beitrag ausländischer Unternehmen in China kommen auch indirekte Effekte, wie spezifische Formen von Wissenstransfer, zum Tragen, die einheimische Firmen $\mathrm{zu}$ höheren F\&E-Ausgaben bewegen.

\section{Abb. 13: Beiträge ausländischer Unternehmen zur F\&E-Quote, 2007}

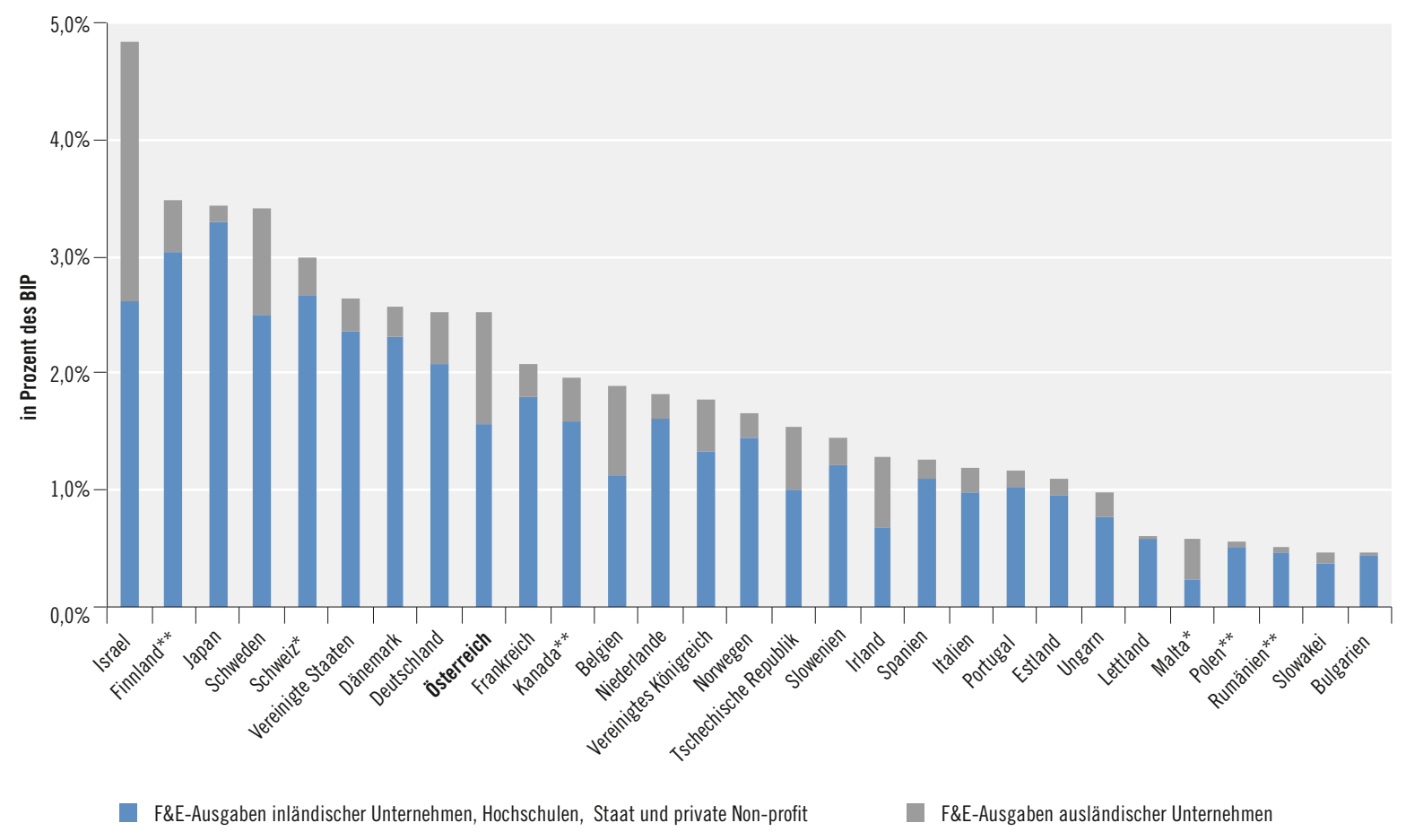

Anm.: * 2008 statt 2007, ** 2006 statt 2007.

Quelle: OECD, Eurostat, nationale statistische Ämter. Berechnungen AIT.

25 Vgl. Dachs et al. (2012). 
Ausländische Unternehmen leisten einen besonders hohen Beitrag zur F\&E-Quote in Schweden, Österreich, Belgien, der Tschechischen Republik und Irland. Mit der Ausnahme von Schweden konnten alle diese Länder auch innerhalb der letzten 15 Jahre ihren Anteil an den F\&E-Ausgaben der EU-27 deutlich erhöhen. Schweden verzeichnete zwar keinen solchen Anstieg, weist aber die zweihöchste F\&E-Quote innerhalb der EU-27 auf. F\&E-Ausgaben ausländischer Unternehmen haben somit einen relativ geringen Einfluss auf die globale Verteilung der F\&E-Ausgaben, spielen aber gerade in kleineren und mittleren Ländern eine bedeutende und weiter zunehmende Rolle für die Entwicklung der F\&E-Quote. In Österreich entfällt mittlerweile rund ein Drittel der gesamten F\&E-Ausgaben auf die F\&EAusgaben von Unternehmen im ausländischen Besitz - im internationalen Vergleich ein außerordentlich hoher Wert. ${ }^{25 a}$

Die Internationalisierung von F\&E wird maßgeblich durch Unternehmen im Sachgüterbereich vorangetrieben und ist besonders stark in einigen exportintensiven Mittelhoch- und Hochtechnologie-Sektoren ausgeprägt ${ }^{26}$. Tab. 3 zeigt die F\&E-Ausgaben ausländischer Unternehmen in Österreich nach Herkunftsland und vergleicht diese mit den entsprechenden Ausgaben dieser Länder in der gesamten EU-27. Die F\&E-Ausgaben deutscher Unternehmen sind dabei mit einem Anteil von über der Hälfte der gesamten F\&E-Ausgaben ausländischer Unternehmen für Österreich von besonderer Bedeutung. Österreich ist aber auch innerhalb der EU-27 der wichtigste Auslandsstandort deutscher Unternehmen, 38 \% dieser Ausgaben konzentrieren sich auf Österreich. Darüber hinaus tragen auch Unternehmen aus der Schweiz, den Vereinigten Staaten, Kanada und der Niederlande maßgeblich zu den F\&E-Ausgaben in Österreich bei. Vor allem kanadische und Schweizer Unternehmen konzentrieren sich mit $28 \%$ bzw. $11 \%$ ihrer EUweiten Forschungsaktivitäten auf Österreich.

\section{Resümee}

Während in globaler Perspektive schnell wachsende asiatische Volkswirtschaften, insbesondere China, in den letzten Jahren deutlich ihre Anteile an den gesamten globalen F\&E-Ausgaben zu

Tab. 3: Österreich als Standort für F\&E ausländischer Unternehmen (2007, in Mio. €)

\begin{tabular}{|l|c|c|c|}
\multirow{2}{*}{\multicolumn{1}{c|}{ Herkunftsland }} & \multicolumn{2}{c|}{ F\&E ausländischer Firmen in } & $\begin{array}{c}\text { Anteil Österreich } \\
\text { an EU-27 }\end{array}$ \\
\cline { 2 - 3 } Deutschland & Österreich & EU-27 & $38 \%$ \\
Schweiz & 1,456 & 3,786 & $11 \%$ \\
Vereinigte Staaten & 309 & 2,941 & $2 \%$ \\
\hline Kanada & 228 & 13,535 & $28 \%$ \\
\hline Niederlande & 180 & 632 & $4 \%$ \\
Schweden & 177 & 4,442 & $4 \%$ \\
Großbritannien & 40 & 893 & $1 \%$ \\
Frankreich & 32 & 2,365 & $1 \%$ \\
\hline Belgien & 26 & 3,276 & $6 \%$ \\
Japan & 18 & 300 & $1 \%$ \\
Italien & 16 & 1,133 & $2 \%$ \\
Finnland & 14 & 705 & $4 \%$
\end{tabular}

Anm.: Nur Länder mit zumindest 10 Mio. € F\&E-Ausgaben in Österreich berücksichtigt. Quelle: OECD, Eurostat, nationale statistische Ämter. Berechnungen AIT.

25a siehe dazu Fußnote 1a, S. 9.

26 Ebenda. 
Lasten der USA, Japans und der EU-27 erhöhen konnten, gelang es Österreich als eines der wenigen EU-Länder, seinen Anteil stabil zu halten. Da dies in einem Umfeld massiv steigender globaler F\&E-Ausgaben geschah, ging dieser stabile Anteil Österreichs mit einer beträchtlichen absoluten Steigerung der Ausgaben einher. Der Anteil Österreichs an den F\&E-Ausgaben der EU-27 sowie der OECD stieg dadurch deutlich.

Trotz der Verschiebungen der globalen Verteilung der F\&E-Ausgaben in Richtung Asien sind Europa und Nordamerika im Jahr 2009 immer noch für über $60 \%$ der globalen F\&E-Ausgaben verantwortlich. Innerhalb der EU-27 konnte zwar ein Rückgang des Anteils der vier größten Volkswirtschaften festgestellt werden, zum Stand 2010 entfielen dennoch über $65 \%$ der EU27 F\&E-Ausgaben auf diese vier Länder. Neben Österreich konnte eine Reihe weiterer Länder relativ an Bedeutung gewinnen. Neben Spanien ist das in erster Linie eine Reihe kleinerer und mittlerer EU-Staaten inklusive der beiden Innovation Leader Dänemark und Finnland, aber auch Belgien, Irland und die Tschechische Republik.

Während der Anstieg der F\&E-Ausgaben in China nur zu einem sehr geringen Teil durch ausländische F\&E-Investitionen zu erklären ist, ist ihre Bedeutung in kleineren und mittleren EUStaaten deutlich größer. Im Fall von Österreich tragen ausländische Unternehmen bereits rund ein Drittel zu den gesamten F\&E-Ausgaben bei und sind somit ein maßgeblicher Treiber des festgestellten starken Anstiegs der F\&E-Ausgaben. Einen besonderen Stellenwert nimmt dabei das Nachbarland Deutschland ein. Nach den USA ist Österreich das zweitwichtigste Zielland für grenzüberschreitende F\&E-Ausgaben deutscher Unternehmen. Der Anteil deutscher Unternehmen an ausländischen F\&E-Investitionen in Österreich beträgt mehr als $50 \%$. 


\section{Die großen Förderagenturen des Bundes}

\subsection{Wissenschaftsfonds (FWF)}

Der FWF steht in Österreich für die Förderung der Grundlagenforschung, indem die vom FWF finanzierten Projekte ihren "Wert" aus ihrer Bedeutung für die Entwicklung der Wissenschaft, der Erweiterung des wissenschaftlichen Kenntnisstandes und des Grundlagenwissens definieren. Demgemäß ruht die Arbeit des FWF auf drei Säulen:

- Stärkung der wissenschaftlichen Leistungsfähigkeit Österreichs im internationalen Vergleich sowie seiner Attraktivität als Wissenschaftsstandort, vor allem durch Förderung von Spitzenforschung einzelner Personen bzw. Teams, aber auch durch Beiträge zur Verbesserung der Konkurrenzfähigkeit der Forschungsstätten und des Wissenschaftssystems in Österreich;

- Qualitative und quantitative Ausweitung des Forschungspotentials nach dem Prinzip „Ausbildung durch Forschung";

- Verstärkte Kommunikation und Ausbau der Wechselwirkungen zwischen Wissenschaft und allen anderen Bereichen des kulturellen, wirtschaftlichen und gesellschaftlichen Lebens, wobei insbesondere die Akzeptanz von Wissenschaft durch systematische Öffentlichkeitsarbeit gefestigt werden soll.

Der Qualitätsbenchmark des FWF ist in allen seinen Arbeitsbereichen die internationale wissenschaftliche Community. Die Qualität der vom FWF geförderten Forschungen wird mit einem durchgängigen Peer Review System gesichert: Nur ExpertInnen aus dem Ausland, deren Unbe- fangenheit überprüft wird, sind in den Begutachtungsprozess der an den FWF gestellten Förderanträge eingebunden. Jährlich werden über 5.000 derartige internationale Gutachten eingeholt.

Tab. 4 und Tab. 5 geben einen Überblick über die Förderungen des FWF im Jahr 2012. Das Jahr 2012 markiert mit einem Antragsvolumen von 676,7 Mio. $€$ einen neuen Höchstwert. Im Jahr 2012 lag das Bewilligungsvolumen des FWF mit 196,4 Mio. € knapp unter der 200 Mio. € Marke. Auch das stellt ein Rekordbewilligungsvolumen dar, allerdings beträgt die Steigerung gegenüber 2011 lediglich 0,6\%. Die Anzahl der entschiedenen Anträge war mit 2.216 im Jahr 2012 praktisch unverändert, die Anzahl der bewilligten Projekte mit 684 leicht rückläufig. Die Bewilligungsquote des FWF insgesamt hat sich 2012 nur marginal verändert. Gemessen an der Zahl der bewilligten Neuanträge sank die Quote von 30,6\% (2011) auf 30,2\%. Das Verhältnis der Neubewilligungssumme zu den beantragten Mitteln sank von 24,8\% im Jahr 2011 auf 24,2\% im Jahr 2012.

Die Antragssummen stiegen über die Jahre hinweg kontinuierlich: Ein Vergleich mit dem Jahr 2000 zeigt, dass sich die Zahl der eingereichten Projekte mehr als verdoppelt, die Höhe der beantragten Summen sich aber rund verfünffacht hat. Dieser Nachfrage konnten die Bewilligungen nicht entsprechen: Im gleichen Zeitraum stieg die Zahl der bewilligten Projekte um 30\%, die bewilligten Summen hatten sich lediglich verdoppelt. Somit klafft die Schere zwischen Nachfrage und Bewilligungsmöglichkeiten immer stärker auseinander. 
Tab. 4: Anzahl der Förderungen im Jahr 2012

\begin{tabular}{|c|c|c|c|c|c|c|c|}
\hline \multirow[t]{3}{*}{ Förderungsprogramm } & \multicolumn{2}{|c|}{ Anträge entschieden } & \multicolumn{2}{|c|}{ Neubewilligungen } & \multicolumn{3}{|c|}{ Bewilligungsquote in $\%$} \\
\hline & \multicolumn{2}{|c|}{2012} & \multicolumn{2}{|c|}{2012} & \multicolumn{3}{|c|}{2012} \\
\hline & Anzahl & $\%$-Frauen & Anzahl & $\%$-Frauen & Rate & Frauen & Männer \\
\hline Einzelprojekte & 1.080 & $25,6 \%$ & 334 & $26,0 \%$ & $30,9 \%$ & $31,5 \%$ & $30,7 \%$ \\
\hline Internationale Programme & 311 & $15,4 \%$ & 83 & $15,7 \%$ & $26,7 \%$ & $27,1 \%$ & $26,6 \%$ \\
\hline Spezialforschungsbereiche (SFBs) & 65 & $16,9 \%$ & 27 & $11,1 \%$ & $12,5 \%$ & $0,0 \%$ & $15,0 \%$ \\
\hline SFBs Verlängerungen & 42 & $16,7 \%$ & 35 & $11,4 \%$ & $83,3 \%$ & $57,1 \%$ & $88,6 \%$ \\
\hline Nationale Forschungsnetzwerke Verlängerungen & 6 & $16,7 \%$ & 4 & $25,0 \%$ & $66,7 \%$ & $100,0 \%$ & $60,0 \%$ \\
\hline START & 53 & $20,8 \%$ & 7 & $28,6 \%$ & $13,2 \%$ & $18,2 \%$ & $11,9 \%$ \\
\hline START Verlängerungen & 6 & $16,7 \%$ & 6 & $16,7 \%$ & $100,0 \%$ & $100,0 \%$ & $100,0 \%$ \\
\hline Wittgenstein & 21 & $9,5 \%$ & 2 & $0,0 \%$ & $9,5 \%$ & $0,0 \%$ & $10,5 \%$ \\
\hline Doktoratskollegs (DKs) & 5 & $20,0 \%$ & 2 & $0,0 \%$ & $12,5 \%$ & $0,0 \%$ & $15,4 \%$ \\
\hline DKs Verlängerungen & 3 & $0,0 \%$ & 2 & $0,0 \%$ & $66,7 \%$ & $0,0 \%$ & $66,7 \%$ \\
\hline Schrödinger & 135 & $33,3 \%$ & 68 & $30,9 \%$ & $50,4 \%$ & $46,7 \%$ & $52,2 \%$ \\
\hline Meitner & 123 & $39,0 \%$ & 40 & $40,0 \%$ & $32,5 \%$ & $33,3 \%$ & $32,0 \%$ \\
\hline Firnberg & 52 & $100,0 \%$ & 15 & $100,0 \%$ & $28,8 \%$ & $28,8 \%$ & - \\
\hline Richter & 57 & $100,0 \%$ & 15 & $100,0 \%$ & $26,3 \%$ & $26,3 \%$ & - \\
\hline Translational Research & 78 & $14,1 \%$ & 21 & $9,5 \%$ & $26,9 \%$ & $18,2 \%$ & $28,4 \%$ \\
\hline Klinische Forschung (KLIF) & 123 & $30,1 \%$ & 17 & $52,9 \%$ & $13,8 \%$ & $24,3 \%$ & $9,3 \%$ \\
\hline Programm zur Entwicklung und Erschließung der Künste (PEEK) & 56 & $48,2 \%$ & 6 & $66,7 \%$ & $10,7 \%$ & $14,8 \%$ & $6,9 \%$ \\
\hline Gesamt & 2.216 & $28,7 \%$ & 684 & $28,2 \%$ & $30,2 \%$ & $30,2 \%$ & $30,2 \%$ \\
\hline Konzeptanträge für SFBs & 24 & $16,7 \%$ & 6 & $16,7 \%$ & & & \\
\hline Konzeptanträge für DKs & 16 & $18,8 \%$ & 5 & $20,0 \%$ & & & \\
\hline
\end{tabular}

Quelle: FWF.

Gemessen an den Anteilen an den Fördermitteln weisen die Förderungen von Einzelprojekten das größte Gewicht auf. Im Jahr 2012 wurden in Summe Einzelprojekte mit 319,7 Mio. € gefördert, das entspricht 47,2 \% der gesamten Fördersumme des FWF. Es folgen die internationalen Programme mit 71,8 Mio. $€$ (Anteil von 10,6 \%) und START mit 57,8 Mio. $€$ (Anteil von 8,5 \%).

Der größte Teil der FWF-Förderungen fließt in die Finanzierung von wissenschaftlichem Personal. Somit trägt der FWF also wesentlich zum Ausbau des wissenschaftlichen Humanpotenzials bei. Im Vergleich zum Jahr 2000 hat sich die Zahl des über FWF-Projekte finanzierten Forschungspersonals rund verdoppelt: Im Jahr 2012 standen 3.852 Personen auf der Payroll des FWF. Setzt man das in Beziehung zur Gesamtheit des wissenschaftlich-künstlerischen Personals der
Universitäten - laut Unidata zum Stichtag 31.12.2012 insgesamt 20.104,9 VZÄ - ist das ein erhebliches Potenzial (siehe Tab. 6).

Besonders stark gestiegen ist die Zahl der sogenannten FWF-Fellows. Das sind ForscherInnen, die ein FWF-Projekt leiten und ihr eigenes Gehalt aus dem Projekt finanzieren. Ohne FWF-Projekt hätten diese, fast ausschließlich jungen, WissenschaftlerInnen große Probleme, ihre Forschungsarbeiten durchzuführen, da die Forschungsstätten ihnen meist keine Karriereperspektiven eröffnen können. Ihr Anteil an den ProjektleiterInnen der Einzelprojekte stieg bewilligungsseitig in den letzten fünf Jahren von 16\% (2007) auf 20\% (2012). Eine weitere Herausforderung im Bereich des wissenschaftlichen Personals stellt aus Sicht des FWF der, mit rund 30\% nach wie vor zu ge- 
Tab. 5: Fördersummen nach Programm im Jahr 2012

\begin{tabular}{|c|c|c|c|c|c|c|c|}
\hline \multirow[t]{3}{*}{ Förderungsprogramm } & \multirow{2}{*}{\multicolumn{2}{|c|}{$\begin{array}{c}\text { Anträge entschieden } \\
2012\end{array}$}} & \multirow{2}{*}{\multicolumn{2}{|c|}{$\begin{array}{c}\text { Neubewilligungen } \\
2012\end{array}$}} & \multicolumn{3}{|c|}{ Bewilligungsquote in \% } \\
\hline & & & & & & 2012 & \\
\hline & Summe & $\%$-Frauen & Summe & $\%$-Frauen & Rate & Frauen & Männer \\
\hline Einzelprojekte & $€ 319,7$ & $25,9 \%$ & $€ 97,6$ & $26,2 \%$ & $29,8 \%$ & $30,4 \%$ & $29,6 \%$ \\
\hline Internationale Programme & $€ 71,8$ & $13,4 \%$ & $€ 16,2$ & $15,2 \%$ & $21,9 \%$ & $24,8 \%$ & $21,4 \%$ \\
\hline SFBs & $€ 25,9$ & $17,9 \%$ & $€ 12,0$ & $8,0 \%$ & $10,2 \%$ & $4,6 \%$ & $11,6 \%$ \\
\hline SFBs Verlängerungen & $€ 18,2$ & $15,5 \%$ & $€ 14,0$ & $13,0 \%$ & $77,0 \%$ & $64,5 \%$ & $79,2 \%$ \\
\hline NFNs Verlängerungen & $€ 3,7$ & $24,6 \%$ & $€ 2,6$ & $32,0 \%$ & $54,0 \%$ & $68,7 \%$ & $49,2 \%$ \\
\hline START & $€ 57,8$ & $19,8 \%$ & $€ 4,4$ & $27,5 \%$ & $7,4 \%$ & $10,3 \%$ & $6,7 \%$ \\
\hline START Verlängerungen & $€ 3,3$ & $18,1 \%$ & $€ 3,3$ & $18,1 \%$ & $99,8 \%$ & $100,0 \%$ & $99,7 \%$ \\
\hline Wittgenstein & $€ 31,5$ & $9,5 \%$ & $€ 3,0$ & $0,0 \%$ & $9,5 \%$ & $0,0 \%$ & $10,5 \%$ \\
\hline DKs & $€ 11,9$ & $18,0 \%$ & $€ 6,5$ & $1,3 \%$ & $14,4 \%$ & $0,0 \%$ & $17,7 \%$ \\
\hline DKs Verlängerungen & $€ 7,1$ & $0,0 \%$ & $€ 4,1$ & $0,0 \%$ & $58,6 \%$ & $0,0 \%$ & $58,6 \%$ \\
\hline Schrödinger & $€ 13,3$ & $33,5 \%$ & $€ 7,3$ & $30,0 \%$ & $52,9 \%$ & $46,6 \%$ & $56,0 \%$ \\
\hline Meitner & $€ 15,1$ & $39,8 \%$ & $€ 5,9$ & $39,2 \%$ & $33,6 \%$ & $33,5 \%$ & $33,6 \%$ \\
\hline Firnberg & $€ 11,0$ & $100,0 \%$ & $€ 3,3$ & $100,0 \%$ & $28,9 \%$ & $28,9 \%$ & - \\
\hline Richter & $€ 15,6$ & $100,0 \%$ & $€ 4,7$ & $100,0 \%$ & $26,7 \%$ & $26,7 \%$ & - \\
\hline Translational Research & $€ 25,9$ & $13,3 \%$ & $€ 6,1$ & $7,8 \%$ & $23,0 \%$ & $13,2 \%$ & $24,5 \%$ \\
\hline KLIF & $€ 28,4$ & $27,1 \%$ & $€ 3,3$ & $52,8 \%$ & $11,5 \%$ & $22,5 \%$ & $7,4 \%$ \\
\hline PEEK & $€ 16,4$ & $52,4 \%$ & $€ 2,0$ & $69,3 \%$ & $12,2 \%$ & $16,3 \%$ & $7,8 \%$ \\
\hline Gesamt & $€ 676,7$ & $25,8 \%$ & $€ 196,4$ & $25,3 \%$ & $24,2 \%$ & $24,5 \%$ & $24,0 \%$ \\
\hline Konzeptanträge für SFBs & $€ 104,9$ & $18,9 \%$ & $€ 24,6$ & $13,1 \%$ & & & \\
\hline Konzeptanträge für DKs & $€ 35,5$ & $18,7 \%$ & $€ 12,1$ & $17,8 \%$ & & & \\
\hline
\end{tabular}

Quelle: FWF.

Tab. 6: Durch den FWF finanziertes Forschungspersonal im Jahr 2012

\begin{tabular}{|l|l|r|r|r|}
\hline & & 2010 & 2011 & 2012 \\
\hline Postdocs & Alle & 1.197 & 1.229 & 1288 \\
& Frauen & 554 & 575 & 517 \\
\hline \multirow{3}{*}{ DoktorandInnen } & Männer & 643 & 654 & 771 \\
& Alle & 1.683 & 1.771 & 1935 \\
& Frauen & 710 & 745 & 819 \\
& Männer & 973 & 1.026 & 1116 \\
\hline \multirow{3}{*}{ Technisches Personal } & Alle & 122 & 137 & 173 \\
& Frauen & 82 & 98 & 118 \\
& Männer & 40 & 39 & 55 \\
\hline \multirow{3}{*}{ Sonstiges Personal } & Alle & 403 & 405 & 456 \\
& Frauen & 193 & 213 & 215 \\
& Männer & 210 & 192 & 241 \\
\hline Summe & Alle & 3.405 & 3.542 & 3852 \\
\cline { 2 - 5 } & Frauen & 1.539 & 1.631 & 1669 \\
\cline { 2 - 5 } & Männer & 1.866 & 1.911 & 2183
\end{tabular}

Quelle: FWF. ringe, Anteil an Frauen bei den AntragstellerInnen von FWF Projekten dar. Erfreulich ist, dass der Frauenanteil bei den Neubewilligungen von 25,9\% im Jahr 2011 auf 28,2\% im Jahr 2012 stieg. Auf eine Erhöhung des Frauenanteils bei Projektleitungen zielt auch eine Zielvorgabe in den Doktoratskollegs wie auch in den Spezialforschungsbereichen: Hier soll ein 30\% Anteil von Frauen in der Faculty bzw. im Team der Projektteilleitungen erreicht werden.

Im Hinblick auf die Aufteilung der FWF-Förderungen auf Wissenschaftsdisziplinen zeigen sich auf höher aggregierter Ebene die Strukturen über die Jahre hinweg vergleichsweise stabil. Grob gesprochen kann man drei Bereiche beschreiben: Life Sciences, Naturwissenschaft und Technik, Geistes- und Sozialwissenschaften. Im Berichtsjahr 2012 flossen - bezogen auf die Gesamtbewilligungssumme $-73,8$ Mio. $€$ in den Be- 
Abb. 14: Bewilligungen nach Wissenschaftsdisziplinen (Gesamtbetrachtung aller FWF-Programme)

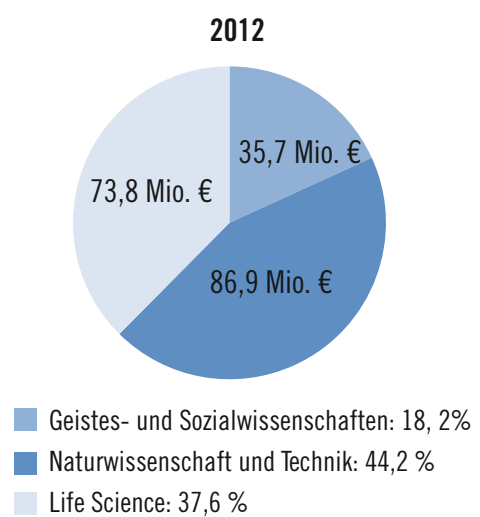

Quelle: FWF.

reich der Life Sciences, 86,9 Mio. $€$ in den Bereich Naturwissenschaft und Technik sowie 35,7 Mio. $€$ in den Bereich Geistes- und Sozialwissenschaften.

Diese Verteilung beruht nicht auf Quoten des FWF, sondern ergibt sich aus der Antragslage. Allerdings gibt es zwei Bereiche mit Rahmenbedingungen, denen mit den allgemeinen Verfahrensmechanismen des FWF offenbar tatsächlich nur ungenügend entsprochen werden kann, und denen der FWF daher mit eigenen Ausschreibungen Rechnung getragen hat: Im Bereich der künstlerischen Forschung (PEEK) ging 2012 die vierte und im Bereich der klinischen Forschung (KLIF) die zweite Ausschreibung erfolgreich über die Bühne. In beiden Fällen setzte der FWF eine internationale Jury ein, um Fachkompetenzen einzubringen. Im Falle von KLIF hat sich jedoch herausgestellt, dass dies nicht mehr notwendig ist, sodass die für diesen Bereich notwendigen, speziellen Verfahrensschritte im Rahmen der üblichen FWF-Verfahren abgedeckt werden können. Die KLIF-Projekte werden infolgedessen nach einer letzten Ausschreibung 2012/13 analog zur FWF-Einzelprojektförderung behandelt werden.

Ebenfalls positiv entwickelte sich die Abgeltung von Overheadkosten seit ihrer Wiedereinführung im Jahr 2011 bei den Einzelprojekten und beim Programm zur Entwicklung und Erschließung der Künste (PEEK): Im Jahr 2012 zahl-
Durchschnitt 2007-2010

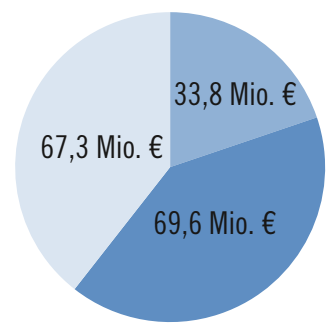

Geistes- und Sozialwissenschaften: $18,8 \%$

Naturwissenschaft und Technik: 40,8\%

Life Science: $39,4 \%$ te der FWF mit 5,6 Mio. $€$ um 4,3 Mio. $€$ mehr Overheads an österreichische Forschungsstätten als im Vorjahr. Dieser Betrag ist nicht Bestandteil des FWF-Bewilligungsvolumens, sondern kommt gleichsam „on-top" dazu. Angesichts des stringenten FWF-Budgets war es bis dato nicht möglich, eine Ausweitung der Overhead-Zahlungen auf alle Programme des FWF zu erreichen. Die Folge sind unerwünschte Verzerrungen in Programmen, wie z.B. in den internationalen Projekten, SFBs, DKs und Frauenprogrammen, wo WissenschaftlerInnen ohne Abgeltung von Overheadkosten durchaus einen Wettbewerbsnachteil an den Forschungsstätten erfahren.

\subsection{Forschungsförderungsgesellschaft (FFG)}

Die Österreichische Forschungsförderungsgesellschaft (FFG) bietet ein differenziertes Portfolio unterschiedlichster Instrumente zur Förderung von Forschungsvorhaben von Unternehmen und Forschungseinrichtungen. Die Bandbreite der Instrumente reicht dabei von niederschwelligen Einstiegsformaten bis hin zur Förderung von Spitzenforschung. Im Jahr 2012 kam es innerhalb der FFG $z u$ einigen organisatorischen Änderungen. So konnte die bereits früher eingeleitete Umsetzung des Themen- und Portfoliomanagements-Konzepts weitgehend abgeschlossen werden. Die FFG verfügt damit über 
ein standardisiertes Set an Förderungsinstrumenten, ein durchgängiges Themenmonitoring, das zeitnah die Allokation der Förderungsmittel über Themen zeigt, sowie programmübergreifend aufgesetzte Thementeams, die einen kontinuierlichen Erfahrungsaustausch zwischen Programmen und eine Abstimmung des Instrumenteneinsatzes entlang von Themen sicherstellen. Auf Instrumentenebene wurde im Jahr 2012 mit der Initiative Markt.Start eine Neuerung durchgeführt. Mit dieser Initiative adressiert die FFG im Auftrag des BMVIT einen neuralgischen Punkt im österreichischen Innovationssystem: Die Umsetzung erfolgreich abgeschlossener F\&E-Projekte am Markt. Mit diesem Instrument bietet die FFG jungen Unternehmen, die ein Entwicklungsprojekt in den Basisprogrammen erfolgreich abgeschlossen haben, eine Darlehensfinanzierung für die anschließende Markterschließungsphase. Das Finanzierungsvolumen kann bis zu 1 Mio. $€$ umfassen. Zusätzlich ist noch zu erwähnen, dass mit 01.01.2013 die FFG mit der Begutachtung der für die Forschungsprämie geltend gemachten F\&E-Aufwendungen betraut ist.

Tab. 7 gibt einen Überblick über die Zahl der Projekte, Beteiligungen, die eingebundenen Akteure und die im Jahr 2012 vertraglich zugesicherten Fördermittel. Im Jahr 2012 konnte insgesamt ein Fördervolumen (inklusive Haftungen und Darlehen) von 483,3 Mio. $€$ zugesichert werden, was einem Barwert von 361 Mio. $€$ entspricht. Gegenüber dem Jahr 2011 entspricht dies einer Zunahme des Barwerts der Förderungen von 3,4 \% (2011: 349 Mio. $€$ Barwert). Mit diesem Fördervolumen konnten Forschungsvorhaben im Gesamtausmaß von 979,3 Mio. $€$ (plus 8,4 \% gegenüber 2011) gefördert werden. Die Gesamtzahl der geförderten Projekte betrug 2.913, die 5.125 Beteiligungen und 2.876 verschiedene Akteure umfassten. Die Zahl der von der FFG geförderten Akteure stieg dabei gegenüber dem Vorjahr (2.758 Akteure) um 4,3\%, was auf eine weiterhin laufende Verbreiterung der Forschungsbasis Österreichs hindeutet.
Gemessen am Barwert wie auch an der Anzahl der Projekte und den induzierten Gesamtkosten der Forschungsprojekte weist der Programmbereich der Basisprogramme das größte Gewicht innerhalb des umfangreichen Portfolios der FFG auf. Innerhalb dieses Bereichs sticht die Programmlinie Basisprogramm, das Kernprogramm der bottom-up orientierten unternehmensorientierten Forschungsförderung der FFG, mit einem Anteil von ca. $42 \%$ am Barwert (und $43 \%$ an den Projekten) hervor. Mit 634 geförderten Projekten ist deren Zahl gegenüber dem Vorjahr (2011: 607 geförderte Projekte) in der Programmlinie Basisprogramm um 4,4 \% gestiegen. Besonders zu erwähnen ist auch der mittlerweile bewährte Innovationsscheck. In dieser Programmlinie, die darauf abzielt, kleinen und mittleren Unternehmen den Einstieg in eine kontinuierliche F\&E-Tätigkeit zu erleichtern, wurden insgesamt 486 Projekte gefördert.

Den zweitgrößten Programmbereich (gemessen am zugesagten Förderbarwert) der FFG umfassen die unterschiedlichen Strukturprogramme, die in Summe 111,4 Mio. $€$ Barwert umfassen. Hervorzuheben ist hier das Kompetenzzentrumprogramm COMET. Im Jahr 2012 entfallen 79,9 Mio. $€$ auf dieses Programm. Diese hohe Summe ist dadurch bedingt, dass es im Jahr 2012 zu vertraglichen Verlängerungen von mehreren bestehenden Kompetenzzentren gekommen ist. Das Programm COMET ist das Nachfolgeprogramm der K-Programme (K-plus, K-ind, K-net) und verfolgt das Ziel, die Kooperationskultur zwischen Industrie und Wissenschaft weiter zu stärken und den Aufbau gemeinsamer Forschungskompetenzen und deren Verwertung zu forcieren. Das Programm orientiert sich besonders an Exzellenz, der Einbindung von internationalem Forschungs-Know-how sowie dem Aufbau und der Sicherung der Technologieführerschaft von Unternehmen zur Stärkung des österreichischen Forschungsstandorts.

Der dritte quantitativ bedeutsame Programmbereich des Förderportfolios der FFG ist der Bereich der thematischen Programme. Ziel dieser 
Tab. 7: FFG-Förderstatistik 2012 [Beiträge in $1.000 €$ ]

\begin{tabular}{|c|c|c|c|c|c|c|c|}
\hline & Programm & Projekte & Beteiligung & Akteure & $\begin{array}{l}\text { Gesamt- } \\
\text { kosten }\end{array}$ & $\begin{array}{l}\text { Förderung inkl. } \\
\text { Haftungen u. } \\
\text { Darlehen }\end{array}$ & Barwert \\
\hline \multirow[t]{2}{*}{ ALR } & ASAP & 6 & 11 & 9 & 1.574 & 934 & 934 \\
\hline & & 6 & 11 & 9 & 1.574 & 934 & 934 \\
\hline \multirow[t]{8}{*}{ BP } & Programmlinie Basisprogramm & 634 & 656 & 515 & 382.757 & 208.798 & 98.060 \\
\hline & Programmlinie Dienstleistungsinnovationen & 36 & 38 & 37 & 12.427 & 6.567 & 5.397 \\
\hline & Programmlinie Headquarter & 20 & 22 & 22 & 56.113 & 24.963 & 17.883 \\
\hline & Programmlinie Hightech Start-up & 19 & 19 & 19 & 14.265 & 9.979 & 6.650 \\
\hline & BRIDGE & 59 & 197 & 183 & 21.997 & 14.662 & 14.662 \\
\hline & EUROSTARS & 12 & 12 & 11 & 6.713 & 3.906 & 3.906 \\
\hline & Innovationsscheck & 486 & 971 & 730 & 3.048 & 2.840 & 2.840 \\
\hline & & 1.266 & 1.915 & 1.427 & 497.319 & 271.714 & 149.398 \\
\hline \multirow[t]{2}{*}{ EIP } & TOP.EU & 20 & 20 & 9 & 1.838 & 1.378 & 1.378 \\
\hline & & 20 & 20 & 9 & 1.838 & 1.378 & 1.378 \\
\hline \multirow[t]{7}{*}{$S P$} & AplusB & 5 & 5 & 5 & 39.216 & 12.900 & 12.900 \\
\hline & COIN & 16 & 97 & 90 & 8.848 & 5.169 & 5.169 \\
\hline & COMET & 7 & 488 & 439 & 248.213 & 79.850 & 79.850 \\
\hline & FoKo & 14 & 203 & 189 & 5.432 & 4.336 & 4.336 \\
\hline & Strat. Impulszentren & 18 & 33 & 31 & 2.767 & 2.171 & 2.171 \\
\hline & Talente & 1.191 & 1.243 & 671 & 11.329 & 7.006 & 7.006 \\
\hline & & 1.251 & 2.069 & 1.301 & 315.805 & 111.431 & 111.431 \\
\hline \multirow[t]{15}{*}{ TP } & AT:net & 4 & 4 & 4 & 1.138 & 284 & 284 \\
\hline & benefit & 31 & 61 & 54 & 10.945 & 6.656 & 6.656 \\
\hline & ENERGIE DER ZUKUNFT & 45 & 142 & 110 & 17.773 & 8.902 & 8.902 \\
\hline & FIT-IT & 36 & 60 & 41 & 29.435 & 10.979 & 10.979 \\
\hline & GEN-AU & 3 & 3 & 3 & 27 & 27 & 27 \\
\hline & IEA & 13 & 20 & 11 & 1.054 & 1.054 & 1.054 \\
\hline & Intelligente Produktion & 34 & 98 & 84 & 14.768 & 10.569 & 10.569 \\
\hline & IV2Splus & 92 & 272 & 185 & 22.762 & 15.821 & 15.821 \\
\hline & KIRAS & 19 & 107 & 70 & 12.058 & 8.229 & 8.229 \\
\hline & Leuchttürme eMobilität & 2 & 26 & 25 & 13.205 & 5.567 & 5.567 \\
\hline & NANO & 10 & 23 & 17 & 2.071 & 1.698 & 1.698 \\
\hline & NANO-EHS & 4 & 9 & 7 & 588 & 573 & 573 \\
\hline & Neue Energien 2020 & 74 & 279 & 209 & 36.544 & 25.415 & 25.415 \\
\hline & TAKE OFF & 3 & 6 & 6 & 430 & 430 & 430 \\
\hline & & 370 & 1.110 & 675 & 162.798 & 96.204 & 96.204 \\
\hline \multicolumn{2}{|c|}{ FFG Förderungen und Aufwendungen } & 2.913 & 5.125 & 2.876 & 979.335 & 481.661 & 359.345 \\
\hline \multicolumn{2}{|c|}{ FFG-Beauftragungen } & & & & 1.654 & 1.654 & 1.654 \\
\hline \multicolumn{2}{|c|}{ FFG Operative Mittel 2012 gesamt } & & & & 483.315 & 483.315 & 360.999 \\
\hline
\end{tabular}

Anm.: Die quantitativen Angaben beziehen sich auf die im Jahr 2012 zugesagten Fördermittel.

Quelle: FFG. 
Programme ist es, in ausgewählten Themen fokussiert Schwerpunkte zu setzen, um in strategischen Zukunftsfeldern auch international sichtbare kritische Massen der Forschung zu erreichen. Insgesamt entfallen auf die thematischen Programme 96,2 Mio. $€$ Barwert. Konkret umfassen diese Schwerpunktsetzungen Themen wie Energie, IKT, Produktion oder Sicherheitsforschung. Etliche der Themen sind daher im besonderen Ausmaß anschlussfähig an die in HORIZON 2020 von der EU definierten „Grand Challenges" wie auch an die „Key Enabling Technologies" (KET). Somit sind die thematischen Programmlinien Teil einer neuen Missionsorientierung $^{27}$ der österreichischen Forschungs- und Technologiepolitik, wie sie auch in der 2011 beschossenen FTI-Strategie der österreichischen Bundesregierung gefordert wird.

Die FTI-Initiative „Produktion der Zukunft" fördert neue technologische Entwicklungen im Bereich der Material- und Produktionstechnologien. Mit dem Programm "IKT der Zukunft" werden Innovationen in verschiedenen Bereichen der Informations- und Kommunikationstechnologien, darunter auch in der Mikro- und Nanoelektronik, unterstützt. Schließlich werden Schlüsseltechnologieentwicklungen in wichtigen Anwendungsfeldern auch in Förderprogrammen der Bereiche Energie und Mobilität angesprochen, wie z.B. das Forschungs- und Demonstrationsprogramm „Technologische Leuchttürme der Elektromobilität", das Förderprogramm „Energieeffiziente Fahrzeugtechnologien" des Klima- und Energiefonds, das österreichische Luftfahrttechnologieforschungsprogramm TAKE OFF und das Programm "Smart Cities".

Angesichts der guten Position Österreichs bei Schlüsseltechnologien (siehe Kapitel 4.5) kann erwartet werden, dass das Land von den verstärkten forschungs- und technologiepolitischen Anstrengungen der Europäischen Kommission in diesem Bereich, u.a. im Rahmen des Programms HORIZON 2020, profitieren wird. Die nationale
Förderung sollte die europäische Förderung an einigen Punkten gezielt ergänzen. Hierzu zählt erstens die weitere Stärkung der Verbindung zwischen industrieller und öffentlicher Forschung in den einzelnen Schlüsseltechnologien, denn in kaum einem anderen Bereich ist die Entwicklung neuer Technologie so stark auf neue wissenschaftliche Forschungsergebnisse angewiesen wie hier. Neben kooperativen Forschungseinrichtungen wie den Kompetenzzentren sollten auch bilaterale Forschungsprojekte zwischen Wissenschaft und Unternehmen mit ausreichenden Zeithorizonten unterstützt werden. Zweitens sollten die Potenziale im Schnittfeld von zwei oder mehreren Schlüsseltechnologien besser genutzt werden. Drittens könnte die Verknüpfung von Schlüsseltechnologien und künftigen Anwendungsfeldern über die Programme Smart Cities und Elektromobilität hinaus auch auf andere Anwendungsgebiete, wie etwa Medizin/Gesundheitswirtschaft, nachhaltige Energienutzung oder neue Produktions- und Logistikkonzepte, ausgeweitet werden.

Das breite Förderangebot der FFG spiegelt sich auch bei Betrachtung der Förderungen nach Organisationstyp wider (Tab. 8). Auf Basis der 2012 zugesagten Mittel beträgt der Anteil der Unternehmen $52 \%$. Gegenüber 2011 hat sich die Zahl der Beteiligungen von Unternehmen um $10 \%$ erhöht (2011: 2.688 Beteiligungen). Die Zahl der unterschiedlichen Akteure macht bei den Unternehmen 1.991 aus, ein prozentueller Anstieg gegenüber dem Vorjahr (mit damals 1.934 Akteuren) von 2,9 \%. Dieser Anstieg kann wiederum als ein zusätzlicher Hinweis über die anhaltende Dynamik der Forschungsaktivitäten der österreichischen Wirtschaft und die laufende Verbreiterung der unternehmerischen Forschungsbasis interpretiert werden.

In Bezug auf den Anteil am zugesagten Förderbarwert liegen die Forschungseinrichtungen mit $31 \%$ an zweiter Stelle, gefolgt von den Hochschulen mit $11 \%$. Die Veränderung des Anteils

27 Siehe zur sogenannten „neuen Missionsorientierung“ der Technologiepolitik Gassler et al. (2006). 
der Forschungseinrichtungen (im Vorjahr betrug der diesbezügliche Anteil $21 \%$ ) ist auf die im Jahr 2012 erfolgten Vertragsverlängerungen im Rahmen des Kompetenzzentrumprogramms COMET zurückzuführen. Nach entsprechenden Zwischenevaluierungen gab es hier im Jahr 2012 Verlängerungen und somit Förderzusagen, die es im Jahr 2011 aufgrund des mehrjährigen Rhythmus dieser Vertragsverlängerungen nicht gegeben hat.

Die Förderung der FFG im Jahr 2012 nach den unterschiedlichen Technologiefeldern ist in Abb. 15 dargestellt, wobei die Technologiefelder auf Basis des „CORDIS Subject Index Classification Codes $^{\prime \prime 28}$ definiert sind. Zugunsten der Übersichtlichkeit sind hierfür einige der Technologiefelder (z.B. alle IKT-orientierten Technologiefelder, ebenso die drei Biotechnologiefelder, nämlich landwirtschaftliche, industrielle und medizinische, sowie einige kleinere, eng verwandte Felder) zusammengefasst. Zu beachten ist, dass die "Breite" dieser Technologiefelder sehr unterschiedlich ist, die einzelne Reihung der Technologiefelder lässt also nicht auf ein entsprechendes Über- oder Untergewicht einer bestimmten Technologie in Bezug auf die Förderung durch die FFG schließen. Nichtsdestotrotz ist eine Betrachtung der Mittelflüsse nach diesen Technologiefeldern von Interesse, zeigt es doch, dass sich mit der Werkstofftechnik (umfasst Stahl- und Metallverarbeitung, Kunststoff- und Gummiindustrie etc.) und der industriellen Fertigung (Pro- duktionstechnik, Werkzeugbau, industrielle Prozesse etc.) zwei zentrale Technologiefelder der Sachgüterproduktion zwei große Stärkefelder der österreichischen Industriestruktur auch in der Förderungslandschaft der FFG prominent wiederfinden. Auf diese beiden Technologiefelder entfallen mit 88,7 Mio. $€$ immerhin ein Viertel der gesamten zugesagten Fördermittel der FFG.

Weitere quantitativ bedeutsame Technologiefelder sind Energie, Informations- und Kommunikationstechnologien (IKT) sowie Elektronik/ Mikroelektronik. Mit den beiden Letztgenannten sind somit auch zwei zentrale Themenschwerpunkte auf EU-Ebene (in HORIZON 2020) in besonderem Maße im Förderportfolio der FFG abgedeckt. Mit den Technologiefeldern Energie (das auch die erneuerbaren Energietechniken beinhaltet) sowie Umwelt \& Nachhaltigkeit sind auch zentrale "Missionen" der österreichischen FTI-Strategie bzw. einige der "Grand Challenges" von HORIZON 2020 in einem quantitativ besonders relevanten Ausmaß vertreten.

\subsection{Austria Wirtschaftsservice (aws)}

Die Austria Wirtschaftsservice GmbH (aws) wurde im Jahr 2002 mit dem Ziel gegründet, zur Steigerung des österreichischen Wirtschaftswachstums beizutragen, wobei die Eigentümervertreter das BMWFJ und das BMVIT darstellen. Als spezielle Förderbank des Bundes bietet die aws dazu alle Formen der Unternehmensfinanzie-

Tab. 8: FFG-Förderungen nach Organisationstyp 2012 [in $1.000 €$ ]

\begin{tabular}{|lrr|rrr|r}
\multicolumn{1}{c|}{ Organisationstyp } & Akteure & Beteiligungen & Gesamtförderung & Barwert & Anteile am Barwert \\
\hline Unternehmen & 1991 & 2.959 & 310.378 & 188.308 & $52 \%$ \\
\hline Forschungseinrichtungen & 151 & 771 & 112.157 & 111.910 & $31 \%$ \\
\hline Hochschulen & 473 & 1.046 & 39.709 & 39.709 & $11 \%$ \\
\hline Intermediäre & 52 & 77 & 16.286 & 16.286 & $5 \%$ \\
\hline Sonstige & 209 & 272 & 3.132 & 3.132 & $1 \%$ \\
\hline Gesamt & 2876 & 5.125 & 481.661 & 359.345 & $100 \%$ \\
\hline
\end{tabular}

Quelle: FFG.

28 Siehe http://cordis.europa.eu/guidance/sic-codes_en.html 
Abb. 15: FFG-Förderung nach Technologiefeldern 2012

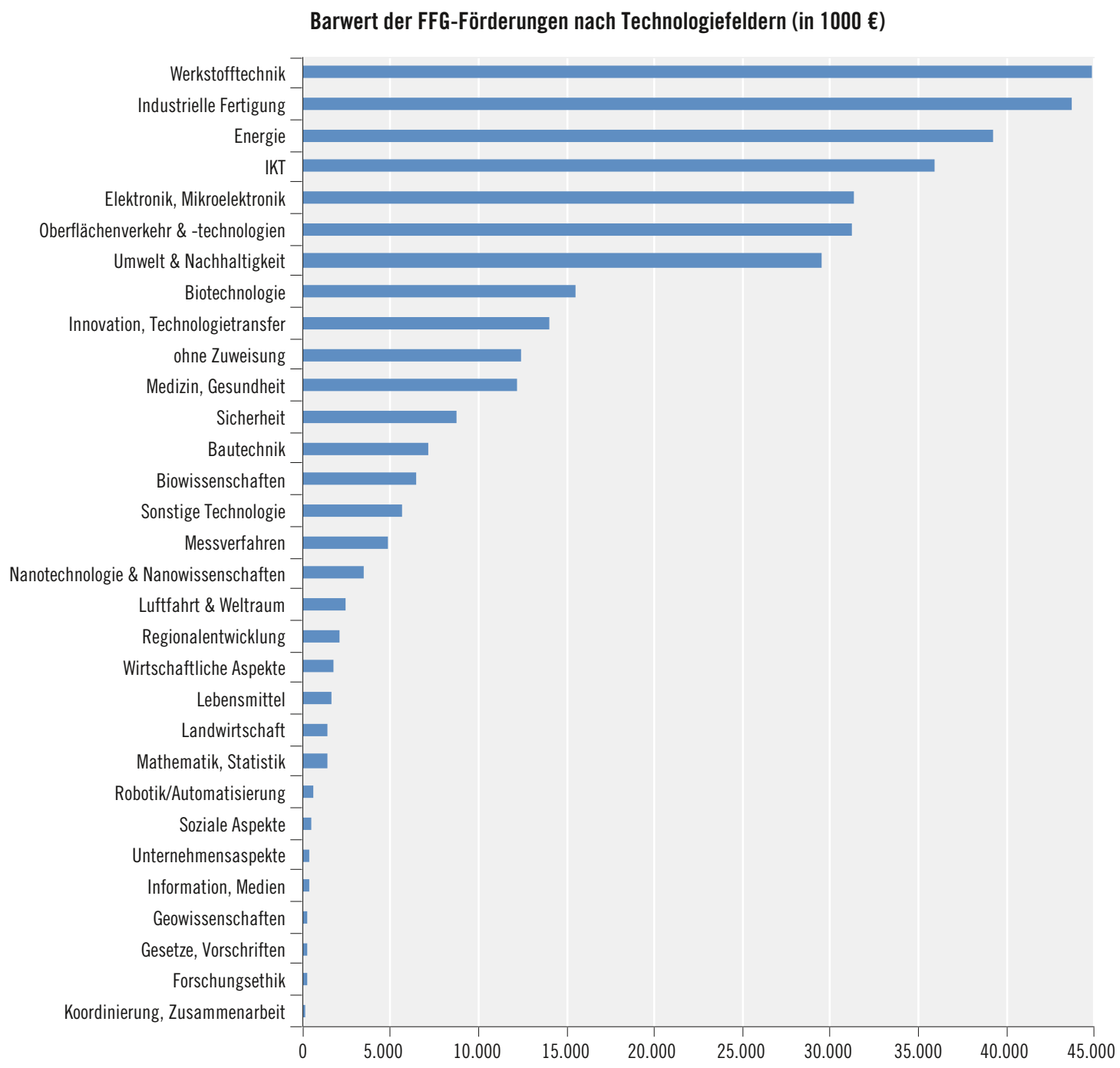

Quelle: FFG.

rung an - von Garantien und Krediten über $\mathrm{Zu}$ schüsse bis zu Eigenkapitalinstrumentarien. Je nach Unternehmensphase und Finanzierungsanlass wird dabei ein Finanzierungsmix, der die Verteilung von öffentlichem und privatem Risiko berücksichtigt, erarbeitet. Neben monetären Leistungen werden begleitend Coachingmaßnahmen angeboten, die von spezifischen Schulungen für High-Tech UnternehmensgründerInnen bis hin zu IP-Beratung und Verwertung sowie Tech- nologie- und Marktrecherchen reichen. Durch eine stark wachstums- und innovationsorientierte Förderungslogik wird dadurch ein breites Themenspektrum von der Gründungsvorbereitung über die Markteinführungsphase bis hin zu größeren Wachstumssprüngen, wie Internationalisierungen in späteren Unternehmensphasen, abgedeckt.

Tab. 9 gibt einen Überblick über die Förderleistungen im Jahr 2012 im Bereich der Finanzie- 
Tab. 9: AWS-Förderungsleistung 2012

\begin{tabular}{|l|r|r|r|r|r|r|} 
& \multicolumn{2}{|c|}{ Förderungszusagen [Anzahl] } & \multicolumn{2}{c|}{ Gesamtprojektvolumen [Mio. $€$ ] } & \multicolumn{2}{|c|}{ Förderungsleistung [Mio. $€$ ] } \\
\cline { 2 - 7 } & $\mathbf{2 0 1 2}$ & $\mathbf{2 0 1 1}$ & $\mathbf{2 0 1 2}$ & $\mathbf{2 0 1 1}$ & $\mathbf{2 0 1 2}$ & $\mathbf{2 0 1 1}$ \\
\hline Garantien & $\mathbf{7 5 9}$ & 672 & 458,0 & 863,0 & 199,9 & 195,3 \\
Kredite & 1.068 & 1.044 & $1.454,2$ & 445,0 & 558,2 & 530,4 \\
Zuschüsse & 2.567 & 2.305 & $1.643,2$ & $1.066,0$ & 103,5 & 101,5 \\
\hline Eigenkapital & 5 & 7 & 20,8 & 35,0 & 11,2 & 19,2 \\
\hline Gesamtergebnis & 4.399 & 4.028 & $* 2.609,1$ & $2.409,0$ & 872,8 & 846,4 \\
\hline
\end{tabular}

Anm.: * Gesamtergebnis um Mehrfachzählungen bereinigt.

Quelle: aws.

rungsinstrumente. Allein über die monetären Instrumente werden jährlich rund 4.000 Unternehmen und deren Wachstums- und Innovationsprojekte mit einem Gesamtvolumen von mehr als 2,6 Mrd. € unterstützt. Die im Jahr 2012 mit allen Finanzierungsinstrumenten gemeinsam erbrachte Förderungsleistung beläuft sich auf mehr als 872 Mio. $€$. Dies bedeutet eine Steigerung der Förderleistung um knapp 3,2 \% gegenüber dem Vorjahr. Darüber hinaus unterstützen die Coaching- und Awarenessmaßnahmen der aws die FTI-politische Zielsetzung der Bundesregierung, Wertschöpfung durch Technologieverwertung am Markt zu verwirklichen.

Mehr als ein Viertel der gesamten Förderleistung der aws entfiel 2012 mit rund 200 Mio. $€$ auf die Übernahme von Garantien, wobei die Internationalisierung innovativer österreichischer Unternehmen einen Schwerpunkt bildete. Im Kreditbereich wurde eine Nachfragesteigerung von mehr als $5 \%$ des Kreditvolumens auf 558 Mio. $€$ deutlich, was darauf hindeutet, dass sich die Investitionsbereitschaft österreichischer Unternehmen wieder erhöht. Zudem ist ein Trend in Richtung größerer Projekte (mehr als 5 Mio. $€$ Gesamtinvestitionsvolumen pro Unternehmen) erkennbar. Die Eigenkapitalinstrumentarien der aws gewinnen angesichts von mangelndem privatem Risikokapital in Österreich, insbesondere für wissensintensive Unternehmungen, zunehmend an Bedeutung. So wurden im Rahmen der Venture Capital Initiative im Jahr 2012 drei Beteiligungen an (internationalen) Fonds eingegan- gen, die sich verpflichten, in österreichische High- und Cleantech-Unternehmen zu investieren. Schlussendlich konnte die aws auch im $\mathrm{Zu}-$ schusssektor eine leichte Steigerung verzeichnen.

Die aws agiert vorwiegend in der Rolle einer „Finanzierungspartnerin" für - aus Sicht und im Verhältnis zur Finanzierungskraft des geförderten Unternehmens vergleichsweise große - Innovationsvorhaben. Substanzielle Finanzierungsbeiträge beruhen einerseits auf mittels Krediten und Zuschuss ausgereichten Unterstützungen. Andererseits entstehen sie durch Hebeleffekte der eingesetzten Eigenkapital- und Garantieinstrumente, indem sie zusätzliche private Finanzierungsquellen eröffnen, wie z.B. kommerziell ausgereichte Kredite oder zusätzliches privates Eigenkapital.

In den aufgelegten Programmen zeigt sich bei der Selektion förderungswürdiger Vorhaben eine starke Innovationsorientierung der aws, wobei über reine F\&E-Aktivitäten hinausreichende Realinvestitionen einen bedeutenden Ansatzpunkt bilden (siehe Abb. 16). Konsequenterweise liefern die umgesetzten Projekte maßgebliche Beiträge zum Wissenstransfer sowie zur wirtschaftlichen Verwertung und Diffusion kommerziell tragfähiger Innovationen im unternehmerischen Kontext. Aus der Durchführung der Innovationsprojekte selbst folgen wiederum substanzielle Beiträge $z u$ jenen Unternehmensstrategien, die sowohl eine Realisierung von Wachstumschancen ermöglichen als auch eine Verbesserung der 
Abb. 16: Ansatzpunkte zur Förderung innovativer Investitionsprojekte

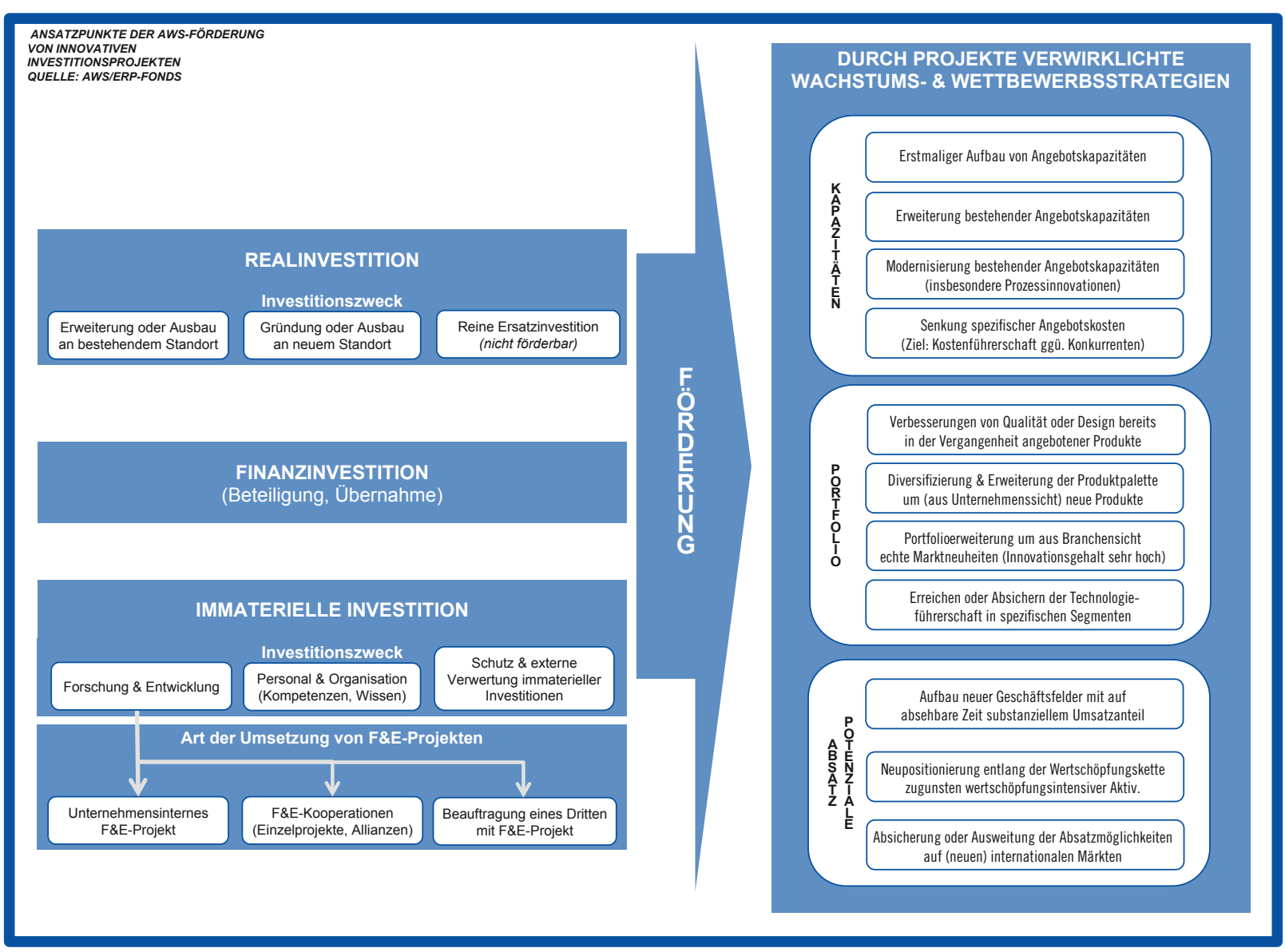

Quelle: aws.

Wettbewerbsposition der Fördernehmer bewirken.

Die FTI-politische Bedeutung des aws-Instrumentariums beruht nicht nur auf einem breit gewählten Ansatz zur finanziellen Unterstützung von Innovationsvorhaben, sondern auch auf Programmen, die Lösungen für spezifische Problemlagen ausgewählter Zielgruppen bereitstellen. Ein Kernstück der aws-Tätigkeit stellen Förderungsangebote dar, die - wie insbesondere PreSeed, Seedfinancing, Impulse und die Venture Capital Initiative - bei technologie- und wissensintensiven Gründungsvorhaben die Chancen auf einen erfolgreichen Marktauftritt bereits in frühen Phasen erhöhen (siehe Tab. 10).
Tab. 10: Übersicht der monetären aws-Programme zur Steigerung der wissensintensiven Gründungen 2012

\begin{tabular}{lcc|c|c}
$\mathbf{2 0 1 2}$ & $\begin{array}{c}\text { Projekte } \\
\text { [Anzahl] }\end{array}$ & $\begin{array}{c}\text { Gesamtprojekt- } \\
\text { volumen } \\
\text { [Mio. } € \text { ] }\end{array}$ & $\begin{array}{c}\text { Förderungs- } \\
\text { leistung } \\
\text { [Mio. } € \text { ] }\end{array}$ \\
\hline PreSeed & 27 & 6,1 & 4,1 \\
Seedfinancing & 18 & 124,9 & 12,3 \\
Management auf Zeit & 3 & 12,0 & 0,1 \\
\hline Kreativwirtschaft (Impulse) & 75 & 9,8 & 4,6 \\
Venture Capital Initiative (VCI) & 5 & 32,6 & 16,3 \\
Summe & 128 & 185,4 & 37,4
\end{tabular}

Quelle: aws.

Im Zusammenhang mit Angeboten, die auf spezifische Zielgruppen fokussieren, wurden zusätz- 
lich wirkungserhöhende Instrumente entwickelt: Das Angebot an monetären Unterstützungen findet Ergänzungen um Coaching- und Vermittlungsdienstleistungen, welche die Fördernehmer von der Ideenfindung über Konzeption, Planung und Implementierung bis hin zur Verwertung von Projektergebnissen unterstützen (siehe Tab. 11).

Tab. 11: Übersicht zu Awareness-, Coaching- und Vermittlungsdienstleistungen sowie Schulungsmaßnahmen 2012

\begin{tabular}{|l|r|}
\hline \multicolumn{1}{|c|}{2012} & $\begin{array}{r}\text { Projekte } \\
\text { [Anzahl] }\end{array}$ \\
\hline Awareness- und Coaching-Dienstleistungen \\
\hline \multicolumn{2}{|l|}{ Jugend Innovativ - Wettbewerb } \\
\hline Staatspreis Innovation & 539 \\
\hline Businessplan Wettbewerb - Best of Biotech (BOB) & 627 \\
\hline Intellectual Property (IP) - Beratung und Verwertung & 80 \\
\hline aws-Bonitätsanalyse & 237 \\
\hline Summe & 6 \\
\hline Vermittlungsdienstleistungen & 1.489 \\
\hline Business Angels - Börse (I²) & 178 \\
\hline Teilnehmerlnnen Schulungs- und Ausbildungsmaßnahmen & \\
\hline Life Science & 310 \\
\hline Kreativwirtschaft & 855 \\
\hline Summe & 1.165 \\
\hline
\end{tabular}

Quelle: aws.

Die Betreuungsintensität ist in der Vorgründungs- und Gründungsphase besonders hoch und nimmt mit der zunehmenden Etablierung im Unternehmenslebenszyklus ab. Genau daran orientieren sich die Awareness- und Coachingange- bote der aws. Beginnend von Maßnahmen zur Steigerung des „entrepreneurial spirit" etwa durch Jugend Innovativ, den SchülerInnenwettbewerb, bei dem 2012539 Projektideen eingereicht wurden, oder auch den Businessplan Wettbewerb BOB (Best of Biotech), an dem 201280 Projekte teilgenommen haben, und nicht zuletzt durch den Staatspreis Innovation setzen die nicht-monetären Unterstützungsleistungen vor allem in der Gründungsphase von wissensintensiven Unternehmen an. Speziell auf die Wachstumsfelder Life Sciences und Kreativwirtschaft abgestimmt werden für Start-ups spezifische Ausbildungsmodule angeboten, die 2012 von insgesamt 1.165 Personen angenommen wurden.

Das Coaching erstreckt sich für Start-ups und KMU auch auf den Aufbau, die Nutzung und die Verwertung von geistigem Eigentum (IP). Die aws bietet daher KMU gemeinsam mit dem Österreichischen Patentamt das Service "discover. $\mathrm{IP}^{\prime \prime}$ an, um das Wissen österreichischer Kleinund Mittelunternehmen über die optimale Nutzung ihres geistigen Eigentums zu fördern und zu vertiefen. Gemeinsam mit IP-Verwertungsberatungen der aws wurden 2012 diese Leistungen von 237 Unternehmen in Anspruch genommen.

Einschlägige Maßnahmen wurden im Jahr 2012 auch bei der Dienstleistungsorientierung gegenüber den Förderansuchenden gesetzt. So konnte die Zeitspanne von der Einreichung bis zum Vertragsabschluss um ein Drittel reduziert werden. 


\section{Wissenschaftliche Forschung und tertiäre Bildung}

Kein Sektor ist stets so vielen mannigfaltigen Veränderungen ausgesetzt wie der Hochschulsektor. So wurden mit dem Hochschulplan und der Hochschulkonferenz neue wichtige Governance-Instrumente für die Koordination und Steuerung des gesamten österreichischen Hochschulraums geschaffen, die Leistungsvereinbarungen 2013 bis 2015 mit den Universitäten abgeschlossen und im Zuge dessen die Hochschulstrukturmittel eingeführt sowie die laufende Umsetzung des neuen Kollektivvertrages für die ArbeitnehmerInnen an den Universitäten evaluiert. Darüber hinaus wurde eine sektorenübergreifenden Agentur für Qualitätssicherung und Akkreditierung Austria, die AQ Austria, etabliert und die Österreichische Akademie der Wissenschaften neu positioniert. Nicht nur national, sondern auch international zieht - alle wissenschaftlich tätigen Institutionen betreffend - das Thema Open Access mit dem Ziel, wissenschaftliche Publikationen im Internet weitgehend öffentlich zugänglich zu machen, große Aufmerksamkeit auf sich und findet sich bereits jetzt in verschiedensten Ansätzen von Maßnahmen und Initiativen hochschulpolitischer Akteure wieder.

\subsection{Hochschulplan und Hochschulkonferenz als neue Governance-Instrumente für den öster- reichischen Hochschulraum}

Die im Jahr 2011 eingeleitete Entwicklung des Hochschulplans ist ein wichtiges wissenschaftsund bildungspolitisches Vorhaben mit dem Ziel, durch abgestimmte Kooperation, Profilbildung und der Bündelung von Ressourcen die höchste Qualität von Forschung und Lehre sicherzustellen. Die zunehmende Ausdifferenzierung der Akteure, die Internationalisierung der Forschung, veränderte Finanzierungssysteme, Initiativen und Politiken auf europäischer Ebene und ambitionierte forschungspolitische Ziele erfordern, dass sich die Hochschulpartner und die Politik abstimmen und durch die Bündelung von Ressourcen Profile geschärft und weiterentwickelt werden. Ein erster Hochschulplan - dieser versteht sich als rollierend weiterzuentwickelndes Planungsinstrument - wurde im Dezember 2011 präsentiert und war Ergebnis der Arbeiten und Abstimmungsprozesse zwischen der Universitätenkonferenz, der Fachhochschulkonferenz, der Senate der Universitäten, des Wissenschaftsrates, einer internationalen ExpertInnengruppe und des Bundesministeriums für Wissenschaft und Forschung (BMWF). Der Hochschulplan stützt sich auch auf Ergebnisse des Hochschuldialogs des BMWF und einen ExpertInnenbericht von drei international renommierten HochschulforscherInnen.

Um die Realisierung der im Hochschulplan formulierten Ziele zu unterstützen und die koordinierenden Maßnahmen umzusetzen, wurde im Frühjahr 2012 die Hochschulkonferenz konstituiert. Es handelt sich dabei um ein koordinierendes und beratendes Gremium, das Stellungnahmen zu wichtigen wissenschaftspolitischen Themen erarbeitet, Problembereiche priorisiert und Empfehlungen und Lösungsvorschläge als Input für den Hochschulplan und den Bundesminister einbringt. Die Hochschulkonferenz besteht aus Mitgliedern des BMWF, der Universitätenkonferenz, der Fachhochschulkonferenz, des Wissenschaftsrates, den Senaten der Universitäten sowie der Hochschülerschaft. Sie hat sich am 03.05.2012 konstituiert und seitdem im Rahmen von unterschiedlichen Arbeitsgruppen kontinuierlich Fragestellungen bearbeitet, um akkordierte Positio- 
nen zwischen allen Akteuren zu gewinnen. Die Hochschulkonferenz folgt dabei dem Prinzip einer partnerschaftlichen Auseinandersetzung zwischen den Hochschulen und ihren Interessensgruppen. Die Koordination zwischen den Arbeitsgruppen der Hochschulkonferenz erfolgt über das Generalsekretariat des Ministeriums und einer dafür eingerichteten Geschäftsstelle. Im Sinne einer breiteren Kommunikation und Abstimmung mit anderen Interessensgruppen ist vorgesehen, dass andere Akteure, wie Ministerien, Forschungsförderungsfonds, Forschungsgesellschaften, UniversitätslehrerInnenverband oder die Landeshauptleutekonferenz, Stellungnahmen zu wichtigen Themen einbringen. Die Hochschulkonferenz ist nicht gesetzlich formalisiert, nimmt jedoch eine wichtige Position in der Governance der österreichischen Hochschulen ein. Sie realisiert die Vorstellungen moderner Governancekonzepte, die postulieren, dass die Möglichkeiten zentraler politischer Steuerung des Hochschulsystems vor dem Hintergrund der größeren Autonomie, aber auch dem gestiegenen Wettbewerb zwischen den Hochschulen, limitiert sind und eine Abstimmung zwischen den einzelnen Akteuren erforderlich ist. Eine wichtige Leitmaxime des Hochschulplans und somit auch der Hochschulkonferenz ist die Förderung der Differenzierung und Profilbildung sowie die Kooperation bei gleichzeitiger Wahrung der Autonomie der Akteure. Die Etablierung der Hochschulkonferenz als koordinierende Instanz kann demzufolge als wichtige Weiterentwicklung des österreichischen Wissenschafts- und Universitätssystems verstanden werden.

Im Rahmen der Erstellung des Hochschulplans wurden verschiedene Arbeitsgruppen gebildet und vier wesentliche Themen bearbeitet:

- Koordinierungsmaßnahmen

- Studienplatzfinanzierung

- Bauleitplan

- Forschungsinfrastrukturplan

Für die Umsetzung des Ziels einer besseren Koordination der nationalen Hochschulpolitik wurde, wie oben angeführt, das Koordinierungsgremium der Hochschulkonferenz etabliert. Unter Einbeziehung wichtiger Akteure des Hochschulraums werden seit 2011 zentrale Fragestellungen für die Hochschulentwicklung diskutiert. Die Koordination im Bereich Profilbildung, Lehre und Forschung ist dabei die zentrale Herausforderung. Sowohl im Bereich der Schwerpunktbildung in der Forschung als auch bei der Planung des Fächerangebots ist hier eine Abstimmung und ein Abgleich zwischen den Universitäten und im Besonderen auch zwischen den Standorten notwendig.

Die Einführung der Studienplatzfinanzierung ist ein prioritäres Ziel der nationalen Hochschulpolitik und ein wichtiges Vorhaben im aktuellen Regierungsprogramm. Im Rahmen des Hochschulplans wurden erste Eckpunkte eines derartigen Modells durch eine von Universitätenkonferenz und BMWF beschickte Arbeitsgruppe definiert. Mit dem Ende 2012 publizierten Entwurf zur kapazitätsorientierten studierendenbezogenen Universitätsfinanzierung hat der Gesetzgeber nach einer einjährigen Verhandlungsphase der Regierung erste Konkretisierungen des Modells vorgenommen und nun dessen Umsetzung in die Wege geleitet. Ziel ist eine transparente, sowie stärker als bisher auf Qualität und Kapazität hin ausgerichtete Gestaltung der Finanzierung der Universitäten. Die Finanzierung der Universitäten soll demzufolge zukünftig auf Basis von drei separaten Finanzierungsströmen erfolgen: Das Globalbudget wird sich zukünftig aus einem Teilbetrag für Lehre, einem Teilbetrag für Forschung und einem Teilbetrag für Infrastruktur und klinischen Mehraufwand zusammensetzen. Damit folgt Österreich dem internationalen Trend der Trennung der Finanzierung von Forschung und Lehre. Entsprechend einem speziell für Österreich entwickelten Modell stellt die Anzahl der angebotenen und betreuten Studienplätze, gewichtet nach Fächergruppen, dabei das wesentliche Kriterium für die Finanzierung der Lehre, aber auch einen Indikator für die Finanzierung der Forschung dar. Dabei wird diejenige Forschung, die zur Aufrechterhaltung der Qualität der forschungsgeleiteten Lehre erforderlich ist, 
auf Basis der Anzahl der Studienplätze bemessen und als Forschungszuschlag bezeichnet. Ein derartiges Modell wird auf internationaler Ebene beispielsweise auch von der Schweiz umgesetzt. Zusätzlich gibt es sowohl für die Lehre als auch für die Forschung eine strategische Komponente. Für die Finanzierung der universitären Forschung sollte zusätzlich ein Subbetrag auf Basis eines wettbewerbsorientieren Forschungsindikators dotiert werden.

Die Umsetzung bis zum Vollausbau ist in mehreren Phasen, entsprechend den gesetzlichen und budgetären Rahmenbedingungen, bis zum Jahr 2021 geplant. Der erste Schritt erfolgt mit der schrittweisen Optimierung der Studienbedingungen, die mit der Möglichkeit von Zugangsregeln in fünf besonders stark nachgefragten Studienfächern und der zusätzlichen Schaffung von 95 ProfessorInnenstellen in diesen Studienfeldern einhergeht.

Im Rahmen des Bauleitplans wurden für die drei Planungsregionen Ost, Süd und West wichtige Bauvorhaben priorisiert. Der Bauleitplan legt die Abfolge des Baugeschehens an den 22 staatlichen Hochschulen und der Österreichischen Akademie der Wissenschaften fest. Diese abgestimmte Gesamtplanung diente auch als Vorlage für die Leistungsvereinbarungen.

Mit Hilfe des Forschungsinfrastrukturplans sollen Investitionen in Großforschungsinfrastruktur geplant und abgestimmt werden. Dabei wird eine Prioritätensetzung für die Finanzierung und den Ausbau der kostenintensiven Forschungsinfrastruktur vorgenommen. In diesem Zusammenhang kann auch auf die in den Jahren 2011 und 2012 durchgeführte systematische Erfassung der gesamten Forschungsinfrastruktur an österreichischen Universitäten mit einem Anschaffungswert von mehr als $100.000 €$ hingewiesen werden, die eine wichtige Basis für die Entwicklung einer ForschungsinfrastrukturRoadmap liefert.

Die Förderung der Internationalisierung ist ein weiteres wichtiges Ziel des Hochschulplans und wird als Querschnittthema verstanden. Im Hochschulplan wurde die Entwicklung von Internatio- nalisierungsstrategien an allen Universitäten als wichtige Maßnahme definiert. Derartige Strategien müssen internationale, nationale als auch regionale Entwicklungen in Einklang bringen und strategische Partnerschaften zwischen Hochschulen, außeruniversitären Forschungseinrichtungen und der Wirtschaft bündeln. Um sich erfolgreich an internationalen und europäischen Initiativen, Programmen und Netzwerken zu beteiligen, ist eine Abstimmung unter den nationalen Akteuren notwendig. In diesem Zusammenhang empfiehlt der Hochschulplan ebenfalls die Formulierung von regionalen Spezialisierungsstrategien, international auch als "Smart Specialisation" bezeichnet. Erklärtes Ziel ist es, dass die Hochschulen und außeruniversitären Forschungseinrichtungen im Hinblick auf die zukünftige Ausrichtung der EU-Forschungspolitik in enger Kooperation mit den jeweiligen regionalen Kontaktstellen der Förderungsagenturen regionale Spezialisierungsstrategien entwickeln. Ferner wird in diesem Zusammenhang im Hochschulplan angeregt, dass Forschungsservicestellen verstärkt ausgebaut werden sollen, auch um Projektmanagementaktivitäten von (international und europäisch finanzierten) Drittmittelprojekten zu übernehmen und zu professionalisieren.

Die Notwendigkeit einer verstärkten Abstimmung auf regionaler Ebene demonstrieren auch die ins Leben gerufenen Hochschulkonferenzen der Bundesländer (derzeit in Tirol, Steiermark, Burgenland und Salzburg), deren Zielsetzungen jener der Hochschulkonferenz des Bundes entsprechen, wie z. B. die Abstimmung inhaltlicher Positionierungen und die Förderung von Kooperationen unter Beibehaltung eigenständiger Profile. So soll z.B. in der Steiermark der Ressourceneinsatz des gemeinsamen Forschungs- und Lehrraums optimiert und die Infrastruktur bestmöglich genutzt werden. Auch im Bereich der Intellectual Property Rights und der Forschungsservices soll zukünftig verstärkt zusammengearbeitet werden.

Mit dem Hochschulplan und der Hochschulkonferenz wurden 2011 und 2012 wichtige politische Zielsetzungen und Positionen des zustän- 
digen Ressorts definiert. Die Abstimmung bei der Profil- und Schwerpunktsetzung im Bereich Forschung, die Erstellung eines Forschungsinfrastrukturplans sowie Maßnahmen zur Einführung einer neuen Universitätsfinanzierung können an dieser Stelle als besonderes relevant für die weitere Entwicklung des österreichischen Forschungs- und Innovationsstandorts charakterisiert werden. Die im Rahmen des Hochschulplans erarbeiteten Ziele und Strategien lieferten eine wichtige Basis für die Ausarbeitung der Leistungsvereinbarungen für die Jahre 2013 bis 2015. Die Leistungsvereinbarungen sind eines der zentralen Instrumente zur Umsetzung von Hochschul- und wissenschaftspolitischen Zielsetzungen auf Basis von budgetären Anreizmechanismen. Auf die im Jahr 2012 zwischen dem BMWF und den Universitäten abgeschlossenen Leistungsvereinbarungen wird im Folgenden eingegangen.

\subsection{Die Finanzierung der Universitäten in den Jahren 2013 bis 2015}

Die Finanzierung der Universitäten für die Jahre 2013 bis 2015 ist durch zwei wesentliche Merkmale geprägt. Zum einen gehen die Universitäten eine neue dreijährige Leistungsvereinbarungsperiode ein, zum anderen wird das formelgebundene Budget durch das neue Instrument der Hochschulraum-Strukturmittel abgelöst. Im Folgenden werden zunächst die wesentlichen Schwerpunkte und forschungspolitischen Strategien, die in den Leistungsvereinbarungen definiert wurden, beschrieben. Dabei wird im Besonderen auf die Schaffung kritischer Größen durch nationale Kooperationen, auf Schwerpunktsetzungen im Bereich Forschung und auf den Ausbau der Großforschungsinfrastruktur eingegangen. Wenngleich die Bereitstellung von qualifizierten AbsolventInnen zweifelsohne einen wichtigen Beitrag für den Forschungs-, Innovations- und Wirtschaftsstandort Österreich darstellt, soll auf die Entwicklungen im Bereich Lehre und Weiterbildung im Forschungs- und Technologiebericht nicht detaillierter eingegan- gen werden. Auf Basis der Leistungsvereinbarungen der öffentlichen Universitäten soll schließlich der Frage nachgegangen werden, i) wie weit österreichische Universitäten bei der Profilbildung fortgeschritten sind, ii) welche grundsätzlichen Strategien eingeschlagen werden und iii) in welchem Umfang Forschungsschwerpunkte definiert werden.

\subsubsection{Abschluss der Leistungsvereinbarungen 2013 bis 2015}

Die Leistungsvereinbarungen wurden mit dem UG 2002 eingeführt und sind das zentrale Instrument für die Finanzierung und Steuerung der österreichischen öffentlichen Universitäten. Mit Hilfe der Leistungsvereinbarungen werden über eine Periode von drei Jahren Globalbudgets für Universitäten allokiert, deren Höhe an Ziele und Leistungen gekoppelt sind. 2012 erfolgten die Verhandlungen für die dritte Leistungsvereinbarungsperiode 2013 bis 2015. Als Ende April die Entwürfe von Seiten der Universitäten vorlagen, erfolgte eine erste Verhandlungsrunde und eine schriftliche Stellungahme von Seiten des zuständigen Ressorts. Dazu wurden für die von den Universitäten formulierten Vorhaben und Ziele quantitative Analysen und Bewertungen vom BMWF durchgeführt. Bis Ende Dezember konnten mit allen Universitäten die Verhandlungen erfolgreich finalisiert werden. Der Abschluss der neuen Leistungsvereinbarungen baute auch auf den Erfahrungen der beiden ersten Leistungsvereinbarungen auf, berücksichtigte Empfehlungen des Rechnungshofes und des Österreichischen Wissenschaftsrates und erfolgte auf Grundlage eines adaptierten Verhandlungsleitfadens.

Der Hochschulplan, aber auch die FTI-Strategie des Bundes stellen den zentralen strategischen Rahmen von Seiten des BMWF für die Steuerung der Universitäten dar. Die wichtigsten Ziele und Themen im Bereich Forschung für das Ministerium waren, i) die Fortführung der Schwerpunkt- und Profilbildung, ii) die Intensivierung der Kooperationen, iii) der Ausbau der Internationalisierung, iv) der strategische Aus- 
bau der Forschungsinfrastruktur sowie v) die weitere (quantitative oder qualitative) Entwicklung der Drittmitteleinwerbung. Weiterhin waren hochschulpolitische Vorgaben die verstärkte Koordinations- und Kapazitätsorientierung, die im Lichte des Hochschulplans und der zukünftigen studierendenbezogenen Steuerung und Finanzierung eine zusätzliche Abstimmung zwischen den Akteuren erfordert. Beinahe alle Universitäten nehmen bei angestrebten Zielen und Maßnahmen folglich auch explizit zum Österreichischen Hochschulplan Bezug.

Die Formulierung der Leistungsvereinbarungen von Seiten der Universitäten war begleitet durch die Erstellung bzw. Adaptierung der Entwicklungspläne. Diese bilden die Grundlage für die Erstellung der Leistungsvereinbarungsentwürfe durch die Universitäten.

\section{Schwerpunkte und Strategien im Bereich Forschung}

Die Bündelung und Ausrichtung der Forschungstätigkeiten auf Forschungsschwerpunkte ist ein wichtiges und längerfristiges Ziel der Hochschulund Forschungspolitik. Die Schwerpunkt- und Profilentwicklung ist dabei ein Vorhaben, das besondere Kompetenzen der österreichischen Universitäten bzw. Fakultäten stärken soll. Universitäten sollten dabei von ihren Ressourcen ausgehen und festlegen, worin ihre besonderen Stärken liegen und was sie an vorhandenen Kapazitäten ausbauen können, sodass sie nachhaltig in der Scientific Community und am Forschungsmarkt reüssieren können bzw. als einzigartig gesehen werden. Bereits im Rahmen der Erstellung der ersten Entwicklungspläne und Leistungsvereinbarungen in den Jahren 2004 und 2005 begannen Universitäten mit der Formulierung von Forschungsschwerpunkten. Mit Beginn 2006 hatten alle öffentlichen Universitäten auf Ebene der Fakultäten bzw. Departments Forschungsschwerpunkte formuliert und ein Großteil der Hochschulen hatte auch bereits auf gesamtuniversitärer Ebene gemeinsam prioritär zu bearbeitende Forschungsthemen definiert. Die gesamtuniversitären „Forschungsschwerpunkte" werden von den Universitäten explizit als Forschungsschwerpunkte (z.B. Universität Innsbruck), aber auch als Forschungscluster (z.B. Medizinische Universität Wien), Forschungsfelder (Medizinische Universität Graz), Fields of Expertise (Technische Universität Graz), Kompetenzfelder (z.B. Universität für Bodenkultur), Profillinien (z.B. Veterinärmedizinische Universität Wien) oder Profilschwerpunkte (Universität für künstlerische und industrielle Gestaltung Linz) bezeichnet. Zusätzlich haben einige Universitäten auch noch Forschungsplattformen oder Forschungszentren definiert, an denen mehrere Fakultäten gemeinsam beteiligt sind. Insgesamt zeigt sich, dass die als Forschungsschwerpunkte bezeichneten Forschungsthemen häufig enger definiert sind, während Kompetenzfelder, Forschungscluster oder Forschungsfelder breiter gefasst sind. Für die vollständige Liste aller von den Universitäten verfolgten Forschungsschwerpunkte auf gesamtuniversitärer Ebene siehe auch Anhang III.

Der Prozess der Schwerpunktbildung war und ist für die Universitäten ein herausfordernder und teilweise schwieriger Prozess, gilt es doch, über Instituts-, Fakultäts- und Disziplinengrenzen hinweg gemeinsame Themen zu definieren und dabei auch angestammte Pfade zu verlassen. Die Schwerpunkte bzw. Themenfelder haben im Allgemeinen fast immer einen interdisziplinären Charakter. Damit versuchen die Universitäten die optimale Kombination aus erfolgreicher Einzelforschung, fakultären Forschungsschwerpunkten, interfakultären Forschungsschwerpunkten und universitären Schwerpunkten $\mathrm{zu}$ finden. Universitäten stehen dabei in einem Spannungsfeld: Sie wollen und müssen sich einerseits auf ausgewählte Forschungsthemen konzentrieren, wollen aber anderseits die notwendige Breite für innovative Entwicklungen aufrechterhalten. Dabei ist die fachliche Breite vor allem für kleinere Universitäten zur Realisierung der forschungsgeleiteten Lehre notwendig, wie dies etwa die Universität Linz bekräftigt. Zugleich liegt die Einsicht vor, dass in der Vielfalt auch eine Stärke liegt und neue innovative Forschungsfelder vor allem durch die Vernetzung von unterschiedli- 
chen Disziplinen entstehen. Die Universität Graz bekennt sich in diesem Zusammenhang etwa zur Methoden- und Themenvielfalt, definiert aber zugleich in den Kernbereichen ihrer Wissenschaftsdisziplinen Forschungsschwerpunkte. Auch die Universität Wien betrachtet die auf disziplinärer Exzellenz beruhende interdisziplinäre Forschung im besonderen Maße profilbildend. Interdisziplinäre Forschung wird dabei als etwas verstanden, das - immer basierend auf hochqualifizierter disziplinärer Forschung - innerhalb einer Fakultät, zwischen den Fakultäten bzw. Zentren oder in der Zusammenarbeit zwischen Universitäten und außeruniversitärer Forschungseinrichtungen erfolgt. Dies illustriert die allgemeine Herausforderung für Universitäten, die geeignete Balance zwischen Differenzierung und Fokussierung zu finden. In diesem Zusammenhang weisen einige Universitäten auch auf ihre einzigartige Kombination von Fächern hin, so hebt etwa die Universität für Bodenkultur die Verknüpfung von Natur-, Ingenieur- sowie Sozial- und Wirtschaftswissenschaften hervor oder die Universität Linz die Verschränkung der Natur-, Ingenieur-, Sozial-, Wirtschafts- und Rechtswissenschaften.

Im Rahmen der rollierenden Entwicklungsplanung und der neu verhandelten Leistungsvereinbarung zeigt sich nun, dass beinahe alle österreichischen Universitäten explizit gesamtuniversitäre "Forschungsschwerpunkte" definiert haben, die - neben anderen forschungsstrategischen Zielen der Universitäten - prioritär entwickelt werden sollen. So haben etwa die Wirtschaftsuniversität Wien oder die Universität Klagenfurt 2012 ihren internen Schwerpunktbildungsprozess erfolgreich abgeschlossen; letztere hat die "Profilbildung durch fakultätsübergreifende Forschungsthemen" als Vorhaben in der Leistungsvereinbarung definiert und will hierfür Koordinationsmechanismen etablieren.

Insgesamt handelt es sich bei der Profil- und Schwerpunktbildung jedoch um einen kontinuierlichen Prozess. So waren in den letzten Jahren einige Universitäten bestrebt, die Anzahl der Forschungsschwerpunkte zu reduzieren, eine Erwartung, die teilweise auch von den Universitätsrä- ten eingefordert wurde. So hat etwa die Technische Universität Graz in den vergangenen Jahren die Anzahl der Forschungsschwerpunkte, dort auch Fields of Expertise genannt, von sieben auf fünf reduziert und vermarket diese auch als „Fingerprint" der Universität. Die Universität Linz fokussiert sich von vormals acht auf nun sechs sogenannte Exzellenzschwerpunkte. In diesem Prozess werden in manchen Fällen auch inhaltlich verwandte Themen stärker gebündelt. Einige Universitäten (Bsp. Medizinische Universität Graz) sprechen indes explizit davon, dass keine Zusammenlegung von Forschungsschwerpunkten vorgenommen wurde oder zukünftig geplant ist. Zugleich haben fallweise Universitäten neue vielversprechende Themen als zukünftig prioritär zu verfolgen definiert. So möchte die Montanuniversität Leoben in den nächsten Jahren die Energietechnik verstärkt ausbauen und das "Zentrum am Berg" - ein Forschungs-, Sicherheits- und Ausbildungszentrum unter realen Betriebsbedingungen für Fachgebiete wie Geotechnik, Rohstoffgewinnung, Berg- und Tunnelbau oder Petroleum Engineering - realisieren. Die Wirtschaftsuniversität will zukünftig den neuen Schwerpunkt "Global Transformations and Sustainability: People, Businesses and Policies" hier als Ausbaubereich bezeichnet - neu etablieren und entwickeln. Die Medizinische Universität Innsbruck plant, den neuen Forschungsschwerpunkt „Genetik, Epigentik, Genomik“ zu forcieren und argumentiert etwa, dass erfolgreiche GEN-AU Projekte dazu beigetragen haben, dieses Thema auszubauen. Die Veterinärmedizinische Universität Wien will den Forschungsschwerpunkt „Tierverhalten und Mensch-TierBeziehung" etablieren. Die Definition derartiger Forschungsschwerpunkte erfolgt etwa bei der Medizinischen Universität Innsbruck nach besonders stringenten Kriterien, wie die Anzahl der bereits vorhandenen Publikationen im Themengebiet, die am Thema beteiligten WissenschaftlerInnen und die Möglichkeiten, erfolgreich Drittmittel einzuwerben.

Große Anstrengungen unternehmen die Universitäten auch, um die gesamtuniversitären 
Schwerpunkte nachhaltig zu verankern und auszubauen. Dazu zählen die Ernennung von Koordinatoren, die zusätzliche Finanzierung aus universitären Mitteln, die Nachbesetzung bestehender und Schaffung neuer Professuren in den Forschungsschwerpunkten, der Aufbau von Doktoratskollegs, der Ausbau von Postdoc-Programmen sowie die Förderung einer gezielten internationalen Publikationsstrategie. Einzelne Universitäten haben die in den frühen Phasen noch häufig sehr allgemein abgegrenzten Themen weiter spezifiziert und mit den Strategien der sich beteiligenden Institute oder Fakultäten akkordiert. Die Technische Universität Wien spezifiziert in diesem Zusammenhang etwa die „TU Forschungsmatrix". Die Weiterentwicklung des gesamtuniversitären Schwerpunktbildungsprozesses ist entsprechend häufig als explizites Vorhaben innerhalb der Leistungsvereinbarungen definiert.

Insgesamt wurden von den Universitäten vielfach erste Analysen, Bewertungen und Evaluierungen der verfolgten Schwerpunkte durchgeführt. So werden etwa von den Medizinischen Universitäten die Anzahl der Publikationen und sogar der Zitationen auf Ebene der einzelnen Forschungsschwerpunkte ausgewertet. Aber auch Drittmitteleinnahmen (wie z.B. FWF, Sonderforschungsbereiche, EU-Projekte, COMET) und die Etablierung von strukturierten Doktoratsprogrammen bzw. Graduiertenkollegs werden als wichtig erachtet. Die Einwerbung von Drittmitteln im Bereich der Forschungsschwerpunkte wird dabei als Indikator für den Erfolg eines Forschungsschwerpunktes gewertet. Einige Universitäten verfolgen auch eine Strategie, bei der mittelfristig erfolgreiche Schwerpunkte oder Plattformen längerfristig institutionalisiert werden, etwa in Form von Forschungszentren.

Gleichzeitig wollen die Universitäten neue riskantere Themen abseits der definierten Forschungsschwerpunkte fördern. An der Universität Graz wird etwa in diesem Zusammenhang argumentiert, dass das Forschungsprofil von Universitäten zu Recht auf bestehenden Stärken aufbaut und dazu kritische Massen gebildet werden.
Es wird jedoch argumentiert, dass es für Universitäten unerlässlich ist, Freiräume zu schaffen, in denen Forschung, die vollkommen neue Wege beschreitet und sich abseits des Etablierten entwickelt, entstehen kann. Ziel ist es entsprechend an der Universität Graz, die Entwicklung von „radikal (im Sinne von unorthodoxen) innovativen Forschungsansätzen" zu fördern, vor allem durch die Bildung von interdisziplinären Teams aus verschiedenen Wissenschaftskulturen.

Neben der expliziten Forcierung der Profilentwicklung sind die Erhöhung des Drittmittelanteils, die Erhöhung der Anzahl der Publikationen, der Ausbau der Doktoratsprogramme und die weitere Internationalisierung wichtige Vorhaben, die in den Leistungsvereinbarungen definiert wurden. Hier wurden auch konkrete Zielwerte innerhalb der Leistungsvereinbarungen festgelegt. Zur quantitativen oder qualitativen Weiterentwicklung der Drittmittel haben Universitäten Drittmittelstrategien definiert, auch in Zusammenhang mit der Internationalisierung und dem Verweis auf die Bedeutung der EU-Forschungsrahmenprogramme und HORIZON 2020. Die Wirtschaftsuniversität Wien will in diesem Zusammenhang die Schaffung einer eigenen EU-Stelle zur weiteren Professionalisierung der Forschungsservices etablieren und finanzielle Mittel (Matching Grants) zur Unterstützung der Anbahnung und Abwicklung großer EU-Projekte zur Verfügung stellen.

Des Weiteren zeigt die Analyse der neuen Entwicklungspläne und Leistungsvereinbarungen, dass die Ausrichtung der Forschung zur Lösung gesellschaftlicher Herausforderungen an Bedeutung gewinnt. Die EU hat hierfür den Begriff der "Grand Challenges" definiert, der bereits vielfach innerhalb der neuen Strategiedokumente der Hochschulen rezipiert wird. Die Universität Wien argumentiert in diesem Zusammenhang etwa: „Die Europäische Kommission wird im kommenden Rahmenprogramm „Horizon 2020“ ein verstärktes Augenmerk auf die Förderung von Forschungsaktivitäten setzen, die sich mit großen gesellschaftlichen Herausforderungen beschäftigen. Auch deshalb ermutigt die Uni- 
versität Wien ihre WissenschafterInnen dazu, sich verstärkt an Projekten in diesen Bereichen zu beteiligen und hat bereits beachtliche Vorarbeiten in entsprechenden Gebieten vorzuweisen." Dabei wird auch die Ausrichtung auf gesellschaftliche Problemstellungen als profilbildend angesehen. Um die gesellschaftlichen Herausforderungen $\mathrm{zu}$ adressieren, formuliert die Universität für Bodenkultur Wien etwa das „Drei-Säulen-Modell" - Naturwissenschaften, Technik sowie Sozial- und Wirtschaftswissenschaften - in Lehre und Forschung, das eine umfassende Bearbeitung gesellschaftsrelevanter Problemstellungen ermöglichen soll.

\section{Strategien im Bereich Kooperation, Forschungs- infrastruktur und Internationalisierung}

Neben der Schwerpunktbildung auf universitärer Ebene ist es ein wichtiges forschungspolitisches Ziel von Seiten des BMWF, die Kooperation und Abstimmung der Forschungstätigkeiten zwischen den Universitäten zu fördern. Ein weiterer Kernpunkt für die Verhandlungen von Seiten des Ministeriums war eine stärkere strategische Steuerung bei der Planung von Investitionen in Forschungsinfrastruktur und eine entsprechende Abstimmung zwischen den Universitäten. In der Leistungsvereinbarung 2013 bis 2015 mussten die Universitäten vor diesem Hintergrund - auch erstmals in einem eigenen Unterabschnitt im Kapitel Forschung - ihre Investitionen in nationale und internationale Großforschungsinfrastruktur planen und den Bezug zur Forschungsstrategie darstellen. Beide Ziele sind, wie oben angeführt, zentrale Vorgaben und Empfehlung des Hochschulplans.

Die Kooperation im Bereich der Forschung zwischen den heimischen Universitäten wurde in den letzten Jahren sukzessive ausgebaut und war teilweise auch durch die Notwendigkeit vorangetrieben, eine immer investitions- und kostenintensivere Forschungsinfrastruktur gemeinsam zu nutzen. Infrastrukturen werden zunehmend kooperativ genutzt; die Universitäten sprechen von fakultärer, interfakultärer und interuniversitärer gemeinsamer Nutzung von Forschungsinfrastrukturen. So nennt etwa die Universität Wien in diesem Zusammenhang je ein Beispiel und führt aus, dass das Massenspektrometriezentrum gemeinsam von allen Instituten der Fakultät Chemie genutzt wird, das NMRSpektroskopie-Zentrum in Kooperation zwischen der Fakultät für Chemie und Lebenswissenschaften und das Fakultätszentrum Nanostrukturforschung nicht nur von der Fakultät für Physik und Chemie, sondern auch in Kooperation mit der Technischen Universität Wien und der Universität für Bodenkultur genutzt wird. Bekannte erfolgreiche Kooperationen, wie die Max Perutz Laboratories in Wien (Universität Wien und Medizinische Universität Wien), die NAWI Graz (Universität Graz und Technische Universität Graz) oder das High Performance Computing in Wien (Universität Wien, Technische Universität Wien und Universität für Bodenkultur) sollten alle gemäß Leistungsvereinbarung weiter ausgebaut werden. Daneben können beispielhaft neuere Vorhaben wie der Wassercluster Lunz (Universität Wien, Universität für Bodenkultur und Donau-Universität Krems), das Vienna Center for Computational Materials Science (Universität Wien und Technische Universität Wien), der Forschungsbereich Regenerative Biologie (Universität Salzburg und Paracelsus Medizinische Privatuniversität) oder Kooperationen im Bereich des Biobanking (Medizinische Universitäten) angeführt werden. Neben Kooperationen zwischen Universitäten wurden im Rahmen der Leistungsvereinbarungen auch Kooperationsvorhaben mit außeruniversitären Forschungseinrichtungen geplant. Auch Vorhaben im Bereich der Beteiligung an internationalen Großforschungsinfrastrukturen werden angeführt. So beteiligt sich etwa die Technische Universität Wien am CERN, die Medizinische Universität am INSTRUCT, einer Initiative europäischer Strukturbiologen, - Leistungen, die einen entsprechenden finanziellen Aufwand von Seiten der Universitäten erfordern und innerhalb der Leistungsvereinbarungen Berücksichtigung finden. 
Im Kontext einer stärker abgestimmten Planung der Forschungsaktivitäten zwischen Universitäten und anderen Forschungsakteuren an den einzelnen Standorten folgen Universitäten häufig einer "Smart Specialisation"-Strategie. Derartige Strategien sind derzeit in vielen Bundesländern in Ausarbeitung, häufig auch in Kombination mit einer gleichzeitig entwickelten Internationalisierungsstrategie. So soll am Standort Graz zukünftig verstärkt die Lipidforschung zwischen den drei Grazer Universitäten ausgebaut werden. Die Veterinärmedizinische Universität Wien hat etwa die Bereiche „Biomed" und „Biomedtech" innerhalb des Life Science Clusters Wien als passende Bereiche für die Einbindung in die Smart Specialisation-Strategie definiert.

Die Eingliederung von Instituten der Akademie der Wissenschaften ist ebenfalls ein wichtiges Vorhaben an einigen österreichischen Universitäten (siehe hierzu auch Kapitel 3.5) und entsprechend als Vorhaben in der Leistungsvereinbarung definiert. Dies erfordert vielerorts die Umsetzung integrativer Maßnahmen und die Definition von neuen Forschungsstrategien.

Ein weiterer Schwerpunkt ist die Kooperation im Bereich der Forschungsservices. Vor dem Hintergrund der bereits bewährten Kooperation in der Steiermark (NAWI Graz, BioTechMed und Steirische Hochschulkonferenz) ist etwa dort geplant, auch forschungs- und technologierelevanten Serviceleistungen der fünf Universitäten vermehrt aufeinander abzustimmen. Auch im Bereich der Patentaktivitäten und -verwertung soll verstärkt zusammengearbeitet werden.

Die Entwicklung von „Internationalisierungsstrategien" an allen Universitäten wurde im Hochschulplan als eine wesentliche Maßnahme zur Etablierung der europäischen und internationalen Orientierung formuliert. Die internationale Forschungsorientierung der Universitäten wird auch durch die Orientierung am HORIZON 2020 ersichtlich und dem bereits angeführten geplanten Ausbau der Forschungsservices für internationale Projekte und Programme an vielen Universitäten. Einige Universitäten wollen sich auch an Joint Programming Initiatives (JPIs) beteiligen und haben dies als explizites Vorhaben definiert, so etwa die Medizinischen Universitäten Wien und Graz.

Insgesamt wurden im Leistungsbereich „Forschung" 291 Vorhaben und 101 Ziele formuliert. Dabei wurden von Seiten des BMWF, aber auch von Seiten der Universitäten selbst vermehrt Indikatoren genutzt, um die Zielerreichung und den Erfolg der Umsetzung von Vorhaben zu quantifizieren und messbar zu machen. Des Weiteren wurden für die Umsetzung der Vorhaben in stärkerem Umfang als in den letzten Leistungsvereinbarungen Meilensteine definiert. Was die ausverhandelten Zielwerte betrifft, hat man hier durchwegs konservative Zielwerte angesetzt. Besonders ambitionierte und vermutlich unrealistische Ziele will man vor dem Hintergrund eines schwierigeren Umfelds, was die Finanzierung und den steigenden Wettbewerb betrifft, nicht vereinbaren. „Konstant halten", „Stabilisierung", oder "moderater Anstieg" sind typische Formulierungen in diesem Zusammenhang, die vermutlich aber allesamt das Ziel einer „belastbareren" Vereinbarungsbasis verfolgen.

Zusammenfassend kann an dieser Stelle der Befund gestellt werden, dass der Profilbildungsprozess an den öffentlichen Universitäten eine hohe Kontinuität bei den definierten und über die Jahre verfolgten Forschungsschwerpunkten aufweist. Daneben zeigt sich ein starker Entwicklungstrend in Richtung einer stärkeren Bündelung der Ressourcen und der Nutzung von gemeinsamen Infrastrukturen im Rahmen von gemeinsam definierten Forschungsthemen und Forschungszentren. Alle Universitäten bekennen sich dazu, die Aktivitäten im Bereich Kooperationen unter Berücksichtigung der Empfehlungen des Hochschulplans strategisch fortzuführen. Die Universitäten wollen zur Stärkung der Kooperation zukünftig auch gemeinsame Anträge für eine Finanzierung aus den HochschulraumStrukturmitteln stellen, sobald die entsprechende Ausschreibung vorliegt. 


\subsubsection{Die Hochschulraum-Strukturmittel}

Im Rahmen einer Novelle des UG wurde im Sommer 2012 das Instrument der Hochschulraum-Strukturmittel eingeführt. Damit wurde die leistungsorientierte Finanzierung in Form des Formelbudgets durch einen neuen Allokationsmechanismus abgelöst und der zur Finanzierung der Universitäten zur Verfügung stehende Gesamtbetrag um die Hochschulraum-Strukturmittel ergänzt. Das Formelbudget wurde mit dem UG 2002 eingeführt und für die Finanzierung einer eigenen Budgetkomponente im Zeitraum von 2004 bis 2012 angewendet. Gemeinsam mit dem Grundbudget bildet das formelgebundene Budget das Globalbudget, das jeweils für die dreijährige Leistungsvereinbarungsperiode im Voraus festgelegt wird. Diese Budgetkomponente machte insgesamt rund $20 \%$ des Globalbudgets der Universitäten aus. Insgesamt wurden im Formelbudget elf Indikatoren verwendet, die unterschiedlich gewichtet wurden. Die Lehre wurde durch vier Indikatoren bemessen und insgesamt mit $45 \%$ gewichtet, die Forschung bzw. Erschließung und Entwicklung der Künste (EEK) wurden mit zwei Indikatoren bemessen, die in Summe ebenfalls mit $45 \%$ gewichtet wurden. Des Weiteren wurden die Frauenförderung (zwei Indikatoren) mit $7 \%$ und die Studierendenmobilität (zwei Indikatoren) mit $3 \%$ innerhalb der Gesamtformel berücksichtigt. Mit der Anzahl der prüfungsaktiven Studierenden, der Anzahl der Studienabschlüsse und den Einnahmen aus F\&E-Projekten wurden beim Formelbudget drei Indikatoren verwendet, die in adaptierter Form nun auch beim Verteilungsmodell der Hochschulraum-Strukturmittel zur Anwendung kommen.

Ziel der neuen Regelung ist es, die bisherige komplexe indikatorenbezogene Finanzierung über das formelgebundene Budget an Hand von wenigen Indikatoren nachvollziehbar zu berechnen. Der den Universitäten zur Verfügung stehende Gesamtbetrag setzt sich für die LV-Periode 2013 bis 2015 aus einem Teilbetrag für die Grundbudgets und einem Teilbetrag für die
Hochschulraum-Strukturmittel zusammen. Neben dem Grundbudget, das weiterhin auf Basis von Leistungsvereinbarungen definiert wird, werden für die LV-Periode 2013 bis 2015 Hochschulraum-Strukturmittel in der Höhe von 450 Mio. $€$ verteilt. Die Gesamtsumme wird in fünf Teilbeträge mit folgenden Anteilen geteilt: 1 . Teilbetrag für prüfungsaktiv betriebene ordentliche Studien $(60 \%)$, 2. Teilbetrag für AbsolventInnen ordentlicher Studien $(10 \%), 3$. Teilbetrag für Wissenstransfer $(14 \%), 4$. Teilbetrag für private Spenden (2\%) sowie 5 . Teilbetrag für Kooperationen $(14 \%)$. Die entsprechenden Indikatoren sind wie folgt definiert: Indikator 1: „Anzahl der prüfungsaktiv betriebenen ordentlichen Bachelor-, Diplom- und Masterstudien mit Gewichtung nach Fächergruppen“, Indikator 2: „Anzahl der AbsolventInnen ordentlicher Bachelor-, Diplom- und Masterstudien mit Gewichtung nach Fächergruppen“, Indikator 3: „Erlöse aus F\&EProjekten/Projekten der Entwicklung und Erschließung der Künste in $€$ " sowie Indikator 4: Erlöse aus privaten Spenden in $€$ (gemäß Wissensbilanzverordnung 2010). Der Teilbetrag für Kooperationen wird auf Basis von qualitativen Kriterien von Ausschreibungen verteilt.

Die Indikatoren 1 und 2 werden jeweils nach Fächergruppen gewichtet. Dabei wurden sämtliche von österreichischen Universitäten angebotene Studien sieben Fächergruppen zugeordnet, die jeweils mit einem Faktor von 1 bis maximal 5 gewichtet werden. Auch im früheren Formelmodell gab es bereits eine solche Gewichtung, allerdings wurde hier nur zwischen drei nach Studienrichtungen gegliederten Fächergruppen differenziert. Die im Hochschulraum-Strukturmittel-Modell vorgenommene Differenzierung in sieben Gruppen ist insofern relevant, da sie in ihrer Logik mit der geplanten Einführung der Studienplatzfinanzierung einhergeht. Bei der Festlegung dieser Gewichte gab es zahlreiche Konsultationsprozesse mit den Universitäten, um unterschiedliche Kosten, die den Universitäten für die Durchführung der Lehre in unterschiedlichen Fächern entstehen, möglichst adäquat abzubilden. Die zwischen dem Ministeri- 
um und der Universitätenkonferenz konstituierte Arbeitsgruppe zur kapazitätsorientierten Studienplatzfinanzierung hat im Jahr 2011 umfangreiche Abschätzungen der Kosten für die Lehre in unterschiedlichen Fächern unter Berücksichtigung von angestrebten Zielwerten im Bereich der Betreuungsverhältnisse durchgeführt, die eine wichtige Grundlage für die Definition der sieben Fächergruppen waren.

Sowohl die Regelung zur Verteilung der Hochschulraum-Strukturmittel als auch die Studienplatzfinanzierung verfolgen zur Schaffung eines transparenteren Kalkulations-, Budgetierungsund Controlling-Rahmens eine Strategie der getrennten Ausweisung der Finanzierung von Forschung und Lehre. Vor dem Hintergrund der oben angeführten Struktur werden für die LV-Periode 2013 bis 2015 im Kontext der HochschulraumStrukturmittel 315 Mio. $€$ (Indikator 1 und 2) für die Lehre verteilt. Für die Komponente Wissenstransfer stehen insgesamt 72 Mio. $€$ (Indikator 3 und 4) zur Verfügung. Für die Komponente „Kooperationen“ stehen 63 Mio. $€$ zur Verfügung. Der oben angeführte Indikator „Erlöse aus F\&E-Projekten/Projekten der Entwicklung und Erschließung der Künste in $€^{\prime \prime}$ beruht auf der Kennzahl I.C.2 „Erlöse aus F\&E-Projekten/Projekten der Entwicklung und Erschließung der Künste in $€^{\prime \prime}$ der Wissensbilanz-Verordnung (WBV 2010). Hier werden jedoch nur jene Erlöse berücksichtigt, die von FWF, Jubiläumsfonds der OENB, EU, Ländern (inkl. deren Stiftungen und Einrichtungen), Gemeinden und Gemeindeverbänden (ohne Wien), Unternehmen sowie Privaten (Stiftungen, Vereine etc.) als Förderung oder Auftrag lukriert werden.

Wie oben angeführt wird ein weiterer Teil der Hochschulraum-Strukturmittel im Rahmen von Ausschreibungsverfahren für Kooperationsprojekte vergeben. Die Kooperationsprojekte sollen folgende drei Bereiche des universitären Leistungsspektrums abdecken: Lehre, Forschung bzw. Entwicklung und Erschließung der Künste sowie Verwaltung. Es sollen jedenfalls Projekte aus allen drei Bereichen gefördert werden, wobei im Sinn der rechtlichen Vorgaben exzellenzför- dernde bzw. strukturentwickelnde Kooperationsprojekte (insbesondere Cluster- \& Schulbildung) vorrangig Berücksichtigung finden sollen. Mit dem Teilbetrag für Kooperationen werden die Kosten einzelner Projekte bis zu einem Drittel finanziert. Die Projektmittel dienen der Anschubfinanzierung. Zur Abwicklung des Ausschreibungs- und Vergabeverfahrens wird eine Kommission eingesetzt. Die Kooperationsprojekte werden 2013 eingereicht. Einige Universitäten haben bereits in den aktuellen Leistungsvereinbarungen mögliche Projekte definiert, die als Projekte eingereicht werden sollen.

Wie auch schon in der Vergangenheit ist das vom BMWF zugewiesene Globalbudget für die LV-Periode 2013 bis 2015 frei disponierbar. Dabei werden an den österreichischen Universitäten in der Regel intern Zielvereinbarungen zwischen dem Rektorat und den Fakultäten bzw. Departments definiert und einige Universitäten verwenden in Analogie zum Formelbudget bzw. den Hochschulraum-Strukturmitteln Indikatorenbezogene Modelle zur internen Allokation der finanziellen Ressourcen.

Mit den Hochschulraum-Strukturmitteln wurde ein neues, transparentes und leistungsorientiertes Finanzierungsmodell realisiert, welches in Bezug auf die Forschung Anreize setzt, dass Universitäten Drittmittel akquirieren und eine entsprechende Ko-Finanzierung vornehmen.

\subsection{Umsetzung des neuen Kollektivvertrages für die ArbeitnehmerInnen an den Universitäten}

Nach mehrjährigen Verhandlungen zwischen dem Dachverband der Universitäten und der Gewerkschaft Öffentlicher Dienst wurde der erste Universitäts-Kollektivvertrag (KV) - gültig für alle ArbeitnehmerInnen der Universitäten, welche nach dem 31. Dezember 2003 in ein Dienstverhältnis mit der Universität eingetreten sind abgeschlossen. Der KV ist per 01.10.2009 in Kraft getreten, womit nun mehrere Jahre vergangen sind und die Frage nach dem Umsetzungsgrad Anlass war, den KV im Rahmen einer Studie des 
Instituts für Wissenschaftskommunikation und Hochschulforschung der Universität Klagenfurt näher zu betrachten. ${ }^{29}$ Die Studie zeigt, dass die Umstellung auf den Kollektivvertrag an den heimischen Universitäten weit fortgeschritten ist. Im WS 2011 sind mehr als drei Viertel aller wissenschaftlich-künstlerischen MitarbeiterInnen auf Basis des KV beschäftigt. Dabei ist zu beachten, dass der KV auf unterschiedlichen Personalstrukturen der Universitäten bzw. Universitätstypen $^{30}$ aufsetzt und dass die unterschiedlichen Anteile der bestehenden (unbefristeten) „Nicht-KV-Beschäftigungsverhältnisse" ein Faktor sind, der den "Spielraum" der Universitäten bei der Umsetzung mitbestimmt und universitätsspezifische Unterschiede mitbedingt.

UniversitätsassistentInnen gemäß KV stellen - wie Abb. 17 veranschaulicht - mit etwa einem Drittel den größten Anteil des wissenschaftlichen-künstlerischen Stammpersonals an den österreichischen Universitäten im WS 2011 dar, gefolgt von den UniversitätsdozentInnen und ProfessorInnen. Insbesondere die technisch-naturwissenschaftlichen Universitäten haben mit einem Anteil von 41,4 \% an UniversitätsassistentInnen gemäß KV an Stammpersonal, gefolgt von den Volluniversitäten mit einem Anteil von $37,1 \%$, weitgehend auf die neuen Assistenzen umgestellt. Die neuen Kategorien Senior Lecturers und Senior Scientists sind hingegen gering vertreten, mit Ausnahme an Kunstuniversitäten, wo der Anteil an Senior Lecturers (20,4 \%) am Stammpersonal im Vergleich $\mathrm{zu}$ den anderen Universitäten überdurchschnittlich hoch ist. Ein Grund dafür ist, dass der Stellenwert der Lehre (vor allem in Form des künstlerischen Einzelunterrichts) an Kunstuniversitäten besonders hoch ist, und daher die Lehrenden verstärkt ins
Stammpersonal integriert sind. Anders ist die Situation an den Medizinischen Universitäten; hier ist der Anteil an UniversitätsdozentInnen unbefristet beschäftigte habilitierte MitarbeiterInnen aus älteren Vertragsverhältnissen - am Stammpersonal $(37,5 \%)$ noch überdurchschnittlich hoch. Darüber hinaus haben die Medizinischen Universitäten die Laufbahnstellen (7,4 \% an Stammpersonal) bislang am besten ausgebaut, gefolgt von den Volluniversitäten (5,6 \%).

Die Laufbahnstellen und damit das Instrument der Qualifizierungsvereinbarung bilden das Kernstück des Kollektivvertrags. Die Motivation hierfür ist, ähnlich dem US-amerikanischen tenure track ein Laufbahnmodell an Österreichs Universitäten zu schaffen, welches mit Erfüllen einer Qualifizierungsvereinbarung (in der Regel innerhalb von sechs Jahren) in ein dauerhaftes Dienstverhältnis als "Assoziierte Professorin bzw. assoziierter Professor" mündet. Der wesentliche Unterschied zum amerikanischen Modell ist, dass in Österreich eine Bewerbung auf eine Laufbahnstelle durchaus auch universitätseigenen MitarbeiterInnen offen ist, während im US-amerikanischen System ausschließlich extern kompetitiv rekrutiert wird. Darüber hinaus führt der tenure track im US-amerikanischen System direkt in die Universitätsprofessur, während in Österreich die (durch das Universitätsgesetz „organisationsrechtlich" geprägte) Professur im Sinne des UG 2002 nach wie vor ausschließlich über ein Berufungsverfahren zu erlangen ist.

Dass es in Österreich den Universitäten frei steht, Laufbahnstellen auch intern zu vergeben, zeigt die Vergabepraxis der vergangenen Jahre: $68 \%$ der im WS 2010 neu geschaffenen Laufbahnstellen wurden an ehemalige interne UniversitätsassistentInnen vergeben. Universitäten

29 Siehe Pechar et al. (2012).

30 Zu den Universitätstypen zählen „Volluniversitäten“, welche die Universitäten Wien, Graz, Innsbruck, Salzburg, Linz und Klagenfurt umfassen, „Technisch-naturwissenschaftliche Universitäten“, welche die Technischen Universitäten Wien und Graz, sowie die Universität für Bodenkultur Wien und die Montanuniversität Leoben umfassen, „Medizinische Universitäten“, welche die drei humanmedizinischen Universitäten Wien, Graz und Innsbruck sowie die Veterinärmedizinische Universität Wien umfassen, „Kunstuniversitäten", welche die Akademie der bildenden Künste, die Universität für angewandte Kunst Wien, die Universität für Musik und darstellende Kunst Wien, die Universität Mozarteum Salzburg, die Universität für Musik und darstellende Kunst Graz sowie die Universität für künstlerische und industrielle Gestaltung Linz umfassen. Die Wirtschaftsuniversität Wien lässt sich anhand dieser Typologie nicht zuordnen und wird daher in der Kategorie „Alle Universitäten“ berücksichtigt. 
Abb. 17: Struktur des wissenschaftlich-künstlerischen Stammpersonals (Kopfzahlen) in Prozent, wS 2011

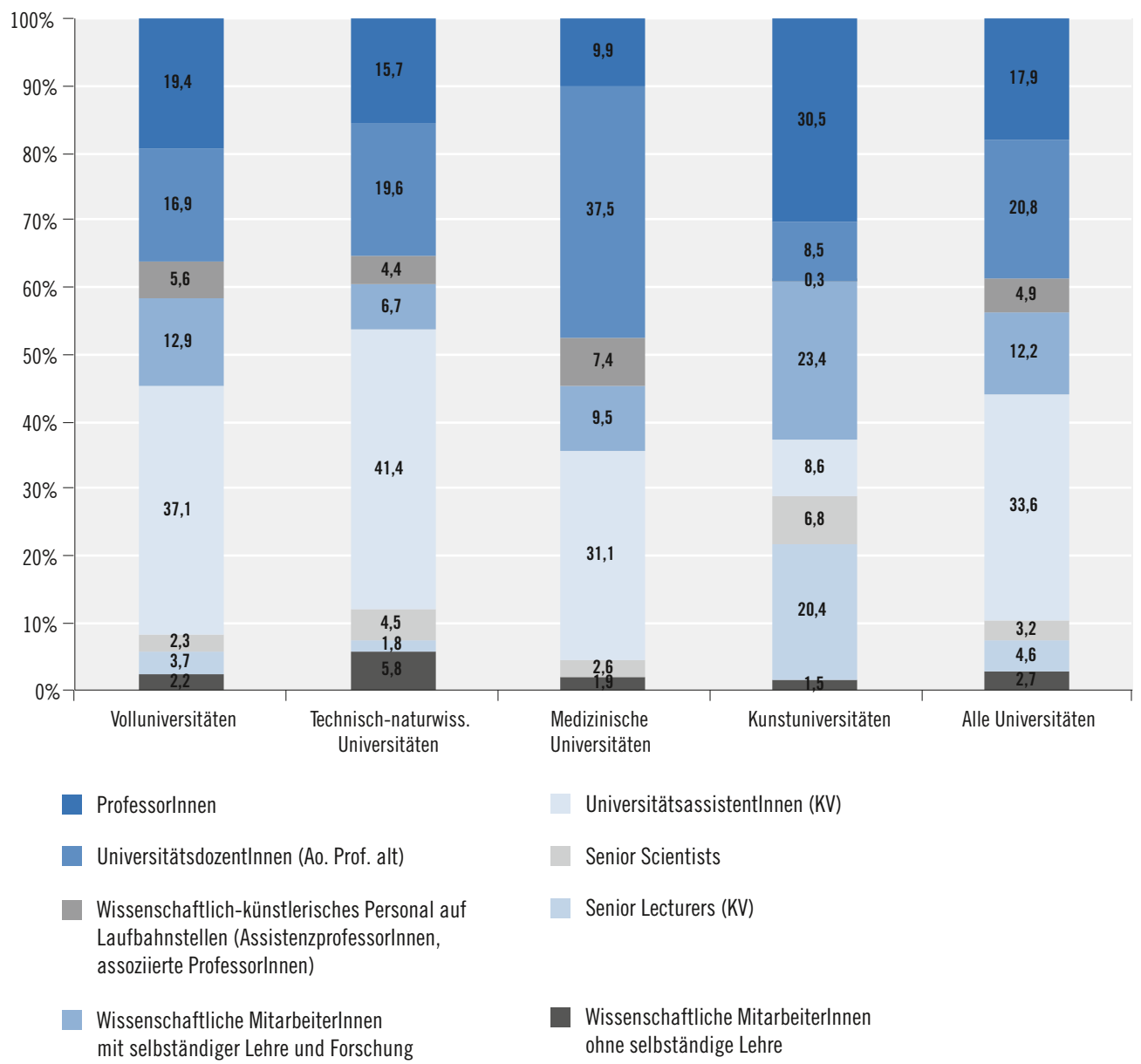

Quelle: Pechar et al. (2012), adaptiert.

begründen dies damit, dass sie im Sinn einer bewussten Nachwuchsförderung bereits hoch qualifizierte, im Haus beschäftigte MitarbeiterInnen nicht verlieren wollten und ihnen daher ein Angebot auf eine Laufbahnstelle gemacht haben; in Zukunft sollen Laufbahnstellen jedoch verstärkt - dem internationalen Standard entsprechend kompetitiv vergeben werden. Mit Abstand die meisten Laufbahnstellen eingerichtet hat bislang die Medizinische Universität Wien (152 Lauf- bahnstellen im WS 2011), gefolgt von der Universität Innsbruck (78 Laufbahnstellen) und Universität Graz (57 Laufbahnstellen). Laufbahnstellen, in deren Rahmen in der Regel eine wissenschaftliche Qualifikation in Form einer Habilitation erworben wird, haben jedoch nicht für jeden Typus von Universität denselben Stellenwert. So werden von den künstlerischen Universitäten Anstellungen auf Zeit statt Laufbahnstellen bevorzugt. ${ }^{31}$ Dies spiegeln auch die Ergebnisse in 
Abb. 17 wider. Einige Universitäten setzen Laufbahnstellen gezielt zur Förderung von Frauen ein, beispielsweise die TU Graz, die in der Leistungsvereinbarungsperiode 2010 bis 2012 jährlich zwei Laufbahnstellen explizit für hoch qualifizierte Wissenschaftlerinnen geschaffen hat.

Eine weitere merkliche Veränderung betrifft die Entwicklung der unbefristet Beschäftigten an Österreichs Universitäten. Wie Abb. 18 veranschaulicht ist der Anteil der unbefristet Beschäftigten an der Gesamtzahl des wissenschaftlichen Personals (inklusive drittfinanzierte Mitarbeite-
rInnen) in den letzten Jahren sukzessive zurückgegangen - auch mitbedingt durch die Übergangszeit nach Inkrafttreten des UG bis zum Inkrafttreten des KV. ${ }^{32}$ Im WS 2011 sind im Durchschnitt aller Universitäten $24,4 \%$ des wissenschaftlichen Personals unbefristet angestellt. Die Universitäten, welche den niedrigsten Anteil an unbefristet beschäftigten wissenschaftlichen MitarbeiterInnen haben, sind dabei vor allem die Volluniversitäten (19,4\% im WS 2011) und die Technisch-naturwissenschaftlichen Universitäten $(21,5 \%$ im WS 2011).

Abb. 18: Anteil unbefristet beschäftigter wissenschaftlicher MitarbeiterInnen am wissenschaftlichen Personal ${ }^{33}$ (Kopfzahlen) nach Universitätstyp in \%, WS 2005-2011

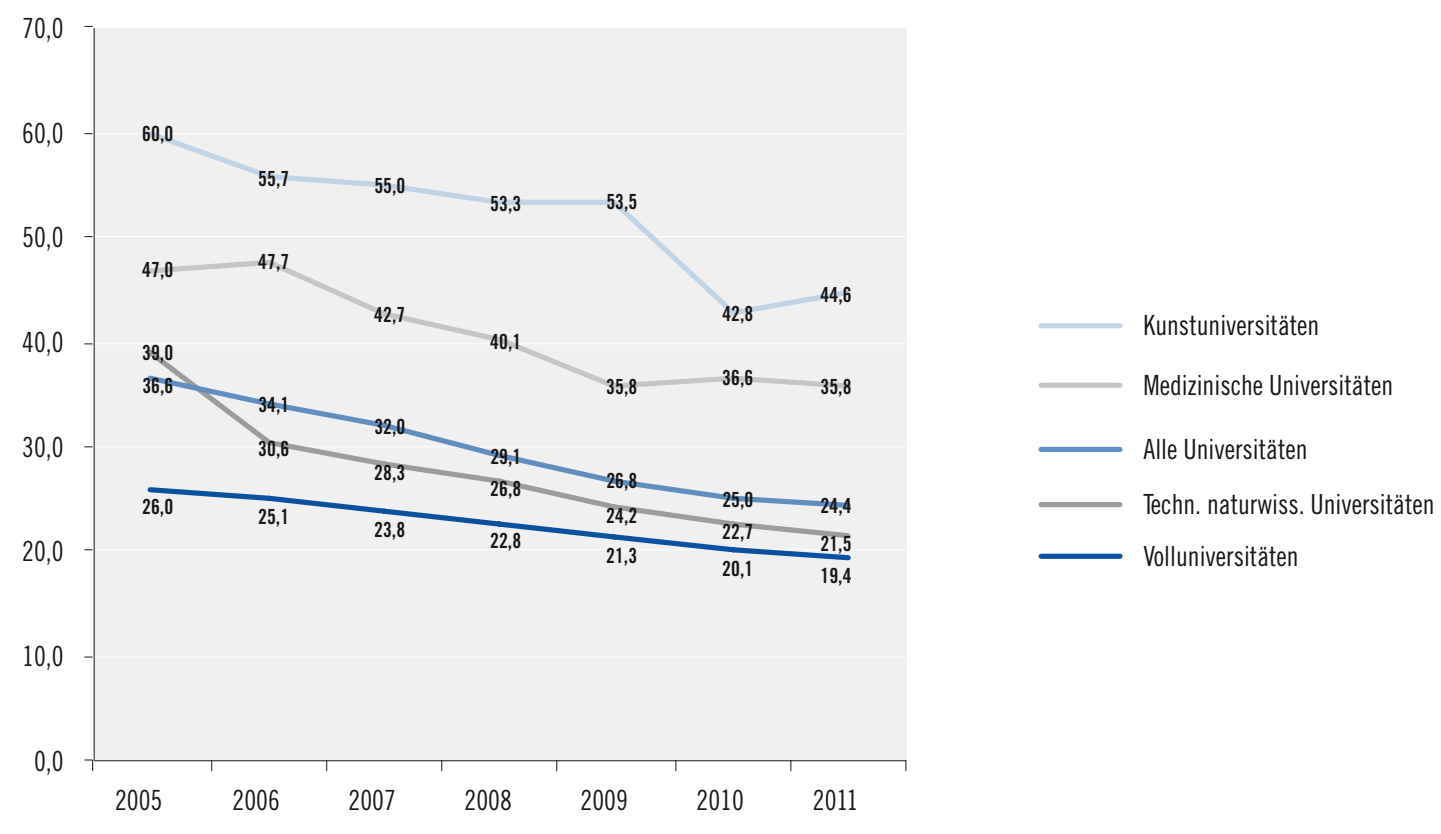

Quelle: Pechar et al. (2012), adaptiert.

32 So wurden seit Inkrafttreten des UG bei Neuaufnahmen von wissenschaftlich-künstlerischen MitarbeiterInnen bis zum Inkrafttreten des KV vor dem Hintergrund der gesetzlichen Übergangsregelung (die Universitäten hatten sich beim Abschluss von Arbeitsverträgen mit wissenschaftlich-künstlerischen MitarbeiterInnen am Vertragsbedienstetengesetz zu orientieren) fast ausschließlich befristete Vertragsverhältnisse begründet.

33 Die Kategorie „wissenschaftliches Personal" umfasst sämtliche Mitglieder des wissenschaftlichen Stammpersonals, studentische MitarbeiterInnen, drittmittelfinanzierte ProjektmitarbeiterInnen sowie LektorInnen. 
Bei den befristeten Beschäftigungen ist auffällig, dass insbesondere der Anteil von Prädoc-Stellen an allen Universitäten tendenziell zugenommen hat. Diese Arbeitsverhältnisse, die bei UniversitätsassistentInnen gemäß KV zur Vertiefung und Erweiterung der fachlichen und wissenschaftlichen bzw. künstlerischen Bildung dienen, erfüllen primär eine Ausbildungsfunktion und führen dazu, dass eine größere Zahl von DoktorandInnen institutionell integriert wird. Postdoc-Stellen - befristet auf vier bis sechs Jahre ohne Aussicht auf Qualifizierungsvereinbarung - verlieren hingegen zunehmend an Attraktivität und werden z.B. an der Universität Salzburg gar nicht mehr ausgeschrieben. Dies ist u.a. auch darauf zurückzuführen, dass insbesondere Drittmittelanstellungen in den vergangenen Jahren enorm zugenommen haben. Vor allem an den technischnaturwissenschaftlichen Universitäten stellen die extern finanzierten ProjektmitarbeiterInnen eine wichtige, teils sogar die größte Personalgruppe dar. Ein Großteil der Drittmittelstellen wird dabei als Prädoc-Stellen eingerichtet, die auch als Ausbildungsstellen fungieren. Ein nahtloser Übergang von einer Prädoc- auf eine Postdoc-Stelle ist an einigen österreichischen Universitäten, wie z.B. an der Universität Wien, Universität Graz und der Technischen Universität Graz, heute nicht mehr möglich, sodass nach dem Doktorat ein Institutionenwechsel vorzunehmen ist bzw. ein solcher Wechsel im Zuge einer wissenschaftlichen Karriere heute auch generell empfohlen wird.

Das Karrieremodell des KV bringt es mit sich, dass die Universitäten in ihrer Personalplanung zwischen Rotations- bzw. Fluktuationsstellen und Laufbahnstellen unterscheiden. Damit bietet sich den Universitäten ein Gestaltungs- und Handlungsspielraum, aber es eröffnet sich ihnen zugleich auch ein Spannungsfeld, mit welchem es umzugehen gilt: So sollen einerseits ausreichend Rotationsstellen zur Verfügung stehen, um die
Ausbildung von qualifiziertem wissenschaftlichen Nachwuchs sicherzustellen; andererseits sollen genug Laufbahnstellen vorhanden sein, um dem hoch qualifizierten Nachwuchs Karriereperspektiven bieten zu können. ${ }^{34}$ Was die Größenordnung des adäquaten Verhältnisses von Laufbahnstellen zu Rotationsstellen betrifft, sind in den Zielvorstellungen der einzelnen österreichischen Universitäten durchaus Unterschiede festzustellen.

\subsection{Externe Qualitätssicherung und Agentur für Qualitätssicherung und Akkreditierung Austria}

Das externe Qualitätssicherungssystem für den Hochschulbereich war in Österreich bislang historisch bedingt - sektorspezifisch geregelt. Angesichts der Entwicklungen im Bereich Qualitätssicherung auf nationaler und europäischer Ebene (wie z.B. Beschlussfassung der European Standards und Guidelines) hat Österreich die Weiterentwicklung des externen Qualitätssicherungssystems in das Regierungsprogramm für die 24. Gesetzgebungsperiode (2008 bis 2013) aufgenommen. Ziel war es, ein sektorenübergreifendes System zu schaffen und die drei bislang getrennt agierenden Qualitätseinrichtungen, nämlich den Österreichischen Fachhochschulrat (FHR), den Österreichischen Akkreditierungsrat für Privatuniversitäten (ÖAR) sowie die Österreichische Qualitätssicherungsagentur (AQA), in eine zentrale Einrichtung $\mathrm{zu}$ integrieren. Die Rechtsgrundlage für dieses neue System und eine neue, sektorenübergreifende Qualitätssicherungseinrichtung wurden mit dem HochschulQualitätssicherungsgesetz (HS-QSG), das seit 01.03.2013 vollständig in Kraft ist, geschaffen.

Die neue Qualitätssicherungseinrichtung - die Agentur für Qualitätssicherung und Akkreditierung Austria (AQ Austria) ${ }^{35}$ - hat ihre operativen Tätigkeiten im März 2012 aufgenommen und agiert als eine eigenständige Einrichtung mit vol-

34 Siehe hierzu auch Universitätsbericht (2011), S. 87.

35 www.aq.ac.at 
ler Rechtsfähigkeit, für deren Rechtsaufsicht und Finanzierung der Staat verantwortlich ist. Die AQ Austria - bestehend aus vier Organen, dem Kuratorium, der Generalversammlung, dem Board und der Beschwerdekommission - ist somit die zentrale Anlaufstelle für alle Agenden der externen Qualitätssicherung für öffentliche und private Universitäten sowie Fachhochschulen.

Das HS-QSG beinhaltet neben den Regelungen zur Organisation und Aufgaben der AQ Austria auch die Qualitätssicherungsverfahren und deren Rahmenbedingungen. Explizites Ziel der externen Qualitätssicherung ist, nicht nur die Strukturen und Verfahren der Qualitätssicherung im Bereich Studium und Lehre zu erfassen, sondern grundsätzlich alle Aufgabenbereiche der Hochschulen (wie auch Forschung, Personalentwicklung etc.) zu berücksichtigen. Damit soll die externe Qualitätssicherung einen doppelten Zweck erfüllen: Sie soll zum einen dem Staat als Lenkungsinstrument wie auch zum anderen den Hochschulen selbst als Hilfestellung für ihre Weiterentwicklung dienen.

$\mathrm{Zu}$ den zentralen Aufgaben der Agentur zählen die beiden Qualitätssicherungsverfahren, Audits und Akkreditierungen. Audits dienen der verpflichtenden externen Überprüfung des hochschulinternen Qualitätsmanagementsystems an öffentlichen Universitäten - mit dem Ziel, dass eine Zertifizierung durch das Audit den Aufbau eines Qualitätsmanagementsystems gemäß $\S 14$ Universitätsgesetz 2002 bestätigt. Auch an bestehenden Fachhochschulen, die bereits eine institutionelle Evaluierung nach den Bestimmungen des Fachhochschul-Studiengesetzes durchlaufen haben, sind Audits durchzuführen. Für neue Fachhochschulen sind institutionelle Akkreditierungen und für neue Studiengänge Programmakkreditierungen anzuwenden. Was die Privatuniversitäten betrifft, so werden institutionelle Akkreditierungsverfahren angewandt. Neue Studiengänge unterliegen auch hier wiederum der Programmakkreditierung.
Eine wesentliche Neuerung des HS-QSG ist, dass die Hochschulen bei Audit-Verfahren Wahlfreiheit bezüglich der Qualitätssicherungsagenturen haben. Das bedeutet, dass öffentliche Universitäten für die Durchführung von Audits die nationale Agentur (AQ Austria) oder eine ausländische Qualitätssicherungsagentur beauftragen können. Letztere hat jedoch die Bedingung zu erfüllen, dass die betreffende Agentur eingetragenes Mitglied im European Quality Assurance Register for Higher Education (EQAR) oder eine international anerkannte und unabhängige Qualitätsagentur sein muss. Damit soll sichergestellt werden, dass die Audits den Prüfbereichen gemäß HS-QSG und gängigen europäischen Verfahrensund Professionalisierungsstandards entsprechen. Zugleich soll aber auch ein gewisser Grad an Offenheit des Systems signalisiert werden.

Diese Offenheit des Systems schlägt sich auch in der verstärkten Einbeziehung von Studierenden in die externe Qualitätssicherung nieder. So sieht das HS-QSG erstmalig vor, dass Studierende im obersten Entscheidungsgremium der AQ Austria, im Board vertreten sind. Studierende sollen darüber hinaus als Teil von Gutachtergruppen agieren sowie u.a. von den neuen Bestimmungen zur Transparenz (wie z.B. Veröffentlichungspflicht der Verfahrensergebnisse) profitieren. Des Weiteren wurde eine weisungsfreie "Ombudsstelle für Studierende" als Ombuds-, Informations- und Servicestelle im BMWF eingerichtet, welche die bislang bestehende "Studierendenanwaltschaft" integriert. Eine weitere Neuerung umfasst die Registrierungspflicht grenzüberschreitender Studien. Ausländische Anbieter von Studienprogrammen müssen sich in Zukunft beim BMWF registrieren lassen, um damit nicht nur einen adäquaten Überblick über die verschiedenen Angebote in Österreich gewährleisten, sondern auch entsprechende Hintergrundinformationen bereitstellen zu können. ${ }^{36}$

36 Dieses Kapitel basiert auf der Sonderausgabe von Austria Innovativ (6a/2012) sowie auf Erlinger-Schacherbauer (2012). 


\subsection{Neupositionierung der Österreichischen Akademie der Wissenschaften}

Die Österreichische Akademie der Wissenschaften (ÖAW) hat eine lange Tradition. Im Jahr 1847 gegründet, besteht sie heute aus einer Gelehrtengesellschaft, einer Forschungsträgereinrichtung sowie einer Nachwuchsförder- und Serviceeinrichtung. Für das BMWF nimmt die ÖAW die Abwicklung von Forschungsprogrammen wahr, entwickelt in dieser Rolle Stipendienprogramme, verwaltet und vergibt Stipendien aus zweckgebundenen öffentlichen Mitteln, aus Drittmitteln sowie mit Unterstützung durch private SponsorInnen.

Seit 2000 ist insbesondere die Forschungsträgerorganisation beachtlich gewachsen. Darunter sind zahlreiche wissenschaftlich höchst erfolgreiche Forschungsinstitute, welche in Gebieten wie der Molekularbiologie, Biomedizin, Physik, angewandten Mathematik, Weltraumforschung, Materialwissenschaften sowie den Geistes-, Sozial- und Kulturwissenschaften tätig sind. $\mathrm{Zu}$ den renommiertesten Forschungsgesellschaften bzw. -einrichtungen zählen u.a. das Institut für Molekulare Biotechnologie (IMBA), das Gregor Mendel Institut für Molekulare Pflanzenbiologie (GMI), das Forschungszentrum für Molekulare Medizin (CeMM), das Johann Radon Institute for Computational and Applied Mathematics (RICAM), das Institut für Quantenoptik und Quanteninformation (IQOQI), das Institut für Demographie, das Institut für Mittelalterforschung und das Institut für Iranistik.

Den wissenschaftlichen Erfolg der ÖAW belegen die Performance-Indikatoren der letzten Jahre: So wurden im Jahr 2012 im MINT-Bereich 1.103 wissenschaftliche Publikationen - davon 46 Beiträge in hochrangigen Fachzeitschriften wie "Nature", "Science" oder "Cell" und deren Schwesterjournalen - sowie im GSK-Bereich 1.106 wissenschaftliche Publikationen veröffentlicht.

2012 wurden insgesamt 75 Preise und Auszeichnungen an ÖAW-MitarbeiterInnen vergeben. Als Highlight ist die Verleihung des mit 7,4
Mio. US \$ dotierten „Innovator Award“ zu nennen, mit dem das US-amerikanische Verteidigungsministerium die Forschung am ÖAW-eigenen IMBA unterstützt. Zu zwei ÖAW-MitarbeiterInnen 2012 zuerkannten START-Preisen kommen 16 ERC-Grants (neun Starting Grants, sechs Advanced Grants, sowie ein Proof of Concept Grant), die mit einem Fördervolumen von insgesamt mehr als 24,3 Mio. € 2012 an ÖAW-Instituten laufen. Die Relation der von ÖAW-Forschungseinrichtungen im Jahr 2012 eingeworbenen Drittmittel zum Basisbudget von knapp 96,59 Mio. $€$ ergibt eine Drittmittelquote von 45,39 \% (MINT 47,76 \%, GSK 38,68\%). Zahlreiche ÖAW-Forschungsergebnisse fanden rasch ihren Weg in die Anwendung - 2012 waren 39 Patente eingereicht - , in die wissenschaftsbasierte Beratung, z.B. anhand demographischer Studien, und durch Symposien, Vorträge und Ausstellungen auch in die Öffentlichkeit.

Im Bereich der Nachwuchsförderung wurde 2012 erstmals das von der ÖAW in Zusammenarbeit mit dem FWF entwickelte und von der Nationalstiftung mit 8 Mio. $€$ für fünf Jahre finanzierte Impulsprogramm "New Frontiers Groups" ausgeschrieben, mit dem selbständige Nachwuchsgruppen mit selbstgewählter wissenschaftlicher Thematik an ÖAW-Instituten eingerichtet werden, die neue Impulse in die ÖAW-Forschung einbringen. All dies spiegelt die hohe (nationale und internationale) Wettbewerbsfähigkeit der ÖAW wider, deren Attraktivität für wissenschaftliche Spitzenkräfte in Zukunft weiterhin erhöht werden soll, indem internationalen Standards folgend auch an der ÖAW die Möglichkeit eines Tenure Track - eines transparenten, leistungsgerechten wissenschaftlichen Karrierepfads - geschaffen wird.

Der enorme Ausbau des wissenschaftlich sehr erfolgreichen ÖAW-Forschungsträgers machte es notwendig, die Managementstrukturen der ÖAW $\mathrm{zu}$ modernisieren und anzupassen. Eine neue Satzung und Geschäftsordnung traten 2011 in Kraft, welche die Implementierung moderner und transparenter Strukturen auf der Managementebene nach sich zogen: 
- Ausweitung der Kompetenzen des Präsidiums,

- Einrichtung eines Akademierats mit Aufsichtsfunktion,

- Etablierung eines Direktors/einer Direktorin für Finanzen und Administration sowie

- Erweiterung der Befugnisse der LeiterInnen von Forschungseinrichtungen.

Als wesentlicher Schritt wurde im November 2011 erstmals in der Geschichte der ÖAW eine Leistungsvereinbarung - geltend für die Periode 2012 bis 2014 - zwischen dem Bundesministerium für Wissenschaft und Forschung (BMWF) und der ÖAW abgeschlossen. Darin hat die Akademie ihre für 2012 bis 2014 geplanten Leistungen dargelegt und im Gegenzug eine dreijährige finanzielle Planungssicherheit in Form eines Globalbudgets von 223,8 Mio. $€$ seitens des Bundes erhalten. Als größter außeruniversitärer Forschungsträger mit über 1.100 MitarbeiterInnen (VZÄ) verfolgt die ÖAW das Ziel, ihre führende Rolle in der anwendungsoffenen Grundlagenforschung in Österreich wie auch international zu halten sowie im Rahmen von Schwerpunktsetzungen auszubauen. Die ÖAW hat daher eine Priorisierung in ihrem Forschungsportfolio vorgenommen, um sich auf ausgewiesene disziplinäre und interdisziplinäre Stärken zu konzentrieren, und ihre Forschungsgebiete anhand von sechs Schwerpunkten spezifiziert: (1) Europäische Identitäten sowie Wahrung und Interpretation des kulturellen Erbes, (2) Demographischer Wandel, Migration und Integration von Menschen in heterogenen innovativen Gesellschaften, (3) Biomedizinische Grundlagenforschung, (4) Molekulare Pflanzenbiologie, (5) Angewandte Mathematik inklusive Modellierung und Bioinformatik, sowie (6) Quantenoptik und Quanteninformation.

Darüber hinaus hält die Leistungsvereinbarung 2012 bis 2014 weitere von der ÖAW in ihrer Funktion als Forschungsträger zu erfüllende Ziele fest:

- Ausschließliches Betreiben von Forschungseinheiten mit fachspezifisch kritischer Größe,

- Weiterführung hochspezialisierter und/oder langfristiger Forschungsvorhaben zur Wah- rung und Interpretation des kulturellen Erbes,

- Beitrag zur Profilbildung und strategischen Positionierung innerhalb der österreichischen Forschungslandschaft in Kooperation mit Universitäten und außeruniversitären Einrichtungen sowie

- Beteiligung an regionalen, europäischen und internationalen Forschungsinfrastrukturen im Rahmen der European Roadmap for Research Infrastructures.

Vorrangiges Ziel ist es, die Spitzenforschung der ÖAW-Forschungseinrichtungen in Zukunft auf einem international wettbewerbsfähigen Niveau auszubauen. Ebenfalls unterstützt wird diese Entwicklung vom Österreichischen Hochschulplan, welcher explizit auf die für den Forschungsstandort Österreich in Zukunft essentielle Fokussierung auf exzellente Forschungsbereiche verweist und die Profilbildung an Hochschulund Forschungseinrichtungen postuliert. Die ÖAW hat in diesem Sinne eine wichtige Maßnahme zur künftigen Schwerpunktsetzung und Profilbildung gesetzt und einzelne ÖAW-Einrichtungen bzw. -Forschungsgruppen an österreichische Universitäten transferiert. Folgende Übertragungen sind erfolgt:

Übertragung an die Universität Innsbruck

- Institut für Limnologie (ILIM)

- Institut für Biomedizinische Alternsforschung (IBA)

Übertragung an die Universität Salzburg

- Geographic Information Science (GIS)

- Kommission zur Herausgabe des Corpus der lateinischen Kirchenväter (CSEL)

- Institut für Realienkunde des Mittelalters und der frühen Neuzeit (IMREAL)

Übertragung an die Universität für Weiterbildung Krems

- Institut für Integrierte Sensor-Systeme (IISS) (rückwirkend mit 1.1.2013)

Übertragung an die Medizinische Universität Graz, Technische Universität Graz und Universität Graz

- Institut für Biophysik und Nanosystemforschung (IBN) 
Übertragung an die Universität Graz

- Institut für europäisches Schadenersatzrecht (ESR) (Forschungsgruppe)

Übertragung an die Montanuniversität Leoben

- Erich-Schmid-Institut für Materialwissenschaft (ESI) (Forschungsgruppe)

Übertragung an die Universität Wien

- Kommission für Rechtsgeschichte Österreichs

- Institut für europäische Integrationsforschung

- Kommission für Linguistik und Kommunikationsforschung (Forschungsgruppe)

Übertragung an die Universität Klagenfurt

- Kommission für vergleichende Medien- und Kommunikationsforschung (Forschungsgruppe)

Die Fokussierung auf Schwerpunktfelder wurde nicht nur mittels Übertragungen von Forschungseinrichtungen an Universitäten unterstützt, sondern auch innerhalb der ÖAW forciert, indem nach einschlägigen Evaluierungen - Forschungseinheiten zusammengelegt bzw. in bestehende Institute eingegliedert wurden. Statt 63 Forschungseinheiten Anfang des Jahres 2012 sind nun 28 Institute unter dem Dach der ÖAW versammelt. Das durch die Übertragungen und Zusammenlegungen freiwerdende Budget steht der ÖAW zur Investition in ihre Schwerpunktfelder zur Verfügung, um damit ihre wissenschaftliche Exzellenz auch in Zukunft zu sichern.

\subsection{Open Access}

Eine Aufgabe von WissenschaftlerInnen ist es, Wissen und Informationen zu schaffen und diese zu verbreiten. In den meisten Disziplinen stellen dabei Publikationen das wichtigste Medium zur Verbreitung wissenschaftlicher Erkenntnisse dar. Dabei werden in der Regel externe (anonyme) Begutachtungsverfahren eingesetzt, um die Einhal- tung von Qualitätsstandards zu garantieren. Auf dieser Grundlage entscheiden die herausgebenden Verlage über die Veröffentlichung der Publikation und diese wird, im Fall der positiven Beurteilung, kommerziell angeboten.

Diese Struktur der Verbreitung wissenschaftlicher Erkenntnisse hat in den letzten Jahren zu einer Konzentration der Anbieter sowie zu einer erheblichen Preiserhöhung seitens der Verlagshäuser geführt. In manchen Wissenschaftsdisziplinen (wie Medizin, Naturwissenschaften und Technik) haben sich die Preise in den letzten 20 Jahren vervierfacht, sodass die Budgets der wissenschaftlichen Bibliotheken den Kostensteigerungen der großen Verlage kaum Schritt halten konnten. ${ }^{37}$ Die möglichen und erhofften Wirkungen durch den Einsatz digitaler Distributionsmöglichkeiten via Internet sind jedoch bislang in diesem Bereich ausgeblieben. Vor diesem Hintergrund sind daher Rufe nach neuen Modalitäten und Strukturen der Distribution wissenschaftlicher Erkenntnisse laut geworden. Im Zentrum steht dabei der Begriff Open Access (OA), „...den freien Zugang zu wissenschaftlichen Resultaten (Publikationen und Forschungsdaten) im Internet".38

Gemäß dem Prinzip des offenen Zugangs (Open Access-Paradigma) sollen Wissen und Informationen in Zukunft umfassend frei zugänglich werden, um nicht zuletzt damit auch einen Beitrag zur besseren Transparenz, Nachhaltigkeit und Interaktivität in einer Wissensgesellschaft zu leisten.

Befürworter des Open Access führen mehrere Gründe für diesen Paradigmenwechsel an:

- Mit dem Internet wurden technische Voraussetzungen geschaffen, wissenschaftliche Resultate jederzeit und von jedem Ort zugänglich zu machen;

- Die Verlage erhalten die wissenschaftlichen

37 Siehe hierzu auch http://open-access.net/at_de/general_information/gruende_und_vorbehalte/gruende_F\&Er_oa/

38 Reckling (2013), S. 1. Das folgende Kapitel basiert im Wesentlichen auf einer von Falk Reckling im Februar 2013 verfassten Publikation mit dem Titel „Open Access - Aktuelle internationale und nationale Entwicklungen“. An dieser Stelle sei Falk Reckling auch für seine umfassende Unterstützung für das vorliegende Kapitel gedankt. 
Publikationen von den in der Regel mit öffentlichen Geldern finanzierten AutorInnen meist kostenlos;

- Externe GutachterInnen bieten ihre Leistungen für die Qualitätssicherung in den meisten Fällen ebenfalls kostenlos an;

- Schließlich haben die enorm gestiegenen Kosten von Zeitschriftenabonnements und -lizenzen (getrieben durch die Preispolitik großer renommierter Verlage) dazu geführt, dass nur noch wenige Bibliotheken in der Lage sind, alle erforderlichen bzw. gewünschten Publikationen anzuschaffen.

Neben dem finanziellen Argument wird auch das Postulat angeführt, dass die Erkenntnisse öffentlich finanzierter Forschung sehr wohl frei zugänglich sein sollten, nicht nur, um den Wissensaustausch mit der Scientific Community sicherzustellen, sondern auch um gegenüber der Gesellschaft einen Mehrwert zu schaffen. Der erst jüngst in Großbritannien veröffentlichte FinchReport - mit dem Ziel, eine Basis für eine nationale OA-Strategie zu schaffen - nennt angesichts der aktuellen Diskussionen folgende Argumente für eine in Zukunft wegweisende OA-Politik:

- Verbesserte Transparenz und Zugänglichkeit, mehr Verantwortungsbewusstsein, sowie ein erhöhtes öffentliches Interesse an Forschung,

- Verbesserter Wissenstransfer insbesondere zwischen Forschung und Innovation, und damit mehr Wirtschaftswachstum und Wohlstand,

- Höhere Effizienz, was den Forschungsprozess selbst durch den erweiterten und erleichterten Zugang zu Wissen, die Wissenssuche durch den geringeren Zeitaufwand und den breiteren und verbesserten Einsatz von Analysemethoden betrifft, sowie
- Verbesserte Legitimation gegenüber der Öffentlichkeit, insbesondere, wenn die Forschung aus öffentlichen Mitteln finanziert wird. ${ }^{39}$

Maßgeblich für die Verbreitung des Open AccessParadigmas war die Berliner Erklärung über den offenen Zugang zu wissenschaftlichem Wissen (2004), welche weltweit von 249 Institutionen (darunter von drei österreichischen Institutionen wie dem Österreichischen Wissenschaftsfonds, der Österreichischen Rektorenkonferenz und der Universität Wien) unterzeichnet wurde..$^{40}$ Mittlerweile gibt es fast 400 institutionelle Unterzeichner. ${ }^{41}$ Darin ist festgehalten, dass Open AccessVeröffentlichungen im Wesentlichen zwei Voraussetzungen zu erfüllen haben:

1. Dass die Urheber und Rechteinhaber „....allen Nutzern unwiderruflich das freie, weltweite Zugangsrecht zu diesen Veröffentlichungen “ gewähren und ihnen erlauben, „....diese Veröffentlichungen - in jedem beliebigen digitalen Medium und für jeden verantwortbaren Zweck - zu kopieren, zu nutzen, zu übertragen und öffentlich wiederzugeben sowie Bearbeitungen davon zu erstellen und zu verbreitern, sofern die Urheberschaft korrekt angegeben wird"; sowie

2. Dass „eine vollständige Fassung der Veröffentlichung sowie aller ergänzenden Materialien, einschließlich einer Kopie der oben erläuterten Rechte [...] in einem geeigneten elektronischen Standardformat in mindestens einem Online-Archiv hinterlegt (und damit veröffentlicht)..." wird. ${ }^{42}$

Open Access als erstrebenswertes Ziel bedeutet aber nicht, dass Publizieren in Open Access in Zukunft "gratis" sein wird. Im Gegenteil, die Kosten für das wissenschaftliche Publizieren

\footnotetext{
39 Vgl. Finch (2012).

40 Neben der Berliner Erklärung sind noch die Budapest-Open-Access-Initiative (2002), welche sowohl von Einzelpersonen als auch von Institutionen unterstützt wurde, sowie die Wiener Erklärung (2005) als weitere an die internationale Community adressierte proaktive Initiativen zu nennen.

41 Siehe Liste der Signatoren unter: http://oa.mpg.de/lang/de/berlin-prozess/signatoren/

42 Berliner Erklärung, S. 3.
} 
werden in der Umbruchphase steigen, wobei prinzipiell zwei - jeweils zu finanzierende - Wege für die Umsetzung von OA beschritten werden können: Green Road und Gold Road.

\subsubsection{Green Road}

Die Grundprinzipien der Green Road sind, dass jeder wissenschaftliche Beitrag parallel zur Publikation in einer Zeitschrift von den AutorInnen in einer frei zugänglichen Datenbank - einem Repositorium - archiviert wird. Archiviert werden können Preprints und Postprints. ${ }^{43}$ Unter Preprints werden die Manuskripte wissenschaftlicher Beiträge verstanden, die bei Zeitschriften eingereicht wurden, aber zumeist noch keine Qualitätssicherungsverfahren durchlaufen haben. Die AutorInnen verfügen in der Regel daher noch über die Nutzungsrechte, sodass der Selbstarchivierung meist keine rechtlichen Regelungen entgegenstehen. Bei den Postprints stellt sich die Situation anders dar, da diese bereits den Reviewprozess durchlaufen haben und zur Veröffentlichung akzeptiert wurden. Hier können rechtliche Probleme auftreten, da die Verlage auf unterschiedliche Weise mit dieser Art der Zweitveröffentlichung umgehen. Viele Wissenschaftsverlage definieren sogenannte Embargozeiten, d.h. sie gestatten eine zeitlich verzögerte Publikation von Postprints. Da es sich bei den Embargozeiten um Zeitperioden von zwischen 6 bis zu 36 Monaten handelt, schränkt dies sowohl die Zitierfähigkeit als auch die Aktualität der Veröffentlichung massiv ein.

Von Seiten der WissenschaftlerInnen werden die Politiken (wie z.B. unterschiedliche Bestimmungen zu Embargozeiten, Formate, Archivierungsorte) der einzelnen Verlage als oft undurchsichtig, kompliziert und daher zu zeitintensiv wahrgenommen. Dennoch ist in einigen Disziplinen, wie zum Beispiel der Mathematik, Physik oder Wirtschaftswissenschaft, die Selbstar- chivierung durch die WissenschaftlerInnen bereits schon sehr weit verbreitet, wenn auch meist nur in Form von Preprints.

\subsubsection{Gold Road}

Als Gold Road wird eine Vorgehensweise verstanden, bei der die wissenschaftliche Originalarbeit direkt in einer OA-Publikation erfolgt. Dabei wird in der Regel ein Peer Review-Verfahren durchlaufen und die AutorInnen schließen mit dem Verlag meist einen Publikationsvertrag ab, der die Nutzungsrechte und -bedingungen regelt. Auf der Internetplattform Directory of Open Access Journals sind bereits über 8.700 OA-Zeitschriften gelistet, bei denen ein Peer Review Verfahren Voraussetzung für eine Veröffentlichung ist. ${ }^{44}$ Die Finanzierung kann dabei über zwei mögliche Wege erfolgen:

- Die erste Finanzierungsform basiert darauf, dass die AutorInnen in Form von Gebühren pro Artikel, über sogenannte Article Processing Charges (APC), die Kosten für die Publikationen übernehmen. Dieses Modell hat sich vor allem im Bereich Life Sciences durchgesetzt. Um es weiter zu fördern, haben Förderorganisationen begonnen, wie es auch der Österreichische Wissenschaftsfonds seit Anfang 2004 praktiziert, die Publikationskosten für ihre FördernehmerInnen zu übernehmen.

- Die zweite Finanzierungsform sieht vor, dass sich Förderorganisationen, Forschungsstätten, Fachgesellschaften und Bibliotheken neben den kommerziellen Verlagen wieder stärker im wissenschaftlichen Publikationswesen engagieren; d.h. WissenschaftlerInnen werden die technischen und finanziellen Ressourcen zur Verfügung gestellt, welche eine Herausgabe von Zeitschriften oder Buchreihen erlauben, ohne dass dabei Kosten von den AutorInnen getragen werden müssen. Diese Vorgehensweise hat bislang vor allem in kleineren

43 Neben Preprints und Postprints wird auch zwischen institutionellen und fachspezifischen Repositorien unterschieden. In institutionellen Repositorien werden die wissenschaftlichen Aktivitäten von Institutionen gebündelt, während in den disziplinären Repositorien die wissenschaftlichen Beiträge disziplinenübergreifend zusammengeführt werden.

http://www.doaj.org/doaj?func=browse\&uiLanguage=en 
Disziplinen und den Geistes- und Sozialwissenschaften verstärkt Anwendung gefunden. ${ }^{45}$

Bei beiden Finanzierungsformen werden die Kosten von den Lesern auf die AutorInnen der wissenschaftlichen Beiträge bzw. auf die Mitglieder der Wissenschaftsorganisationen verschoben. Somit ist die Gold Road keineswegs kostengünstiger als das konventionelle System, dennoch können Effizienzgewinne entstehen, wenn durch eine verstärkte Konkurrenz die Gesamtkosten reduziert werden.

Nach jüngsten Schätzungen liegt der Anteil der nach der Gold Road veröffentlichten referierten Artikel bei 10 bis $16 \%$. Offensichtlich weist auch dieser Weg Probleme auf, welchen die WissenschaftlerInnen beim Publizieren gegenüberstehen. So zählt es als Nachteil, dass sich OAPublikationsorgane erst in den letzten zehn Jahren systematisch etabliert haben und heute vergleichsweise noch wenige OA-Publikationsorgane Renommee ausweisen, welches für die WissenschaftlerInnen Anreiz ist, in solchen Organen zu publizieren. Ebenfalls engagieren sich bisher nur wenige Forschungsstätten und Förderer bei der finanziellen Unterstützung der Gold Road, sei es entweder als Träger von OA Publikationsorganen oder sei es über die Bereitstellung der Mittel für APCs für die WissenschaftlerInnen. Hinzu kommt, dass der neue Markt Anbieter hervorgebracht hat, welche zu relativ hohen Kosten eine geringe Qualität anbieten.

Folglich bieten zahlreiche Verlage neben der Veröffentlichung in OA-Journalen sogenannte Hybrid-Modelle an. Es handelt sich dabei um Hybrid-Zeitschriften, welche nicht Open Access sind, den WissenschaftlerInnen aber die Option anbieten, ihre Artikel „freizukaufen" und somit diese in Open Access zu publizieren. De facto übernehmen die AutorInnen bzw. Förderorganisationen die Gebühren, um die Publikationen den LeserInnen frei zugänglich zu machen. Was die
Verlage betrifft, so bieten heute international tätige Großverlage generell die Option des Publizierens in Hybrid-Zeitschriften an, was die Kritik mit sich bringt, dass diese Verlage zwar steigende Open Access-Beiträge von konventionellen Zeitschriften einfordern, jedoch die Abonnementkosten bislang noch nicht gesenkt haben; d.h. die Verlage nutzen die OA-Entwicklungen zu ihren Gunsten. ${ }^{46}$

\subsubsection{Die Entwicklung von Open Access Journals}

Die ersten Open Access Journale (OAJ) wurden bereits 1989 gegründet und, wie Abb. 19 veranschaulicht, ist die Anzahl der OAJ in den letzten zehn Jahren exponentiell gewachsen. Insgesamt geht man davon aus, dass heute von mehr als 30.000 referierten wissenschaftlichen Fachzeitschriften etwa ein Viertel OAJ sind.

\section{Abb. 19: Entwicklung von Open Access Journals (OAJ) von 2003-2012}

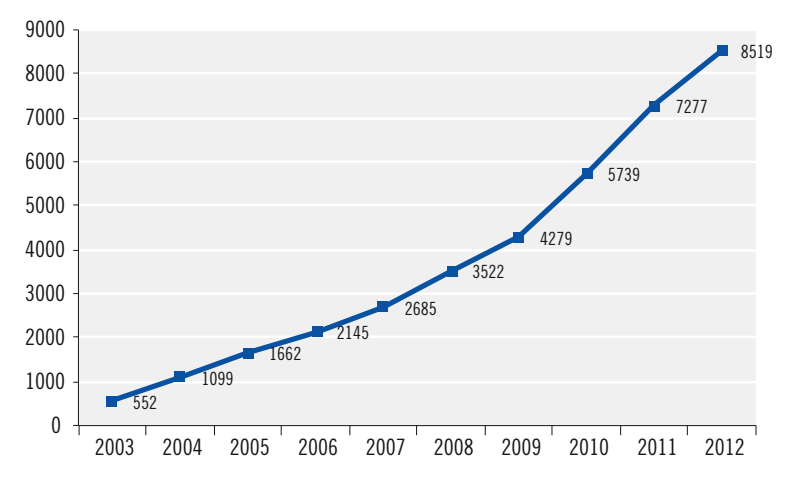

Quelle: Falk (2013), übernommen von: www.doaj.org.

Open Access Journals werden im Directory of OA Journals (DOAJ) registriert, wo aktuell Journals aus 121 Ländern gelistet sind. Weltweit führend ist die USA mit insgesamt 1.270 registrierten OAJ, gefolgt von Ländern wie Brasilien (801), Großbritannien (575), Indien (463), Spanien (442),

45 Vgl. Reckling et al. (2012).

46 Vgl. Bauer, Stieg (2010). Eine Übersicht der Verlage, welche Hybridmodelle anbieten, bietet das Subverzeichnis der SHERPA/RoMEOSeite „Publishers with Paid Options for Open Access" (http://www.sherpa.ac.uk/romeo/PaidOA.html). 
Ägypten (350), Deutschland (259) und Kanada (255). Gemessen an der Einwohnerzahl ist die Schweiz (125) derzeit der größte Produzent von OAJ. Demzufolge investieren vor allem wissenschaftsstarke Nationen in diesen neuen Markt, aber auch wissenschaftlich aufstrebende Länder wie Brasilien, Indien und Ägypten nutzen offenbar die kostengünstige Möglichkeit für einen Markteintritt. Nach Disziplinen haben die Life Sciences mit etwa $35 \%$ den höchsten Anteil am gesamten Markt, gefolgt von den Sozialwissenschaften $(25 \%)$, den Geisteswissenschaften $(15 \%)$, den Naturwissenschaften (12\%), sowie den Technik- und Ingenieurswissenschaften (10\%). Was die Entwicklung von OAJ in Österreich betrifft, so wurden Ende 2012 insgesamt 40 OAJ publiziert, wobei zu den renommiertesten Journals vor allem Living Reviews in European Governance, European Integration online Papers, Myrmecological News oder Vienna Yearbook of Population Research zählen.

Getrieben wurde der Markt der OAJ vor allem durch die technischen Möglichkeiten des Internets, welche einen Markteintritt nicht nur wesentlich preiswerter gestalten, sondern auch eine Reihe von frei verfügbaren Softwarelösungen allen voran das von einem Verbund amerikanischer und kanadischer Universitäten finanzierte Open Journal System - zur Verfügung stellen. Weitere Antreiber für die rasante Entwicklung von OAJ waren die nicht-kommerzielle Public Library of Science (PLoS) und der kommerzielle BioMedCentral Verlag.

Als weiteren Schritt hat die Public Library of Science ein Publikationsmodell namens PLoSOne, ein sogenanntes Megajournal, etabliert, das sich innerhalb weniger Jahre zur größten Fachzeitschrift der Welt entwickelt hat. Mit knapp 24.000 Beiträgen im Jahr 2012 folgt PLoSOne drei Prinzipien: (1) umgehend zu publizieren, (2) die Beiträge aller naturwissenschaftlichen Disziplinen zu akzeptieren sowie (3) keine editorische Selektion etwa nach der wissenschaftlichen Be- deutung der Beiträge zu machen. Damit geht einher, dass etwa $70 \%$ aller eingereichten Beiträge akzeptiert werden, die Kosten für den editorischen Aufwand sehr gering und somit auch die APC pro Artikel (etwa $1.000 €$ ) niedrig gehalten werden. Auch diesem Modell folgten zahlreiche Nachahmer bei kommerziellen Verlagen wie z.B. SageOpen, Forum of Mathematics, OpenBiology, GigaScience oder die erst jüngst lancierte Initiative Open Library of Humanities.

\subsubsection{Internationale Entwicklungen}

In den USA wurde erst jüngst am 14. Februar 2013 die überparteiliche Gesetzesinitiative Fair Access to Science and Technology Research Act (FASTR) in den Kongress sowie in das Repräsentantenhaus eingebracht. Demnach sollen wissenschaftliche Publikationen, die von öffentlichen Fördergebern (mit einem Jahresbudget von insgesamt mehr als 100 Mio. US \$) finanziert werden, entsprechend der Green Road mit einer maximalen Embargozeit von sechs Monaten in OA archiviert werden. Auf europäischer Ebene startete die EU-Kommission bereits im August 2008 ein Open-Access-Pilotprojekt, welches sich auf Teile des 7. Europäischen Rahmenprogramms (FP7) und European Research Council (ERC) bezieht. Open Access wird dabei in ausgewählten Bereichen wie z.B. Energie, Umwelt, Gesundheit und IKT gefördert, indem alle FördernehmerInnen angehalten sind, ihre wissenschaftlichen (peerreviewed) Publikationen in einem Repositorium abzulegen und nach einer Embargozeit von sechs bzw. zwölf Monaten diese frei zugänglich zu machen. Gefördert werden sämtliche Publikationskosten inklusive AutorInnengebühren beim Goldenen Weg. Dieses Pilotprojekt wird begleitet von einem Monitoring-Projekt namens Ope$n A I R E$, welches den stetigen Austausch mit dem Europäischen Parlament, den Mitgliederstaaten und den Stakeholdern übernommen hat. ${ }^{47}$ Pro aktiv unterstützt wird OA ebenfalls vom Euro-

47 Siehe „Open Access in FP7“, http://ec.europa.eu/research/science-society/index.cfm? fuseaction=public.topic\&id=1300\&lang=1 
pean Research Council (ERC). So ist der ERC der weltweit größten Volltextdatenbank für wissenschaftliche Publikationen in den Life Sciences, dem Repositorium Europe PubMedCentral, beigetreten. Mit über 2,6 Mio. Papers ist Europe PubMedCentral das mit Abstand bedeutendste Postprint-Repositorium, zu dessen Aufbau die Politiken tragender Förderorganisationen wie NIH, Wellcome Trust, MRC, BBSRC, FWF und ERC wesentlich beigetragen haben. Alle genannten Förderorganisationen verpflichten ihre ProjektnehmerInnen, in Europe PubMedCentral zu archivieren.

In Zukunft soll das Publizieren in OA weiterhin forciert werden. Es sollen alle Publikationen, die im Rahmen der EU-Förderung entstehen, im Open Access zugänglich gemacht werden. Für HORIZON 2020 bedeutet dies, dass alle geförderten Publikationen (inklusive Forschungsdaten) zu OA nach der Green Road oder der Gold Road verpflichtet werden sollen. ${ }^{48}$ Darüber hinaus kommuniziert die EU-Kommission das Ziel, dass alle Mitgliedsländer mittels entsprechender Maßnahmen bis 2016 zumindest $60 \%$ aller wissenschaftlichen Publikationen in OA veröffentlichen sollen. Die Förderorganisationen werden dabei insbesondere in der Verantwortung gesehen, sich der Langzeitarchivierung wissenschaftlicher Daten anzunehmen. Um diese Entwicklung zu beschleunigen, hat die EU-Kommission per 1. Februar 2013 den Mitgliedsländern empfohlen, entsprechend den europäischen Zielen, OAMaßnahmen für öffentlich finanzierte Forschung auf nationaler Ebene zu setzen sowie eine klare Politik hinsichtlich einer rascheren Umsetzung von Open Access und Open Data zu definieren. ${ }^{49}$ Bislang praktizieren die EU-Mitgliedsländer durchaus unterschiedliche Ansätze zur Umsetzung von Open Access, wobei zu den absoluten Vorreitern sicherlich Großbritannien zählt. Großbritannien hat als erstes Land der Welt an- gekündigt, die Ökonomie des wissenschaftlichen Publikationssystems in den kommenden Jahren vollständig auf OA umzustellen. Wegweisend hierfür ist der sogenannte Finch Report, an dessen Erstellung sämtliche Interessensgruppen beteiligt waren und in welchem Szenarien des Umstiegs entwickelt wurden. Für den Umstieg selbst stellen die UK Research Councils in den nächsten Jahren rund 3 Mrd. Pfund zur Verfügung und ändern ihre Politiken. Per 1. April 2013 müssen alle wissenschaftlichen Publikationen, welche durch Research Councils gefördert werden, in OA zugänglich gemacht werden. Die sogenannten Article Processing Charges (APC) sollen dabei von eigens zur Verfügung gestellten Mitteln finanziert werden. Darüber hinaus wird von den Universitäten und Hochschuleinrichtungen erwartet, dass sie selbst Fonds zwecks Unterstützung des OA-Publishing aufsetzen. ${ }^{50}$

Unter den nordischen Ländern hat Dänemark in den letzten Jahren Schritte in Richtung OA unternommen. Nach einem Prozess der Abstimmung mit allen relevanten Stakeholdern hat Dänemark eine nationale OA-Strategie erarbeitet und aufgesetzt. Schweden hat eigens ein OA-Programm, welches vom National Library's Department for National Cooperation koordiniert wird, ins Leben gerufen. Darüber hinaus sind die Universitäten gesetzlich verpflichtet worden, der Öffentlichkeit Zugang zu ihren Forschungsergebnissen inklusive "research results for commercial exploitation" zu gewähren. Publizieren in OA ist - begleitet von zahlreichen Verpflichtungen und Regelungen seitens der Universitäten auch in Finnland gängige Praxis geworden. Selbiges gilt in Norwegen, wo OA auf nationaler Ebene im White Paper on Research als übergeordnetes Ziel festgehalten wird. Demnach müssen alle öffentlich finanzierten wissenschaftlichen Publikationen grundsätzlich frei zugänglich gemacht werden. In den Niederlanden zeigen sich sowohl

48 Bei der Green Road sollen die wissenschaftlichen Publikationen nach einer Embargozeit von sechs Monaten, in den Geistes- und Sozialwissenschaften nach einer Embargozeit von zwölf Monaten frei zugänglich gemacht werden.

49 Siehe Council of the European Union (2013).

50 Vgl. Osborn (2013). 
die Scientific Community als auch die Bibliotheken als durchaus aktiv hinsichtlich der Umsetzung von OA, wenn auch drastische Budgetkürzungen keine zusätzlichen Investitionen in diesem Bereich in Zukunft erwarten lassen. ${ }^{51}$

Deutschland zählt zu jenen Ländern, welche keine nationale OA-Strategie verfolgen. Es wird vielmehr ein Bottom-up-Ansatz verfolgt; d.h. ausschlaggebend für eine Weiterentwicklung von OA sind vor allem einzelne Stakeholder-Initiativen. Forschungsorganisationen wie die Deutsche Forschungsgemeinschaft (DFG) haben bereits eine eigene OA-Politik definiert. Ähnlich ist die Strategie in der Schweiz. Der Schweizer Nationalfonds verpflichtete bereits seit 2007 seine FördernehmerInnen, in OA zu publizieren. Zwecks weiterer Unterstützung von Open Access hat die Schweiz nun einen Dialog zwischen Wissenschaft und Gesellschaft initiiert. Sowohl der Verbund der vier schweizerischen Akademien der Wissenschaften als auch die Konferenz der Universitätsbibliotheken empfehlen dabei, wissenschaftliche Publikationen frei zugänglich zu machen bzw. haben Projekte zum Thema OA gestartet. So gibt es Pilotprojekte wie z.B. an der Hauptbibliothek Universität Zürich, welche ab 2012 Open Access Publikationen im Bereich Geistes- und Sozialwissenschaften mit einem Publikationsfonds unterstützt.

\subsubsection{Status-quo in Österreich}

Wie in anderen europäischen Ländern gibt es auch in Österreich keine national abgestimmte OA-Politik bzw. zentral organisierte Initiativen. So sind es in Österreich einzelne Institutionen und WissenschaftlerInnen, welche das Publizieren in Open Access pro-aktiv vorantreiben. Mitunter hat die Österreichische Rektorenkonferenz bereits 2004 die Berlin Declaration on Open Ac- cess to Knowledge in the Sciences and Humanities unterzeichnet und nach einer Veröffentlichung der Recommendations from the EUA Working Group on Open Access im Jahr 2008 eine Arbeitsgruppe zum Thema Open Access gegründet. Ergebnis dieser Arbeitsgruppe sind Empfehlungen der Österreichischen Universitätenkonferenz (uniko) zu einer Open Access-Politik der Universitäten im Jänner 2010. Darin wird den Universitäten empfohlen, den Übergang zum Open Access-Paradigma aktiv zu unterstützen, indem sie eine Green Road-Strategie verfolgen im Bewusstsein, dass die Benutzung von bestehenden bzw. die Errichtung von Repositorien zusätzlicher (mitunter drittmittelfinanzierter) Ressourcen bedarf. Darüber hinaus werden die Universitätsleitungen angehalten, eine Open Access Politik zu verabschieden. ${ }^{52}$

Auf nationaler Ebene wurde Open Access als (Zukunfts-)Thema insofern verankert, als dass der Rat für Forschung und Technologieentwicklung (RFTE) Open Access in der Strategie 2020 wie folgt verankert hat: "Der Rat betrachtet es als Bringschuld von Wissenschaft und FTI - vor allem jener, die mit öffentlichen Mitteln finanziert wird -, die Gesellschaft breit und umfassend über ihr Tun und Handeln sowie über ihre Erkenntnisse und Entwicklungen zu informieren. Dazu gehört unter anderem, dass bis zum Jahr 2020 alle öffentlichen Forschungsergebnisse in Österreich (vor allem Publikationen, Forschungsprimärdaten etc.) frei im Internet zugänglich sind - Stichwort: Open Access. ${ }^{153}$ Dementsprechend sind heute verschiedene Stakeholder zwecks Umsetzung und Verbreitung von OA in Österreich aktiv bzw. sind bereits auch zahlreiche Maßnahmen seitens der Förderorganisationen, Forschungsgesellschaften und Universitäten hierfür implementiert worden.

51 Vgl. Europäische Kommission (2011b).

52 Siehe uniko (2010).

53 RFTE (2009): Strategie 2020, S. 31. 


\section{Maßnahmen des Österreichischen Wissenschaftsfonds (FWF)}

Der FWF, einer der Erstunterzeichner der Berliner Erklärung, betreibt seit 2003 eine konsequente OA Politik, welche aktuell folgende Maßnahmen umfasst:

- Seit 2003 übernimmt der FWF Finanzierungen von Publikationen nach der Gold Road. Das waren im Jahr 2012 knapp 900 Zeitschriftenund Buchpublikationen mit einem Finanzumfang von etwa 1,6 Mio. $€$. Gemessen am Gesamtbudget ist diese Summe neben dem Wellcome Trust ${ }^{54}$ einer der höchsten Werte einer Förderorganisation weltweit.

- Alle FördernehmerInnen gehen seit 2006 die Verpflichtung ein, wann immer rechtlich möglich, ihre Publikationen nach Gold Road oder Green Road frei zugänglich zu machen.

- Seit März 2010 beteiligt sich der FWF über das Partnerrepositorium UKPubMedCentral an PubMedCentral; das bedeutet, dass alle ProjektleiterInnen im Bereich Life Sciences ein Projektkonto bei UKPubMedCentral erhalten, um darin ihre Publikationen $\mathrm{zu}$ archivieren. Bislang wurden knapp 3.000 Publikationen aus FWF-Projekten im Bereich Life Sciences über diese Initiative frei zugänglich gemacht.

- Der FWF hat zudem die Förderung von OpenAccess-Veröffentlichungen von Büchern ausgeweitet: Mit der Etablierung der FWF E-BookLibrary wird ein Repositorium, welches vom PHAIDRA-Team der Universität Wien technisch unterstützt wird, zur Verfügung gestellt, in welchem alle seit Dezember 2011 beim FWF geförderten Publikationen zeitgleich mit dem Erscheinen des Buches, des E-Books oder Ähnlichem frei zugänglich gemacht werden. Außerdem bietet der FWF an, alle vom FWF geförderten und seit 2000 erschienen Bücher $\mathrm{zu}$ digitalisieren und ebenfalls in der FWF EBook-Library zu veröffentlichen.
- Gemeinsam mit dem BMWF hat der FWF im Oktober 2012 eine Interessensbekundung für die Anschubfinanzierung von OAJ in den Geistes- und Sozialwissenschaften ausgeschrieben. Damit sollen 2013 fünf bis zehn international hochklassige OAJ gefördert werden.

- International engagiert sich der FWF über Science Europe (eine Dachorganisation der europäischen Forschungsförderer und Forschungsträgerorganisationen) für gemeinsame europäische Standards in $\mathrm{OA}$, welche im Frühjahr 2013 publiziert werden sollen.

- In Österreich hat der FWF in jüngster Zeit eine Diskussion um eine University/Academic Press angestoßen. Die Idee ist, dass Forschungsstätten und Verlage gemeinsam eine Publikationsplattform gründen, welche die technischen Voraussetzungen für international sichtbare Publikationen mit Qualitätssicherungsverfahren internationalen Standards (wie z.B. Peer Review, Fachlektorate) und OA schaffen.

\section{Maßnahmen der wissenschaftlichen Institutionen ÖAW und IST Austria}

Implizit verfolgt die österreichische Akademie der Wissenschaft (ÖAW) bereits seit 2005 eine Open Access-Politik, formell beschlossen wurde diese im Sommer 2011. So empfiehlt die Akademie den ÖAW-WissenschaftlerInnen, ihre Forschungsergebnisse frei zugänglich zu machen, indem diese möglichst zeitnah, in nach Fachgebieten differenzierter Form - sofern vertraglich möglich - gemäß der Green Road-Strategie archiviert werden. Die ÖAW-AutorInnen haben dabei das Recht, eine digitale Kopie ihrer wissenschaftlichen Publikation in einem Respositorium der Öffentlichkeit zugänglich zu machen. $\mathrm{Zu}$ diesem Zweck stehen ihnen das (seit 2006 etablierte) institutionelle Repositorium EPUB.OEAW, welches

54 Der Wellcome Trust mit Sitz in London ist die weltweit zweitreichste Stiftung, die medizinische Forschung fördert. 
ebenso als elektronische Plattform des ÖAW-Verlags fungiert, oder auch andere fachspezifische Dienste zur Verfügung. Der ÖAW-Verlag ist ein sogenannter Green Publisher; d.h., dass Zeitschriftenartikel als Manuskript (nicht jedoch im Verlagslayout) auf der Homepage des Wissenschaftlers oder im Repositorium der Forschungsinstitution bereits vor der Verlagsveröffentlichung online gestellt werden dürfen. Die ÖAW behält sich lediglich ein nicht exklusives Recht zur Veröffentlichung, was für die AutorInnen bedeutet, dass sie ihre wissenschaftlichen Arbeiten ohne weitere Einschränkungen weiterverwerten können. Diese Regelung hat ihre Gültigkeit für Zeitschriften, nicht aber für Bücher und Datenbanken. Darüber hinaus bietet die ÖAW im Rahmen des Programms Author's Choice die Option an, gegen Bezahlung einer OA-Gebühr Zeitschriftenartikel oder Beiträge in Sammelbänden bereits zum Zeitpunkt der Publikation im Internet frei zugänglich zu machen. Diese Option ist durchaus als eine Möglichkeit für die Gold Road zu sehen, wenn auch die ÖAW in der Regel (vor allem aus Kostengründen) die Green Road zu beschreiten empfiehlt; für die Gold Road empfiehlt sie prinzipiell eine Drittmittelfinanzierung (wie z.B. durch den FWF). Dass die ÖAW auch in Zukunft, ihr OA-Angebot forcieren wird, ist mitunter in der Leistungsvereinbarung 2012 bis 2014 zwischen ÖAW und BMWF festgelegt. Publizieren in OA soll somit auch seitens der ÖAW in Zukunft ausgebaut werden. ${ }^{55}$

Ebenfalls hat das Institute of Science and Technology Austria (IST Austria) ein eigenes Repositorium nach dem Open Access-Prinzip aufgebaut. Ausschlaggebend hierfür war die Evaluierung des IST Austria, welche u.a. auf sämtliche Publikationen des Instituts eingehen sollte. Beim Aufbau selbst war ein Hauptaspekt, dass der Aufwand für die Eingabe von Daten möglichst gering gehalten werden sollte. Ziel war es, dass Daten und Files von der Publikationsdatenbank einfach in das Repository übertragen werden können. Für die WissenschaftlerInnen sollte der Aufwand gering sein, indem sie ihren Content einmalig in das System laden, alle weiteren notwendigen Schritte von den Diensten der Bibliothek übernommen werden. Insgesamt forciert daher auch die OA-Politik des IST Austria - mittels Repositorium basierend auf EPrints - die Green Road. Zusätzlich hat IST Austria aber auch erst jüngst einen Publikationsfonds zur Förderung der Gold Road eingerichtet. ${ }^{56}$

\subsubsection{Maßnahmen der österreichischen Universitäten}

Die österreichischen Universitäten haben bereits begonnen, Maßnahmen und Initiativen zur Umsetzung von OA zu setzen. Am weitesten gediehen sind die Open-Access-Aktivitäten der Universität Wien, welche Services und Infrastruktur zur Selbstarchivierung (Green Road) der wissenschaftlichen Publikationen und primären Forschungsdaten anbietet und zugleich WissenschafterInnen bei ihren Publikationsvorhaben technisch, finanziell und mit bibliometrischer Begleitung unterstützt. ${ }^{57}$ Seit 2008 hat die Universität Wien das gesamtuniversitäre Digital Asset Management System Phaidra (Permanent Hosting, Archiving and Indexing of Digital Resources and Assets) etabliert. Phaidra bietet als Repositorium für Verwaltung, Forschung und Lehre die Möglichkeit der langfristigen Verwaltung von digitalen Inhalten in unterschiedlichen Formaten, so dass wertvolle digitale Bestände dauerhaft gesichert und weltweit zugänglich gemacht werden können. Der NutzerInnenkreis hat sich nach Inbetriebnahme von Phaidra rasch über die Grenzen der Universität Wien ausgebreitet. So nutzt heute eine Reihe von Institutionen Phaidra entweder als eigenständige Installation oder als Hosting-Angebot, etwa die Kunstuniversitäten Graz und Linz,

55 Vgl. Nentwich et al. (2012).

56 Vgl. Rossini (2012).

57 Siehe http://openaccess.univie.ac.at. 
die Universität für Angewandte Kunst in Wien, der Österreichische Wissenschaftsrat, die Österreichische Forschungsgemeinschaft, das Forum Universitätsbibliotheken Österreichs oder der Wissenschaftsfonds. ${ }^{58}$ Die Wirtschaftsuniversität Wien verfügt ebenfalls über ein institutionelles Repositorium. Seit zehn Jahren betreibt sie den Open Access-Publikationsserver ePubwu, welcher wissenschaftliche Arbeiten kostenfrei und dauerhaft online verfügbar macht. Aktuell sind etwa 1.300 Dokumente abrufbar, die im Rahmen der urheberrechtlichen Bestimmungen $\mathrm{zu}$ wissenschaftlicher und privater Nutzung kopiert, ausgedruckt und zitiert werden können.

Auch andere Universitäten wie z.B. die Technische Universität Graz oder die Universität Graz planen ein Repositorium zur Umsetzung der Green Road. So ist es Ziel der Universität Graz, einen Publikationsserver zu installieren, in welchem Publikationen wie (1) Diplomarbeiten, Dissertationen und Habilitationen, (2) Aufsätze, Proceedings, Research Papers, Reports und Sonderdrucke, sowie (3) digitale Objekte aller Art wie Bilder (z.B. Handschriften), Videoclips, Audio-Dateien, CD-ROMs, Begleitmaterialien etc. gesammelt und über eine Online-Plattform der Öffentlichkeit zur Verfügung gestellt werden können. Ähnlich soll an der Universität Mozarteum Salzburg ein Ausbau zur hybriden Bibliothek erfolgen. Publikationsfonds zur Förderung der Gold Road haben darüber hinaus die Technische Universität Graz, die Universität Wien und die Universität für Bodenkultur eingerichtet.

Insgesamt engagiert sich die überwiegende Mehrheit der österreichischen Universitäten sehr aktiv für eine Einführung und Entwicklung von Open Access-Strategien bzw. für eine Weiterführung von Open Access-Initiativen. Die Universitäten haben ihr Engagement hierzu u.a. auch in den Leistungsvereinbarungen 2013 bis 2015 verankert. In Zukunft kommt dabei der Weiterentwicklung von OA im Universitätenverbund eine besondere Relevanz zu. So gibt es
Kooperations- und Koordinationsaktivitäten im Bereich Open Access in Zusammenarbeit mit dem FWF und der österreichischen Bibliothekenverbund- und Service $\mathrm{GmbH}$, an welchen sich alle österreichischen Universitäten beteiligen. Ziel ist es, eine österreichweite Lösung für Open Access sowie für den Aufbau von Institutional Repository für Preprints zu erarbeiten. Darüber hinaus entwickeln sich auch auf regionaler Ebene Kooperationen wie z.B. die gemeinsame steirische Open Access-Plattform. An dieser Plattform beteiligen sich die Universität Graz, Technische Universität Graz, Medizinische Universität Graz, Montanuniversität Leoben und Kunstuniversität Graz - mit dem Ziel, bis 2015 ein universitäres Repositorium inklusive einer elektronischen Langzeitarchivierung und eines repräsentativen Forschungsinformationssystems aufzusetzen.

Eine weitere Initiative nimmt sich der Finanzierung des österreichischen Anteils an der internationalen Initiative $\mathrm{SCOAP}^{3}$ (Sponsoring Consortium for Open Access Publishing in Particle Physics) an. Ziel ist es, dass ab 2014 alle relevanten Fachzeitschriften der Hochenergiephysik in OA erscheinen. Um dies zu organisieren, haben sich die Universität Wien, die Technische Universität Wien, die Universität Graz, die Universität Innsbruck, die ÖAW, der FWF, das BMWF und die Österreichische Bibliothekenverbund- und Service GmbH (OBVSG) zusammengeschlossen. Schließlich wurde Ende November 2012 auf Initiative der UNIKO und des FWF das OA Netzwerk Austria (OANA) konstituiert. Das Netzwerk strebt in Zukunft an, (1) die OA-Maßnahmen der österreichischen Forschungsstätten, Fördergeber und der Forschungspolitik abzustimmen, (2) einheitliche Positionen gegenüber den Informationsanbietern (v.a. gegenüber den Verlagen) zu formulieren, sowie (3) Ansprechpartner und Informationsquelle für die (Forschungs-)Politik zu sein.

58 Einen Überblick zu den Kooperationen liefert die 2012 eingerichtete Website http://phaidra.org 


\subsubsection{Weitere Entwicklungen des Open Access-Paradigmas}

Das Internet hat die Art und Weise, wie WissenschaftlerInnen arbeiten und publizieren, wie die Communities recherchieren und kommunizieren, nachhaltig verändert. Der Zugang zu Informationen hat sich verändert. Eine Wissenschaft ist heute ohne Zugriff auf elektronische Publikationen, Datenbanken oder Präsenz im Internet nicht mehr denkbar. Damit geht auch einher, dass die Transparenz innerhalb der Forschung steigt. Der „Elfenbeinturm“ der Wissenschaften ist aufgebrochen - mittels Zugang zu Publikationen, aber auch mittels Zugang zu Forschungsdaten und integrativen Datenbanken wie z.B. STAR METRICS in den USA. Letztere tragen durch eine Verlinkung von Publikationen, Daten, Patenten, Forschungsinstitutionen, Drittmittelgebern etc. wesentlich zum Fortschritt in OA bei.

\section{Open Data}

Der freie Zugang zu Forschungsdaten geht wie OA ebenfalls mit der Forderung nach mehr interner Transparenz in der Forschung einher, allerdings ist dieser ungleich schwieriger umzusetzen, weil sich hier anders als bei Publikationen die technischen Anforderungen und Standards zwischen den Disziplinen sehr unterscheiden, und diese oft nur national und für jede Disziplin einzeln gelöst werden können. Insofern befindet sich die Diskussion um Open Data noch am Beginn, obgleich sich einige allgemeine Prinzipien abzeichnen: So herrscht in der Community Konsens, dass der freie Zugang zu öffentlich geförderten Forschungsdaten ein essentieller Bestandteil von Wissenschaft ist, da dieser erst die Reproduzierbarkeit wissenschaftlicher Erkenntnisse ermöglicht. Die Nutzung und Wiederverwertung der Daten sollen daher - unter Berücksichtigung ethischer und rechtlicher Grenzen - in Zukunft möglichst rest- riktionsfrei erfolgen, und die Archivierung eine nachhaltige Verwertbarkeit erlauben.

Auf nationaler Ebene gibt es bereits vereinzelt Ansätze, für die Forschung relevante Daten der Öffentlichkeit zur Verfügung zu stellen. So wird eine Reihe von forschungsrelevanten Daten und Materialien von öffentlich finanzierten Einrichtungen wie Museen, Bibliotheken, Statistikämtern oder meteorologischen Anstalten verwaltet und auf eigens eigenrichteten Portalen publiziert. Allerdings ist der Zugang (wie z.B. zu den Mikrodaten) oft nur sehr begrenzt oder muss wiederum freigekauft werden. Weiter fortgeschritten ist im Gegensatz hierzu der Datenzugang in der öffentlichen Verwaltung, auch Open Government Data genannt. Open Government Data bezieht sich auf "Datenbestände des öffentlichen Sektors, die von Staat und Verwaltung im Interesse der Allgemeinheit ohne jedwede Einschränkung zur freien Nutzung, zur Weiterverbreitung und zur freien Weiterverwendung frei zugänglich gemacht werden. ${ }^{159}$ Angesichts des gesteigerten Interesses an Open Access werden in jüngster Zeit auch in Österreich verstärkt Initiativen gesetzt, Daten der öffentlichen Verwaltung frei zugänglich zu machen. ${ }^{60}$

\section{STAR METRICS}

Open Access hat auch im Bereich der öffentlichen Forschungsförderung, im Speziellen bezugnehmend auf die Abwicklung durch Förderorganisationen, deutlich an Relevanz gewonnen. Vorreiter sind hier wiederum die USA. So wurde im Jahr 2009 STAR METRICS (Science and Technology in America's Reinvestment - Measuring the Effects of Research on Innovation, Competitiveness and Science) als ein Kooperationsprojekt zwischen dem US-amerikanischen Office of Science and Technology Policy sowie der National Science Foundation und den National Institutes for Health initiiert. Ziel von STAR METRICS ist es, eine einheitliche Dateninfrastruktur als Grundlage stan- 
dardisierter Evaluationsmethoden zur Analyse von Forschungsprojekten aufzubauen. Der Aufbau des STAR METRICS-Programms gliedert sich hierbei in zwei Stufen: In der ersten Stufe geht es um die Ermittlung direkter Effekte von öffentlichen Forschungsinvestitionen auf die Beschäftigungssituation im Wissenschaftssektor; in der zweiten Stufe sollen wissenschaftliche, soziale und ökonomische Effekte von Forschungsinvestitionen weitgehend erfasst werden. Ähnlich kohärente Dateninfrastrukturen haben bereits Belgien und Brasilien aufgebaut, auch für andere europäische Länder ist der Aufbau von Datenbanken zur Dokumentation öffentlicher Forschungsförderung sowie zur Sichtbarmachung wissenschaftlicher Ergebnisse und Auswirkungen öffentlicher Forschung eine unerlässliche Herausforderung. So empfiehlt z.B. auch die deutsche Expertenkommission Forschung und Innovation im Jahr 2012 - bezugnehmend auf STAR METRICS - eine Initiierung ähnlicher Projekte in Deutschland. ${ }^{61}$

\section{Resümee: Open Access als ein in Zukunft gelebtes Paradigma}

In einer Prospektion der Steigerungsraten sind einige ExpertInnen zum Ergebnis gekommen, dass sich Open Access als dominantes Publikationsmodell in den nächsten 10-15 Jahren durchgesetzt haben wird. ${ }^{62} \mathrm{Ob}$ dies eintrifft, wird sich zeigen müssen. Gleichwohl hat $\mathrm{OA}$ schon jetzt eine Transformation des Rollenverständnisses der am Forschungsprozess Beteiligten eingeläutet:

- Förderorganisationen werden Kosten für die Publikationen, die als Ergebnis aus ihren Förderungen hervorgehen, in ihre Budgets einkalkulieren.

- Auf der einen Seite werden Forschungsstätten und Bibliotheken die Mittel für die Publikationen teilweise auf die WissenschaftlerInnen übertragen. Auf der anderen Seite werden sie aber verstärkt die WissenschaftlerInnen durch
Verlagsinfrastrukturen und Repositorien aktiver im Publikationsprozess unterstützen. ${ }^{63}$

- Von den WissenschaftlerInnen wird verstärkt erwartet werden, die Kosten für Publikationen in ihren strategischen Planungen zu berücksichtigen.

- Den Verlagen muss bewusst sein, dass sie sich nicht in einem „normalen“ Markt bewegen, wo durch private Investitionen und Leistungen legitimer Weise Gewinne erzielt werden. Vielmehr beruht ihr Geschäft größtenteils auf öffentlich finanzierten Leistungen. Daher muss die Preisbildung auch davon abhängig gemacht werden, welchen Service und welche Qualität das entsprechende Publikationsorgan erbringt.

- Die (Forschungs-)Politik wird für die Rahmenbedingungen (wie z.B. FWF-Politik, Verankerung der OA-Politik der Universitäten in den Leistungsvereinbarungen, Schaffung von Repositorien) sorgen müssen, dass die öffentlichen Güter, welche die Wissenschaft produziert, durch einen freien Zugang auch einen öffentlichen Mehrwert erzeugen.

Wie bei jeder Reform werden auch beim Paradigmenwechsel hin zu OA-Publikationen in der Übergangsphase die Kosten wahrscheinlich steigen, bevor sich Effizienzgewinne einstellen. Die Kosten werden dabei von den AutorInnen, nicht mehr von der Leserschaft getragen. In den Geisteswissenschaften wird unterdessen dieser Paradigmenwechsel nicht so merklich sein, weil bislang die AutorInnen über Druckkostenzuschüsse auch immer an den Publikationskosten beteiligt waren; anders in den Naturwissenschaften (wo hauptsächlich in Form von Artikeln, nicht in Form von Monographien publiziert wird), wo OA $\mathrm{zu}$ einer weitgehenden Kostenentlastung der Wirtschaft durch die öffentliche Hand führen wird. Mittelfristig dürfte eine freiverfügbare Wissenschaft allerdings kaum abschätzbare Innovationspotentiale eröffnen.

61 Vgl. EFI (2012).

62 Vgl. Lewis (2012).

63 Das erst jüngst vorgestellte Konzept EpiSciences geht sogar davon aus, dass die Scientific Community auf der Basis von PreprintServern völlig ohne kommerzielle Anbieter auskommen könnte. 


\section{Die Rolle der Industrie im Innovationssystem}

Dieses Kapitel stellt die Rolle der Industrie im Innovationssystem dar, wobei unter Industrie im Wesentlichen der Abschnitt der Herstellung von Waren (Abschnitt C der ÖNACE-Gliederung) verstanden wird. Während die Beschäftigungsfunktion der Industrie zwar nach wie vor bedeutsam, aber rückgängig ist, erweist sich ihr Beitrag zur Innovations- und damit zur Wachstumsperformance einer Volkswirtschaft längerfristig von entscheidenderer Bedeutung. ${ }^{64}$ Hierzu wird zunächst ein kurzer Überblick über die Ursachen der industriepolitischen Renaissance gegeben. Anschließend setzt eine Darstellung des globalen und österreichischen Strukturwandels den Rahmen, vor dem industrielle Entwicklungen interpretiert werden müssen. Schließlich werden die vielfältigen Beiträge der Industrie zum technischen Fortschritt dargestellt, wobei hier explizit eine breite Perspektive eingenommen wird. Deshalb erfolgt neben der Darstellung und Interpretation von innovationsbezogenen Indikatoren auch eine Analyse von Produktivitätskennzahlen, die letztlich das Ergebnis von Innovationsprozessen und von herausragender Bedeutung für die Wohlstandsentwicklung einer Volkswirtschaft sind. Zuletzt wird die Performance der österreichischen Industrie bei Schlüsseltechnologien und im Export von potenziellen Umweltschutzgütern dargestellt. Diese beiden Themen sind von zentraler Bedeutung für die zukünftige Entwicklung und internationale Wettbewerbsfähigkeit der österreichischen Industrie.

Jede empirische Analyse der Industrie und de- ren Vergleich mit der Gesamtwirtschaft bzw. dem Dienstleistungssektor hat einige Veränderungen dieser Sektoren zu berücksichtigen. ${ }^{65}$ Der Verbund mit dem Dienstleistungssektor ist enger als je zuvor und es kommt zu einem Anstieg des Anteils von Dienstleistungsaktivitäten innerhalb des Industriesektors. Die Auslagerung von Dienstleistungsaktivitäten durch Industrieunternehmen sorgt für ein Wachstum des Dienstleistungssektors auf Kosten der Industrie, ohne grundsätzliche Änderung der Aktivitäten. Viele Industriegüter werden heute als Güterbündel verkauft, welches neben dem physischen Gut vor allem auch Servicekomponenten enthält. Das Organisieren von Prozessen und die Integration von Systemen entlang von internationalen Wertschöpfungsketten sind zentrale Aufgaben einer modernen Industrie, die den Einsatz von hochqualifizierten TechnikerInnen verlangen. All dies deutet darauf hin, dass die Trennung in einen Industrie- und Dienstleistungssektor gemäß herkömmlicher Branchenstatistik zunehmend an inhaltlicher Substanz verliert. Weiterhin ist zu bedenken, dass weder der Industrie- noch der Dienstleistungssektor homogene Aggregate sind. Vielmehr sind die Unterschiede innerhalb der Sektoren größer als zwischen diesen. Allerdings zeigen sich bei einer empirischen Analyse nach wie vor deutliche Unterschiede zwischen diesen beiden Sektoren. Gleichzeitig ist aber auch Vorsicht bei der Interpretation der Daten geboten und viele Vergleiche haben notwendigerweise den Charakter einer pointierten Darstellung.

64 Vgl. Helper et al. (2012).

65 Vgl. Pilat et al. (2006), Mc Kinsey (2012). 


\subsection{Die Renaissance der Industriepolitik}

Die weltweite Wirtschafts- und Finanzkrise hat zu einer Neubeurteilung wirtschaftspolitischer Optionen und des Strukturwandels geführt. Im Zentrum dieser global beobachtbaren Re-Orientierung steht insbesondere der industrielle Sektor. Galt die Industrie lange Zeit als Auslaufmodell und ein großer Industriesektor als Zeichen eines verzögerten Strukturwandels, so hat sich dieses Urteil mittlerweile geradezu in sein Gegenteil verkehrt. Neben einer Renaissance der Industriepolitik ist die Industrie durch einen parallel ablaufenden technologischen Paradigmenwandel gekennzeichnet, der von einigen AutorInnen als „Dritte industrielle Revolution" bezeichnet wird. ${ }^{66}$ Diese ist vor allem durch eine Konvergenz verschiedener Technologien wie etwa Materialtechnologien, Internet, 3D-Printing oder Technologien im Zusammenhang mit erneuerbaren
Energien gekennzeichnet. Als Folge davon könnte es zusammen mit steigenden Produktionskosten in China zu einer teilweisen Rückwanderung von industriellen Fertigungsprozessen in OECD-Staaten kommen. ${ }^{67}$

Worin liegen die Ursachen für das neue Interesse der Wirtschaftspolitik an der Industrie? Zunächst einmal ist festzustellen, dass bereits vor der Krise eine Popularitätszunahme der Industriepolitik etwa im Zusammenhang mit dem Aufstieg Chinas oder der Lissabon-Strategie zu beobachten war. ${ }^{68}$ Die Krise selbst hat dann vor allem durch den Gegensatz zwischen dem offenbar an seine Grenzen geratenen angelsächsischen, finanzmarktgeriebenem Wachstumsmodell und dem erstaunlich robusten, industriegetriebenem Geschäftsmodell Deutschland für eine wirtschaftspolitische Neuausrichtung gesorgt. ${ }^{69}$ Großbritannien, die USA, aber auch Frankreich waren mit einer starken Deindustrialisierung in

Abb. 20: Industrieanteil und Leistungsbilanzsaldo

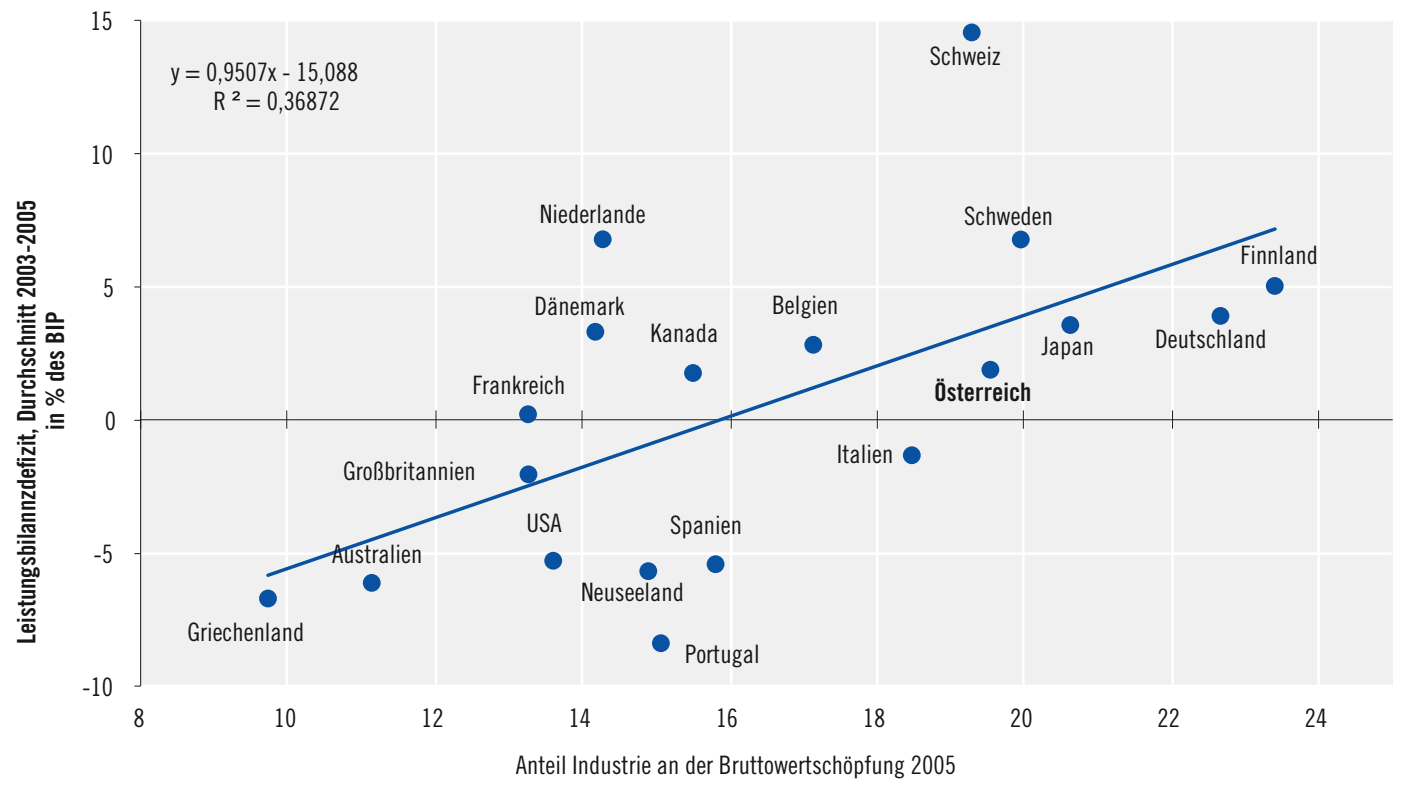

Quelle: OECD, Weltbank. Berechnungen JOANNEUM RESEARCH.

66 Vgl. Rifkin (2011), Reiner (2012), Marsh (2012).

67 Vgl. Fishman (2012), The Economist (2012).

68 Vgl. Aiginger (2006), Riess, Välilä (2006).

69 Vgl. Rattner (2011), Rürup, Heilmann (2012). 
den Jahren vor der Krise konfrontiert. ${ }^{70}$ Die Befürchtung besteht, dass die Verlagerung sowie das Schrumpfen der Industrie letztlich zu einer Erosion des Innovationspotenzials der Volkswirtschaft führen. ${ }^{71}$ US-Präsident Obama hat die Erneuerung und Expansion der Industrie zu einem wichtigen Ziel seiner Amtszeit auserkoren. ${ }^{72}$ In Europa versuchen die südeuropäischen Staaten ihre Leistungsbilanzdefizite in den Griff zu bekommen, was ebenfalls nach einer eine Umlenkung volkswirtschaftlicher Ressourcen in den Industriesektor verlangt. Aber auch Großbritannien oder die USA sehen sich angesichts stark negativer Handels- bzw. Leistungsbilanzen mit der Herausforderung konfrontiert, ihren Exportsektor zu stärken. Abb. 20 zeigt, dass ein hoher
Industrieanteil mit einem Leistungsbilanzüberschuss einhergeht, während der Wegfall von industriellen Exportkapazitäten offenbar keineswegs automatisch durch steigende Dienstleistungsexporte substituiert werden kann.

Der Paradigmenwechsel in den nationalen Wirtschaftspolitiken zeigt sich auch auf Ebene der supranationalen Organisationen. Beispielsweise vertrat die OECD lange Zeit eine ablehnende Haltung gegenüber Industriepolitik unter der liberalen Devise "deregulate and wait". In einer aktuellen Ausgabe des OECD Observer wurde jedoch argumentiert, dass ,industrial policy can be made to work ${ }^{\prime \prime}{ }^{73}$ Die EU hat ebenfalls neue industriepolitische Akzente mit ihrer 2010 erschienen Strategie einer „integrierten Indus-

\section{Abb. 21: Industrieanteil an der Wertschöpfung in der EU-27 und der Schweiz, Japan und USA}

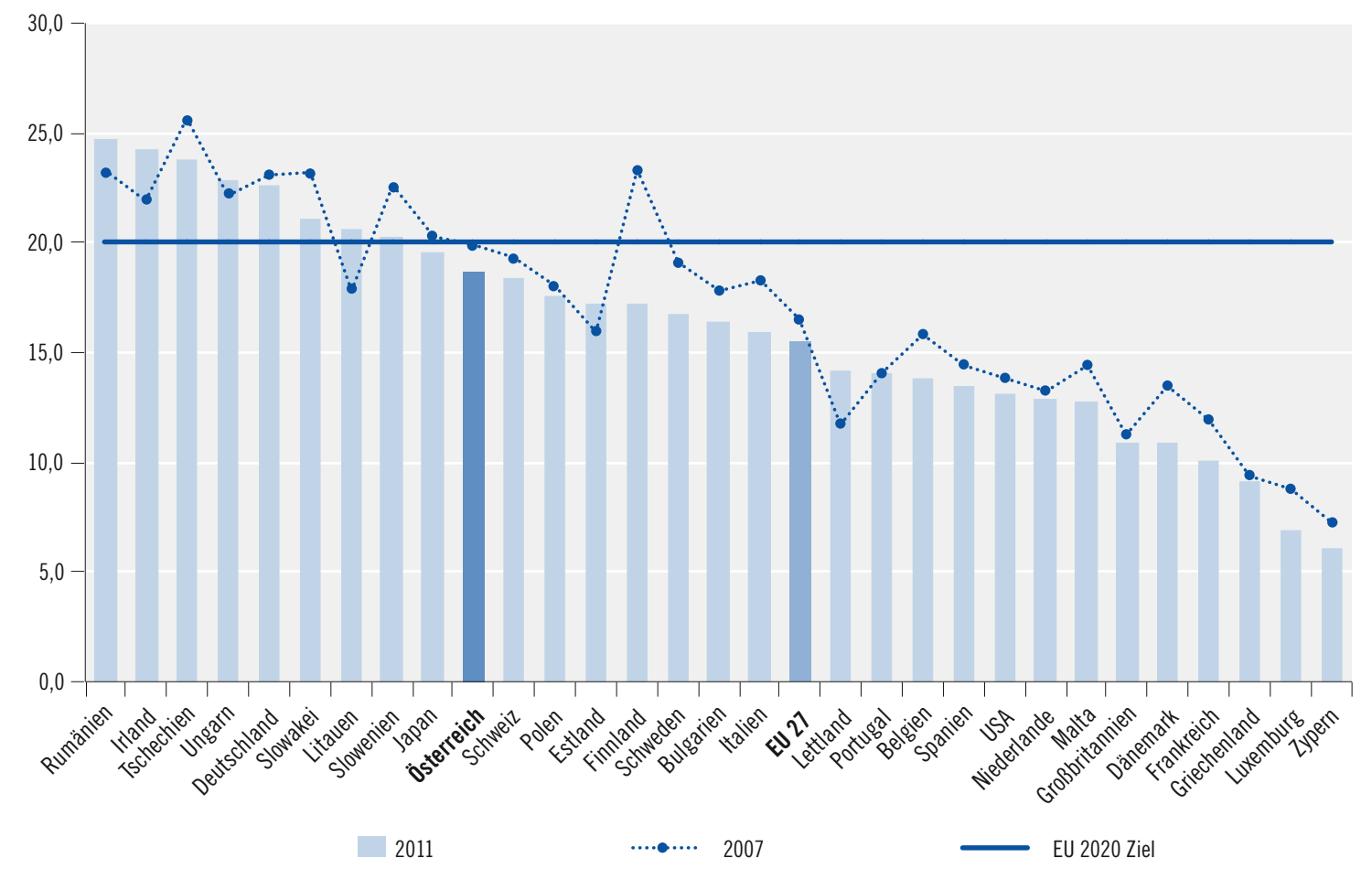

Quelle: AMECO, EU Commission, COM(2012) 582.

70 Vgl. Shanmugalingam et al. (2010), Spence, Hlatshwayo (2011).

71 Vgl. Pisano, Shih (2009), Tassey (2010).

72 Vgl. The White House (2012).

73 Vgl. Pilat (2012). 
triepolitik" als Teil von Europa 2020-Strategie gesetzt. ${ }^{74}$ Diese wurde bereits zwei Jahre später mit der Veröffentlichung eines „Updates" dieser Strategie überarbeitet und akzentuiert. ${ }^{75}$ In diesem „Update" hat sich die EU das Ziel gesetzt, einen Industrieanteil von $20 \%$ an der Wertschöpfung zu erreichen. Als Motivation hierfür werden vor allem die wichtige Rolle der Industrie für Innovation und Exporte sowie der Standortwettbewerb mit den USA und Asien um die Ansiedlung zukunftsträchtiger Industrien genannt. Wie Abb. 21 zeigt, liegt der aktuelle Industrieanteil der EU-27 bei ca. $16 \%$. Das bedeutet nicht mehr und nicht weniger, als dass Europa nach einer substanziellen Reindustrialisierung strebt, nachdem Jahre lang der Strukturwandel vor allem durch eine Deindustrialisierung gekennzeichnet war. Österreich gehört mit 18,7 \% (2011) zur Gruppe der Länder mit einem hohen Industrieanteil. Vor der Krise lag der Anteil noch bei 19,9\% (2007). Betrachtet man nur Länder mit ähnlichem Entwicklungsniveau, so ist Österreich nach Deutschland das Land mit dem höchsten Industrieanteil in der EU.

Generell dürfte das simultane Aufkommen neuer industriepolitischer Initiativen den Wettbewerb um Industrieinvestitionen weiter erhöhen. Ähnliches trifft auch für die Standortkonkurrenz um die rasch wachsenden Umweltindustrien zu. Hier ist die Wirtschaftspolitik in besonderem Maße gefordert, einen klugen Mittelweg zwischen industriepolitischen Initiativen und dem Wirkenlassen der Marktkräfte zu finden.

\subsection{Globale Verschiebungen von industriellen Produktionskapazitäten}

Die letzten Jahrzehnte waren von einem tiefgreifenden Umbruch in der räumlichen Organisation der globalen Industrieproduktion gekennzeichnet. Niemals zuvor in der Wirtschaftsgeschichte gab es so viele Staaten, die industrielle Kapazitäten und Fähigkeiten aufweisen und im Standortwettbewerb um Investitionen und Produktionsaufträge konkurrieren. Insbesondere die Reorganisation der Industrie entlang von kontinentalen und globalen Wertschöpfungsketten hat in einigen Schwellenländern zu einer dramatischen $\mathrm{Zu}-$ nahme der Industrie geführt.

Die Verschiebungen im weltweiten Industriegefüge lassen sich anhand der Veränderung von Produktionsanteilen und absoluten Wertschöpfungsgrößen darstellen. In Anteilen betrachtet nahm der Anteil der Entwicklungsländer an der globalen industriellen Wertschöpfung von $24 \%$ im Jahr 1990 - über $27 \%$ - um 2000 auf $42 \%$ im Jahr 2012 zu. $^{76}$ Abb. 22 stellt die Entwicklung der industriellen Wertschöpfung der zehn bedeutsamsten Produktionsländer weltweit im Jahr 2011 in Mrd. US \$ dar. Die bedeutsamste Entwicklung im betrachteten Zeitraum von 1970 bis 2011 ist zweifelsohne die beeindruckende Performance von China, das 2010 die USA als Nation mit der bislang höchsten Industriewertschöpfung überholte und das einzige Land ist, welches über zwei Billionen US \$ an industrieller Wertschöpfung pro Jahr erreicht. Deutlich stagnierend erscheint demgegenüber die Entwicklung Japans, das seit 1990 mit bislang nicht überwundenen makroökonomischen Problemen kämpft. Auffallend ist auch die deutsche Entwicklung, die nach einer längeren Null- bzw. Negativwachstumsphase seit dem Jahr 2002 wieder deutlich an Dynamik gewonnen hat. Deutlich wird anhand von Abb. 22 vor allem auch die hohe Konzentration der Industriewertschöpfung auf die vier wichtigsten Produktionsländer China, USA, Japan und Deutschland. Diese vier Staaten sind deutlich abgesetzt von den weiteren sechs wichtigsten Industriestandorten - Brasilien, Russland oder Indien sind gegenüber China von untergeordneter Bedeutung.

\footnotetext{
74 Vgl. Europäische Kommission (2010b).

75 Vgl. Europäische Kommission (2012a).

76 Vgl. Marsh (2012).
} 
Abb. 22: Bruttowertschöpfung im Industriesektor in den 10 Hauptproduktionsnationen, Mrd. US \$, 1970-2011

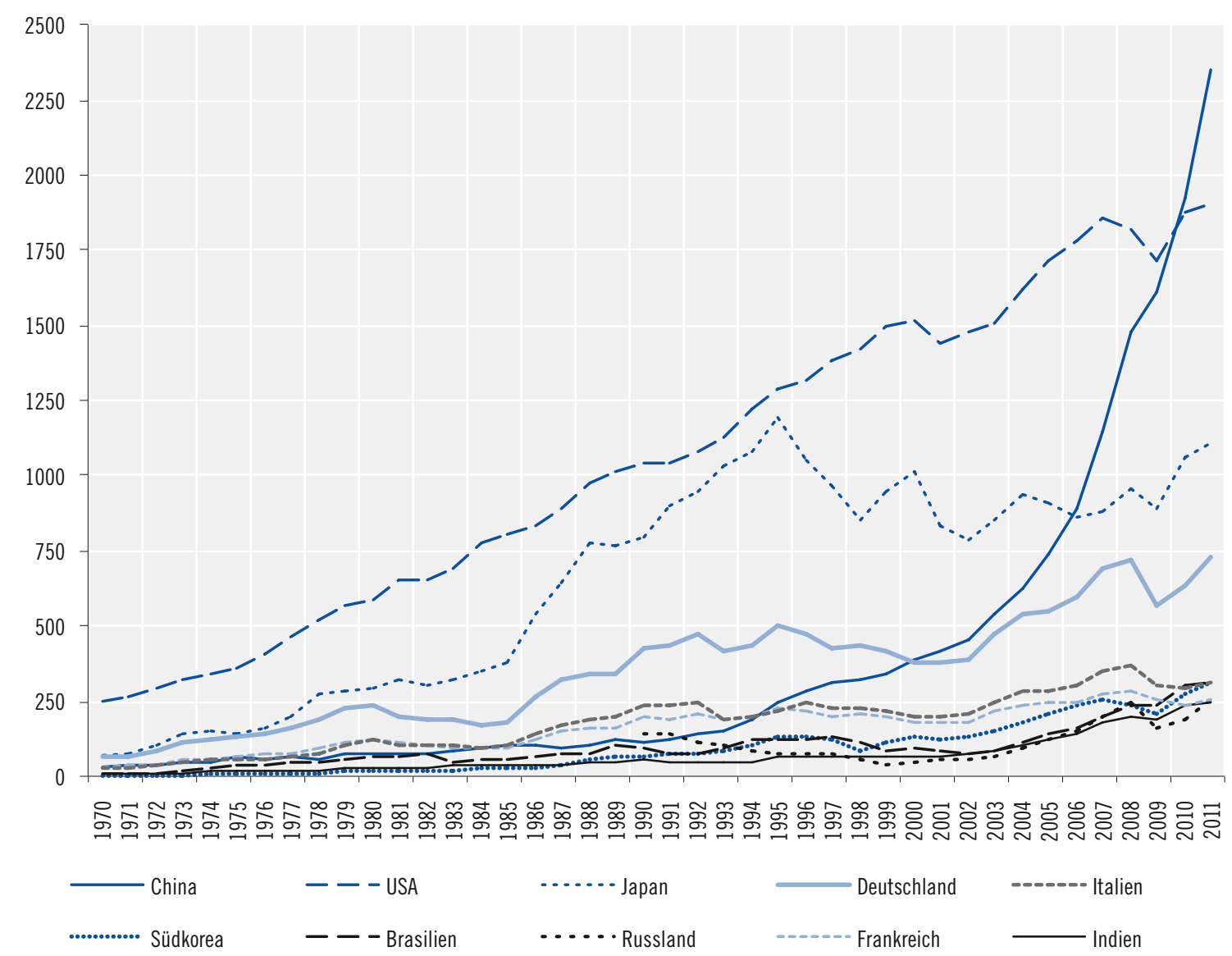

Quelle: UN National Accounts. Berechnungen JOANNEUM RESEARCH.

Abb. 23 zeigt die Daten zur industriellen Wertschöpfung für die weltweit 25 wichtigsten Industriestandorte in Mrd. US \$ für 2011. In diesen Staaten erfolgt ca. $87 \%$ der weltweiten Industriewertschöpfung. Die vier Länder China, USA, Japan und Deutschland sind mit 54 \% Wertschöpfungsanteil für mehr als die Hälfte des weltweiten Industrieoutputs verantwortlich. Österreich liegt an 24. Stelle und gehört damit noch knapp zu den 25 wichtigsten Industrieproduzenten.

Während die Abb. 22 und Abb. 23 die Bedeutung Chinas und weiterer Schwellenländer deutlich werden lässt, ergibt sich ein gänzlich anderes Bild, wenn man die Industrieintensität einer Gesellschaft im Sinne der industriellen Wertschöp- fung pro Einwohner als Maßstab heranzieht. Abb. 24 zeigt dies für die gleichen Staaten wie in Abb. 23. China, aber auch Indien, Brasilien oder Russland erscheinen nunmehr als Staaten mit einer (noch) sehr niedrigen Industrieintensität. Beispielsweise ist die Industriewertschöpfung pro Einwohner in der "Weltfabrik" China mit ca. 1.700 US \$ weniger als halb so hoch wie im stark deindustrialisierten Großbritannien mit ca. 3.700 US \$. Die Schweiz ist mit Abstand das industrieintensivste Land der Welt, gefolgt von Deutschland, Japan, Österreich und Schweden. Der wesentliche Erklärungsfaktor für diese Verteilung der Industrieintensität sind Produktivitätsunterschiede des Industriesektors zwischen den OECD-Staaten und den Schwellenländern. 
Abb. 23: Industrielle Bruttowertschöpfung der 25 größten Industrieproduzenten, in Mrd. US \$, 2011

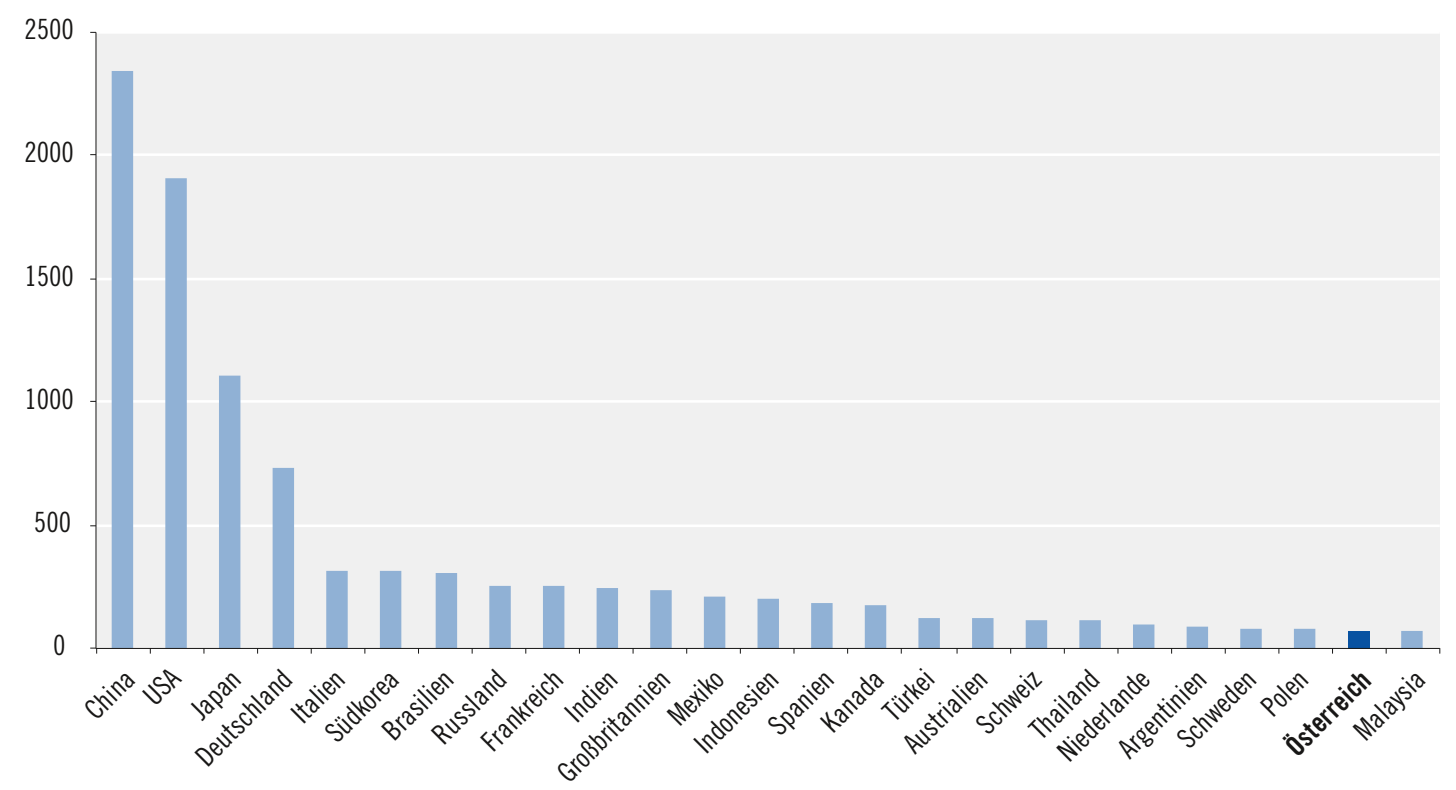

Quelle: UN National Accounts, World Bank. Berechnungen JOANNEUM RESEARCH.

Abb. 24: Industrielle Wertschöpfung in US \$ pro Einwohner der 25 größten Industrieproduzenten, 2011

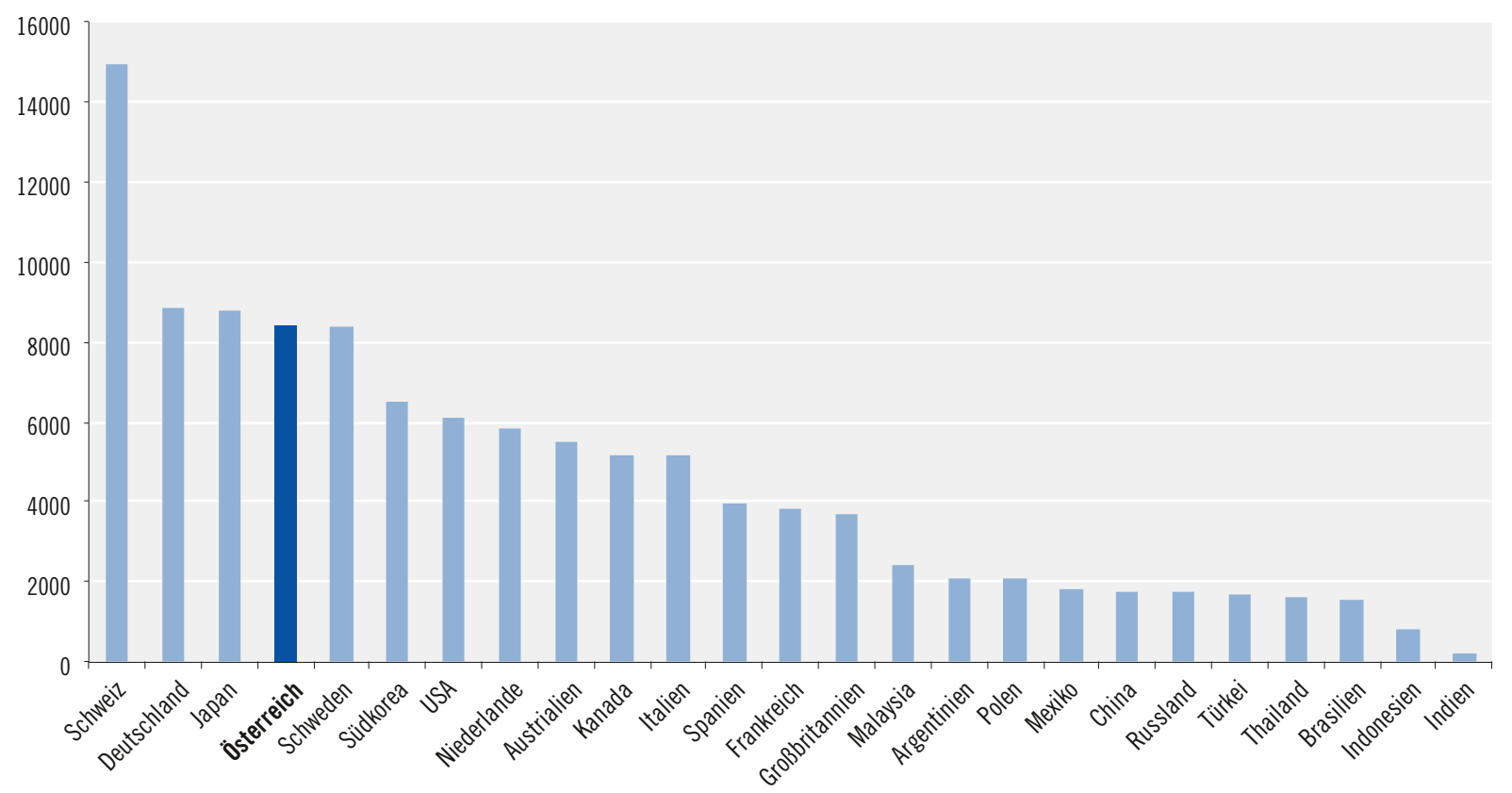

Quelle: UN National Accounts, World Bank. Berechnungen JOANNEUM RESEARCH. 


\subsection{Wandel und Struktur der österreichischen Industrie im internationalen Vergleich}

Österreich ist so wie alle entwickelten Volkswirtschaften von einem langfristigen Deindustrialisierungsprozess gekennzeichnet. Eine genauere Analyse bringt jedoch die Erkenntnis, dass der Strukturwandel keineswegs einheitlich verläuft und dass die Deindustrialisierung einzelner Volkswirtschaften kein Naturgesetz ist. ${ }^{77}$ Vor allem die letzten 10 bis 15 Jahre haben gezeigt, dass einzelne reiche Volkswirtschaften einen hohen Industrieanteil halten können, während andere Länder wie z.B. Großbritannien oder Frankreich durch eine teils sehr rasche Deindustrialisierung gekennzeichnet waren.

Eine Darstellung der langfristigen Entwicklung der österreichischen Industrie zeigt Abb. 25.
Dabei werden die typischen Merkmale des Strukturwandels der Industrie deutlich. Zunächst ergibt sich ein säkularer Rückgang der Industrie an der nominellen Wertschöpfung von 1960 bis 2011 von ca. $29 \%$ auf $19 \%$. Allerdings wird auch eine deutliche Abnahme des Rückgangs der Industrie und eine annähernde Stabilisierung des Industrieanteils seit den frühen 1990er Jahren erkennbar. Dies ist insofern bemerkenswert, als dies genau jene Zeit ist, in der es zu einer deutlichen Zunahme der Konkurrenz durch den Abbau von Marktsegmentierungen kam (Ostöffnung, EU-Beitritt). Demgegenüber nahm der Beschäftigungsanteil stetig von $25 \%$ (1976) auf $15 \%$ (2011) ab. Die steigende Kurve in Abb. 25 stellt den Anteil der realen Industriewertschöpfung an der realen Gesamtwertschöpfung dar. Der reale Anteil nahm aufgrund der relativ höheren Pro-

Abb. 25: Österreich: Industrieanteil an der Bruttowertschöpfung (nominell, real) und Beschäftigung, 1960-2011

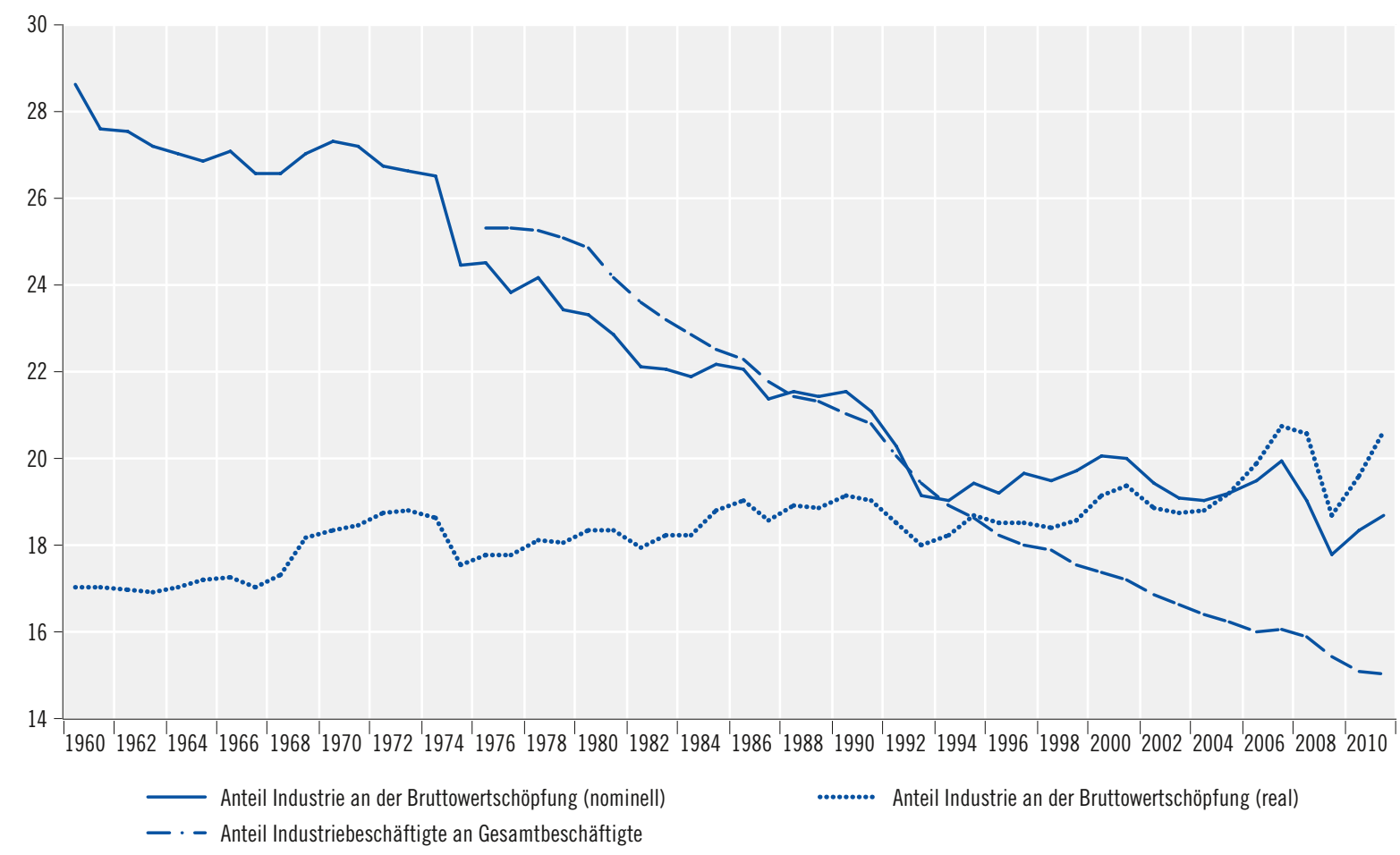

Quelle: AMECO, Volkswirtschaftliche Gesamtrechnung. Berechnungen JOANNEUM RESEARCH.

77 Siehe Rürup und Heilmann (2012). 
duktivitätssteigerungen in der Industrie zu. Dies ist eine Folge der hohen Wettbewerbsintensität auf Industriegütermärkten, welche die Unternehmen dazu zwingt, die Produktivitätsgewinne in Form von niedrigeren Preisen an die Nachfrager weiterzugeben. Die geringeren Preissteigerungsraten im Industriesektor führen zwangsläufig dazu, dass - unter sonst gleichen Umständen - eine Abnahme des Anteils an der Wertschöpfung gemessen in nominellen Preisen stattfindet. Das hohe industrielle Produktivitätswachstum erklärt auch den stärkeren Rückgang der Beschäftigung im Vergleich zur nominellen Wertschöpfung.

Einen internationalen Vergleich der österreichischen Entwicklung zeigt Tab. 12 anhand von Indikatoren zur absoluten und relativen Deindustrialisierung. Dabei wurden aggregierte Ländergruppen gebildet, die jeweils unterschiedliche sektorale Entwicklungspfade repräsentieren. In Tab. 12 wird einerseits zwischen Wertschöpfungs- und Beschäftigungsindikatoren (Spalte 2 und 3) und anderseits zwischen Volumensgrößen und Wertgrößen (Zeile 2 und 4) unterschieden. Letzteres lässt Rückschlüsse darauf zu, ob es sich um eine absolute oder relative Deindustrialisierung handelt. Von absoluter Deindustrialisierung spricht man, wenn die Indikatoren in absoluten Größen (Volumina) abnehmen, von relativer Deindustrialisierung, wenn die Anteile abnehmen. Die Volumina der industriellen Produktion, gemessen in Veränderung der realen Wertschöpfung, nahmen in allen Ländergruppen und auch in Österreich zu. Die höchsten Zunahmen weisen die nordischen Volkswirtschaften auf, die niedrigsten Zunahmen zeigen die südeuropäischen Staaten. Es existiert demnach keine absolute Deindustrialisierung bei der Wertschöpfung. Das Gegenteil ergibt sich bei den Beschäftigungsvolumina. Diese nehmen in allen Ländergruppen und auch in Österreich ab, wobei der Rückgang in Österreich im Vergleich zu den Ländergruppen am schwächsten ausfällt. In Österreich kam es vor allem zwischen 2000 und 2007 nur zu einem sehr geringen Rückgang der Beschäftigung. Der stärkste Beschäftigungsrückgang zwischen 1995 und 2007/2010 fand in den angelsächsischen Staaten statt.

Bei den Wertschöpfungsanteilen der Industrie wird die Sonderrolle der kontinentaleuropäischen Staaten Deutschland, Schweiz und Österreich deutlich. Hier kam es zwischen 1995 und 2007 sogar zu einer leichten Zunahme der Industrie an der Wertschöpfung. Eine rasche Deindustrialisierung zeigen die angelsächsischen und südeuropäischen Staaten, deren Industrieanteil an der Wertschöpfung nunmehr bei ca. $14 \%$ liegt. Damit ergibt sich ein uneinheitliches Bild bei der relativen Deindustrialisierung gemessen am Wertschöpfungsanteil der Industrie und für die Jahre 2007 bzw. 2010 ergibt sich eine deutliche Differenzierung zwischen den Ländergruppen. Bei den Beschäftigungsanteilen bestätigt sich demgegenüber eine stetige relative Deindustrialisierung für alle Ländergruppen. Erneut weisen die kontinentaleuropäischen Staaten mit etwa $15 \%$ den höchsten und die angelsächsischen Staaten mit ca. $10 \%$ den niedrigsten Industrieanteil auf.

Die Unterschiede in der Industriedynamik sind das Ergebnis eines komplexen Zusammenwirkens unterschiedlicher Faktoren. ${ }^{78}$ Neben angebotsseitigen Ursachen wie Produktivitätsfortschritt oder Outsourcing der Industrie von Dienstleistungsaktivitäten spielen auch nachfrageseitige Faktoren wie eine vermehrte Verwendung der Einkommen für Dienstleistungen eine Rolle. Letzteres stellt im Übrigen keinen Sättigungseffekt dar, sondern ist vor allem ein Resultat der Konsumentenreaktion auf die Änderung von relativen Preisen zwischen Industrie- und Dienstleistungsgütern. ${ }^{79}$ Eine zunehmende Aufmerksamkeit wird dem Einfluss der Importe aus Niedriglohnländern zuteil und der Verlagerung

78 Siehe Nickel et al. (2008), Schettkat (2006).

79 Siehe Debande (2006). 
Tab. 12: Absolute und relative Deindustrialisierung: Volumen und Anteile industrieller Wertschöpfung und Beschäftigung $(1995=100)$

\begin{tabular}{|c|c|c|c|c|c|c|c|c|}
\hline & \multicolumn{4}{|c|}{ Volumen industrieller Wertschöpfung } & \multicolumn{4}{|c|}{ Volumen industrieller Beschäftigung } \\
\hline & 1995 & 2000 & 2007 & 2010 & 1995 & 2000 & 2007 & 2010 \\
\hline Österreich & 100 & 120 & 153 & 144 & 100 & 97 & 96 & 92 \\
\hline Kontinentaleuropäische Staaten & 100 & 111 & 137 & 126 & 100 & 96 & 90 & 88 \\
\hline Nordische Staaten & 100 & 131 & 171 & 157 & 100 & 102 & 94 & 83 \\
\hline Angelsächsische Staaten & 100 & 128 & 156 & 146 & 100 & 99 & 82 & 75 \\
\hline \multirow[t]{3}{*}{ Südeuropäische Staaten } & 100 & 113 & 121 & 108 & 100 & 103 & 98 & 86 \\
\hline & \multicolumn{4}{|c|}{ Anteile industrieller Wertschöpfung } & \multicolumn{4}{|c|}{ Anteile industrieller Beschäftigung } \\
\hline & 1995 & 2000 & 2007 & 2010 & 1995 & 2000 & 2007 & 2010 \\
\hline Österreich & 19,4 & 20,1 & 19,9 & 18,4 & 18,7 & 17,4 & 16,0 & 15,1 \\
\hline Kontinentaleuropäische Staaten & 21,5 & 21,6 & 22,4 & 20,8 & 20,8 & 19,2 & 17,7 & 16,9 \\
\hline Nordische Staaten & 20,6 & 20,5 & 18,4 & 15,4 & 17,8 & 17,1 & 14,9 & 13,4 \\
\hline Angelsächsische Staaten & 18,0 & 15,9 & 13,4 & 12,8 & 16,0 & 14,7 & 11,3 & 10,2 \\
\hline Südeuropäische Staaten & 18,0 & 17,3 & 14,4 & 12,8 & 18,4 & 17,3 & 14,7 & 13,5 \\
\hline
\end{tabular}

Anm.: Als Beschäftigungsdaten für die angelsächsischen Staaten für 2010 wurden aufgrund mangelnder Datenverfügbarkeit Durchschnittswerte von 2008 und 2009 verwendet.

Kontinentaleuropäische Staaten: Deutschland, Österreich und Schweiz; Nordische Staaten: Schweden, Finnland und Dänemark; Angelsächsische Staaten: USA und Großbritannien; Südeuropäische Staaten: Griechenland, Spanien, Frankreich, Italien und Portugal.

Quelle: AMECO. Berechnungen JOANNEUM RESEARCH.

von Industrieaktivitäten aus OECD-Staaten in Schwellenländer. ${ }^{80}$ Generell dürften diese Faktoren für die verschiedenen Industriebranchen sehr unterschiedliche Bedeutung aufweisen. Dennoch zeigen empirische Studien sehr deutlich, dass die quantitativ wichtigsten Effekte interne Faktoren sind, wobei das Produktivitätswachstum von entscheidender Bedeutung sein dürfte. Damit schrumpft die Industrie nicht zuletzt gerade deshalb, weil sie so erfolgreich in der Steigerung ihrer Effizienz ist. Trotzdem hat der Einfluss der Importkonkurrenz zur Erklärung der Deindustrialsierung in den letzten Jahren an Bedeutung gewonnen. ${ }^{81}$

Es bleibt jedoch die Frage offen, warum einige Staaten trotz hoher Produktivitätsgewinne in der Industrie einen relativ hohen Industrieanteil halten können. Offenbar schaffen es diese Industrie- standorte, den Produktivitätsgewinn durch eine starke Expansion ihres Absatzes zumindest teilweise zu kompensieren. Anders formuliert: Eine hohe preisliche und vor allem nicht-preisliche Wettbewerbsfähigkeit sowie eine vorteilhafte Spezialisierung und Positionierung in internationalen Wertschöpfungsketten von Industriestandorten sind wichtige Elemente zum Erhalt eines starken industriellen Kerns. Diese Elemente dürften auch den Erfolg der deutschen oder österreichischen Industrie erklären, deren Angebotsstruktur mit einem Schwerpunkt auf hoch spezialisierte Investitionsgüter in Nischenmärkten einen Qualitätsaufschlag ermöglicht und ausgezeichnet zum rezenten Globalisierungszyklus und der damit einhergehenden Nachfrage nach diesen Gütern aus den Schwellenländern passt. ${ }^{82}$ Im Gegensatz zur US-amerikanischen Industrie,

80 Siehe Debande (2006), Dachs et al. (2006).

81 Siehe Rowthorn, Ramaswamy (1998), Rowthorn, Coutts (2004).

82 Siehe Rürup, Heilmann (2012). 
welche durch massives Outsourcing nach Asien zusammenschrumpfte, erhöhten Deutschland oder Österreich durch den Aufbau europäischer Wertschöpfungsketten unter Nutzung von osteuropäischen Lohnkostenvorteilen die Wettbewerbsfähigkeit der heimischen Produktion unter Beibehaltung eines deutlich größeren Industriesektors als in anderen Staaten. ${ }^{83}$ Für die Jahre vor der Krise konnte die österreichische Industrie von einer Lohnmoderation profitieren, welche wenn auch in geringerem Ausmaß - wie in Deutschland die preisliche Wettbewerbsfähigkeit erhöhte. ${ }^{84}$ In Summe dürften jedoch Produktivitätssteigerungen und der technische Fortschritt bedeutsamer für die Erklärung der hohen Wettbewerbsfähigkeit und Dynamik der österreichischen Industrie sein. ${ }^{85}$ Darüber hinaus fungierten die osteuropäischen Staaten aber auch als dynamische Exportmärkte für die österreichische Industrie. ${ }^{86}$ Allerdings zeigen neuere Studien, dass zunehmend qualifikationsintensive Aktivitäten aufgrund von Humankapitalengpässen in Deutschland und Österreich nach Osteuropa ausgelagert werden. ${ }^{87}$

Die Struktur der österreichischen Industrie ist traditionell durch einen geringen Anteil von technologie- und qualifikationsintensiven Industriesegmenten gekennzeichnet. So lag 2007 so- wohl der Anteil der Hochtechnologieindustrie als auch jener der qualifikationsintensiven Sektoren (inklusive einiger Dienstleistungsbranchen) unter dem Durchschnitt der EU-25 (ohne Rumänien und Bulgarien), während für den Anteil der niedrigtechnologischen und qualifikationsextensiven Industrien das Gegenteil gilt: Hier hat Österreich einen höheren Anteil als die EU25 (Europäische Kommission 2011c). Ein ähnliches Bild ergibt sich auch bei der Exportstruktur (Reinstaller und Sieber 2012). Dieser vielfach als negativ angesehene Befund für die Wirtschaftsstruktur bei gleichzeitig guter makroökonomischer Performance war und ist Anlass zur Diskussion. ${ }^{88}$ Tab. 13 zeigt zunächst den Anteil der Industriesektoren nach F\&E-Intensitäten im Zeitverlauf (siehe Anhang I). In Summe ergibt sich im Zeitraum von 1980 bis 2007 ein deutliches technologisches Upgrading der österreichischen Industrie. Die beiden weniger technologieintensiven Segmente verlieren, Mittelhochtechnologie und Hochtechnologie gewinnen. Allerdings ist der Hauptgewinner des Strukturwandels nicht die Hochtechnologie, sondern die Mittelhochtechnologie, und der Anteil der Mittelniedrigtechnologie bleibt annähernd konstant. Beispiele für das Segment der Mittelhochtechnologie sind der Maschinenbau und der Fahrzeug-

Tab. 13: Intersektoraler Strukturwandel: Wertschöpfungsanteile der österreichischen Industrie nach Technologieintensität in \% der industriellen Wertschöpfung insgesamt

\begin{tabular}{l|cc|c|c|c|c|c|} 
& $\mathbf{1 9 8 0}$ & $\mathbf{1 9 8 5}$ & $\mathbf{1 9 9 0}$ & $\mathbf{1 9 9 5}$ & $\mathbf{2 0 0 0}$ & $\mathbf{2 0 0 7}$ & $\begin{array}{c}\text { Veränderung 1980/2007 } \\
\text { (in Prozentpunkten) }\end{array}$ \\
\hline Hochtechnologie Industrie & 7,3 & 8,6 & 10,1 & 10,2 & 10,9 & 9,6 & 2,3 \\
\hline Mittelhochtechnologie Industrie & 21,9 & 22,8 & 24,6 & 24,3 & 26,7 & 32,9 & 11,0 \\
\hline Mittelniedrigtechnologie Industrie & 29,5 & 30,6 & 27,8 & 27,9 & 28,1 & 28,2 & $-1,3$ \\
\hline Niedrigtechnologie Industrie & 41,2 & 38,0 & 37,4 & 37,6 & 34,2 & 29,2 & $-12,0$
\end{tabular}

Quelle: STAN Datenbank OECD. Berechnungen JOANNEUM RESEARCH.

83 Siehe Wolfmayr et al. (2007).

84 Siehe Tichy (2010).

85 Siehe Ragacs et al. (2011)

86 Siehe Wolfmayr et al. (2007).

87 Siehe Marin (2008), (2010).

88 Siehe Peneder (2008), Dachs (2009), Janger (2012). 
bau (siehe Anhang I). Damit erhöhte sich vor allem der Anteil der Industrien mittleren Technologieniveaus, auf die nunmehr ca. $61 \%$ der industriellen Wertschöpfung entfallen; 1980 betrug dieser Anteil noch etwa $51 \%$.

Angesichts von Niedriglohnkonkurrenz aus Osteuropa und Asien stellt sich die Frage, warum Österreich trotz dieser offenbar niedrigen Technologie- und Qualifikationsintensität einen relativ großen und wettbewerbsfähigen Industriesektor aufweist. Einige Hinweise hierzu enthält Tab. 14. Diese zeigt den Anteil der Industrien im mittleren Technologiesegment und anschließend die F\&E-Intensität dieser Branchen im internationalen Vergleich. Österreich weist demnach nach Deutschland den zweithöchsten Anteil im Segment der Mittelhochtechnologie auf. Der Anteil Deutschlands ist mit $45 \%$ noch wesentlich höher und verweist auf die außergewöhnlich starke Spezialisierung der deutschen Industrie auf $\mathrm{Ma}$ schinen- und Fahrzeugbau. Der Anteil Österreichs an mittelniedrigtechnologischen Industrien ist mit $28 \%$ demgegenüber wesentlich höher als in Deutschland und gleichauf mit Ländern wie Italien oder Spanien.

Die Besonderheit der österreichischen Industrien im mittleren Technologiesegment stellt der untere Teil von Tab. 14 dar. Hier zeigt sich, dass die österreichische mitteltechnologische Indus- trie überdurchschnittlich F\&E-intensiv ist. Während der österreichische Industriesektor insgesamt eine wesentlich geringere F\&E-Intensität als Deutschland, Finnland oder die USA aufweist, ist der besonders dynamische österreichische Mittelhochtechnologiesektor F\&E-intensiver als dessen Pendant in den genannten Staaten. Einzig die schwedische und italienische Industrie ist hier F\&E-intensiver als die österreichische Industrie. Ein ähnlicher Befund ergibt sich für die Mittelniedrigtechnologie. Mit 2,8 \% F\&E-Intensität ist dieser österreichische Industriesektor überdurchschnittlich F\&E-intensiv. Erneut zeigt Italien hier eine höhere F\&E-Intensität. Im Vergleich zu Italien wird aber deutlich, dass F\&E nur einen Teil der Industrieperformance ausmacht. Während die österreichische Industrie im Zeitraum von 1995-2007 hohe Produktivitätsgewinne erzielte, kam es zu einem Rückgang der Produktivität in der italienischen Industrie. ${ }^{89}$ Ein Vergleich der F\&E-Intensitäten mit Spanien oder Slowenien macht deutlich, dass die in der OECDKlassifikation zusammengefassten Branchen in Wirklichkeit sehr große Unterschiede zwischen den Staaten aufweisen: Der österreichische, deutsche oder Schweizer Maschinenbauer dürfte sich signifikant von einem spanischen oder slowenischen Maschinenbauer unterscheiden.

Tab. 14: Internationaler Performancevergleich von Industrien im mittleren Technologiesegment, 2007

\begin{tabular}{|c|c|c|c|c|c|c|c|c|c|}
\hline & \multicolumn{9}{|c|}{ Anteile an der industriellen Wertschöpfung } \\
\hline & Österreich & Deutschland & Finnland & Schweden & USA & Großbritannien & Italien & Spanien & Slowenien \\
\hline Mittelhochtechnologie Industrie & 32,9 & 45,1 & 23,0 & 31,8 & 22,9 & 24,2 & 27,0 & 26,6 & 26,9 \\
\hline \multirow[t]{3}{*}{ Mittelniedrigtechnologie Industrie } & 28,2 & 22,8 & 24,0 & 23,3 & 25,3 & 21,9 & 30,9 & 32,9 & 29,2 \\
\hline & \multicolumn{9}{|c|}{ F\&E-Intensität } \\
\hline & Österreich & Deutschland & Finnland & Schweden & USA & Großbritannien & Italien & Spanien & Slowenien \\
\hline Mittelhochtechnologie Industrie & 11,2 & 9,8 & 7,4 & 15,0 & 9,8 & 7,2 & 11,8 & 4,1 & 4,1 \\
\hline Mittelniedrigtechnologie Industrie & 2,9 & 1,9 & 3,1 & 2,7 & k.A. & 2,1 & 3,7 & 1,3 & 1,5 \\
\hline
\end{tabular}

Quelle: STAN Datenbank OECD. Berechnungen JOANNEUM RESEARCH.

89 Siehe Reiner (2012). 
Zusammenfassend ergibt sich für Österreich das Bild einer reifen, aber dynamischen Industrienation, die in Nischen im mittleren Technologiesegment offenbar sehr erfolgreich auf den internationalen Märkten reüssieren kann. Die Industrien mittleren Technologieniveaus verlangen möglicherweise keine außergewöhnlich hohen F\&Eund AkademikerInnenquoten, um international wettbewerbsfähig zu sein. ${ }^{90}$ Der kumulative Aufbau von implizitem Erfahrungswissen in der Produktion, die Verfügbarkeit von gut ausgebildeten FacharbeiterInnen und ein flexibles und intelligentes Suchen nach Marktnischen dürften eine ebenso wichtige Rolle spielen. ${ }^{91}$ Darüber hinaus zeigt sich aber auch, dass gerade die mitteltechnologischen Industriesegmente Österreichs F\&Eintensiver sind als in wichtigen Vergleichsstaaten. Dieser Effekt bestätigt sich auch in dem Befund, dass Österreich eine höhere F\&E-Quote aufweist als dies aufgrund der Wirtschaftsstruktur zu erwarten wäre. ${ }^{92}$ Möglicherweise sind diese mitteltechnologischen Branchen mit einem hohen Anteil an kumulativem Erfahrungsaufbau und implizitem Wissen imitationsresistenter als manche Hochtechnologieindustrie. ${ }^{93}$ Dies könnte sich im zunehmenden Standortwettbewerb um industrielle Kapazitäten als Vorteil erweisen. Voraussetzung hierfür ist jedoch die beständige Suche nach weiteren Verbesserungen und ein flexibles Reagieren auf sich verändernde Weltmarktstrukturen. Dies schließt freilich auch eine weitere Steigerung der F\&E-Intensität, als eine neben anderen Strategien zur weiteren Verbesserung der Wettbewerbsfähigkeit, mit ein.

\subsection{Industrie, Wachstum und Innovation}

Eine Darstellung der Rolle der Industrie im Innovationssystem ist primär aus wachstumspolitischen Gründen von Interesse. Innovation und
F\&E sind aus ökonomischer Perspektive Mittel zum Zweck der Verbesserung der Wachstumsperformance und der Wohlfahrtszunahme. Daher ist zunächst nach dem generellen Wachstumsbeitrag der Industrie $\mathrm{zu}$ fragen. Von herausragender Bedeutung in diesem Zusammenhang ist die Entwicklung der Produktivität und der unterschiedlichen Beiträge der Sektoren hierzu. Ökonomienobelpreisträger Paul Krugman (1994, 13) hat dies folgendermaßen auf den Punkt gebracht: "Productivity isn't everything, but in the long run it is almost everything." Dahinter steht die wachstumstheoretische Erkenntnis, dass eine Steigerung des Wohlstands letztlich nur durch Produktivitätswachstum erreicht werden kann.

Die Produktivität und damit das Wachstum und der Wohlstand einer Ökonomie werden - unter anderem - wesentlich von der Struktur der Wirtschaft bzw. des Unternehmenssektors bestimmt. Die verschiedenen Branchen oder Sektoren weisen unterschiedliche Potenziale hinsichtlich ihres Beitrags zur Steigerung der ökonomischen Performance einer Volkswirtschaft auf. Dabei sind vor allem jene Segmente der Wirtschaft als wachstumsförderlich anzusehen, die zum Aufbau von Wissen beitragen und damit positive Externalitäten durch die Diffusion dieses Wissen generieren, dynamische Skaleneffekte aufweisen und Potenzial für Produktdifferenzierung bieten. ${ }^{94}$ Diese Eigenschaften dürften - bei aggregierter Betrachtung - in besonderem Ausmaß für den Industriesektor zutreffen.

Aus theoretischer Sicht gibt es zwei Ansätze, welche die Rolle der Industrie im Wachstumsprozess analysieren. Der erste Ansatz stammt von Nicholas Kaldor $(1967,1972)$. Die Industrie weist aufgrund von hohen Fixkosten, Lern- und Spezialisierungseffekten in der Produktion sinkende Durchschnittskosten auf. Beides zusammen trägt zu einer überproportionalen Produkti-

90 Siehe Tidd et al. (1997), Janger (2012).

91 Siehe Malerba (2005), von Tunzelmann, Acha (2005).

92 Siehe Reinstaller, Unterlass (2012).

93 Siehe Tichy (2010).

94 Siehe Janger (2012). 
vitätssteigerung im Industriesektor bei und führt dazu, dass die Industrie gleichsam die Wachstumsmaschine einer Volkswirtschaft bildet. Dieser Zusammenhang, der auch als Kaldor's Wachstumsgesetz bezeichnet wird, ist in empirischen Studien bestätigt worden. ${ }^{95}$

Die zweite Theorie wurde von William Baumol (1967) in seinem Beitrag über die „Kostenkrankheit der Dienstleistungen" entwickelt. Die geringeren Produktivitätssteigerungen der Dienstleistungsunternehmen bewirken unter bestimmten Umständen eine beständige Zunahme des Dienstleistungssektors an der Wertschöpfung und Beschäftigung und damit letztlich eine „Wachstumskrankheit": Das gesamtwirtschaftliche Produktivitätswachstum stagniert und Wohlstandszuwächse bleiben aus. Auch diese Entwicklung wird in empirischen Untersuchungen nachgewiesen. ${ }^{96}$ Es muss aber auch festgestellt werden, dass einige Studien zum Schluss kommen, dass keine Wachstumsverlangsamung mit der Tertiärisierung der Wirtschaft einhergeht. ${ }^{97}$

\subsubsection{Industrie und Produktivitätswachstum}

Die Industrie weist ein höheres Produktivitätswachstum als der Dienstleistungssektor auf. Dies ist ein äußerst robustes Ergebnis von zahlreichen empirischen Untersuchungen..$^{98}$ Allerdings gibt es auch Dienstleistungsbranchen, die ein überdurchschnittliches Produktivitätswachstum aufweisen, und die unterschiedlichen Bereiche der Industrie entwickeln sich ebenfalls keineswegs einheitlich. ${ }^{99}$ In Summe gilt jedoch, dass der Strukturwandel in Richtung Dienstleistungsindustrie mit einem Abstieg auf der Produktivitätsleiter verbunden ist (structural change burden), während umgekehrt der Wandel von einer Agrarökonomie in eine Industriegesellschaft mit einem Aufstieg auf der Produktivtätsleiter einhergeht (structural change bonus).

Anschaulich berichtet Rodrik (2011) die negativen Wachstumseffekte durch die Deindustrialisierung in den USA und Großbritannien. Seit 1990 verzeichnen in den USA persönliche und soziale Dienstleistungen die höchste Beschäftigungszunahme, während die Industrie stark schrumpft. Aufgrund des positiven Produktivitätsdifferenzials zugunsten der Industrie kam es in weiterer Folge zu einem um 0,3 Prozentpunkte niedrigeren jährlichen Produktivitätswachstum der gesamten US-Volkswirtschaft, da das relative Gewicht der Industrie geringer wurde. Das entspricht etwa einem Sechstel der tatsächlich realisierten Produktivitätsgewinne in den USA. In Großbritannien ist der Einfluss noch bedeutsamer. Zwischen 1990 und 2005 hat der Strukturwandel in Richtung weniger produktiver Dienstleistungen einen negativen Effekt von 0,5 Prozentpunkten auf das jährliche Produktivitätswachstum; das entspricht einem Viertel der gesamten Produktivitätsgewinne.

Für Österreich zeigt Abb. 26 die Entwicklung der Arbeitsproduktivität von Industrie und Gesamtwirtschaft für den Zeitraum von 1996 bis 2009. Dabei zeigt sich im Einklang mit der Theorie und den Ergebnissen für andere Staaten ein höheres Produktivitätswachstum der Industrie als für die Gesamtwirtschaft. Im Durchschnitt wuchs die Industrie im betrachtenen Zeitraum mit einer jährlichen durchschnittlichen Rate von ca. 2,9\% beinahe doppelt so rasch wie die Gesamtwirtschaft, deren Produktivität um 1,5\% expandierte. Auffällig ist in Abb. 26 die höhere Volatilität des industriellen Produktivitätswachstums. Im Krisenjahr 2009 zeigte sich - so wie etwa auch in Deutschland - eine drastische Abnahme der Arbeitsproduktivität. Diese Abnahme ist durch die Hortung von Arbeitskräften trotz mangelnder

\footnotetext{
Siehe Necmi (1999), Wienert (2009), McCasland, Theodossiou (2012), Oh et al. (2012).

Siehe Peneder (2003), Hartwig (2012).

7 Siehe Maroto-Sanchez, Cuadrado-Roura (2009).

98 Siehe OECD (2005), Pilat et al. (2006).

99 Siehe Jorgensen, Timmer (2011).
} 
Abb. 26: Jährliches Wachstum der österreichischen Arbeitsproduktivität in Industrie und Gesamtwirtschaft in \%

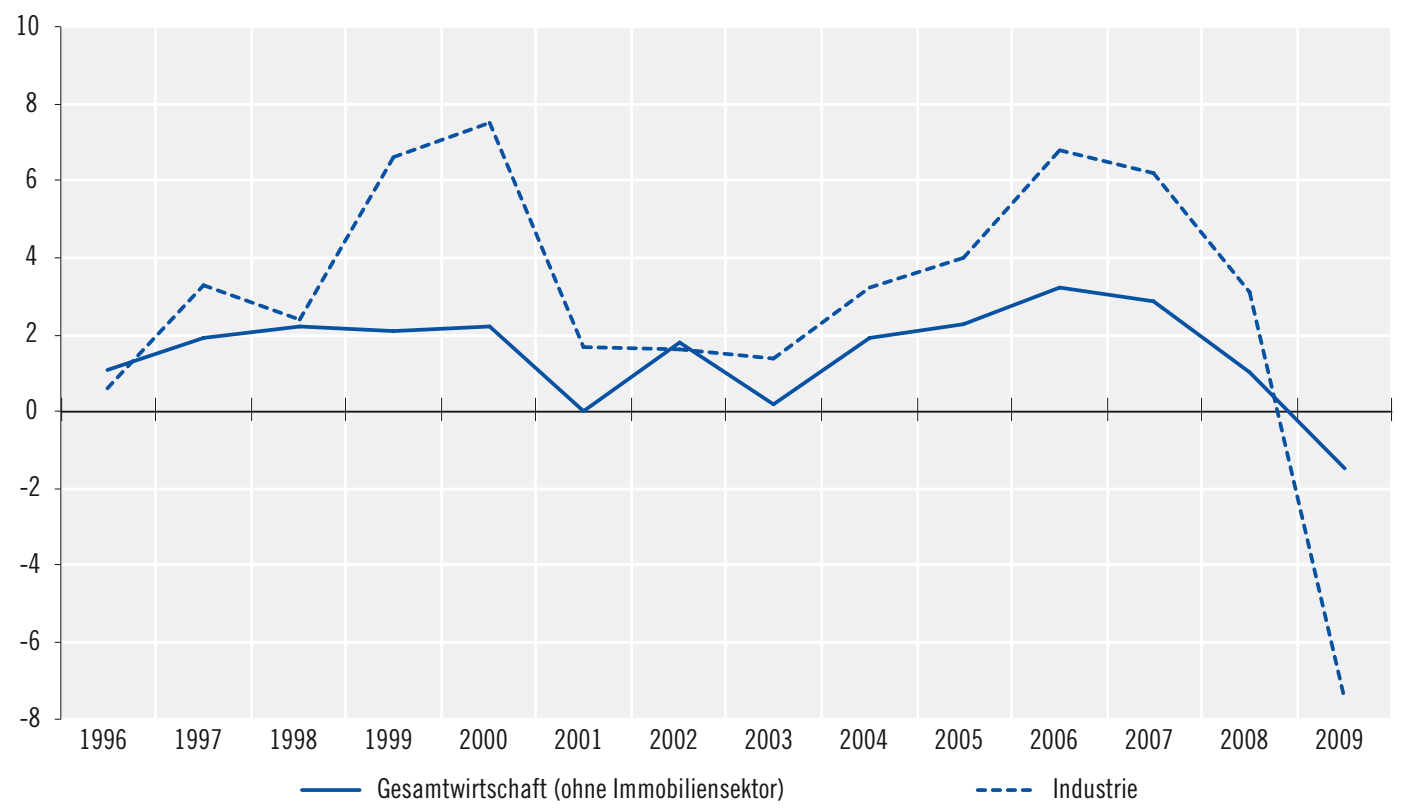

Quelle: OECD Productivity Database. Berechnungen JOANNEUM RESEARCH.

Auslastung begründet. ${ }^{100}$ Damit wurde aber gleichzeitig die Basis für einen raschen Aufschwung im Folgejahr geschaffen, als die internationale Nachfrage nach Industriegütern wieder zunahm.

Durch eine Zerlegung der Beiträge zum gesamtwirtschaftlichen Produktivitätswachstum kann die relative Bedeutung der Sektoren dargestellt werden. Dabei zeigen die Produktivitätsanalysen der OECD, dass die österreichische Industrie in den letzten zwei Jahrzehnten für ca. 40-50 \% des gesamtwirtschaftlichen Produktivitätswachstums verantwortlich war. Während dieser Wert für Länder wie Schweden oder Finnland noch höher wie für Österreich ist, sind Griechenland oder Großbritannien eher durch ein dienstleistungsgetriebenes Produktivitätswachstum gekennzeichnet. ${ }^{101}$ Der Beitrag der österreichischen Industrie zum Wachstum des Sozial- produkts liegt demgegenüber für den Durchschnitt der Jahre 1995 bis 2010 bei rund $30 \% .{ }^{102}$ Die Arbeitsproduktivität wird wesentlich von der Höhe der Faktoreinsätze Arbeit und Kapital bestimmt. Um die Effizienz der Produktion und deren Veränderung über die Zeit isoliert von diesen Einflüssen zu erfassen, wird die so genannte Multifaktorproduktivität berechnet. ${ }^{103}$ Diese kann als ein Maß verstanden werden, welches angibt, mit welcher Effizienz Inputs in Outputs umgewandelt werden. Beispielsweise können zwei Unternehmen mit gleich viel Kapital und ArbeiterInnen sehr unterschiedliche Produktivität aufweisen. Die Ursache hierfür liegt in einem unterschiedlichen Niveau der Multifaktorproduktivität begründet. Diese fasst so unterschiedliche Aspekte wie Technologie, Innovation, Anreizsysteme oder Organisationseffekte zusam-

100 Siehe Rattner (2011).

101 Siehe Pilat et al. (2006), OECD (2008).

102 Siehe Ragacs et al. (2011).

103 Siehe Syverson (2011). 
men. Damit stellt die Multifaktorproduktivität in einem sehr umfassenden Sinne technologische und nicht-technologische Innovationen in einer Maßzahl dar.

Wachstumszerlegungen für Österreich zeigen, dass die Steigerung der Multifaktorproduktivität den mit Abstand bedeutsamsten Beitrag zum Wachstum des Sozialprodukts leistet. Kurz gesagt: Österreich wird nicht so sehr reicher, weil mehr Kapital eingesetzt wird, sondern weil die Ressourcen immer intelligenter und effizienter eingesetzt werden. ${ }^{104}$ Das gilt im Übrigen für so gut wie alle OECD-Länder. ${ }^{105}$ Eine sektorale Differenzierung des Wachstums der Multifaktorproduktivität zeigt Abb. 27. ${ }^{106}$ Dargestellt sind der Industriesektor und ausgewählte große Dienst- leistungsbranchen. Zunächst zeigt sich, dass die Multifaktorproduktivität der Gesamtwirtschaft zwischen 1980 und 2007 um etwas mehr als $25 \%$ zunahm. Im Gegensatz hierzu stieg die Effizienz der Industrie mit $125 \%$ mehr als fünfmal so rasch an. Betrachtet man die Dienstleistungsbranchen, so stieg die Effizienz im Groß- und Einzelhandel zwar ebenfalls rascher als in der Gesamtwirtschaft, aber deutlich langsamer als in der Industrie. Die Multifaktorproduktivität in den großen Dienstleistungsbranchen der sozialen und persönlichen Dienste nahm ebenso ab wie in den unternehmensnahen Dienstleistungen und der Finanzbranche. Damit zeigt dieser Indikator noch eindrücklicher die höhere Dynamik der Industrie und deren überproportionalen Beitrag zum ge-

\section{Abb. 27: Entwicklung der Multifaktorproduktivität für Gesamtwirtschaft, Industrie und ausgewählte Dienstleistungsbranchen} $(1980=100)$

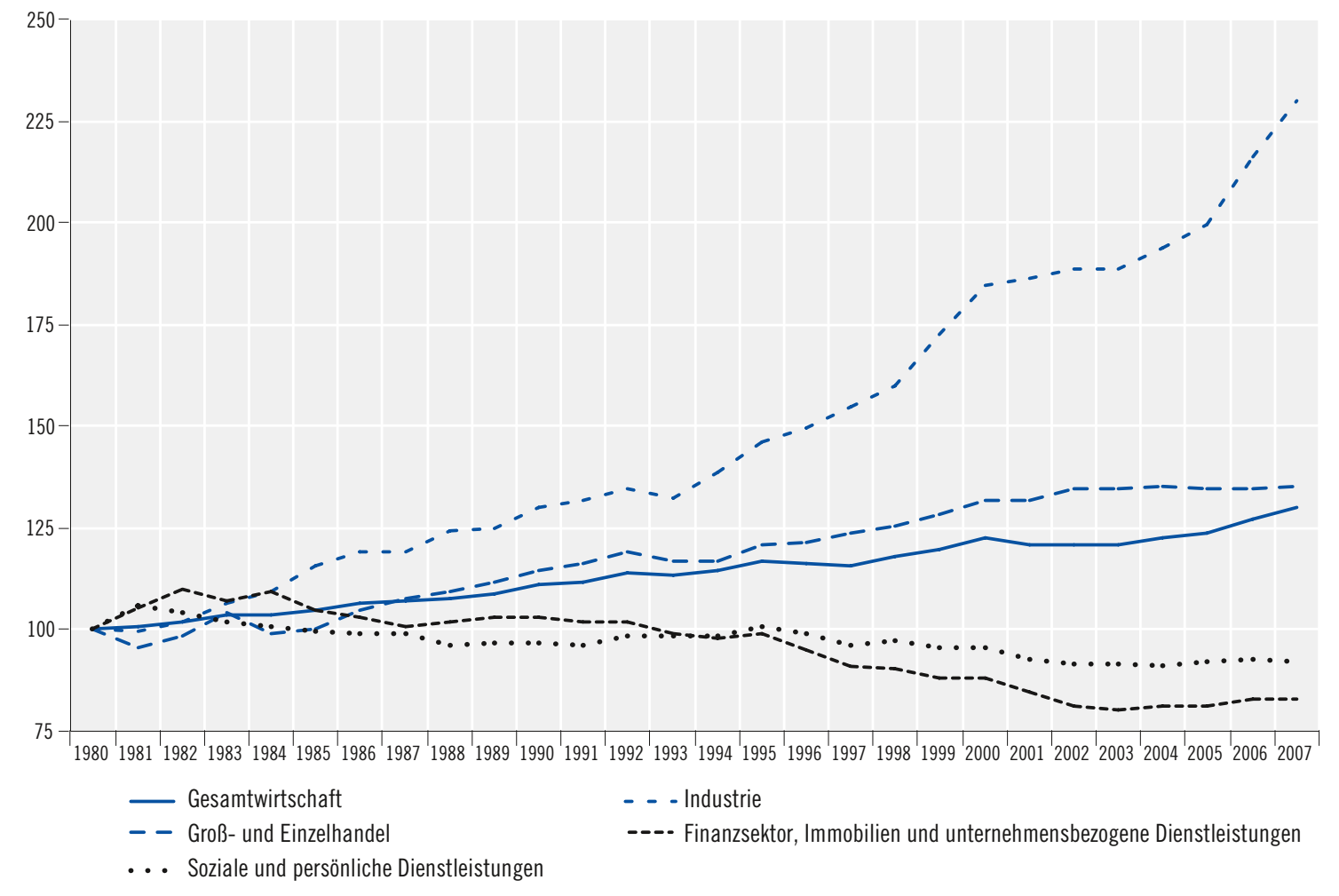

Quelle: EU KLEMS Datenbank. Berechnungen JOANNEUM RESEARCH.

104 Siehe Gnan et al. (2004).

105 Siehe Blanchard, Illing (2010).

106 Siehe O'Mahony, Timmer (2009). 
samtwirtschaftlichen Produktivitätswachstum.

Im Zusammenhang mit den hier vorgetragenen Argumenten zum Produktivitätswachstum wird oftmals auf Mess- und Erfassungsprobleme im Dienstleistungssektor hingewiesen. Demnach könnten die Produktivitätssteigerungen im Dienstleistungssektor höher sein als es die verfügbaren Daten zeigen. Es gibt aber auch einige Hinweise, dass die Produktivitätsentwicklung im Industriesektor unterschätzt wird. Eine Ursache hierfür liegt etwa in der mangelnden Erfassung von Qualitätsverbesserungen und Preisentwicklungen von innovativen Produkten, insbesondere im IKT-Bereich. ${ }^{107}$ Demgegenüber dürfte eine Überschätzung der Produktivitätsentwicklung im Dienstleistungssektor insbesondere für den Finanzsektor zutreffen, einem der dynamischsten Sektoren in den Jahren vor der Wirtschaftskrise. ${ }^{108}$ Weiterhin ist anzumerken, dass die zunehmende Bedeutung von vor- und nachgelagerten Dienstleistungsaktivitäten im Industriesektor zu den gleichen Erfassungsproblemen wie im Dienstleistungssektor führen. Kurzum, in der wirtschaftswissenschaftlichen Literatur ist die Frage, ob die Messprobleme im Industrieoder Dienstleistungssektor gravierender sind, nicht eindeutig beantwortet. ${ }^{109}$ Durch die Verwendung von Wachstumsgrößen, anstatt des Vergleichs von Niveaus, werden jedenfalls mögliche Verzerrungen reduziert. ${ }^{110}$

\subsubsection{Hohe Industrieintensität bei den Innovation Leaders}

Die Rolle und Bedeutung der Industrie für das Innovationssystem einer Volkswirtschaft kann in einer ersten Annäherung durch eine Analyse des Zusammenhangs zwischen Industrieintensität und Innovationsperformance erörtert werden. Letztere soll dabei zunächst sehr breit anhand von synthetischen Indikatoren zweier Innovationsrankings definiert werden.

Abb. 28 zeigt die Durchschnittswerte der EU27 Staaten nach den unterschiedlichen Innovationsklassen gemäß Innovation Union Scoreboard (2011). Dabei wurden z.B. für die vier Innovation Leaders Schweden, Deutschland, Finnland und Dänemark Durchschnittswerte des Industrieanteils und des BIP pro Kopf berechnet. Betrachtet man zunächst den Industrieanteil an der Wertschöpfung, so zeigt sich, dass die Innovation Leaders mit beinahe $20 \%$ den höchsten Industrieanteil an der Wertschöpfung aufweisen, während die Innovation Followers /ohne Luxemburg) mit 15,5 \% einen deutlich niedrigeren Industriealisierungsgrad zeigen. ${ }^{11}$ Die Moderate Innovators und Modest Innovators zeigen wieder etwas höhere Industrieanteile von etwa $17,5 \%$; diese sind aber ebenfalls niedriger als in der Gruppe der Innovation Leaders. Die Tatsache, dass die Innovation Leaders den höchsten durchschnittlichen Industrieanteil aufweisen, ist umso bemerkenswerter, als eine Betrachtung der unterschiedlichen Entwicklungsniveaus das genau gegensätzliche Ergebnis erwarten ließe. Der typischerweise postulierte negative Zusammenhang zwischen Niveau des Sozialprodukts und dem Industrieanteil gilt hier jedenfalls nicht. Österreich weist nach Abb. 28 einen gleich hohen Industrieanteil wie die Innovation Leaders und ein etwas höheres BIP pro Kopf auf; beide Werte liegen deutlich über dem Durchschnitt der EU-27.

Neben dem Innovation Union Scoreboard der EU ist der deutsche Innovationsindikator ${ }^{112}$ ein ebenfalls vielzitierter synthetischer Indikator

\footnotetext{
107 Siehe Blanchard, Illing (2010).

108 Siehe den Haan (2011).

109 Siehe Schettkat (2006).

110 Siehe Schettkat (2010).

111 Bei Berücksichtigung von Luxemburg ergäben sich ein durchschnittlicher Industrieanteil von ca. 14,8 \% und ein wesentlich höheres BIP pro Kopf von 31.689.

112 Siehe Innovationsindikator (2012).
} 
Abb. 28: Durchschnittlicher Industrieanteil an der Wertschöpfung (2007) und BIP pro Kopf (2012) von unterschiedlich innovativen Ländergruppen gemäß IUS 2011

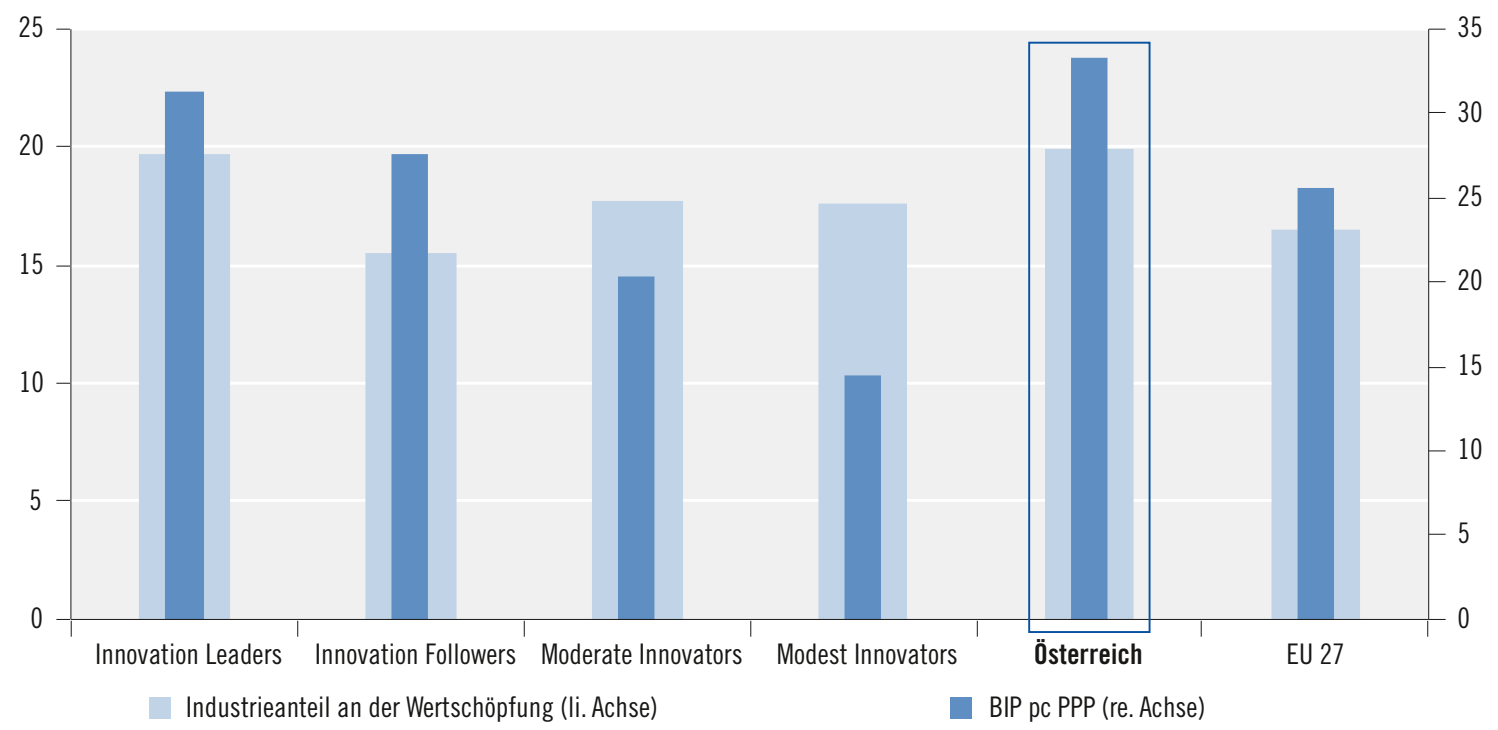

Quelle: AMECO, IUS. Berechnungen JOANNEUM RESEARCH.

zur Einschätzung der relativen Innovationsperformance von Volkswirtschaften. Im aktuellsten Ranking von 2012 werden die ersten fünf Plätze von folgenden Ländern eingenommen: Schweiz, Singapur, Schweden, Deutschland und Finnland. All diese Staaten sind durch einen hohen Industrieanteil gekennzeichnet, wobei sich ein Durchschnittswert von 18,5\% (2007) ergibt. Fasst man alle restlichen, im Ranking berücksichtigten Länder mit Ausnahme der BRICS-Staaten zusammen, so weisen diese einen durchschnittlichen Industrieanteil an der Wertschöpfung von 16,8 \% auf. Erneut ergibt sich also das Bild, dass die innovativsten Volkswirtschaften einen durchschnittlich höheren Industrieanteil aufweisen als die weniger innovativen Ökonomien.

Zusammengenommen unterstützen diese Ergebnisse die Vermutung, dass die Industrie einen positiven Einfluss auf die Innovationsperformance hat. Ebenso beachtet werden muss aber auch die umgekehrte Wirkungsrichtung: Eine starke Innovationsperformance ermöglicht auch in Hochlohnländern den Erhalt eines substanziellen industriellen Kerns trotz Niedriglohnkonkurrenz.

\subsubsection{Industrie und Forschung und Entwicklung (F\&E)}

F\&E-Investitionen und die F\&E-Intensität des Unternehmenssektors gelten als wichtige Bestimmungsgründe des volkswirtschaftlichen Wachstums und der Wettbewerbsfähigkeit einer Volkswirtschaft. ${ }^{113}$ Eine Analyse der F\&E-Ausgaben nach Sektoren ergibt, dass die Industrie der mit Abstand bedeutsamste Investor in F\&E ist. Abb. 29 zeigt dabei für EU- und OECD-Staaten den Anteil der Industrie an den Gesamtausgaben des Unternehmenssektors für F\&E und den Anteil an der Industrie an der Wertschöpfung. Für Österreich liegt der Industrieanteil etwas unter $20 \%$, aber gleichzeitig ist die Industrie für $70 \%$

113 Siehe Aiginger, Falk (2005). 
Abb. 29: Anteil der F\&E-Ausgaben der Industrie an den gesamten F\&E-Ausgaben des Unternehmenssektors (2010) in \% und Industrieanteil an der Wertschöpfung (2010)*

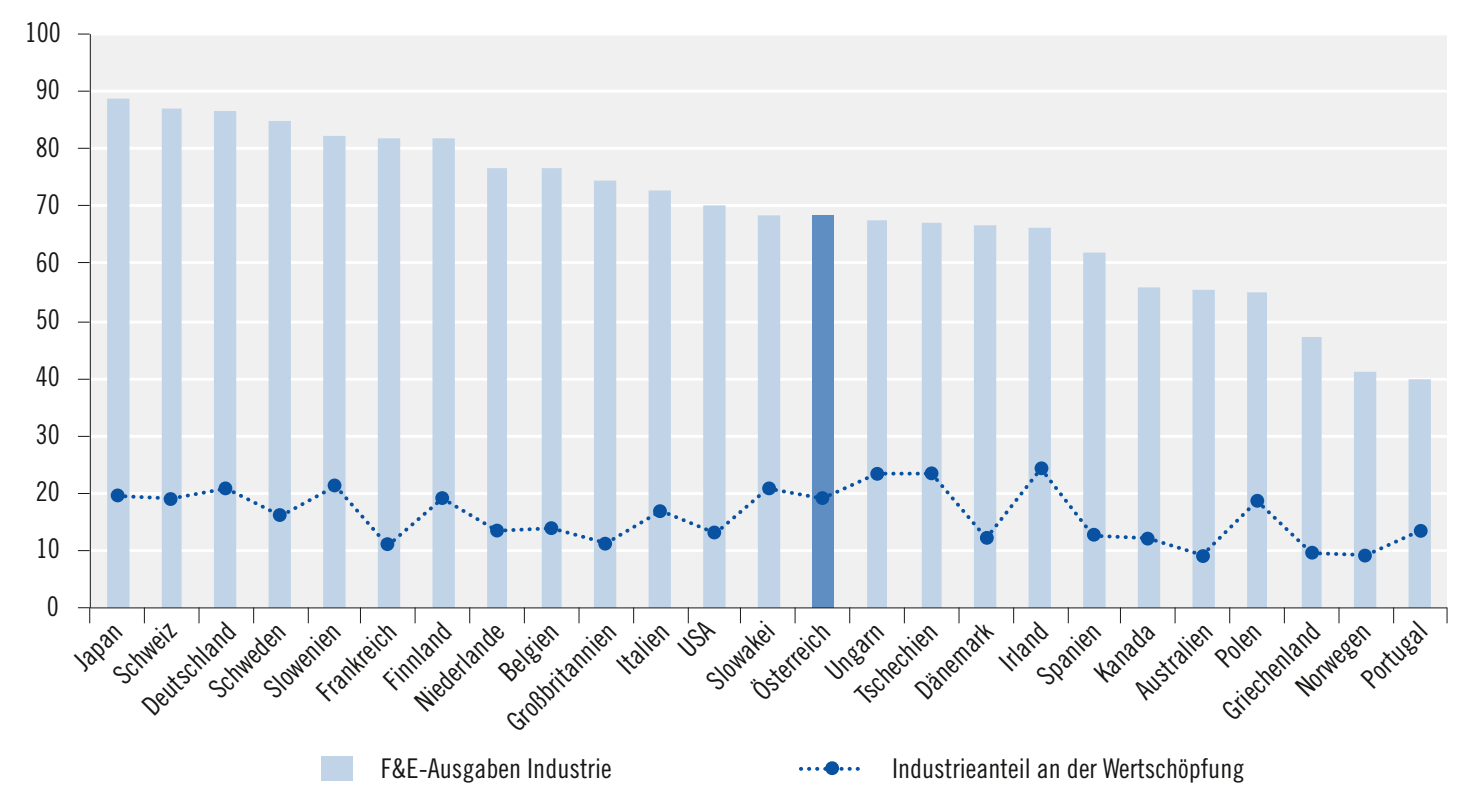

Anm.: 'Die Berechnung der F\&E-Ausgaben der Industrie erfolgte indirekt über den Anteil der F\&E-Dienstleistungsausgaben. Dabei können kleine Unschärfen auftreten, weil etwa auch die Bauwirtschaft F\&E-Ausgaben tätigt, allerdings sind diese gering.

Quelle: OECD STAN-Datenbank, AMECO.

der F\&E-Ausgaben des Unternehmenssektors verantwortlich. Selbst in Großbritannien oder den USA, die als klassische Dienstleistungsökonomien gelten und deren Industrieanteil gering ist, liegt der Industrieanteil an den F\&E-Ausgaben sogar über jenen Österreichs. Die Innovation Leader Deutschland, Schweden oder Finnland weisen Industrieanteile bei den F\&E-Ausgaben von über $80 \%$ auf.

Neben dem Anteil an den Ausgaben ist die F\&E-Intensität von Sektoren von Interesse. Diese ist in Abb. 30 im intersektoralen und internationalen Vergleich dargestellt. Die F\&E-Intensität ist dabei als Anteil der F\&E-Ausgaben an der sektoralen Wertschöpfung berechnet. Neben dem Industriesektor sind der Dienstleistungssektor insgesamt sowie die unternehmensbezogenen Dienstleistungen dargestellt. Zunächst ergibt sich erneut das Bild eines wesentlich F\&Eintensiveren Industriesektors in allen dargestell- ten Ländern. Weiterhin ist der Industriesektor, der ja auch niedrigtechnologische Industrien wie die Bekleidungs- und Textilindustrie umfasst, auch wesentlich F\&E-intensiver als die Gruppe der hochwertigen Dienstleistungen. Für Österreich beträgt die F\&E-Intensität der Industrie $7,4 \%$, jene des Dienstleistungssektors 0,9\% und die der unternehmensbezogenen Dienstleistungen etwa $2,2 \%$. Im internationalen Vergleich hat die österreichische Industrie in Summe damit eine deutlich geringere F\&E-Intensität als die Innovation Leaders. Dieser Befund gilt jedoch nicht für die österreichische Industrie im mittleren Technologiesegment (siehe oben). Dänemark und Deutschland, die noch am ehesten eine vergleichbare Industriestruktur aufweisen, geben mit 9,0\% und 8,1\% F\&E-Intensität relativ mehr für $\mathrm{F} \& \mathrm{E}$ aus. Die Industriesektoren Finnlands und Schwedens sind wiederum deutlich technologieintensiver als die Industrie der USA. 
Abb. 30: F\&E-Intensität (F\&E-Ausgaben in \% der Wertschöpfung) nach Branchen (2009 bzw. aktuellste verfügbare Daten)

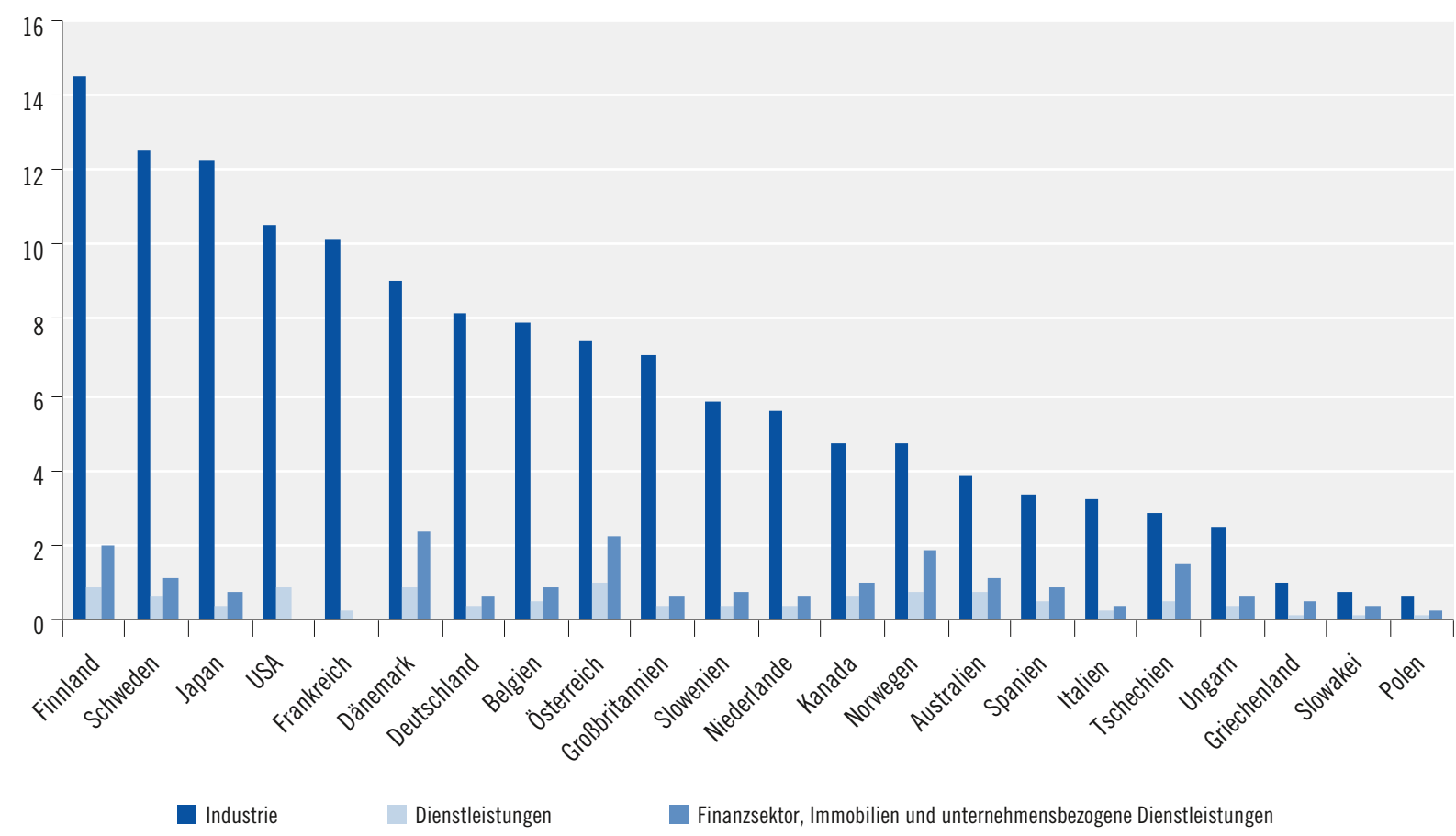

Quelle: OECD STAN-Datenbank. Berechnungen JOANNEUM RESEARCH

Die hohe Bedeutung der Industrie für F\&E und die relativ niedrige F\&E-Intensität des Dienstleistungssektors legt die Vermutung nahe, dass die Tertiärisierung mit einer Abnahme der F\&E-Intensität und F\&E-Quote verbunden sein könnte. Tatsächlich zeigen empirische Analysen, dass der Strukturwandel für zahlreiche Länder einen negativen Effekt auf die F\&E-Quote hat (Struktureffekt) (Dachs 2009). Demgegenüber steht jedoch eine Zunahme der F\&E-Intensität in den Branchen (Intensitätseffekt). Es ist diese Zunahme der F\&E-Aktivitäten innerhalb von bestehenden Branchen, welche die negativen Effekte des Strukturwandels auf die F\&E-Quote überkompensieren. Allerdings ist diese Kompensation kein $\mathrm{Na}$ turgesetz und Großbritannien ist ein Beispiel dafür, wie die Deindustrialisierung auch mit einem Rückgang der F\&E-Ausgaben des Unternehmenssektors verbunden sein kann (Abb. 31).

\subsubsection{Innovationen und Innovationskooperationen im Industrie- und Dienstleistungssektor}

Dienstleistungsunternehmen weisen - bei aller Heterogenität - technologisch bedingt unterschiedliche Innovationsmuster als Industrieunternehmen auf. Typischerweise wird dabei angenommen, dass Dienstleistungen primär nichttechnologische Innovationen (Marketinginnovation, organisatorische Innovation) durchführen, während die Industrie vor allem technologische Innovationen (Produktinnovation, Prozessinnovation) aufweist. Wenngleich diese Beschreibung im Sinne einer Charakterisierung der relativen Spezialisierung der beiden Sektoren zutreffend ist, so verdeckt sie doch die Tatsache, dass die Industrie letztlich in allen Innovationskategorien eine höhere Innovationsintensität aufweist. ${ }^{114}$ Dieses Ergebnis ist insofern interessant, als es

114 Siehe Miles (2005). 
Abb. 31: Beispiel Großbritannien: Deindustrialisierung und Rückgang der unternehmerischen F\&E-Ausgaben am BIP

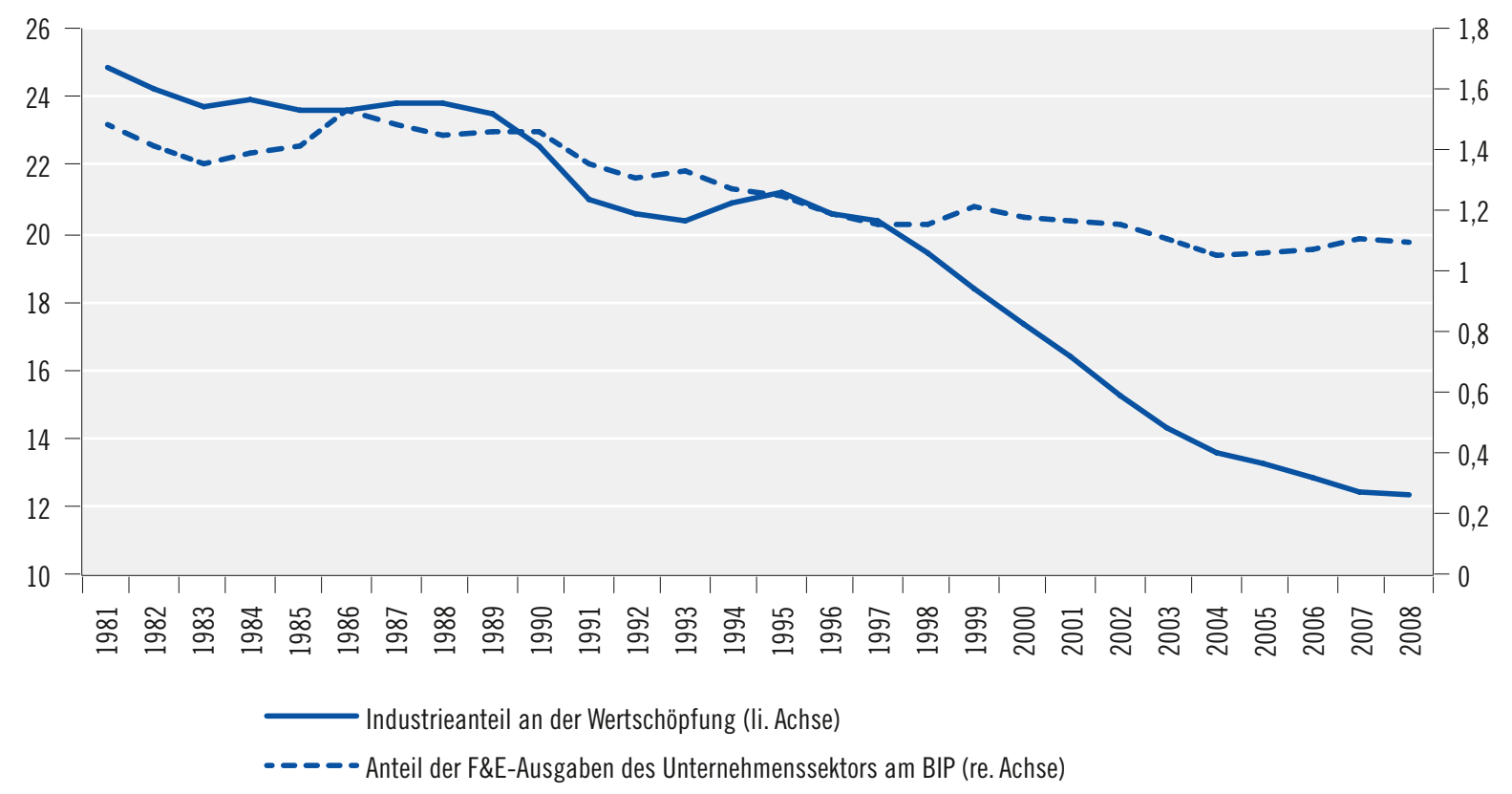

Quelle: OECD, AMECO. Berechnungen JOANNEUM RESEARCH.

die Ergebnisse des Vergleichs der F\&E-Intensitäten bestätigt und nicht konterkariert.

Tab. 15 zeigt die Ergebnisse der aktuellen österreichischen Innovationserhebung. Im Sachgütersektor innovierten ca. $61 \%$ aller Unternehmen in den Jahren von 2008 bis 2010, während der entsprechende Wert für den Dienstleistungssektor bei 53 \% liegt. Der Anteil der innovativen Unternehmen im wissensintensiven Bereich liegt mit $64 \%$ etwas höher als im Segment der mittelniedrigtechnologischen Industrie $(61 \%)$, aber deutlich unter den Anteilen der Hochtechnologie oder Mittelhochtechnologie.

Bei den technologischen Innovationen ergibt sich das zu erwartende Bild eines deutlich höheren Anteils von technologisch innovativen Industrieunternehmen im Vergleich zum Dienstleistungssektor. Allerdings gaben auch mehr als die Hälfte der Unternehmen des wissensintensi- ven Dienstleistungssektors an, dass sie im technologischen Sinne in den Jahren 2008 bis 2010 innovativ waren.

Die letzte Spalte von Tab. 15 zeigt den Anteil der Unternehmen mit nicht-technologischen Innovationen in den jeweiligen Sektoren. Bei einem aggregierten Vergleich ergibt sich für die Industrie ein etwas höherer Anteil als für den Dienstleistungssektor. Allerdings ist anzumerken, dass bei der letzten Innovationserhebung der Dienstleistungssektor einen höheren Anteil an nicht-technologisch innovativen Unternehmen aufwies. ${ }^{115}$ Eindeutig ist jedoch der wesentlich höhere Anteil von nicht-technologischen Innovationen in der Hochtechnologieindustrie. Die aktuellen Daten weisen auch das Segment der Mittelhochtechnologie als innovativer im nicht-technologischen Sinne als die Dienstleistungen aus. Diese Ergebnisse dürften primär eine Folge der Komplemen-

115 Siehe CIS (2008), Statistik Austria (2010). 
Tab. 15: Innovation im Industrie- und Dienstleistungssektor

\begin{tabular}{|c|c|c|c|}
\hline & $\begin{array}{l}\text { Innovationsaktive } \\
\text { Unternehmen } \\
\text { in \% aller Unternehmen }\end{array}$ & $\begin{array}{l}\text { Innovationsaktive Unternehmen mit } \\
\text { technologischen Innovationen } \\
\text { in \% aller Unternehmen }\end{array}$ & $\begin{array}{l}\text { Innovationsaktive Unternehmen mit } \\
\text { nicht-technologischen Innovationen } \\
\text { in \% aller Unternehmen }\end{array}$ \\
\hline Herstellung von Waren & 60.6 & 50.4 & 45.1 \\
\hline Hochtechnologie & 95.9 & 89.3 & 79.9 \\
\hline Mittelhochtechnologie & 80.4 & 73.8 & 57.8 \\
\hline Mittelniedrigtechnologie & 61.1 & 50.4 & 43.7 \\
\hline Niedrigtechnologie & 51.1 & 39.6 & 39.1 \\
\hline Dienstleistungen & 53.4 & 38.9 & 43.6 \\
\hline Wissensintensive Dienstleistungen & 63.9 & 51.6 & 51.6 \\
\hline Weniger wissensintensive Dienstleistungen & 48.2 & 32.5 & 39.7 \\
\hline
\end{tabular}

Quelle: CIS 2010. Berechnungen JOANNEUM RESEARCH.

tarität von technologischen und nicht-technologischen Innovationen sein. Die Einführung neuer Produkte mag eine Marketinginnovation zu ihrer Vermarktung nach sich ziehen und ein neuer Prozess selten ohne simultane organisatorische Neuerungen eingeführt werden.

Damit bestätigt sich grosso modo das Bild eines innovativeren Industriesektors. Dieses Ergebnis wird weiter verstärkt, wenn man bedenkt, dass ein wichtiger Teil der Dienstleistungsinnovationen über den Zukauf von Industriegütern stattfindet. Nach der adaptierten Innovationstaxonomie von Pavitt (Tidd et al. 1997) zählen weite Bereiche der Dienstleistungsökonomie zu den sogenannten zulieferdominierten Branchen, deren Innovationsprozess vorwiegend darin besteht, externe Technologien zu erwerben. Auch der Finanzsektor oder der Handel, die zu den informationsintensiven Sektoren zählen, sind wesentlich auf den Zukauf von Technologien aus anderen Sektoren angewiesen. Man denke hier beispielsweise an die Bankautomaten, die das Geschäftsmodell der Banken in der Kundenbetreuung radikal verändert haben. Allerdings ist gerade die Finanzbranche ein gutes Beispiel für eine Dienstleistungsbranche mit einem hohen Anteil an endogenen Innovationen, wenngleich deren Nutzen im Nachhinein betrachtet zum Teil fragwürdig erscheint. Diese Neigung des Dienstleistungssektors zum Zukauf industrieller Technologie zeigt sich jedenfalls auch in den Innovationsausgaben. Die anteilsmäßigen Ausgaben für den Erwerb von Maschinen und Sachmitteln für Innovation liegen im Dienstleistungssektor stabil und signifikant über jenen der Industrie. Die Industrie investiert demgegenüber mehr in unternehmensinterne F\&E. ${ }^{116}$ Damit trägt der Industriesektor indirekt wesentlich zur Innovation und Produktivitätssteigerung im Dienstleistungssektor bei.

Freilich ist aber auch die umgekehrte Relation zu beachten: Ein leistungsfähiger Dienstleistungssektor ist eine wesentliche Voraussetzung für eine dynamische Industrieentwicklung. Allerdings zeigen Studien, dass die Industrie durch ihre Intermediärnachfrage wesentlich zur Entstehung und Wettbewerbsfähigkeit unternehmensbezogener Dienstleistungen beiträgt. ${ }^{117}$ Dies dürfte einer der Gründe sein, warum es im Entwicklungsprozess von Volkswirtschaften keine einfache Abkürzung von einer Agrarökonomie in eine hochwertige Dienstleistungswirtschaft geben kann. ${ }^{118}$

Die Leistung eines Innovationssystems wird unter anderem vom Kooperationsverhalten der

116 Siehe Statistik Austria (2012), (2010).

117 Siehe Guerri, Maliciai (2005).

118 Siehe Paque (2009). 
Akteure beeinflusst. Üblicherweise wird davon ausgegangen, dass intensivere Kooperationsmuster aufgrund der damit verbundenen Nutzung von Komplementaritäten sowie der Diffusion von Wissen vorteilhafter als fragmentierte Innovationssysteme sind. Damit jedoch die Innovationsfähigkeit dauerhaft erhalten bleibt, muss die Offenheit von regionalen und nationalen Innovationssystemen gewährleistet sein. Tab. 16 stellt das durchschnittliche Innovationskooperationsverhalten von Industrie- und Dienstleistungsunternehmen dar. Während $25 \%$ der Industrieunternehmen Innovationskooperationen aufweisen, beträgt der entsprechende Anteil von Dienstleistungsunternehmen $20 \%$. Dieser Unterschied vergrößert sich bei einer Betrachtung der Innovationskooperationen mit wissenschaftlichen Einrichtungen. Damit sorgen Industrieunternehmen in überproportionalem Maße für die Diffusion von wissenschaftlichem Wissen in den Unternehmenssektor und dessen anschließende Verwertung. Die beiden letzten Spalten in Tab. 16 verweisen auf die intensivere Vernetzung der Industrieunternehmen im Innovationssystem, weil diese in höherem Ausmaß mit einer Vielzahl von unterschiedlichen Kooperationspartnern in Innovationsbeziehungen stehen.

Die in diesem Kapitel dargestellte Innovationsperformance der Industrie eröffnet wichtige Einblicke in die Ursachen der unterschiedlichen
Produktivtätsperformance von Industrie und Dienstleistungen. Die beiden genannten Theorien vom Wachstumsmotor Industrie ${ }^{119}$ und von der Kostenkrankheit der Dienstleistungen ${ }^{120}$ betonen beide das höhere Potenzial für Produktivitätssteigerungen in der Industrie. Darüber hinaus ist aber aus innovationsökonomischer Perspektive zu betonen, dass die Industrie auch eine höhere F\&E-Intensität und Innovationsintensität aufweist. Dies fördert wiederum den sektoralen technischen Fortschritt und damit das Produktivitätswachstum.

Warum investieren aber Industrieunternehmen mehr in F\&E als Dienstleistungsunternehmen, warum sind sie - jedenfalls laut verfügbaren Statistiken - im Durchschnitt innovativer? Darauf können im Wesentlichen zwei Antworten gegeben werden: Erstens dürften F\&E-Investitionen und Innovationsaktivitäten einen höheren Ertrag für Industrieunternehmen als für Dienstleistungsunternehmen erbringen. Dies ist letztlich wieder eine Folge produktionstechnologischer Unterschiede zwischen den Sektoren. Zweitens ist der Wettbewerbsdruck in der Industrie aufgrund der Handelbarkeit von Gütern und geringerer Marktsegmentierung durch Regulierungen wesentlich höher als in vielen Dienstleistungsbranchen. Dies wiederum zwingt die Industrie zur ständigen Suche nach Differenzierungsmöglichkeiten gegenüber der Konkurrenz mit-

Tab. 16: Innovationskooperationen von Industrie und Dienstleistungen im Vergleich

\begin{tabular}{|c|c|c|c|c|}
\hline & \multirow{2}{*}{$\begin{array}{l}\text { Anteil von Unternehmen mit } \\
\text { Innovationskooperation } \\
\text { in \% aller Unternehmen }\end{array}$} & \multicolumn{3}{|c|}{$\begin{array}{c}\text { Anteil von Unternehmen mit ... } \\
\text { an allen Unternehmen mit Innnovationskooperation in \% }\end{array}$} \\
\hline & & $\begin{array}{l}\text {... Kooperation mit wissen- } \\
\text { schaftlicher Einrichtung (z.B, } \\
\text { Universität, Fachhochschule) }\end{array}$ & $\begin{array}{l}\text {... vier Arten von } \\
\text { Kooperationspartnern }\end{array}$ & $\begin{array}{l}\text {... fünf und mehr Arten von } \\
\text { Kooperationspartnern }\end{array}$ \\
\hline Gesamtwirtschaft & 22,4 & 42,6 & 12,1 & 23,1 \\
\hline Herstellung von Waren & 25,5 & 48,7 & 13,3 & 26,5 \\
\hline Dienstleistungen & 19,8 & 35,7 & 10,5 & 19,4 \\
\hline
\end{tabular}

Quelle: CIS 2010. Berechnungen JOANNEUM RESEARCH.

119 Kaldor (1967).

120 Siehe Baumol (1967). 
tels Innovationsaktivitäten und F\&E-Investitionen. ${ }^{121}$ Zusammengenommen sind Technologie und Konkurrenz die beiden zentralen Faktoren zur Erklärung der sektoralen Innovationsunterschiede zwischen Industrie und Dienstleistungen.

\section{5 Österreichs Position bei Schlüsseltechnologien}

Neue Technologien sind eine entscheidende Triebkraft für Innovationen. Sie tragen wesentlich zu Wettbewerbsvorsprüngen und Produktivitätssteigerungen bei. Unter der Vielzahl neuer technologischer Entwicklungen sind jene von besonderem Interesse, die auf viele Anwendungsbereiche ausstrahlen und damit die Innovationsfähigkeit einer Volkswirtschaft in der Breite beeinflussen können. Solche breitenwirksamen neuen Technologien werden häufig als Schlüsseltechnologien bezeichnet, da sie den Weg zu ganz neuen Produkten, Dienstleistungen und Geschäftsmöglichkeiten eröffnen.

Die Bedeutung von Schlüsseltechnologien für die industrielle Entwicklung wurde jüngst von der Europäischen Kommission (2012b) in ihrer Kommunikation "A European Strategy for Key Enabling Technologies - A bridge to growth and jobs" betont. Gerade für die heute anstehenden Herausforderungen etwa im Bereich der Energieversorgung, des Umweltschutzes und der Gesundheit können Schlüsseltechnologien entscheidende Beiträge für Innovationsdurchbrüche leisten. Mit Hilfe neuer Materialien, neuer Verfahren und neuer Komponenten sind wesentliche Fortschritte etwa bei der Steigerung der Energieeffizienz, beim Klimaschutz oder bei der Sicherung eines hohen Gesundheitsstands bei einer alternden Bevölkerung zu erreichen. Die Nutzung von Schlüsseltechnologien gilt gleichzeitig als eine Voraussetzung, um Europa als Industriestandort in einer globalisierten Welt zu erhalten. Denn sie ermöglichen zum einen die Modernisierung der Produktionsbasis und tragen zum anderen zur Entstehung neuer, wettbewerbsfähiger Produkte und Industriesegmente bei.

Die EU-Kommission definiert industrielle Schlüsseltechnologien („Key Enabling Technologies" - KETs) als "knowledge and capital-intensive technologies associated with high research and development intensity, rapid and integrated innovation cycles, high capital expenditure and highly-skilled employment [...] of systemic relevance, multidisciplinary and trans-sectorial, cutting across many technology areas with a trend towards convergence, technology integration and the potential to induce structural change $^{\text {"122 }}$ und hat sechs industrielle Schlüsseltechnologiebereiche identifiziert: ${ }^{123}$

- Werkstofftechnologie („Neue Materialien“)

- Industrielle („,weiße ") Biotechnologie

- Optische Technologien („Photonik“)

- Nanotechnologie

- Mikro- und Nanoelektronik

- Produktionstechnologien ${ }^{124}$

Die EU-Kommission sieht bei Schlüsseltechnologien ein großes Potenzial, um den Industriestandort Europa zu stärken. Denn Europa steht in vielen Schlüsseltechnologiefeldern in der Wissenschaft und bei technischen Erfindungen weltweit an der Spitze. Andererseits sieht die EUKommission auch große Defizite bei der Umsetzung von F\&E-Ergebnissen in neue Produkte. Deshalb schlägt die EU-Kommission die Förderung von Schlüsseltechnologien entlang der In-

121 Siehe Schumpeter (2005), Syverson (2011).

122 Siehe Europäische Kommission (2009a).

123 Eine Definition und Beschreibung der sechs Schlüsseltechnologien findet sich in Europäische Kommission (2009b) und Aschhoff et al. (2010). Vgl. auch den Bericht der High-level Expert Group on Key Enabling Technologies (2011) und des EU-Wettbewerbsfähigkeitsberichts 2010 (Europäische Kommission 2010).

124 In der jüngsten EU-Kommunikation aus dem Jahr 2012 (Europäische Kommission 2012) wurden die Produktionstechnologien auf fortgeschrittene Produktionstechniken, die in einer der fünf anderen Schlüsseltechnologien zum Einsatz kommen, eingeschränkt. In der hier vorliegenden Analyse wird der ursprüngliche, weitere Produktionstechnikbegriff zugrunde gelegt, der alle fortgeschrittenen Produktionsverfahren unabhängig von ihren Einsatzbereichen umfasst. 
novationskette vor, d.h. von der Grundlagenforschung bis zu Pilotanlagen und groß dimensionierten Demonstrationsprojekten. Hierfür sollen Mittel des HORIZON-2020-Programms (alleine über 6,6 Mrd. $€$ für industrielle Anwendungen von Schlüsseltechnologien), der Europäischen Investitionsbank, der EIT KICs und der Strukturfonds genutzt sowie neue Public-Private-Partnerships angestoßen werden.

Für die österreichische FTI-Politik stellt sich die Frage, welche Rolle Österreich derzeit im Bereich der industriellen Schlüsseltechnologien sowohl bei F\&E als auch in der industriellen Produktion einnimmt und wie sich die nationalen FTI-Maßnahmen (die teilweise in direkter, teilweise in indirekter Verbindung zu Schlüsseltechnologien stehen) gegenüber den (geplanten) EUAktivitäten zur Förderung von KETs positionieren. Mit dem Ziel der Österreichischen Bundesregierung, Österreich als Innovation Leader zu etablieren, erhält auch das Thema der industriellen Schlüsseltechnologien eine neue Bedeutung. Denn um führend bei Innovationen zu sein, muss ein Land nicht nur in der Lage sein, aktuelle technologische Trends aufzugreifen, sondern es muss auch selbst solche Trends setzen können. Es wird daher untersucht, welche Position Österreich derzeit bei der Entwicklung und Vermarktung von industriellen Schlüsseltechnologien einnimmt, und wie sich die Performance Österreichs in den zurückliegenden zehn Jahren verändert hat.

\subsubsection{Bedeutung von Schlüsseltechnologien für industrielle Innovationen}

Industrielle Schlüsseltechnologien sind eine Form von Querschnittstechnologien, ${ }^{125}$ die eine wesentliche Grundlage für technische Innovationen bilden. Als Bausteine für innovative, integrierte Lösungen eröffnen sie vielfältige Möglichkeiten für Produkt- und Prozessinnovationen in einer großen Zahl von Branchen und Anwendungsfeldern. Im Unterschied zu anderen Querschnittstechnologien wie Informations- und Kommunikationstechnologien ${ }^{126}$ beschränken sich die direkten Innovationswirkungen von industriellen Schlüsseltechnologien allerdings im Wesentlichen auf den Produktionssektor; denn neue Werkstoffe, biotechnologische oder nanotechnologische Verfahren sowie optische oder mikroelektronische Komponenten sind primär für Innovationen bei Materialen und Herstellungsverfahren nutzbar. Gleichwohl bieten sich auch in Dienstleistungssektoren indirekte Innovationspotenziale beim Einsatz von auf Schlüsseltechnologien basierenden Produkten, insbesondere im Gesundheitsbereich (Medizintechnik), im Transportgewerbe (energieeffizientere und leisere Fahrzeuge) und in den Informationsund Kommunikationsdienstleistungen (leistungsfähigere Informations- und Kommunikationstechnik).

Entscheidend für die Wirkung von industriellen Schlüsseltechnologien - wie von Querschnittstechnologien generell - auf Innovation und Wettbewerbsfähigkeit ist nicht nur die Entwicklung der grundlegenden Technologien, sondern vor allem ihre Nutzung in komplexen Produkten und Gesamtlösungen. Dabei geht es sowohl um die Geschwindigkeit der Diffusion neuer Technologien als auch um die Erschließung neuer Einsatzbereiche. Eine große Herausforderung ist es, die technologischen Möglichkeiten, die diese neuen Technologien bieten, mit den Anforderungen der Nutzer, des Marktes und von rechtlichen Vorgaben, den Fähigkeiten der weiterverarbeitenden Unternehmen und einer kosteneffizienten Produktion in Einklang zu bringen. Eine direkte Zusammenarbeit zwischen Akteuren auf verschiedenen Stufen von Innovationsprozessen und Wertschöpfungsketten, insbesondere zwischen den Technologieproduzenten und den Anwendern der Basistechnologien, kann

125 Vgl. Helpman (1998).

126 Vgl. Ark, Piatkowski (2004). 
solche Abstimmungsprozesse wesentlich unterstützen. ${ }^{127}$ Vor diesem Hintergrund ist es nicht gleichgültig, wo neue industrielle Schlüsseltechnologien hervorgebracht werden. Eine starke einheimische technologische Basis erleichtert es auch der weiterverarbeitenden Industrie, innovative Lösungen durch die Nutzung industrieller Schlüsseltechnologien zu entwickeln.

Vor diesem Hintergrund zielen viele Aktivitäten zur Förderung von Schlüsseltechnologien und zur Nutzung ihrer Innovationspotenziale nicht nur auf die Forschungsförderung innerhalb der Schlüsseltechnologiebranchen ab, sondern auch auf Kooperationen zwischen diesen Branchen und der Wissenschaft, den Anwenderindustrien und den Endnutzern. Österreich folgt diesem Weg z.B. mit den Kompetenzzentren-Programmen (COMET) und der Förderung von Anwendungsfeldern neuer Technologien im Bereich Smart Cities und Elektromobilität.

Die Erwartungen an die kurzfristigen Innovationswirkungen von industriellen Schlüsseltechnologien sollten allerdings nicht $\mathrm{zu}$ hoch geschraubt werden. Denn bei vielen Innovationsideen steht die Umsetzung erst am Anfang. Und je höher der Innovationsanspruch ist, desto länger sind die Zeiträume, bis sich die Innovationsideen in alltagstaugliche Produkte umsetzen lassen, und desto höher sind die notwendigen Investitionen und die Barrieren, die überwunden werden müssen. Eine breite Diffusion setzt meist erst ein, wenn sich ein technischer Standard herausgebildet hat und der Nutzen gegenüber bestehenden Produkten und Lösungen klar ersichtlich und bewertbar ist. Erst dann wird die Nachfrage ausreichend hoch sein, um über Skaleneffekte die Produktionskosten zu senken und damit niedrigere Produktpreise $z u$ ermöglichen. Insofern haben sich viele Erwartungen an kurzfristig realisierbare hohe Umsatzpotenziale der neuen industriellen Schlüsseltechnologien, etwa im Bereich nanotechnologischer oder biotechnologischer Anwendungen ${ }^{128}$ als zu optimistisch erwiesen. Andere Bereiche der industriellen Schlüsseltechnologien, die in ihrem technologischen Entwicklungspfad bereits weiter fortgeschritten sind, wie etwa die Mikro- und Nanoelektronik, die optischen Technologien oder verschiedene Felder der Werkstofftechnologien zeigen jedoch, wie groß die Ausstrahlungseffekte von Schlüsseltechnologien auf Innovationen in den unterschiedlichsten Industriebranchen in längerfristiger Perspektive sein können.

\subsubsection{Position Österreichs bei Patentanmeldungen im Bereich von Schlüsseltechnologien}

Die Position Österreichs bei der Entwicklung neuer Technologien in den einzelnen Feldern der industriellen Schlüsseltechnologien kann anhand der Patentstatistik nachgezeichnet werden. Hierfür wird auf eine Zuordnung von Patentklassifikationsnummern der Internationalen Patentklassifikation (IPC) zu den sechs Schlüsseltechnologiefeldern zurückgegriffen, die von der EUKommission für ein Monitoringsystem entwickelt wurde ${ }^{129} .130 \mathrm{Um}$ die Dynamik der technologischen Entwicklung in den sechs Feldern zu untersuchen, werden alle Patentanmeldungen von österreichischen Anmeldern bzw. ErfinderInnen im Zeitraum seit 2000 betrachtet und zu Patentfamilien aggregiert (d.h. Patente, die sowohl an österreichischen als auch an ausländischen oder internationalen Ämtern angemeldet wurden, werden nur einmal gezählt). Für die Positionierung Österreichs im internationalen Vergleich werden nur Patentanmeldungen am Europäischen Patentamt (EPO) und über das Verfah-

127 Vgl. Fagerberg (1995), Porter (1990).

128 Vgl. Europäische Kommission(2010).

129 Vgl. van de Velde et al. (2013).

130 Für die Produktionstechnologien wird nicht auf die in dieser Studie vorgeschlagene Abgrenzung zurückgegriffen, da diese nur Produktionstechnologien erfasst, die in direktem Bezug zu einer der fünf anderen Schlüsseltechnologien stehen. Stattdessen wird eine Abgrenzung aus einer früheren Studie der Europäischen Kommission verwendet, die Produktionstechnologien breiter abgrenzt (vgl. Aschhoff et al., 2010). 
ren nach dem Patentkooperationsvertrag (patent cooperation treaty - PCT) der World Intellectual Property Organisation betrachtet, um so ein höheres Maß an Vergleichbarkeit zwischen verschiedenen Ländern zu erreichen. Die Analysen beruhen auf der Patstat-Datenbank des Europäischen Patentamts (Ausgabe November 2012). Aufgrund des Zeitverzugs zwischen Patentanmeldung und Patentveröffentlichung von $18 \mathrm{Mo-}$ naten liegen vollständige Anmeldezahlen nur bis zum Jahr 2010 vor. Für EPO/PCT-Anmeldungen ist der Zeitverzug aufgrund des Abstands zwischen Erstanmeldung und Anmeldung an weiteren Patentämtern noch größer, sodass nur bis zum Jahr 2009 internationale Vergleichszahlen vollständig verfügbar sind.
Im Zeitraum 2000 bis 2010 wurden von Organisationen und Privatpersonen mit Sitz in Österreich $^{131}$ insgesamt 32.838 Patente (im Sinn von Patentfamilien) angemeldet. Von diesen sind 7.101 Patente $(21,6 \%)$ dem Bereich der industriellen Schlüsseltechnologien zuzuordnen. Dieser Anteil stieg von gut $18 \%$ Anfang der 2000er Jahre auf über $24 \%$ am Ende des Jahrzehnts signifikant an. Die Patentdynamik war somit in den Schlüsseltechnologien deutlich höher als im Durchschnitt aller Technologiefelder. Während die Anzahl der Patentanmeldungen insgesamt von 2000 bis 2010 um $38 \%$ zunahm, betrug das Wachstum in den industriellen Schlüsseltechnologien $72 \%$. Die höchste Dynamik innerhalb der Schlüsseltechnologien wiesen die Photonik so-

Abb. 32: Entwicklung der Anzahl der Patentanmeldungen in industriellen Schlüsseltechnologien in Österreich 2000-2010

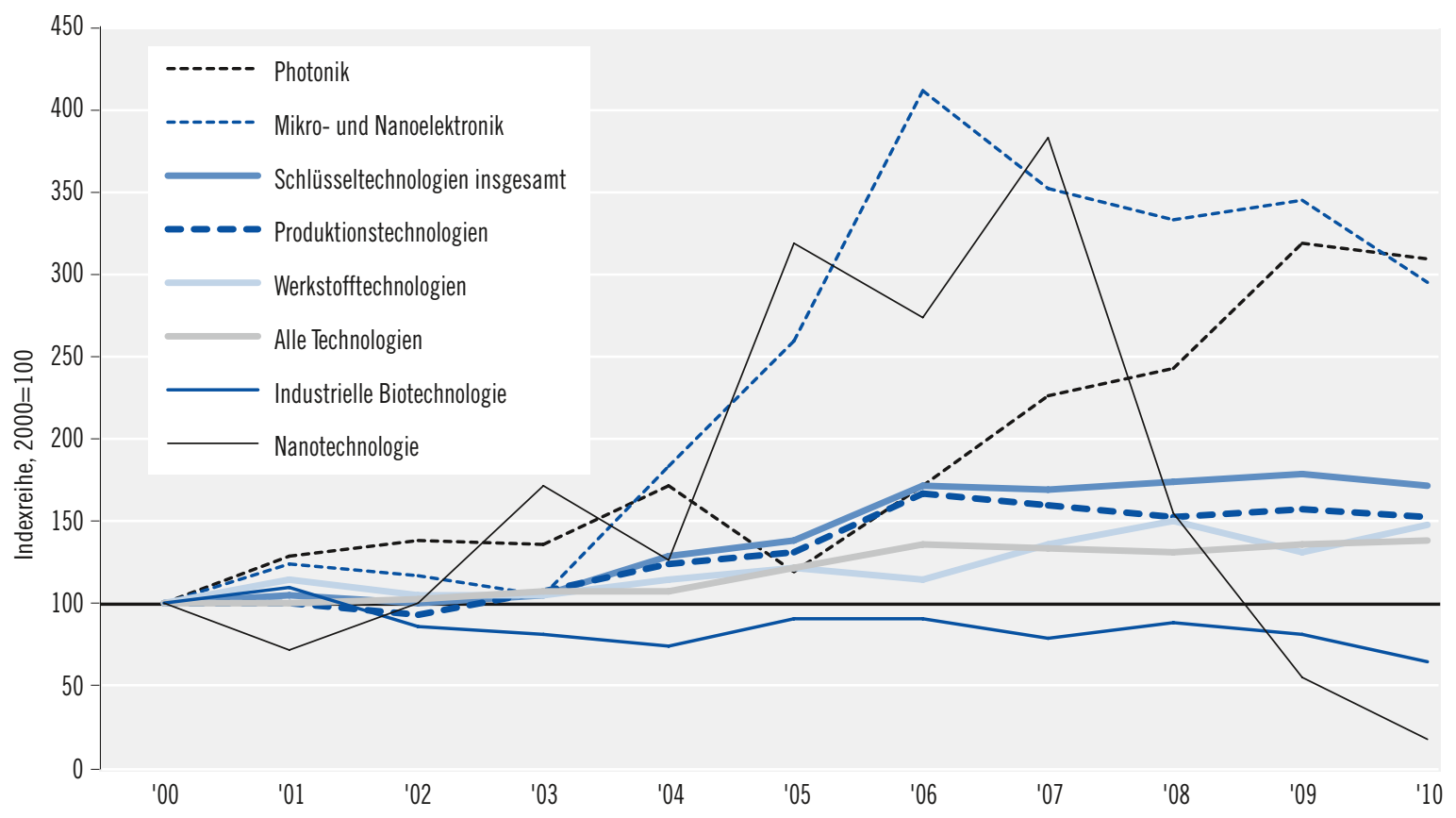

Quelle: Patstat. - Schlüsseltechnologiedefinition aus van de Velde et al. (2013). - Berechnungen des ZEW.

131 Eine Patentanmeldung wird aus Österreich kommend gewertet, wenn zumindest eine der anmeldenden Organisationen bzw. Privatpersonen ihren Sitz in Österreich hat. Diese Vorgangsweise wird einer Zuordnung über den Wohnsitz der ErfinderInnen vorgezogen, da erstens dadurch besser erfasst werden kann, wo die Entscheidungen über die kommerzielle Verwertung der patentierten Erfindungen erfolgt. Zweitens führt eine Zuordnung über den ErfinderInnensitz in grenznahen Regionen mit vielen grenzüberschreitenden ArbeitspendlerInnen zu unscharfen Ergebnissen. Dabei ist zu beachten, dass bei Patentanmeldungen von Unternehmen in Österreich, die Tochtergesellschaften von ausländischen Unternehmen sind, in den meisten Fällen das Tochterunternehmen aus Österreich als Anmelder (mit) aufscheint. Eine Auswertung auf Basis der ErfinderInnensitze erbringt im Übrigen sehr ähnliche Ergebnisse bei insgesamt etwas höheren Patentanmeldezahlen. 
wie die Mikro- und Nanoelektronik auf, in beiden Feldern verdreifachte sich die Anzahl der pro Jahr angemeldeten Patente im Verlauf des vergangenen Jahrzehnts (vgl. Abb. 32). Angesichts von nur wenigen Patentanmeldungen pro Jahr in der Nanotechnologie verlief die Patentdynamik dort sehr sprunghaft. Nach stärkeren Patentaktivitäten von 2005 bis 2007 gab es jüngst nur mehr vereinzelt Patentanmeldungen in diesem Technologiefeld. In den Produktionstechnologien lag das Patentwachstum leicht über dem Mittel aller Technologiefelder, bei den Werkstofftechnologien entsprach es etwa der durchschnittlichen Veränderung der Patenanmeldezahlen in Österreich. Der einzige industrielle Schlüsseltechnologiebereich mit einem unterdurchschnittlichen Wachstum während des vergangenen Jahrzehnts ist die industrielle Biotechnologie.

Patente im Bereich industrieller Schlüsseltechnologien werden überwiegend international angemeldet. Im Zeitraum 2000 bis 2010 wurden über $85 \%$ aller Schlüsseltechnologiepatente (auch) an Patentämtern außerhalb Österreichs angemeldet (Abb. 33). Bei $36 \%$ der Patentanmeldungen wurden Schutzrechte in außereuropäischen Regionen geltend gemacht, bei weiteren $40 \%$ in europäischen Ländern außerhalb Österreichs und Deutschlands. $11 \%$ der Patente wurden auf internationaler Ebene einzig in Deutschland angemeldet. $14 \%$ aller Schlüsseltechnologiepatente haben lediglich für Österreich Schutz angesucht. Im Vergleich zu allen Patentanmeldungen durch österreichische Anmelder sind Schlüsseltechnologiepatente deutlich stärker international orientiert. Dies weist auf eine hohe Exportorientierung der Unternehmen hin, die Erfindungen im Bereich der Schlüsseltechnologien hervorbringen. Über die Zeit haben die Anmeldungen in außereuropäischen Ländern an Bedeutung gewonnen. Dahinter steht u.a. auch die verstärkte Nutzung des PCT-Verfahrens als Anmeldeweg.

\section{Abb. 33: Verteilung der Patentanmeldungen in industriellen Schlüsseltechnologien in Österreich 2000-2010 nach Anmeldeämtern}
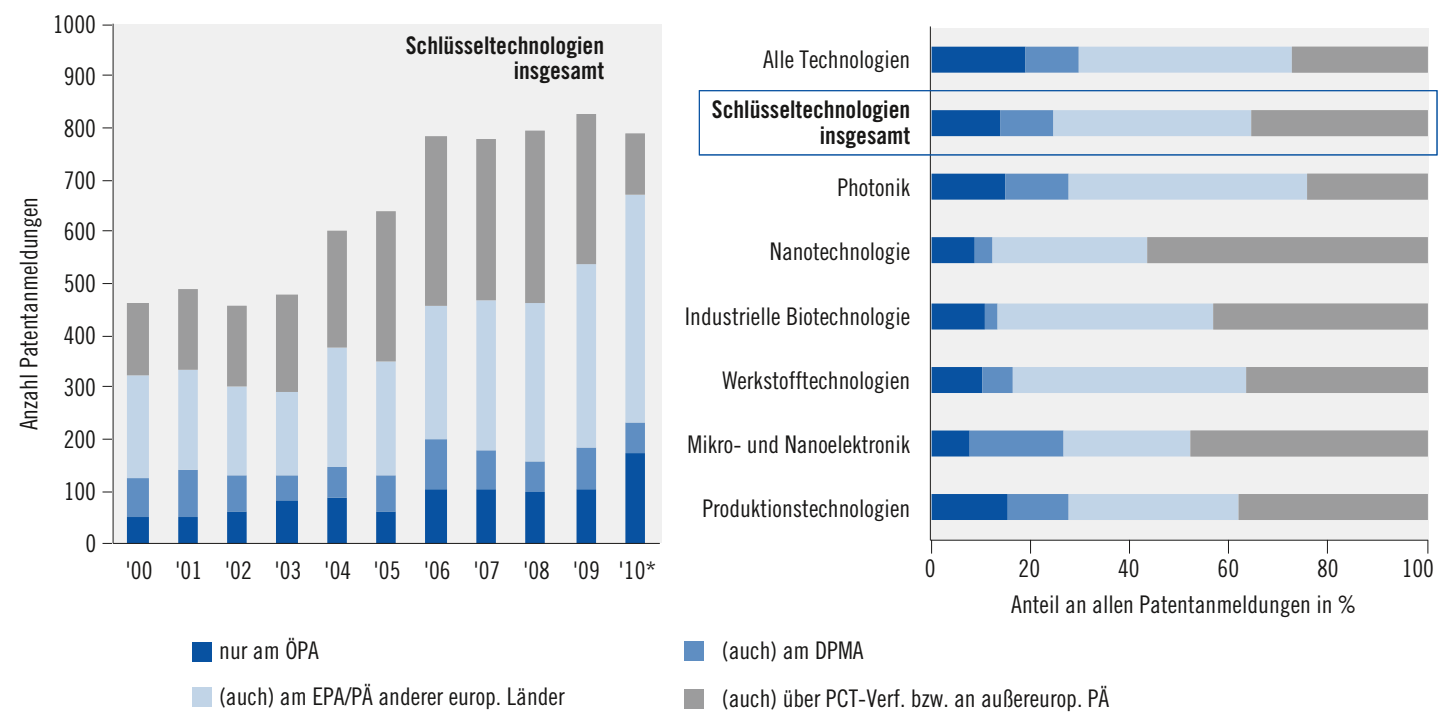

Anm.: * 2010 Untererfassung der Patentanmeldungen über PCT-Verfahren bzw. an außereuropäischen Patentämtern und Überfassung der drei anderen Anmeldewege aufgrund der oft erst späteren Anmeldung von Patenten an außereuropäischen Ämtern.

ÖPA: Österreichisches Patentamt, DPMA: Deutsches Patent- und Markenamt, EPA: Europäisches Patentamt, PÄ: Patentämter, PCT: Patent Cooperation Treaty.

Quelle: Patstat. - Schlüsseltechnologiedefinition aus van de Velde et al. (2013). - Berechnungen des ZEW. 
Abb. 34: Verteilung der Patentanmeldungen in industriellen Schlüsseltechnologien in Österreich 2000-2010 nach Sektorzugehörigkeit der Anmelder

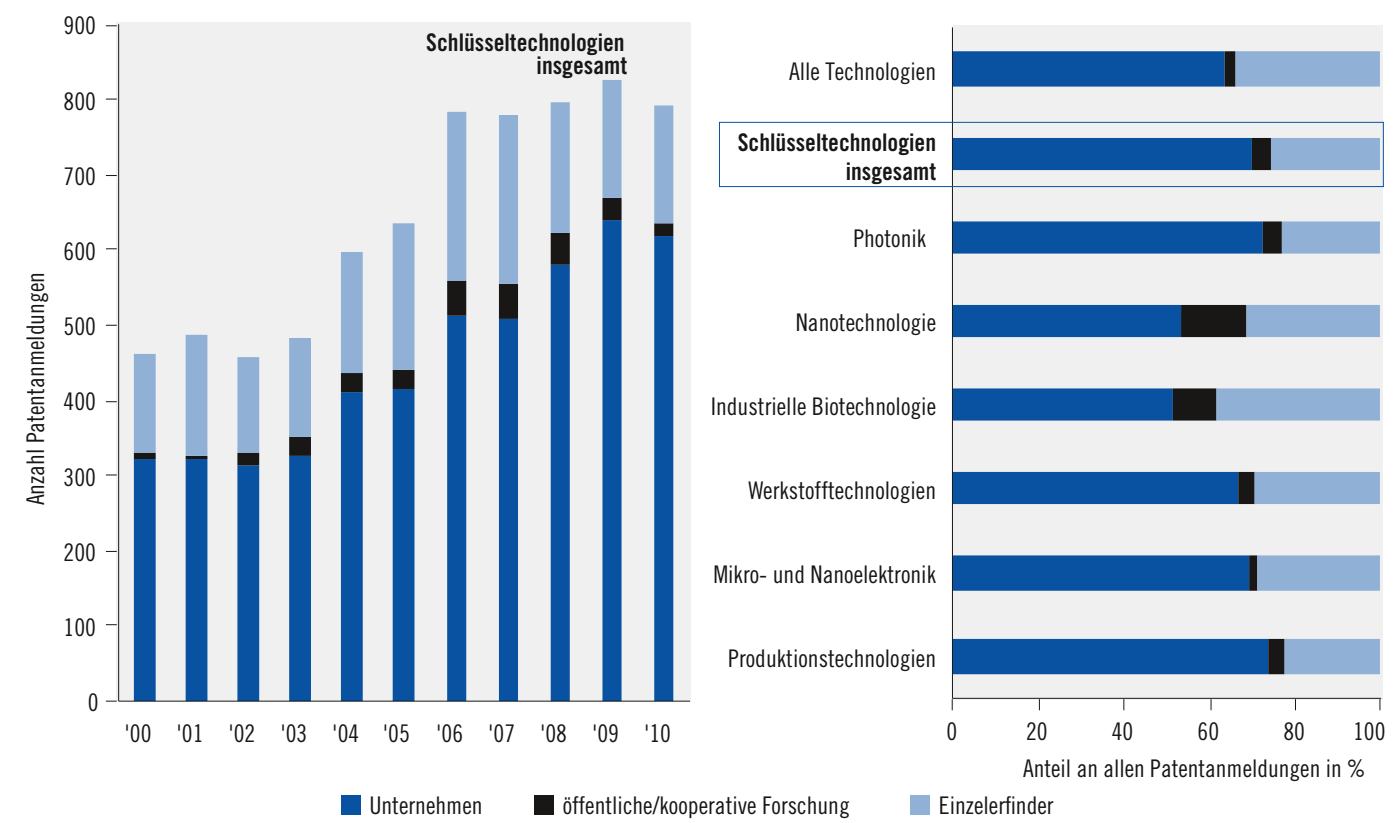

Quelle: Patstat. - Schlüsseltechnologiedefinition aus van de Velde et al. (2013). - Berechnungen des ZEW.

$70 \%$ der industriellen Schlüsseltechnologiepatente wurden von Unternehmen angemeldet, $4 \%$ von Hochschulen, öffentlichen oder kooperativen Forschungseinrichtungen und $26 \%$ von EinzelerfinderInnen. ${ }^{132}$ Die Bedeutung der Unternehmen als Patentanmelder ist in den Schlüsseltechnologien höher als im Mittel aller Technologiefelder (Abb. 34). Besonders hoch ist der Anteil der Unternehmen in der Photonik und in den Produktionstechnologien. Die öffentliche Forschung spielt in der Nanotechnologie und in der industriellen Biotechnologie eine vergleichsweise große Bedeutung. Der Anstieg der Patentanmeldungen im Bereich der Schlüsseltechnologien im vergangenen Jahrzehnt geht im Wesentlichen auf das Konto der Unternehmen zurück, aber auch die öffentliche Forschung hat ihre Pa- tentaktivitäten - von sehr niedrigem Niveau aus - merklich gesteigert.

Eine Zuordnung der Anmelder aus dem Bereich der Unternehmen und der kooperativen Forschung zu Wirtschaftszweigen zeigt, dass der größte Teil der Schlüsseltechnologiepatente aus den Branchen Elektronik/Messtechnik/Optik (auf die 28,6 \% der Patentanmeldungen durch Unternehmen im Zeitraum 2000 bis 2010 entfiel), Maschinenbau (15,0\%), Elektrotechnik $(11,8 \%)$, Chemie/Mineralöl (9,8 \%), Forschung und Entwicklung $(7,9 \%)$ und Metallerzeugung $(4,2 \%)$ stammt (Tab. 17). Die Branche Elektronik/Messtechnik/Optik ist in der Nanotechnologie, der Mikro-/Nanoelektronik und den Produktionstechnologien der größte Patentanmelder. In der industriellen Biotechnologie stammen über

132 Bei Patentanmeldungen, an denen sowohl Unternehmen, Hochschulen, öffentliche oder kooperative Forschungseinrichtungen einerseits und EinzelerfinderInnen andererseits beteiligt waren, wurden EinzelerfinderInnen nicht gezählt, da es sich bei ihnen fast immer um Eigentümer oder Beschäftigte des Unternehmens bzw. der Forschungsorganisation handelt. Der Anteil der Patentanmeldungen durch EinzelerfinderInnen bezieht sich somit auf Patente, die ausschließlich durch EinzelerfinderInnen angemeldet wurden. Bei EinzelerfinderInnen kann es sich um selbstständig tätige Personen, Eigentümer von Unternehmen oder Beschäftigte handeln. 
$45 \%$ der Patentanmeldungen von Unternehmen aus der Pharma- und Chemiebranche, in den Werkstofftechnologien dominieren die Chemieindustrie und die Metallerzeugung, und in der Photonik kommen über drei Viertel der Patente aus der Elektrotechnik und Elektronik/Messtechnik/Optik.

Vergleicht man den Anteil einer Branche an den Patentanmeldungen in allen Technologiefeldern mit dem Anteil an Schlüsseltechnologiepatenten, so erweisen sich Elektronik/Messtechnik/Optik, die Chemie- und Mineralölindustrie, die Metallerzeugung und die Elektrotechnik als besonders schlüsseltechnologieaffin. Ihr Patentportfolio ist überdurchschnittlich stark auf Schlüsseltechnologien ausgerichtet. Die Unternehmen der Branche F\&E, die auch die kooperative Forschung einschließt, patentieren dagegen überproportional häufig außerhalb der Schlüsseltechnologien.

$75,8 \%$ der von Unternehmen 2000 bis 2010 angemeldeten Schlüsseltechnologiepatente stammen von Großunternehmen (mit 250 oder mehr Beschäftigten) und 24,2 \% von KMU. KMU sind im Bereich der Schlüsseltechnologiepatente insofern unterrepräsentiert, als ihr Anteil an allen Patentanmeldungen durch Unternehmen bei 30,4 \%

Tab. 17: Verteilung der Patentanmeldungen aus dem Unternehmenssektor (inkl. kooperative Forschung) in industriellen Schlüsseltechnologien in Österreich 2000-2010 nach Branchen

\begin{tabular}{|c|c|c|c|c|c|c|c|c|c|c|}
\hline Branche & ÖNACE & Alle & ST & PH & NT & IB & WS & ME & PT & F\&E \\
\hline Nahrungsmittel/Getränke & $10-12$ & 0,6 & 0,2 & 0,0 & 0,0 & 2,3 & 0,2 & 0,0 & 0,1 & 0,6 \\
\hline Textil/Bekleidung/Leder & $13-15$ & 1,0 & 0,4 & 0,3 & 0,0 & 2,7 & 0,5 & 0,0 & 0,4 & 0,3 \\
\hline Holz/Möbel & 16,31 & 2,5 & 1,3 & 0,9 & 0,0 & 1,0 & 2,2 & 0,1 & 1,2 & 0,7 \\
\hline Papier/Druck & $17-18$ & 1,3 & 0,7 & 0,6 & 1,1 & 0,5 & 1,7 & 0,0 & 0,4 & 0,7 \\
\hline Chemie/Mineralöl & $19-20$ & 4,3 & 9,8 & 0,0 & 6,9 & 15,0 & 35,2 & 0,2 & 2,7 & 3,7 \\
\hline Pharma & 21 & 8,4 & 3,7 & 1,8 & 7,6 & 35,5 & 5,7 & 0,0 & 1,5 & 3,8 \\
\hline Gummi/Kunststoff & 22 & 3,3 & 1,6 & 0,4 & 0,0 & 0,6 & 4,1 & 0,4 & 1,0 & 2,2 \\
\hline Glas/Keramik/Steinwaren & 23 & 2,2 & 2,2 & 1,7 & 0,0 & 0,0 & 6,3 & 0,4 & 1,1 & 1,4 \\
\hline Metallerzeugung & 24 & 2,0 & 4,2 & 0,4 & 1,1 & 0,0 & 12,8 & 1,6 & 4,9 & 2,6 \\
\hline Metallverarbeitung & 25 & 7,1 & 3,6 & 1,9 & 1,6 & 1,6 & 7,2 & 0,6 & 3,6 & 2,5 \\
\hline Elektronik/ Messtechn./ Optik & 26 & 14,3 & 28,6 & 33,8 & 45,7 & 4,2 & 2,7 & 77,4 & 33,2 & 10,4 \\
\hline Elektrotechnik & 27 & 7,5 & 11,8 & 42,4 & 15,2 & 2,1 & 2,6 & 9,9 & 5,4 & 16,2 \\
\hline Maschinenbau, Reparatur & 28,33 & 19,0 & 15,0 & 4,7 & 3,3 & 3,1 & 9,3 & 3,0 & 22,6 & 11,1 \\
\hline Automobilbau & 29 & 4,8 & 2,4 & 1,2 & 0,0 & 0,0 & 1,0 & 1,6 & 3,3 & 7,2 \\
\hline Bahn-/Flugzeugbau & 30 & 1,6 & 0,5 & 0,5 & 0,0 & 0,0 & 0,3 & 0,2 & 0,7 & 2,1 \\
\hline Medizintechnik/Sportwaren & 32 & 3,2 & 0,9 & 0,4 & 1,1 & 0,0 & 0,7 & 0,2 & 1,2 & 1,8 \\
\hline Bergbau/Ver-/Entsorgung & $5-9,35-39$ & 0,5 & 0,4 & 0,9 & 0,0 & 1,0 & 0,2 & 0,1 & 0,4 & 0,3 \\
\hline Bau/Immobilien & $41-43,68$ & 0,9 & 0,5 & 0,0 & 0,0 & 2,1 & 0,4 & 0,0 & 0,7 & 0,6 \\
\hline Handel/Verkehr/Logistik & $45-53$ & 1,6 & 0,8 & 0,6 & 0,0 & 0,5 & 1,1 & 0,0 & 0,9 & 5,2 \\
\hline Software/Telekommunik. & $61-63$ & 1,5 & 0,9 & 1,5 & 0,0 & 0,4 & 0,2 & 0,3 & 1,0 & 4,1 \\
\hline Architektur-/Ingenieurbüros & 71 & 2,5 & 1,9 & 0,9 & 1,1 & 4,8 & 0,8 & 0,1 & 2,8 & 7,5 \\
\hline$F \& E$ & 72 & 8,8 & 7,9 & 4,1 & 15,3 & 20,1 & 4,2 & 3,6 & 9,9 & 12,6 \\
\hline sonstige Wirtschaftszweige & $1-3,55-60,64-66,69-70,73-96$ & 1,3 & 0,8 & 1,0 & 0,0 & 2,3 & 0,6 & 0,2 & 0,9 & 2,2 \\
\hline
\end{tabular}


Tab. 18: Verteilung der Patentanmeldungen aus dem Unternehmenssektor in industriellen Schlüsseltechnologien in Österreich 2000-2010 nach der Unternehmensgröße

\begin{tabular}{|l|c|c|c|c|c|c|c|c|c|}
\multicolumn{1}{c|}{ Größenklasse } & Alle & ST & PH & NT & IB & WS & ME & PT & F\&E \\
\hline KMU (bis 250 Beschäftigte) & 30,4 & 24,2 & 29,6 & 41,4 & 32,2 & 15,4 & 18,6 & 26,6 & 28,7 \\
Großunternehmen (250 und mehr Beschäftigte) & 69,6 & 75,8 & 70,4 & 58,6 & 67,8 & 84,6 & 81,4 & 73,4 & 71,3 \\
\hline
\end{tabular}

Alle: Alle Technologiefelder; ST: Schlüsseltechnologien insgesamt; PH: Photonik; NT: Nanotechnologie; IB: industrielle Biotechnologie; WS: Werkstofftechnologien; ME: Mikro-/Nanoelektronik; PT: Produktionstechnologien; F\&E: F\&E-Ausgaben im Jahr 2009.

Quelle: Patstat. - Statistik Austria.- Schlüsseltechnologiedefinition aus van de Velde et al. (2013). - Berechnungen des ZEW.

Tab. 19: Verteilung der Patentanmeldungen aus dem Unternehmenssektor und der öffentlichen und kooperativen Forschung in industriellen Schlüsseltechnologien in Österreich 2000-2010 nach Bundesländern

\begin{tabular}{|lrr|rrr|r|r|r|r|r|} 
& Bundesland & Alle & ST & PH & NT & IB & WS & ME & PT & F\&E \\
\hline Burgenland & 0,6 & 0,7 & 0,8 & 0,0 & 0,7 & 0,3 & 1,3 & 0,6 & 0,6 \\
\hline Kärnten & 4,9 & 11,3 & 2,9 & 0,9 & 0,4 & 4,7 & 44,3 & 14,0 & 2,7 \\
\hline Niederösterreich & 10,9 & 14,0 & 13,1 & 20,1 & 8,8 & 31,6 & 5,7 & 8,8 & 8,9 \\
Oberösterreich & 19,1 & 18,7 & 6,1 & 20,9 & 13,8 & 21,5 & 7,3 & 23,2 & 16,0 \\
\hline Salzburg & 5,7 & 3,5 & 2,0 & 0,0 & 2,9 & 3,8 & 1,1 & 4,9 & 3,7 \\
\hline Steiermark & 18,5 & 21,2 & 11,8 & 11,1 & 15,1 & 17,4 & 27,0 & 24,5 & 19,9 \\
\hline Tirol & 13,6 & 8,2 & 8,4 & 4,5 & 28,4 & 14,7 & 3,3 & 5,3 & 9,1 \\
Vorarlberg & 10,6 & 11,0 & 41,2 & 2,7 & 0,0 & 1,5 & 3,7 & 6,7 & 2,7 \\
\hline Wien & 16,0 & 11,4 & 13,9 & 39,6 & 30,0 & 4,5 & 6,3 & 12,1 & 34,0
\end{tabular}

Alle: Alle Technologiefelder; ST: Schlüsseltechnologien insgesamt; PH: Photonik; NT: Nanotechnologie; IB: industrielle Biotechnologie; WS: Werkstofftechnologien; ME: Mikro-/Nanoelektronik; PT: Produktionstechnologien; F\&E: F\&E-Ausgaben im Jahr 2009.

Quelle: Patstat. - Statistik Austria.- Schlüsseltechnologiedefinition aus van de Velde et al. (2013). - Berechnungen des ZEW.

und ihr Anteil an den gesamten F\&E-Ausgaben der Unternehmen bei 28,7 \% liegt. Der Anteil von KMU an den Patentanmeldungen ist in der Nanotechnologie mit $41,4 \%$ und in der industriellen Biotechnologie mit 32,2 \% vergleichsweise hoch und in den Werkstofftechnologien und der Mikro- und Nanoelektronik sehr niedrig.

Die regionale Verteilung der Anmelder von Schlüsseltechnologiepatenten (ohne Privatanmelder) weicht signifikant von der regionalen Verteilung der F\&E-Kapazitäten (Unternehmen inkl. kooperative Forschung, Hochschulen, Staat, private gemeinnützige Einrichtungen) ab. ${ }^{133}$ Während im Durchschnitt der Periode 2000 bis 2009 auf Wien $34 \%$ der gesamten F\&E-Ausgaben entfielen, kamen aus Wien nur 11,4 \% der Schlüsseltechnologiepatente. Dieser Unterschied er- klärt sich primär daraus, dass die industriellen F\&E-Aktivitäten der Wiener Wirtschaft überwiegend auf Bereiche außerhalb der Schlüsseltechnologien (wie z.B. Pharmazie/rote Biotechnologie, Nachrichtentechnik) ausgerichtet sind und dass die Patentaktivitäten der öffentlichen Forschung (Hochschulen, Staat), die den größten Teil der F\&E-Kapazitäten Wiens stellt, generell gering sind, da wissenschaftliche Publikationen den Hauptverwertungsweg in der Wissenschaft darstellen. Die beiden Länder mit den größten Anteilen an den gesamten Patentanmeldungen im Bereich der Schlüsseltechnologien sind die Steiermark $(21,2 \%)$ und Oberösterreich $(18,7 \%)$. Relativ hohe Anteile weisen außerdem die Länder Niederösterreich $(14,0 \%)$, Kärnten $(11,3 \%)$ und Vorarlberg $(11,0 \%)$ auf. Innerhalb der einzel-

133 Patente von Anmeldern mit mehreren Standorten in Österreich wurden jenem Standort zugeordnet, an dem der überwiegende Teil der F\&E-Aktivitäten des Anmelders erfolgt. 
nen Schlüsseltechnologiefelder ist die regionale Verteilung der Patentanmeldungen teilweise stark konzentriert. So kamen über $71 \%$ der 2000-2010 angemeldeten Patente in der Mikround Nanoelektronik aus Kärnten oder der Steiermark, über $80 \%$ der Nanotechnologiepatente wurden von Unternehmen oder Einrichtungen aus Wien, Niederösterreich oder Oberösterreich angemeldet, und über $58 \%$ der Patente in der industriellen Biotechnologie stammen aus Wien oder Tirol. In der Photonik ist Vorarlberg das Bundesland mit dem höchsten Anteil. In den Werkstofftechnologien dominieren Niederösterreich und Oberösterreich, und in den Produktionstechnologien Oberösterreich und die Steiermark. Die regionale Verteilung wird insgesamt stark durch die Aktivitäten einzelner Großunternehmen bestimmt.

Um die Entwicklung der SchlüsseltechnologiePatentanmeldungen in Österreich im internatio- nalen Vergleich einzuordnen, werden die sogenannten „internationalen Patentanmeldungen " betrachtet. Dabei handelt es sich um Anmeldungen am EPO oder über das PCT-Verfahren an der WIPO. Diese Anmeldewege werden üblicherweise dann genommen, wenn ein Patent in vielen Ländern geschützt und international vermarktet werden soll. Aufgrund des höheren Aufwands, der mit EPO bzw. PCT-Anmeldungen einhergeht, gelten diese Patente auch als wertvoller. Der Anteil Österreichs an den weltweiten internationalen Patentanmeldungen stieg seit Mitte der 2000er Jahre deutlich an. Dies gilt für die Patentanmeldungen quer über alle Technologiefelder und noch mehr für Patente in industriellen Schlüsseltechnologien. Entfielen im Jahr 2002 $0,66 \%$ der weltweiten internationalen Patentanmeldungen in Schlüsseltechnologien auf österreichische Anmelder, so stieg dieser Anteil bis 2009 auf $1,73 \%$ (Abb. 35). Damit war der Bedeutungs-

\section{Abb. 35: Anteil Österreichs an den weltweiten internationalen Patentanmeldungen in industriellen Schlüsseltechnologien} 2000-2009

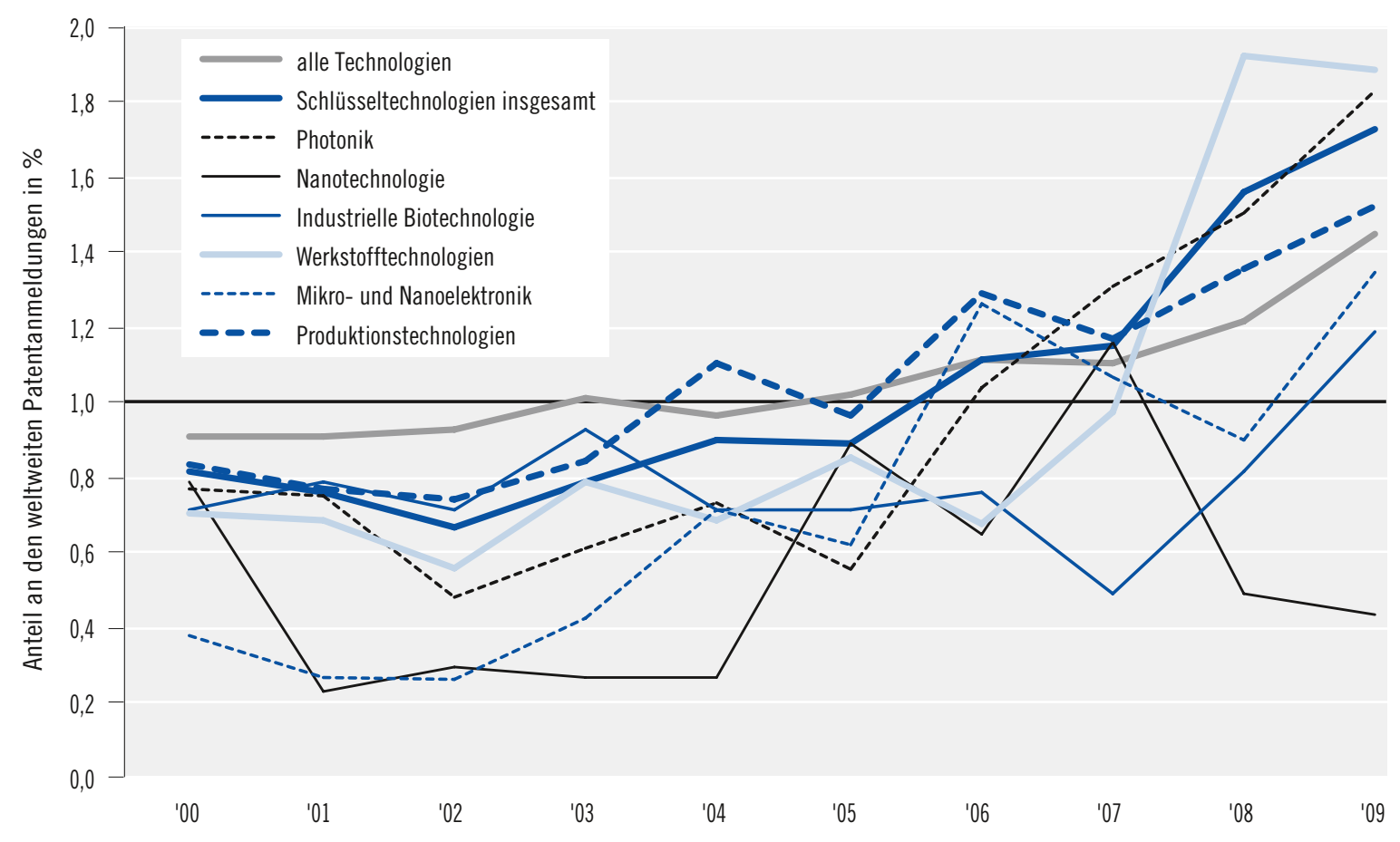

Anm.: Internationale Patentanmeldungen: Anmeldungen am EPO oder über das PCT-Verfahren.

Quelle: Patstat. - Schlüsseltechnologiedefinition aus van de Velde et al. (2013). - Berechnungen des ZEW. 
gewinn Österreichs in den Schlüsseltechnologien merklich höher als im Mittel aller Technologien.

Besonders stark war der Anteilsgewinn Österreichs an den weltweiten Patentaktivitäten in den Werkstofftechnologien, der Photonik, den Produktionstechnologien und der Mikro- und Nanoelektronik. In der industriellen Biotechnologie ist nur in den Jahren 2008 und 2009 ein Anstieg des Anteilswerts festzustellen, bei allerdings weltweit rückläufigen Patentaktivitäten in diesem Feld. In der Nanotechnologie ist der Beitrag Österreichs zur weltweiten Anzahl internationaler Patentanmeldungen in nahezu jedem Jahr unterdurchschnittlich.
Österreich weist bei fast allen industriellen Schlüsseltechnologien eine höhere Patentdynamik als viele europäische Vergleichsländer auf (Abb. 36). Mit einer Zunahme der Anzahl internationaler Patentanmeldungen in den Schlüsseltechnologien zwischen den Perioden 2000 bis 2003 und 2006 bis 2009 um 50 \% liegt Österreich deutlich vor Belgien, Finnland, Dänemark, Schweden und der Schweiz, die auf Zuwachsraten zwischen 6 und $19 \%$ kommen. In Deutschland und in der EU-27 blieb die Zahl der Schlüsseltechnologie-Patentanmeldungen zwischen den beiden Perioden jeweils konstant, in den Niederlanden ging sie um 14 \% zurück.

Abb. 36: Dynamik der internationalen Patentanmeldungen in industriellen Schlüsseltechnologien in den 2000er Jahren in ausgewählten Ländern

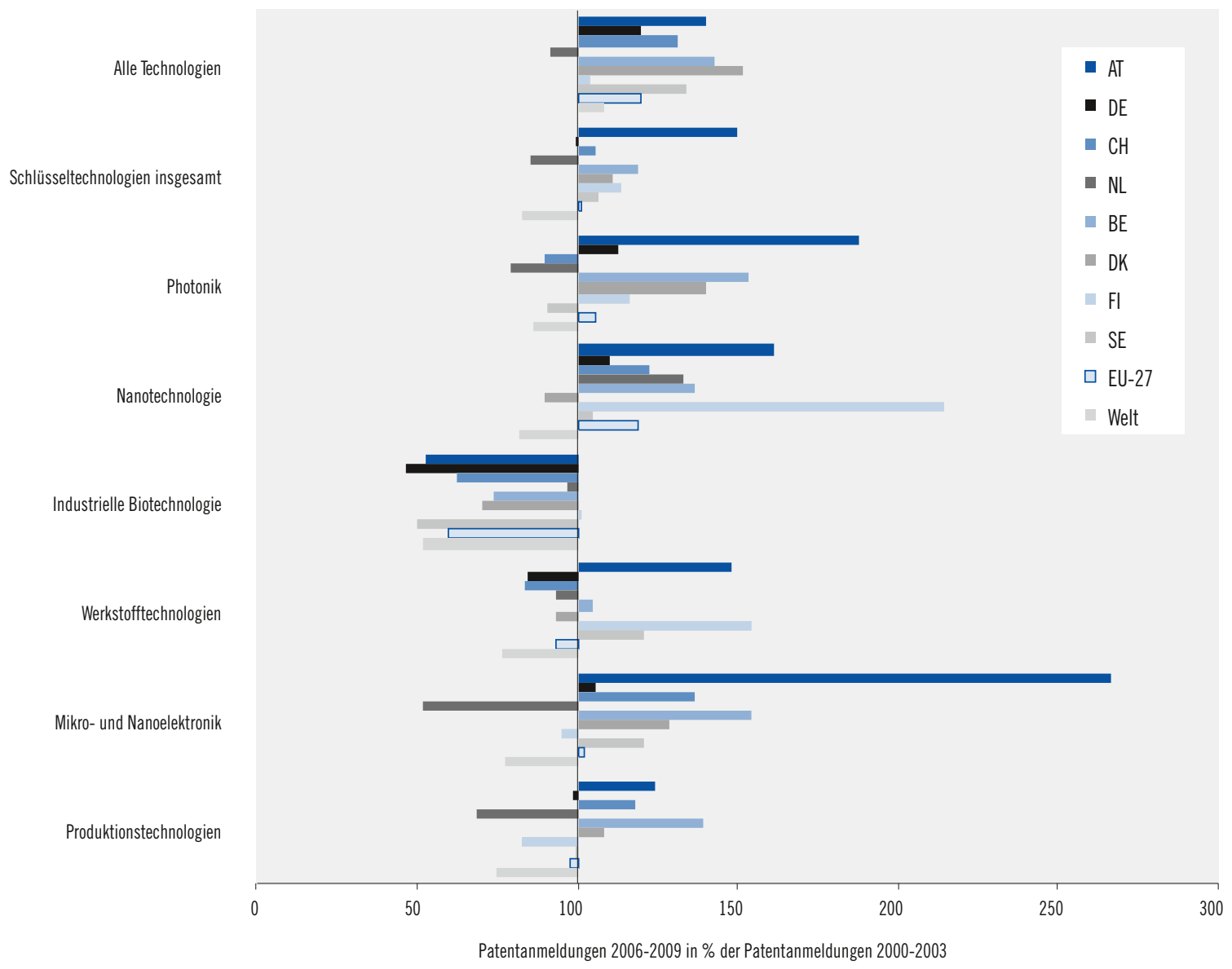

Anm.: Internationale Patentanmeldungen: Anmeldungen am EPO oder über das PCT-Verfahren.

Quelle: Patstat. - Schlüsseltechnologiedefinition aus van de Velde et al. (2013). - Berechnungen des ZEW. 
Die im internationalen Vergleich hohe Patentdynamik Österreichs zeigt sich in fünf der sechs Schlüsseltechnologien, einzig in der industriellen Biotechnologie sind die Patentanmeldezahlen - wie in fast allen anderen Ländern auch rückläufig. Im Bereich der Photonik und der Mikro- und Nanoelektronik erreicht kein anderes Land aus der Gruppe der Vergleichsländer ein ähnlich hohes Wachstum wie Österreich. Besonders beeindruckend ist die Zunahme in der Mikro- und Nanoelektronik, hier stieg das Aufkommen an internationalen Patentanmeldungen durch österreichische Anmelder um 166 \%. In den Werkstofftechnologien und der Nanotechnologie liegt Österreich jeweils hinter Finnland an zweiter Stelle der Patentdynamik. Bei Produktionstechnologien nimmt Österreich ebenfalls Rang zwei ein, hier liegt Belgien voran.

Die starke Zunahme der Patentanmeldezahlen im Verlauf der 2000er Jahre hat dazu geführt, dass Österreich gegen Ende des Jahrzehnts eine positive Spezialisierung auf Schlüsseltechnologien aufweist. Anfang des Jahrzehnts lag der Anteil der Patentanmeldungen in Schlüsseltechnologien in Österreich dagegen noch niedriger als im Mittel aller Länder. Die positive Spezialisierung

Abb. 37: Spezialisierung bei internationalen Patentanmeldungen in industriellen Schlüsseltechnologien 2006-2009: relativer Patentanteil für ausgewählte Länder

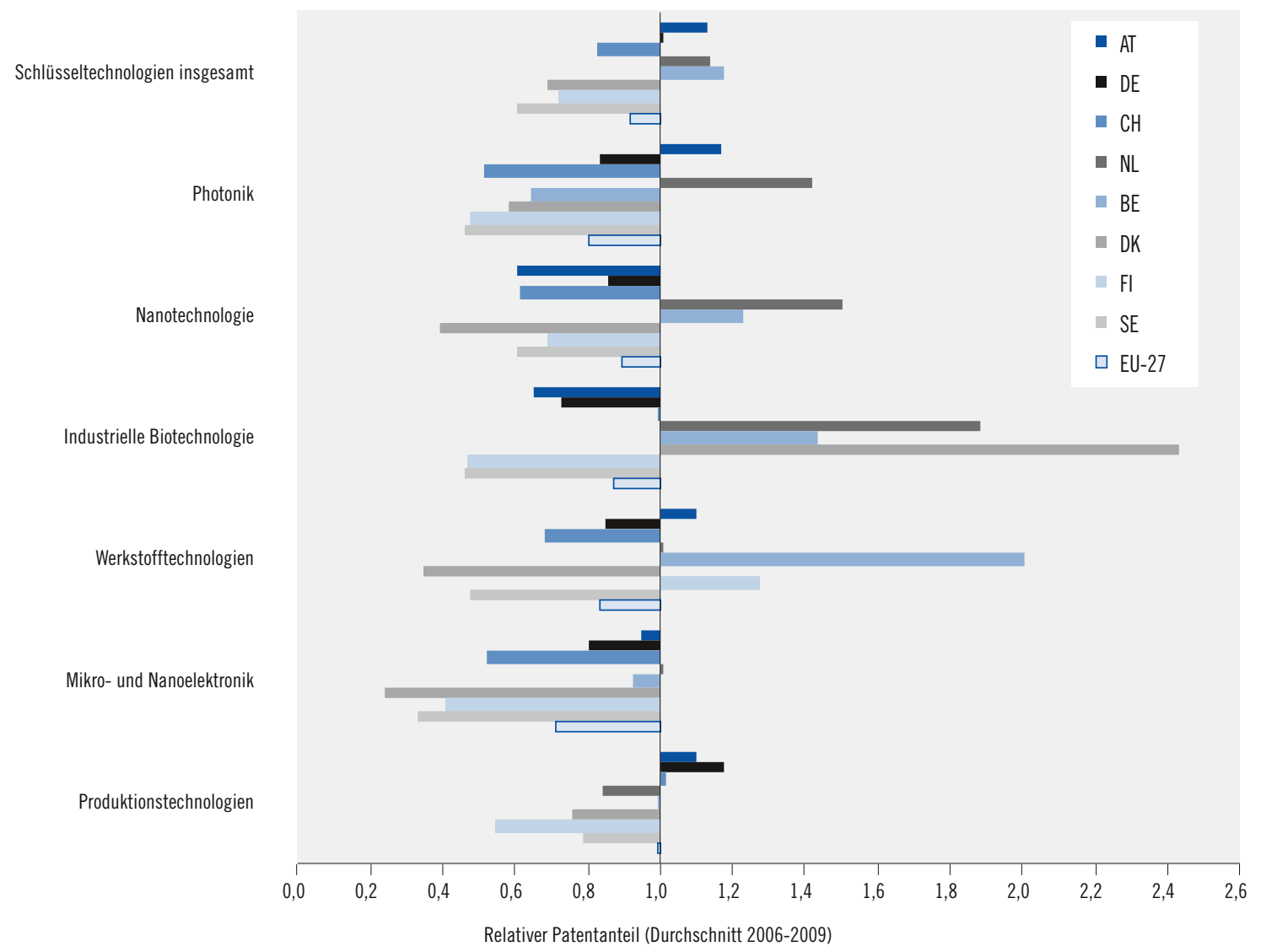

Anm.: Relativer Patentanteil: Anteil der Patentanmeldungen 2006-2009 im jeweiligen Technologiefeld an den gesamten Patentanmeldungen 2006-2009 eines Landes, geteilt durch den Anteil der Patentanmeldungen 2006-2009 im jeweiligen Technologiefeld weltweit an den gesamten Patentanmeldungen 2006-2009 weltweit. Ein Wert größer 1,0 zeigt an, dass das jeweilige Land in diesem Feld überdurchschnittlich viel patentiert.

Quelle: Patstat. - Schlüsseltechnologiedefinition aus van de Velde et al. (2013). - Berechnungen des ZEW. 
ergibt sich aus überproportionalen Patentaktivitäten in der Photonik, den Werkstofftechnologien und den Produktionstechnologien (Abb. 37). In der Mikro- und Nanotechnologie ist trotz der hohen Patentdynamik die Spezialisierung noch immer leicht unterdurchschnittlich. Vergleichsweise niedrig sind weiterhin die Patentaktivitäten in der industriellen Biotechnologie und in der Nanotechnologie. Insgesamt weist Österreich recht ausgewogene Patentaktivitäten in den sechs Schlüsseltechnologien auf. Andere Länder ähnlicher Größe sind wesentlich stärker auf einzelne Technologiefelder ausgerichtet. So weist Dänemark eine ausgesprochene Spezialisierung auf die industrielle Biotechnologie auf, während es in den meisten anderen Feldern kaum aktiv ist. Das eher "flache" Profil Österreichs ähnelt eher größeren Ländern wie z.B. Deutschland. Angesichts des Umstands, dass es zwischen den sechs Schlüsseltechnologien hohe Spillover-Potenziale und viele Überschneidungsbereiche für gemeinsame oder aufeinander aufbauende technologische Entwicklungen gibt, kann ein breites und ausgewogenes Schlüsseltechnologieportfolio als vorteilhaft bewertet werden.

\subsubsection{Position Österreichs im Außenhandel mit schlüsseltechnologiebasierten Produkten}

Eine Bestimmung der wirtschaftlichen Leistungsfähigkeit eines Landes im Bereich der industriellen Schlüsseltechnologien ist weitaus schwieriger als die Bestimmung der technologischen Leistungsfähigkeit. Denn die Produktionsund Außenhandelsstatistiken erlauben, im Gegensatz zur Patentstatistik, i.d.R. keine eindeuti- ge Zuordnung der produzierten und gehandelten Güter zu den einzelnen Technologiefeldern, da in diesen Statistiken Güter nach ihrer Verwendung oder den überwiegend eingesetzten Materialien, nicht aber nach der in der Produktion eingesetzten Technologie oder bestimmten verwendeten technologischen Komponenten klassifiziert werden. In einem Projekt der EU-Kommission $^{134}$ wurde gleichwohl der Versuch unternommen, in den gängigen Güterklassifikationen ${ }^{135}$ jene Produkte zu identifizieren, die als überwiegend schlüsseltechnologiebasiert angesehen werden können. Auf Basis dieser Zuordnung kann die Entwicklung des Außenhandels in den sechs Schlüsseltechnologiefeldern international vergleichend untersucht werden. ${ }^{136}$ Produktionsstatistische Auswertungen sind dagegen aufgrund von Geheimhaltungen auf der Ebene einzelner Güter in den zugrundeliegenden Statistiken nicht möglich.

Der Anteil Österreichs an den weltweiten ${ }^{137}$ Exporten von schlüssteltechnologiebasierten Produkten ist in allen Technologiefeldern mit Ausnahme der Produktionstechnologien unterdurchschnittlich (Abb. 38). Während Österreich im Jahr 2011 einen Anteil von 1,5 \% am gesamten Warenhandel erreicht hat, lagen die Anteile in fünf Schlüsseltechnologien mit 0,4 \% (Photonik) bis $1,0 \%$ (Werkstofftechnologien) deutlich darunter. In den Produktionstechnologien erreichte Österreich 2011 einen überdurchschnittlichen Welthandelsanteil von 1,8\%. Mitte der 2003er Jahre lag er zum Teil sogar doppelt so hoch, wofür einzelne Großaufträge im Bereich der Metallurgietechnik und von Anlagen für die Halbleitertechnik wesentlich verantwortlich waren. In der in-

134 Siehe van de Velde et al. (2013).

135 Das ist die CPA (Classification of Products by Activity) und die darauf aufbauende Prodcom-Klassifikation der Europäische Kommissionim Bereich der Produktionsstatistik sowie das HS (Harmonised Commodity Description and Coding System) und die davon abgeleitete CN (Combined Nomenclature) der EU-Kommission im Bereich der Außenhandelsstatistik.

136 Hierfür wird auf eine eingegrenzte und von ExpertInnen validierte Liste zurückgegriffen, die nur den Kernbereich der einzelnen Technologiefelder abdeckt und nur jene Gütergruppen berücksichtigt, für die eine Zuordnung zu einem Schlüsseltechnologiefeld eindeutig möglich ist. Aufgrund von Umstellungen in der Güterklassifikation im Jahr 2002 können nur Werte ab dem Jahr 2002 ausgewiesen werden.

137 „Weltweit“ ist hier auf eine Gruppe von 39 Ländern eingeschränkt, die für den Handel mit Schlüsseltechnologieprodukten besonders bedeutend sind: EU-27-Länder, Schweiz, Norwegen, Island, Kroatien, Mazedonien, USA, Japan, Südkorea, Kanada, China, Russland und Israel. 
dustriellen Biotechnologie, der Photonik, der Mikro- und Nanoelektronik und den Produktionstechnologien hat Österreichs Welthandelsanteil in den vergangenen zehn Jahren tendenziell abgenommen, was auch dem Trend für alle verarbei- tenden Industriewaren entspricht. In der Nanotechnologie und den Werkstofftechnologien konnte Österreich seinen Welthandelsanteil dagegen stabil halten. Da gleichzeitig mit der weltwirtschaftlichen Integration der Schwellenländer

Abb. 38: Welthandelsanteil Österreichs in industriellen Schlüsseltechnologien 2002-2011

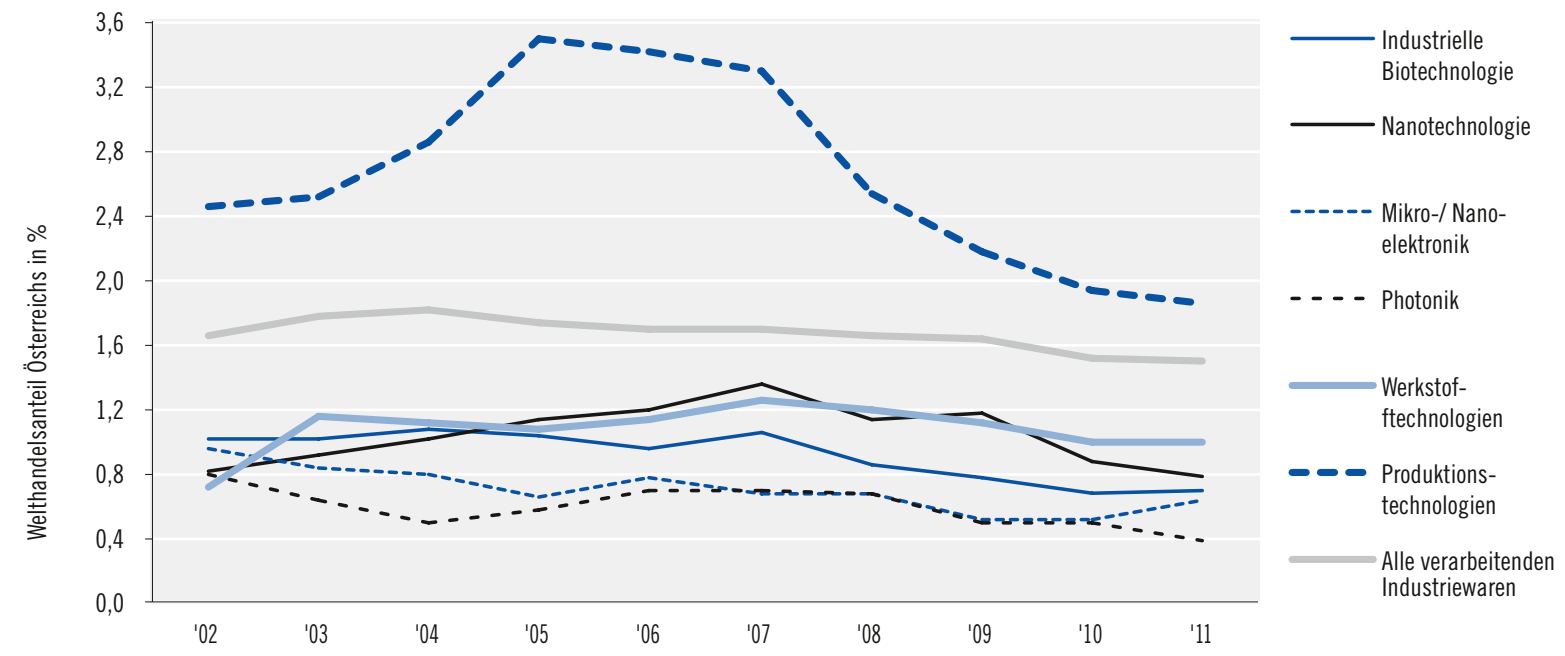

Anm.: Welthandelsanteil: Anteil der Exporte Österreichs an den gesamten Exporten von 39 den Welthandel dominierenden Ländern (EU-27, Schweiz, Norwegen, Island, Kroatien, Mazedonien, USA, Japan, Südkorea, Kanada, China, Russland, Israel).

Quelle: Comtrade. - Schlüsseltechnologiedefinition aus van de Velde et al. (2013). - Berechnungen des NIW und ZEW.

Abb. 39: Dynamik der Exporte Österreichs in industriellen Schlüsseltechnologien 2002-2011

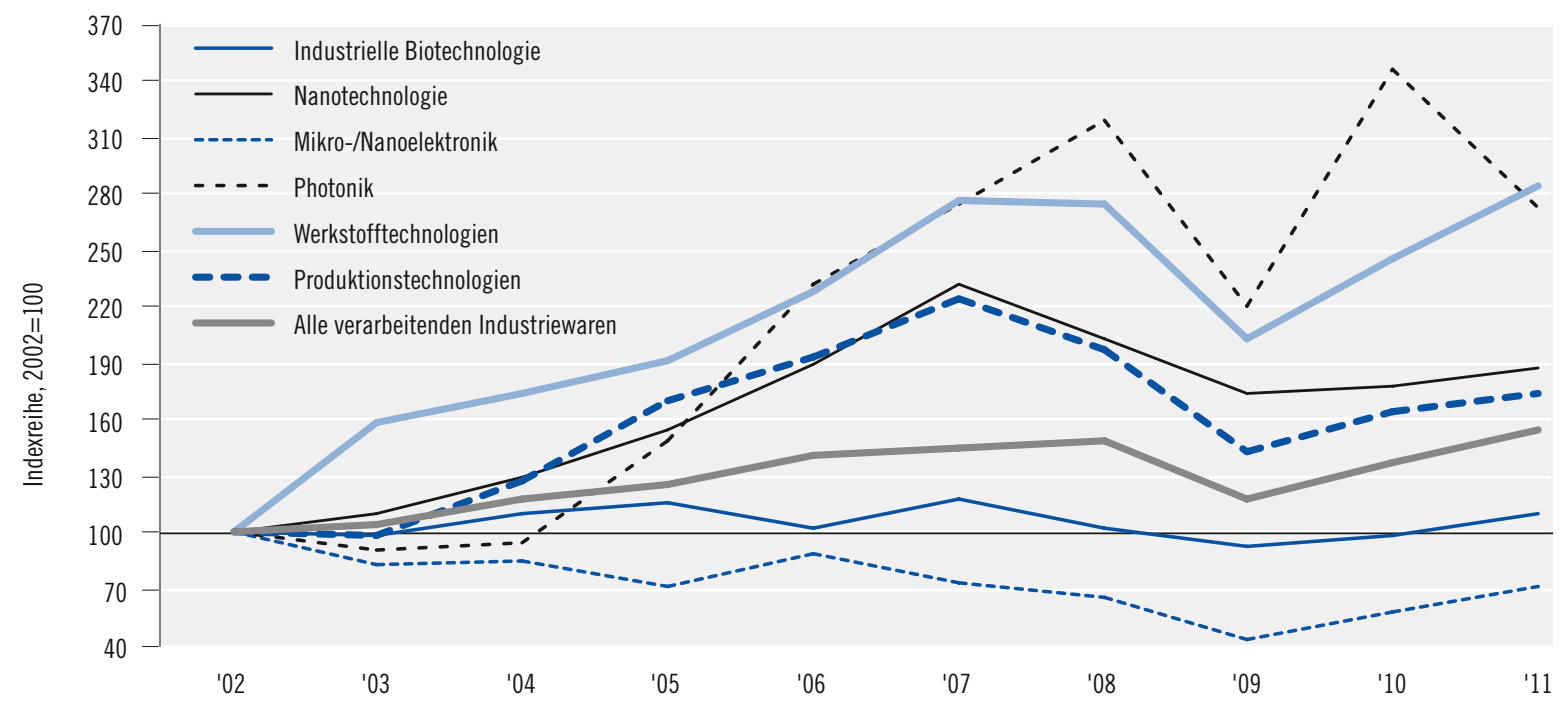

Anm.: Indexreihe auf Basis der Exportvolumina in €.

Quelle: Comtrade. - Schlüsseltechnologiedefinition aus van de Velde et al. (2013). - Berechnungen des NIW und ZEW. 
der Wettbewerb auf den Weltmärkten zugenommen hat, stellt ein stabiler Welthandelsanteil eine beachtenswerte Leistung dar.

Die Exporte Österreichs stiegen in vier Schlüsseltechnologien deutlich rascher als im Durchschnitt aller Warengruppen (Abb. 39). In der Photonik und in den Werkstofftechnologien lagen die nominellen Exporte 2011 um mehr als zweieinhalb Mal so hoch wie 2002, in der Nanotechnologie und in den Produktionstechnologien überstiegen die Exporte des Jahres 2011 das Niveau von 2002 um 87 (bzw. 74 \%) (zu laufenden Preisen gerechnet). In der industriellen Biotechnologie gab es dagegen während der 2000er Jahre keinen merklichen Anstieg der Exportvolumina. In der
Mikro- und Nanoelektronik lagen die Exporte 2011 sogar deutlich unter dem Wert von 2002.

Die Exportdynamik Österreichs im Bereich der industriellen Schlüsseltechnologien unterscheidet sich in einigen Technologiefeldern deutlich von der weltweiten Außenhandelsdynamik und weicht häufig auch von der durchschnittlichen Exportdynamik in der EU-27 ab. Vergleicht man das Volumen der Exporte im Zeitraum 2007 bis 2011 mit dem der Jahre 2002 bis 2006, so weist Österreich in den Werkstofftechnologien eine besonders starke Zunahme auf, die sowohl über dem weltweiten wie dem EU-Wert liegt als auch den Exportzuwachs in jedem der Vergleichsländern übersteigt (Abb. 40). In der Photonik und

Abb. 40: Exportdynamik in industriellen Schlüsseltechnologien 2002-2011 in ausgewählten Ländern

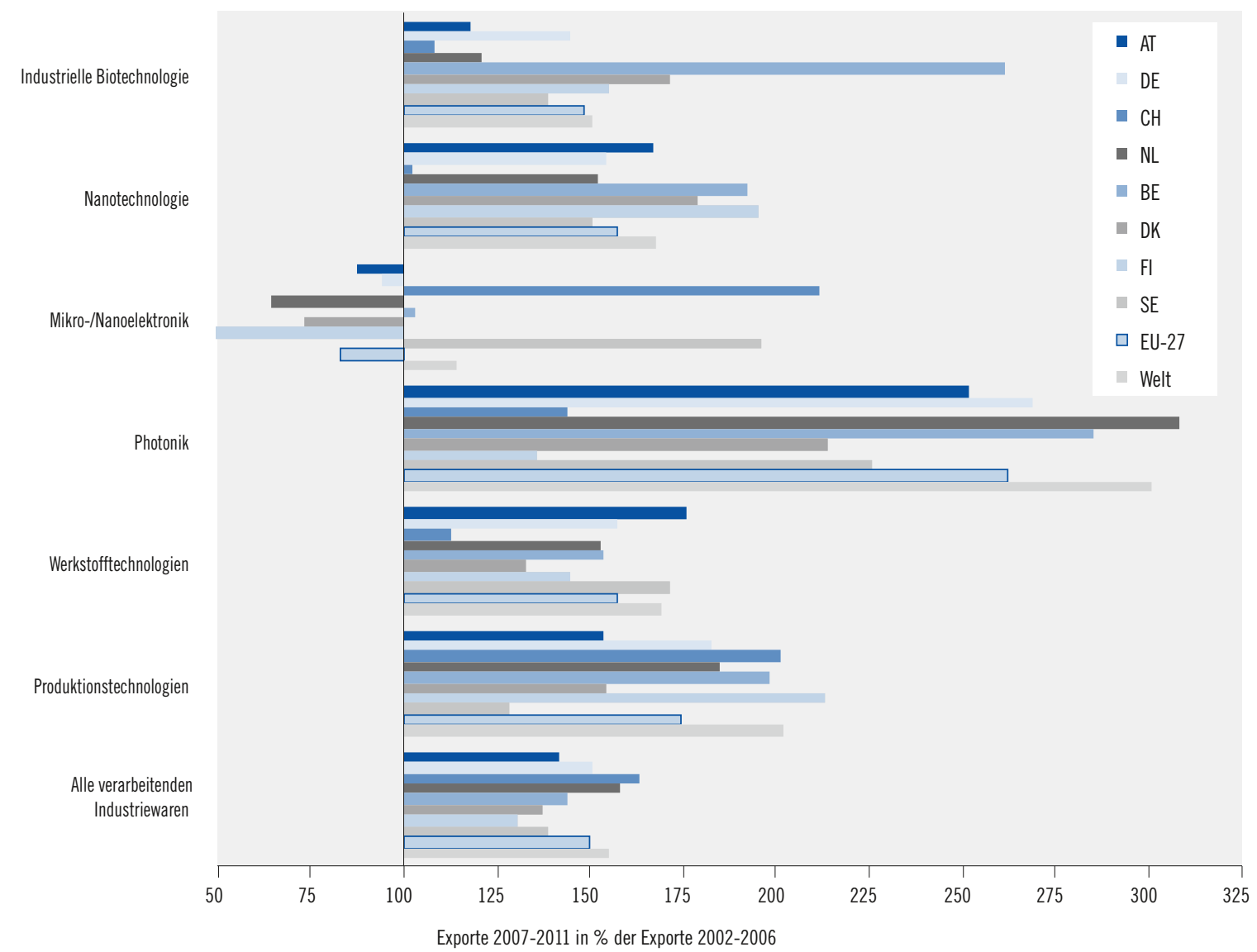

Anm.: Exporte in US-\$ zu laufenden Preisen.

*EU-27, Schweiz, Norwegen, Island, Kroatien, Mazedonien, USA, Japan, Südkorea, Kanada, China, Russland und Israel.

Quelle: Comtrade. - Schlüsseltechnologiedefinition aus van de Velde et al. (2013). - Berechnungen des NIW und ZEW. 
der Nanotechnologie werden ebenfalls vergleichsweise hohe Zuwachsraten verzeichnet. Unterdurchschnittlich war das Exportwachstum im Bereich der Produktionstechnologien und der industriellen Biotechnologie. Für alle fünf genannten Schlüsseltechnologien gilt, dass es im Laufe der 2000er Jahre zu einer erheblichen Ausweitung des internationalen Handels gekommen war. In der Nanotechnologie, der Photonik, den Werkstofftechnologien und den Produktionstechnologien lag die Handelsexpansion deutlich über dem Durchschnitt für alle Industriewaren, in der industriellen Biotechnologie nahm das Exportvolumen etwa im Tempo der gesamten Handelsausweitung zu.

Eine Ausnahme stellt die Mikro- und Nanoelektronik dar. Hier nahm der Welthandel im vergangenen Jahrzehnt nur leicht zu und in einigen Industrieländern zum Teil deutlich ab. Österreich reiht sich mit einem Rückgang der Exporte um $12 \%$ im Vergleich von 2007/11 mit 2002/06 in diese Gruppe ein. In der EU-27 nahmen die Exporte um 17 \% zwischen den beiden
Perioden ab, weltweit stiegen sie um $14 \%$ an. Hintergrund dieser Außenhandelsentwicklung ist die zunehmende Verlagerung der Produktion mikroelektronischer Bauteile in die Regionen, in denen diese in weiteren Verarbeitungsstufen eingesetzt werden. Dadurch verringerten sich die Ausfuhren in den früheren Hauptexportländern Japan, USA, Großbritannien und Deutschland. Stark wachsende Ausfuhren melden ost- und südostasiatische Länder, darunter Südkorea, Taiwan und China.

Neben der Exportdynamik ist die Außenhandelsbilanz ein zweiter wichtiger Indikator für die Beurteilung der wirtschaftlichen Leistungsfähigkeit im Bereich von industriellen Schlüsseltechnologien. In zwei Feldern - Produktionstechnologien und Mikro-/Nanoelektronik - weist Österreich in allen Jahren seit 2002 eine positive Handelsbilanz auf, wobei die Überschüsse in den Produktionstechnologien besonders hoch sind (Abb. 41). In der industriellen Biotechnologie wurden bis 2007 Überschüsse erzielt, seither überwiegen die Importe die Exporte. Umgekehrt

Abb. 41: Außenhandelsbilanz Österreichs in industriellen Schlüsseltechnologien 2002-2011

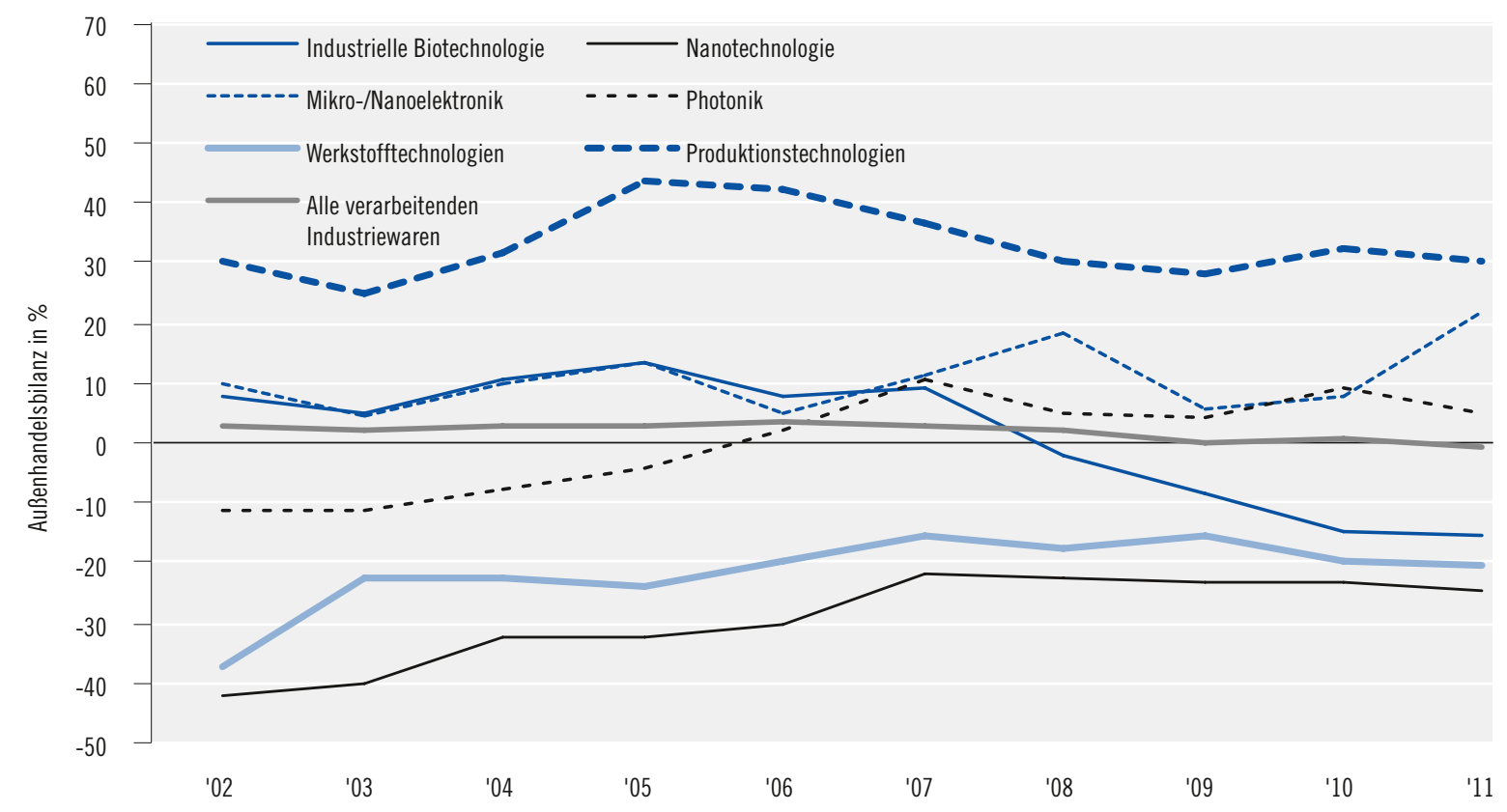

Anm.: Außenhandelsbilanz: Exporte minus Importe in \% des gesamten Außenhandelsvolumens (Exporte plus Importe).

Quelle: Comtrade. - Schlüsseltechnologiedefinition aus van de Velde et al. (2013). - Berechnungen des NIW und ZEW. 
Abb. 42: Außenhandelsspezialisierung in industriellen Schlüsseltechnologien 2007-2011 in ausgewählten Ländern

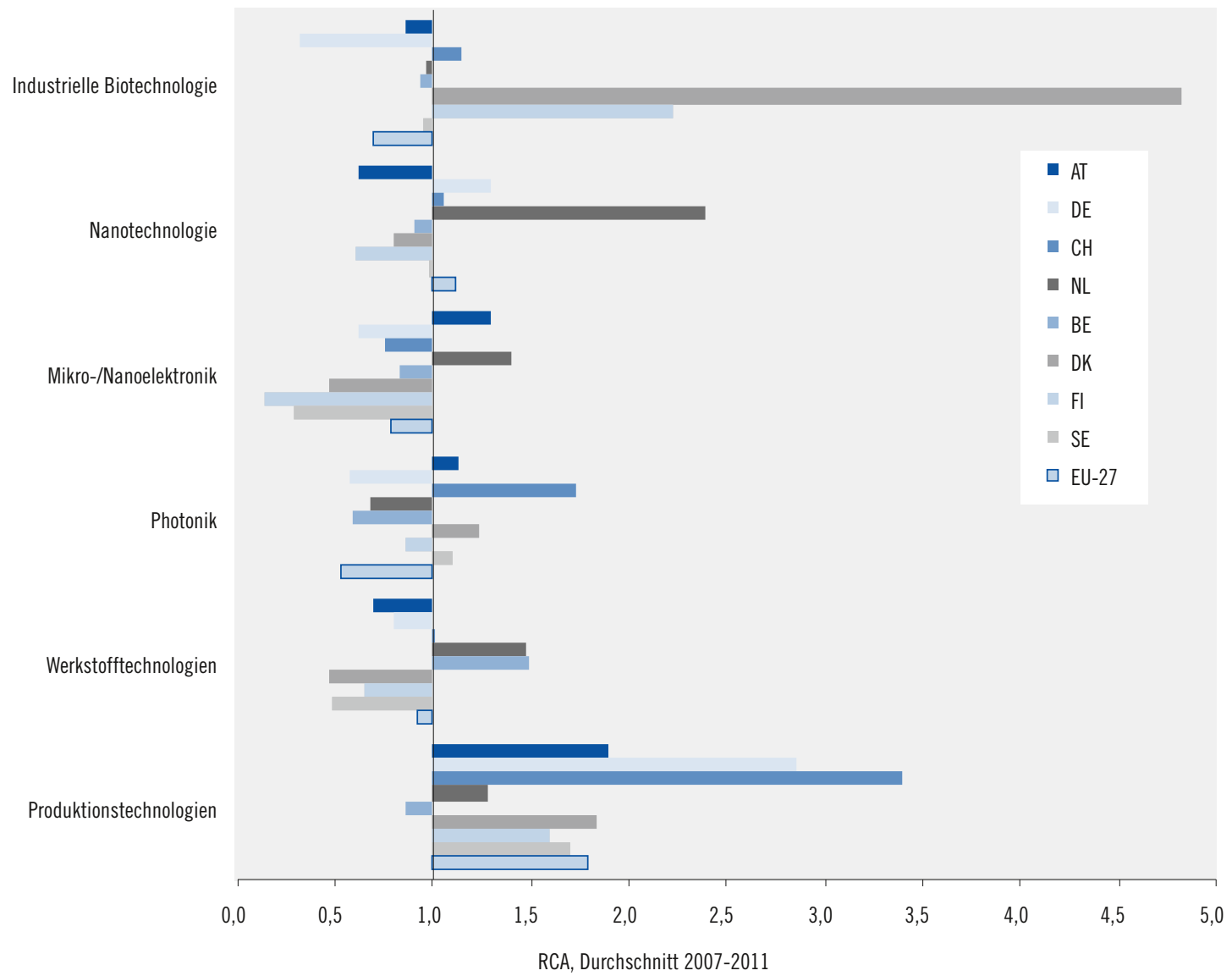

Anm.: RCA: Revealed Comparative Advantage: Export-Import-Relation in der jeweiligen Schlüsseltechnologie in Relation zur Export-Import-Relation für alle verarbeitenden Industriewaren.

Quelle: Comtrade. - Schlüsseltechnologiedefinition aus van de Velde et al. (2013). - Berechnungen des NIW und ZEW.

war die Entwicklung in der Photonik, hier werden seit 2006 leichte Exportüberschüsse erzielt. Eine negative Außenhandelsbilanz weisen die Werkstofftechnologien und die Nanotechnologie auf, wobei sich das Defizit Ende der 2000er Jahre merklich geringer als noch zu Beginn des Jahrzehnts ausfiel.

Die aktuell positive Handelsbilanz bei Produktionstechnologien, Mikro-/Nanoelektronik und Photonik bei einer im Gesamthandel ausgeglichenen Handelsbilanz bedeutet, dass Österreich auf den Handel mit diesen drei Schlüsseltechnologien spezialisiert ist. Am höchsten ist die Spezialisierung in den Produktionstechnolo- gien (Abb. 42). In der Mikro-/Nanoelektronik ist sie leicht positiv, was im Vergleich zu anderen hochentwickelten europäischen Industrieländern gleichwohl eine besondere Situation darstellt, da neben Österreich nur noch die Niederlande eine positive Spezialisierung in dieser Schlüsseltechnologie aufweist. In der Photonik zeigen neben Österreich noch die Schweiz, Schweden und Dänemark positive Spezialisierungswerte. Die stärkste negative Spezialisierung zeigt Österreich in der Nanotechnologie.

Im internationalen Vergleich fällt das Spezialisierungsmuster Österreichs vergleichsweise flach aus, d.h. Österreich ist in allen sechs 
Schlüsseltechnologien vertreten und fokussiert nicht nur auf einzelne Felder. Andere Länder vergleichbarer Größe weisen demgegenüber für einzelne Schlüsseltechnologien deutlich höhere Spezialisierungskennzahlen auf. So sind Dänemark und Finnland sehr stark auf die industrielle Biotechnologie ausgerichtet, die Niederlande auf die Nanotechnologie, die Schweiz auf die Photonik sowie die Schweiz und Deutschland auf die Produktionstechnologien.

Zusammenfassend kann konstatiert werden, dass Österreich somit sowohl hinsichtlich seinen F\&E- wie von seinen Produktions- und Vermarktungskapazitäten her gut bei Schlüsseltechnologien positioniert ist. Österreich hat in den vergangenen zehn Jahren seine Patentaktivitäten im Bereich industrieller Schlüsseltechnologien deutlich ausgeweitet und sein Patentportfolio in Richtung Schlüsseltechnologien verschoben. Dadurch hat Österreich an Gewicht innerhalb der weltweiten Produktion neuen technologischen Wissens in diesen für die künftige Wettbewerbsfähigkeit der Industrie besonders wichtigen Technologiefeldern gewonnen. Die Stärken Österreichs liegen dabei vor allem in den Produktionstechnologien, den Werkstofftechnologien und der Photonik. Im Gegensatz zu vielen anderen kleineren hochentwickelten Volkswirtschaften verfügt Österreich jedoch in allen sechs Schlüsseltechnologiefeldern über signifikante F\&E-Kapazitäten. Mit der Stärkung der Position bei Patentanmeldungen ging auch eine kräftige Ausweitung der Exporte Österreichs von schlüsseltechnologiebasierten Produkten einher. Besonders hohe Wachstumsraten verzeichneten die Photonik, die Werkstofftechnologien die Nanotechnologie und die Produktionstechnologien. Allerdings ist der Welthandelsanteil Österreichs bei Schlüsseltechnologien mit Ausnahme der Produktionstechnologien unterdurchschnittlich. Wachstumspotenziale sind somit noch vorhanden. Auch im Außenhandel bestätigt sich, dass Österreich in allen sechs
Schlüsseltechnologiefeldern gut vertreten ist. Diese positiven Entwicklungen wurden von den österreichischen Industrieunternehmen getragen und von der FTI-Politik unterstützt.

\subsection{Wachstumsfaktor Umweltindustrie}

Ökonomisches Wachstum ist die Voraussetzung für Beschäftigung, die Finanzierung sozialer Sicherungssysteme und damit auch steigender Wohlfahrt. Die Wirtschafts- und Finanzkrise macht gerade am Beispiel von Krisenstaaten wie Griechenland oder Spanien deutlich, welch negative Folgen fehlendes Wachstum haben kann. Gleichwohl stellt Wachstum um jeden Preis keine vernünftige wirtschaftspolitische Option dar. Steigende Rohstoffpreise, ökologische Beschränkungen und Gefahrenpotenziale (Klimawandel) sowie soziale Nutzeneinbußen durch Umweltdegradation unterstreichen die Relevanz von Strategien, die auf intelligentes, d.h. vor allem nachhaltiges Wachstum setzen. ${ }^{138}$

\subsubsection{Marktentwicklung und Marktpotenzial für Umweltschutzgüter}

Die Industrie spielt zur Realisierung eines intelligenten Wachstums eine zentrale Rolle, weil der Wandel in Richtung einer umweltverträglichen und nachhaltigen Wirtschaftsweise mit einem massiven Investitionsbedarf verbunden ist. Es geht dabei um den Umbau der materiellen Welt durch das Ersetzen herkömmlicher, nicht nachhaltiger Technologien mit Investitionsgütern, die eine ökologisch verträgliche Lebens- und Wirtschaftsweise im Sinne von "low energy low carbon-low distance ${ }^{\prime 139}$ sicherstellen. Diese Phase der Transformation wird aller Voraussicht nach einen bedeutsamen globalen Investitionszyklus auslösen und einen Nachfrageschub für jene Industriegüter nach sich ziehen, die in der Lage sind, die entsprechenden technologischen

138 Siehe Aghion et al. (2009).

139 Kletzan-Slamanig, Köppl (2009). 
Anforderungen zu erfüllen. ${ }^{140}$ Allerdings ist dieser Wandel keineswegs eine alleine durch Marktkräfte erreichbare Vision. Starke Netzwerkeffekte von herkömmlichen Technologien behindern vielfach den Umstieg auf alternative Systeme der Energieversorgung oder Mobilität. ${ }^{141}$ Ebenfalls offen scheint noch die Frage, welche Staaten von dieser Transformation durch die Zunahme "grüner" Industrien profitieren können. Im Moment herrscht jedenfalls ein intensiver Standortwettbewerb um die Erlangung von "first mover advantages" und Agglomerationsvorteilen. Generell scheint die Verteilung der industriellen Schwerpunkte noch nicht entschieden. Eine intelligente Industriepolitik kann hier positive Akzente setzen und somit neue Wachstumschancen fördern, die aber letztlich von den Unternehmen genützt und realisiert werden müssen.
Die zu erwartende Dynamik ist bereits in einigen Segmenten der Umwelttechnikindustrie erkennbar. Abb. 43 zeigt die globalen Investitionen in erneuerbare Technologien, die sich im Jahr 2011 auf rund 206 Mrd. US \$ beliefen. Bemerkenswert ist aber vor allem die hohe Dynamik der Investitionen, sowohl in den Industrie- als auch in den Entwicklungsländern. Diese Dynamik wird durch einen Vergleich der Wachstumsraten der Investitionen in erneuerbare Energien mit der Wachstumsrate der Weltwirtschaft deutlich. Abb. 43 zeigt, dass die Investitionen, abgesehen vom Krisenjahr 2009, stets wesentlich rascher wuchsen als die Weltwirtschaft insgesamt. Diese dürfte auch in der absehbaren Zukunft anhalten und innovativen Industrieunternehmen neue Expansionsmöglichkeiten bieten. ${ }^{142}$

\section{Abb. 43: Niveau bzw. Wachstum der globalen Investitionen in erneuerbare Energien in Industrie- und Entwicklungsländern} in Mrd. US \$ bzw. Prozent

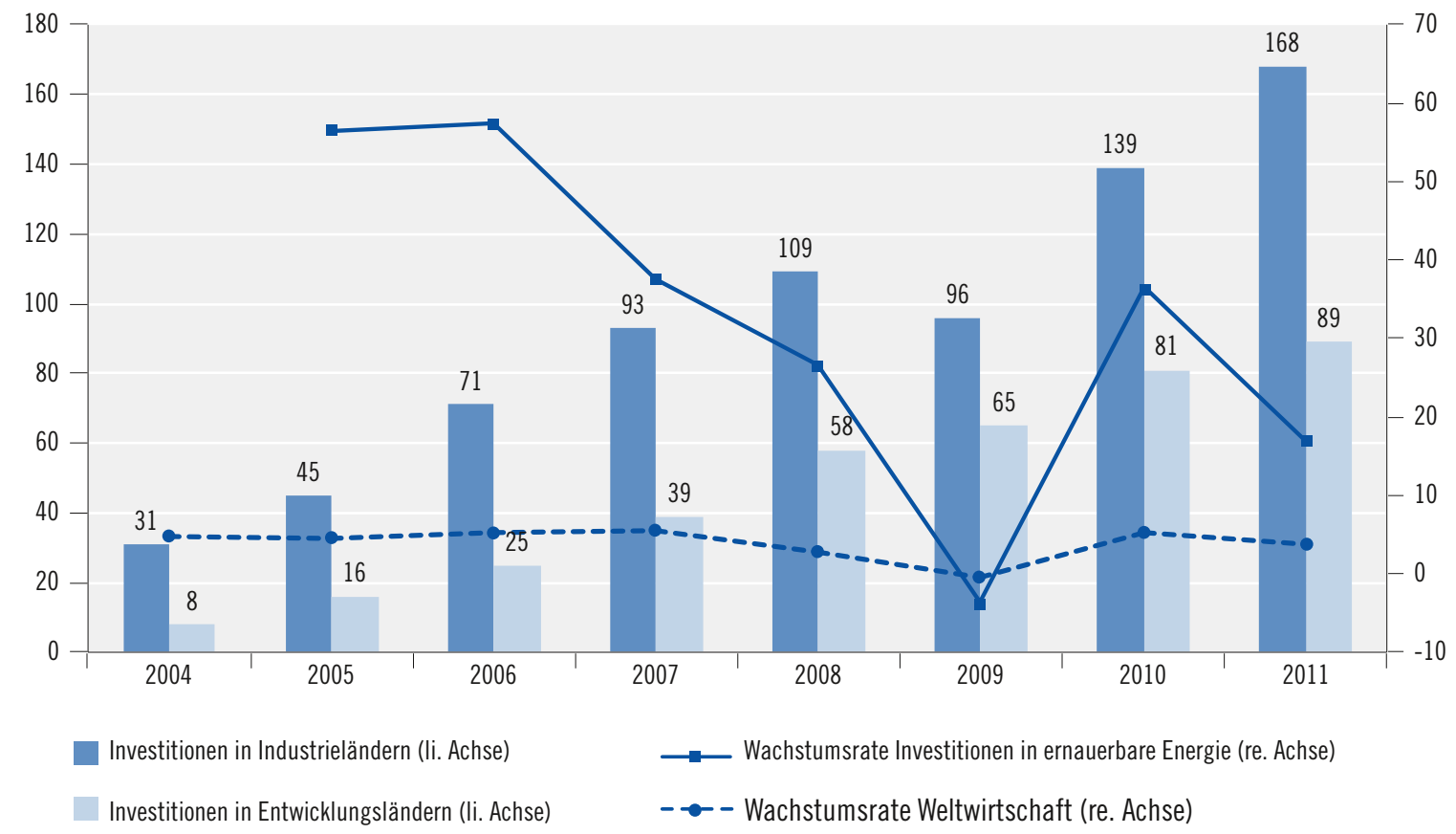

Quelle: Bloomberg 2012, IMF World Economic Outlook Database. Berechnungen JOANNEUM RESEARCH.

140 Siehe Rifkin (2011).

141 Siehe OECD $(2011 \mathrm{~b})$.

142 Ebenda. 


\subsubsection{Wettbewerbsfähigkeit der österreichischen Umweltindustrie}

Die bisherigen Analysen zu Struktur und Dynamik der österreichischen Umwelttechnikindustrie zeigen eine positive Performance und Wettbewerbsfähigkeit. Dies drückt sich etwa in steigenden Exporten und einer hohen Technologie- und Innovationsintensität aus. Zahlreiche Unternehmen der Branche können dem Hochtechnologiesegment zugerechnet werden. ${ }^{143}$

Betrachtet man die Ausgangsposition Österreichs im Bereich Öko-Innovationen im internationalen Vergleich, so zeigt sich, dass Österreich eine generell hohe Wettbewerbsfähigkeit sowie eine dynamische Entwicklung aufweist. Abb. 44 zeigt dies am Beispiel des Gesamtindex des Eco Innovation Scoreboards, der 16 Variablen berücksichtigt, die primär Inputs und Outputs von Ökoinnovationen abbilden. Beispiele für verwendete Indikatoren sind etwa die Staatsausgaben für Umwelt- und Energieforschung, Ressourcenproduktivität, oder Umsatz in Ökoindustrien. Demnach ist Österreich an sechster Stelle in der EU-27.
Länder wie Deutschland, Großbritannien, Niederlande oder Belgien, die im allgemeinen Innovation Scoreboard der EU vor Österreich platziert sind, zeigen bei Öko-Innovationen eine schlechtere Performance als Österreich. Offensichtlich weist die österreichische Volkswirtschaft gute Voraussetzungen für die Realisierung und Vermarktung von Öko-Innovationen auf.

Die Entwicklung der Exporte ist einer der zentralen Indikatoren für die Wettbewerbsfähigkeit von Branchen und von zentraler Bedeutung für kleine offene Volkswirtschaften wie Österreich. Im Folgenden wird auf Basis einer Klassifikation des Niedersächsischen Instituts für Wirtschaftsforschung von potenziellen Umweltschutzgütern die Exportperformance und Spezialisierung Österreichs in diesem Segment dargestellt (siehe Anhang: Klassifikation von potenziellen Umweltschutzgütern (Gehrke et al. 2012)). Bei den potenziellen Umweltschutzgütern handelt es sich ausschließlich um Sachgüter, die in Gruppen gemäß ihrem Verwendungskontext - wie etwa Abfall oder Klimaschutz - eingeteilt werden.

Tab. 20 zeigt die Entwicklung der Exporte von

\section{Abb. 44: Österreichs Öko-Innovationsperformance im internationalen Vergleich} (Gesamtindex Eco-Innovation Scoreboard 2011)

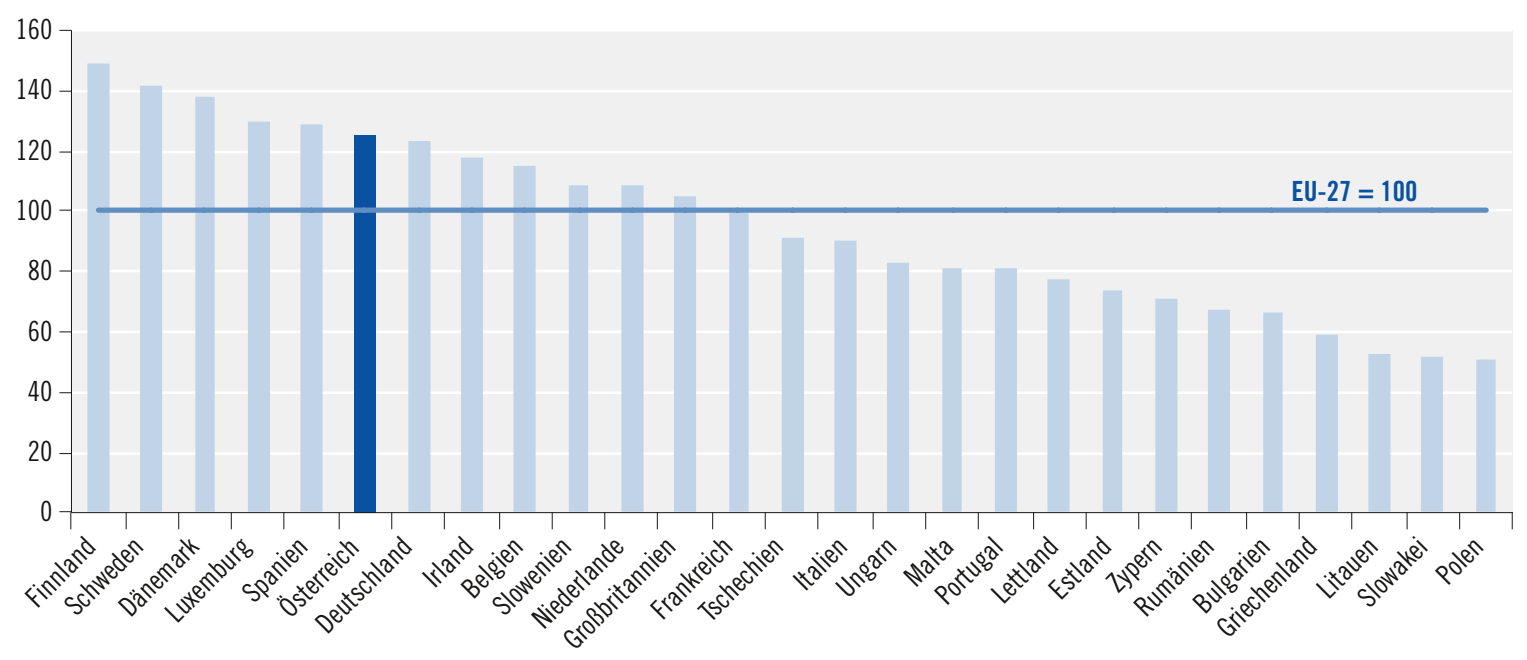

Quelle: http://www.eco-innovation.eu/

143 Siehe Kletzan-Slamanig, Köppl (2009). 
Tab. 20: Österreichische Exporte von potenziellen Umweltschutzgütern in Mrd. € 2002-2011

\begin{tabular}{|c|c|c|c|c|c|c|c|c|c|c|}
\hline & 2002 & 2003 & 2004 & 2005 & 2006 & 2007 & 2008 & 2009 & 2010 & 2011 \\
\hline Abfall & 0,4 & 0,4 & 0,5 & 0,6 & 0,7 & 0,8 & 0,8 & 0,7 & 0,7 & 0,8 \\
\hline Wasser & 0,8 & 0,9 & 1,0 & 1,2 & 1,3 & 1,6 & 1,7 & 1,2 & 1,3 & 1,6 \\
\hline Luft & 0,1 & 0,1 & 0,1 & 0,1 & 0,1 & 0,2 & 0,2 & 0,2 & 0,2 & 0,2 \\
\hline MSR & 0,2 & 0,2 & 0,3 & 0,3 & 0,3 & 0,5 & 0,4 & 0,3 & 0,4 & 0,5 \\
\hline Lärm & 0,1 & 0,1 & 0,1 & 0,1 & 0,2 & 0,2 & 0,3 & 0,2 & 0,2 & 0,2 \\
\hline Klimaschutz & 1,1 & 1,3 & 1,5 & 1,7 & 1,9 & 2,2 & 2,6 & 2,3 & 2,5 & 2,7 \\
\hline Rationelle Energieverwendung & 0,5 & 0,6 & 0,6 & 0,6 & 0,6 & 0,8 & 0,8 & 0,8 & 0,8 & 0,9 \\
\hline Rationelle Energieumwandlung & 0,3 & 0,3 & 0,3 & 0,4 & 0,3 & 0,3 & 0,4 & 0,4 & 0,3 & 0,5 \\
\hline Erneuerbare Energiequellen & 0,4 & 0,5 & 0,6 & 0,7 & 1,0 & 1,1 & 1,3 & 1,1 & 1,3 & 1,3 \\
\hline Exporte pot. Umweltschutzgüter insgesamt & 2,7 & 3,0 & 3,4 & 3,8 & 4,4 & 5,2 & 5,8 & 4,6 & 5,1 & 5,7 \\
\hline Importe pot. Umweltschutzgüter insgesamt & 2,6 & 2,8 & 3,1 & 3,5 & 3,9 & 4,2 & 4,3 & 3,6 & 4,1 & 4,6 \\
\hline Saldo Exporte-Importe pot. Umweltschutzgüter (in Mrd. €) & 0,1 & 0,2 & 0,3 & 0,4 & 0,5 & 1,0 & 1,5 & 1,0 & 1,0 & 1,1 \\
\hline Anteil Umweltgüterexporte an Industriewarenexporten (in \%) & 4,0 & 4,3 & 4,3 & 4,7 & 4,8 & 5,2 & 5,6 & 5,7 & 5,4 & 5,4 \\
\hline
\end{tabular}

Anm.: ${ }^{1} U m$ Doppelzählungen bereinigt.

Quelle: OECD, ITCS - International Trade By Commodities, Rev. 3 (versch. Jgge.). - COMTRADE-Datenbank, Statistik Austria. Berechnungen des NIW.

potenziellen Umweltschutzgütern in Mrd. €. Zunächst zeigt sich, dass alle Gütergruppen im Zeitraum von 2002 bis 2011 steigende Exportwerte aufweisen. Die bereits 2002 bedeutsamste Gütergruppe der potenziellen Klimaschutzgüter sorgte 2011 für einen Exportumsatz von 2,7 Mrd. Euro, wobei etwa die Hälfte hiervon auf erneuerbare Energiequellen entfiel. Eine Betrachtung der Exportwachstumsraten zeigt, dass die Güter für erneuerbare Energiequellen das dynamischste Wachstum aufweisen; von 2002 bis 2011 expandierten diese Exporte um 230 \%, jene der Klimaschutzgüter insgesamt um ebenfalls beachtliche 140 \%. Geringere Wachstumsraten weisen Güter im Zusammenhang mit Luft und Lärm auf, auch wenn diese bereits im Jahr 2002 von einem niedrigeren Niveau gekennzeichnet waren. Der Gesamtexport an potenziellen Umweltschutzgütern stieg von 2,7 Mrd. $€$ um $109 \%$ auf 5,7 Mrd. $€$ im Jahr 2011. Im Vergleich hierzu stiegen die Gesamtausfuhren an Industriewaren im gleichen Zeitraum um ca. 53 \% an. Als Folge davon nahm der Anteil der potenziellen Umweltschutzgüter am Export von Industriewaren von $4 \%$ auf 5,4 \% zu. Weiterhin zeigt Tab. 20, dass der Handel mit Umweltgütern einen positiven Beitrag zur Aktivierung der Warenhandelsbilanz leistet und 2011 einen positive sektorale Handelsbilanz in der Höhe von 1,1 Mrd. $€$ realisiert wurde.

Diese gute Performance zeigt sich auch bei einer Analyse der komparativen Vorteile Österreichs im Vergleich mit anderen Volkswirtschaften. Dabei wird wie üblich die Maßzahl des Revealed Comparative Advantage (RCA) verwendet, die neben den Exporten auch die Importkonkurrenz berücksichtigt. Dazu wird die Import/ Exportrelation einer bestimmten Gütergruppe mit der Import/Exportrelation für alle Industriewaren normiert. Positive Werte zeigen einen komparativen Vorteil, negative Werte einen komparativen Nachteil im Außenhandel für eine bestimmte Gütergruppe an. ${ }^{144} \mathrm{Abb} .45$ stellt die Entwicklung der Spezialisierung bzw. der komparativen Vorteile Österreichs im Export von potenziellen Umweltschutzgütern dar. Für den gesamten Zeitraum von 2002 bis 2011 sind die

144 Siehe Siebert, Lorz (2007). 


\section{Abb. 45: Spezialisierung ausgewählter Länder (RCA-Werte) bei potenziellen Umweltschutzgütern}
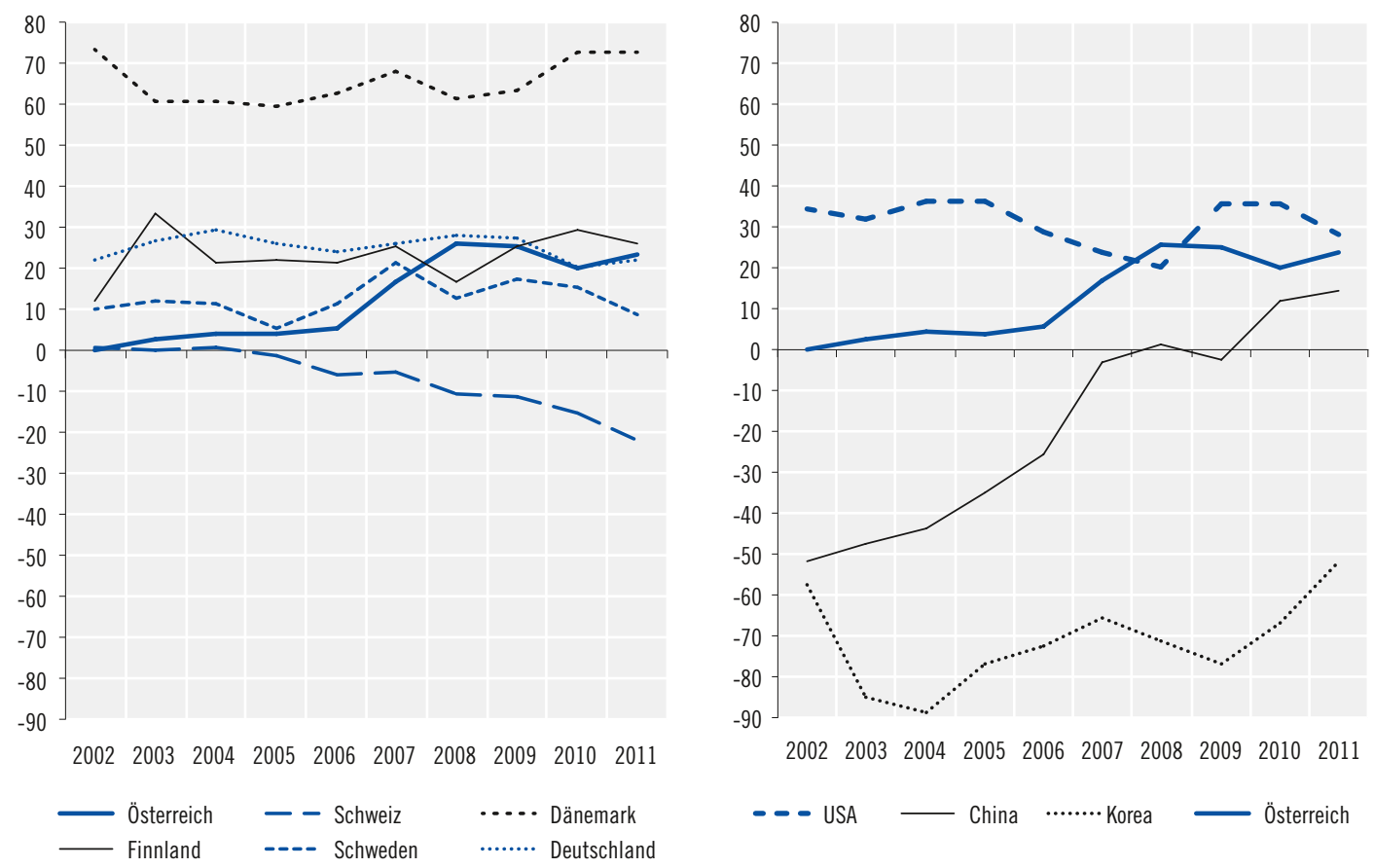

Anm.: RCA: Positives Vorzeichen bedeutet, dass die Export/Import-Relation bei dieser Produktgruppe höher ist als bei Verarbeiteten Industriewaren insgesamt. Quelle: OECD, ITCS - International Trade By Commodities, Rev. 3 (versch. Jgge.). - COMTRADE-Datenbank. Berechnungen des NIW.

RCA-Werte positiv; damit zeigen diese Daten, dass Österreich einen komparativen Vorteil bei potenziellen Umweltschutzgütern besitzt. Weiterhin wird deutlich, dass dieser in den Jahren vor der Krise deutlich zugenommen hat. Im Vergleich zu anderen Ländern wie Deutschland oder Finnland hat dieses Wachstum zu einem catching-up geführt, während Dänemark aufgrund von Weltmarktführungspositionen wie etwa in der Windindustrie noch wesentlich stärkere Spezialisierungsvorteile aufweist. Die Schweiz zeigt einen zunehmenden komparativen Nachteil. Der rechte Teil von Abb. 45 zeigt Österreich im Vergleich mit den USA, China und Korea. Diese beiden asiatischen Staaten investieren massiv in Maßnahmen zur Förderung von Umwelttechnikindustrien. Tatsächlich ergibt sich insbesondere im Falle Chinas eine außerordentlich dynamische Entwicklung, wenngleich diese erst kürzlich von einem komparativen Nachteil in einen komparativen Vorteil mündete. In jedem Fall aber dürfte mit einem Anhalten dieser Dynamik der chinesischen Exporte von potenziellen Umweltschutzgütern in der mittleren Frist zu rechnen sein.

Während Abb. 45 die komparativen Vorteile beim Handel mit potenziellen Umweltschutzgütern insgesamt im Vergleich zu anderen Staaten zeigt, stellt Abb. 46 die komparativen Vorteile (RCA) (rechte Seite) sowie den relativen Welthandelsanteil (RXA) (linke Seite) für die einzelnen Untergruppen der potenziellen Umweltschutzgüter aus Österreich dar. Ein positiver RXA-Wert zeigt, dass Österreich bei der jeweiligen Gütergruppe einen höheren Exportanteil an den österreichischen Gesamtexporten aufweist als der Exportanteil dieser Gütergruppe an den weltweiten Exporten ausmacht. Demgegenüber wird beim RCA nur der nationale Exportsektor berücksichtigt. Der RXA misst also die Abweichungen der österreichischen Exportstruktur von der durchschnittlichen Weltexportstruktur 
und ist damit ein Maß für die Exportspezialisierung. Ein positiver RXA-Wert weist auf eine Exportspezialisierung hin. Abb. 46 zeigt bei beiden Indikatoren für die potenziellen Umweltschutzgüter insgesamt komparative Vorteile bzw. eine positive Exportspezialisierung; diese Vorteile und die Spezialisierung nehmen über den Zeitraum 2001-2011 zu. Deutlich zeigt sich eine Zweiteilung bei den potenziellen Umweltschutzgütern in Abfall, Abwasser, Lärm und Klimaschutz einerseits sowie MSR (Mess-, Steuer- und Regelungstechnik) und Luft andererseits. Bei der ersten Gruppe weist Österreich komparative Vorteile und eine positive Exportspezialisierung auf, bei der anderen Gruppe nicht.

Wenngleich der Handel kein Nullsummenspiel darstellt, ist die Verschiebung von Welthandelsanteilen ein weiterer wichtiger Indikator für die Wettbewerbsfähigkeit von Branchen. Die Entwicklung der Welthandelsanteile von potenziellen Umweltschutzgütern ist in Tab. 21 dargestellt, wobei die Staaten in absteigender Reihenfolge nach ihren Marktanteilsveränderungen geordnet sind. Die letzte Spalte zeigt, dass von den Vergleichsländern vor allem China und etwas weniger Korea Marktanteile gewinnen konnten. Österreich ist die einzige hochentwickelte Volkswirtschaft, die keine Verluste an Weltmarktanteilen hinnehmen musste, sondern ihren Anteil bei ca. 1,7\% stabilisieren konnte. Vor allem die USA verlieren stetig an Weltmarktanteilen.

Eine regionale Verteilung der Exporte von potenziellen Umweltschutzgütern ist in Tab. 22 dargestellt. Die Entwicklung entspricht dabei weitgehend dem wirtschaftspolitischen Ziel einer Diversifizierung der Exportmärkte sowie ei-

Abb. 46: Außenhandelsspezialisierung Österreichs bei potenziellen Umweltschutzgütern, RCA (links) und RXA (rechts)

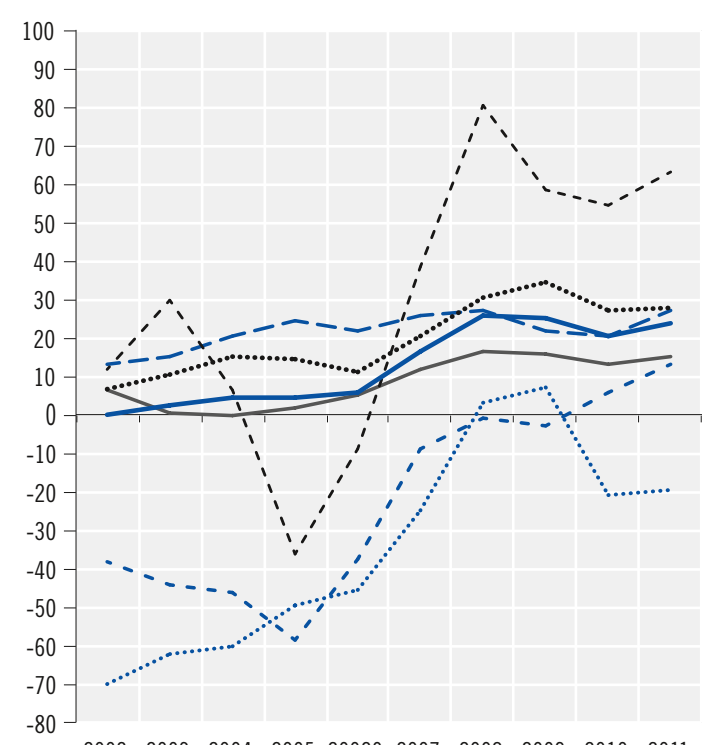

20022003200420052002020072008200920102011

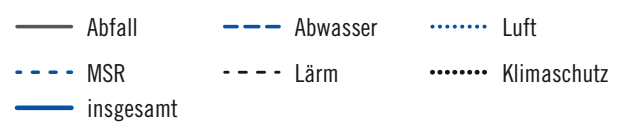

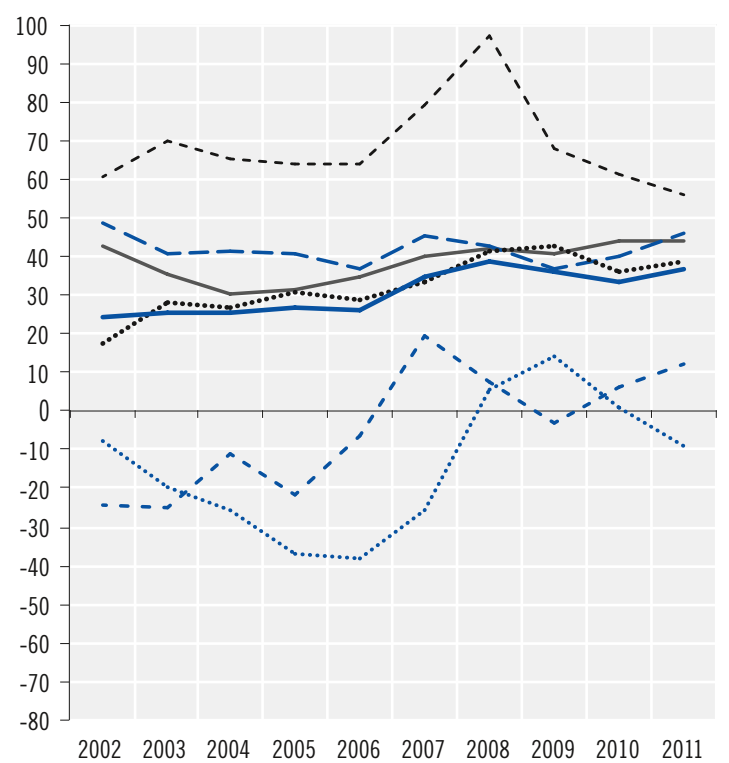

$\begin{array}{llll}\text { — Abfall } & \text { - - } \text { Abwasser } & \text {........ Luft } \\ \text {-..- MSR } & \text {-.-. Lärm } & \text {........ Klimaschutz }\end{array}$

Anm.: RXA: Positives Vorzeichen bedeutet, dass der Anteil am Welthandel bei dieser Produktgruppe höher ist als bei Verarbeiteten Industriewaren insgesamt. RCA: Positives Vorzeichen bedeutet, dass die Export/Import-Relation bei dieser Produktgruppe höher ist als bei Verarbeiteten Industriewaren insgesamt. MSR: Mess-, Steuer- und Regelungstechnik.

Quelle: OECD, ITCS - International Trade By Commodity Statistics, Rev. 3 (versch. Jgge.). - COMTRADE-Datenbank. Berechnungen und Schätzungen des NIW. 
Tab. 21: Welthandelsanteile und deren Veränderung bei potenziellen Umweltschutzgütern

\begin{tabular}{|c|c|c|c|c|c|c|c|c|c|c|c|}
\hline & 2002 & 2003 & 2004 & 2005 & 2006 & 2007 & 2008 & 2009 & 2010 & 2011 & $\begin{array}{l}\text { Veränderung 2002-2011 } \\
\text { in Prozentpunkten }\end{array}$ \\
\hline China & 4 & 4,7 & 5,7 & 7 & 8,3 & 9,7 & 10,9 & 11,5 & 14 & 14,5 & 10,5 \\
\hline Korea & 1,7 & 1,3 & 1,4 & 1,6 & 1,7 & 1,7 & 1,7 & 2,1 & 2,1 & 2,4 & 0,7 \\
\hline Österreich & 1,7 & 1,8 & 1,9 & 1,8 & 1,8 & 2 & 2 & 1,9 & 1,7 & 1,7 & 0 \\
\hline Finnland & 1,2 & 1,4 & 1,1 & 1,1 & 1,1 & 1,2 & 1,1 & 0,8 & 0,9 & 0,9 & $-0,3$ \\
\hline Deutschland & 15,5 & 15,5 & 15,5 & 15,7 & 15,8 & 15,6 & 16,1 & 15,7 & 15 & 15,2 & $-0,3$ \\
\hline Schweden & 2 & 2,1 & 2,1 & 1,8 & 1,8 & 1,9 & 1,9 & 1,7 & 1,6 & 1,6 & $-0,4$ \\
\hline Schweiz & 1,9 & 1,8 & 1,8 & 1,7 & 1,5 & 1,6 & 1,6 & 1,7 & 1,5 & 1,4 & $-0,5$ \\
\hline Dänemark & 2,1 & 2 & 1,8 & 1,8 & 1,8 & 1,9 & 1,9 & 1,9 & 1,7 & 1,5 & $-0,6$ \\
\hline USA & 14,4 & 12,4 & 11,8 & 12 & 11,3 & 10,6 & 10,4 & 11,3 & 11 & 10,8 & $-3,6$ \\
\hline
\end{tabular}

Anm.: Anteil der Ausfuhren eines Landes an den Weltausfuhren in \%. Die Weltausfuhren sind berechnet aus den Exporten der OECD-Länder, Chinas und Hongkongs zuzüglich der Importe aus den nicht genannten Ländergruppen.

Quelle: OECD, ITCS - International Trade By Commodities, Rev. 3 (versch. Jgge.). - COMTRADE-Datenbank. Berechnungen des NIW.

Tab. 22: Regionale Struktur der Exporte von potenziellen Umweltschutzgütern

\begin{tabular}{|l|r|r|r|r|r|r|r|r|r|r|} 
& $\mathbf{2 0 0 2}$ & $\mathbf{2 0 0 3}$ & $\mathbf{2 0 0 4}$ & $\mathbf{2 0 0 5}$ & $\mathbf{2 0 0 6}$ & $\mathbf{2 0 0 7}$ & $\mathbf{2 0 0 8}$ & $\mathbf{2 0 0 9}$ & $\mathbf{2 0 1 0}$ & $\mathbf{2 0 1 1}$ \\
\hline EU-27 & 74,1 & 73,2 & 72,1 & 69,0 & 69,8 & 68,0 & 68,3 & 64,9 & 66,5 & 63,8 \\
\hline USA & 4,5 & 3,4 & 3,1 & 3,5 & 3,8 & 3,5 & 3,7 & 3,5 & 4,5 & 6,1 \\
BRIC & 2,6 & 3,2 & 3,9 & 6,1 & 5,5 & 6,4 & 6,6 & 7,0 & 7,0 & 7,0 \\
\hline übrige Regionen & 18,8 & 20,2 & 20,8 & 21,4 & 20,8 & 22,1 & 21,4 & 24,6 & 22,0 & 23,1 \\
\hline Welt & 100 & 100 & 100 & 100 & 100 & 100 & 100 & 100 & 100 & 100
\end{tabular}

Quelle: OECD, ITCS - International Trade By Commodities, Rev. 3 (versch. Jgge.). - COMTRADE-Datenbank. Berechnungen des NIW.

ner steigenden Präsenz in den BRIC-Staaten, um an deren Wachstumsvorsprung zu partizipieren. Der 2002 mit ca. 74 \% dominante Anteil der EU27 ging bis zum Jahr 2011 um etwa 10 Prozentpunkte auf ca. $64 \%$ zurück. Im Vergleich hierzu belief sich der Anteil der gesamten Warenexporte in die EU-27 Staaten auf ca. $70 \%$ im Jahr 2011. In Bezug auf die BRIC-Staaten ist das Bild genau umgekehrt: Mit $7 \%$ ist der Export von potenziellen Umweltschutzgütern in diesen Ländern höher als im Vergleich zum gesamten Warenexport $(6,3 \%) .{ }^{145}$

Zusammenfassend ergibt sich damit ein positives Bild der Wettbewerbsfähigkeit der österreichischen Umweltindustrie. Es zeigen sich zunehmende komparative Vorteile und positive
Exportspezialisierungen sowie ein überdurchschnittliches Wachstum der Exporte bei einer deutlich positiven sektoralen Handelsbilanz. Österreich konnte seine Position auf den Weltmärkten bislang trotz massiver Aufholprozesse von China und anderen aufstrebenden Volkswirtschaften halten und seine Exportstruktur bei potenziellen Umweltschutzgütern vorteilhaft diversifizieren. Die gute Positionierung im Eco-Innovation Scoreboard verweist auf attraktive Rahmenbedingungen. Damit besitzt Österreich eine ausgezeichnete Ausgangsposition im Wettbewerb um industrielle Kapazitäten von Umwelttechnikindustrien. Diese gilt es zu nützen und in Wertschöpfung und Exporterfolge umzusetzen.

145 Siehe BMWFJ (2012). 


\subsection{Resümee}

Die Rolle der Industrie und deren Beitrag für Innovation, Exporte und Beschäftigung stehen erneut im Zentrum der wirtschaftspolitischen Debatten. Zahlreiche Länder und auch die Europäische Kommission verfolgen eine Strategie der Reindustrialisierung.

Die globale Verschiebung industrieller Produktionskapazitäten zeigt für China in den letzten Jahren ein außergewöhnlich dynamisches Industriewachstum. Gleichzeitig ist der Output der Industrie pro Kopf der Bevölkerung nach wie vor in den OECD-Staaten wesentlich höher als in China, Indien oder Brasilien.

Österreich gehört im internationalen Vergleich zur Gruppe der Länder mit den höchsten Industrieanteilen. Dennoch hat der Industrieanteil im langfristigen Vergleich abgenommen, wenngleich seit etwa 15 Jahren - abgesehen von der Krise - ähnlich wie in Deutschland oder der Schweiz eine Stabilisierung des Industrieanteils an der Wertschöpfung stattgefunden hat. Die Beschäftigung hingegen fällt sowohl nach der absoluten Zahl als auch nach dem Anteil an der Gesamtbeschäftigung. In Bezug auf den technologischen Wandel innerhalb der Industrie ergibt sich eine starke Zunahme von Industrien im mittleren Technologiesegment. Wenngleich der Anteil der Hochtechnologie nach wie vor niedrig ist, so stellt sich diese mitteltechnologische Industrie in Österreich als relativ F\&E-intensiver als in relevanten Vergleichsstaaten dar. Generell erlebte die österreichische Industrie seit 1990 einen äußerst erfolgreichen Prozess der Internationalisierung, der Ausdruck ihrer hohen Wettbewerbsfähigkeit ist und der ein weiteres Abschmelzen des industriellen Kerns verhindert hat.

Die Industrie trägt in einem deutlich überproportionalen Ausmaß zum technologischen Wandel einer Volkswirtschaft bei. Ein Großteil der F\&E- und Innovationsaktivitäten wird von Industrieunternehmen - freilich oftmals in enger Kooperation mit Dienstleistungsunternehmen durchgeführt. Unterschiedliche Technologien und Wettbewerbsintensitäten dürften die wesentlichen Erklärungsfaktoren hierfür sein. Diese überlegene Innovationsperformance setzt sich in dem Maße fort, als die Industrie ein Motor des Produktivitätswachstums ist. Ähnliches lässt sich auch für den Exportsektor feststellen. Die Rolle der Industrie für F\&E sowie Innovation wird auch dadurch deutlich, dass die Innovation Leaders über durchwegs höhere Industrieanteile als die Innovation Followers, Moderate Innovators und Modest Innovators verfügen, obwohl diese durch ein niedrigeres Entwicklungsniveau gekennzeichnet sind.

Österreich ist sowohl hinsichtlich seiner F\&Esowie seiner Produktions- und Vermarktungskapazitäten gut bei Schlüsseltechnologien positioniert. In den vergangenen zehn Jahren haben die österreichischen Industrieunternehmen ihre Patentaktivitäten im Bereich industrieller Schlüsseltechnologien deutlich ausgeweitet. Dadurch hat Österreich an Gewicht innerhalb der weltweiten Produktion neuen technologischen Wissens in diesen für die künftige Wettbewerbsfähigkeit der Industrie besonders wichtigen Technologiefeldern gewonnen. Mit der Stärkung der Position bei Patentanmeldungen ging auch eine kräftige Ausweitung der Exporte Österreichs von schlüsseltechnologiebasierten Produkten einher.

Eine empirische Analyse der österreichischen Umweltindustrie zeigt eine positive Dynamik und eine über die Zeit zunehmende Wettbewerbsfähigkeit dieser Branchen. Durch die hohen Wachstumserwartungen auf den Weltmärkten für Umweltschutzgüter eröffnen sich hier realistische Optionen für eine signifikante Expansion moderner und ökologisch nachhaltiger industrieller Aktivitäten.

Die Industrie wird auch in Zukunft eine wichtige Rolle für die Performance von Innovationssystemen in hochentwickelten Ländern wie Österreich spielen. Diese These folgt vor allem aus dem Argument, dass Produkt- und Prozessinnovationen gerade in den technologisch progressiven Branchen oftmals zueinander komplementär sind. Daraus ergibt sich die Notwendigkeit der räumlichen Nähe zwischen F\&E- und Designak- 
tivitäten sowie Produktionsstandorten. ${ }^{146}$ Anders formuliert: Eine Abwanderung der Produktion kann mittelfristig eine Abwanderung von F\&EAbteilungen nach sich ziehen. ${ }^{147}$

Die österreichische Industrie ist eine Erfolgsgeschichte. Ihr Erfolg beruht wesentlich auf motivierten leistungsbereiten und kompetenten UnternehmerInnen und FacharbeiterInnen. Hinzu kommen Rahmenbedingungen die insbesondere durch die Sozialpartnerschaft und die europäische Integration vorteilhaft gestaltet sind. ${ }^{148}$ Die Ostöffnung und der Binnenmarkt haben durch den steigenden Wettbewerbsdruck zu einer Dynamisierung der heimischen Industrieunternehmen beigetragen. In Zukunft wird eine weitere Verbesserung des Humankapitals von entscheidender Bedeutung sein. Schulen und Lehrlingsausbildung, aber auch die Ausbildung von NaturwissenschaftlerInnen und IngenieurInnen an Universitäten und Fachhochschulen ist besondere Aufmerksamkeit zu schenken. Die Industriepolitik, die in Österreich ja im Wesentlichen und richtigerweise Innovationspolitik ist, kann Impulse und Anreize für eine weitere Verbesserung der internationalen Wettbewerbsfähigkeit der Industrie setzen. Dafür sind die notwendigen Instrumente und Institutionen vorhanden. Es gilt also vor allem den erfolgreichen Weg der letzten Jahre weiter zu gehen und flexibel und koordiniert auf neue Herausforderungen für die österreichische Industrie zu reagieren.

146 Siehe Tassey (2010), Aghion et al. (2011), Psiano, Shih (2012). 


\section{Innovation auf Unternehmensebene}

Die kontinuierliche Umsetzung von Innovationen ist die treibende Kraft für einen dauerhaften Unternehmenserfolg und somit letztlich für wirtschaftliches Wachstum und Beschäftigung. Diesbezüglich werden die Auswirkungen von F\&EAusgaben auf die Beschäftigungsentwicklung und Exportorientierung von Unternehmen in Österreich untersucht. Darüber hinaus werden die Daten der europäischen Innovationserhebung (Community Innovation Survey - CIS) genutzt, um das unternehmerische Innovationsverhalten in Österreich zu analysieren und international zu vergleichen. Der vorliegende Beitrag widmet sich dabei zunächst der Innovationsperformance österreichischer Unternehmen im internationalen Vergleich, bevor er tiefergehend branchen- und vor allem größenspezifische Unterschiede im Innovationsverhalten analysiert. Schließlich werden schnell wachsende Jungunternehmen in Österreich und Deutschland hinsichtlich der Nachhaltigkeit ihres Wachstumspfades untersucht. Hintergrund ist, dass diesen Unternehmen eine wesentliche Rolle für den Strukturwandel und die Generierung von Beschäftigung zugeschrieben wird. Weswegen auch die EU-Kommission im Rahmen von „Europe 2020" dem Thema „schnell wachsende Unternehmen" eine verstärkte Aufmerksamkeit schenkt und ab 2013 einen entsprechenden Indikator einführen wird.

\subsection{Die Wirkung von F\&E auf die Beschäftigung}

Spätestens seit Beginn der 1990er Jahre ist es evident, dass Forschung, technologische Entwicklung und Innovation mit Wachstum verbunden sind. Auch auf europäischer Ebene ist es Common Sense, dass Innovationen für den Wirtschaftsstandort Europa als wichtiger Wachstumsmotor angesehen wird: „Europe's competitivness, our capacity to create millions of new jobs to replace those lost in the crisis and overall, our future standard of living depends on our ability to drive innovation in products, services, business and social processes and models. "149

Zwar wird proklamatisch immer wieder der positive Zusammenhang zwischen F\&E, Innovation, Wachstum und Beschäftigung postuliert, aber die empirischen Evidenzen sind mitunter schwierig zu deuten. Insbesondere auf gesamtwirtschaftlicher Ebene ist evident, dass der Wirkungszusammenhang zwischen Inputs (Forschung) und Outputs (Wachstum, Beschäftigung) komplex und mehrdimensional ist. ${ }^{150}$ Neben der Frage nach dem Einfluss der konjunkturellen Entwicklung auf die F\&E-Ausgaben sind diese wohl noch mehr als alle anderen Investitionen von Zukunftserwartungen abhängig und verhalten sich daher nicht unbedingt parallel zum Konjunkturzyklus. Denn die Effekte von Forschung, technologischer Entwicklung und Innovation (FTI) auf die Schaffung neuer Arbeitsplätze hängen insbesondere vom betrachteten Zeitraum ab, von den jeweiligen neuen Technologien sowie von der Wettbewerbssituation des entsprechenden Marktes und dem Strukturanpassungspotenzial des Arbeitsmarktes. Es ist daher kaum möglich, von einem direkten linearen Zusammenhang zwischen FTI und neuen Arbeitsplätzen in der Gesamtwirtschaft zu sprechen. Dennoch kommen die meisten gesamtwirtschaftlichen

149 Europäische Kommission (2010a), S. 2.

150 Siehe dazu Schibany, Gassler (2010). 
Modelle zur Beschäftigungswirkung von FTI zu einem positiven Zusammenhang, da technischer Fortschritt unterm Strich wachstumsstärkend wirkt, und davon auch die Beschäftigung über den Zusammenhang von Outputwachstum und Beschäftigung betroffen ist - trotz der komplexen Wirkungskanäle einer kleinen offenen Volkswirtschaft. ${ }^{151}$

Ähnlich wie die Abschätzung von FTI auf Beschäftigung und Wachstum auf gesamtwirtschaftlicher Ebene liegen auch theoretische Annahmen hinter den Modellen des Zusammenhangs von FTI und Beschäftigung auf Unternehmensebene. Allerdings ist es auf Firmenebene nur in eingeschränktem Ausmaß möglich, Verdrängungseffekte abzuschätzen. Es kann nur schwer unterschieden werden, ob die positiven Umsatz- und Beschäftigungssteigerungen eines innovativen und F\&E-betreibenden Unternehmens auf eine reine Marktexpansion zurückzuführen ist, oder ob diese Entwicklung die Folge eines Verdrängungseffektes ist. ${ }^{152}$

Dennoch lassen sich auf Unternehmensebene viel deutlicher die Wirkungszusammenhänge von FTI und Beschäftigung sowohl theoretisch als auch empirisch erfassen. Natürlich ist auch auf der Unternehmensebene die Entscheidung, in $\mathrm{F} \& \mathrm{E} z \mathrm{u}$ investieren, von vielen Einflussfaktoren abhängig, wie die jeweilige Nachfrage- und Marktentwicklung, die volkswirtschaftlichen Rahmenbedingungen oder ob diese Investition tatsächlich die gewünschten Erträge bringt. Der Tenor der meisten Studien lautet jedoch, dass sich FTI für Unternehmen jedenfalls lohnt und dass dies auch deutlich positive Auswirkungen auf die Beschäftigung hat.

Für Österreich haben in einer jüngst veröffentlichten Studie Falk et al. (2013) die Auswirkun- gen von F\&E-Ausgaben auf die Beschäftigungsentwicklung und Exportorientierung untersucht. Auf Firmenebene darf diese Studie - in der insbesondere auf die Entwicklung vor und den Zeitraum nach der Wirtschafts- und Finanzkrise eingegangen wird - zu den fundiertesten Studien zur Wachstumsdynamik von F\&E-betreibenden Unternehmen gezählt werden.

\section{Datenbasis}

Datenbasis sind die Firmendaten der Forschungsförderungsgesellschaft (FFG) mit Informationen zu ca. $700 \mathrm{~F} \& \mathrm{E}$-betreibenden Unternehmen für den Zeitraum 2009 bis 2011. Diese Daten, welche aus dem Förderansuchen der Basisprogramme stammen, enthalten Angaben zu Umsatz, Beschäftigung, F\&E-Ausgaben, F\&E-Personal, Firmenalter, Exportquote, Cash-Flow und regionale Zugehörigkeit. Die Daten sind von hoher Qualität und umfassen insbesondere auch Kleinstunternehmen mit weniger als zehn Beschäftigten sowie auch Dienstleistungsunternehmen.

\subsubsection{F\&E-Intensität und Beschäftigungswachstum vor und nach der Krise}

Falk et al. (2013) gehen zunächst der Frage nach, ob Unternehmen mit hoher F\&E-Intensität eine höhere Beschäftigungsintensität aufweisen. Dabei wird die Höhe der F\&E-Aktivitäten als Anteil der F\&E-Ausgaben am Umsatz gemessen und die Unternehmen in drei Klassen unterteilt: (i) weniger als $3 \%$, (ii) drei bis einschließlich $15 \%$, sowie (iii) mehr als $15 \%$. Die folgende Abb. 47 lässt ein eindeutiges Bild erkennen. Das Beschäftigungswachstum steigt mit der F\&E-Intensität kontinuierlich an.

\footnotetext{
151 "The long-run economic impact of innovation on employment is clearly not negative; many decades, and even centuries, of innovation in advanced economies have been accompanied by employment growth instead oft he ever-decreasing levels of jobs that many predicted." (Harrison 2008, S. 2).

152 Vgl. Harrison (2008).
} 
Abb. 47: Jährliche Beschäftigungszuwächse nach F\&E-Intensität in \%, 2006-2011

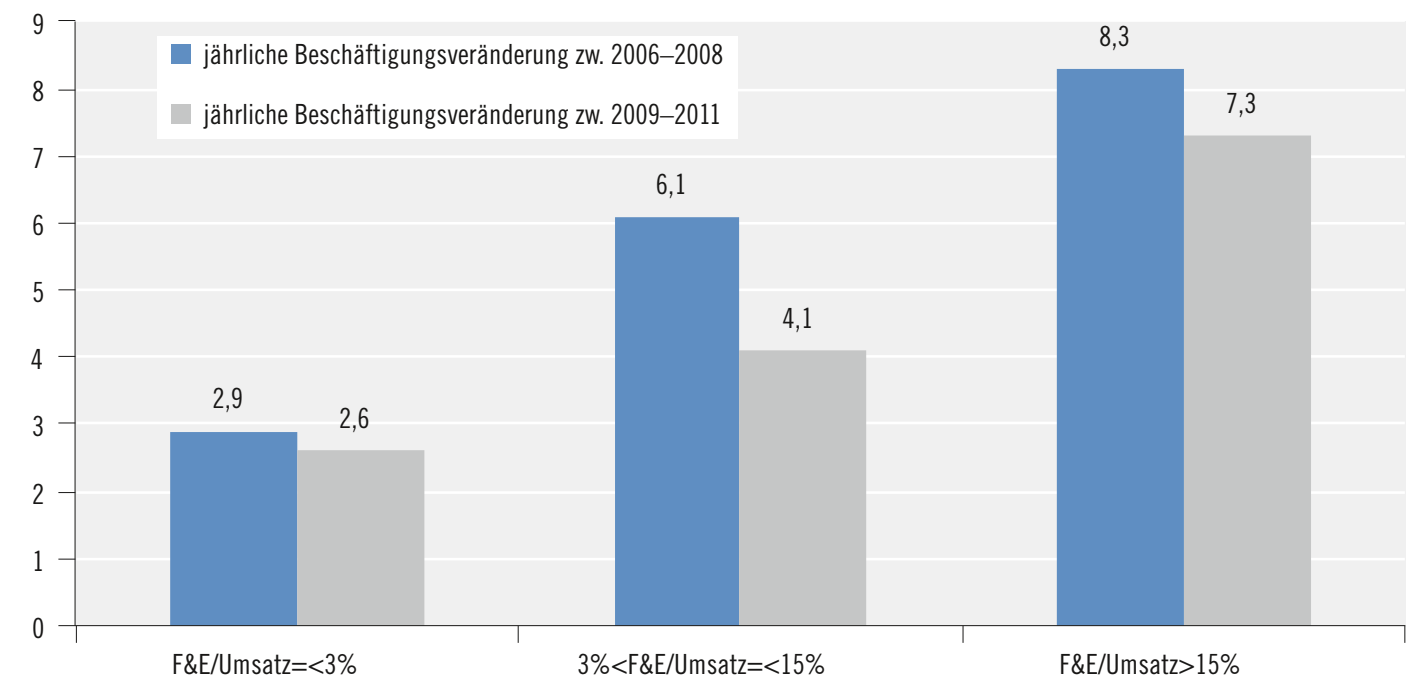

Anm.: Auswertung basiert auf 1.752 (2006-2008) und 752 (2009-2011) Firmenbeobachtungen.

Quelle: FFG-Stammdaten. Berechnungen WIFO, Falk et al. (2013).

Zwischen 2006 und 2008 wiesen Unternehmen mit geringer F\&E-Intensität ein durchschnittliches jährliches Beschäftigungswachstum von 2,9\% auf. Im gleichen Zeitraum erzielten Unternehmen mit mittleren F\&E-Ausgaben ein mehr als doppelt so hohes Beschäftigungswachstum von $6,1 \%$. Einen noch höheren Wert erreichten Unternehmen mit einer F\&E-Intensität von über $15 \%$. Zwar bremste die Finanz- und Wirtschaftskrise die Beschäftigungsdynamik deutlich ein, jedoch fiel bei Unternehmen mit einer hohen F\&E-Intensität der dämpfende Effekt schwächer aus.

\subsubsection{F\&E-Intensität und Beschäftigungswachstum nach Unternehmensgröße}

Um ein detailliertes Bild über den Zusammenhang von F\&E-Ausgaben und Beschäftigungswir- kung zu erhalten, werteten Falk et al. (2013) die Daten auch getrennt nach Unternehmensgrößenklassen aus. Dabei wurden die Unternehmen hinsichtlich ihrer Firmengröße in drei Gruppen unterteilt: (i) weniger als 50 Beschäftigte, (ii) zwischen 50 und 249 Beschäftigte und (iii) 250 Beschäftigte und mehr. Neugegründete und junge Unternehmen, welche im Jahr 2006 oder später gegründet wurden, sind in die Analyse nicht einbezogen worden, weil diese Unternehmen häufig durch ein rasches Beschäftigungswachstum charakterisiert sind und somit nicht mit den etablierten Unternehmen vergleichbar sind. Auch bei diesem Selektionskriterium bestätigt die deskriptive Statistik einen positiven Zusammenhang zwischen F\&E-Intensität und Beschäftigungsentwicklung in allen Unternehmensgrößenklassen. 
Abb. 48: Jährlicher Beschäftigungszuwachs nach F\&E-Intensität und Firmengröße in \%, 2009-2011

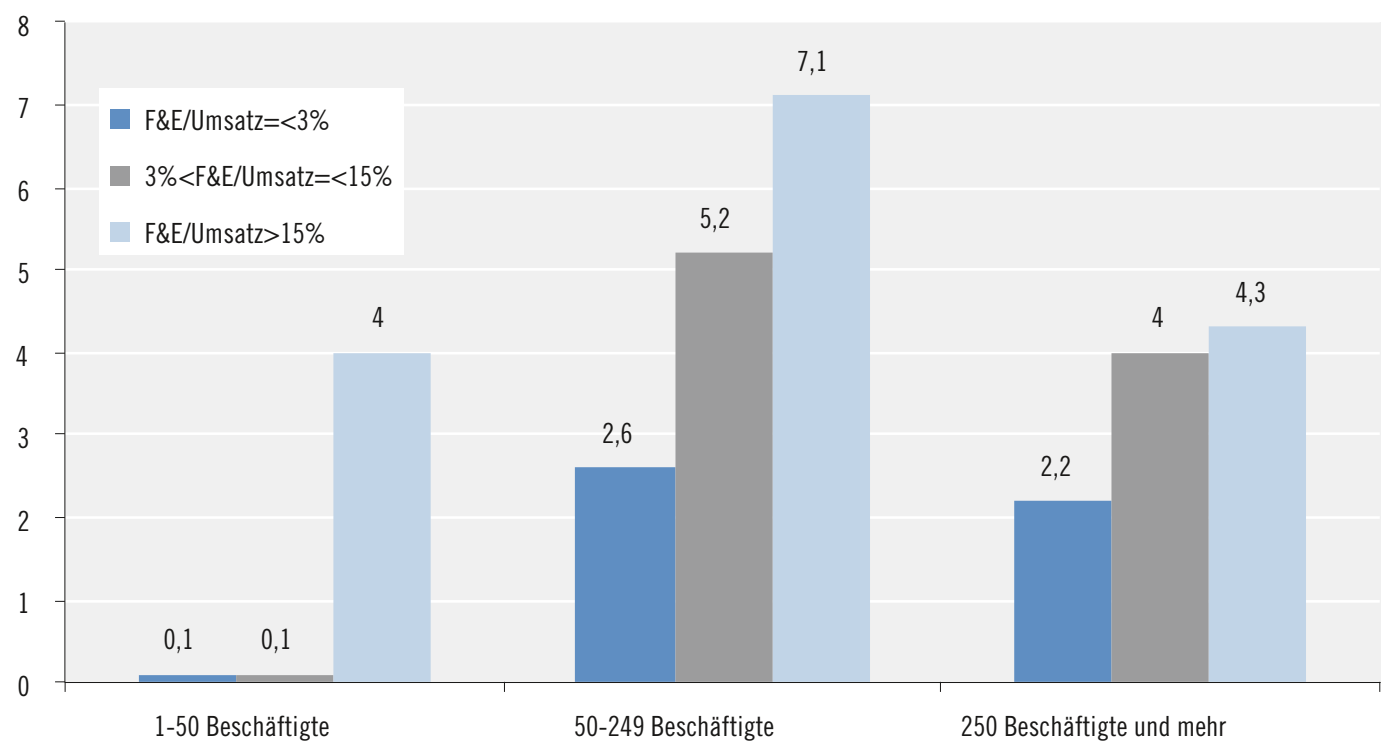

Anm.: Anzahl der Beobachtungen: 615. Stichprobe enthält keine jungen Unternehmen (Gründungsjahr 2006 oder jünger). Durchschnitt ist als Median über alle Unternehmen gemessen.

Quelle: FFG-Stammdaten. Berechnungen WIFO, Falk et al. (2013).

Die Ergebnisse zeigen, dass zwischen 2009 und 2011 Unternehmen mit mittlerer und höherer F\&E-Intensität, unabhängig von der Beschäftigtenzahl, ein beträchtlich höheres Beschäftigungswachstum aufwiesen als Unternehmen mit niedrigerer F\&E-Intensität. Der höchste Beschäftigungszuwachs konnte bei forschungsintensiven Unternehmen mit 50 bis 249 Beschäftigten beobachtet werden. Unternehmen in dieser Größenklasse, die im Jahr 2009 zwischen drei und $15 \%$ bzw. mehr des Umsatzes in F\&E-Aktivitäten investierten, erzielten im Zeitraum 2009 bis 2011 mit 5,2 \% bzw. 7,1 \% Jahresdurchschnitt die höchsten Beschäftigungszuwächse. Auffallend ist insbesondere der Vergleich zwischen den Kleinunternehmen mit geringer F\&E-Intensität und jenen mit einer F\&E-Intensität von über $15 \%$. Innerhalb dieser Gruppe wurde das Beschäftigungswachstum somit ausschließlich von den innovativsten, schnell wachsenden Unternehmen („Gazellen“) getragen.
Als Ergänzung zu den deskriptiven Ergebnissen führten Falk et al. (2013) auch eine Regressionsanalyse auf Basis von Querschnittsdaten durch. Die empirischen Ergebnisse auf Basis der Medianregression zeigen, dass die F\&E-Personalintensität (definiert als Anteil der F\&E-MitarbeiterInnen an der Gesamtbeschäftigung) der Unternehmen zu Beginn eines Zeitraumes (2006 und 2009) einen positiven und signifikanten Einfluss auf das Beschäftigungswachstum in den nächstfolgenden Jahre hat. Das heißt laut Falk et al. (2013), dass F\&E-intensive Unternehmen bei vergleichbarer Größe und vergleichbarem Unternehmensalter schneller wachsen als nicht F\&Eintensive Unternehmen. Für den jüngsten Zeitraum 2009 bis 2011 zeigen die Ergebnisse, dass Unternehmen mit einer um zehn Prozentpunkte höheren F\&E-Personalintensität eine um 0,9 Prozentpunkte höhere Wachstumsrate der Beschäftigung aufweisen. 


\subsubsection{F\&E-Intensität und Beschäftigungswachstum nach Unternehmensalter}

Bei der Frage nach dem möglichen Einfluss des Unternehmensalters auf die Beschäftigungswirkung lässt sich auf noch höherem Niveau dieser positive Zusammenhang feststellen. Denn sehr forschungsintensive Jungunternehmen (im Jahr 2006 oder später gegründet) weisen jährliche Beschäftigungsraten von $22,5 \%$ auf, was doppelt so hoch ist verglichen mit jungen Unternehmen mit mittleren F\&E-Ausgaben. Auch bei Jungunternehmen mit niedrigen $\mathrm{F} \& \mathrm{E}$-Ausgaben kam es mit $+4,5 \%$ zu einem überraschend starken $\mathrm{Zu}$ wachs der Beschäftigten, während die Beschäftigung im Durchschnitt aller Altersklassen um jährlich -0,3\% sank. Freilich zeigt dieses Ergebnis gleichsam auch ein natürliches Ergebnis, weil junge und neu gegründete Unternehmen zunächst nur Arbeitsplätze schaffen und noch keine abbauen können, solange sie noch keine unselbstständigen Beschäftigten aufweisen. Dies ist bei etablierten und großen Unternehmen naturgemäß anders.

Werden die Jungunternehmen weiter nach ihrem Gründungsdatum unterschieden, so zeigt sich die zunehmende Bedeutung der F\&E-Intensität speziell bei jungen Unternehmen. Gleichzeitig deutet es auch auf einen Verjüngungsprozess der österreichischen Wirtschaft hin, wie Falk et al. (2013) betonen (siehe Abb. 49).

Unternehmen, die im Jahr 2008 oder später gegründet wurden, wiesen im Zeitraum 2009 bis 2011 durchgängig eine höhere Beschäftigungsdynamik auf als Unternehmen, die zwei bzw. vier Jahre früher gegründet wurden. Besonders stark zeigt sich dieser Effekt bei Jungunternehmen mit F\&E-Ausgaben von mehr als $15 \%$. Sie erzielten mit einem durchschnittlichen Beschäftigungswachstum von 41,4\% einen beinahe doppelt so hohen Wert wie die nur zwei Jahre älteren Unternehmen mit ähnlicher F\&E-Intensität. Es zeigt, dass besonders junge forschungsintensive Unternehmen in den letzten Jahren ihre Beschäftigten-

Abb. 49: Jährlicher Beschäftigungszuwachs nach F\&E-Intensität und Unternehmensalter zwischen 2009-2011

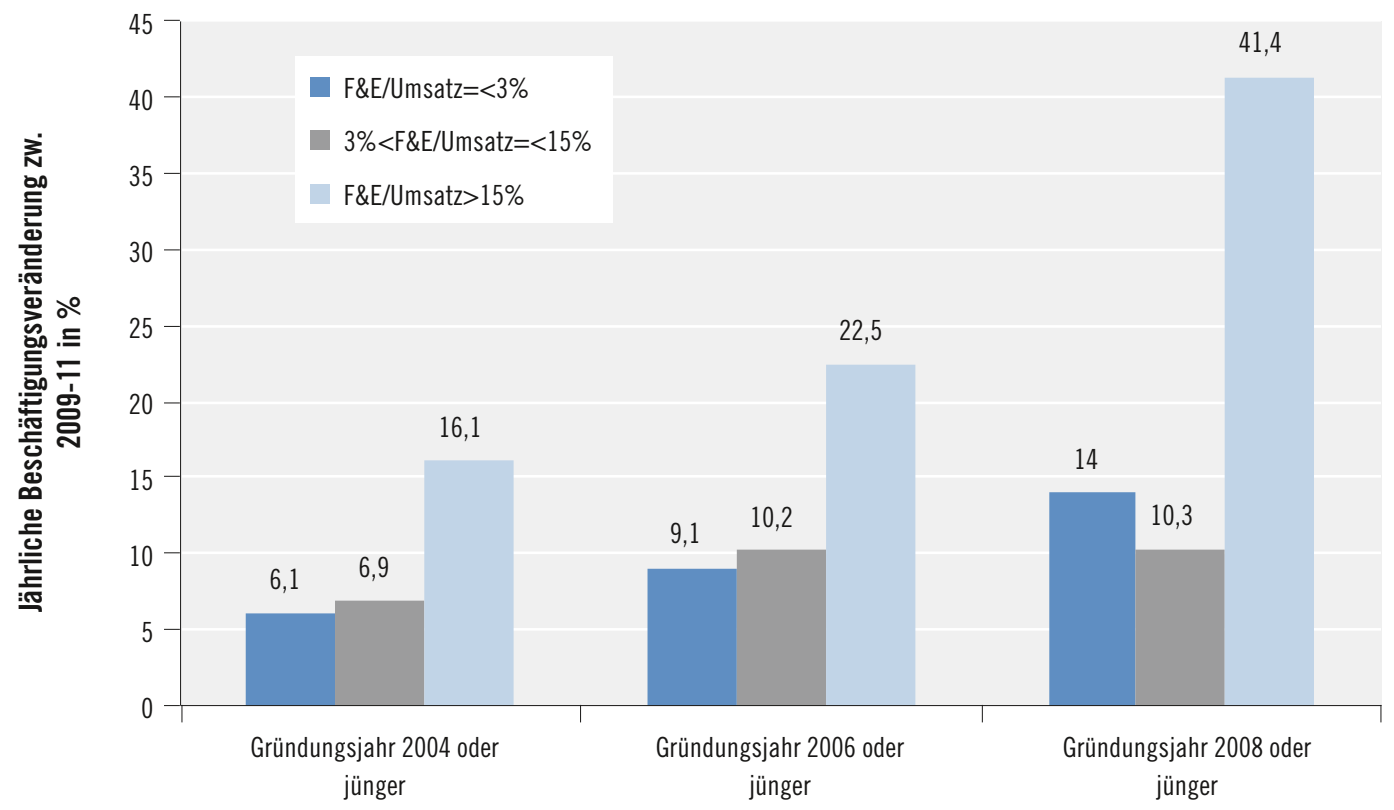

Anm.: Durchschnitt ist als Median über alle Unternehmen gemessen. Anzahl der Unternehmen: 227 (Gründungsjahr 2004 oder jünger), 161 (Gründungsjahr 2006 oder jünger) und 87 (Gründungsjahr 2008 oder jünger).

Quelle: FFG-Bilanzdaten. WIFO Berechnungen, Falk et al. (2013). 
zahl substantiell steigern konnten und damit einen wichtigen Platz im strukturellen Wandel der österreichischen Wirtschaft einnehmen.

\subsubsection{F\&E-Intensität und Exportquote nach Unternehmensgröße}

Die folgende Abb. 50 zeigt ebenfalls einen engen Zusammenhang zwischen F\&E-Intensität und Exportneigung. Die Berechnungen von Falk et al. (2013) ergeben einerseits einen positiven Zusammenhang zwischen Forschungsintensität und Exportneigung sowie andererseits die Unternehmensgröße als entscheidenden Faktor in Sachen Export. Mittelgroße Unternehmen erzielten im Beobachtungszeitraum 2009 bis 2011, selbst bei geringer F\&E-Intensität von weniger als $3 \%$, eine beträchtlich höhere Exportquote von $53 \%$ und damit mehr als das Eineinhalbfache der forschungsstärksten Kleinbetriebe. Bei einer F\&EIntensität von zwischen 3 und $15 \%$ finden diese Unternehmen schon $68 \%$ der Nachfrage im Ausland. Selbiges gilt für Großunternehmen mit 250 Beschäftigten und mehr. In dieser Kategorie exportieren jene Unternehmen mit mittlerer F\&E-Intensität 89 \% ihrer Güter ins Ausland.
Falk et al. (2013) zeigen auf Basis eines quadratischen Regressionsmodells einen nicht-linearen Zusammenhang zwischen Exportquote und der F\&E-Personalintensität. Das heißt, es steigt die Exportquote zunächst mit Zunahme der F\&EPersonalintensität kontinuierlich an, dieser positive Effekt des F\&E-Einsatzes auf die Exportquote schwächt sich aber mit zunehmender Personalintensität $\mathrm{ab}$. Ab einer F\&E-Personalintensität von $45 \%$ kommen Unternehmen bei der durchschnittlichen Exportquote über den Sättigungspunkt von $52 \%$ nicht mehr hinaus - die Kurve flacht ab. Selbst nahezu reine Forschungsunternehmen mit drei Viertel F\&E-Beschäftigten erreichen im Durchschnitt keinen höheren Wert.

Falk et al. (2013) weisen auch auf die einschränkenden Faktoren in den vorliegenden Analysen hin. Denn die Datenbasis umfasst nur einen gewissen Teil der österreichischen Betriebe, das bedeutet solche F\&E-betreibenden Unternehmen, welche in der Datenbasis der FFG erfasst sind. In der Analyse wurden die Faktoren Firmengröße, Gründungsdatum und Beobachtungszeitraum berücksichtigt, um die Effekte der F\&E-Intensität auf die Beschäftigungszuwächse und Exportquoten statistisch zu erfassen. Daher

Abb. 50: Exportquote und F\&E-Intensität 2009-2011

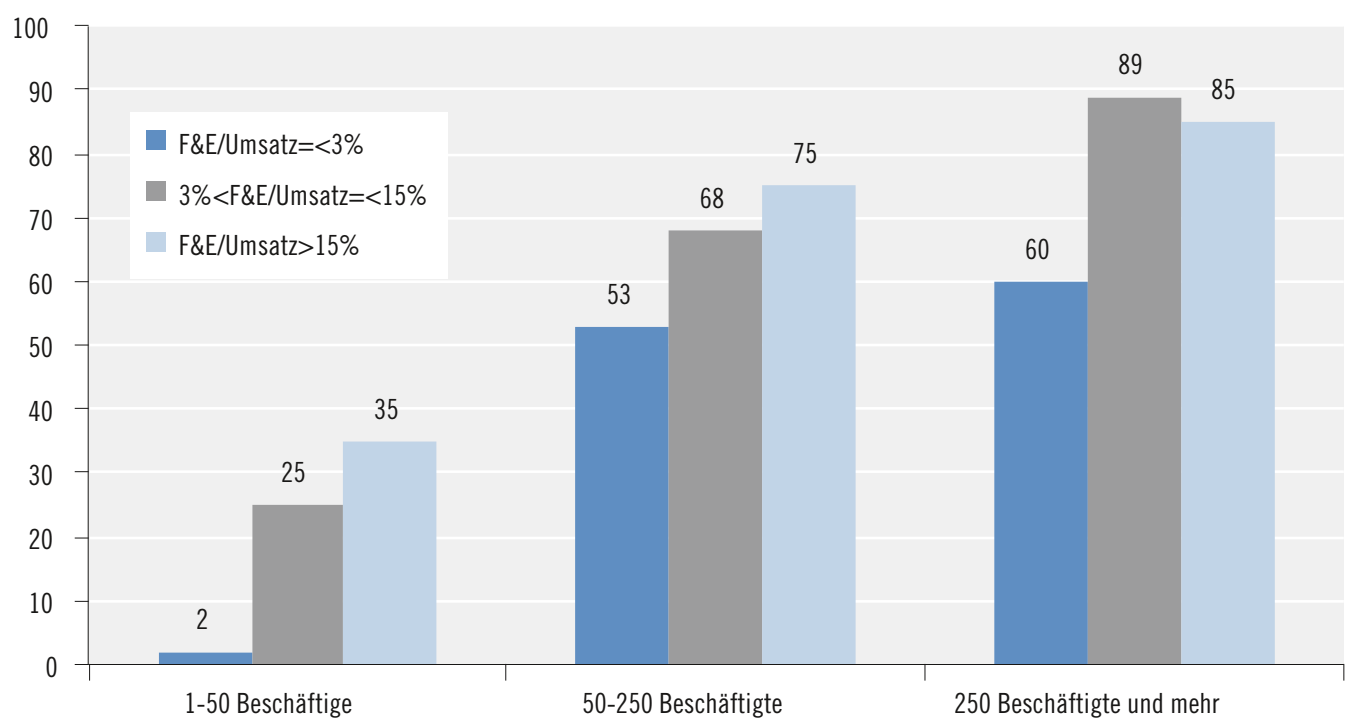

Quelle: FFG-Bilanzdaten. Berechnungen WIFO, Falk et al. (2013). 
bleiben andere Einflussfaktoren unberücksichtigt. Dennoch kann durch eine derartige Analyse der generelle Befund, dass in einer entwickelten Volkswirtschaft der technologische Wandel einer der wichtigsten Wachstumstreiber ist, ausreichend belegt werden.

\subsection{Innovationsaktivitäten im Unternehmens- sektor und die Rolle von KMU}

Im November 2012 wurden die Ergebnisse der siebten Innovationserhebung (CIS 2010) publiziert. Diese Ergebnisse bilden die Datengrundlage dieses Kapitels in dem einerseits die Innovationsperformance österreichischer Unternehmen im europäischen Vergleich (d.h. mit ausgewählten Ländern) positioniert wird und andererseits für eine Reihe von Indikatoren einige österreichspezifische Ergebnisse im Detail (z.B. auf Branchenebene) dargestellt werden.

Anzumerken ist, dass im Rahmen der Europäischen Innovationserhebung ein subjektiver Innovationsbegriff angewendet wird, d.h. das befragte Unternehmen entscheidet aus seiner subjektiven Sicht heraus, ob und inwieweit Innovationsaktivitäten gesetzt wurden. Damit werden also auch jene Innovationen erfasst, die zumindest für das Unternehmen neu sind, auch wenn es sich dabei um keine eigentliche Marktneuheit handelt. Zudem wird ein - wie in Innovationserhebungen mittlerweile allgemein üblich - breites Innovationsverständnis verwendet. Neben technologischen Innovationen werden auch nicht-technologische Innovationen wie organisatorische und Marketinginnovationen erfasst.

\subsubsection{Die Innovationsperformance im europäi- schen Vergleich}

In Abb. 51 ist die Innovatorenquote (Anteil innovierender Unternehmen an allen Unternehmen) für die teilnehmenden Länder dargestellt, wobei zwischen den unterschiedlichen Innovationsty- pen (bzw. Kombinationen davon, da Unternehmen im Beobachtungszeitraum auch Innovationstätigkeiten in den verschiedensten Bereichen durchführen können) differenziert wird. Im europäischen Vergleich zeigen sich ausgesprochen große Disparitäten hinsichtlich der Innovatorenquote; Die Spannweite reicht von einem Anteil innovierender Unternehmen um die 80 \% beim Spitzenreiter Deutschland bis zu lediglich rund $25 \%$ beim Schlusslicht Bulgarien, der europäische Durchschnittwert liegt bei $53 \%$. Österreich kann auf eine Innovatorenquote von $56 \%$ verweisen und liegt somit über dem europäischen Durchschnitt.

Betrachtet man die unterschiedlichen Innovationstypen, lässt sich feststellen, dass - in praktisch allen Ländern - der Gutteil der Unternehmen gleichzeitig sowohl technologische als auch nicht-technologische Innovationsaktivitäten durchführt. Deren Anteil an allen innovierenden Unternehmen bewegt sich zwischen $30 \%$ und $60 \%$. In Österreich fallen $55 \%$ aller innovierenden Unternehmen in jene Gruppe, die sowohl technologische als auch nicht-technologische Innovationsaktivitäten durchführt. Damit zeigt sich, dass Innovationsprozesse mehrdimensional sind, wobei technologische und organisatorische Veränderungen miteinander verknüpft sind. Ein Umstand, der von der Innovationsforschung in den vergangenen Jahren immer wieder betont wurde und letztlich auch Niederschlag in diversen innovationspolitischen Maßnahmen gefunden hat, die vielfach nicht mehr ausschließlich auf „harte" Technologien abzielen.

Der Vergleich zur sechsten Innovationserhebung $^{153}$ zeigt, dass diese Strukturen über die Zeit robust sind. Dies gilt auch für Österreich. Nur wenige Länder verzeichnen wesentliche Abweichungen: So stieg die Innovatorenquote in den Niederlanden von $45 \%$ auf $57 \%$ und in Schweden von $54 \%$ auf $60 \%$, während sie in der Tschechischen Republik von $56 \%$ auf $52 \%$ sank.

153 Siehe CIS 2008, vgl. FTB (2012). 
Abb. 51: Unternehmen mit Innovationsaktivitäten (in \% aller Unternehmen) im Jahr 2010

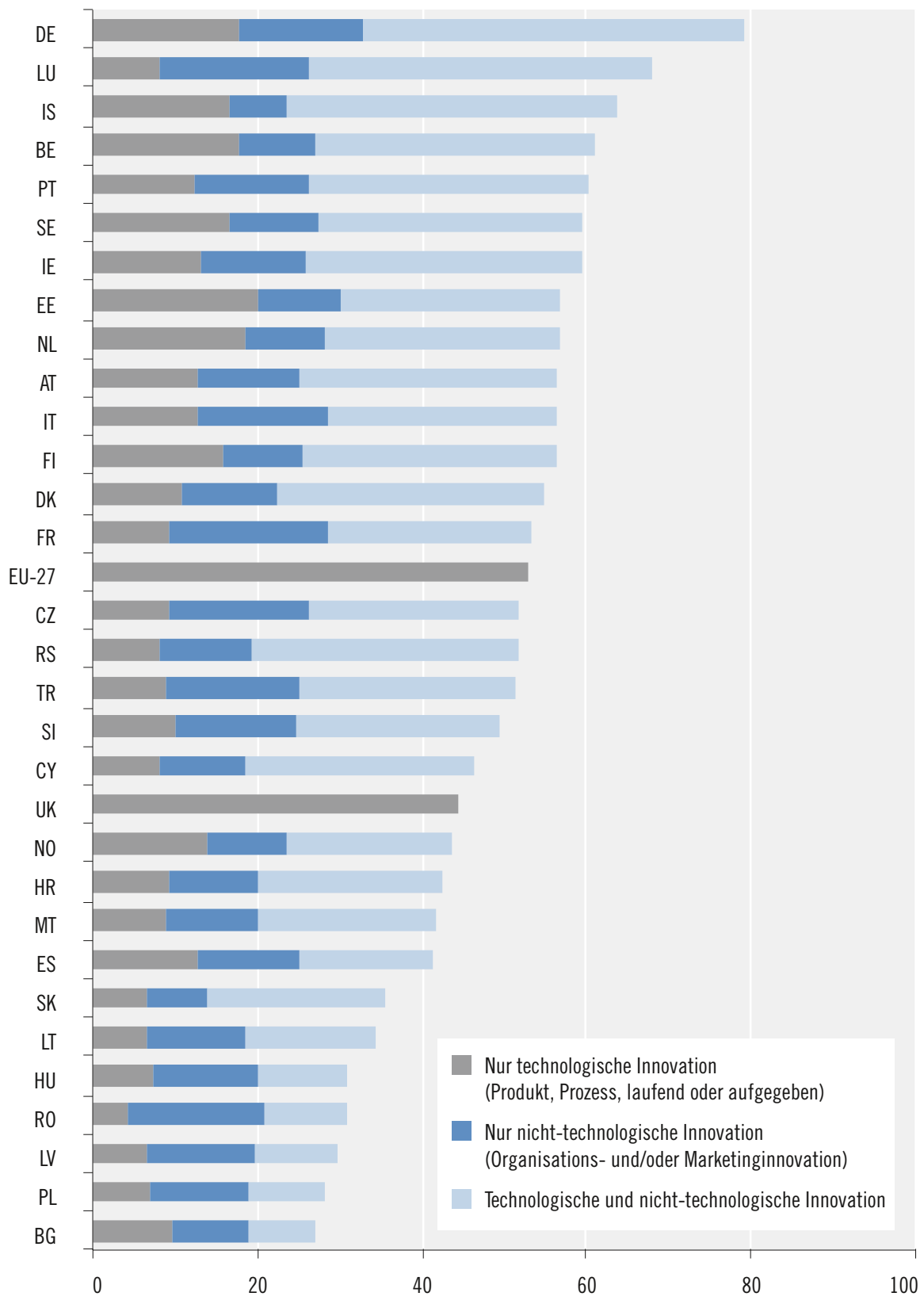

Anm.: Für Großbritannien sowie für die EU-27 insgesamt ist keine Differenzierung nach Innovationstypen möglich.

Quelle: Eurostat CIS 2010. Berechnungen JOANNEUM RESEARCH. 
Für die Innovationsaktivitäten für Produktund Prozessinnovationen (also für die technologischen Innovationsprozesse) kann zwischen verschiedenen Tätigkeitsarten differenziert werden, wobei die Gewichtung nach den monetären Aufwendungen für die einzelnen Tätigkeiten erfolgt. Konkret wird zwischen (i) unternehmensinterner Forschung und experimenteller Entwicklung (interne F\&E), (ii) Vergabe von F\&E-Aufträgen an Dritte (externe F\&E), (iii) Erwerb von Maschinen, Ausrüstung und Software sowie (iv) Erwerb von externem Wissen ${ }^{154}$ unterschieden. In Abb. 52 sind die Ergebnisse für ausgewählte Länder dargestellt. Im Großteil der hier angeführten Vergleichsländer (darunter auch in Österreich) hat die interne F\&E das größte Gewicht im Rahmen der technologischen Innovationsaktivitäten: Rund die Hälfte der Innovationsausgaben entfällt auf interne F\&E; in Österreich sogar zwei Drittel (69 \%). Die zweitwichtigste Ausgabenkategorie sind Investitionen in Maschinen, Ausrüstung und Software, die für knapp ein Drittel der Ausgaben (in Österreich ein Fünftel) verantwortlich sind. Dieser embodied technological change ist insbesondere für aufholende Innovationssysteme wie in Ungarn oder Tschechien von großer Bedeutung. Dies galt auch für Österreich bis Mitte der 1990er Jahre. Hingegen spielen für "reife" Innovationssysteme unternehmensinterne F\&EAnstrengungen eine größere Rolle. Diesbezüglich weist Österreich unter den Vergleichsländern inzwischen gar den höchsten Anteil von F\&E-Ausgaben an allen Innovationsaufwendungen auf und hat - im Vergleich zum CIS 2008 damit den ersten Rang von Finnland übernommen. Grundsätzlich sind aber auch die Ausgabestrukturen über die Zeit hinweg sehr konstant. ${ }^{155}$

\section{Innovationskooperationen}

Für die Leistungsfähigkeit eines Innovationssystems ist nicht nur die Innovationskraft der einzelnen Akteure, sondern auch deren Zusammenspiel in Form von Kooperationsnetzwerken von großer Bedeutung. Intensive Kooperationsbeziehungen zwischen Unternehmen sowie zwischen Unternehmen und (öffentlichen) Forschungsinstitutionen generieren positive Netzwerkeffekte,

\section{Abb. 52: Aufteilung der Innovationsausgaben nach Tätigkeitsarten (in \%, für Unternehmen mit technologischen} Innovationstätigkeiten) im Jahr 2010

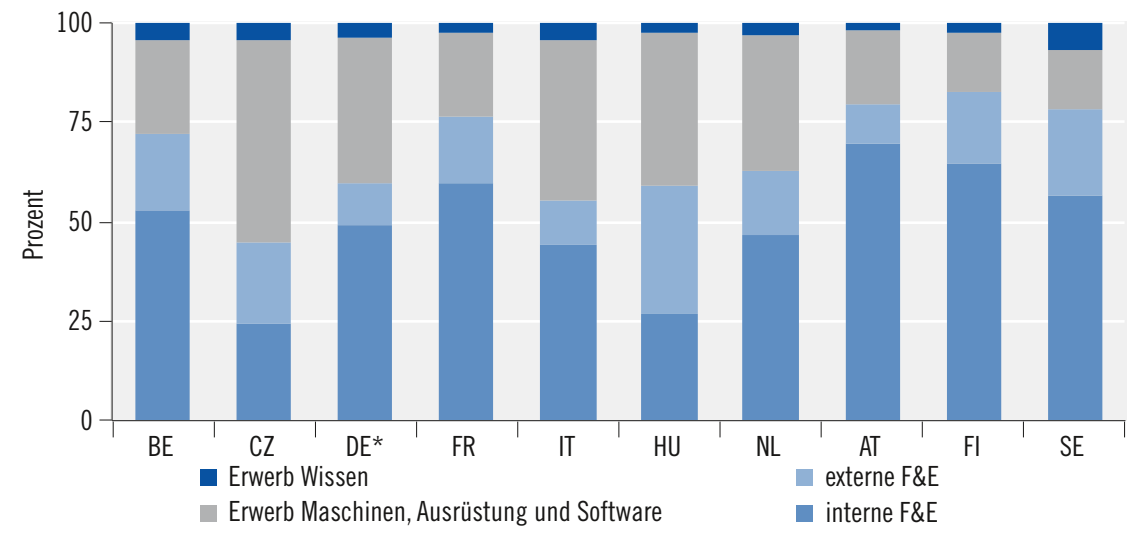

Quelle: Eurostat CIS 2010; außer * CIS 2008, da für Deutschland im CIS 2010 keine Differenzierung der Innovationsausgaben durch Eurostat publiziert ist. Berechnungen JOANNEUM RESEARCH.

154 Hierzu zählen der Ankauf von Patenten, Lizenzen etc. 155 Vgl. FTB (2012). 
indem neu generiertes Wissen kontinuierlich zwischen den Akteuren fließt, was zur raschen Diffusion neuer Innovationen beiträgt. Letztlich führen derartige Effekte zum Entstehen innovativer Milieus, die durch eine hohe Innovationskraft und intensive Austauschbeziehungen gekennzeichnet sind. In Abb. 53 sind die Anteile der kooperierenden Unternehmen an allen Unternehmen mit technologischen Innovationen ${ }^{156}$ angegeben. In Österreich kooperiert jedes zweite dieser Unternehmen - ein europäischer Spitzenwert. Zudem ist der Anteil der kooperierenden Unternehmen somit seit dem CIS 2008 um rund 10 Prozentpunkte gestiegen.

Diese Kooperationen finden mit unterschiedlichen Akteuren statt. Der CIS unterscheidet dabei folgende Gruppen: andere Unternehmen innerhalb der eigenen Unternehmensgruppe, Zulieferer, Auftraggeber/Kunden, Mitbewerber, Beratungsfirmen/private F\&E-Einrichtungen, Universitäten/Fachhochschulen sowie öffentliche außeruniversitäre Forschungsinstitutionen.

Die Kooperationshäufigkeit mit diesen unterschiedlichen Akteursgruppen ist in Abb. 54 für eine Reihe von ausgewählten Ländern dargestellt. ${ }^{157}$ Auch wenn die Kooperationsdichte zwischen den Ländern stark schwankt, lässt sich ein einheitliches Muster hinsichtlich der relativen Bedeutung der jeweiligen Akteursgruppen für Innovationskooperationen erkennen. Vor allem Zulieferer und oftmals auch Kunden sind die mit Abstand wichtigsten Kooperationspartner. In der

Abb. 53: Innovationskooperationen im europäischen Vergleich (in \% aller Unternehmen mit technologischer Innovationstätigkeit) im Jahr 2010

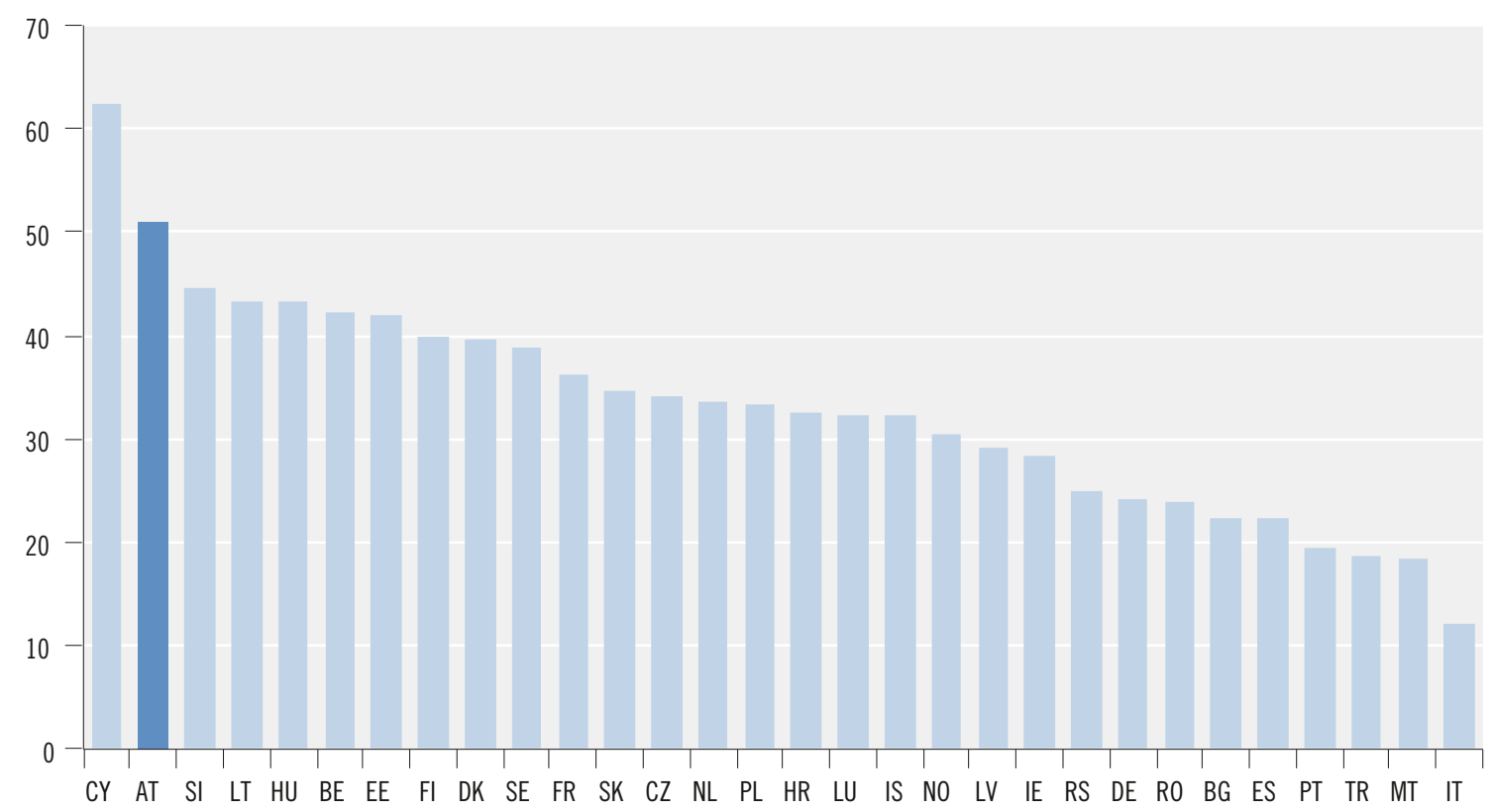

Quelle: Eurostat CIS 2010. Berechnungen JOANNEUM RESEARCH.

156 Die Frage nach Kooperationspartnern wird im CIS nur Unternehmen mit Produkt- oder Prozessinnovationen sowie andauernden und abgebrochenen technologischen Innovationsaktivitäten gestellt.

157 Unterschiede in den Ländermustern zwischen Abb. 42 und Abb. 43 erklären sich durch die Art der jeweiligen Frage: Abb. 42 zeigt den Anteil der Unternehmen, die irgendeine Form von Innovationskooperation angegeben haben. Abb. 43 weist hingegen den Anteil der Kooperationen mit unterschiedlichen Partnern auf, wobei Mehrfachantworten möglich sind. Beispielsweise kooperieren in Finnland zwar nur knapp 40 \% der Unternehmen, jedoch arbeiten diese mit vielen unterschiedlichen Partnern bei Innovationen zusammen. 
Abb. 54: Kooperationspartner nach Akteursgruppen (in \% der Unternehmen mit technologischen Innovationsaktivitäten) im Jahr 2010

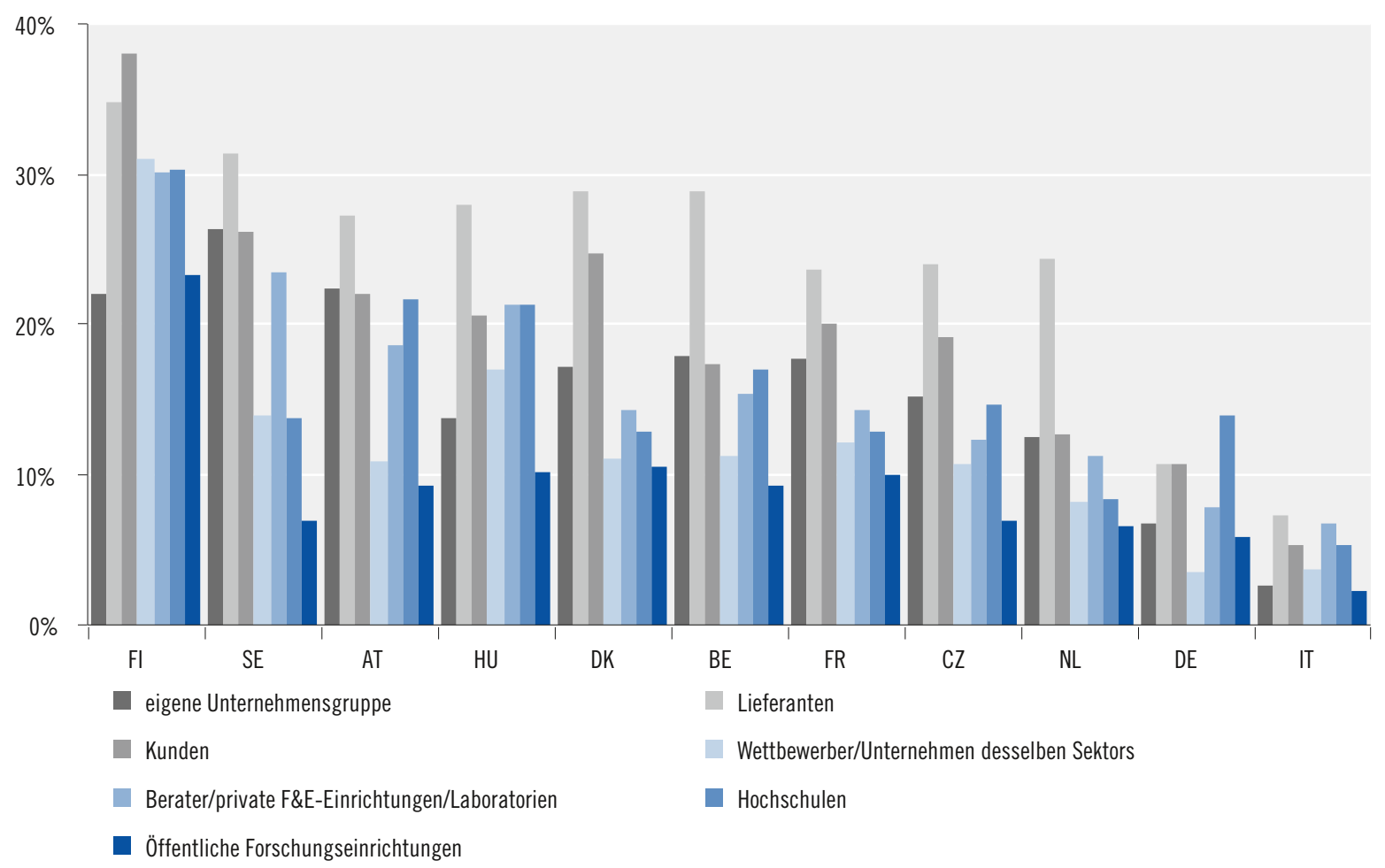

Quelle: Eurostat CIS 2010. Berechnung JOANNEUM RESEARCH.

modernen, hochgradig arbeitsteiligen Wirtschaft sind auch die Innovationsprozesse entlang der arbeitsteiligen Wertschöpfungsketten interaktiv organisiert. ${ }^{158}$ Des Weiteren finden Innovationsprozesse vielfach in Zusammenarbeit mit Beratern/privaten Forschungseinrichtungen oder öffentlichen Hochschulen statt, wenn deren Bedeutung auch nicht an die der vertikalen Kooperationsbeziehungen heranreicht. "Horizontale" Kooperationen mit Mitbewerbern oder Unternehmen der gleichen Branchen spielen hingegen durchgängig eine geringere Rolle. Noch seltener wird mit den - vergleichsweise wenigen - öffentlichen Forschungseinrichtungen zusammengearbeitet.
Österreich weicht in seinen Kooperationsbeziehungen nicht von diesem allgemeinen Muster ab, wenngleich die Kooperationsintensität Österreichs nicht ganz an Länder wie Finnland oder Schweden heranreicht. Gut ein Viertel der österreichischen Unternehmen mit technologischen Innovationen kooperiert mit Lieferanten, mehr als ein Fünftel mit Kunden oder Unternehmen der eigenen Unternehmensgruppe. Zum Vergleich: In Finnland arbeiten $35 \%$ mit Lieferanten und $38 \%$ mit Kunden zusammen. Bemerkenswert ist die relativ hohe Kooperationsdichte mit Universitäten und Hochschulen, die in Österreich mit $22 \%$ deutlich höher ist als in den meisten Vergleichsländern (Finnland als eines der

158 Die Akteursgruppe „Andere Unternehmen innerhalb der Unternehmensgruppe“ kann ebenfalls zu diesen Akteuren hinzugerechnet werden, da die verschiedenen Tochterunternehmen innerhalb einer Unternehmensgruppe vielfach arbeitsteilig organisiert sind, d.h. z.B. Tochterunternehmen A ist Zulieferer von Tochterunternehmen B der gleichen Unternehmensgruppe. 
Abb. 55: Innovationsförderung im europäischen Vergleich (in \% aller Unternehmen mit technologischen Innovationsaktivitäten), 2008/2010

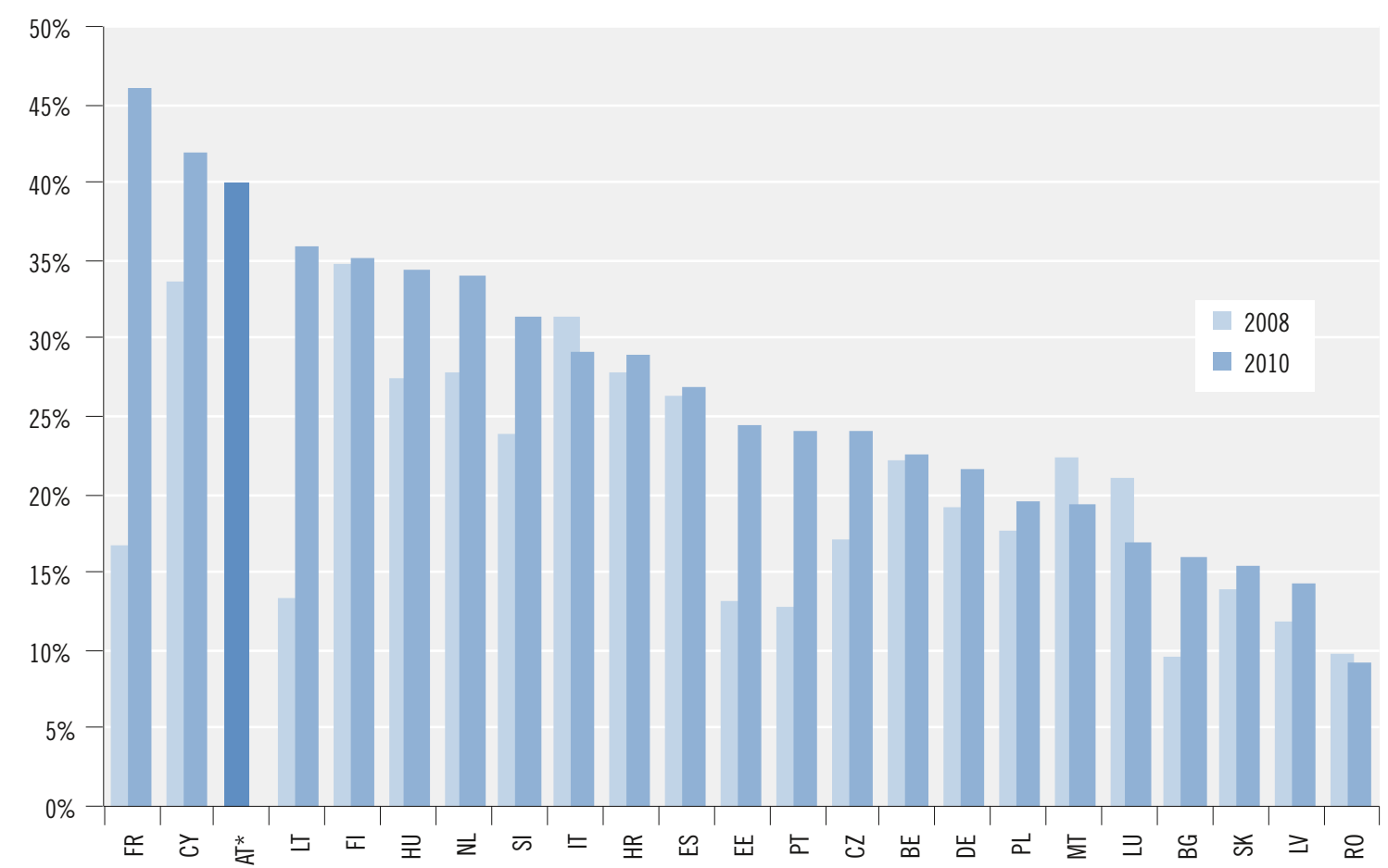

Quelle: Eurostat CIS $2010 / 2008,{ }^{*}$ nur CIS 2008. Berechnung JOANNEUM RESEARCH.

Spitzenländer erreicht $30 \%)$. Offensichtlich ist das österreichische Innovationssystem mittlerweile durch eine vergleichsweise intensive Austauschbeziehung des Unternehmenssektors mit dem Universitätssektor gekennzeichnet, die seitens der FTI-Politik seit längerem intensiv gefördert wird. ${ }^{159}$

Außeruniversitäre Forschungseinrichtungen spielen in Österreich als Kooperationspartner in unternehmerischen Innovationsprozessen eine deutlich geringere Rolle als Universitäten; nur $9 \%$ der Unternehmen gaben entsprechende Arrangements an. Dies ist international nicht unüblich (der einzige Ausreißer ist erneut Finnland mit $23 \%$ ). Zudem ist zu berücksichtigen, dass der außeruniversitäre Sektor in Österreich relativ klein ist.

\section{Innovationsförderung}

Die direkte Förderung von unternehmerischen Innovationsaktivitäten ist eine der zentralen Säulen der Technologiepolitik. Dabei stellt sich die Frage, welche "Reichweite" - unabhängig vom monetären Rahmen ${ }^{160}$ - die entsprechenden Förderinstrumente aufweisen, d.h. kommen sie einer kleinen Gruppe von Unternehmen zu Gute oder gelingt es mit diesen Instrumenten viele innovative Unternehmen anzusprechen. Die entsprechenden Ergebnisse im europäischen Ver-

159 Siehe hierzu die Ausführungen im FTB 2012.

160 Im Gegensatz zum öffentlich geförderten Anteil an den unternehmerischen F\&E-Ausgaben gibt es keine Informationen hinsichtlich des Förderanteils an den gesamten Innovationsausgaben des Unternehmenssektors. Beim Förderanteil an den F\&E-Ausgaben ist Österreichs Fördersystem mit $1 \%$ jedenfalls mit an der Spitze der europäischen Länder. 
gleich sind in Abb. 55 angegeben. In Österreich gaben 2008 ca. $40 \%$ aller Unternehmen mit technologischen Innovationsaktivitäten an, einschlägige Fördermaßnahmen von Seiten der öffentlichen Hand erhalten zu haben. Damit lag Österreich an der Spitze aller europäischen Länder. Im CIS 2010 wurde diese Frage in Österreich nicht mehr gestellt. Die aktuellen Daten zeigen, dass inzwischen auch Länder wie Frankreich eine ähnliche Quote aufweisen, nachdem hier die Förderung massiv ausgeweitet wurde ${ }^{161}$. Dennoch ist der Anteil der mit öffentlichen Mitteln geförderten Unternehmen in Österreich weiterhin vergleichsweise hoch.

\subsubsection{Branchenspezifische Ergebnisse für Österreich}

Im Folgenden werden ausgewählte Ergebnisse der österreichischen Innovationserhebung auf Branchenebene dargestellt. Unterschiede nach Unternehmensgröße werden im Detail im darauffolgenden Kapitel betrachtet.

Abb. 56 zeigt die Innovatorenquote in den einzelnen Branchen, differenziert nach Art der Innovationsaktivität (technologisch versus nichttechnologisch). Dabei ist festzustellen, dass der Anteil innovationsaktiver Unternehmen in allen Branchen bei rund $50 \%$ oder darüber liegt - die

\section{Abb. 56: Innovatorenquote in Österreich nach Branchen (innovationsaktive Unternehmen in \% aller Unternehmen)} im Jahr 2010

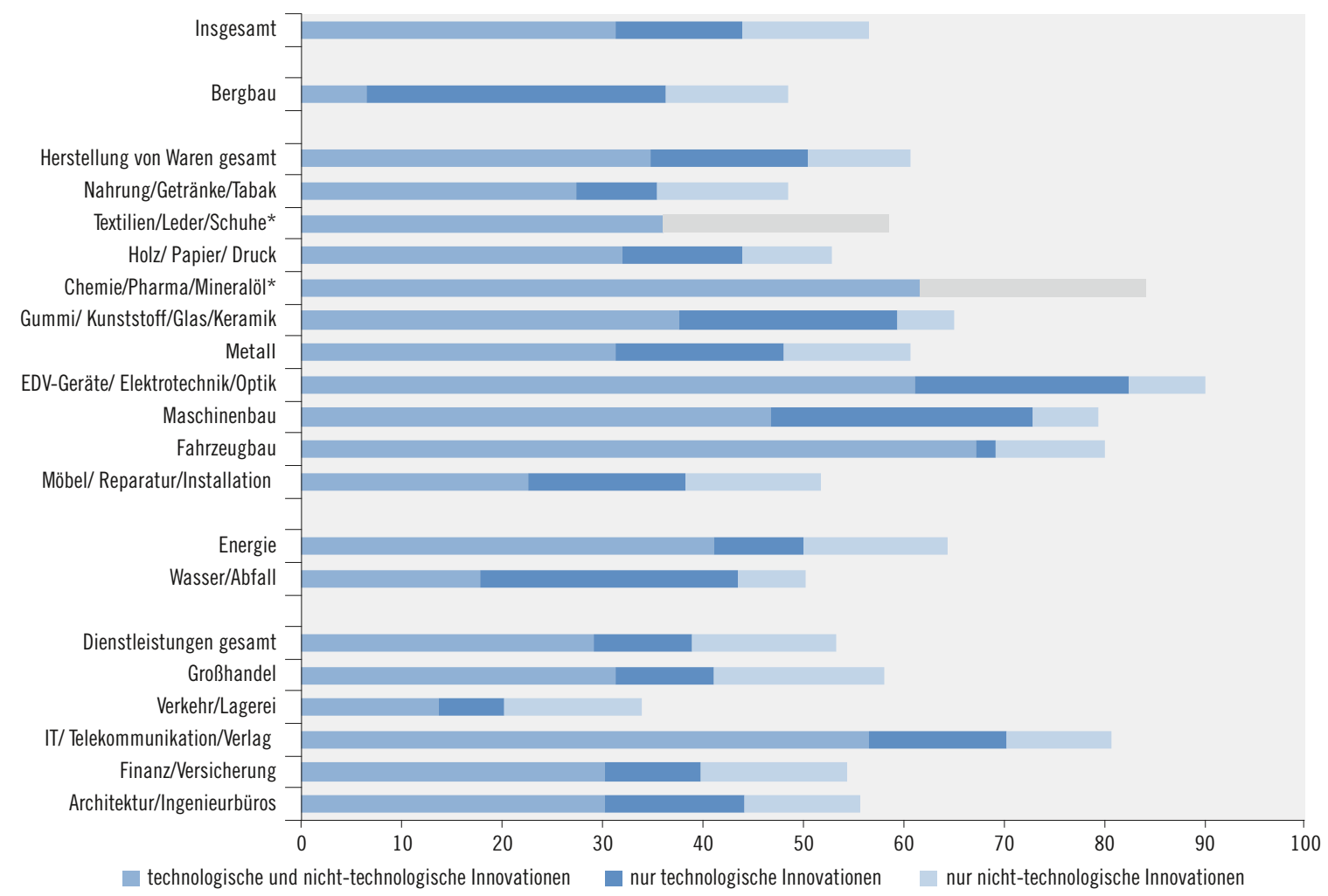

Anm.: * Aus Geheimhaltungsgründen werden von der Statistik Austria keine Werte für nur (nicht) technologische Innovatoren in den Branchen Textilien/ Leder/ Schuhe und Chemie/ Pharma/ Mineralöl veröffentlicht.

Quelle: Statistik Austria CIS 2010. Berechnung JOANNEUM RESEARCH.

161 Hierzu zählen die Stärkung bzw. Neuerung in Bereichen der indirekten F\&E-Förderung, der Clusterpolitik, der Innovationsförderung für KMU durch die OESO sowie zusätzliche Fördermittel, die im Rahmen des „Investments for the future“ Programms zur Verfügung gestellt wurden (vgl. ERAWATCH Country Reports für Frankreich, div. Jahre). 
einzige Ausnahme ist Verkehr und Lagerei mit $34 \%$. Besonders herausragend sind die "klassischen" Technologiebranchen EDV/Elektrotechnik/Optik (hier erreicht die Innovatorenquote $90 \%$ ), die Chemie- bzw. Pharmabranche (84\%) sowie der Maschinenbau und die Fahrzeugindustrie (je etwa $80 \%$ ). Der Dienstleistungssektor weist insgesamt eine etwas niedrigere Innovatorenquote $(53 \%)$ als die Sachgüterproduktion $(61 \%)$ auf. Allerdings ist die Heterogenität innerhalb der Dienstleistungen sehr hoch: So umfasst sie neben dem erwähnten Schlusslicht Verkehr/ Lagerei auch die IT-Branche (IT/Telekommunikation/Verlag), die mit $81 \%$ eine der höchsten Innovatorenquote aller Branchen aufweist.

Bemerkenswert ist, dass in den meisten Branchen der Anteil der dualen Innovatoren, also jener Unternehmen, die sowohl technologische als auch nicht-technologische Innovationaktivitä- ten durchführen, überwiegt. Somit sind die Innovationsprozesse zumeist mehrdimensional und kombinieren die Entwicklung neuer Produkte oder Prozesse mit organisatorischen Innovationen und Neuerungen im Marketing.

Neben der Innovatorenquote stellt sich auch die Frage nach der Intensität von Innovationsprozessen und inwieweit sich diese Intensität zwischen Branchen unterscheidet. Ein taugliches $\mathrm{Maß}$ zur Erfassung der Intensität von Innovationsprozessen ist der Anteil der Innovationsausgaben am Umsatz, wie in Abb. 57 dargestellt ist. Diesbezüglich zeigen sich deutliche Unterschiede zwischen den einzelnen Branchen. Während Innovationsaktivitäten also relativ gleich häufig in den jeweiligen Branchen vorzufinden sind, ist das relative Gewicht dieser Innovationsaktivitäten doch sehr unterschiedlich verteilt. Spitzenreiter sind Architektur- und Ingenieurbüros mit

\section{Abb. 57: Innovationsintensität nach Branchen (Anteil der Innovationsausgaben am Umsatz - Unternehmen mit technologischen Innovationsaktivitäten) im Jahr 2010}

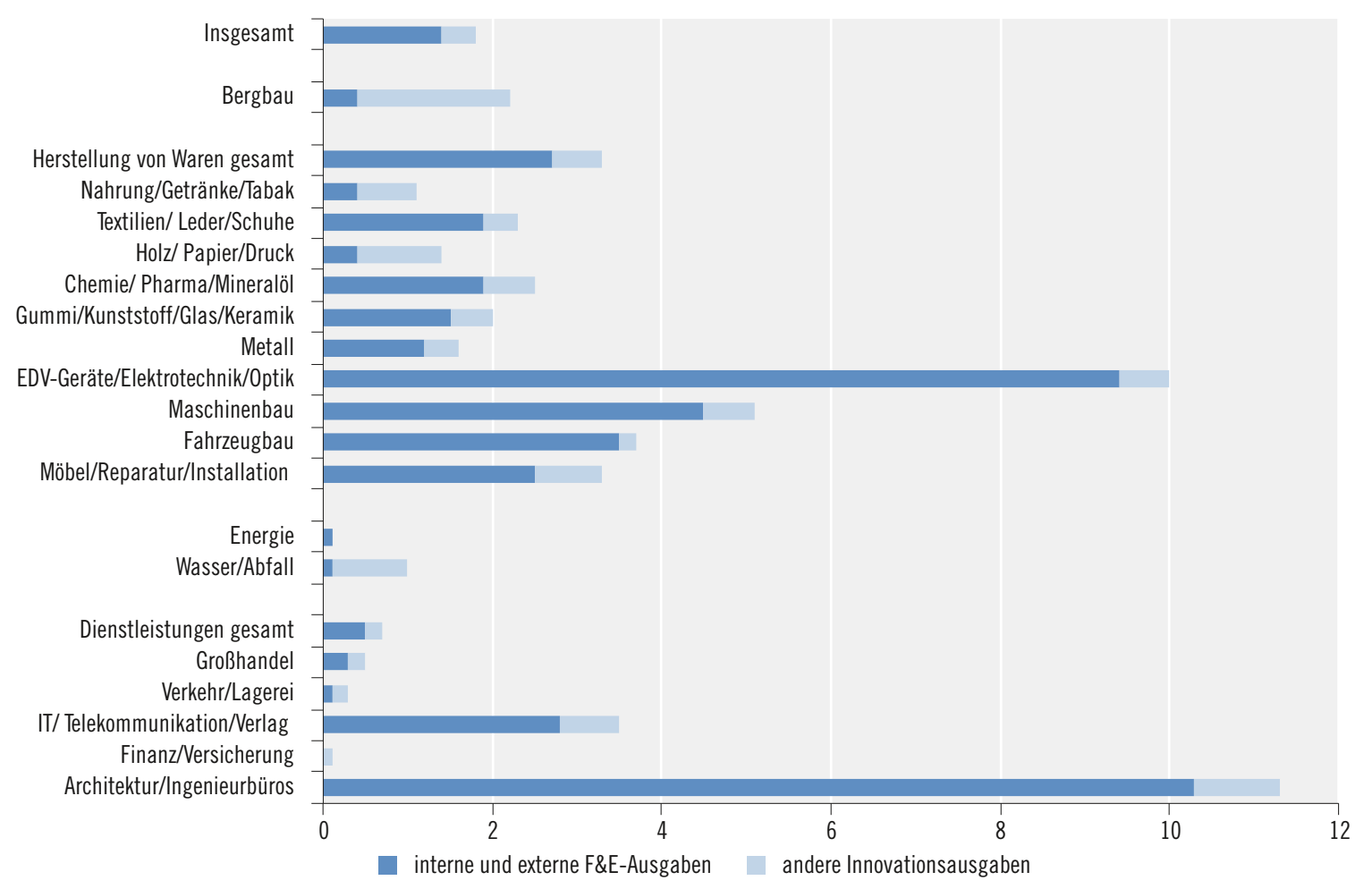


$11 \%$ vor der Herstellung von EDV-Geräten/Elektrotechnik/Optik mit $10 \%$ des Umsatzes für Innovationsausgaben. In diesen beiden Branchen dominieren interne und externe F\&E-Aufwendungen die Innovationsausgaben. Dies ist in den meisten Branchen mit hoher Innovationsintensität der Fall. Hingegen überwiegen in einigen der weniger innovationsintensiven Branchen die sonstigen Innovationsausgaben, so bei Nahrung/ Getränke/Tabak, Holz/Papier/Druck, Wasser/ Abwasser, Verkehr/Lagerei, Finanz/Versicherung, aber auch im Bergbau. Überdurchschnittliche F\&E-Intensitäten finden sich traditionell zudem in den Branchen Maschinenbau (knapp 5 \%) und Fahrzeugbau (ca. 4 \%). Generell ist die durchschnittliche Innovationsintensität in der Sachgüterproduktion mit ca. 3,3 \% deutlich höher als im Dienstleistungssektor $(0,7 \%)$, wobei erneut auf die bereits erwähnte Heterogenität im Dienstleistungsbereich hinzuweisen ist. Ebenso sei erneut die hohe Strukturpersistenz betont.

\subsubsection{Innovationsstrategien der kleinen und mittleren Unternehmen}

Kleine und mittlere Unternehmen (KMU) sind von besonderer Bedeutung für die österreichische Volkswirtschaft. Laut der Definition von Eurostat zählen dazu alle Unternehmen, die zwischen einem und 249 Beschäftigte ${ }^{162}$ haben. Diese Gruppe macht $97 \%$ aller österreichischen Unternehmen aus, beschäftigt zwei Drittel aller Beschäftigten und erwirtschaftet knapp $60 \%$ der Bruttowertschöpfung (Tab. 23). Die Innovationsperformance aller KMU kann allerdings nicht untersucht werden, da die europäische Innovationserhebung (CIS) keine Kleinstunternehmen mit weniger als zehn Beschäftig- ten berücksichtigt. Somit konzentriert sich die vorliegende Analyse auf kleinere und mittlere Unternehmen mit zehn bis 249 MitarbeiterInnen (die im Folgenden als KMU bezeichnet werden). Aufgrund der Vielzahl von Kleinstunternehmen machen die so definierten KMU zwar nur $12 \%$ aller Unternehmen aus; jedoch sind sie immer noch für $42 \%$ aller Beschäftigten und $41 \%$ der Bruttowertschöpfung verantwortlich und bilden damit das Rückgrat der österreichischen Wirtschaft (Tab. 23). ${ }^{163}$ Aufgrund dieser hohen Bedeutung stellt sich die Frage, inwieweit das Innovationsverhalten der österreichischen Unternehmen größenabhängig ist und inwiefern KMU ein spezifisches Innovationsmuster aufweisen. ${ }^{164}$ Bei der folgenden Analyse ist allerdings zu berücksichtigen, dass KMU über die Branchen nicht gleichverteilt sind. So weisen einige Branchen einen niedrigen Anteil der Unternehmen mit 10 bis 249 Beschäftigten auf: In der IKT-Branche (J) liegt dieser beispielsweise bei $7 \%$, im Maschinenbau (C25) bei $29 \%$ und in der Papierindustrie (C17) bei $52 \%$. Das beobachtbare Innovationsverhalten der KMU ist somit nicht alleine durch ihre Größe, sondern auch durch ihre spezifische Branchenzugehörigkeit geprägt. Der Einfluss dieser Branchenzugehörigkeit kann auf Basis der hier zunächst durchgeführten sekundärstatistischen Auswertung der veröffentlichten Gesamtergebnisse der österreichischen Innovationserhebung von 2012 nicht kontrolliert bzw. separat erfasst werden. Allerdings werden im folgenden Abschnitt 5.2.4 ergänzende ökonometrische Analysen der unveröffentlichten Einzeldaten durchgeführt, die es erlauben, den Einzeleffekt der Unternehmensgröße - auch im internationalen Vergleich - zu ermitteln.

162 Dazu zählen Kleinstunternehmen (1-9 Beschäftigte), kleine Unternehmen (10-49 Beschäftigte) und mittlere Unternehmen (50-249 Beschäftigte).

163 Hinzu kommt, dass in Österreich im internationalen Vergleich - und zwar auch gegenüber anderen kleineren Ländern wie der Schweiz, Finnland, Niederlande oder Schweden - auch die Großunternehmen relativ „klein“ sind und es kaum herausragende Großkonzerne gibt.

$164 \mathrm{Zu}$ Recht verweisen Kaufmann und Tödtling (2002) darauf, dass der KMU-Sektor sehr heterogen ist und sich „einfache Verallgemeinerungen" somit verbieten. Nichtsdestotrotz soll auf Basis vorhander Daten ein erster Eindruck vermittelt werden. 
Tab. 23: Anteil der Unternehmensgrößenklassen an der Wirtschaftsleistung Österreichs 2010 (in Prozent)

\begin{tabular}{|c|c|c|c|c|}
\hline & \multirow{2}{*}{$\begin{array}{c}\text { Anzahl } \\
\text { Unternehmen }\end{array}$} & \multicolumn{2}{|c|}{ Beschäftigte im Jahresdurchschnitt } & \multirow{2}{*}{$\begin{array}{l}\text { Bruttowertschöpfung } \\
\text { zu Faktorkosten }\end{array}$} \\
\hline & & insgesamt & unselbstständige & \\
\hline Großunternehmen ( $>=250$ Beschäftigte) & 0,3 & 33,1 & 36,8 & 42,0 \\
\hline KMU (1-249 Beschäftigte) & 97,3 & 66,9 & 63,2 & 58,0 \\
\hline \multicolumn{5}{|l|}{ davon } \\
\hline Kleinstunternehmen (< 10 Beschäftigte) & 87,4 & 24,8 & 17,3 & 17,3 \\
\hline kleine und mittlere Unternehmen (10-249 Beschäftigte) & 12,2 & 42,2 & 45,9 & 40,7 \\
\hline
\end{tabular}

Quelle: Statistik Austria: Hauptergebnisse der Leistungs- und Strukturstatistik 2010 nach Gruppen (3-Stellern) der ÖNACE 2008 (Abschnitte B-N, S95) und nach Beschäftigtengrößenklassen. Berechnung JOANNEUM RESEARCH.

\section{Innovationsverhalten von KMU - deskriptive Analysen}

Abb. 58 zeigt, dass ohne Berücksichtigung der Branche ein ausgeprägter Zusammenhang zwischen Unternehmensgröße und Innovation besteht: Je größer ein Unternehmen ist, desto eher werden Innovationsaktivitäten durchgeführt.

Zwar ist auch die Hälfte der Kleinunternehmen (10 bis 49 Beschäftigte) innovativ, der Wert für Großunternehmen liegt jedoch bei fast $90 \%$.
Einer der Hauptunterschiede hierbei ist, dass KMU seltener duale Innovatoren sind, also sowohl technische als auch nicht-technische (Organisations- und/oder Marketing-) Innovationen hervorbringen.

Zudem zeigen die Ergebnisse der Statistik Austria, dass kleinere und mittlere Unternehmen insgesamt deutlich weniger finanzielle Mittel für Innovationen aufwenden als größere Unternehmen (Abb. 59). Dies gilt zum einen absolut, zum anderen aber auch relativ, wenn die In-

Abb. 58: Innovationsformen und Unternehmensgröße im Jahr 2010

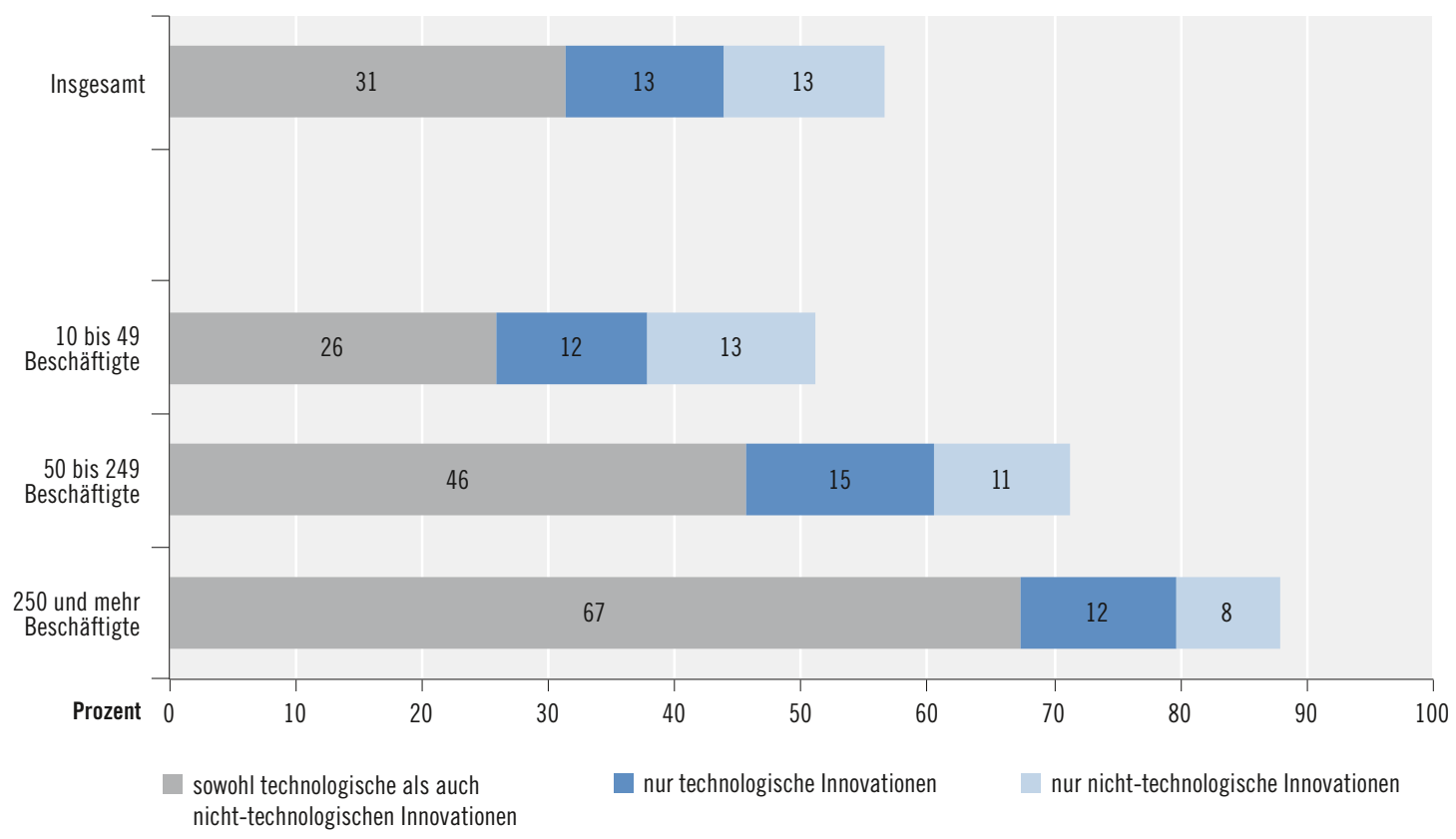

Quelle: Statistik Austria CIS 2010. Berechnung JOANNEUM RESEARCH. 
novationsausgaben in Bezug zum Umsatz gesetzt werden: Die Innovationsintensität ist mit 1,3\% (kleine) bzw. 1,2\% (mittlere) deutlich geringer als bei Großunternehmen mit 2,2 \% (Abb. 60).

Strukturell ist interne F\&E zwar auch bei KMU der wichtigste Kostenfaktor und somit die wichtigste Aktivität für den Innovationsprozess. Im Vergleich zu Großunternehmen zeigt sich jedoch, dass andere Innovationsaktivitäten einen verhältnismäßig hohen Stellenwert genießen. Dies gilt insbesondere für den Erwerb von Maschinen und Sachmitteln für Innovationen.

Die relativ geringere Bedeutung von F\&E für kleine und mittlere Unternehmen wird auch dadurch deutlich, dass mit $49 \%$ der Anteil F\&Etreibender KMU (an allen KMU mit technologischen Innovationsaktivitäten) klar unter dem
Anteil der F\&E-treibenden Großunternehmen (71 \%) liegt. Zudem wird interne F\&E von KMU seltener kontinuierlich (25\%) durchgeführt als von großen Unternehmen (57 \%). Analysen von Rammer et al. (2004) zeigen, dass F\&E- und Innovationsaktivitäten auch in deutschen KMU weniger kontinuierlich sind und enger mit dem Konjunkturverlauf schwanken als die Aktivitäten von Großunternehmen.

Auf die geringere Bedeutung von F\&E in KMU weisen auch die Zahlen der F\&E-Erhebung $2009^{165}$ hin, wonach zwar $86 \%$ aller F\&E-durchführenden Einheiten KMU sind ${ }^{166}$, diese aber nur $29 \%$ aller F\&E-Aufwendungen tragen ${ }^{167}$ und $37 \%$ der F\&E-Beschäftigten ${ }^{168}$ angestellt haben.

Hintergrund hierfür ist, dass die Durchführung von (kontinuierlicher) F\&E KMU vor eine große

\section{Abb. 59: Innovationsausgaben und -aktivitäten und Unternehmensgröße im Jahr 2010}

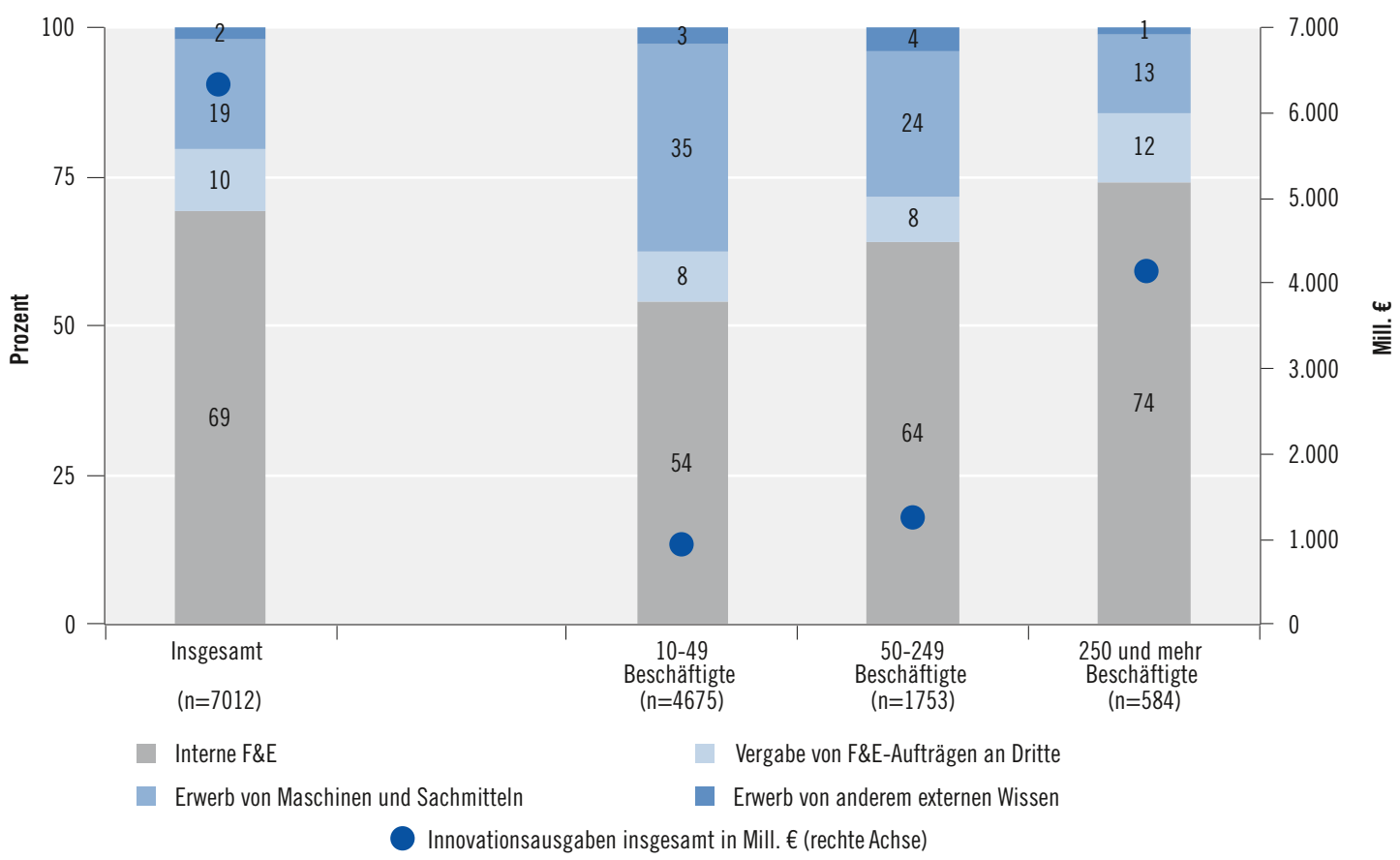

Quelle: Statistik Austria CIS 2010. Berechnung JOANNEUM RESEARCH.

165 Siehe Statistik Austria (2011).

166 Bei der F\&E-Erhebung werden auch Kleinstunternehmen berücksichtigt. Kleinst- und Kleinunternehmen machen 59 \%, mittlere Unternehmen $26 \%$ aus.

167 Kleinst- und Kleinunternehmen $11 \%$, mittlere Unternehmen $18 \%$.

168 Kleinst- und Kleinunternehmen $16 \%$, mittlere Unternehmen $21 \%$. 
Abb. 60: Anteil der Innovationsausgaben am Umsatz nach Beschäftigungsgrößenklassen im Jahr 2010

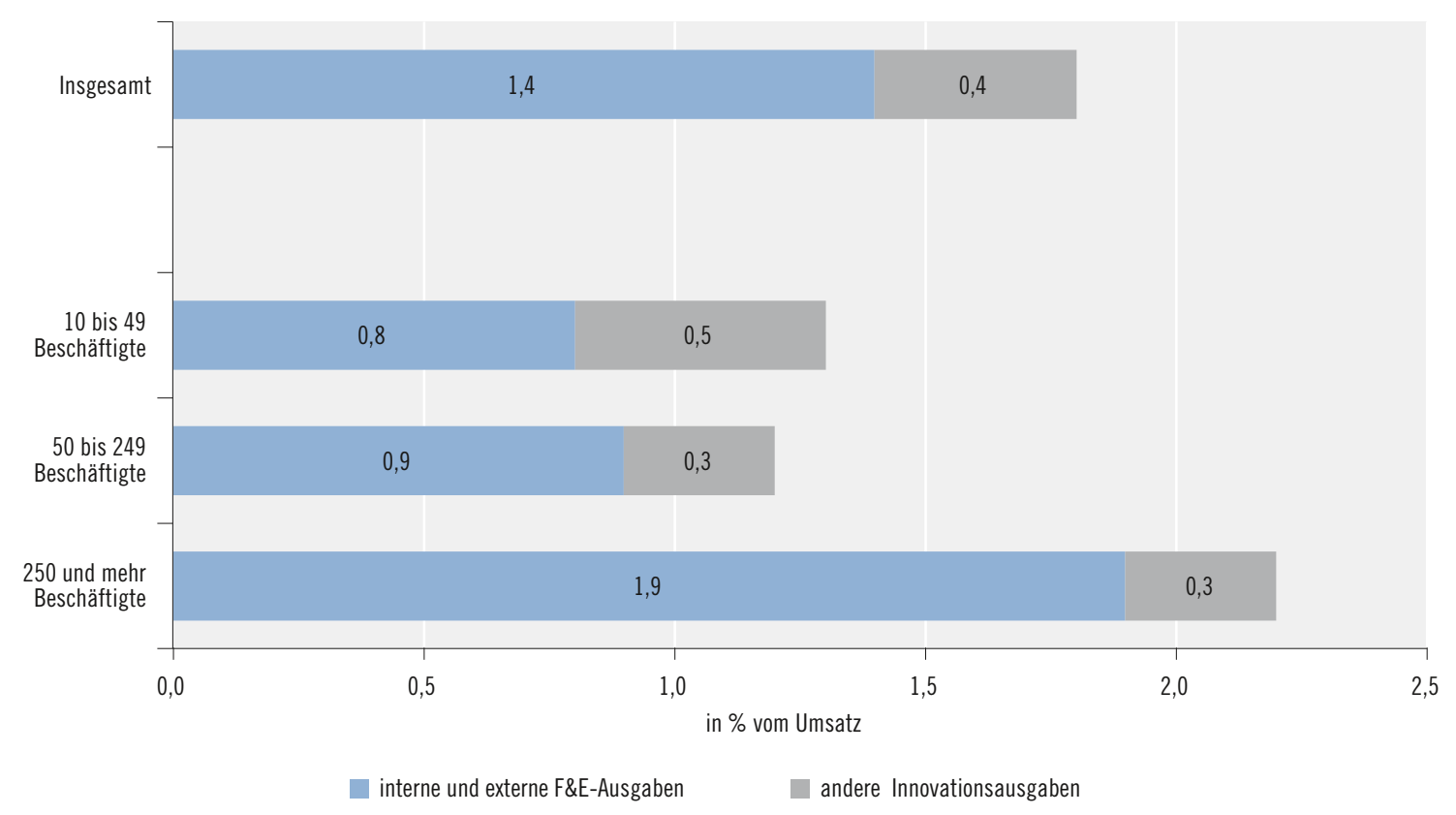

Quelle: CIS 2010. Berechnungen JOANNEUM RESEARCH.

Herausforderung stellt. Hierzu zählen insbesondere Kosten (sunk costs), die durch den Unterhalt der F\&E-Infrastruktur, die Mindestgröße und Laufzeit von F\&E-Projekten entstehen sowie die finanziellen Risiken, die durch die hohe technologische bzw. wirtschaftliche Unsicherheit von F\&E-Projekten hervorgehen und die für KMU durchaus existenzgefährdend sein können. ${ }^{169}$

Die vergleichsweise geringere Bedeutung von (interner) F\&E für den Innovationsprozess heißt jedoch nicht, dass KMU Neuentwicklungen überwiegend auslagern würden. Zwar ist die Entwicklung von neuen Produkten oder Prozessen durch andere bei kleineren Unternehmen etwas üblicher als bei größeren, aber doch über $70 \%$ der KMU haben Produkte und über 50 \% Prozesse im eigenen Unternehmen entwickelt. Ein Anteil, der auch unter Großunternehmen nicht viel höher ist (80 \% bzw. 64 \%; siehe hierzu Tab. 24).
Eine Voraussetzung bzw. ein Erfolgsfaktor für unternehmensinterne Innovationsaktivitäten ist dabei die Qualität und das Qualifikationsniveau der Beschäftigten. Hinsichtlich der Humankapitalausstattung - gemessen am Anteil der HochschulabsolventenInnen - zeigt sich der KMUSektor stärker geteilt als die Gruppe der Großunternehmen. So gibt es einen relativ großen Anteil an KMU, die wenige bis gar keine HochschulabsolventInnen beschäftigen: Allein ein Drittel der kleinen Unternehmen weist keine HochschulabsolventInnen unter der Belegschaft auf. Auf der anderen Seite ist der Anteil der Unternehmen, bei denen jeder zweite Beschäftigte einen Hochschulabschluss vorweist, bei kleinen Unternehmen mit $8 \%$ deutlich höher als bei mittleren und großen Unternehmen (Abb. 61).

Für die Teilgruppe des F\&E-Personals weisen Zahlen der F\&E-Erhebung $2009^{170}$ auf relativ ge-

169 Siehe Rammer et al. (2008).

170 Siehe Statistik Austria (2011). 
Tab. 24: Wer hat die Produkt-/ Prozessinnovationen der Jahre 2008-2010 entwickelt? (in \% aller Unternehmen mit technologischen Innovationen, untergliedert nach Unternehmensgröße)

\begin{tabular}{|c|c|c|c|c|c|}
\hline & \multirow[b]{2}{*}{ Beschäftigte } & \multicolumn{4}{|c|}{ Entwicklung der Produkt-/ Prozessinnovation(en) durch ... } \\
\hline & & $\begin{array}{l}\text {... das eigene } \\
\text { Unternehmen }\end{array}$ & $\begin{array}{c}\ldots \text { das eigene } \\
\text { Unternehmen zusammen } \\
\text { mit anderen }\end{array}$ & $\begin{array}{l}\text {... eigene Anpassung/ } \\
\text { Veränderung von } \\
\text { Entwicklungen anderer }\end{array}$ & $\begin{array}{l}\text {... durch andere } \\
\text { Unternehmen/ } \\
\text { Einrichtungen }\end{array}$ \\
\hline \multirow{4}{*}{ Produkt } & Insgesamt & 72,7 & 42,2 & 13,8 & 10,3 \\
\hline & $10-49$ & 71,8 & 37,9 & 13,1 & 11,9 \\
\hline & 50-249 & 71,6 & 46,2 & 15,0 & 8,7 \\
\hline & 250 und mehr & 80,2 & 58,1 & 15,1 & 4,5 \\
\hline \multirow{4}{*}{ Prozess } & Insgesamt & 54,4 & 45,7 & 17,8 & 15,2 \\
\hline & $10-49$ & 54,6 & 39,9 & 16,0 & 15,8 \\
\hline & $50-249$ & 50,5 & 52,7 & 20,1 & 14,9 \\
\hline & 250 und mehr & 63,7 & 64,4 & 24,1 & 11,5 \\
\hline
\end{tabular}

Quelle: Statistik Austria CIS 2010. Berechnung JOANNEUM RESEARCH.

ringe strukturelle Unterschiede hin: So sind sowohl in Kleinst- und Kleinunternehmen wie auch in Großunternehmen etwa 60 \% des F\&EPersonals WissenschaftlerInnen und IngenieurInnen. Lediglich in mittleren Unternehmen liegt deren Anteil bei knapp 50 \%. Der Anteil des höherqualifizierten nichtwissenschaftlichen Personals liegt entsprechend bei rund $35 \%$ für Kleinstund Kleinunternehmen bzw. $45 \%$ für mittlere Unternehmen.

Abb. 61: Innovationsaktive Unternehmen nach dem Anteil der Beschäftigten mit Universitäts- oder Fachhochschulabschluss an den gesamten Beschäftigten in \% im Jahr 2010

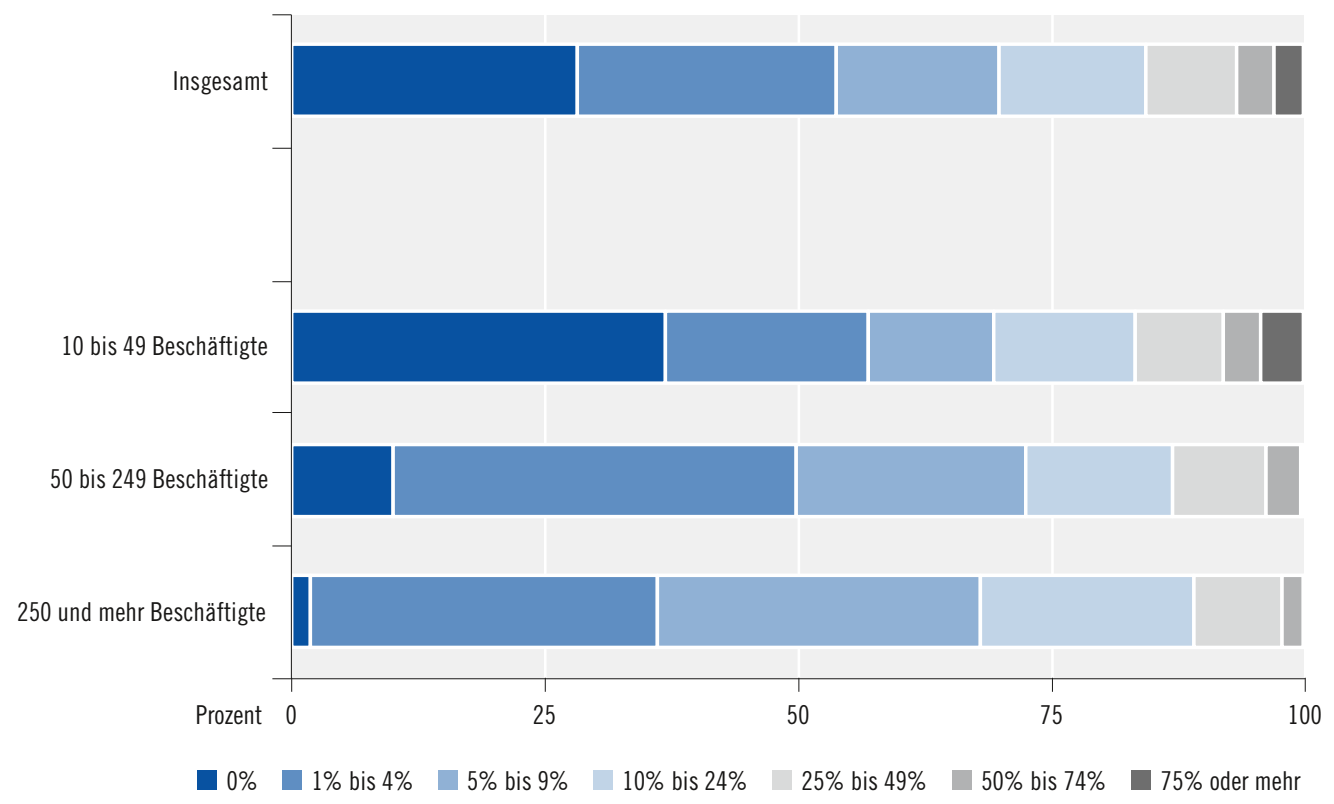

Quelle: Statistik Austria CIS 2010. Berechnung JOANNEUM RESEARCH. 


\section{Innovationskooperationen}

Innovative kleinere und mittlere Unternehmen nehmen seltener an Innovationskooperationen teil als größere Unternehmen. Dies ist vor allem darin begründet, dass kleinere Unternehmen üb- licherweise eine schlechtere Ressourcenausstattung aufweisen, so dass für sie die Organisation von Kooperationsbeziehungen vergleichsweise aufwändiger ist. Dies gilt vor allem bei der Durchführung einer Zusammenarbeit mit ausländischen Kooperationspartnern (Tab. 25).

Tab. 25: Anteil der Unternehmen mit Innovationskooperationen (a) in \% aller Unternehmen mit technologischen Innovationsaktivitäten und (b) in \% aller Unternehmen mit Innovationskooperationen) im Jahr 2010

\begin{tabular}{l|c|c|c|} 
& \multirow{2}{*}{ Beschäftigte } & (a) Unternehmen mit & \multicolumn{2}{|c|}{ (b) davon mit } \\
\cline { 3 - 4 } & Innovationskooperationen & nationalen Partnern & internationalen Partnern \\
\hline Insgesamt & $\mathbf{5 1 , 0}$ & $\mathbf{8 5 , 6}$ & $\mathbf{6 0 , 7}$ \\
\hline 10-49 & 44,7 & 83,5 & 55,5 \\
\hline $50-249$ & 59,1 & 87,4 & 61,7 \\
250 und mehr & 77,1 & 91,3 & 82,9
\end{tabular}

Quelle: CIS 2010. Berechnungen JOANNEUM RESEARCH.

Abb. 62: Innovationskooperationen mit unterschiedlichen Akteuren (in \% aller Unternehmen mit Innovationskooperationen, nach Beschäftigungsgrößenklassen) im Jahr 2010

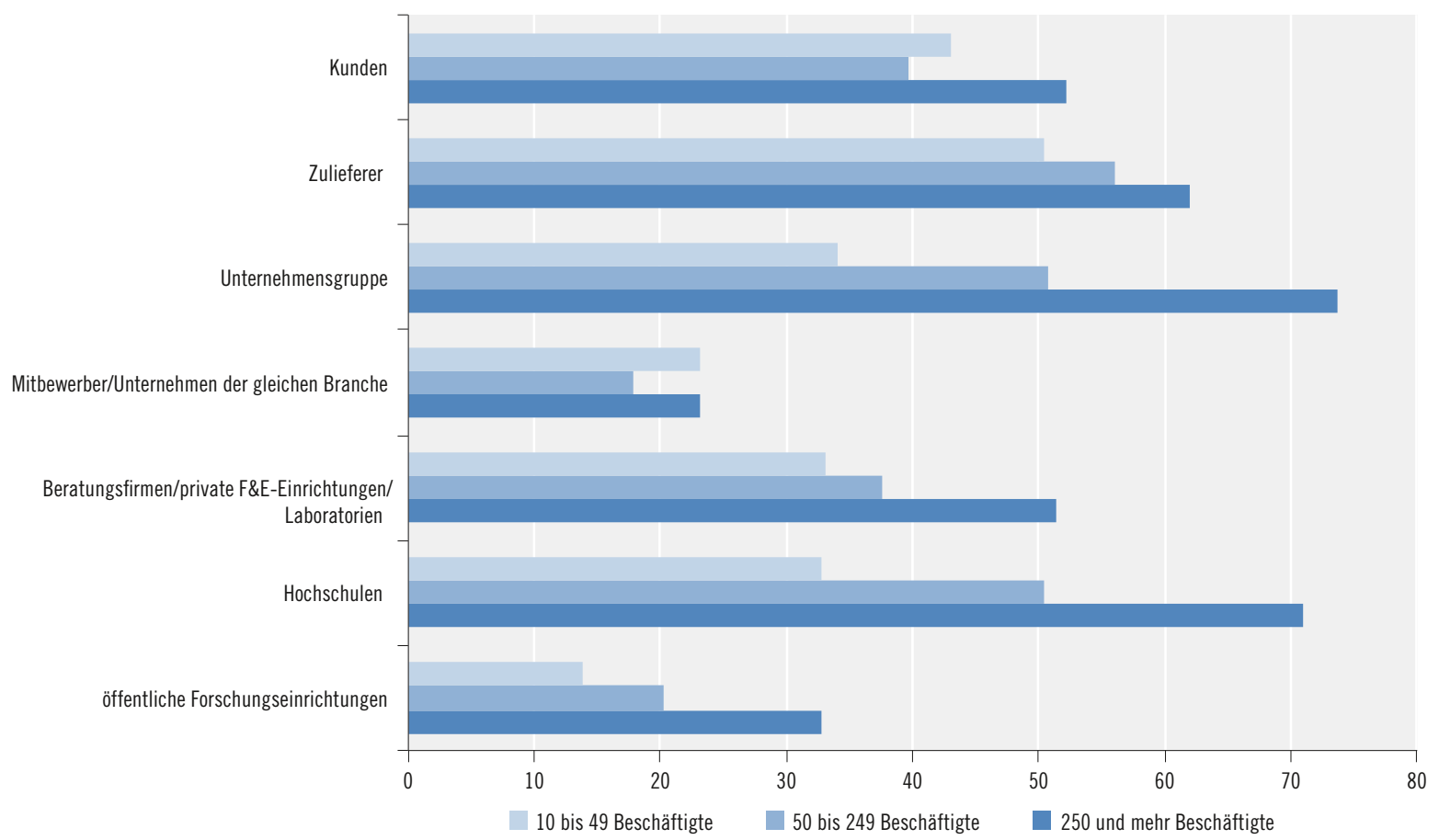

Quelle: CIS 2010. Berechnungen JOANNEUM RESEARCH. 
Hinsichtlich der Art der Kooperationspartner zeigt sich, dass KMU interaktive Innovationsaktivitäten mit Kunden oder Zulieferern nahezu so häufig suchen wie Großunternehmen (Abb. 62). Seltener sind hingegen Kooperationen innerhalb einer Unternehmensgruppe. Dies ist im Wesentlichen der Tatsache geschuldet, dass KMU seltener Teil einer solchen Unternehmensgruppe ${ }^{171}$ sind: Zu einer Gruppe gehören nur $11 \%$ der kleinen, immerhin $33 \%$ der mittleren, jedoch $62 \%$ der großen Unternehmen. Eine weitere Auffälligkeit ist, dass KMU nicht so oft mit Hochschulen zusammenarbeiten. Kooperationen mit außeruniversitären Forschungseinrichtungen sind allgemein selten. Ursache hierfür dürften die Grundlagenorientierung und Fristigkeit entsprechender Kooperationen mit Forschungseinrichtungen sein, die teilweise zu hohe Anforderungen an die Ressourcen der KMU stellen.

Diese Erklärung gilt auch für die Fokussierung auf wenige Kooperationspartner (bzw. -typen): Die Daten belegen, dass KMU häufiger mit nur einer Art oder zwei Arten von Kooperationspartnern (kleine: $61 \%$, mittlere: $44 \%$; große: $21 \%$ aller Unternehmen mit Innovationskooperationen) zusammenarbeiten, während Großunternehmen oft auch Kooperationsbeziehungen zu fünf oder mehr Arten von Kooperationspartnern unterhalten (können) (kleine: $15 \%$; mittlere: $27 \%$; große: $51 \%$ ).

\section{Innovationsoutput}

Als Ergebnis der Innovationsprozesse erzielen Großunternehmen 14 \% ihres Umsatzes mit innovativen Produkten. Mittelgroße Unternehmen erreichen mit $12 \%$ ein ähnliches Niveau, während kleine Unternehmen lediglich auf $8 \%$ Umsatzanteil kommen. Für alle Unternehmensgrö- ßenklassen sind Marktneuheiten dabei für einen kleineren Umsatzanteil verantwortlich als Produktinnovationen, die nur neu für das Unternehmen sind (Abb. 63).

\subsubsection{Besonderheiten des Innovationsverhaltens von KMU in Österreich}

Um festzustellen, inwieweit sich das Innovationsverhalten der KMU in Österreich von dem der KMU in anderen Ländern unterscheidet, werden regressionsanalytische multivariate Verfahren herangezogen. Dabei wird der Einfluss der Unternehmensgröße auf die einzelnen oben dargestellten Innovationsindikatoren bei gleichzeitiger Kontrolle für die Branchenzugehörigkeit und andere unternehmensspezifische Einflussfaktoren ${ }^{172}$ untersucht. Dieses Verfahren hat - im Vergleich zu einer deskriptiven Analyse auf gesamtwirtschaftlicher Ebene - den Vorteil, dass Gleiches mit Gleichem verglichen wird, nämlich das Innovationsverhalten von Unternehmen, die unter ähnlichen Rahmenbedingungen agieren. Für diese Analysen ist der Zugriff auf die Originaldaten (Mikrodaten) der CIS-Erhebungen in Österreich sowie in Vergleichsländern nötig. Eurostat stellt einen solchen Datenzugang zur Verfügung, allerdings sind darin die Mikrodaten aus Österreich nicht enthalten. Diese können nur durch die Statistik Austria analysiert werden. ${ }^{173}$ Dadurch sind direkte regressionsanalytische Vergleiche des Innovationsverhaltens österreichischer KMU mit KMU aus anderen Ländern nicht möglich. Daher wird auf einen Ansatz zurückgegriffen, bei dem für jedes Land ermittelt wird, inwieweit sich KMU in ihren Innovationsaktivitäten von den Großunternehmen in ihrem Land unterscheiden. Um differenzierte Aussagen zu

171 Die Eingruppierung als KMU erfolgt auf Basis der Beschäftigtenzahl des befragten Unternehmens ohne Berücksichtigung etwaiger Kapitalverflechtungen mit anderen Unternehmen. Die Frage nach der Zugehörigkeit zu einer Unternehmensgruppe basiert zudem ausschließlich auf der Selbstauskunft des befragten Unternehmens im Fragebogen.

172 Hierfür stehen in den CIS-Daten nur wenige Informationen zur Verfügung. In dieser Analyse werden drei Gruppen von Variablen herangezogen: die Zugehörigkeit zu einer Unternehmensgruppe, die geographische Marktausrichtung (regionale, landesweite, europäische, außereuropäische Absatzmärkte; diese Information steht allerdings nicht für die Unternehmen aus Finnland zur Verfügung) sowie die relative Produktivität (Umsatz je Beschäftigten in Relation zum Branchendurchschnitt) als Maß für Effizienzunterschiede, die sich u.a. in einer unterschiedlichen Finanzierungssituation niederschlagen.

173 An dieser Stelle sei Statistik Austria für die Durchführung der Datenanalysen gedankt. 
Abb. 63: Umsätze mit Produktinnovationen (in \% des Umsatzes, nach Beschäftigungsgrößenklassen) im Jahr 2010

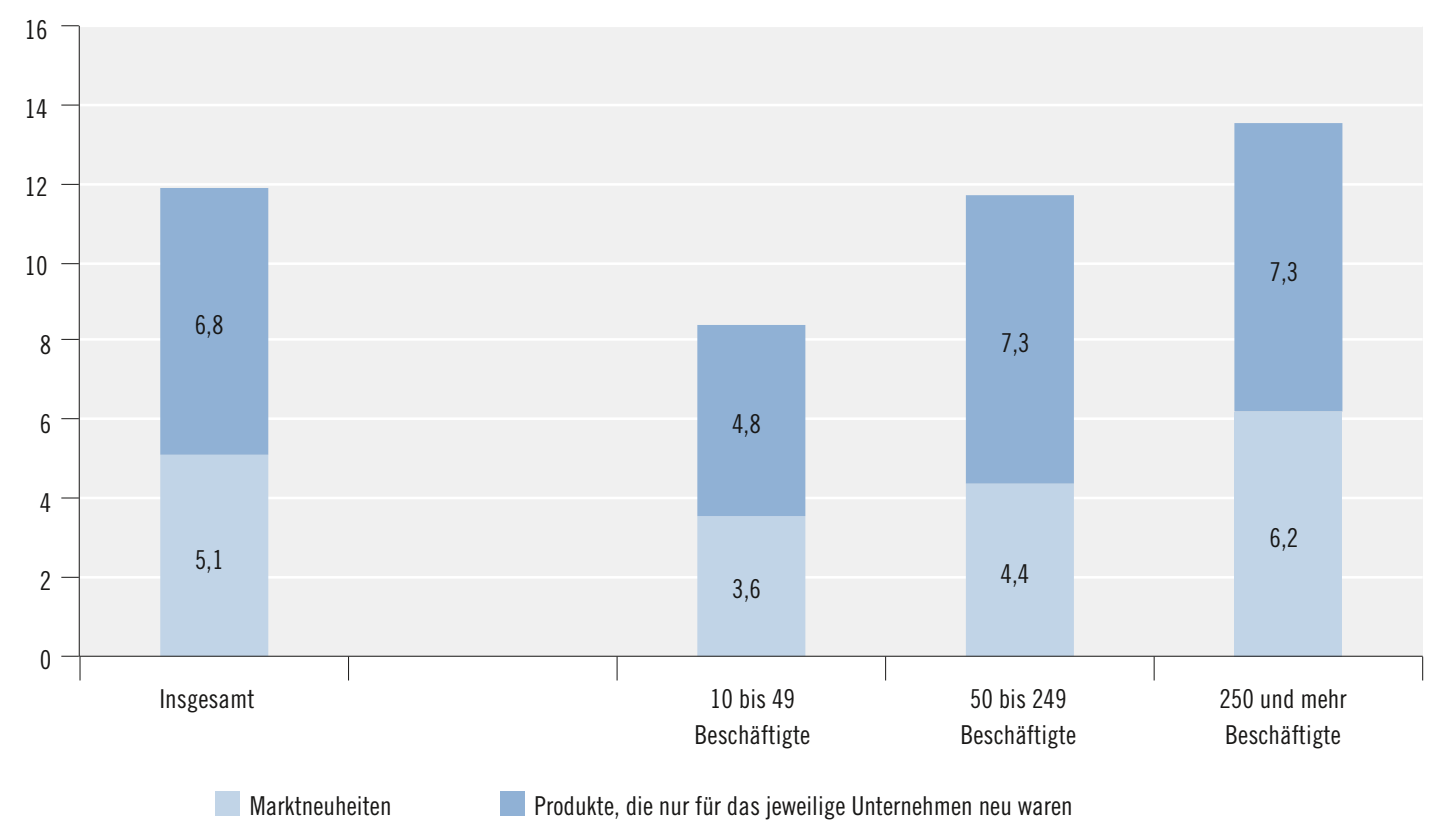

Quelle: CIS 2010. Berechnungen JOANNEUM RESEARCH.

ermöglichen, werden vier Gruppen von KMU unterschieden: sehr kleine Unternehmen (10-19 Beschäftigte), kleine Unternehmen (20-49 Beschäftigte), mittelkleine Unternehmen (50-99 Beschäftigte) und mittlere Unternehmen (100-249 Beschäftigte). Die folgenden Analysen beruhen auf den Daten des CIS 2008, da zum Analysezeitpunkt die Mikrodaten des CIS 2010 noch nicht zur Verfügung standen. Als Vergleichsländer dienen Deutschland, Frankreich, die Niederlande, Norwegen, Schweden und Finnland, d.h. Länder mit einem ähnlichen Entwicklungsstand, in denen Innovationsaktivitäten eine ähnlich hohe Bedeutung für die Wettbewerbsfähigkeit der Unternehmen wie in Österreich besitzen. ${ }^{174}$

Der internationale Vergleich zeigt einige Besonderheiten des Innovationsverhaltens von KMU in Österreich:

- Die Innovationsbeteiligung der mittleren Unternehmen (100 bis 249 Beschäftigte) unterscheidet sich weniger stark von jener der
Großunternehmen als in den meisten anderen Ländern. Dies gilt insbesondere für technologische Innovationen sowie für Marketinginnovationen.

- Der Abstand in den F\&E-Aktivitäten zwischen KMU und Großunternehmen ist in Österreich vergleichsweise gering. Dies gilt für alle Größenklassen innerhalb der KMU. Mittlere Unternehmen, die F\&E unternehmensintern betreiben, weisen eine ähnlich hohe Bereitschaft auf, F\&E kontinuierlich zu betreiben (d.h. Personal eigens für $\mathrm{F} \& \mathrm{E}$ abzustellen bzw. eine eigene F\&E-Abteilung zu unterhalten) wie Großunternehmen. Ähnlich geringe Unterschiede wie Österreich weisen auch Frankreich und die Niederlande auf. In beiden Ländern existiert ebenso wie in Österreich eine steuerliche F\&E-Förderung, welche die breite Masse der KMU erreicht und dazu beiträgt, dass ein größerer Anteil der KMU F\&E-Aktivitäten durchführt.

174 Daten zu Dänemark und Belgien, die sich ebenfalls als Vergleichsländer anbieten würden, liegen nicht vor. 
- Bei der Inanspruchnahme einer finanziellen öffentlichen Förderung für Innovationsaktivitäten sind die Unterschiede zwischen KMU und Großunternehmen in Österreich niedriger als in Deutschland und Finnland, jedoch gröBer als in Frankreich und den Niederlanden. Berücksichtigt man aber zusätzlich die unterschiedliche F\&E-Neigung von KMU und Großunternehmen, so gibt es in Österreich im Gegensatz zu Frankreich und den Niederlanden - keinen statistisch signifikanten Unterschied in der Inanspruchnahme öffentlicher Förderungen. Dies spiegelt den einfachen $\mathrm{Zu}-$ gang für F\&E-betreibende Unternehmen zur steuerlichen F\&E-Förderung über die Forschungsprämie wider. Dadurch erhalten faktisch alle F\&E betreibenden KMU in Österreich eine finanzielle Förderung.

- Keine Besonderheiten zeigen sich beim Kooperationsverhalten der KMU, d.h. hier ist der Abstand zu den Großunternehmen in Österreich ähnlich hoch wie in den meisten der Vergleichsländer.

- Die Innovationsausgaben in Relation zum
Umsatz sind in kleineren Unternehmen aus Österreich signifikant höher als in Großunternehmen (wobei jeweils nur Unternehmen mit technologischen Innovationsaktivitäten betrachtet werden). Dieser Effekt zeigt sich in keinem der Vergleichsländer. In Österreich waren es im Jahr 2008 vor allem vergleichsweise hohe Ausgaben für interne F\&E und für den Erwerb von Maschinen und Software, die $\mathrm{zu}$ einer höheren Innovationsintensität der kleineren Unternehmen geführt haben.

- Beim Innovationserfolg zeigen mittlere Unternehmen aus Österreich, die Produktinnovationen eingeführt haben, eine ähnlich hohe Wahrscheinlichkeit, Marktneuheiten einzuführen wie Großunternehmen. In den Vergleichsländern (ausgenommen Norwegen) sind dagegen Großunternehmen signifikant häufiger in der Lage, Marktneuheiten einzuführen. Beim Umsatzanteil mit neuen Produkten erreichen KMU höhere Anteile als Großunternehmen. Dies gilt für österreichische KMU ebenso wie für KMU aus den meisten Vergleichsländern.

Tab. 26: Unterschiede in der Innovationsbeteiligung zwischen KMU und Großunternehmen in ausgewählten Ländern im Jahr 2008

\begin{tabular}{|c|c|c|c|c|c|c|c|c|}
\hline Innovationsindikator & \multirow{2}{*}{$\begin{array}{l}\text { Größen- } \\
\text { variable }\end{array}$} & AT & DE & $\mathbf{F R}$ & NL & NO & SE & FI \\
\hline (Basis: alle Unternehmen) & & \multicolumn{7}{|c|}{ Abweichung von Unternehmen mit 250 u.m. Beschäftigten in \%-Punkten } \\
\hline \multirow{4}{*}{$\begin{array}{l}\text { Anteil der Unternehmen mit } \\
\text { technologischen Innovationen }\end{array}$} & $10-19$ & -26 & -27 & -23 & -21 & -15 & -32 & -32 \\
\hline & $20-49$ & -27 & -26 & -21 & -16 & -12 & -27 & -24 \\
\hline & $50-99$ & -21 & -22 & -15 & -11 & -11 & -24 & -23 \\
\hline & $100-249$ & -9 & -17 & -8 & -6 & -10 & -17 & -14 \\
\hline \multirow{4}{*}{$\begin{array}{l}\text { Anteil der Unternehmen mit } \\
\text { nicht-technologischen Innovationen }\end{array}$} & $10-19$ & -26 & -22 & -22 & -25 & -13 & -32 & -33 \\
\hline & $20-49$ & -19 & -14 & -17 & -21 & -11 & -24 & -23 \\
\hline & $50-99$ & -19 & -11 & -14 & -15 & -10 & -17 & -19 \\
\hline & $100-249$ & -9 & -13 & -7 & -9 & I & -15 & -14 \\
\hline \multirow{4}{*}{$\begin{array}{l}\text { Anteil der Unternehmen mit } \\
\text { internen F\&E-Aktivitäten }\end{array}$} & $10-19$ & -17 & -28 & -17 & -16 & -20 & -30 & -39 \\
\hline & $20-49$ & -16 & -27 & -15 & -12 & -19 & -24 & -32 \\
\hline & 50-99 & -12 & -26 & -10 & -8 & -11 & -21 & -29 \\
\hline & $100-249$ & -6 & -21 & -6 & -5 & -11 & -16 & -18 \\
\hline
\end{tabular}

Anm.: Abweichung in \%-Punkten bei Kontrolle für Branchenzugehörigkeit, Zugehörigkeit zu einer Unternehmensgruppe, regionaler Marktausrichtung und relativer Produktivität.

"/“: kein statistisch signifikanter Unterschied bei $<5 \%$ Fehlerwahrscheinlichkeit. Werte in Klammern: Unterschied zwischen 5 und $<10 \%$ Fehlerwahrscheinlichkeit signifikant.

Quelle: CIS 2008. Berechnungen ZEW/Statistik Austria. 
Tab. 27: Unterschiede zwischen KMU und Großunternehmen im Innovationsverhalten von Unternehmen mit technologischen Innovationsaktivitäten in ausgewählten Ländern im Jahr 2008

\begin{tabular}{|c|c|c|c|c|c|c|c|c|}
\hline Innovationsindikator & \multirow{2}{*}{$\begin{array}{l}\text { Größen- } \\
\text { variable }\end{array}$} & AT & DE & $\mathbf{F R}$ & NL & No & SE & $\mathbf{F I}$ \\
\hline $\begin{array}{l}\text { (Basis: Unternehmen mit technologischen } \\
\text { Innovationsaktivitäten) }\end{array}$ & & \multicolumn{7}{|c|}{ Abweichung von Unternehmen mit 250 u.m. Beschäftigten in \%-Punkten } \\
\hline \multirow[t]{4}{*}{ Anteil Unternehmen mit Kooperationen } & $10-19$ & -21 & -18 & -18 & -25 & -12 & -28 & -36 \\
\hline & $20-49$ & -26 & -22 & -17 & -24 & -12 & -23 & -33 \\
\hline & $50-99$ & -22 & -17 & -15 & -19 & -14 & -25 & -30 \\
\hline & $100-249$ & -18 & -20 & -13 & -10 & -14 & -11 & -25 \\
\hline \multirow{4}{*}{$\begin{array}{l}\text { Anteil Unternehmen mit Inanspruchnahme } \\
\text { offentlicher Förderung }\end{array}$} & $10-19$ & -18 & -28 & -5 & -12 & n.v. & n.v. & -26 \\
\hline & $20-49$ & -15 & -26 & -3 & -9 & n.v. & n.v. & -23 \\
\hline & $50-99$ & -14 & -28 & -4 & -7 & n.v. & n.v. & -21 \\
\hline & $100-249$ & I & -29 & -3 & $(-4)$ & n.v. & n.v. & -19 \\
\hline \multirow{4}{*}{$\begin{array}{l}\text { Anteil der Unternehmen mit Inanspruchnahme } \\
\text { öffentlicher Förderung bei Kontrolle für F\&E- } \\
\text { Aktivität }\end{array}$} & $10-19$ & I & -23 & -3 & $(-7)$ & n.v. & n.v. & -19 \\
\hline & $20-49$ & I & -20 & I & $(-4)$ & n.v. & n.v. & -16 \\
\hline & 50-99 & I & -23 & -3 & $(-4)$ & n.v. & n.v. & -16 \\
\hline & $100-249$ & I & -26 & $(-3)$ & I & n.v. & n.v. & -16 \\
\hline \multirow{4}{*}{$\begin{array}{l}\text { Anteil der Innovationsausgaben insgesamt am } \\
\text { Umsatz }\end{array}$} & $10-19$ & +13 & I & I & I & I & I & I \\
\hline & $20-49$ & +5 & I & I & I & I & I & I \\
\hline & 50-99 & I & I & I & I & I & I & I \\
\hline & $100-249$ & 1 & I & I & I & I & I & I \\
\hline
\end{tabular}

Anm.: Abweichung in \%-Punkten bei Kontrolle für Branchenzugehörigkeit, Zugehörigkeit zu einer Unternehmensgruppe, regionaler Marktausrichtung und relativer Produktivität.

,"“: kein statistisch signifikanter Unterschied bei $<5 \%$ Fehlerwahrscheinlichkeit. Werte in Klammern: Unterschied zwischen 5 und $<10 \%$ Fehlerwahrscheinlichkeit signifikant.

Quelle: CIS 2008. Berechnungen ZEW/Statistik Austria.

Tab. 28: Unterschiede zwischen KMU und Großunternehmen im Innovationserfolg mit Produktinnovationen in ausgewählten Ländern im Jahr 2008

\begin{tabular}{|c|c|c|c|c|c|c|c|c|}
\hline Innovationsindikator & \multirow{2}{*}{ Größen-variable } & AT & $\mathbf{D E}$ & FR & NL & NO & SE & FI \\
\hline (Basis: Unternehmen mit Produktinnovationen) & & \multicolumn{7}{|c|}{ Abweichung von Unternehmen mit 250 u.m. Beschäftigten in \%-Punkten } \\
\hline \multirow{4}{*}{$\begin{array}{l}\text { Anteil der Unternehmen, die Marktneuheiten } \\
\text { eingeführt haben }\end{array}$} & $10-19$ & $(-9)$ & -13 & -9 & I & I & -8 & -13 \\
\hline & $20-49$ & $(-9)$ & $(-7)$ & -9 & I & I & -13 & I \\
\hline & $50-99$ & -16 & -15 & -8 & -10 & I & -8 & -21 \\
\hline & $100-249$ & l & -14 & -11 & $(-5)$ & I & $(-9)$ & -13 \\
\hline \multirow[t]{4}{*}{ Umsatzanteil mit neuen Produkten } & $10-19$ & +10 & +9 & +10 & +11 & +8 & +7 & +6 \\
\hline & $20-49$ & I & +7 & +7 & +4 & +6 & +5 & I \\
\hline & $50-99$ & l & +5 & l & +4 & l & / & I \\
\hline & $100-249$ & I & $(+3)$ & +3 & +3 & I & I & I \\
\hline \multirow{4}{*}{$\begin{array}{l}\text { Umsatzanteil mit Marktneuheiten (nur Unter- } \\
\text { nehmen mit Marktneuheiten) }\end{array}$} & $10-19$ & +11 & +9 & +9 & +10 & +9 & +8 & +7 \\
\hline & $20-49$ & +6 & +8 & +7 & +5 & +7 & +8 & $(+4)$ \\
\hline & $50-99$ & I & +4 & I & +4 & I & +4 & +6 \\
\hline & $100-249$ & 1 & 1 & +4 & +3 & I & +3 & I \\
\hline
\end{tabular}

Anm.: Abweichung in \%-Punkten bei Kontrolle für Branchenzugehörigkeit, Zugehörigkeit zu einer Unternehmensgruppe, regionaler Marktausrichtung und relativer Produktivität.

„/“" kein statistisch signifikanter Unterschied bei $<5 \%$ Fehlerwahrscheinlichkeit. Werte in Klammern: Unterschied zwischen 5 und $<10 \%$ Fehlerwahrscheinlichkeit signifikant.

Quelle: CIS 2008. Berechnungen ZEW/Statistik Austria. 
Zusammengefasst unterstreichen die Ergebnisse die Bedeutung eines breitenwirksamen Innovationsfördersystems für die Verringerung des $\mathrm{Ab}$ stands zwischen KMU und Großunternehmen in der Innovationsleistung. In Österreich konnten dabei vor allem die mittleren Unternehmen an die Großunternehmen "herangeführt" werden. Dies ist angesichts der Unternehmensstruktur in Österreich mit dem im internationalen Vergleich eher geringen Anteil von Großunternehmen wichtig, um eine weitere Verbesserung der Innovationsleistung der österreichischen Wirtschaft zu erreichen. Denn um den Aufstieg zu den Innovation Leaders zu schaffen, muss gerade auch der KMU-Sektor wichtige Beiträge leisten. Deshalb sollte auch der Weg einer breit angelegten Innovationsförderung fortgesetzt werden.

\section{Resümee}

Die Analyse der Europäischen Innovationserhebung zeigt, dass Österreich im europäischen Vergleich eine gute (bis sehr gute) Position einnimmt. Der Anteil innovierender Unternehmen liegt in Österreich deutlich über dem Schnitt der EU-27 und die Innovatorenquote ist durchgängig in allen Branchen hoch. Gleichzeitig lässt sich aus der Struktur der Innovationsausgaben mit dem hohen Gewicht der F\&E-Ausgaben auf ein "reifes", modernes Innovationssystem schließen, dessen Unternehmen laufend neues Wissen generieren und am Markt in Form von neuen Produkten bzw. Dienstleistungen platzieren. Dabei sind Österreichs Unternehmen gut mit ihren Zulieferern und Kunden, aber auch mit Universitäten bzw. Hochschulen in Innovationsnetzwerke eingebunden. Die österreichische Wirtschaftspolitik hat bereits seit langem den hohen Stellenwert von unternehmerischen Innovationen anerkannt und fördert das unternehmerische Innovationsverhalten mit entsprechenden Instrumenten. Dabei hat Österreichs Fördersystem eine hervorragende Reichweite, d.h. Innovation wird "flächig" adressiert, der Anteil der Unternehmen, die in den Genuss von innovationsspezifischen Fördermaßnahmen kommen, zählt zu den höchsten innerhalb der EU. Diese Breitenwirkung der Förderung trägt wesentlich dazu bei, dass die Unterschiede zwischen KMU und Großunternehmen im Innovationsverhalten in Österreich geringer als in den meisten anderen hoch entwickelten europäischen Industrieländern sind.

\subsection{Schnell wachsende junge Unternehmen}

In der wissenschaftlichen und auch in der politischen Diskussion wird der Gründungsdynamik im Unternehmenssektor einer Volkswirtschaft hohe Bedeutung für die Wettbewerbsfähigkeit, für die Innovationstätigkeit, für den Strukturwandel und für die Generierung von Beschäftigung zugeschrieben. Hierbei wird häufig auf die sogenannten innovativen Gründungen abgestellt, und von diesen insbesondere auf die schnell wachsenden Jungunternehmen, denen eine wesentliche Rolle für den Strukturwandel und die Generierung von Beschäftigung zugeschrieben wird. Mit der Bedeutung schnell wachsender kleiner und junger Unternehmen für die Beschäftigungsentwicklung hat sich als einer der ersten D.L. Birch auseinandergesetzt, ${ }^{175}$ von ihm wurde auch der Begriff "Gazellen" ${ }^{\prime 176}$ für Unternehmen mit hohen Wachstumsraten eingeführt. In jüngerer Zeit allerdings, wie beispielsweise von Shane (2008), wird angezweifelt, dass sich durch die Stimulierung des Gründungsgeschehens insgesamt die Anzahl schnell und nachhaltig wachsender Unternehmen erhöhen lässt. Er plädiert vielmehr für eine Konzentration auf wenige neu aufkommende Branchen.

Gleichwohl wird die EU-Kommission dem Thema "schnell wachsende Unternehmen“ eine verstärkte Aufmerksamkeit widmen. So soll ab

175 Siehe Birch (1979), Birch, Medoff (1994), Birch et al. (1995).

176 Birch definierte als Unternehmen mit schnellem Wachstum solche, die über einen Zeitraum von mindestens fünf Jahren ein jährliches Umsatzwachstum von mindestens 20 Prozent aufweisen. 
2013 ein neuer Headline Indikator zur Messung der Fortschritte im Rahmen von „Europe 2020" den Anteil der schnell wachsenden jungen Unternehmen in den Ländern der Europäischen Union ausweisen. Zusammen mit Indikatoren zur Arbeitsproduktivität, zum Patentverhalten der Unternehmen, zur Beschäftigung in wissensintensiven Bereichen und zum Beitrag des Handels mit innovationsbezogenen Gütern zur gesamten Handelsbilanz, soll der neue Indikator ein Bild der Entwicklungen im Unternehmenssektor zeichnen.

Es hat sich gezeigt, dass sich Wachstum (aber auch Innovationsverhalten) von jungen Unternehmen auch innerhalb von Branchengruppen erheblich unterscheiden kann. Eine Klassifizierung auf der Ebene der Branchen, orientiert an den jeweiligen Durchschnittswerten, birgt somit gewisse Risiken der Fehlinterpretation. Differenzierende Analysen auch innerhalb von Sektoren und Branchengruppengrenzen sind sinnvoll. Über die Unterschiede hinsichtlich des Wachstums ist gegenwärtig noch wenig bekannt, hinsichtlich des Innovationsverhaltens zeigen Untersuchungen, dass auch in Branchen der Spitzentechnik nur 15 bis $20 \%$ der Unternehmen tatsächlich sehr hohe F\&E-Intensitäten aufweisen. Auch in im Durchschnitt weniger innovativen Branchen finden sich Unternehmen mit hohem Wachstum und auch sehr hohen F\&E-Intensitäten.

Für den Wachstumspfad, den junge Unternehmen tatsächlich einschlagen, sind neben ihrem Engagement hinsichtlich der Innovationsaktivitäten noch zahlreiche andere Faktoren und Umstände verantwortlich. Hierfür können beispielhaft genannt werden:

- Die strategische Ausrichtung der jungen Unternehmen im Hinblick auf das Thema „Wachstum": Viele Gründer, auch solche von Hochtechnologieunternehmen, suchen häufig nicht die maximal mögliche Expansion ihres Unternehmens. Sie gründen vielmehr mit einer bestimmten Geschäftsidee und wollen selbstbestimmt arbeiten. Eine schnelle Expansion ihres Unternehmens, womöglich in $\mathrm{Zu}$ - sammenhang mit einer Gewährung von Einflussmöglichkeiten an externe Finanziers, würde ihr Gründungskonzept konterkarieren.

- Die Markt- und Wettbewerbsbedingungen auf dem Absatzmarkt: Nicht alle Märkte bieten die Möglichkeiten für eine schnelle und starke Expansion junger Unternehmen. Hierfür sind neben der Nachfrageintensität auch die Schutzmöglichkeiten der eigenen Leistungen vor Imitation oder Abwandlung (beispielsweise durch Patentschutz) ausschlaggebend. Genauso spielt die regionale Größe eines ökonomisch einheitlichen Heimatmarktes eine wichtige Rolle. Trotz des europäischen Binnenmarktes sind diesbezüglich noch erhebliche Unterschiede für in Europa ansässige Unternehmen im Vergleich zu solchen auf dem US-amerikanischen Markt zu verzeichnen.

- Die „individuellen“ Bedingungen der jungen Unternehmen: Die unternehmerischen, technologischen und kaufmännischen Fähigkeiten im Gründungsteam eines Unternehmens müssen ausreichen, um starkes Wachstum auch strukturell - managen zu können. Da genügend qualifizierte MitarbeiterInnen akquiriert werden müssen, um einen Wachstumsprozess zu realisieren, muss die Personalpolitik entsprechend ausgerichtet sein. Eine häufig kaum zu überspringende Hürde stellt sich hinsichtlich der Finanzierung eines Wachstumsprozesses, insbesondere dann, wenn es erst zu sehr zeitverzögerten Rückflüssen aus Investitionsaufwendungen kommt.

Diese Überlegungen verdeutlichen, dass die Ursachen für das Wachsen - und insbesondere auch für das Nicht-Wachsen - von jungen Unternehmen sehr divergent und vielfältig sein können. Inwieweit sie durch politische Maßnahmen oder staatlich-administrative Interventionen adressiert werden können, ist völlig offen. Gerade deshalb ist es von besonderem Interesse, inwieweit sich die Tendenz als junges Unternehmen einen Wachstumskurs zu steuern, schon in der ersten Phase am Markt zeigt und von welcher Persistenz sie ist. Ebenso von Interesse ist es, inwie- 
weit sich Unterschiede hinsichtlich einer frühen Wachstumsorientierung zwischen jungen Unternehmen zeigen, die Branchengruppen mit unterschiedlicher Technologieorientierung oder Wissensintensität zuzurechnen sind. Genau diesen Fragen wird im Folgenden nachgegangen.

\subsubsection{Beschäftigungsentwicklung junger Unternehmen in Österreich}

Datenbasis für die Untersuchung bilden die Daten der Kreditauskunftei Creditreform. Seit 1996 übermittelt Creditreform dem Zentrum für Europäische Wirtschaftsforschung (ZEW) mit Sitz in Mannheim zweimal jährlich einen Komplettabzug seiner umfangreichen Datenbank mit Informationen $\mathrm{zu}$ wirtschaftsaktiven Unternehmen mit Sitz in Österreich (darunter auch Informationen $\mathrm{zu}$ bereits geschlossenen Unternehmen) für wissenschaftliche Zwecke. Die Informationen $\mathrm{zu}$ den Unternehmen werden in der Regel mehrfach recherchiert und in der Datenbank jeweils aktualisiert. Informationen über die Schließung von Unternehmen, sei es im Rahmen von Insolvenzverfahren oder auch durch sogenannte freiwillige Schließungen, werden in der Datenbank erfasst. Ebenfalls ermittelbar sind Fusionen oder Unternehmensübernahmen. Gegenwärtig liegen in dem hier verwendeten Datenbestand Angaben zu knapp 700.000 Unternehmen mit Sitz in Österreich vor, rund 350.000 sind als gegenwärtig sicher am Markt aktiv (nicht geschlossen) anzusehen. Es ist davon auszugehen, dass die hier verwendete Datenbasis gewisse Untererfassungen von Einzelunternehmen und Freiberuflern aufweist, über deren Umfang gegenwärtig noch keine vollständige Klarheit besteht. Aus diesem Grund werden Auswertungen basierend auf den Creditreformdaten mit analogen Berechnungen auf Basis von Daten der Arbeitsmarktdatenbank (AMDB) des Arbeitsmarktservice Österreich (AMS) gespiegelt, um die Tendenz der Aussagen abzusichern.

Kann aus der Beschäftigungsentwicklung von Gründungen in jungen Jahren (zumindest tendenziell) auf die spätere Beschäftigungsentwick- lung geschlossen werden? Das ist im Kern die Frage, der in den folgenden Betrachtungen nachgegangen wird. Dazu wird die Beschäftigtenentwicklung der österreichischen Gründungen der Gründungsjahrgänge 2002 und 2003 in den Jahren 2007 und 2011 betrachtet und analysiert, inwieweit sich systematische Zusammenhänge zwischen der Beschäftigungsentwicklung in den ersten vier bis fünf Lebensjahren und der weiteren vier Lebensjahre der jungen Unternehmen identifizieren lassen.

\subsubsection{Gründungen aller Branchen}

Zunächst werden alle österreichischen Gründungen der Jahrgänge 2002 und 2003 in die Untersuchung einbezogen, unabhängig davon, welchen Branchen sie zuzurechnen sind. Die überlebenden Unternehmen der beiden genannten Gründungskohorten haben im Jahr 2007 auf Basis der Creditreformdaten im Durchschnitt 5,8 Beschäftigte (vgl. Tab. 29). Dabei werden sowohl die sozialversicherungspflichtigen als auch die sonstigen Beschäftigten mitgezählt. Die Zählung erfolgt nach „Köpfen“, nicht in Vollzeitäquivalenten. Wird das Mannheimer Unternehmenspanel (MUP) des ZEW zugrundgelegt - das auf Creditreformdaten zu Unternehmen mit Standort in Deutschland basiert - dann zeigt sich, dass die deutschen Unternehmen der Gründungsjahrgänge 2002 und 2003 mit 4,6 Beschäftigten im Jahr 2007 im Durchschnitt etwas kleiner sind als die Gründungen Österreichs (vgl. Tab. 29).

Um abschätzen zu können, inwieweit „frühes" Beschäftigungswachstum sich auf "späteres" Beschäftigungswachstum niederschlägt, wird die Population der österreichischen Unternehmen, die in den Jahren 2002 und 2003 gegründet wurden, anhand unterschiedlicher Kriterien (Beschäftigungsschwellen) jeweils in zwei Gruppen aufgeteilt. Durch die Wahl dieser Schwellen soll eine schrittweise Annäherung an "schnell wachsende" Gründungen erfolgen. Betrachtet wird dann jeweils die weitere Beschäftigtenentwicklung der so separierten Gruppen bis zum Jahr 2011. 
Zunächst werden die Gründungen danach aufgeteilt, ob sie 2007 einen überdurchschnittlichen Beschäftigtenstand aufwiesen oder ob sie nur über eine mindestens durchschnittliche Anzahl von Beschäftigten verfügten $(>5,8$ versus $\leq 5,8)$. Die Verteilung der 2002er und 2003er Gründungen über die Beschäftigtenzahlen 2007 ist deutlich rechtsschief. So fallen nur 10,4 \% der Gründungen in die Gruppe derer mit überdurchschnittlicher Beschäftigtenzahl in 2007 (17,8 \% in Deutschland), entsprechend 89,6 \% der Gründungen haben höchstens eine durchschnittliche Beschäftigung. Die weitere Entwicklung der Beschäftigung in den Unternehmen ist nicht unabhängig davon, welcher dieser beiden Gruppen die Unternehmen zuzurechnen sind. So weisen die Unternehmen mit überdurchschnittlicher MitarbeiterInnenzahl 2007 im Jahr 2011 in Durchschnitt 12,7 Beschäftigte auf, die Gruppe mit der höchsten durchschnittlichen Beschäftigtenzahl 2007 hat 2011 dagegen durchschnittlich nur drei MitarbeiterInnen (siehe Tab. 29). Gründungen der beiden betrachteten Gründungsjahrgänge, die zwischen 2007 und 2011 aus dem Markt ausscheiden, werden 2011 mit der MitarbeiterInnenzahl von Null berücksichtigt. Die aus dem MUP für deutsche Gründungen der Jahre 2002 und 2003 analog errechneten durchschnittlichen Beschäftigtenzahlen für 2011, separiert nach dem gleichen Kriterium (2007 über- bzw. höchstens durchschnittliche MitarbeiterInnenzahl) deuten zum einen in die gleiche Richtung und sind zum anderen den Werten für österreichische Gründungen sehr ähnlich (siehe Tab. 29) ${ }^{177}$.

Die Gruppe der Gründungen mit „hohem“ Beschäftigungswachstum wird in einem zweiten Schritt weiter eingegrenzt. Nicht die Durchschnittsbeschäftigtenzahl dient hier als die Gruppen trennende Beschäftigungsschwelle, sondern die MitarbeiterInnenzahl von 10 im Jahr 2007 (>10 versus $\leq 10$ ). Nur $4,5 \%$ der österreichischen Gründungen der Jahre 2002 und 2003 weisen mehr als 10 MitarbeiterInnen im Jahr 2007 auf 15,3 \% der deutschen Gründungen der beiden Jahre). Diese Gruppe der Unternehmen hat im Jahr 2011 im Durchschnitt deutlich mehr Beschäftigte $(18,8)$ als die Unternehmen, die 2007 höchstens 10 Personen beschäftigten $(4,2)$. Tab. 29 zeigt, dass auch für diese Beschäftigungsschwelle die Auswertungen für deutsche Unternehmen die gleiche Tendenz und ähnliche durchschnittliche Beschäftigungszahlen ergeben.

\section{Tab. 29: Durchschnittlicher Beschäftigtenstand aller Gründungen der Jahre 2002/2003 in 2011 nach unterschiedlichen Beschäftigtenschwellen 2007}

\begin{tabular}{|c|c|c|c|}
\hline $\begin{array}{l}\text { Gründungskohorten } 2002 \text { und } 2003 \\
\text { Beschäftigtendurchschnitt } 2007\end{array}$ & $\begin{array}{l}\text { Creditreformdaten Österreich } \\
5,8\end{array}$ & $\begin{array}{c}\text { AMDB } \\
3,4\end{array}$ & $\begin{array}{l}\text { MUP-Deutschland } \\
4,6\end{array}$ \\
\hline \multicolumn{4}{|l|}{ Beschäftigtendurchschnitt 2011} \\
\hline 2007 überdurchschnittlich & 12,7 & 9,7 & 11,0 \\
\hline 2007 höchstens durchschnittlich & 3,0 & 1,9 & 3,0 \\
\hline \multicolumn{4}{|l|}{ Beschäftigtendurchschnitt 2011} \\
\hline 2007 mehr als 10 & 18,8 & 19,4 & 19,2 \\
\hline 2007 höchstens 10 & 4,2 & 3,0 & 4,2 \\
\hline \multicolumn{4}{|l|}{ Beschäftigtendurchschnitt 2011} \\
\hline 2007 mehr als 20 & 35,5 & 31,1 & 33,3 \\
\hline 2007 höchstens 20 & 5,9 & 3,8 & 5,2 \\
\hline
\end{tabular}

Quellen: Creditreformdaten Österreich, Mannheimer Unternehmenspanel - Berechnungen des ZEW. Arbeitsmarktdatenbank (AMDB) des AMS Berechnungen von JOANNEUM RESEARCH.

177 Die Kerndichtefunktionen der Verteilungen der Beschäftigung im Jahr 2011 in den jeweils durch die unterschiedlichen Beschäftigungsschwellen definierten Gruppen zeigen, dass die in Tab. 29 dargelegten Unterschiede der Durchschnittswerte der jeweiligen Gruppen nicht auf wenige „Ausreißer" zurückgehen, sondern auch in den Verteilungsfunktionen ihren Niederschlag finden. 
In einem dritten Schritt wird ein noch strengeres Kriterium als Beschäftigungsschwelle angewendet. Die Unternehmen werden danach differenziert, ob sie 2007 mehr als 20 oder mindestens 20 MitarbeiterInnen aufwiesen (>20 versus $\leq 20$ ). Die Unternehmen in der so definierten Gruppe mit "hohem" Beschäftigungswachstum haben in den ersten vier bis fünf Lebensjahren einen MitarbeiterInnenstand von über 20 erreicht. Eine Entwicklung, die man durchaus mit dem Begriff „schnell wachsend" belegen kann. Dieses Kriterium erfüllen allerdings nur sehr wenige Gründungen der Kohorten 2002 und 2003. Nur 0,7 \% von ihnen haben im Jahr 2007 mehr als 20 Beschäftigte $1,1 \%$ der deutschen Gründungen dieser Kohorten). Die weitere Entwicklung, die diese Unternehmen bis zum Jahr 2011 nahmen, ist beachtlich. Im Durchschnitt hatten sie 2011 35,5 Beschäftigte, die Gründungen mit höchstens 20 MitarbeiterInnen in 2007 wiesen dagegen nur durchschnittlich 5,9 MitarbeiterInnen auf (vgl. Tab. 29). Auch für diese Beschäftigtenschwelle zeigen die analogen Berechnungen für Deutschland sehr ähnliche Werte.

Vergleichbare Auswertungen aus der Arbeitsmarktdatenbank des Arbeitsmarktservice Österreich ergeben qualitativ die gleichen Ergebnisse (vgl. Tab. 29, mittlere Spalte). Hier werden nur die sozialversicherungspflichtigen Beschäftigten berücksichtigt, auch ist die Erhebungseinheit hier eine einzelne Betriebsstätte und nicht, wie in den Creditreformdaten für Österreich und im MUP, ein Unternehmen. Aus diesem Grund kann es nicht verwundern, dass die konkret errechneten Werte von den Werten auf Basis der Creditreformdaten abweichen. Die Tendenz der Ergebnisse und auch in etwa die Relationen der Unterschiede hinsichtlich der MitarbeiterInnenzahlen in 2011 für Gruppen, definiert nach unterschiedlichen Beschäftigungsschwellen in 2007, werden deutlich bestätigt.

Für die Gründungen insgesamt kann resümiert werden, dass Gründungen, die nach den ersten Lebensjahren eine "hohe“ Beschäftigtenzahl erreichen, auch in der nächsten Lebensphase der Unternehmen mit erheblich größerer
Wahrscheinlichkeit deutlich an Beschäftigung zulegen als solche, die zunächst klein bleiben. Der überwiegende Anteil der nach einigen Jahren noch „kleinen“ Unternehmen bleibt auch weiterhin klein.

\subsubsection{Gründungen der wissensintensiven Branchen}

Unter dem Gesichtspunkt des Wachstumspotenzials wird häufig den Gründungen aus den Branchen der forschungs- und wissensintensiven Branchen aus der Sachgüterproduktion und dem Dienstleistungsbereich besondere Bedeutung zugemessen. Um zu einer Einschätzung zu gelangen, ob das Einschlagen eines „frühen Wachstumspfades" für Gründungen dieser Branchen ebenfalls - oder gar ausgeprägter als im Durchschnitt aller Gründungen - einen Zusammenhang zur Entwicklung späterer Beschäftigtenzahlen zulässt, wird die Betrachtung nun auf die Gründungen der forschungs- und wissensintensiven Branchen der Jahre 2002 und 2003 sowie auf den Verlauf ihrer MitarbeiterInnenzahlen beschränkt.

Das hierfür gewählte Vorgehen entspricht dem, das im vorstehenden Abschnitt für die Analyse der Beschäftigtenentwicklung für die Gründungen aller Branchen vorgenommen wurde einschließlich des Vergleichs mit analogen Auswertungen für die Gründungen in den forschungs- und wissensintensiven Branchen in Deutschland.

Die 2002er und 2003er Gründungen in den forschungs- und wissensintensiven Branchen Österreichs weisen mit 6,8 MitarbeiterInnen nach vier bzw. fünf Jahren im Durchschnitt einen Beschäftigten mehr auf, als dies für den Durchschnitt aller Gründungen der Fall ist (vgl. Tab. 29). Die Anteile der Unternehmen der forschungs- und wissensintensiven Branchen der Gründungsjahrgänge 2002 und 2003, die 2007 die drei gewählten Beschäftigungsschwellen übertrafen, unterscheiden sich nicht nennenswert von den Anteilen bei Betrachtung aller Gründungen dieser Jahrgänge. Diesbezüglich zeigt sich keine stärkere „Wachstumsorientierung" der Gründungen dieser avan- 
cierten Wirtschaftszweige. So wiesen 2007 $11,1 \%$ der Gründungen der forschungs- und wissensintensiven Branchen Österreichs der betrachteten Gründungskohorten einen für diese Branchengruppen überdurchschnittlichen Beschäftigungsstand auf (gegenüber 10,4 \% bei allen Gründungen), 6,2 \% der Gründungen der avancierten Branchen hatten mehr als zehn MitarbeiterInnen (gegenüber 5,9 \% bei allen Gründungen) und mehr als 20 Beschäftigte hatte ein Anteil von 1,5\% der jungen Unternehmen dieser Branchen (gegenüber 0,7 \% bei allen Gründungen). Die deutschen Gründungen der Jahre 2002 und 2003 in den forschungs- und wissensintensiven Branchen sind 2007 mit 4,8 MitarbeiterInnen im Durchschnitt deutlich kleiner als die Österreichs (vgl. Tab. 30). Die Anteile von Unternehmen mit mehr als 10 und mit mehr als 20 Beschäftigten im Jahr 2007 unterscheiden sich mit $6,4 \%$ und $1,5 \%$ kaum von den österreichischen Werten (auf einen Vergleich der Anteile von Gründungen mit überdurchschnittlichen MitarbeiterInnenzahlen wird wegen der unterschiedlichen Durchschnittwerte verzichtet).

Anders als in Deutschland, wo sich die nach den unterschiedlichen Beschäftigungsschwellen separierten durchschnittlichen Beschäftigungswerte der Gründungen der forschungs- und wissensintensiven Branchen für das Jahr 2011 kaum von denen im Durchschnitt aller Branchen un- terscheiden, sind in Österreich hier deutliche Unterschiede zwischen den Gründungen der avancierten Branchen und allen Gründungen zu verzeichnen (vgl. Tab. 30). Die durchschnittlichen Beschäftigtenzahlen des Jahres 2011 der jeweiligen Gruppen mit über den 2007 er-Beschäftigtenschwellen liegender Mitarbeiterzahl sind erheblich höher, als dies bei Betrachtung aller Gründungen der Fall ist. So wachsen österreichische Gründungen der forschungs- und wissensintensiven Branchen der Jahre 2002 und 2003 im Durchschnitt auf 20,3 MitarbeiterInnen im Jahr 2011, wenn sie 2007 eine überdurchschnittliche Beschäftigung zu verzeichnen hatten (gegenüber 12,7 MitarbeiterInnen bei allen Gründungen). Hatten die jungen Unternehmen der hier betrachteten Branchen 2007 mehr als 10 MitarbeiterInnen, dann stieg die Durchschnittsbeschäftigung 2011 auf 27,5 Personen (gegenüber 18,8 bei allen Branchen), und hatten sie mehr als $20 \mathrm{Be}-$ schäftigte, erreichten sie $2011 \mathrm{im}$ Durchschnitt einen MitarbeiterInnenstand von immerhin 64,9 (gegenüber 35,5 bei allen Gründungen). In den forschungs- und wissensintensiven Branchen aus dem Industrie- und Dienstleistungssektor weisen die jungen österreichischen Unternehmen, die schon zu Beginn ihrer Marktaktivitäten auf einen Wachstumskurs setzen auch im weiteren Verlauf ihrer Geschäftstätigkeiten eine deutliche Wachstumsorientierung auf. Für die Unterneh-

\section{Tab. 30: Durchschnittlicher Beschäftigtenstand der Gründungen in den forschungs- und wissensintensiven Branchen der Jahre 2002 und 2003 in 2011 nach unterschiedlichen Beschäftigtenschwellen 2007}

\begin{tabular}{|c|c|c|}
\hline $\begin{array}{l}\text { Gründungskohorten } 2002 \text { und } 2003 \\
\text { Beschäftigtendurchschnitt } 2007\end{array}$ & $\begin{array}{l}\text { Creditreformdaten Österreich } \\
\qquad 6,8\end{array}$ & $\begin{array}{c}\text { MUP-Deutschland } \\
4,8\end{array}$ \\
\hline \multicolumn{3}{|l|}{ Beschäftigtendurchschnitt 2011} \\
\hline 2007 überdurchschnittlich & 20,3 & 11,3 \\
\hline 2007 höchstens durchschnittlich & 3,5 & 3,1 \\
\hline \multicolumn{3}{|l|}{ Beschäftigtendurchschnitt 2011} \\
\hline 2007 mehr als 10 & 27,5 & 19,3 \\
\hline 2007 höchstens 10 & 4,4 & 4,4 \\
\hline \multicolumn{3}{|l|}{ Beschäftigtendurchschnitt 2011} \\
\hline 2007 mehr als 20 & 64,9 & 30,4 \\
\hline 2007 höchstens 20 & 6,3 & 5,6 \\
\hline
\end{tabular}

Quelle: Creditreformdaten Österreich, Mannheimer Unternehmenspanel - Berechnungen des ZEW. 
men, die nicht über den jeweiligen Beschäftigungsschwellen liegen, zeigen sich kaum Unterschiede zur Betrachtung aller Gründungen. Dies legt nahe, dass es auf die Wachstumsorientierung der Unternehmen, ob sie auch auf mittlere Sicht wachsen, und nicht auf ihre Branchenzugehörigkeit ankommt. ${ }^{178}$

\section{Resümee}

Die hier dargelegten Befunde legen nahe, dass junge Unternehmen, die schon in einer sehr frühen Phase ihrer Marktaktivitäten einen vergleichsweise hohen Beschäftigtenstand aufbauen, auch im weiteren Verlauf ihrer Geschäftsaktivitäten - zumindest im Durchschnitt - deutlich überdurchschnittliche Beschäftigtenzahlen aufweisen werden. Die "Weiche" in einen „Wachstumspfad" stellt sich somit sehr früh. Deutlich wird ebenfalls, dass es nur ein geringer Anteil der jungen Unternehmen ist, der tatsächlich auf einen Wachstumspfad, der dann auch zu nennenswerter Beschäftigung führt, einschwenkt. Beides gilt auch für Gründungen in den sogenannten forschungs- und wissensintensiven Branchen (auch „innovative Branchen“ genannt). Die Tatsache, dass Unternehmen ihr Aktivitätsfeld so wählen, dass sie diesen Branchen zuzurechnen sind, lässt allein noch keine Rückschlüsse auf ihr tatsächliches Beschäftigungswachstum zu. Auch in diesen Branchen sind es nur sehr wenige Unternehmen, die nennenswerte Beschäftigungszunahmen zu verzeichnen haben. Ein hoher Beschäftigtenstand nach wenigen Jahren geht in den forschungs- und wissensintensiven Branchen mit im Durchschnitt noch höherem Beschäfti- gungswachstum in der weiteren Geschäftsphase der Unternehmen einher, als dies schon bei Betrachtung der Gründungen aller Branchen der Fall ist.

Frühes Wachstum ist somit ein guter Indikator dafür, dass junge Unternehmen das Potenzial zu weiterem Wachstum aufweisen und kann als Anhaltspunkt für Investoren oder Fördermaßnahmen gelten. Es stellt sich allerdings die Frage, ob das Fehlen von frühem Beschäftigtenaufbau signalisiert, dass kein Wachstumspotenzial besteht.

Davon kann keinesfalls ausgegangen werden. Es kann viele Ursachen geben, dass Gründungen in den ersten Jahren ihres Bestehens keine große Zahl von MitarbeiterInnen einstellen. Ein ganz wichtiger Grund dafür ist, dass die meisten GründerInnen gar kein Wachstum ihres Unternehmens anstreben. Sie wollen eine bestimmte Geschäftsidee realisieren und möglichst unabhängig arbeiten. ${ }^{179}$ Diese GründerInnen wären auch durch Investmentangebote oder Förderofferten nicht zu einer expansiveren Strategie zu bewegen. Darüber hinaus ist allerdings davon auszugehen, dass Finanzierungsengpässe, zu lange Entwicklungszeiten bis zur Marktreife von Produkten und auch fehlende Qualifikation der GründerInnen für eine Reihe von jungen Unternehmen restringierend wirken und Wachstum verhindern. Wenn eine Identifizierung solcher Unternehmen gelingt - die unternehmensindividuell und unabhängig von der Branchenkategorie erfolgen muss - dann kann frühzeitiges Engagement helfen die "Weiche" in Richtung Wachstum zu stellen.

178 Die Betrachtung der Verteilungen der Beschäftigtenstände im Jahr 2011, separiert nach den drei hier verwendeten Beschäftigungsschwellen für die MitarbeiterInnenzahlen des Jahres 2007 für Gründungen in den avancierten Branchen, unterstützen den Eindruck aus der Betrachtung der Durchschnittswerte.

179 Vgl. Egeln et al. (2012). 


\section{Evaluierungen}

Evaluierungen sind heute sowohl in rechtlicher Hinsicht als auch in der täglichen Praxis ein unabdingbarer Bestandteil im Prozess der Einführung und Implementierung von forschungs- und technologiepolitischen Fördermaßnahmen. In Österreich ist hierfür eine Reihe von Rechtsgrundlagen maßgeblich: das Forschungs- und Technologieförderungsgesetz (FTF-G), das Gesetz zur Errichtung der Österreichischen Forschungsförderungsgesellschaft 2004 (FFG-G), das Forschungsorganisationsgesetz (FOG; Berichtswesen: $\S \S 6-9 \mid$ sowie die auf diesen Gesetzen basierenden Richtlinien zur Forschungsförderung ${ }^{180}$ und zur Förderung der wirtschaftlich-technischen Forschung und Technologieentwicklung, die sogenannten FTE-Richtlinien. ${ }^{181}$ Insbesondere das FTF-G (§ 15 Abs. 2) normiert auf gesetzlicher Ebene die Evaluierungsgrundsätze als Mindesterfordernisse für die Richtlinien. Dabei sehen die Richtlinien vor, dass "für alle auf den FTE-Richtlinien basierenden Förderungsprogrammen und -maßnahmen ein schriftliches Evaluierungskonzept zu erstellen ist, das den Zweck, die Ziele und die Verfahren sowie die Termine zur Überprüfung der Erreichung der Förderungsziele enthält und geeignete Indikatoren definiert". ${ }^{182}$

Nicht zuletzt aufgrund dieser rechtlichen Grundlage werden heute in beinahe allen Forschungs- und Technologieprogrammen Evaluierungen im Zuge der Programmplanung (ex-ante
Evaluierungen), der Programmdurchführung (Monitoring und Interimsevaluierung) sowie zu Programmende (ex-post Evaluierungen) angewandt bzw. für die strategische Weiterentwicklung des österreichischen Forschungsförderportfolios durchwegs als essentiell und richtungsweisend angesehen.

Um daher regelmäßig einen Überblick über die Evaluierungstätigkeiten der österreichischen Forschungsförderprogramme zu geben, werden seit dem Jahr 2009 Ergebnisse rezenter Evaluierungen im Forschungs- und Technologiebericht kurz vorgestellt. Für die Darstellung von Evaluierungen gelten dabei folgende Auswahlkriterien:

- Die Evaluierung weist vornehmlich eine bundespolitische Relevanz auf.

- Ein approbierter Bericht der Evaluierung ist verfügbar.

- Der Evaluationsbericht muss öffentlich zugänglich sein, das bedeutet, der Bericht ist auf der Homepage der Plattform Forschungs- und Technologieevaluierung ${ }^{183}$ veröffentlicht.

Im Folgenden werden daher Ergebnisse einiger seitens der Bundesressorts in Auftrag gegebenen Evaluierungen kurz dargestellt: die Evaluierung der FWF Programme Elise Richter und Hertha Firnberg (im Auftrag des BMWF), die Zwischenevaluierung der Dienstleistungsinitiative (im Auftrag des BMWFJ), die Evaluierung der Strategieprogramme IV2S und IV2Splus (im Auftrag des

180 Richtlinien der Bundesregierung über die Gewährung und Durchführung von Förderungen gemäß §§ 10-12 FOG, BGBl. Nr. 341/1981. 181 Richtlinien zur Förderung der wirtschaftlich-technischen Forschung und Technologieentwicklung (FTE-Richtlinien) gemäß $11 \mathrm{Z} 1$ bis 5 des Forschungs- und Technologieförderungsgesetzes (FTFG) des Bundesministers für Verkehr, Innovation und Technologie vom 27.09.2006 (GZ 609.986/0013-III/I2/2006) und des Bundesministers für Wirtschaft und Arbeit vom 28.9.2006 (GZ $97.005 / 0012 \mathrm{C} 1 / 9 / 2006)$.

182 FTE-Richtlinien, Abschnitt 2.2., S. 4.

183 www.fteval.at 
BMVIT), die Interimsevaluation des österreichischen Sicherheitsforschungsprogramms KIRAS (im Auftrag des BMVIT), sowie die Zwischenevaluierung der vom BMWF beauftragten Regionalen Kontaktstellen (im Auftrag des BMWF).

\subsection{Evaluierung der FWF Programme Elise Richter und Hertha Firnberg}

\section{Ziel der Evaluierung}

Der Schwerpunkt der Evaluierung der Programme Hertha Firnberg und Elise Richter liegt vor allem auf der Frage, welche Wirkungen die Programme auf die geförderten Wissenschaftlerinnen haben, sowie darüber hinaus ob und in welcher Form diese Programme auch Veränderungen an den universitären Einrichtungen bewirken. ${ }^{184}$

\section{Programmziele und Eckdaten}

Beide Förderprogramme, Hertha Firnberg und Elise Richter, sind die Frauenförderprogramme des FWF und zielen speziell darauf ab, hoch qualifizierte Wissenschaftlerinnen in ihrer wissenschaftlichen Karriereentwicklung zu unterstützen. Die Zielgruppe des Hertha Firnberg-Programms sind hoch qualifizierte Universitätsabsolventinnen aller Fachdisziplinen, welche am Beginn der wissenschaftlichen Laufbahn bzw. beim Wiedereinstieg nach der Karenzzeit bestmöglich unterstützt werden sollen. $\mathrm{Zu}$ diesem Zweck sollen die Stipendiatinnen auch in der internationalen Scientific Community etabliert sein, sich an internationalen Forschungskooperationen beteiligen und im Anschluss an die Förderung an der Forschungsstätte angestellt sein. Das Elise Richter-Programm richtet sich komplementär dazu explizit an jene qualifizierten Forscherinnen, die eine Universitätskarriere anstreben. Ziel ist es, nach Abschluss der Förderung eine Qualifikationsstufe zu erreichen, die zur Bewerbung um eine in- oder ausländische Professur befähigt.
Insgesamt wurden im Rahmen des Hertha Firnberg-Programms seit der Einführung im Jahr 1999145 Stipendiatinnen und im Rahmen des Elise Richter-Programms seit der Einführung im Jahr 2006 insgesamt 70 Stipendiatinnen unterstützt. Das Budget für beide Frauenförderprogramme beträgt in den letzten Jahren etwa sechs Mio. $€$ pro Jahr, womit in den Programmen Hertha Firnberg und Elise Richter jeweils etwa 12 bis 13 neue Forschungsstellen pro Jahr genehmigt werden konnten. Die Bewilligungsquoten in den beiden Programmen sind durchaus unterschiedlich hoch. So weist das Hertha Firnberg-Programm im Jahr 2010 eine Bewilligungsquote von $26 \%$ und das Elise Richter-Programm eine Bewilligungsquote von $38,5 \%$ auf. Als Begründung für die höhere Bewilligungsquote bei Elise Richter ist vor allem die bessere Qualität der Anträge, die wiederum in der höheren Erfahrung der Wissenschaftlerinnen begründet ist, zu nennen. In der gesamten Programmlaufzeit wurden bislang die meisten Förderstellen im Rahmen des Hertha Firnberg-Programms im Bereich Life Sciences $(38 \%)$ bewilligt, gefolgt von den Bereichen Naturwissenschaft/Technik (34\%) und Geistes- und Sozialwissenschaften $(28 \%)$. Ein etwas anderes Bild zeigt sich beim Elise Richter-Programm: Hier wurden in der gesamten Laufzeit bislang die meisten Förderstellen in den Geistes- und Sozialwissenschaften $(41 \%)$ bewilligt, gefolgt von den Bereichen Naturwissenschaft/Technik (30\%) und Life Sciences (29\%).

\section{Ergebnisse der Evaluierung}

Die Zustimmung der befragten Stipendiatinnen $z u$ den beiden evaluierten Programmen ist sehr hoch wie es sich z.B. an der Weiterempfehlungsrate ausdrückt: 94 \% der Herta Firnberg (HF) Stelleninhaberinnen und $96 \%$ der Elise Richter (ER) Stelleninhaberinnen sowie MAS geben an, dass sie die Antragstellung einer befreundeten Kollegin bzw. Nachwuchswissenschaftlerin empfehlen

184 Siehe Pohn-Weidinger, Grasenick (2011). 
würden. Die finanzielle Ausstattung wird insgesamt weitgehend als angemessen angesehen. Die Zustimmungswerte (",stimme zu“, ,stimme eher $\mathrm{zu}^{\prime \prime}$ ) liegen bei den Stelleninhaberinnen bei über $80 \%$.

Die Programme unterscheiden sich von anderen Förderprogrammen durch eine Reihe spezifischer Maßnahmen. Hierzu zählt die Betreuung durch MitantragstellerInnen (MAS) ebenso wie jährlich stattfindende zweitägige CoachingWorkshops bzw. die Möglichkeit individuellen Coachings. Die Befragung zeigt, dass diese Maßnahmen mehrheitlich als "sehr wichtig“ bzw. "wichtig" angesehen werden. Im HF Programm ist die Unterstützung durch MAS für $91 \%$ der Befragten ein "sehr wichtiges" bzw. „wichtiges" Element des Programms. Etwa vier Fünftel (HF: 80 \%, ER: $77 \%$ ) geben an, dass ihnen KarriereCoaching-Workshops "sehr wichtig" bzw. „eher wichtig" sind. Dieser Befund wurde auch in den Interviews bestätigt.

Darüber hinaus werden Maßnahmen zur Verbesserung der Integration in internationale Netzwerke als wichtig erachtet. So wünschen sich vier Fünftel der Hertha Firnberg-Stipendiatinnen und etwa $70 \%$ der Elise Richter-Stipendiatinnen Maßnahmen in diesem Bereich - eine Forderung, die sich implizit auch an die Universitäten selbst richtet. Damit verbunden ist auch die Forderung, die Integration der Wissenschaftlerinnen während der Förderlaufzeit an den Universitäten zu erhöhen sowie die beiden Frauenförderprogramme Hertha Firnberg und Elise Richter verstärkt in die karrierefördernden Maßnahmen an den Universitäten zu verankern.

\subsection{Zwischenevaluierung der Dienst- leistungsinitiative}

\section{Ziel der Evaluierung}

Die Zwischenevaluierung der Dienstleistungsinitiative umfasst einen Förderzeitraum von Ende
2009 bis einschließlich Oktober 2012. Neben der Darstellung der Förderkennzahlen war es vor allem Ziel, die Wirkung der Förderung von Innovationsprojekten mit Mitteln der Dienstleistungsinitiative auf die geförderten Unternehmen zu analysieren. Es geht dabei um die Wirkungen auf Ebene der Projekte und der Initiative, des Konzepts, der Prozesse und der Organisation der Dienstleistungsinitiative. ${ }^{185}$

\section{Programmziele und Eckdaten}

Mit der Verabschiedung des Basisdokuments zur Dienstleistungsinitiative wurde Ende 2009 vom BMWFJ ein neuer Weg zur Förderung innovativer Dienstleistungen eingeschlagen: Anstatt ein genuin neues Programm ins Leben zu rufen, wurden existierende Forschungsförderprogramme der FFG um den speziellen Fokus auf Dienstleistungen ergänzt und budgetär ausgeweitet. Parallel dazu wurden Begleitforschungsstudien durchgeführt sowie verschiedene Awareness-Aktivitäten (wie z.B. Veranstaltungen, Beratung) gesetzt. Übergeordnetes Ziel der Dienstleistungsinitiative ist es, durch die verstärkte Förderung von Dienstleistungsinnovationen die Produktivität, Wertschöpfung und Exporte von Dienstleistungsunternehmen sowie von jenen Unternehmen im produzierenden Sektoren, die sich mit produktbegleitenden Dienstleistungen beschäftigen, zu erhöhen und somit auch positive Effekte auf Beschäftigung und Wohlstand zu erzielen. Mitunter sollen insbesondere folgende Ziele erreicht werden:

- Erhöhung der Innovationskraft der österreichischen Dienstleistungswirtschaft,

- Generierung positiver wirtschaftlicher Effekte (vor allem Umsätze und Arbeitsplätze),

- Bewusstseinserweiterung der potentiellen (auch neuen) FFG-KundInnen bezüglich des FFG-Förderangebots,

- Anregung und Generierung neuer Projekte, mit denen die FFG bisher nicht in Verbindung gebracht wurde, 
- Erschließung neuer KundInnenschichten und Steigerung des Anteils an FFG-NeukundInnen,

- Aufbau einer Wissensbasis über Dienstleistungsinnovationen in Österreich (u.a. durch die Begleitforschung),

- Sichtbarmachung spezifischer Eigenschaften von Dienstleistungsinnovationen, um deren stärkere Berücksichtigung im FFG-Förderportfolio zu ermöglichen.

Ein besonderes Merkmal der Dienstleistungsinitiative ist, dass sie im Rahmen von zwei laufenden bottom-up Förderprogrammen der FFG implementiert wurde: zum einen im Rahmen der antragsorientierten Projektförderung in den Basisprogrammen und zum anderen im Rahmen der Programmlinie COIN „Kooperationen \& Netzwerke" der Strukturprogramme. Insgesamt wurden bislang einschließlich Ende November 201278 Projekte in den Basisprogrammen gefördert; von diesen wurden 14 Projekte verlängert. Das Fördervolumen für die Dienstleistungsinitiative in den Basisprogrammen betrug 13,9 Mio. $€$. Darüber hinaus fanden in der Programmschiene COIN Kooperation \& Netzwerke drei Ausschreibungen mit dem Schwerpunkt Dienstleistungsinnovationen statt, mittels derer Projekte mit einem Fördervolumen von insgesamt 8,8 Mio. $€$ gefördert wurden. Zusätzlich wurden im Rahmen der Dienstleistungsinitiative zwei Begleitstudien zur Dienstleistungslandschaft in Österreich finanziert sowie zwei öffentliche Veranstaltungen organisiert.

\section{Ergebnisse der Evaluierung}

Zentrales Ergebnis ist, dass insbesondere bei Kooperation und Netzwerk-Projekten im Rahmen von COIN die Projektförderung durch die Dienstleistungsinitiative mehrheitlich dafür ausschlaggebend war, dass die Projekte überhaupt zustande kamen; bei Dienstleistungsprojekten im Rah- men der Basisförderung ist dies nur für jedes vierte Projekt zutreffend, wenn auch dank der Förderung die Projektvorhaben deutlich größer und konzentrierter durchgeführt werden konnten, als dies andernfalls möglich gewesen wäre. Auch zeigen sich unterschiedliche Ergebnisse hinsichtlich des wirtschaftlichen Nutzens: Verteilen sich in COIN Kooperation \& Netzwerk-Projekten die wirtschaftlichen Effekte auf die unterschiedlichen NetzwerkpartnerInnen, so kommt bei Dienstleistungsprojekten im Basisprogramm der wirtschaftliche Nutzen meist der Projektleiterin bzw. dem Projektleiter zu. Eine Hebelwirkung der Förderung konnte speziell bei Start-Ups nachgewiesen werden; so wurden in der Programmlinie Kooperation \& Netzwerke von COIN immerhin $47 \%$ der FördernehmerInnen als NeukundInnen erfasst. Bemerkenswert ist, dass die geförderten Dienstleistungsinnovationsprojekte (74 \%) bislang eine deutliche sektorielle Konzentration in den Bereichen Informationsdienstleistungen und -technologien aufweisen. Als überaus positiv zu werten ist, dass es innerhalb der FFG gelungen ist, Dienstleistungen als ein Querschnittsthema zu etablieren; das bedeutet, dass seit dem Jahr 2012 alle Projekte dahingehend qualifiziert werden, ob sie Dienstleistungen betreffen oder nicht. Damit geht auch einher, dass die Erfahrungen mit der Implementierung der Dienstleistungsinitiative in zwei Bereichen der bottom-up Förderung der FFG durchwegs positiv sind. Angesichts dessen wird daher auch empfohlen, die Dienstleistungsinitiative mit der gewählten und gegebenen Struktur fortzusetzen. Langfristig soll die Initiative schließlich so angelegt werden, dass sie sich durch ihren Erfolg selbst erübrigt. Um dies zu erreichen, ist es essentiell, die Schnittstelle mit den Basisprogrammen aktiv und transparent zu gestalten, sowie für die künftige Ausrichtung der Dienstleistungsinitiative das Zielgruppenverständnis und die Awarenessmaßnahmen zu überdenken bzw. zu überarbeiten. 


\subsection{Evaluierung des European Space Policy Institute (ESPI)}

\section{Ziel der Evaluierung}

Ziel der Evaluierung ${ }^{186}$ war es den Auf- und Ausbau des European Space Policy Institute (ESPI) seit seiner Gründung im Jahr 2003 (offizielle Eröffnung im Jahr 2005) in Wien nachzuzeichnen, zu bewerten und entsprechende Schlussfolgerungen und Empfehlungen für die weitere Entwicklung abzuleiten.

\section{Programmziele und Eckdaten}

Ziel des ESPI ist es als Denkfabrik („,Think Tank") zu fungieren, in der mittel- bis langfristige Themen der Raumfahrt analysiert und bearbeitet werden und mit den Ergebnissen die strategischen Entscheidungsprozesse für diesen Politikbereich in Europa zu unterstützen. Im Jahr 2002 hat sich der ESA-Rat dafür entschieden, Wien als Standort eines neu zu gründenden Instituts im Bereich der Weltraumpolitik(forschung) zu wählen. Die gründenden Organisationen umfassten dabei einerseits die ESA (European Space Agency) und andererseits die österreichische Agentur für Luft- und Raumfahrt innerhalb der FFG (ALR/FFG), die damit das BMVIT repräsentiert. Mittlerweile ist die Anzahl der Mitglieder auf nunmehr 13 Organisationen (die wichtige Stakeholder der Raumfahrtcommunity umfassen) angewachsen, wobei für die Zukunft eine Fortsetzung dieser langsamen Wachstumsstrategie angestrebt wird.

Gegründet wurde das ESPI als Verein nach österreichischem Privatrecht, wodurch sich etliche Vorteile, wie geringer Kapitalbedarf, Flexibilität bei Mitgliedsänderungen, Status der Gemeinnützigkeit oder große Unabhängigkeit ergeben.

Nach einer Phase des institutionell-organisatorischen Aufbaus waren die Jahre ab 2007 durch intensives Wachstum gekennzeichnet, allein das jährliche Budget stieg von 228.000€ im Jahr 2005 auf $609.000 €$ im Jahr 2011, die Zahl der MitarbeiterInnen erhöhte sich bereits bis zum Jahr 2008 auf die ursprünglich angestrebten zwölf und umfasst derzeit inklusive Leitung und Internships 16 MitarbeiterInnen. Im Zeitraum von 2004 bis 2011 waren im MitarbeiterInnenstab des Instituts insgesamt 25 verschiedene Nationalitäten vertreten. Bemerkenswert ist außerdem die annähernd ausgeglichene Genderbilanz (55 \% männlich, $45 \%$ weiblich).

Der Output des ESPI ist durchaus beachtlich. Bis Ende 2011 wurden 128 unterschiedliche Produkte fertiggestellt, darunter 39 ESPI Reports, 60 Publikationen in wissenschaftlichen Zeitschriften und 11 nicht publizierte Auftragsstudien. Daneben wurden auch zahlreiche Netzwerkaktivitäten sowie Veranstaltungen und Konferenzen durchgeführt. Darüber hinaus wird das ESPI zunehmend von europäischen Universitäten und öffentlichen Forschungseinrichtungen für Vorlesungen und Präsentationen mit thematischem Fokus auf Raumfahrt eingeladen.

\section{Ergebnisse der Evaluierung und Empfehlungen}

Insgesamt bescheinigt das Evaluierungsteam dem ESPI eine bislang erfolgreiche Auf- und Ausbauzeit, die innerhalb der gesteckten Erwartungen bzw. Ziele liegen. Ansatzpunkte für Empfehlungen finden sich beim Prozess der Themenauswahl, der Möglichkeiten bietet, ihn transparenter zu gestalten (z.B. auch durch die intensivere Einbeziehung externer ExpertInnen im Diskussionsprozess). Weitere Empfehlungen beziehen sich vor allem auf die Bereiche Qualitätssicherung einerseits und aktive Politikbeeinflussung durch ESPI andererseits. Hinsichtlich der Qualitätssicherung wird von Seiten der EvaluatorInnen beispielsweise vorgeschlagen, die Balance zwischen senior und junior staff zu optimieren. Auf längere Sicht wäre auch eine verstärkte $\mathrm{Zu}$ sammenarbeit mit Universitäten anzudenken,

186 Siehe Kaufmann, Streicher (2012). 
mit der Absicht internationale Master und/oder PhD Programme gemeinsam anzubieten. Dies ist vor dem Hintergrund zu sehen, dass bereits derzeit die angebotenen Internships international stark nachgefragt werden. Bezüglich der Einbeziehung des ESPI in einschlägige Politikdiskussionen halten die EvaluatorInnen fest, dass der Fokus noch verstärkt auf Weltraumpolitik als solche gelegt, die Einbeziehung in Netzwerke mit supranationalen Netzwerken in den nächsten Jahren weiter ausgebaut sowie auch die Beziehungen mit außereuropäischen Think Tanks mit Schwerpunkt auf Weltraumfragen intensiviert werden sollten. Zusätzlich wären Überlegungen anzustellen, wie das Verhältnis zwischen ESPI und der Europäischen Kommission zur Zukunft $\mathrm{zu}$ definieren ist.

\subsection{Evaluierung der Strategieprogramme IV2S und IV2Splus}

\section{Ziel der Evaluierung}

Ziel der Evaluierung war es den Programmverlauf (Laufzeit IV2S 2002 bis 2006, 2007 Start von IV2Splus) bis Anfang 2012 nachzuzeichnen, wobei der Fokus der Evaluierung auf der Analyse der Wirkungen der durch die Programme geförderten Forschungsprojekte lag. Im Fall von IV2Splus handelt es sich allerdings um eine Zwischenevaluierung, da das Programm und viele der dadurch geförderten Projekte noch im Laufen sind und entsprechende Wirkungen daher erst für die nächsten Jahre zu erwarten sind.

\section{Programmziele und Eckdaten}

Die Strategieprogramme IV2S - Intelligente Verkehrssysteme und Services (Laufzeit 2002 bis 2006) und das Nachfolgeprogramm IV2Splus für den Zeitraum 2007 bis 2012 sind Programme zur strategischen Förderung von Forschung und Entwicklung im Bereich der Mobilitäts- und Verkehrstechnologien. Sie liegen in der Verantwortung des BMVIT und werden operativ von der FFG umgesetzt und abgewickelt. Das Programm zielt darauf ab, mit Hilfe der Förderung von Forschung und Entwicklung zusätzlich auch Impulse für verkehrs- und umweltpolitische Agenden $z u$ setzen. Dementsprechend weist das Programm folgende drei Zieldimensionen auf:

- Gesamtgesellschaftliche Zielsetzungen im Bereich Verkehrs- und Umweltfragen

- Steigerung der unternehmerischen Wettbewerbsfähigkeit durch FTI

- Vernetzung und Kooperation (sowohl national insbesondere zwischen Wissenschaft und Unternehmen als auch europäisch/international)

IV2S war in drei unterschiedliche Programmlinien gegliedert: (i) Bahntechnik, (ii) KFZ-Zulieferindustrie und (iii) Telematik/Logistik. Somit orientiert sich das Programm an Stärkefeldern der österreichischen Industrie und verknüpft gleichzeitig FTI-Fördermaßnahmen mit gesamtgesellschaftlichen Herausforderungen („Mission“) bezüglich Verkehr und Umwelt.

Als Förderinstrument nutzt das Programm nicht rückzahlbare Zuschüsse für F\&E-Projekte auf Grundlage der FTE-Richtlinie. Die Bandbreite von förderbaren Projektkategorien reicht dabei von Grundlagenforschung bis hin zu Demonstrations- und Validationsprojekten, wobei auf Partnerschaftsprojekte (vor allem auch zwischen Wissenschaft und Industrie) fokussiert wurde. Für die operative Umsetzung wurden mehrere Projekt-Calls in Form von Ausschreibungen jeweils pro Programmlinie durchgeführt. Die Auswahl der Themen erfolgte jeweils in enger Abstimmung mit den relevanten Communities.

\section{Ergebnisse der Evaluierung}

Zusammen wurden mit knapp 100 Mio. $€$ Fördermittel in den beiden Programmen bis ca. Anfang 2012 etwa 450 F\&E-Projekte angestoßen. Diese umfassen gesamte F\&E-Aufwendungen von ca. 190 Mio. $€$. Die Gesamtzahl der teilnehmenden Organisationen betrug in IV2S 483, in IV2Splus bis zum Evaluierungszeitpunkt 375 verschiedene Organisationen. Die Community der Programmteilnehmer ist dabei durch einige 
zentrale Akteure aus der Forschung, der Industrie (hier v.a. einige Großunternehmen) sowie der Betreiber charakterisiert. Über 50 \% der Fördermittel konzentrieren sich auf nur $10 \%$ aller teilnehmenden Organisationen. Gleichzeitig ist allerdings das Programm durchaus offen für Newcomer sowie für kleine und mittlere Unternehmen (KMU), wenn auch deren finanzielle Projektanteile deutlich geringeren Umfangs sind.

Hinsichtlich des Projekttyps dominieren eindeutig Forschungsvorhaben im Bereich der angewandten Forschung (industrielle Forschung und experimentelle Entwicklung). Der Anteil der Grundlagenforschung lag in beiden Programmen gemessen an den gesamten Projektkosten unter $10 \%$. Hinsichtlich der realisierten Kooperationen zeigt sich, dass das Ausmaß der Kooperation in beiden Programmen mit jeweils Anteilen kooperativer Projekte von ca. 90 \% sehr hoch war, wobei die durchschnittliche Zahl der Konsortialmitglieder pro Projekt bei drei bis vier Organisationen lag. Zudem finden sich im Großteil der Kooperationsprojekte Teilnehmer sowohl von Seiten der Wissenschaft als auch von Seiten der Wirtschaft. Des Weiteren haben die Programme dazu beigetragen, die Vernetzung zwischen öffentlichen Forschungseinrichtungen und Unternehmen zu verstärken und auch gänzlich neue Kooperationen mit Unternehmen außerhalb des eigentlichen Verkehrsbereichs (z.B. IKT-Unternehmen) geführt.

Die potentiellen Mitnahmeeffekte der Programme können als gering eingeschätzt werden. Eine Analyse der abgelehnten Projektanträge hat gezeigt, dass nur weniger als $10 \%$ aller abgelehnten Projekte dann auch ohne Förderung durchgeführt wurden.

Insbesondere für das Programm IV2S konnten bereits beachtliche wirtschaftliche Wirkungen (wie Umsatzerlöse, Patente, Nutzungslizenzen und Kosten- und Ressourceneinsparungen durch realisierte Effizienzgewinne) und wissenschaftliche Wirkungen (wie Publikationen und Vorträge) identifiziert werden. Naturgemäß ist das Aus- maß der bereits beobachtbaren Wirkungen im Programm IV2Splus geringer, da zum Evaluierungszeitpunkt das Programm noch im Laufen war und viele der geförderten Projekte noch im Bearbeitungsstand waren.

Abschließend ist noch zu erwähnen, dass von Seiten des Evaluierungsteams die Qualität der Programmabwicklung und -umsetzung als hoch eingestuft wurde.

\subsection{Interimsevaluation des österreichischen Sicherheitsforschungsprogramms KIRAS}

\section{Ziel der Evaluierung}

Im Jahr 2009 beauftragte das BMVIT ein Evaluationskonsortium, welches die begleitende Wirkungsevaluation des Sicherheitsforschungsprogramms KIRAS vornimmt. Die Interimsevaluation ist damit ein Baustein der begleitenden Evaluierung und legt den Fokus auf die quantitative und qualitative Analyse der bisherigen Wirkungen und Ergebnisse von KIRAS. ${ }^{187}$

\section{Programmziele und Eckdaten}

Das österreichische Sicherheitsforschungsprogramm KIRAS unterstützt nationale Forschungsvorhaben mit dem Ziel, die Sicherheit Österreichs und seiner Bevölkerung zu erhöhen. Angesichts dessen wird eine Reihe von strategischen Programmzielen von KIRAS angestrebt:

- Erhöhung der Sicherheit und des Sicherheitsbewusstseins der BürgerInnen

- Generierung sicherheitspolitisch erforderlichen Wissens

- Erzielung von Wissens-, Verfahrens- und Technologiesprüngen

- Wachstum der heimischen Sicherheitswirtschaft

- Auf- und Ausbau von Exzellenz im Bereich Sicherheitsforschung 
Um diese strategischen Ziele zu erreichen, verfolgt KIRAS einen integrativen Ansatz, der nicht ausschließlich auf technologische Lösungen, sondern auch auf einer sozial- und geisteswissenschaftlichen Herangehensweise basiert. Diese Herangehensweise soll insbesondere durch die Realisierung des strategischen Querschnittziels - Berücksichtigung gesellschaftlicher Fragestellungen in allen Aspekten der Sicherheitsforschung - unterstützt werden.

Die Programmverantwortung selbst liegt beim BMVIT, welche die FFG mit dem Programm- und Schirmmanagement beauftragt hat. Für die erste Programmphase des Sicherheitsforschungsprogramms KIRAS ist eine Gesamtlaufzeit von 2005 bis 2013 (neun Jahre) geplant, wobei der Zeitraum 2005 bis 2007 als Aufbauphase galt. Bis zur Berichtslegung des Interimsevaluationsberichts wurden durch KIRAS insgesamt 107 Projekte gefördert, was etwa 34 \% der beantragten Projekte entspricht. Der Kostenrahmen pro Projekt beträgt dabei etwa $498.000 €$.

\section{Ergebnisse der Evaluierung}

Alle geförderten KIRAS-Projekte haben bislang durchgehend einen Beitrag zum prioritären Ziel, die öffentliche Sicherheit zu erhöhen, geleistet. Die Projekte adressieren insbesondere Bereiche in denen ein konkretes Bedrohungspotential existiert bzw. ein solches wahrgenommen wird. Neben dem programmspezifischen Fokus auf den Schutz kritischer Infrastruktursektoren hat in letzter Zeit insbesondere der Themenbereich Kriminalität (mit Themen wie "Terrorismus") sowie weitere Themen wie "Unfälle" und „Naturkatastrophen" an Bedeutung gewonnen. Spiegeln diese Themen durchaus die bestehenden Ängste und Bedrohungswahrnehmungen der Bevölkerung wider, so sind die Beiträge der bislang laufenden KIRAS-Projekte zur Erhöhung des Sicherheitsbewusstseins der Bevölkerung eher mo- derat (lediglich in einem Drittel der KIRAS-Projekte sind spezifische Maßnahmen und Aktivitäten zur Bewusstseinsbildung der Bevölkerung vorgesehen). Wesentlich besser sind hingegen die Befunde zur Wissensgenerierung: Rund $45 \%$ der geförderten Unternehmen geben an, dass sie ohne KIRAS-Förderung die Projekte nicht durchgeführt hätten; mit dem Auslaufen der KIRAS-Projektförderung sind etwa $60 \%$ der befragten Einrichtungen bereits an der Konzeption eines Folgeprojekts beteiligt; darüber hinaus sind KIRASProjekte tendenziell technisch komplexer und deutlich kooperationsintensiver, und weisen eine höhere strategische Bedeutung auf als andere Innovations- und Forschungstätigkeiten der beteiligten Einrichtungen. Auch die gesamtwirtschaftlichen Effekte des KIRAS-Programms sind durchaus positiv: So haben das Fördervolumen von 37 Mio. $€$ bzw. die Projektvolumina von insgesamt 53 Mio. $€$ über direkte, indirekte und induzierte Effekte etwa 74 Mio. $€$ an Wertschöpfungsvolumen generiert. Damit wurden insgesamt rund 570 hochqualifizierte Arbeitsplätze gehalten und rund 240 hochqualifizierte Arbeitsplätze neu geschaffen. ${ }^{188}$

Für die zukünftige Ausgestaltung von KIRAS gilt es vor allem, Bedacht darauf zu nehmen, ein besseres gemeinsames Projektverständnis mit den Bedarfsträgern zu generieren. Darüber hinaus sollen die Komplexität und die zeitliche Abhängigkeit von einander aufbauenden KIRASProjekten in der Projektplanung stärker berücksichtigt werden.

\subsection{Zwischenevaluierung der vom BMWF beauf- tragten Regionalen Kontaktstellen (RKS)}

\section{Ziel der Evaluierung}

Die Zwischenevaluierung hatte zum Ziel, die Struktur und Dienstleistungen der vom BMWF beauftragten Regionalen Kontaktstellen - als

188 Die Angaben beziehen sich wiederum auf den Zeitraum bis zur Evaluierung. 
Teil des Beratungs- und Betreuungsnetzwerkes, welches seit dem Beitritt Österreichs zu den EUForschungsprogrammen eingerichtet wurde - im Hinblick auf ihre Effektivität und Effizienz zu analysieren. Darauf basierend sollten Empfehlungen für eine zukünftige Optimierung sowie unter Berücksichtigung der auf EU-Ebene zu erwartenden Veränderungen - Grundlagen für allfällige Folgebeauftragungen erarbeitet werden. Die Evaluierung deckt den Zeitraum 2009 bis 2012 ab. ${ }^{189}$

\section{Programmziele und Eckdaten}

Um potenzielle Antragstellende in den EU-Forschungsrahmenprogrammen und anderen europäischen Programmen vor Ort professionell zu unterstützen, wurde seitens des Bundes unter Federführung des BMWF ein Beratungs- und Betreuungsnetzwerk eingerichtet. Dieses Netzwerk besteht einerseits aus dem bundesweit agierenden Bereich Europäische und Internationale Programme (EIP) in der FFG und andererseits aus den Regionalen Kontaktstellen (RKS) in den Bundesländern. Eine weitere Ebene bilden die Forschungsservicestellen der Universitäten und Forschungseinrichtungen. Der Bereich EIP übernimmt dabei die wichtige Funktion der Koordinierung des Netzwerks - mit dem Ziel, die Qualitätssicherung und inhaltliche Abstimmung im gesamten nationalen Netzwerk sicherzustellen - und ist damit auch direkter Ansprechpartner der Regionalen Kontaktstellen. Finanziert werden die Regionalen Kontaktstellen im Rahmen von Beauftragungsverträgen durch das BMWF, wobei derzeit vier Regionale Kontaktstellen zur Betreuung der ForscherInnen in der jeweili- gen Region zur Optimierung der österreichischen Beteiligung an den EU-Programmen beauftragt sind:

- CATT Innovation Management GmbH (Oberösterreich)

- ITG - Innovations- und Technologietransfer Salzburg GmbH (Salzburg - Beauftragung durch einen Kooperationsvertrag mit dem Land Salzburg)

- SAT - Standortagentur Tirol/Tiroler Zukunftsstiftung (Tirol, Vorarlberg)

- SFG - Steirische Wirtschaftsförderungsgesellschaft (Steiermark, Kärnten) ${ }^{190}$

Ziel der RKS ist es, regionale Stakeholder und Akteure der Wissenschaft, Wirtschaft und Verwaltung in Bezug auf die EU-Forschungsrahmenprogramme und andere europäische Programme bestmöglich $\mathrm{zu}$ informieren, $\mathrm{zu}$ beraten und $\mathrm{zu}$ betreuen. Gemäß Zusagen der Landesregierungen kofinanzieren die Länder die Beauftragungen des BMWF mit 50\%; das BMWF finanziert mit seinem Budget rund 1,15 bis 1,26 VZÄ an den RKS pro Jahr. Bemerkenswert ist, dass alle RKS einen ganzheitlichen Beratungsansatz verfolgen und damit eine Wegweiserfunktion erfüllen. In den letzten Jahren hat sich dabei aber ihre Kundengruppe verändert: Universitäten sind als wichtige Kunden weggefallen, da diese selbst Forschungsservicestellen aufgebaut und ihre Beratung professionalisiert haben. Die Budgets der RKS sind dennoch konstant geblieben, haben sie stattdessen zusätzliche Aufgaben in Form von strategischen Tätigkeiten in Bezug auf Horizon 2020 und im Zusammenhang mit regionalen Wirtschafts- und Innovationsstrategien übernommen.

189 Siehe Good, Radauer (2013).

190 Ebenfalls Teil des regionalen Betreuungsnetzwerkes ist die RKS Niederösterreich - die TIP Technologie- und InnovationsPartner der Wirtschaftskammer Niederösterreich, welche aber nicht vom BMWF finanziert wird und daher nicht Gegenstand der vorliegenden Evaluierung war. 


\section{Ergebnisse der Evaluierung}

Im Gesamtbild zeigt sich, dass die RKS ihre auftragsgemäße Funktion als Wegweiser und Orientierungshilfe in Bezug auf europäische und alternative Förderprogramme weitgehend erfüllen. Auch hat die Integration der RKS in die Landesagenturen eine Professionalisierung mit sich gebracht und ist die Unternehmens- bzw. KundInnenzentrierung (im Gegensatz zur Förderprogrammzentrierung) in der Beratung als überaus positiv zu werten. Die hohe KundInnenzufriedenheit belegt den Erfolg dieses Betreuungsansatzes. Zentrale Erfolgsfaktoren der RKS sind dabei engagierte und geschulte BeraterInnen, die auf Unternehmen eingehen und als AnsprechpartnerInnen mit Kenntnis der regionalspezifischen Gegebenheiten vor Ort sind.

Zum Teil deutliche Unterschiede zwischen den RKS gibt es im Hinblick auf die Art der Zielgruppenansprache, die Größe und Zusammen- setzung der regionalen Zielgruppe, für welche EU-Förderungen relevant ist, und die Intensität der angebotenen Beratungsleistungen. $\mathrm{Zu}$ den Hauptkunden der RKS zählen im Wesentlichen Unternehmen, insbesondere KMU. Darüber hinaus zählen auch Angehörige der außeruniversitären Forschungseinrichtungen, Fachhochschulen, Privatuniversitäten und Intermediäre zum Kundenkreis. Gegenüber früher entfällt bei den meisten RKS die Kundengruppe der Universitäten, womit sie mit einer geschrumpften Zielgruppe, für welche EU-Förderprogramme nur zum Teil von Relevanz sind, konfrontiert sind. Angesichts dieser Entwicklung ist daher die Frage zu stellen, ob der erbrachte und mögliche Nutzen der RKS im Betreuungsnetzwerk den Mitteleinsatz rechtfertigt. Dem Evaluierungsteam ist die Heterogenität der RKS durchaus bewusst, womit sie auch auf die Schwierigkeit, eindeutige Maßnahmenempfehlungen abzugeben, hinweisen. 


\section{Literatur}

Aghion, P., Boulanger, J., Cohen, E. (2011): Rethinking industrial policy. Bruegel Policy Brief, 4.

Aiginger, K. (2006): Industrial policy: a dying breed or a re-emerging phoenix; Journal of Industry, Competition and Trade, 7, 297-323.

Aiginger, K., Falk, M. (2005): Explaining Differences in Economic Growth among OECD Countries; Empirica, 32, 19-43.

Aschhoff, B., Brandes, F., Crass, D., Cremers, K., DiazLopez, F., Grimpe, C., Klein Woolthuis, R., Mayer, M., Montalvo, C., Rammer, C. (2010): European Competitiveness in Key Enabling Technologies. Background Report to the EU Competitiveness Report 2010, Mannheim and Delft: ZEW and TNO.

Austria Innovativ (2012): „Qualitätssicherung: Was das neue Rahmengesetz den heimischen Hochschulen für Änderungen bringt", 6a/2012, Wien.

Bauer, B., Stieg., K. (2010). Eine Übersicht der Verlage, welche Hybridmodelle anbieten, bietet das Subverzeichnis der SHER-PA/RoMEO-Seite "Publishers with Paid Options for Open Access", http://www.sherpa.ac.uk/romeo/PaidOA.html, Zugriff am 23.04.2013.

Baumol, W. (1967): Macroeconomics of unbalanced growth: the anatomy of urban crisis, in: The American Economic Review, 3, 425-426.

Birch, D.L. (1979): The Job Generation Process: a Report, prepared by the Massachusetts Institute of Technology Program on Neighborhood and Regional Change for the Economic Development Administration. US Department of Commerce. Washington and Cambridge / Mass: MIT, Press.

Birch, D.L., Haggerty, A., Parsons ,W. (1995): Who's Creating Jobs?, Boston, Cognetics.

Birch, D.L., Medoff, J. (1994): "Gazelles”, in: L. C. Solomon / A.R. Levenson (eds.): Labor Markets, Employment Policy, and Job Creation, Westview: Boulder Co., pp. 159-168.

Blanchard, O., Illing, G. (2010): Makroökonomie. 5. Auflage, Pearson: München.

Bloomberg (2012): Global trends in renewable energy investment, Frankfurt School of Finance \& Management $\mathrm{GmbH}$
BMWF (2011): Universitätsbericht 2011, dem Nationalrat vom Bundesminister für Wissenschaft und Forschung gemäß $\S 11$ Universitätsgesetz 2002, BGBl. I Nr. 120/2002, Wien.

BMWF, BMVIT, BMWFJ (2012): Österreichischer Forschungs- und Technologiebericht 2012. Bericht der Bundesregierung an den Nationalrat gem. $\S 8(2)$ FOG über die Lage und Bedürfnisse von Forschung, Technologie und Innovation in Österreich.

BMWF, UNIKO (2011): Kapazitätsorientierte Universitätsfinanzierung, Wien, Dezember 2011.

BMWFJ (2012): Österreichs Warenverkehr 2011.

Boston Consulting Group (2009): The Innovation Imperative in Manufacturing. How the United States Can Restore Its Edge, Boston.

Butschek, F. (2012): Österreichische Wirtschaftsgeschichte. Von der Antike bis zur Gegenwart. 2. Auflage, Böhlau: Wien, Köln, Weimar.

Dachs, B. (2009): Strukturwandel und F\&E-Intensität im österreichischen Unternehmenssektor. In: Leitner, K. H., Weber, M., Fröhlich, J. (Hrsg.): Innovationsforschung und Technologiepolitik in Österreich, Studienverlag: Innsbruck, 45-64.

Dachs, B., Hunya, G., Hanzl-Weiss, D., Foster, N., Kampik, F., Leitner, S., Scherngell, T., Stehrer, R., Urban, W., Zahradnik, G. (2012):

Internationalisation of business investments in $R \& D$ and analysis of their economic impact. European Commission, Directorate-General for Research and Innovation, Final Report.

Dachs, B., Webersberger, B., Kinkel, S., Waser, B. (2006): Offshoring of production - a European perspective. European Manufacturing Survey Bulletin, 2.

Debande, O. (2006): De-industrialisation; EIB Papers, $11,65-82$.

Den Haan, W. (2011): Why do we need a financial sector? VOX, http://www.voxeu.org/article/why-dowe-need-financial-sector, Zugriff am 23.04.2013.

Deutsche Telekom Stiftung und BFI (2012): Innovationsindikator 2012, Bonn und Berlin.

Economist Intelligence Unit (2009): A New Ranking of the World's Most Innovative Countries, London: Economist Intelligence Unit. 
EFI (2012): Gutachten 2012: Gutachten zu Forschung, Innovation und technologischer Leistungsfähigkeit Deutschlands, http://www.e-fi.de/fileadmin/ Gutachten/EFI_Gutachten_2012_deutsch.pdf, Zugriff am 23.04.2013.

EFI (2013): Gutachten 2013; Gutachten zu Forschung, Innovation und Technologischer Leistungsfähigkeit Deutschlands, Expertenkommission Forschung und Innovation.

Egeln, J., Fryges, H., Höwer, D., Müller, B., Müller, K. (2012): Wachstumsbedingungen bzw.

Wachstumshemmnisse für junge Unternehmen, Studie zum deutschen Innovationssystem Nr. 142012, http://www.e-fi.de/fileadmin/ Innovationsstudien_2012/StuDIS_14_ZEW_KfW. pdf, Zugriff am 23.04.2013.

Erlinger-Schacherbauer, E. (2012): Positionen des BMWF zur Neuordnung der externen Qualitätssicherung in Österreich, in: Benz, W., Kohler, J., Landfried, K. (Hrsg.): Handbuch Studium \& Lehre, Raabe Verlag.

Europäische Kommission (2009a): Preparing for our future: Developing a common strategy for key enabling technologies in the EU, $\operatorname{COM}(2009)$ 512, Brüssel, 30.09.2009.

Europäische Kommission (2009b): Current situation of key enabling technologies in Europe, Commission Staff Working Document, SEC(2009) 1257, Brüssel, 30.09.2009.

Europäische Kommission (2010a): Europe 2020 Flagship Initiative - Innovation Union; COM (2010) 546 final.

Europäische Kommission (2010b): European Competitiveness Report 2010, SEC(2010) 1276, Brüssel, 28.10.2010.

Europäische Kommission (2011a): Innovation Union Scoreboard 2010; Report für die Generaldirektion Unternehmen und Industrie, Brüssel. http://ec. europa.eu/enterprise/policies/innovation/factsfigures-analysis/innovation-scoreboard/indexen. htm.

Europäische Kommission (2011b): National Open Access and Preservation Policies in Europe: Analysis of a questionnaire to the European research Area Committee, abrufbar unter: http:// ec.europa.eu/research/science-society/document_ library/pdf_06/open-access-report-2011_en.pdf.

Europäische Kommission (2011c): EU industrial structure 2011. Trends and performance.

Europäische Kommission (2012a): A stronger Industry for growth and economic recovery. Industrial policy communication update, COM 582.
Europäische Kommission (2012b): A European strategy for Key Enabling Technologies - A bridge to growth and jobs, $\operatorname{COM}(2012)$ 341, Brüssel, 26.06.2012.

Fagerberg, J. (1995): User-producer interaction, learning and comparative advantage, Cambridge Journal of Economics 19, 243-256.

Falk, M., Spitzlinger, R. (2013): Erfolgsfaktoren für neue Arbeitsplätze von F\&E-durchführenden Unternehmen; WIFO, im Auftrag der FFG, Wien.

Finch, D.J. (2012): Accessibility, sustainability, excellence: how to expand access to research publications. Report of the Working Group on Expanding Access to Published Research Findings.

Fishman, C. (2012): The insourcing boom; The Atlantic Magazine, December 2012.

Gassler, H., Polt, W., Rammer, Ch. (2006): Schwerpunktsetzungen in der Forschungs- und Technologiepolitik - eine Analyse der Paradigmenwechsel seit 1945, in: Österreichische Zeitschrift für Politikwissenschaft (ÖZP), 35. Jg., H. 1, 7-23.

Gehrke, B., Rammer, C., Frietsch, R., Neuhäusler, P. (2010): Listen wissens- und technologieintensiver Güter und Wirtschaftszweige - Zwischenbericht zu den NIW/ISI/ZEW Listen 2010/2011, Berlin, http:// www.e-fi.de/fileadmin/Studien/Studien_2010/ StuDIS_19-2010.pdf, Zugriff am 23.04.2013.

Gehrke, B., Schasse, U. (2012): Wirtschaftsfaktor Umweltschutz: Neuabgrenzung potenzieller Umweltschutzgüter - Liste potenzieller Umweltschutzgüter 2012. Methodenbericht zum Forschungsprojekt „Wirtschaftsfaktor Umweltschutz: Analyse der wirtschaftlichen Bedeutung des Umweltschutzes durch Aktualisierung wichtiger Kenngrößen"im Auftrag des Umweltbundesamtes. Niedersächsisches Institut für Wirtschaftsforschung e.V. (NIW), Hannover, Dezember 2012. Im Erscheinen.

Gnan, E., Janger, J., Scharler, J. (2004): Ursachen des langfristigen Wachstums in Österreich - Plädoyer für eine nationale Wachstumsstrategie; Geldpolitik und Wirtschaft, 1, 25-49.

Good, B., Radauer, A. (2013): Zwischenevaluierung der vom BMWF beauftragten Regionalen Kontaktstellen (RKS), Endbericht, Wien.

Guerri, P., Maliciai, V. (2005): Technology and international competitiveness: The interdependence between manufacturing and producer services. Structural Change and Economic Dynamics, 16, 489-502. 
Harrison, R. (2008): Does Innovation stimulate employment? A firm-level analysis using comparable micro-data from four european countries; in: NBER Working Paper Series 14216.

Hartwig, J. (2012): Testing the growth effects of structural change, in: Structural Change and Economic Dynamics, 23, 11-24.

Helper, S., Krueger, T., Wial, H. (2012): Why does manufacturing matter? Which manufacturing matters? A policy framework. Brookings.

High-level Expert Group on Key Enabling Technologies (2011): Final Report, European Commission, http://ec.europa.eu/enterprise/ sectors/ict/files/kets/hlg_report_final_en.pdf, Zugriff 23.04.2013.

INSEAD (2010): The Global Innovation Index 2009-10, o.O.: INSEAD.

Institut der deutschen Wirtschaft Köln (2012): Innovationsmonitor 2012 - Die Innovationskraft Deutschlands im internationalen Vergleich, Köln.

Janger, J. (2012): Strukturwandel und Wettbewerbsfähigkeit in der EU; WIFI-Monatsberichte, 8, 625640.

Jorgenson, D.W., Timmer, M.P. (2011): Structural Change in Advanced Nations, Scandinavian Journal of Economics 113, 1, 1-29.

Kaldor, N. (1967): Strategic factors in economic development. Ithaca.

Kaldor, N. (1972): The irrelevance of equlibrium economics, 82, 1237-1255.

Kattinger, M. (2012): Gelungene Wandlung des Industriestandortes Steiermark; Neue Zürcher Zeitung, 29.03.2012, 29.

Kaufmann, A., Tödtling, F. (2002): How effective is innovation support for SMEs? An analysis oft he region of Upper Austria, Technovation 22(2002), 147-159.

Kaufmann, K., Streicher, J., Sheikh, S. (2012): Evaluation of the European Space Policy Institute (ESPI), Wien.

Krugman, P. (1994), The Age of Diminished Expectations, MIT Press, Boston.

Lewis, D. (2012): The Inevitability of Open Access, College \& Research Libraries, 73, 5, 493-506.

Loprieno, A., Menzel, E., Schenker-Wicki, A. (2011): Zur Entwicklung und Dynamisierung der österreichischen Hochschullandschaft - eine Außensicht. Rahmenkonzept für einen Hochschulplan, August 2011.

Ludwig, U., Brautzsch, H.U., Loose, B. (2011): Dienstleistungsverbund stärkt Bedeutung der Industrie. Wirtschaftsdienst, 9, 648-650.
Malerba, F. (2005): Sectoral systems. How and why innovation differs across sectors; in: Fagerberf, J., Mowery, D., Nelson, R. (Hrsg.): The Oxford Handbook of Innovation. Oxford University Press: Oxford, 380-406.

Marin, D. (2008): The new corporation in Europe. Bruegel Policy Brief, 7.

Marin, D. (2010): The opening up of Eastern Europe at 20 - jobs, skills and "reverse Maquliadoras" in Austria and Germany. Bruegel Working Paper, 2.

Maroto-Sanchez, A., Cuadrado-Roura, J. (2009): Is growth of services an obstacle to productivity growth? A comparative analysis; Structural Change and Economic Dynamics, 20, 254-265.

Marsh, P. (2012): The new industrial revolution. Consumers, globalization and the end of mass production. Yale University Press: New Haven, London.

McCasland, D., Theodossiou, I. (2012): Is manufacturing still the engine of economic growth? Journal of Post Keynesian Economics, 35, 79-92.

McKinsey (2012): Manufacturing the future: The next era of global growth and innovation.

Miles, I. (2005); Innovation in services; in: Fagerberf, J., Mowery, D., Nelson, R. (Hrsg.): The Oxford Handbook of Innovation. Oxford University Press: Oxford, 4433-458.

Necmi, S. (1999): Kaldor's growth analysis revisited; Applied Economics, 31, 653-660.

Nentwich, M., Stöger, H., Muth, V. (2012): Open Access und die Österreichische Akademie der Wissenschaften, Mitteilungen des VÖB, 65 (2).

Nickel, S., Redding, S., Swaffield, J. (2008): The uneven pace of deindustrialisation in the OECD; The World Economy, 31, 1154-1184.

O'Mahony, M., Timmer, M. (2009): Output, Input and productivity measures at the industry level: the EU KLEMS database; The Economic Journal, 119, 374-403.

Oberholzner, T., Fischl, I., Haefeli, U., Mandl, S. (2012): Evaluierung der Strategieprogramme IV2S und IV2Splus, Wien.

OECD (2002): The Measurement of Scientific and Technological Activities. Proposed Standard Practice for Surveys on Research and Experimental Development. Frascati Manual 2002, OECD, Paris 2002.

OECD (2005): Enhancing the performance of the services sector. Paris.

OECD (2008): OECD Compendium of Productivity Indicators 2008. Paris. 
OECD (2011a): OECD Science, Technology and Industry Scoreboard 2011. Paris.,

OECD (2011b): Fostering innovation for green growth. Paris.

Oh, D., Heshmati, A., Lööf, H. (2012): Technical change and total factor productivity for Swedish manufacturing and service industries; Applied Economics, 44, 2373-2391.

Osborn, A. (2013): Open access publishing takes a step forward, University World News, 02 March 2013, http://www.universityworldnews.com/article. php? story $=20130228122434881$, Zugriff am 23.04.2013.

Paque, K.H. (2009): Die Bilanz. Eine wirtschaftliche Analyse der deutschen Einheit. Hanser: München.

Pechar, H., Park, E., Brechelmacher, A., Ates, G. (2012): Zur Umsetzung des Kollektivvertrags an österreichischen Universitäten, Erhebungen im Auftrag des Bundesministeriums für Wissenschaft und Forschung, Wien.

Penender, M. (2003): Industrial structure and aggregate growth, in: Structural Change and Economic Dynamics, 14, 427-448.

Peneder, M. (2008): Was bleibt vom ÖsterreichParadoxon? Wachstum und Strukturwandel in der Wissensökonomie. WIFO. Wien.

Pfirrmann, O., Heinrich, S., Riesenberg, D., Dinges, M., Haller, R., Hofer, R., Streicher, G., Ecker, B. (2012): Evaluierung des österreichischen Sicherheitsforschungsprogramms KIRAS, Interimsevaluation 2011/2012, Wien.

Pilat, D. (2012): Resurrecting industrial policy; OECD Observer, 29, 9.

Pilat, D., Cimper, A., Olsen, K., Webb, C. (2006): The changing nature of manufacturing in OECD economies. OECD Science, Technology and Industry Working Papers 2006/09.

Pisano, G., Shih, W. (2009): Restoring American competitiveness; Harvard Business Review, JulyAugust, 1-14.

Pohn-Weidinger, S., Grasenick, K. (2011): Elita Evaluierung der FWF Progamme Elise Richter und Hertha Firnberg, Endbericht, Wien.

Porter, M.E. (1990): The Competitive Advantage of Nations, London: Macmillan.

Ragacs, C., Resch, B., Vondra, K. (2011): Wettbewerbsfähigkeit der österreichischen Sachgütererzeugung; Geldpolitik und Wirtschaft, 3, 38-65.

Rammer, C., Czarnitzki, D., Spielkamp, A. (2008): Innovation Success of Non-R\&D-Performers -
Substituting Technology by Management in SMEs, ZEW Discussion Paper No. 08-092, Mannheim

Rammer, C., Penzkofer, H., Stephan, A., Grenzmann, C. (2004): F\&E- und Innovationsverhalten von KMU und Großunternehmen unter dem Einfluss der Konjunktur - Studien zum deutschen Innovationssystem Nr. 22-2004, Berlin.

Rattner, S. (2011): The secrets of Germany's success. What Europe's manufacturing powerhouse can teach America; Foreign Affairs, July/August, 7-11.

Reckling, F., Scherag, E. (2012): FWF-INFO, 10-2012.

Reiner, C. (2012): Play it again, Sam: Die Renaissance der Industriepolitik in der Großen Rezession. Wirtschaft und Gesellschaft, 1, 15-56.

Reinstaller, A., Sieber, S. (2012): Veränderung der Exportstruktur in Österreich und der EU; WIFOMonatsberichte, 657-668.

Reinstaller, A., Unterlass, F. (2012): Strukturwandel und Entwicklung der Forschungs- und Entwicklungsintensität im Unternehmenssektor in Österreich im internationalen Vergleich; WIFOMonatsberichte, 8, 641-656.

RFTE (2009): Strategie 2020, Rat für Forschung und Technologieentwicklung, Wien.

Riess, A., Välilä, T. (2006): Industrial policy: a tale of innovators, champions, and B52s; EIP Papers, 11, 11-34.

Rifkin, J. (2011): The third industrial revolution. Palgrave Macmillan: Hampshire.

Rodrik, D. (2011): The manufacturing imperative. Project Syndicate.

Rossini, M. (2012): Starting a Repository Workshopbericht (Klosterneuburg, 3. Juli 2012), Mitteilungen des VÖB, 65 (2).

Rowthorn, R., Coutts, K. (2004): De-industrialization and the balance of payments in advanced economies. UNCTAD Discussion Paper, 170.

Rowthorn, R., Ramaswamy, R. (1998): Growth, trade and deindustrialization. IMF Working Paper, 98/60.

Rürup, B., Heilmann, D. (2012): Fette Jahre. Warum Deutschland eine glänzende Zukunft hat. Hanser: München.

Schettkat, R. (2006): The shift to services employment: A review of the literature; Structural change and economic dynamics, 17, 127-147.

Schettkat, R. (2010): Dienstleistungen zwischen Kostenkrankheit und Marketization. WISO Diskurs. Friedrich Ebert Stiftung.

Schibany, A., Gassler, H. (2010): Nutzen und Effekte der Grundlagenforschung; POLICIES Research Report 98-2010, Wien. 
Schumpeter, J. (2005): Kapitalismus, Sozialismus und Demokratie. 8. Auflage, Francke: Tübingen, Basel.

Shane, S. A. (2008): The Illusions of Entrepreneurship - The Costly Myth that Entrepreneurs, Investors and Policy Makers live by, New Haven und London, Yale University Press.

Shanmugalingam, S., Puttick, R., Westlake, S. (2010): Rebalancing act. NESTA Research Report.

Shih, W., Pisano, G. (2012): Does America really need manufacturing? Harvard Business Review, March 2012, 94-102.

Siebert, H., Lorz, O. (2007): Außenwirtschaft. 8. Auflage, Lucius \& Lucius: Stuttgart.

Spence, M. Hlatshwayo, S. (2011): The evolving structure of the American Economy and the employment challange. Council on Foreign Relations. Working Paper.

Statistik Austria (2010): Innovation. Ergebnisse der Sechsten Europäischen Innovationserhebung (CIS 2008). Wien.

Statistik Austria (2011): Erhebung über Forschung und experimentelle Entwicklung 2009, http://www. statistik.at/web_de/statistiken/forschung_und_ innovation/f_und_e_in_allen_ volkswirtschaftlichen_sektoren/index.html, Zugriff am 23.04.2013.

Statistik Austria (2012): Innovation 2008-2010 Ergebnisse der Innovationserhebung CIS 2010. Wien.

Syverson, C. (2011): What determines productivity? Journal of Economic Literature, 49, 326-365.

Tassey, G. (2010): Rationales and mechanisms for revitalizing US manufacturing $\mathrm{R} \& \mathrm{D}$ strategies; Journal of Technology Transfer, 35, 283-333.

The Economist (2012): A third industrial revolution. Special Report.

The Information Technology and Innovation Foundation (2009): The Atlantic Century. Benchmarking EU \& U.S. Innovation and Competitiveness, Washington.

The White House (2012): Am America built to last. Washington.

Tichy, G. (2010): Front-Runner-Strategie: Definition und Umsetzung. In: Plattform fteval, 34, 48-67.
Tidd, J., Bessant, J., Pavitt, K. (1997): Managing innovation. Wiley: Chichester.

Titscher, S. (2004): Profilentwicklung an Österreichs Universitäten. in: Höllinger, S. (Hrsg.): Die österreichische Universitätsreform. Zur Implementierung des Universitätsgesetzes 2002. Wien 2004, 264-283.

Unger, M., Dünser, L., Thaler, B., Laimer, A. (2011): Evaluierung des formelgebundenen Budgets der Universitäten, Wien.

UNIDO (2009): Industrial Development Report. uniko (2010): Empfehlungen der Österreichischen Universitätenkonferenz zu einer Open AccessPolitik der Universitäten, Beschluss vom 12. Jänner 2010, http://www.uniko.ac.at/upload/UnikoEmpfehlungen_Open_Access_01_2010.pdf, Zugriff am 23.04.2013.

van de Velde, E., C. Rammer, M. de Heide, F. Pinaud, A. Verbeek, B. Gehrke, K. Mertens, P. Debergh, P. Schliessler, F. van der Zee, M. Butter (2013): Feasibility study for an EU Monitoring Mechanism on Key Enabling Technologies, Brussels: European Commission.

von Lucke, J., Geiger, P. C. (2010): Open Government Data - Frei verfügbare Daten des öffentlichen Sektors, S.3, http://www.zu.de/deutsch/lehrstuehle/ ticc/TICC-101203-OpenGovernmentData-V1.pdf, Zugriff am 23.04.2013.

von Tunzelmann, A., Acha, V. (2005): Innovation in ,low-tech' industries; Fagerberf, J., Mowery, D., Nelson, R. (Hrsg.): The Oxford Handbook of Innovation. Oxford University Press: Oxford, 407-432.

Warta, K., Good, B. (2012): Zwischenevaluierung der Dienstleistungsinitiative des BMWFJ, Endbericht, Wien.

Wienert, H. (2009): ,Wachstumsmotor Industrie? Zur Bedeutung des Verarbeitenden Gewerbes für die Entwicklung des Bruttoinlandprodukts. Beiträge der Hochschule Pforzheim, 130.

Wolfmayr, Y., Mayerhofer, P., Stankovsky, J. (2007): WIFO-Weißbuch: Exporte als Wachstumsmotor; WIFO-Monatsberichte, 3, 249-261. 


\section{Anhang}

\subsection{Länderkürzel}

\author{
AT Österreich \\ $\mathrm{BE}$ Belgien \\ BG Bulgarien \\ CH Schweiz \\ CN China \\ CY Zypern \\ CZ Tschechische Republik \\ DE Deutschland \\ DK Dänemark \\ EE Estland \\ ES Spanien \\ FI Finnland \\ FR Frankreich
}

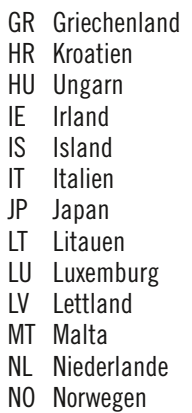

PL Polen

PT Portugal

RO Rumänien

RS Serbien

RU Russland

SE Schweden

SI Slowenien

SK Slowakei

TR Türkei

UK Vereinigtes Königreich

US Vereinigte Staaten

\subsection{Forschungsschwerpunkte an Österreichs Universitäten in der Periode der Leistungsvereinbarungen 2013-2015}

\begin{tabular}{|c|c|}
\hline Universität & „Forschungsschwerpunkte ${ }^{\text {**}^{*}}$ \\
\hline \multirow{17}{*}{$\begin{array}{l}\text { Universität Wien: } \\
\text { Forschungsplattformen }\end{array}$} & Active Ageing \\
\hline & Cognitive Science \\
\hline & $\begin{array}{l}\text { Religion and Transformation in Contemporary } \\
\text { European Society }\end{array}$ \\
\hline & Migration and Integration Research \\
\hline & Characterisation of Drug Involved Mechanisms \\
\hline & $\begin{array}{l}\text { Alternative Solvents as a Basis for Life Supporting } \\
\text { Zones in (Exo)Planetary Systems }\end{array}$ \\
\hline & Wiener Osteuropaforum \\
\hline & $\begin{array}{l}\text { Structural and functional analysis of mRNA } \\
\text { Molecules Targeted by the RNA-binding Protein } \\
\text { Tristetraprolin }\end{array}$ \\
\hline & $\begin{array}{l}\text { Theory and Practice of Subject Didactics/Teaching } \\
\text { Methodologies }\end{array}$ \\
\hline & Translational Cancer Therapy Research \\
\hline & Human Rights in the European Context \\
\hline & Life Science Governance \\
\hline & $\begin{array}{l}\text { Interfakultäre Forschungsplattform und } \\
\text { Dokumentationsstelle für die Kulturgeschichte } \\
\text { Inner- und Südasiens }\end{array}$ \\
\hline & $\begin{array}{l}\text { Neuverortung der Frauen- und Geschlechter- } \\
\text { geschichte im veränderten europäischen Kontext }\end{array}$ \\
\hline & Gödel Research Center \\
\hline & Ethik und Recht in der Medizin \\
\hline & Interdisziplinäre Forschungsplattform Archäologie \\
\hline
\end{tabular}

\begin{tabular}{|c|c|}
\hline \multirow{7}{*}{$\begin{array}{l}\text { Universität Graz: } \\
\text { Forschungsschwerpunkte }\end{array}$} & Lernen - Bildung - Wissen \\
\hline & Heterogenität und Kohäsion \\
\hline & Kultur- und Deutungsgeschichte Europas \\
\hline & Modelle und Simulation \\
\hline & Molekulare Enzymologie und Physiologie (MEP) \\
\hline & Gehirn und Verhalten \\
\hline & Umwelt und Globaler Wandel \\
\hline \multirow{3}{*}{$\begin{array}{l}\text { Universität Innsbruck: } \\
\text { Forschungsschwerpunkte }\end{array}$} & Molekulare Biowissenschaften \\
\hline & Physik \\
\hline & Alpiner Raum - Mensch und Umwelt \\
\hline \multirow{5}{*}{$\begin{array}{l}\text { Medizinische Universität Wien: } \\
\text { Forschungscluster }\end{array}$} & Allergologie/Immunologie/Infektiologie \\
\hline & Krebsforschung/Onkologie \\
\hline & Neurowissenschaften \\
\hline & Kardiovaskuläre Medizin \\
\hline & Imaging (Bildgebung) \\
\hline \multirow{4}{*}{$\begin{array}{l}\text { Medizinische Universität Graz: } \\
\text { Forschungsfelder }\end{array}$} & Neurowissenschaften \\
\hline & Krebsforschung \\
\hline & Kardiovaskuläre Erkrankungen \\
\hline & $\begin{array}{l}\text { Molekulare Grundlagen Lipid-assoziierter } \\
\text { Erkrankungen }\end{array}$ \\
\hline \multirow{4}{*}{$\begin{array}{l}\text { Medizinische Universität } \\
\text { Innsbruck: } \\
\text { Forschungsschwerpunkte }\end{array}$} & Onkologie \\
\hline & Neurowissenschaften \\
\hline & Molekulare und funktionale Bildgebung \\
\hline & $\begin{array}{l}\text { Infektiologie und Immunität \& Organ- und } \\
\text { Gewebeersatz }\end{array}$ \\
\hline
\end{tabular}

* Wie in den Leistungsvereinbarungen bezeichnet. 


\begin{tabular}{|c|c|}
\hline \multirow{4}{*}{$\begin{array}{l}\text { Universität Salzburg: } \\
\text { Forschungsschwerpunkte }\end{array}$} & Biowissenschaften und Gesundheit \\
\hline & Recht, Wirtschaft und Arbeitswelt \\
\hline & Wissenschaft \& Kunst \\
\hline & Salzburg Centre of European Union Studies \\
\hline \multirow{5}{*}{$\begin{array}{l}\text { Technische Universität Wien: } \\
\text { Forschungsschwerpunkte }\end{array}$} & Computational Science and Engineering \\
\hline & Information and Communication Technology \\
\hline & Materials and Matter \\
\hline & Quantum Physics and Quantum Technologies \\
\hline & Energy and Environment \\
\hline \multirow{5}{*}{$\begin{array}{l}\text { Technische Universität Graz: } \\
\text { Fields of Expertise }\end{array}$} & Mobility \& Production \\
\hline & Advanced Materials Science \\
\hline & Sustainability Systems \\
\hline & Information, Communication and Computing \\
\hline & Human- \& Biotechnology \\
\hline \multirow{7}{*}{$\begin{array}{l}\text { Montanuniversität Leoben: } \\
\text { Kernkompetenzbereiche }\end{array}$} & Rohstoffgewinnung und -verarbeitung \\
\hline & Hochleistungswerkstoffe \\
\hline & Umwelttechnik \& Recycling \\
\hline & Prozess- und Produktengineering \\
\hline & Energietechnik \\
\hline & Metallurgie \\
\hline & Kunststofftechnik \\
\hline \multirow{8}{*}{$\begin{array}{l}\text { Universität für Bodenkultur } \\
\text { Wien: Kompetenzfelder }\end{array}$} & Boden- und Landökosysteme \\
\hline & Wasser - Atmosphäre - Umwelt \\
\hline & Lebensraum und Landschaft \\
\hline & $\begin{array}{l}\text { Nachwachsende Rohstoffe und } \\
\text { Ressourcenorientierte Technologien }\end{array}$ \\
\hline & Lebensmittel - Ernährung - Gesundheit \\
\hline & Biotechnologie \\
\hline & Nanowissenschaften und Nanotechnologie \\
\hline & Ressourcen und gesellschaftliche Dynamik \\
\hline \multirow{5}{*}{$\begin{array}{l}\text { Veterinärmedizinische } \\
\text { Universität Wien: Profillinien }\end{array}$} & Physiologische Prozesse \\
\hline & Infektion, Prävention, Schwerpunkt Nutztiere \\
\hline & Lebensmittelsicherheit und Risikobewertung \\
\hline & Tiermodelle und Veterinärbiotechnologie \\
\hline & Tierverhalten und Mensch-Tier-Beziehung \\
\hline \multirow[t]{7}{*}{$\begin{array}{l}\text { Wirtschaftsuniversität Wien: } \\
\text { Forschungsschwerpunkte }\end{array}$} & $\begin{array}{l}\text { Information Systems, Computing and Supply Chain } \\
\text { Management }\end{array}$ \\
\hline & Finance and Accounting \\
\hline & International Business Taxation \\
\hline & Applied Economics and Socioeconomics \\
\hline & Business and Economic Law \\
\hline & International Business, esp. CEE \\
\hline & $\begin{array}{l}\text { Empirically-Focused Research on Management, } \\
\text { Marketing and Strategy }\end{array}$ \\
\hline \multirow{6}{*}{$\begin{array}{l}\text { Universität Linz: } \\
\text { Exzellenzfelder }\end{array}$} & Unternehmensrecht \\
\hline & Soziale Systeme, Märkte \& Wohlfahrtsstaat \\
\hline & Management \& Innovation \\
\hline & Nano-, Bio- \& Polymer-Systems \\
\hline & Computation in Informatics \& Mathematics \\
\hline & Mechatronics and Information Processing \\
\hline
\end{tabular}

\begin{tabular}{|c|c|}
\hline \multirow{7}{*}{$\begin{array}{l}\text { Universität Klagenfurt: } \\
\text { Forschungsthemen }\end{array}$} & Bildungsforschung \\
\hline & Energiemanagement und -technik \\
\hline & Human Centered Computing and Design \\
\hline & Nachhaltigkeit \\
\hline & Selbstorganisierende Systeme \\
\hline & Unternehmertum \\
\hline & Visuelle Kultur \\
\hline \multirow{3}{*}{$\begin{array}{l}\text { Donau-Universität Krems: } \\
\text { Forschungsschwerpunkte }\end{array}$} & Gesundheit und Medizin \\
\hline & Bildung, Kultur und Architektur \\
\hline & Wirtschaft und Globalisierung \\
\hline \multirow{6}{*}{$\begin{array}{l}\text { Akademie der bildenden } \\
\text { Künste Wien: Schwerpunkte in } \\
\text { Forschung, Entwicklung und } \\
\text { Erschließung der Künste }\end{array}$} & Institut für Bildende Kunst \\
\hline & Institut für Kunst und Architektur \\
\hline & Institut für das künstlerische Lehramt \\
\hline & Institut für Konservierung-Restaurierung \\
\hline & Institut für Kunst- und Kulturwissenschaften \\
\hline & $\begin{array}{l}\text { Institut für Naturwissenschaften und Technologie in } \\
\text { der Kunst }\end{array}$ \\
\hline \multirow{9}{*}{$\begin{array}{l}\text { Universität für Angewandte } \\
\text { Kunst Wien: Disziplinen }\end{array}$} & Architektur \\
\hline & Bildende und Medial Kunst \\
\hline & Design \\
\hline & Konservierung und Restaurierung \\
\hline & Kunst- und Kulturwissenschaft \\
\hline & Naturwissenschaften \\
\hline & Sprachkunst \\
\hline & Kunst- und Wissenstransfer \\
\hline & Kunstpädagogik und -vermittlung \\
\hline \multirow{3}{*}{$\begin{array}{l}\text { Universität für Musik und } \\
\text { Darstellende Kunst Wien: Drei } \\
\text { Säulen }\end{array}$} & Säule Konzertfächer \\
\hline & Säule Musikpädagogik \\
\hline & Säule Darstellende Kunst \\
\hline \multirow{5}{*}{$\begin{array}{l}\text { Universität für Musik und } \\
\text { darstellende Kunst Graz: } \\
\text { Schwerpunkte }\end{array}$} & Kammermusik \\
\hline & Jazz \\
\hline & $\begin{array}{l}\text { Bühne (Schauspiel, Musiktheater, } \\
\text { Bühnengestaltung) }\end{array}$ \\
\hline & Zeitgenössische Musik \\
\hline & Forschung \\
\hline \multirow{3}{*}{$\begin{array}{l}\text { Mozarteum Salzburg: } \\
\text { Entwicklungsschwerpunkte }\end{array}$} & Zeitgenössische Kunst \\
\hline & Bühnenkunst \\
\hline & Kammermusik / Ensemblespiel \\
\hline \multirow{3}{*}{$\begin{array}{l}\text { Universität für künstlerische } \\
\text { und industrielle Gestaltung } \\
\text { Linz: Profilschwerpunkte }\end{array}$} & Intermedialität \\
\hline & Raumstrategien \\
\hline & Künstlerisch-wissenschaftliche Forschung \\
\hline
\end{tabular}




\subsection{Liste der Einzelindikatoren des „Innovationsindikators“ der Deutschen Telekom Stiftung und des BDI}

Beschreibung

Anteil der ausländischen Studierenden an allen Studierenden

Beschäftigte mit mind. Sekundarstufe II, ohne Hochschulabschlüsse als Anteil an allen Beschäftigten

Promovierte (ISCED 6) in den MINT-Fächern als Anteil an der Bevölkerung

HochschulabsolventInnen in Relation zu den hoch qualifizierten Beschäftigten im Alter 55 +

Anteil der Beschäftigten mit tertiärer Bildung an allen Beschäftigten

Jährliche Bildungsausgaben (Tertiärstufe einschl. F\&E) je Studentln

Qualität des Bildungssystems (Skala von 1 bis 7 auf Basis von Experteneinschätzungen)

Qualität der mathematisch-naturwissenschaftlichen Bildung (Skala von 1 bis 7 auf Basis von Experteneinschätzungen)

PISA-Index: Wissenschaft, Lesekompetenz, Mathematik (auf offener Skala mit Mittelwert 500 und

Standardabweichung 100)

E-Readiness Indicator (Skala von 1 bis 10)

Bewertung der Erfolgswahrscheinlichkeit von Gründungen (nach Eigenangaben)

Anzahl der Personalcomputer je 100 Einwohner

Anteil Postmaterialisten (Inglehardt) an der Bevölkerung

Staatliche Nachfrage nach fortschrittlichen technologischen Produkten (Skala von 1 bis 7 auf Basis von

Experteneinschätzungen)

Nachfrage der Unternehmen nach technologischen Produkten (Skala von 1 bis 7 auf Basis von Experteneinschätzungen)

Für die Frühphase eingesetztes Venture-Capital in Relation zum Bruttoinlandsprodukt

Ausmaß von Marketing (Skala von 1 bis 7 auf Basis von Experteneinschätzungen)

Anteil der internationalen Co-Patente an allen Anmeldungen von transnationalen Patenten

Anteil der Wertschöpfung in der Hochtechnologie an der gesamten Wertschöpfung

Anteil der Beschäftigten in wissensintensiven Dienstleistungen an allen Beschäftigten

Intensität des einheimischen Wettbewerbs (Skala von 1 bis 7 auf Basis von Experteneinschätzungen)

Bruttoinlandsprodukt (BIP) pro Kopf der Bevölkerung

Patentanmeldungen transnationaler Patente je Einwohner

Patentanmeldungen am USPTO je Einwohner

Wertschöpfung pro Arbeitsstunde (in konstanten PPP - US \$)

Handelsbilanzsaldo bei Hochtechnologien gemessen an der Bevölkerung

Anteil der von Unternehmen finanzierten F\&E-Ausgaben der Hochschulen

Interne F\&E-Ausgaben der Unternehmen als Anteil am BIP

B-Index der steuerlichen F\&E-Förderung: Anteil der F\&E-Ausgaben der Unternehmen, die durch eine steuerliche

F\&E-Förderung finanziert werden

Anteil der staatlich finanzierten F\&E-Ausgaben der Unternehmen am BIP

Anzahl der Forscherlnnen in Vollzeitäquivalente pro 1.000 Beschäftigte

Zahl der wissenschaftlich-technischen Artikel in Relation zur Bevölkerung

Qualität der wissenschaftlichen Forschungseinrichtungen (Skala von 1 bis 7 auf Basis von Experteneinschätzungen)

Zahl der Zitate pro wissenschaftlich-technischer Publikation in Relation zum weltweiten Durchschnitt (gemessen am Durchschnitt der jeweiligen Disziplin)

Anzahl der Patente aus der öffentlichen Forschung je Einwohner

Anteil von internationalen $\mathrm{C}_{0}$-Publikationen an allen wissenschaftlich-technischen Artikeln

Anteil der F\&E-Ausgaben in staatlichen Forschungseinrichtungen und Hochschulen am BIP

Anteil eines Landes an den $10 \%$ am häufigsten zitierten wissenschaftlich-technischen Publikationen
Akteur/ Subsystem Input/ Output

Bildung Input

Bildung Output

Bildung Output

Bildung Input

Bildung Output

Bildung/Staat Input

Bildung/Staat Input

Bildung/Staat Input

Bildung/Staat Input

Gesellschaft Input

Gesellschaft Input

Gesellschaft Input

Input

Staat Input

Wirtschaft Input

Wirtschaft Input

Wirtschaft Input

Wirtschaft Input

Wirtschaft Output

Wirtschaft Input

Wirtschaft Output

Wirtschaft Output

Wirtschaft Output

Wirtschaft Output

Wirtschaft Output

Wirtschaft Input

Wirtschaft Input

Wirtschaft/Staat Input

Wirtschaft/Staat Input

Wissenschaft Input

Wissenschaft Output

Wissenschaft Input

Wissenschaft Output

Wissenschaft Output

Wissenschaft Input

Wissenschaft/Staat Input

Wissenschaft Input 


\subsection{Klassifikation von potenziellen Umweltschutzgütern (Gehrke et al. 2012)}

Die Analyse der internationalen Handelsströme folgt einem angebotsorientierten Potenzialansatz und beruht auf einer in 2012 vom NIW in Zusammenarbeit mit dem Statistischen Bundesamt erstellten neuen Liste von potenziellen Umweltschutzgütern. Potenzialorientiert bedeutet in diesem Zusammenhang, dass diese Güter potenziell zur Nutzung in umweltrelevanten Bereichen eingesetzt werden. Diese umfassen zum einen die Güter zur Nutzung Erneuerbarer Energiequellen und zur Steigerung der Energieeffizienz über rationelle Energieverwendung und -umwandlung (potenzielle Klimaschutzgüter, vgl. Kasten). Hinzu kommen Güter, die vor allem auf den Schutz der klassischen Umweltbereiche (Abfall, Wasser, Luft, Lärm) abzielen sowie - bereichsübergreifend - Instrumente und Komponenten zum Messen, Steuern und Regeln von Umweltschutzanlagen.

Die auf Basis der (achtstelligen) Produktionsstatistik ermittelten Güter wurden zur Durchführung von Außenhandelsanalysen vom NIW in die internationale Außenhandelssystematik des Harmonisierten Systems (HS) auf der tiefstmöglichen (sechsstelligen) Gliederungsebene umgeschlüsselt. Als Datenquellen wurden die Außenhandelsstatistik der OECD sowie die Comtrade-Datenbank der Vereinten Nationen verwendet. Der Analysezeitraum bezieht sich auf die Jahre 2002 bis 2011.

Die quantitativ wichtigste Gütergruppe stellen die potenziellen Klimaschutzgüter dar. Diese sind folgendermaßen unterteilt:

- Erneuerbare Energiequellen

- Windkraft

- Solarenergie

- Solarzellen und -module

- Übrige Solarenergiegüter (Wechselrichter, Spiegel, etc.)

- Wärmepumpen, Biomasse/-gas, Wasserkraft

- Rationelle Energieverwendung

- Im Wesentlichen Isolierung und Wärmedämmung

- Rationelle Energieumwandlung

- Gas- und Dampfturbinen

- Blockheizkraftwerke

\subsection{Abgrenzung von Branchen nach Technologieintensität gemäß OECD-Klassifikation auf Ebene der NACE 2-Steller} (Statistik Austria 2012)

Hochtechnologie: Herstellung von pharmazeutischen Erzeugnissen (ÖNACE 21) sowie Herstellung von Datenverarbeitungsgeräten, elektronischen und optischen Erzeugnissen (ÖNACE 26).

Mittelhochtechnologie: Herstellung von chemischen Erzeugnissen (ÖNACE 20), Herstellung von elektrischen Ausrüstungen (ÖNACE 27), Maschinenbau (ÖNACE 28), Herstellung von Kraftwagen und Kraftwagenteilen (ÖNACE 29) sowie sonstiger Fahrzeugbau (ÖNACE 30).

Mittelniedrigtechnologie: Kokerei und Mineralölverarbeitung (ÖNACE 19), Herstellung von Gummi- und Kunststoffwaren (ÖNACE 22), Herstellung von Glas und Glaswaren, Keramik, Verarbeitung von Steinen und Erden (ÖNACE 23), Metallerzeugung und -bearbeitung (ÖNACE 24), Herstellung von Metallerzeugnissen (ÖNACE 25) sowie Reparatur und Installation von Maschinen und Ausrüstungen (ÖNACE 33).

Niedrigtechnologie: Herstellung von Nahrungs- und Futtermitteln (ÖNACE 10), Getränkeherstellung (ÖNACE 11), Tabakverarbeitung (ÖNACE 12), Herstellung von Textilien (ÖNACE 13), Herstellung von Bekleidung (ÖNACE 14), Herstellung von Leder, Lederwaren und Schuhen (ÖNACE 15), Herstellung von Holz-, Flecht-, Korbund Korkwaren (ohne Möbel) (ÖNACE 16), Herstellung von Papier, Pappe und Waren daraus (ÖNACE 17), Herstellung von Druckerzeugnissen, Vervielfältigung von bespielten Ton-, Bild- und Datenträgern (ÖNACE 18), Herstellung von Möbeln (ÖNACE 31) sowie Herstellung von sonstigen Waren (ÖNACE 32).

Wissensintensive Dienstleistungen: Schifffahrt (ÖNACE 50), Luftfahrt (ÖNACE 51), Verlagswesen (ÖNACE 58), Telekommunikation (ÖNACE 61), Erbringung von Dienstleistungen der Informationstechnologie (ÖNACE 62), Informationsdienstleistungen (ÖNACE 63), Erbringung von Finanzdienstleistungen (ÖNACE 64), Versicherungen, Rückversicherungen und Pensionskassen (ohne Sozialversicherung) (ÖNACE 65), mit Finanz- und Versicherungsdienstleistungen verbundene Tätigkeiten (ÖNACE 66) sowie Architektur- und Ingenieurbüros, technische, physikalische und chemische Untersuchung (ÖNACE 71).

Weniger wissensintensive Dienstleistungen: Großhandel (ohne Handel mit Kraftfahrzeugen und Krafträdern) (ÖNACE 46), Landverkehr und Transport in Rohrfernleitungen (ÖNACE 49), Lagerei sowie Erbringung von sonstigen Dienstleistungen für den Verkehr (ÖNACE 52) sowie Post-, Kurier- und Expressdienste (ÖNACE 53). 


\section{Statistik}

\section{Finanzierung der Bruttoinlandsausgaben für F\&E und die Forschungsquote Österreichs ${ }^{191} 2013$}

Die österreichischen Bruttoinlandsausgaben für Forschung und experimentelle Entwicklung (F\&E) werden nach einer Schätzung von Statistik Austria im Jahr 2013 voraussichtlich 8,96 Mrd. € betragen. Das entspricht einer Forschungsquote (Bruttoinlandsausgaben für F\&E im Verhältnis zum Bruttoinlandsprodukt) von 2,81\%. Gegenüber 2012 wird die Gesamtsumme der österreichischen F\&E-Ausgaben um geschätzte 2,9\% ansteigen.

Von den gesamten prognostizierten Forschungsausgaben 2013 wird mit 43,9 \% (rund 3,93 Mrd. €) auch weiterhin der größte Anteil von österreichischen Unternehmen finanziert werden. Der öffentliche Sektor wird mit rund 3,62 Mrd. $€$ einen Finanzierungsanteil von 40,4 \% an den Gesamtausgaben für F\&E erreichen. $\mathrm{Zu}$ dieser Summe wird der Bund rund 3,09 Mrd. $€$ beitragen, die Bundesländer rund 427 Mio. $€$ und die sonstigen öffentlichen Einrichtungen (Gemeinden, Kammern und Sozialversicherungsträger) rund 105 Mio. €. 15,2 \% (rund 1,36 Mrd. €) der österreichischen Forschungsausgaben werden 2013 vom Ausland finanziert werden. Die Finanzierung durch das Ausland stammt zum überwiegenden Teil von mit heimischen Unternehmen verbundenen ausländischen Unternehmen, deren Tochterunternehmen in Österreich F\&E betreiben. Eingeschlossen sind auch die Rückflüsse aus den EU-Rahmenprogrammen für Forschung, technologische Entwicklung und Demonstration. 0,5\% (rund 49 Mio. $€$ ) werden auf den privaten gemeinnützigen Sektor entfallen.

Die Forschungsfinanzierung durch den Bund steigt nach der Statistik Austria vorliegenden Informationen über die Entwicklung der F\&E-relevanten Budgetanteile und weiterer F\&E-Fördermaßnahmen weiterhin an. Insbesondere aufgrund der Erstattungen an Unternehmen im Rahmen der Forschungsprämie ergibt sich 2012 ein Zuwachs von $14,2 \%$ und nach einem Plus von 2,8 \% im Jahr 2013 werden die Bundesmittel den bisherigen Höchstwert von 3,09 Mrd. € erreichen.

Die Forschungsquote, jener Indikator, der die Bruttoinlandsausgaben für F\&E als Prozentsatz des Bruttoinlandsprodukts (BIP) darstellt, ist für Österreich in den letzten zehn Jahren stark angestiegen. Trotz Wirtschaftskrise gingen die F\&EAusgaben in Österreich nicht bzw. nur geringfügig zurück. 2009 betrug die Forschungsquote 2,71 \%, im Folgejahr stieg sie insbesondere auf-

191 Auf der Grundlage der Ergebnisse der F\&E-statistischen Vollerhebungen sowie sonstiger aktuell verfügbarer Unterlagen und Informationen, insbesondere der F\&E-relevanten Voranschlags- und Rechnungsabschlussdaten des Bundes und der Bundesländer, wird von Statistik Austria jährlich die „Globalschätzung der österreichischen Bruttoinlandsausgaben für F\&E" erstellt. Im Rahmen der jährlichen Erstellung der Globalschätzung erfolgen auf der Basis von neuesten Daten jeweils auch rückwirkende Revisionen bzw. Aktualisierungen. Den Definitionen des weltweit (OECD, EU) gültigen und damit die internationale Vergleichbarkeit gewährleistenden Frascati-Handbuchs entsprechend wird die Finanzierung der Ausgaben der in Österreich durchgeführten Forschung und experimentellen Entwicklung dargestellt. Gemäß diesen Definitionen und Richtlinien ist die ausländische Finanzierung von in Österreich durchgeführter F\&E sehr wohl einbezogen, hingegen österreichische Zahlungen für im Ausland durchgeführte F\&E sind ausgeschlossen (Inlandskonzept). 
grund der vom öffentlichen Sektor eingesetzten F\&E-Mittel auf 2,79\%. 2011 führte der im Vergleich zu den Forschungsausgaben stärkere Anstieg des Bruttoinlandsprodukts zu einem kurzfristigen Absinken der Forschungsquote auf $2,72 \%, 2012$ stieg sie wieder über den Wert von 2010 und erreichte $2,81 \%$. Nach derzeitigem Informationsstand über die Entwicklung des Bruttoinlandsprodukts wird die Forschungsquote voraussichtlich auch 2013 auf diesem Niveau bleiben.

Österreich übertrifft weiterhin deutlich die durchschnittliche Forschungsquote der EU-27 und liegt für das Vergleichsjahr 2011 (dem letzten Jahr, für das internationale Vergleichszahlen verfügbar sind) über dem EU-Durchschnitt von $2,03 \%$. Die Forschungsquoten von Finnland, Schweden und Dänemark liegen über $3 \%$, nach Deutschland $(2,84 \%)$ weist Österreich somit die fünfthöchste Quote innerhalb der EU-27 auf.

In der Schätzung der österreichischen Bruttoinlandsausgaben für F\&E 2013 wurden vorläufige Ergebnisse der F\&E-Erhebung 2011 von Statistik Austria bei Unternehmen, Voranschlags- und Rechnungsabschlussdaten des Bundes und der Bundesländer sowie aktuelle Konjunkturdaten berücksichtigt.

\section{F\&E-Ausgaben des Bundes 2013}

2.1. Die in Tabelle 1 ausgewiesenen Ausgaben des Bundes für in Österreich 2013 durchgeführte F\&E setzen sich wie folgt zusammen: Gemäß jener der F\&E-Globalschätzung zugrunde liegenden Methodik ist das Kernstück die Gesamtsumme des Teils b der Beilage $\mathrm{T}$ des Arbeitsbehelfes zum Bundesfinanzgesetz 2013. Zusätzlich wurden die für 2013 nach derzeitigem Informations- stand zur Auszahlung gelangenden Mittel der Nationalstiftung für Forschung, Technologie und Entwicklung sowie die voraussichtlich 2013 zur Auszahlung gelangenden Forschungsprämien einbezogen. ${ }^{192}$

2.2. Zusätzlich zu den Ausgaben des Bundes für in Österreich durchgeführte F\&E wird der Bund im Jahr 2013 Beitragszahlungen an internationale Organisationen, die Forschung und Forschungsförderung als Ziel haben, in Höhe von 95 Mio. $€$ leisten, die in der Beilage $\mathrm{T} /$ Teil a dargestellt sind, jedoch gemäß dem Inlandskonzept nicht in die österreichischen Bruttoinlandsausgaben für F\&E eingerechnet werden.

2.3. Die in der Beilage $\mathrm{T}$ (Teil a und Teil b) zusammengefassten forschungswirksamen Ausgaben des Bundes, welche die forschungswirksamen Anteile an den Beitragszahlungen an internationale Organisationen (siehe oben Punkt 2.2) einschließen, werden traditioneller Weise unter der Bezeichnung "Ausgaben des Bundes für Forschung und Forschungsförderung " zusammengefasst und entsprechen dem auf Basis des FrascatiHandbuches von OECD und EU angewendeten "GBAORD" -Konzept ${ }^{193}$. Dieses Konzept bezieht sich primär auf die Budgets des Zentral- bzw. Bundesstaates und berücksichtigt im Gegensatz zum Inlandskonzept die forschungsrelevanten Beitragszahlungen an internationale Organisationen, womit es die Grundlage der Klassifizierung von F\&E-Budgetdaten nach sozio-ökonomischen Zielsetzungen für die Berichterstattung an EU und OECD bildet.

Im Jahr 2013 entfallen auf folgende sozio-ökonomischen Zielsetzungen die stärksten Anteile an

192 Quelle: BMF.

193 GBAORD: Government Budget Appropriations or Outlays for R\&D = „Staatliche Mittelzuweisungen oder Ausgaben für Forschung und Entwicklung" (EU-Übersetzung). 
den Ausgaben des Bundes für Forschung und Forschungsförderung:

- Förderung der allgemeinen Erweiterung des Wissens: 30,4 \%

- Förderung von Handel, Gewerbe und Industrie: $27,6 \%$

- Förderung des Gesundheitswesens: 20,8 \%

- Förderung der Erforschung der Erde, der Meere, der Atmosphäre und des Weltraumes: 4,5\%

- Förderung der sozialen und sozio-ökonomischen Entwicklung: 3,5 \%

- Förderung des Umweltschutzes: 3,3\%

- Förderung der Land- und Forstwirtschaft: $2,8 \%$

\section{F\&E-Ausgaben der Bundesländer}

Die als Teilsumme in Tabelle 1 ausgewiesene Forschungsfinanzierung durch die Bundesländer beruht auf den von den Ämtern der Landesregierungen gemeldeten F\&E-Ausgaben-Schätzungen auf Basis der jeweiligen Landesvoranschläge. Die F\&E-Ausgaben der Landeskrankenanstalten werden gemäß einer mit den Landesregierungen vereinbarten Methodik von Statistik Austria jährlich geschätzt.

\section{F\&E-Ausgaben $2010 \mathrm{im}$ internationalen Vergleich}

Die Übersichtstabelle 13 zeigt anhand der wichtigsten F\&E-relevanten Kennzahlen die Position Österreichs im Vergleich zu den anderen Mitgliedsstaaten der Europäischen Union bzw. der OECD. ${ }^{194}$

\section{Beteiligung Österreichs an den Europäischen Rahmenprogrammen für Forschung, technologische Entwicklung und Demonstration}

Die Tabellen 14 bis 16 geben einen Überblick über die Beteiligung Österreichs an den Europäischen Rahmenprogrammen für Forschung und Entwicklung anhand des laufenden Monitoringund Berichtssystems von PROVISO.

\section{Forschungsförderung durch den Wissenschaftsfonds (FWF)}

In den Tabellen 17 bis 19 werden detaillierte Informationen über die Förderungen und die Anzahl der Projekte in den Programmen des FWF bereitgestellt.

\section{Förderungen durch die Forschungs- förderungsgesellschaft (FFG)}

Die Tabellen 20 bis 21 liefern detaillierte Angaben zu den Förderzusagen des Jahres 2012 von Seiten der FFG.

\section{8. aws-Technologieprogramme}

Die Tabelle 22 zeigt einen Überblick bezüglich der erfolgten Förderungen im Rahmen der awsTechnologieprogramme.

\section{Christian Doppler Gesellschaft}

Tabelle 23 bis 25 bezieht sich auf den Stand und die zeitliche Entwicklung der CD-Labors.

194 Quelle: OECD: MSTI 2012-2. 


\section{Tabellenübersicht}

Tabelle 1: Globalschätzung 2013: Bruttoinlandsausgaben für die F\&E-Finanzierung der in Österreich durchgeführten Forschung und experimentellen Entwicklung 1993 bis 2013

Tabelle 2: Globalschätzung 2013: Bruttoinlandsausgaben für F\&E-Finanzierung der in Österreich durchgeführten

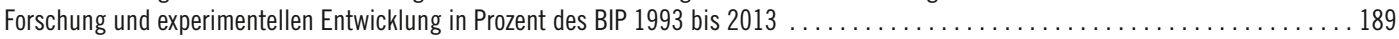

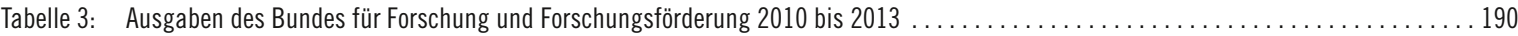

Tabelle 4: $\quad$ Beilage T des Arbeitsbehelfes zum Bundesfinanzgesetz 2013 (Finanzierungsvoranschlag). . . . . . . . . . . . . . . . . 190

Tabelle 5: Ausgaben des Bundes für Forschung und Forschungsförderung nach sozio-ökonomischen Zielsetzungen 1997 bis 2013 . . . . . . . . . 191

Tabelle 6: Ausgaben des Bundes für Forschung und Forschungsförderung nach sozio-ökonomischen Zielsetzungen und Ressorts 2011 . . . . . . 192

Tabelle 7: Ausgaben des Bundes für Forschung und Forschungsförderung nach sozio-ökonomischen Zielsetzungen und Ressorts 2012 . . . . . . . . 193

Tabelle 8: Ausgaben des Bundes für Forschung und Forschungsförderung nach sozio-ökonomischen Zielsetzungen und Ressorts 2013 . . . . . . . 194

Tabelle 9: Allgemeine forschungswirksame Hochschulausgaben des Bundes (General University Funds) 1999 bis 2013 . . . . . . . . . . . . . . 195

Tabelle 10: Forschungsförderungen und Forschungsaufträge des Bundes nach

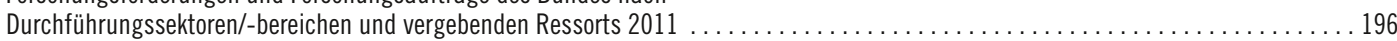

Tabelle 11: Forschungsförderungen und Forschungsaufträge des Bundes nach

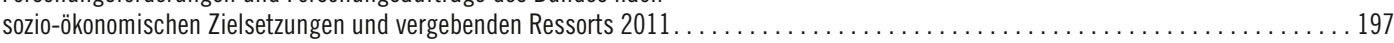

Tabelle 12: Forschungsförderungen und Forschungsaufträge der Bundesdienststellen nach

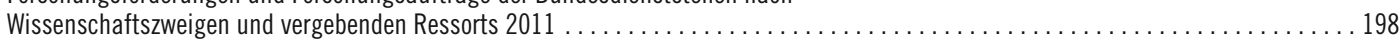

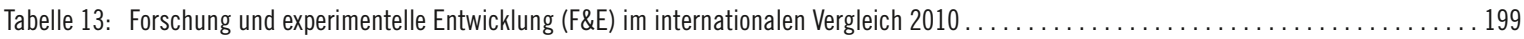

Tabelle 14: Österreichs Pfad vom 4. zum 7. EU-Rahmenprogramm für Forschung, technologische Entwicklung und Demonstration ...........200

Tabelle 15: Ergebnisse Österreichs im 7. EU-Rahmenprogramm für Forschung, technologische Entwicklung und Demonstration ..............200

Tabelle 16: Überblick über Projekte und Beteiligungen im 7. EU-Rahmenprogramm für Forschung,

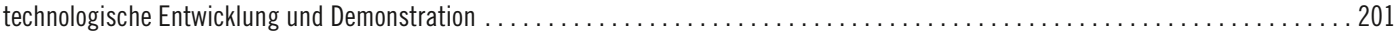

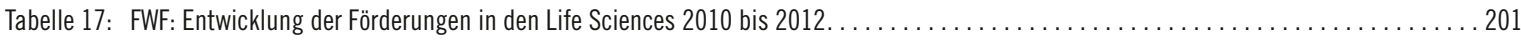

Tabelle 18: FWF: Entwicklung der Förderungen in den Naturwissenschaften und Technik 2010 bis $2012 \ldots \ldots \ldots \ldots \ldots 2$

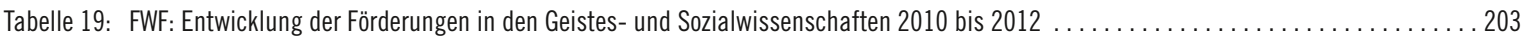

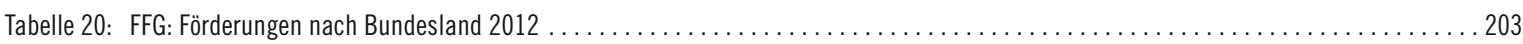

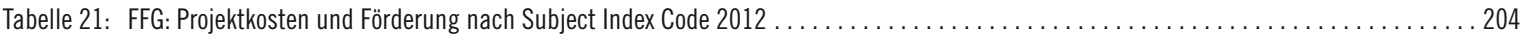

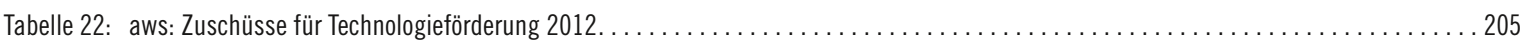

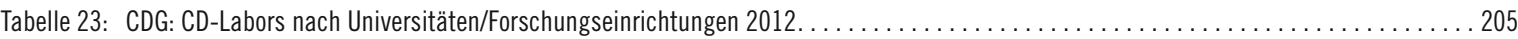

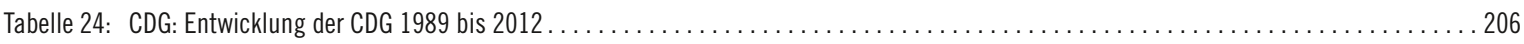

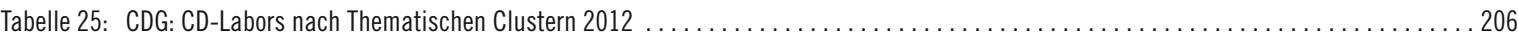



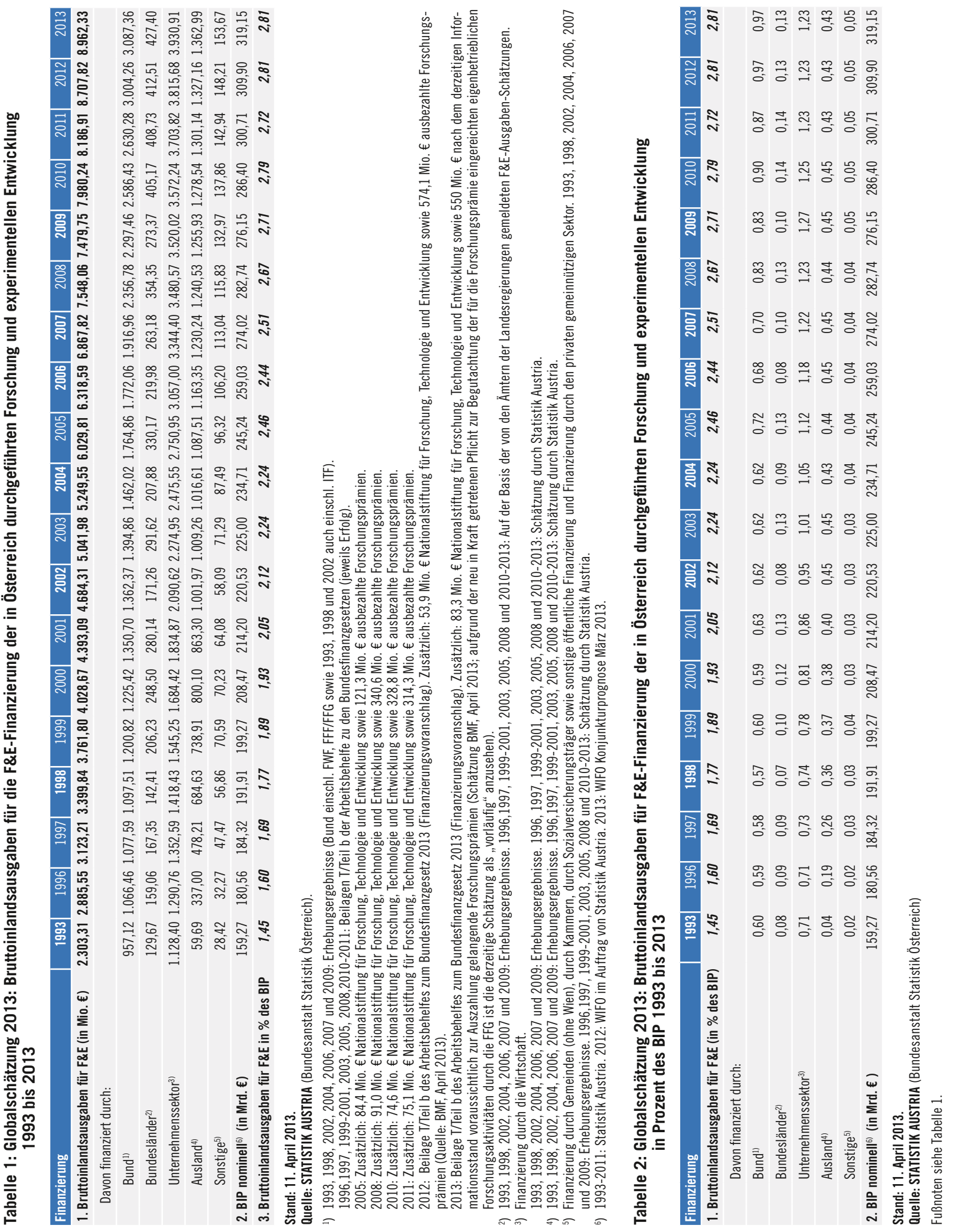
Tabelle 3: Ausgaben des Bundes für Forschung und Forschungsförderung 2010 bis 2013

Aufgliederung der Beilage T der Arbeitsbehelfe zu den Bundesfinanzgesetzen 2012 und 2013

\begin{tabular}{|c|c|c|c|c|c|c|c|c|}
\hline \multirow{3}{*}{ Ressorts ${ }^{1)}$} & \multicolumn{4}{|c|}{ Erfolg } & \multicolumn{4}{|c|}{ Bundesvoranschlag } \\
\hline & \multicolumn{2}{|l|}{$2010^{21}$} & \multicolumn{2}{|l|}{$2011^{3)}$} & \multicolumn{2}{|c|}{$2012^{3)}$} & \multicolumn{2}{|c|}{$2013^{3)}$} \\
\hline & Mio. $€$ & $\%$ & Mio. $€$ & $\%$ & Mio. € & $\%$ & Mio. $€$ & $\%$ \\
\hline Bundeskanzleramt ${ }^{4)}$ & 1,973 & 0,1 & 1,898 & 0,1 & 2,385 & 0,1 & 2,237 & 0,1 \\
\hline Bundesministerium für Inneres & 0,789 & 0,0 & 0,801 & 0,0 & 0,933 & 0,0 & 0,911 & 0,0 \\
\hline Bundesministerium für Unterricht, Kunst und Kultur & 62,380 & 2,7 & 63,436 & 2,7 & 70,793 & 2,9 & 66,842 & 2,6 \\
\hline Bundesministerium für Wissenschaft und Forschung & $1.652,719$ & 72,9 & $1.669,825$ & 71,5 & $1.738,305$ & 70,3 & $1.823,278$ & 71,5 \\
\hline Bundesministerium für Arbeit, Soziales und Konsumentenschutz & 2,232 & 0,1 & 2,736 & 0,1 & 2,171 & 0,1 & 2,501 & 0,1 \\
\hline Bundesministerium für Gesundheit & 4,959 & 0,2 & 3,772 & 0,2 & 5,374 & 0,2 & 4,916 & 0,2 \\
\hline Bundesministerium für europäische und internationale Angelegenheiten & 2,147 & 0,1 & 2,259 & 0,1 & 2,383 & 0,1 & 2,386 & 0,1 \\
\hline Bundesministerium für Justiz & 0,098 & 0,0 & 0,098 & 0,0 & 0,130 & 0,0 & 0,130 & 0,0 \\
\hline Bundesministerium für Landesverteidigung und Sport & 2,440 & 0,1 & 2,079 & 0,1 & 2,589 & 0,1 & 2,380 & 0,1 \\
\hline Bundesministerium für Finanzen & 31,437 & 1,4 & 33,970 & 1,5 & 34,466 & 1,4 & 34,620 & 1,4 \\
\hline Bundesministerium für Land- und Forstwirtschaft, Umwelt und Wasserwirtschaft & 60,927 & 2,7 & 77,425 & 3,3 & 86,609 & 3,5 & 76,048 & 3,0 \\
\hline Bundesministerium für Wirtschaft, Familie und Jugend & 103,200 & 4,5 & 110,489 & 4,7 & 107,076 & 4,3 & 104,338 & 4,1 \\
\hline Bundesministerium für Verkehr, Innovation und Technologie & 344,685 & 15,2 & 366,903 & 15,7 & 417,905 & 16,9 & 428,502 & 16,8 \\
\hline Insgesamt & $2.269,986$ & 100,0 & $2.335,691$ & 100,0 & $2.471,117$ & 100,0 & $2.549,089$ & 100,0 \\
\hline
\end{tabular}

Stand: April 2013.

Quelle: Statistik Austria (Bundesanstalt Statistik Österreich).

$\left.{ }^{1}\right)$ Entsprechend der im jeweiligen Jahr gültigen Fassung des Bundesministeriengesetzes 1986 (BGBI. I Nr. 3/2009).

$\left.{ }^{2}\right)$ Arbeitsbehelf zum Bundesfinanzgesetz 2012.

3) Arbeitsbehelf zum Bundesfinanzgesetz 2013 (Finanzierungsvoranschlag).

$\left.{ }^{4}\right)$ Einschließlich oberste Organe. - Rundungsdifferenzen nicht ausgeglichen.

Tabelle 4: Beilage T des Arbeitsbehelfes zum Bundesfinanzgesetz 2013 (Finanzierungsvoranschlag)

\begin{tabular}{|c|c|c|c|c|c|c|}
\hline \multirow[t]{2}{*}{ Beilage T } & \multicolumn{2}{|c|}{ Bundesvoranschlag 2013} & \multicolumn{2}{|c|}{ Bundesvoranschlag 2012} & \multicolumn{2}{|c|}{ Erfolg 2011} \\
\hline & Insgesamt & Forschung & Insgesamt & Forschung & Insgesamt & Forschung \\
\hline & \multicolumn{6}{|c|}{ Mio. $€$} \\
\hline Teil a* & 110.115 & 95.028 & 108.976 & 94.813 & 107.358 & 94.776 \\
\hline Teil b** & 6.275 .091 & 2.454 .061 & 6.101 .554 & 2.376 .304 & 5.715 .722 & 2.240 .915 \\
\hline Insgesamt & 6.385 .206 & 2.549 .089 & 6.210 .530 & 2.471 .117 & 5.823 .080 & 2.335 .691 \\
\hline
\end{tabular}

Stand: April 2013.

Quelle: Bundesministerium für Finanzen.

* Beitragszahlungen an internationale Organisationen, die Forschung und Forschungsförderung (mit) als Ziel haben.

** Inlandsausgaben des Bundes für Forschung und Forschungsförderung (Bundesbudget Forschung). 


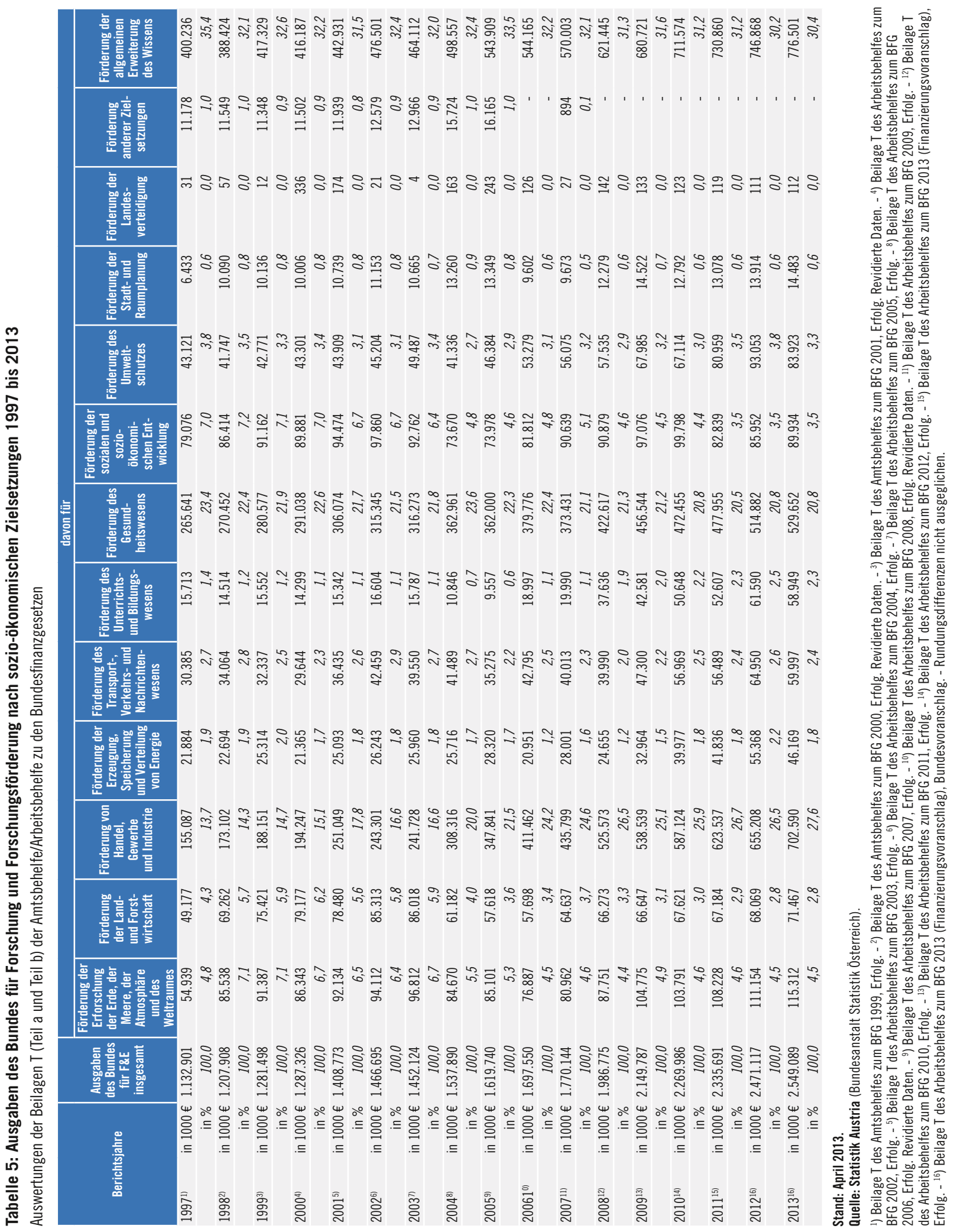




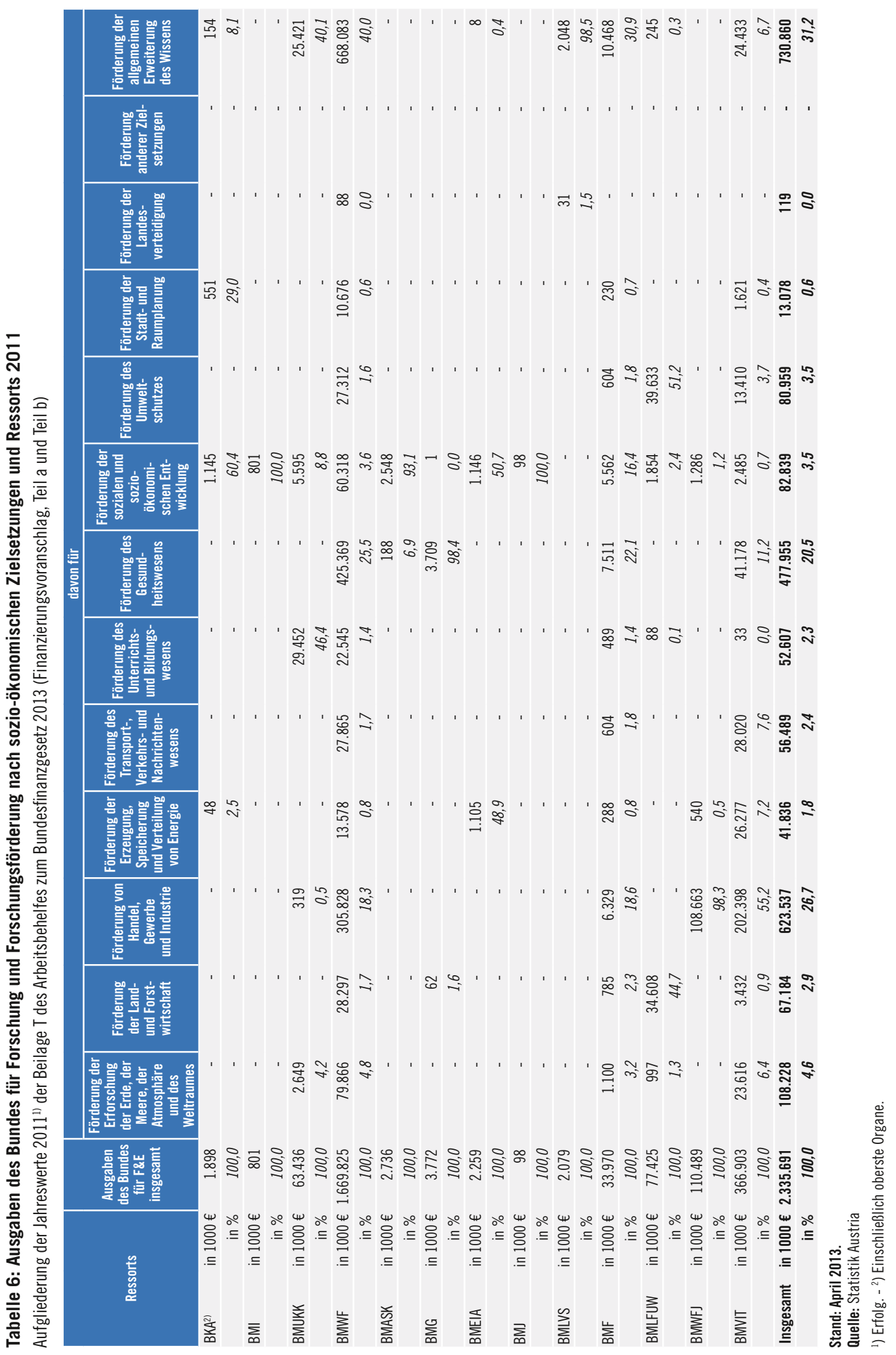




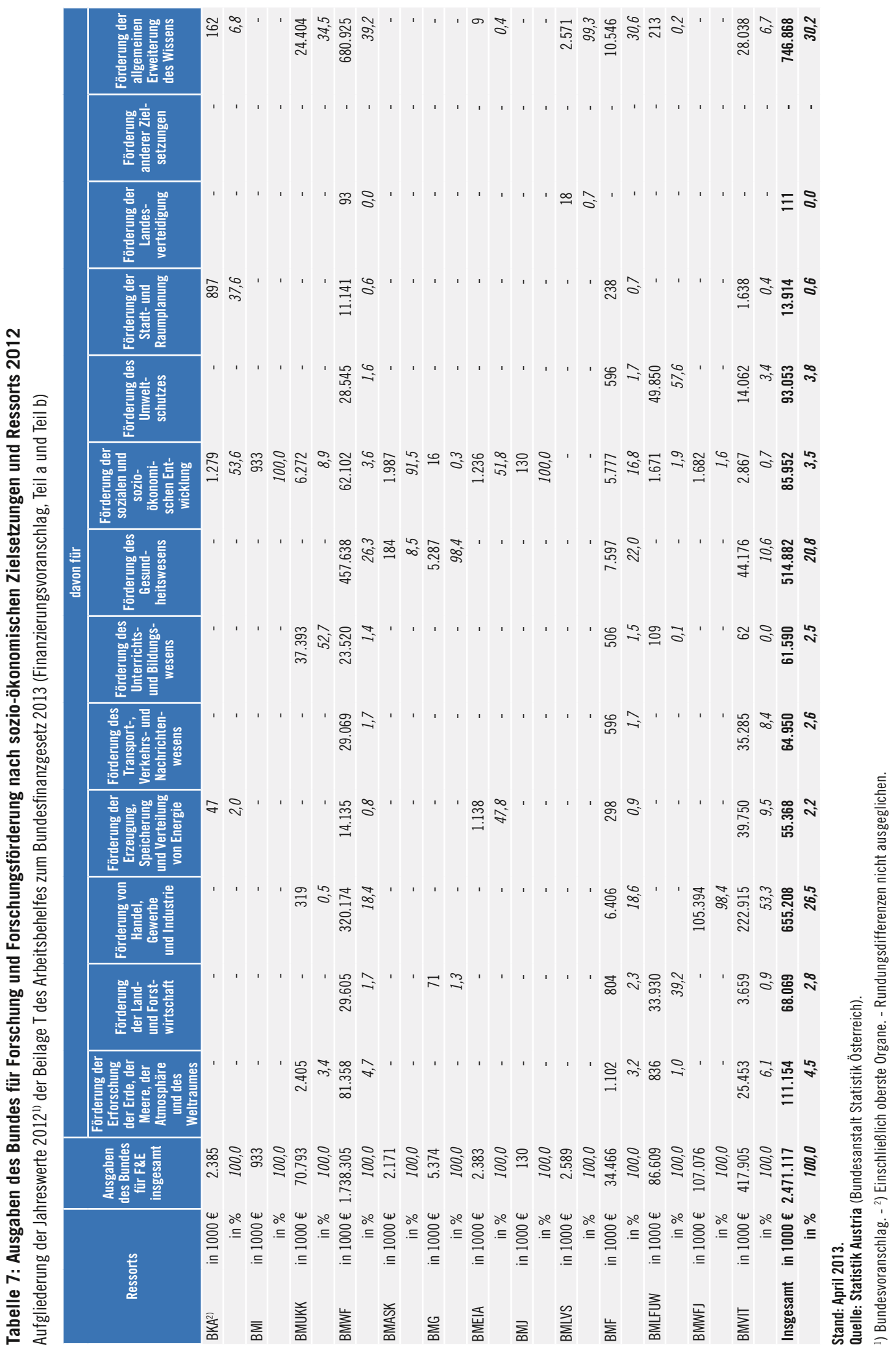




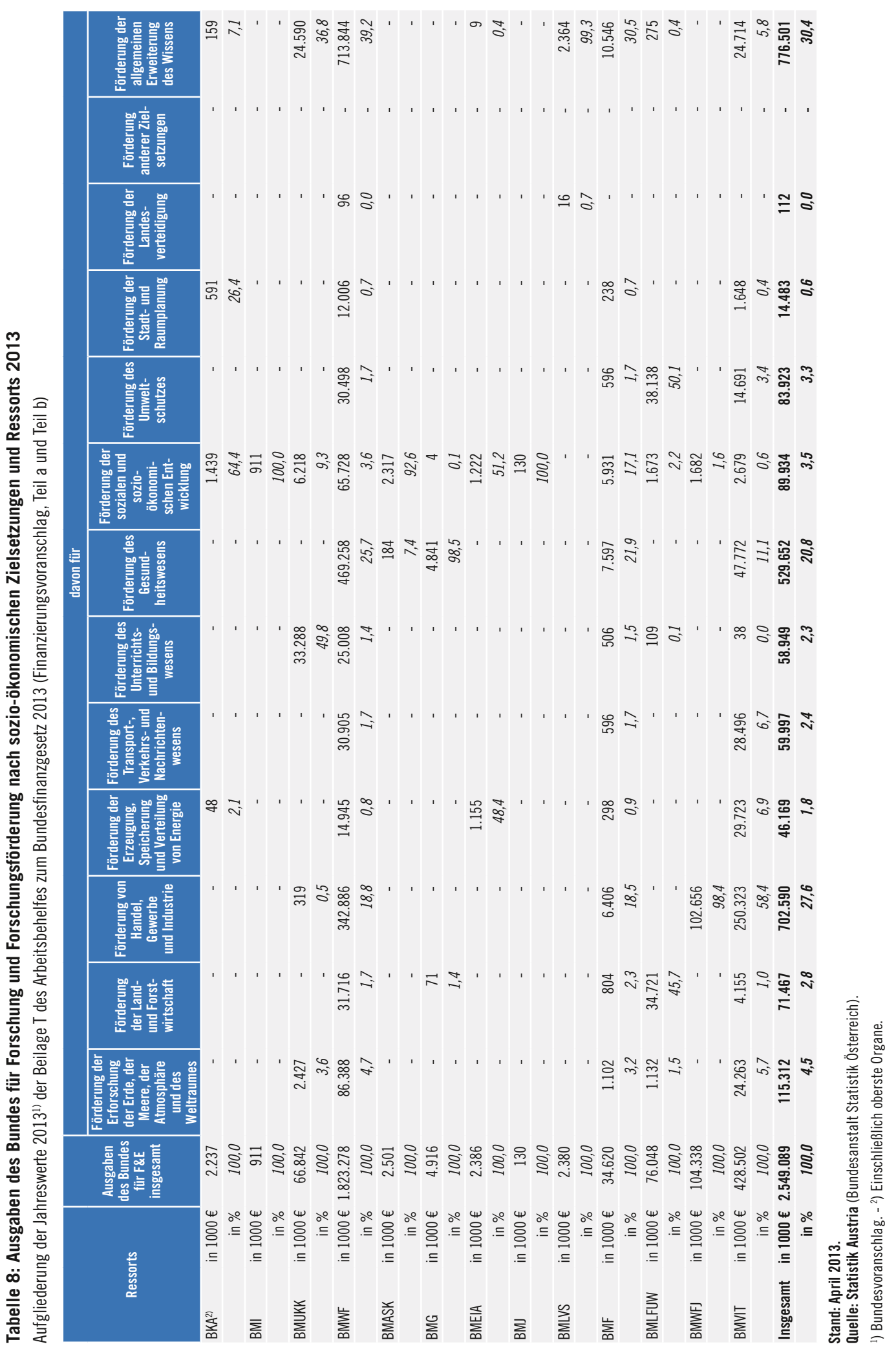


Tabelle 9: Allgemeine forschungswirksame Hochschulausgaben des Bundes (General University Funds) 1999 bis 2013

\begin{tabular}{|c|c|c|}
\hline \multirow{3}{*}{ Jahre } & \multicolumn{2}{|c|}{ Allgemeine Hochschulausgaben ${ }^{1}$} \\
\hline & insgesamt & F\&E \\
\hline & \multicolumn{2}{|c|}{ Mio. $€$} \\
\hline 1999 & $1.960,216$ & 834,529 \\
\hline 2000 & $1.956,167$ & 842,494 \\
\hline 2001 & $2.008,803$ & 866,361 \\
\hline 2002 & $2.104,550$ & 918,817 \\
\hline 2003 & $2.063,685$ & 899,326 \\
\hline 2004 & $2.091,159$ & 980,984 \\
\hline 2005 & $2.136,412$ & $1.014,543$ \\
\hline 2006 & $2.157,147$ & $1.027,270$ \\
\hline 2007 & $2.314,955$ & $1.083,555$ \\
\hline 2008 & $2.396,291$ & $1.133,472$ \\
\hline 2009 & $2.626,038$ & $1.326,757$ \\
\hline 2010 & $2.777,698$ & $1.310,745$ \\
\hline 2011 & $2.791,094$ & $1.307,049$ \\
\hline 2012 & $2.946,922$ & $1.384,819$ \\
\hline 2013 & $3.162,492$ & $1.483,763$ \\
\hline
\end{tabular}

Stand: April 2013.

Quelle: Statistik Austria (Bundesanstalt Statistik Österreich).

1) Auf Basis der Beilagen T der Arbeitsbehelfe zu den Bundesfinanzgesetzen. 


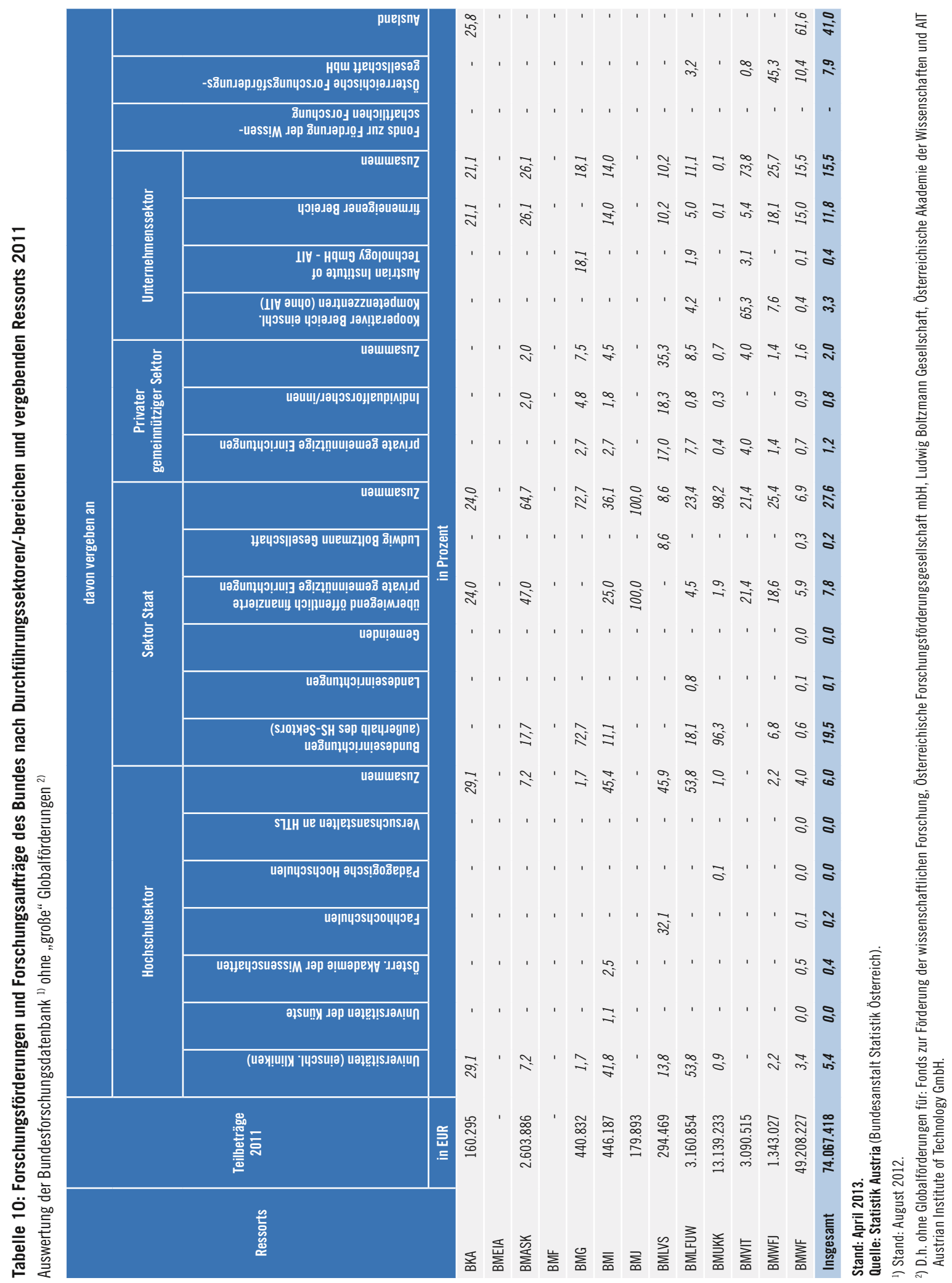




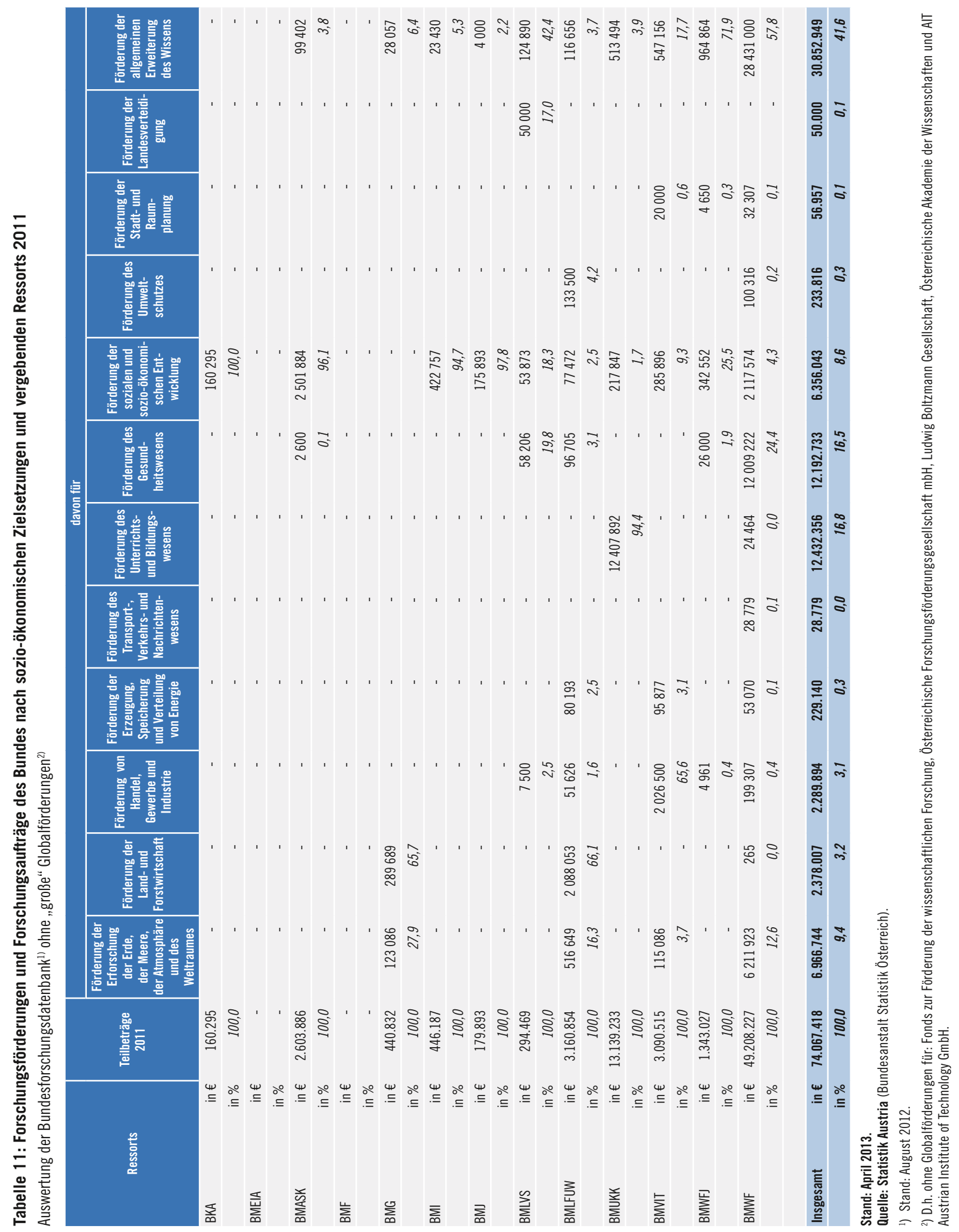


Tabelle 12: Forschungsförderungen und Forschungsaufträge der Bundesdienststellen nach Wissenschaftszweigen und vergebenden Ressorts 2011

Auswertung der Bundesforschungsdatenbank ${ }^{1)}$ ohne „große“ Globalförderungen ${ }^{2)}$

\begin{tabular}{|c|c|c|c|c|c|c|c|c|}
\hline \multirow{2}{*}{ Ressorts } & & \multirow[b]{2}{*}{$\begin{array}{l}\text { Teilbeträge } \\
2011\end{array}$} & \multicolumn{6}{|c|}{ davon für } \\
\hline & & & $\begin{array}{c}1.0 \\
\text { Naturwissen- } \\
\text { schaften }\end{array}$ & $\begin{array}{c}2.0 \\
\text { Technische } \\
\text { Wissenschaften }\end{array}$ & $\begin{array}{c}3.0 \\
\text { Humanmedizin }\end{array}$ & $\begin{array}{c}4.0 \\
\text { Land- und } \\
\text { Forstwirtschaft, } \\
\text { Veterinärmedizin }\end{array}$ & $\begin{array}{c}5.0 \\
\text { Sozialwissen- } \\
\text { schaften }\end{array}$ & $\begin{array}{c}6.0 \\
\text { Geisteswissen- } \\
\text { schaften }\end{array}$ \\
\hline \multirow[t]{2}{*}{ BKA } & in $€$ & 160.295 & - & - & - & - & 160295 & - \\
\hline & in $\%$ & 100,0 & - & - & - & - & 100,0 & - \\
\hline \multirow[t]{2}{*}{ BMEIA } & in $€$ & - & - & - & - & - & - & - \\
\hline & in $\%$ & - & - & - & - & - & - & - \\
\hline \multirow[t]{2}{*}{ BMASK } & in $€$ & 2.603 .886 & - & - & 2600 & - & 2601286 & - \\
\hline & in $\%$ & 100,0 & - & - & 0,1 & - & 99,9 & - \\
\hline \multirow[t]{2}{*}{ BMF } & in $€$ & - & - & - & - & - & - & - \\
\hline & in $\%$ & - & - & - & - & - & - & - \\
\hline \multirow[t]{2}{*}{ BMG } & in $€$ & 440.832 & 35578 & - & - & 405254 & - & - \\
\hline & in $\%$ & 100,0 & 8,1 & - & - & 91,9 & - & - \\
\hline \multirow[t]{2}{*}{ BMI } & in $€$ & 446.187 & - & - & - & - & 362209 & 83978 \\
\hline & in $\%$ & 100,0 & - & - & - & - & 81,2 & 18,8 \\
\hline \multirow[t]{2}{*}{ BMJ } & in $€$ & 179.893 & - & - & - & - & 175893 & 4000 \\
\hline & in $\%$ & 100,0 & - & - & - & - & 97,8 & 2,2 \\
\hline \multirow[t]{2}{*}{ BMLVS } & in $€$ & 294.469 & 172890 & 30133 & 58206 & - & 33240 & - \\
\hline & in $\%$ & 100,0 & 58,7 & 10,2 & 19,8 & - & 11,3 & - \\
\hline \multirow[t]{2}{*}{ BMLFUW } & in $€$ & 3.160 .854 & 742287 & 125000 & - & 2111066 & 182501 & - \\
\hline & in $\%$ & 100,0 & 23,5 & 4,0 & - & 66,7 & 5,8 & - \\
\hline \multirow[t]{2}{*}{ BMUKK } & in $€$ & 13.139 .233 & - & 29500 & - & - & 12710739 & 398994 \\
\hline & in $\%$ & 100,0 & - & 0,2 & - & - & 96,8 & 3,0 \\
\hline \multirow[t]{2}{*}{ BMVIT } & in $€$ & 3.090 .515 & 309886 & 2445877 & - & - & 316752 & 18000 \\
\hline & in $\%$ & 100,0 & 10,0 & 79,2 & - & - & 10,2 & 0,6 \\
\hline \multirow[t]{2}{*}{ BMWFJ } & in $€$ & 1.343 .027 & 137000 & 11961 & 76000 & - & 1092869 & 25197 \\
\hline & in $\%$ & 100,0 & 10,2 & 0,9 & 5,7 & - & 81,3 & 1,9 \\
\hline \multirow[t]{2}{*}{ BMWF } & in $€$ & 49.208 .227 & 42033760 & 1416229 & 1429837 & 145638 & 3431425 & 751338 \\
\hline & in $\%$ & 100,0 & 85,4 & 2,9 & 2,9 & 0,3 & 7,0 & 1,5 \\
\hline \multirow[t]{2}{*}{ Insgesamt } & in $€$ & 74.067 .418 & 43.431 .401 & 4.058 .700 & 1.566 .643 & 2.661.958 & 21.067.209 & 1.281.507 \\
\hline & in $\%$ & 100,0 & 58,7 & 5,5 & 2,1 & 3,6 & 28,4 & 1,7 \\
\hline
\end{tabular}

Stand: April 2013

Quelle: Statistik Austria (Bundesanstalt Statistik Österreich).

1) Stand: August 2012.

2) D.h. ohne Globalförderungen für: Fonds zur Förderung der wissenschaftlichen Forschung, Österreichische Forschungsförderungsgesellschaft mbH, Ludwig Boltzmann Gesellschaft, Österreichische Akademie der Wissenschaften und AIT Austrian Institute of Technology GmbH. 
Tabelle 13: Forschung und experimentelle Entwicklung (F\&E) im internationalen Vergleich 2010

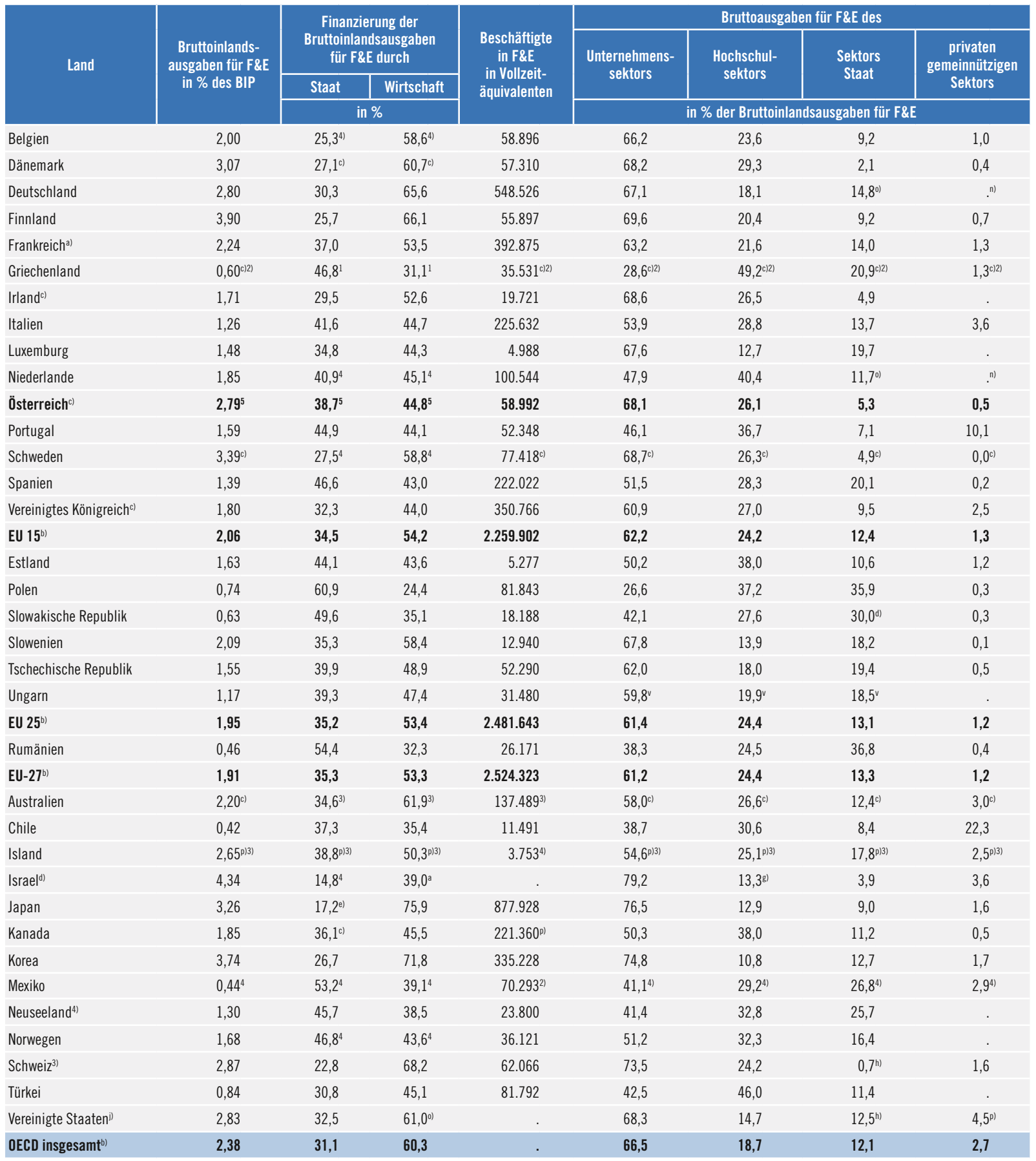

Quelle: OECD (MSTI 2012-2), Statistik Austria (Bundesanstalt Statistik Österreich).

a) Bruch in der Zeitreihe. - b) Schätzung des OECD-Sekretariates (basierend auf nationalen Quellen). - c) Nationale Schätzung, wenn erforderlich vom OECD-Sekretariat den OECDNormen angepasst. - d) F\&E-Ausgaben für Landesverteidigung nicht enthalten. - e) Nationale Erhebungsergebnisse. Vom OECD-Sekretariat den OECD-Normen angepasste Werte. - h)

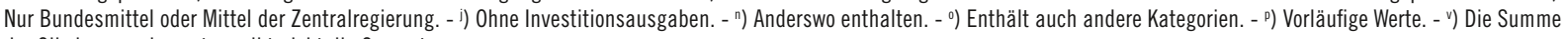
der Gliederungselemente ergibt nicht die Gesamtsumme.

1) 2005. $\left.-{ }^{2}\right)$ 2007. $\left.-{ }^{3}\right)$ 2008. ${ }^{4}$ ) 2009. - 5) Statistik Austria; entsprechend F\&E-Globalschätzung 2013.

Vollzeitäquivalent $=$ Personenjahr. 
Tabelle 14: Österreichs Pfad vom 4. zum 7. EU-Rahmenprogramm für Forschung, technologische Entwicklung und Demonstration

\begin{tabular}{|c|c|c|c|c|}
\hline & 4. RP & 5. RP & 6. RP & 7. RP \\
\hline & 1994-1998 & 1998-2002 & 2002-2006 & $\begin{array}{l}\text { Datenstand } \\
11 / 2012\end{array}$ \\
\hline Anzahl bewilligter Projekte mit österreichischer Beteiligung & 1.444 & 1.384 & 1.324 & 1.882 \\
\hline Anzahl bewilligter österreichischer Beteiligungen & 1.923 & 1.987 & 1.972 & 2.622 \\
\hline Anzahl bewilligter, von österreichischen Organisationen koordinierter Projekte & 270 & 267 & 213 & 300 \\
\hline $\begin{array}{l}\text { Vertraglich gebundene Förderungen für bewilligte österreichische Partnerorganisatio- } \\
\text { nen und Forschende in Mio. } €\end{array}$ & 194 & 292 & 425 & $729^{1}$ \\
\hline $\begin{array}{l}\text { Anteil bewilligter österreichischer Beteiligungen an den insgesamt bewilligten } \\
\text { Beteiligungen }\end{array}$ & $2,3 \%$ & $2,4 \%$ & $2,6 \%$ & $2,5 \%$ \\
\hline $\begin{array}{l}\text { Anteil bewilligter österreichischer Koordinatorlnnen an den insgesamt bewilligten } \\
\text { Koordinatorlnnen }\end{array}$ & $1,7 \%$ & $2,8 \%$ & $3,3 \%$ & $3,4 \%$ \\
\hline Österreichischer Anteil an rückholbaren Fördermitteln (Rückflussindikator, RI) & $1,99 \%$ & $2,38 \%$ & $2,56 \%$ & $2,66 \%$ \\
\hline $\begin{array}{l}\text { Österreichischer Anteil an rückholbaren Fördermitteln (RI) gemessen am österreichi- } \\
\text { schen Beitrag zum EU-Haushalt (Rückflussquote) }\end{array}$ & $70 \%$ & $104 \%$ & $117 \%$ & $126 \%$ \\
\hline
\end{tabular}

Daten: Europäische Kommission. Bearbeitung und Berechnungen: PROVISO.

1) Die zu erwartende Fördersumme für österreichische Beteiligungen aller mit Datenstand 11/2012 bewilligten Projekte beläuft sich auf 832 Mio. € (Hochrechnung, durchschnittliche Kürzungen im Rahmen der Vertragsverhandlungen sind berücksichtigt).

Quelle: Ehardt-Schmiederer, M., Brücker, J., Milovanović, D., Kobel, C., Hackl, F.,Schleicher, L., Antúnez, A., Zacharias, M. (2012): 7. EU-Rahmenprogramm für Forschung, technologische Entwicklung und Demonstration (2007-2013), PROVISO-Überblicksbericht, Herbst 2012, Wien.

Tabelle 15: Ergebnisse Österreichs im 7. EU-Rahmenprogramm für Forschung, technologische Entwicklung und Demonstration

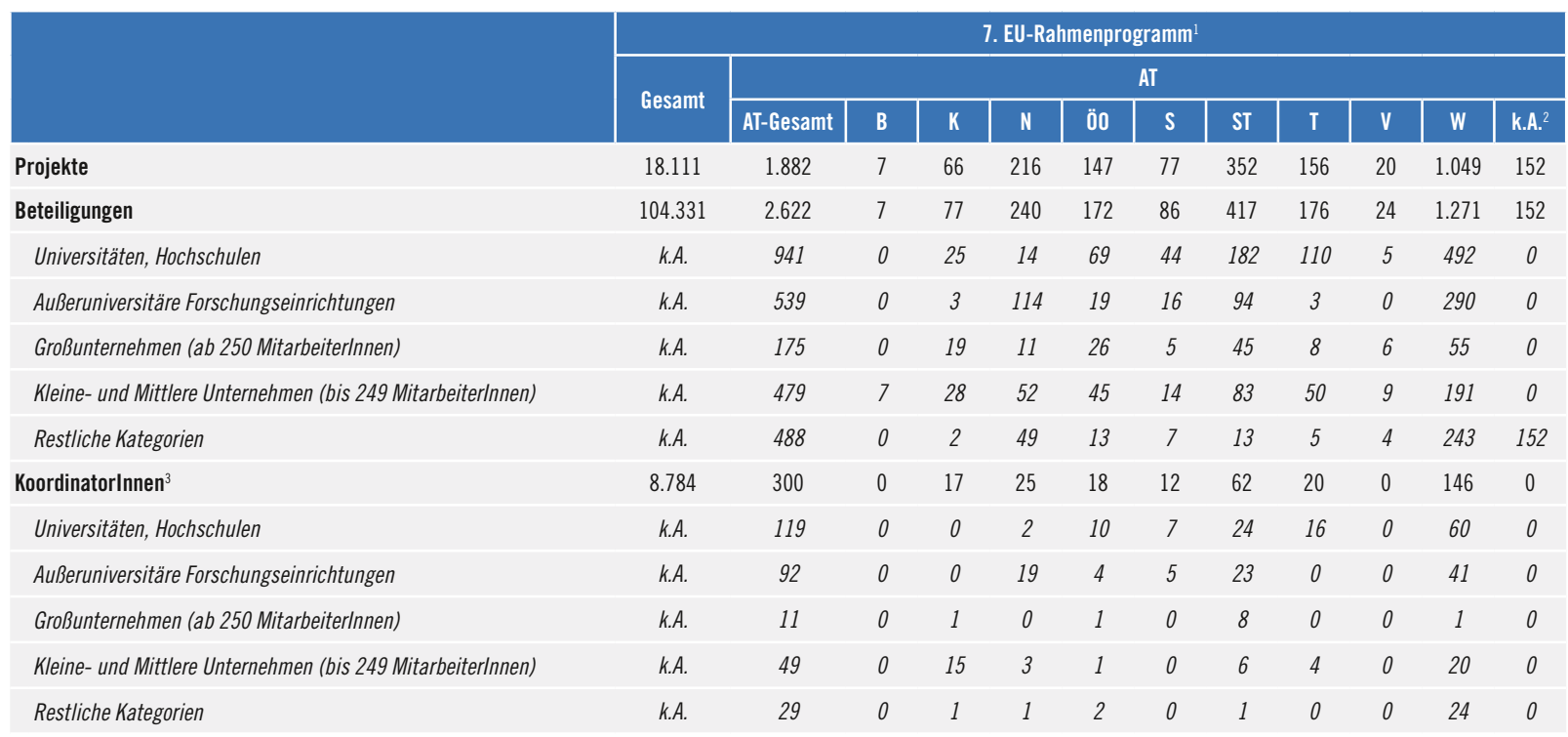

Daten: Europäische Kommission. Bearbeitung und Berechnungen: PROVISO.

1) Mit Datenstand 11/2012 liegen PROVISO nur teilweise Angaben über die Verhandlungsergebnisse der Projekte vor. Da es im Zuge der Vertragsverhandlungen erfahrungsgemäß zu Änderungen kommen kann (z.B. Vertrag über ein bewilligtes Projekt kommt nicht zustande, Konsortien ändern sich innerhalb eines Projektes, Kürzungen der „beantragten“ Fördersummen), verstehen sich die Angaben als Richtwerte.

2) V.a. EinzelforscherInnen der Säule Menschen (Researchers, StipendiatInnen/PreisträgerInnen der Säule Menschen) und der Säule Ideen (Principal Investigators).

$\left.{ }^{3}\right)$ Nicht berücksichtigt sind Projekte der Säule Ideen sowie Individualstipendien und Preise (awards) der Säule Menschen.

Quelle: Ehardt-Schmiederer, M., Brücker, J., Milovanović, D., Kobel, C., Hackl, F.,Schleicher, L., Antúnez, A., Zacharias, M. (2012): 7. EU-Rahmenprogramm für Forschung, technologische Entwicklung und Demonstration (2007-2013), PROVISO-Überblicksbericht, Herbst 2012, Wien. 
Tabelle 16: Überblick über Projekte und Beteiligungen im 7. EU-Rahmenprogramm für Forschung, technologische Entwicklung und Demonstration

\begin{tabular}{|c|c|c|c|}
\hline & $\begin{array}{l}\text { Bewilligte Projekte } \\
\text { (gesamt) }\end{array}$ & $\begin{array}{l}\text { Bewilligte Projekte mit } \\
\text { österreichischer Beteiligung }\end{array}$ & $\begin{array}{l}\text { Anteil bewilligter Projekte mit } \\
\text { österreichischer Beteiligung } \\
\text { an bewilligten Projekten (gesamt) }\end{array}$ \\
\hline Zusammenarbeit & 5.542 & 1.189 & $21,5 \%$ \\
\hline Ideen & 3.189 & 112 & $3,5 \%$ \\
\hline Menschen & 7.770 & 341 & $4,4 \%$ \\
\hline Kapazitäten & 1.610 & 240 & $14,9 \%$ \\
\hline Gesamt & 18.111 & 1.882 & $10,4 \%$ \\
\hline
\end{tabular}

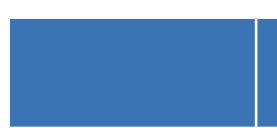

Bewilligte Beteiligungen
(gesamt)

Zusammenarbeit

61.801

Ideen

6.726

Menschen

20.478

Kapazitäten

15.326

104.331

Gesamt

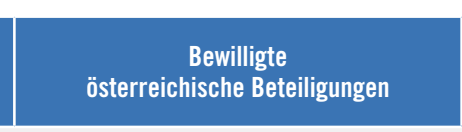

1.730

139

420

333

2.622

Anteil bewilligter

österreichischer Beteiligungen an bewilligten Beteiligungen (gesamt)

Daten: Europäische Kommission. Bearbeitung und Berechnungen: PROVISO. Datenstand: 11/2012.

Quelle: Ehardt-Schmiederer, M., Brücker, J., Milovanović, D., Kobel, C., Hackl, F.,Schleicher, L., Antúnez, A., Zacharias, M. (2012): 7. EU-Rahmenprogramm für Forschung, technologische Entwicklung und Demonstration (2007-2013), PROVISO-Überblicksbericht, Herbst 2012, Wien.

Anmerkung: Mit Datenstand 11/2012 liegen PROVISO nur teilweise Angaben über die Verhandlungsergebnisse der Projekte vor. Da es im Zuge der Vertragsverhandlungen erfahrungsgemäß zu Änderungen kommen kann, verstehen sich die Angaben als Richtwerte.

Tabelle 17: FWF: Entwicklung der Förderungen in den Life Sciences 2010 bis 2012

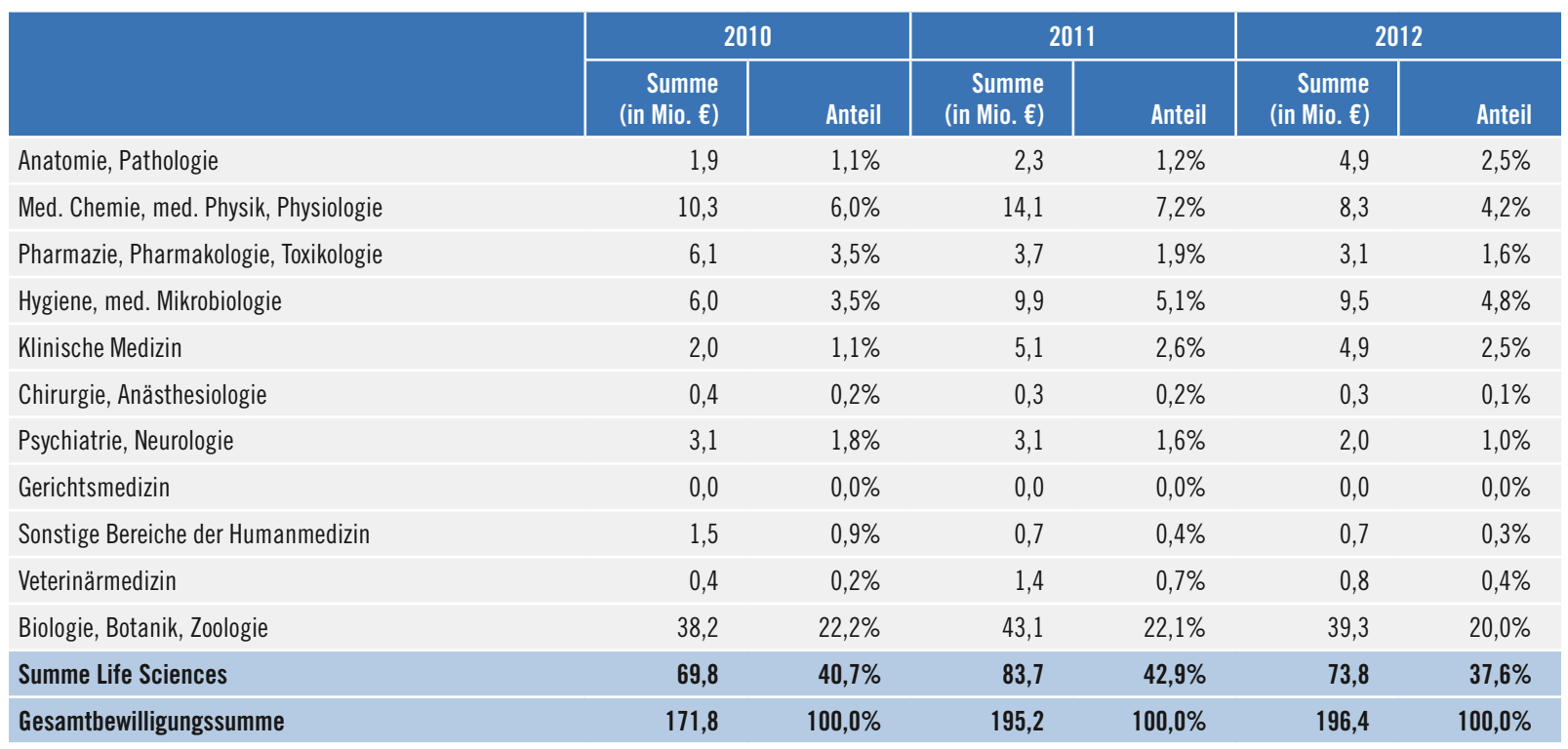


Tabelle 18: FWF: Entwicklung der Förderungen in den Naturwissenschaften und Technik 2010 bis 2012

\begin{tabular}{|c|c|c|c|c|c|c|}
\hline & \multicolumn{2}{|c|}{2010} & \multicolumn{2}{|c|}{2011} & \multicolumn{2}{|c|}{2012} \\
\hline & $\begin{array}{r}\text { Summe } \\
\text { (in Mio. €) }\end{array}$ & Anteil & $\begin{array}{r}\text { Summe } \\
\text { (in Mio. €) }\end{array}$ & Anteil & $\begin{array}{r}\text { Summe } \\
\text { (in Mio. €) }\end{array}$ & Anteil \\
\hline Mathematik, Informatik & 20,2 & $11,8 \%$ & 27,3 & $14,0 \%$ & 31,5 & $16,0 \%$ \\
\hline Physik, Mechanik, Astronomie & 21,2 & $12,3 \%$ & 25,9 & $13,3 \%$ & 26,1 & $13,3 \%$ \\
\hline Chemie & 11,1 & $6,4 \%$ & 10,3 & $5,3 \%$ & 12,0 & $6,1 \%$ \\
\hline Geologie, Mineralogie & 4,4 & $2,6 \%$ & 2,2 & $1,1 \%$ & 1,5 & $0,8 \%$ \\
\hline Meteorologie, Klimatologie & 1,2 & $0,7 \%$ & 1,0 & $0,5 \%$ & 2,2 & $1,1 \%$ \\
\hline Hydrologie, Hydrographie & 0,7 & $0,4 \%$ & 0,7 & $0,4 \%$ & 0,7 & $0,4 \%$ \\
\hline Geographie & 0,9 & $0,5 \%$ & 0,7 & $0,3 \%$ & 1,2 & $0,6 \%$ \\
\hline Sonstige Naturwissenschaften & 1,9 & $1,1 \%$ & 2,1 & $1,1 \%$ & 1,7 & $0,9 \%$ \\
\hline Bergbau, Metallurgie & 0,6 & $0,4 \%$ & 0,6 & $0,3 \%$ & 0,5 & $0,2 \%$ \\
\hline Maschinenbau, Instrumentenbau & 0,2 & $0,1 \%$ & 0,5 & $0,3 \%$ & 0,5 & $0,3 \%$ \\
\hline Bautechnik & 0,8 & $0,5 \%$ & 0,1 & $0,1 \%$ & 0,9 & $0,4 \%$ \\
\hline Architektur & 0,6 & $0,4 \%$ & 0,2 & $0,1 \%$ & 1,0 & $0,5 \%$ \\
\hline Elektrotechnik, Elektronik & 0,9 & $0,5 \%$ & 3,9 & $2,0 \%$ & 2,0 & $1,0 \%$ \\
\hline Technische Chemie, Brennstoff- und Mineralöltechnologie & 0,4 & $0,2 \%$ & 0,4 & $0,2 \%$ & 0,4 & $0,2 \%$ \\
\hline Geodäsie, Vermessungswesen & 0,2 & $0,1 \%$ & 0,4 & $0,2 \%$ & 0,5 & $0,3 \%$ \\
\hline Verkehrswesen, Verkehrsplanung & 0,0 & $0,0 \%$ & 0,0 & $0,0 \%$ & 0,0 & $0,0 \%$ \\
\hline Sonstige Technische Wissenschaften & 1,9 & $1,1 \%$ & 0,9 & $0,5 \%$ & 1,8 & $0,9 \%$ \\
\hline Ackerbau, Pflanzenzucht, -schutz & 0,0 & $0,0 \%$ & 0,2 & $0,1 \%$ & 0,5 & $0,2 \%$ \\
\hline Gartenbau, Obstbau & 0,0 & $0,0 \%$ & 0,0 & $0,0 \%$ & 0,0 & $0,0 \%$ \\
\hline Forst- und Holzwirtschaft & 0,6 & $0,3 \%$ & 0,5 & $0,2 \%$ & 0,5 & $0,3 \%$ \\
\hline Viehzucht, Tierproduktion & 0,3 & $0,2 \%$ & 0,3 & $0,1 \%$ & 0,3 & $0,2 \%$ \\
\hline Sonstige Bereiche der Land- und Forstwirtschaft & 0,3 & $0,2 \%$ & 0,1 & $0,1 \%$ & 0,9 & $0,5 \%$ \\
\hline Summe Naturwissenschaften und Technik & 68,3 & $39,8 \%$ & 78,2 & $40,1 \%$ & 86,9 & $44,2 \%$ \\
\hline Gesamtbewilligungssumme & 171,8 & $100,0 \%$ & 195,2 & $100,0 \%$ & 196,4 & $100,0 \%$ \\
\hline
\end{tabular}


Tabelle 19: FWF: Entwicklung der Förderungen in den Geistes- und Sozialwissenschaften 2010 bis 2012

\begin{tabular}{|c|c|c|c|c|c|c|}
\hline & \multicolumn{2}{|c|}{2010} & \multicolumn{2}{|c|}{2011} & \multicolumn{2}{|c|}{2012} \\
\hline & $\begin{array}{r}\text { Summe } \\
\text { (in Mio. €) }\end{array}$ & Anteil & $\begin{array}{r}\text { Summe } \\
\text { (in Mio. €) }\end{array}$ & Anteil & $\begin{array}{r}\text { Summe } \\
\text { (in Mio. €) }\end{array}$ & Anteil \\
\hline Philosophie & 2,1 & $1,2 \%$ & 1,3 & $0,7 \%$ & 2,1 & $1,1 \%$ \\
\hline Theologie & 0,8 & $0,5 \%$ & 0,8 & $0,4 \%$ & 1,1 & $0,5 \%$ \\
\hline Historische Wissenschaften & 8,0 & $4,7 \%$ & 8,5 & $4,4 \%$ & 8,5 & $4,3 \%$ \\
\hline Sprach- und Literaturwissenschaften & 3,6 & $2,1 \%$ & 3,2 & $1,6 \%$ & 4,0 & $2,0 \%$ \\
\hline Sonstige philologisch und kulturkundliche Richtungen & 1,7 & $1,0 \%$ & 4,1 & $2,1 \%$ & 2,7 & $1,4 \%$ \\
\hline Kunstwissenschaften & 3,8 & $2,2 \%$ & 3,7 & $1,9 \%$ & 4,2 & $2,1 \%$ \\
\hline Sonstige Geisteswissenschaften & 0,8 & $0,5 \%$ & 0,9 & $0,4 \%$ & 0,5 & $0,3 \%$ \\
\hline Politische Wissenschaften & 0,5 & $0,3 \%$ & 0,6 & $0,3 \%$ & 3,6 & $1,8 \%$ \\
\hline Rechtswissenschaften & 0,9 & $0,5 \%$ & 1,1 & $0,6 \%$ & 1,0 & $0,5 \%$ \\
\hline Wirtschaftswissenschaften & 3,7 & $2,2 \%$ & 3,5 & $1,8 \%$ & 1,9 & $1,0 \%$ \\
\hline Soziologie & 1,5 & $0,9 \%$ & 1,3 & $0,7 \%$ & 1,6 & $0,8 \%$ \\
\hline Psychologie & 1,4 & $0,8 \%$ & 2,0 & $1,0 \%$ & 1,6 & $0,8 \%$ \\
\hline Raumplanung & 0,1 & $0,1 \%$ & 0,2 & $0,1 \%$ & 0,2 & $0,1 \%$ \\
\hline Angewandte Statistik & 1,8 & $1,1 \%$ & 0,2 & $0,1 \%$ & 0,1 & $0,1 \%$ \\
\hline Pädagogik, Erziehungswissenschaften & 0,7 & $0,4 \%$ & 0,2 & $0,1 \%$ & 0,6 & $0,3 \%$ \\
\hline Sonstige Sozialwissenschaften & 2,2 & $1,3 \%$ & 1,6 & $0,8 \%$ & 2,1 & $1,1 \%$ \\
\hline Summe Geistes- und Sozialwissenschaften & 33,6 & $19,6 \%$ & 33,2 & $17,0 \%$ & 35,7 & $18,2 \%$ \\
\hline Gesamtbewilligungssumme & 171,8 & $100,0 \%$ & 195,2 & $100,0 \%$ & 196,4 & $100,0 \%$ \\
\hline
\end{tabular}

Tabelle 20: FFG: Förderungen nach Bundesland 2012

\begin{tabular}{|l|r|r|r|r|}
\hline \multicolumn{1}{c|}{ Bundesland } & Beteiligungen & Gesamtförderung & Barwert & Anteile am Barwert \\
\hline Burgenland & 52 & 7.713 & 5.138 & $1,4 \%$ \\
\hline Kärnten & 214 & 30.199 & 20.307 & $5,7 \%$ \\
\hline Niederösterreich & 579 & 28.263 & 23.077 & $6,4 \%$ \\
\hline Oberösterreich & 765 & 113.908 & 70.832 & $19,7 \%$ \\
\hline Salzburg & 201 & 18.780 & 9.093 & $2,5 \%$ \\
\hline Steiermark & 1.160 & 147.146 & 122.170 & $34,0 \%$ \\
\hline Tirol & 244 & 24.139 & 18.483 & $5,1 \%$ \\
\hline Vorarlberg & 117 & 12.405 & 6.708 & $1,9 \%$ \\
\hline Wien & 1.522 & 97.945 & 82.373 & $22,9 \%$ \\
\hline Ausland & 271 & 1.164 & 1.164 & $0,3 \%$ \\
\hline Gesamt & 5.125 & 481.661 & 359.345 & $100,0 \%$ \\
\hline
\end{tabular}


Tabelle 21: FFG: Projektkosten und Förderung nach Subject Index Code 2012

\begin{tabular}{|c|c|c|c|}
\hline Subject Index Code & Gesamtkosten & Gesamtförderung & Barwert \\
\hline Werkstofftechnik & 138.300 & 62.995 & 44.836 \\
\hline Industrielle Fertigung & 136.158 & 63.806 & 43.739 \\
\hline Elektronik, Mikroelektronik & 129.259 & 53.893 & 31.309 \\
\hline Oberflächenverkehr und -Technologien & 67.438 & 39.736 & 31.180 \\
\hline Umwelt & 77.139 & 27.013 & 25.273 \\
\hline Ikt-Anwendungen & 47.883 & 27.349 & 20.604 \\
\hline Regenerative Energieträger & 34.111 & 18.026 & 15.640 \\
\hline Informationsverarbeitung, Informationssysteme & 35.124 & 19.587 & 14.373 \\
\hline Innovation, Technologietransfer & 41.492 & 14.790 & 14.023 \\
\hline Medizinische Biotechnologie & 34.953 & 16.293 & 12.850 \\
\hline Ohne Zuordnung & 19.947 & 12.390 & 12.390 \\
\hline Medizin, Gesundheit & 35.576 & 17.176 & 12.207 \\
\hline Energieeinsparung & 26.195 & 13.996 & 11.764 \\
\hline Energiespeicherung, -Umwandlung und Transport & 15.350 & 9.831 & 9.556 \\
\hline Sicherheit & 12.926 & 8.775 & 8.709 \\
\hline Bautechnik & 16.774 & 9.912 & 7.162 \\
\hline Biowissenschaften & 19.875 & 11.393 & 6.392 \\
\hline Sonstige Technologie & 20.578 & 12.740 & 5.683 \\
\hline Messverfahren & 14.393 & 7.619 & 4.855 \\
\hline Nachhaltige Entwicklung & 6.221 & 3.486 & 3.459 \\
\hline Nanotechnologie und Nanowissenschaften & 5.369 & 3.498 & 3.415 \\
\hline Sonstige Energiethemen & 3.748 & 2.828 & 2.322 \\
\hline Regionalentwicklung & 2.662 & 2.094 & 2.094 \\
\hline Wirtschaftliche Aspekte & 2.708 & 1.913 & 1.690 \\
\hline Lebensmittel & 3.573 & 2.141 & 1.634 \\
\hline Luftverkehr- und Technologien & 2.815 & 1.831 & 1.511 \\
\hline Landwirtschaft & 3.313 & 1.819 & 1.414 \\
\hline Landwirtschaftliche Biotechnologie & 3.577 & 1.516 & 1.374 \\
\hline Mathematik, Statistik & 3.834 & 2.215 & 1.359 \\
\hline Industrielle Biotechnologie & 3.799 & 2.093 & 1.289 \\
\hline Weltraum & 1.574 & 934 & 934 \\
\hline Abfallwirtschaft & 2.247 & 1.319 & 766 \\
\hline Robotik & 2.011 & 1.420 & 597 \\
\hline Netzwerktechnologien & 1.735 & 1.127 & 504 \\
\hline Soziale Aspekte & 671 & 504 & 504 \\
\hline Telekommunikation & 844 & 593 & 388 \\
\hline Unternehmenaspekte & 921 & 644 & 380 \\
\hline Information, Medien & 672 & 336 & 336 \\
\hline Geowissenschaften & 411 & 305 & 305 \\
\hline Gesetze, Vorschriften & 278 & 278 & 278 \\
\hline Forschungsethik & 2.866 & 1.433 & 233 \\
\hline Automatisierung & 13 & 10 & 10 \\
\hline Koordinierung, Zusammenarbeit & 5 & 5 & 5 \\
\hline Gesamtergebnis & 979.335 & 481.661 & 359.345 \\
\hline
\end{tabular}


Tabelle 22: aws: Zuschüsse für Technologieförderung 2012

\begin{tabular}{|c|c|c|c|c|c|}
\hline & Anzahl & $\%$ & [in $1.000 €]$ & [in $1.000 €]$ & $\%$ \\
\hline \multicolumn{6}{|l|}{ PreSeed } \\
\hline LISA PreSeed & 8 & 6,7 & 1.884 & 1.566 & 7,5 \\
\hline PreSeed IKT \& Physical Sciences & 19 & 15,8 & 4.237 & 2.580 & 12,3 \\
\hline \multicolumn{6}{|l|}{ Seedfinancing } \\
\hline LISA Seed & 8 & 6,7 & 75.516 & 6.050 & 28,8 \\
\hline Seedfinancing IKT \& Physical Sciences & 10 & 8,3 & 49.350 & 6.260 & 29,8 \\
\hline \multicolumn{6}{|l|}{ Kreativwirtschaft } \\
\hline Kreativwirtschaft (impulse XL, XS) & 72 & 60,0 & 8.610 & 3.687 & 17,5 \\
\hline Kreativwirtschaft (impulse LEAD) & 3 & 2,5 & 1.231 & 869 & 4,1 \\
\hline Gründungs-Technologiescheck & 5 & 4,2 & 7 & 5 & 0,0 \\
\hline ProTRANS & 21 & 17,5 & 10.080 & 3.151 & 15,0 \\
\hline Summe & 120 & 100,0 & 140.828 & 21.012 & 100,0 \\
\hline
\end{tabular}

Tabelle 23: CDG: CD-Labors nach Universitäten/Forschungseinrichtungen 2012

\begin{tabular}{|c|c|c|}
\hline Universität/Forschungseinrichtung & Anzahl der CD-Labors & Gesamtlaborbudget1 in $€$ \\
\hline Medizinische Universität Graz & 1 & 173.680 \\
\hline Medizinische Universität Innsbruck & 1 & 220.000 \\
\hline Medizinische Universität Wien & 10 & 3.684 .542 \\
\hline Montanuniversität Leoben & 6 & 2.737 .793 \\
\hline Technische Universität Graz & 8 & 2.791 .605 \\
\hline Technische Universität Wien & 9 & 2.411 .204 \\
\hline Universität für Bodenkultur Wien & 8 & 2.745 .729 \\
\hline Universität Graz & 1 & 426.067 \\
\hline Universität Innsbruck & 2 & 631.862 \\
\hline Universität Linz & 7 & 2.753 .317 \\
\hline Universität Salzburg & 3 & 1.205 .000 \\
\hline Universität Wien & 2 & 123.089 \\
\hline Veterinärmedizinische Universität Wien & 2 & 645.888 \\
\hline Research Center for Non Destructive Testing GmbH & 1 & 132.292 \\
\hline Max-Planck-Institut für Eisenforschung GmbH & 1 & 458.000 \\
\hline Technische Universität München & 1 & 322.000 \\
\hline Universität Bochum & 1 & 431.525 \\
\hline Universität Göttingen & 1 & 264.000 \\
\hline University of Cambridge & 1 & 310.878 \\
\hline Summe & 66 & 22.468 .470 \\
\hline
\end{tabular}

Anmerkung: Die Gesamtzahl an CD-Labors beträgt 64; es gibt 2 CD-Labors mit Doppelleitungan an verschiedenen Universitäten.

1) Plan per 07.12.2012 
Tabelle 24: CDG: Entwicklung der CDG 1989 bis 2012

\begin{tabular}{|c|c|c|c|}
\hline Jahr & Ausgaben der CD-Labors in $€$ & Aktive CD-Labors & Aktive Mitgliedsunternehmen \\
\hline 1989 & $247.087,6$ & 5 & \\
\hline 1990 & $1.274 .681,5$ & 7 & \\
\hline 1991 & $2.150 .389,2$ & 11 & \\
\hline 1992 & $3.362 .572,0$ & 16 & \\
\hline 1993 & $2.789 .910,1$ & 17 & \\
\hline 1994 & $3.101 .676,6$ & 18 & \\
\hline 1995 & $2.991 .213,9$ & 14 & \\
\hline 1996 & $2.503 .324,9$ & 15 & 6 \\
\hline 1997 & $2.982 .792,5$ & 16 & 9 \\
\hline 1998 & $3.108 .913,4$ & 17 & 13 \\
\hline 1999 & $3.869 .992,6$ & 20 & 15 \\
\hline 2000 & $3.624 .962,6$ & 18 & 14 \\
\hline 2001 & $4.707 .302,0$ & 20 & 18 \\
\hline 2002 & $7.295 .956,9$ & 31 & 40 \\
\hline 2003 & $9.900 .589,6$ & 35 & 47 \\
\hline 2004 & $10.711 .821,9$ & 37 & 63 \\
\hline 2005 & $11.878 .543,2$ & 37 & 66 \\
\hline 2006 & $12.840 .466,3$ & 41 & 79 \\
\hline 2007 & $14.729 .107,6$ & 48 & 82 \\
\hline 2008 & $17.911 .783,7$ & 58 & 99 \\
\hline 2009 & $17.844 .292,0$ & 65 & 106 \\
\hline 2010 & $19.768 .684,4$ & 61 & 110 \\
\hline 2011 & $20.378 .065,9$ & 61 & 108 \\
\hline $2012^{1)}$ & $22.468 .470,0$ & 64 & 114 \\
\hline
\end{tabular}

1) Plan per 07.12.2012.

Tabelle 25: CDG: CD-Labors nach Thematischen Clustern 2012

\begin{tabular}{l|c|c|}
\hline Thematischer Cluster & Anzahl der CD-Labors & Gesamtlaborbudget in $€$ \\
\hline Chemie & 9 & 3.281 .551 \\
\hline Life Sciences und Umwelt & 12 & 2.963 .702 \\
\hline Maschinen- und Instrumentenbau & 6 & 1.743 .647 \\
\hline Mathematik, Informatik, Elektronik & 13 & 5.199 .644 \\
\hline Medizin & 11 & 3.937 .122 \\
\hline Metalle und Legierungen & 10 & 4.323 .623 \\
Nichtmetallische Werkstoffe & 3 & 1.019 .180 \\
\hline Summe & 64 & 22.468 .470
\end{tabular}



